\title{
Löslichkeit von Gasen in Wasser-Glykol-Kreisläufen energietechnischer Anlagen
}

\author{
Von der Fakultät Maschinenwesen \\ der \\ Technischen Universität Dresden \\ zur \\ Erlangung des akademischen Grades \\ Doktoringenieur (Dr.-Ing.) \\ angenommene Dissertation \\ von \\ Dipl.-Ing. Felix Panitz \\ geb. am 07.04.1983 in Bautzen
}

Gutachter:

Prof. Dr.-Ing. Clemens Felsmann

Prof. Dr.-Ing. habil. Winfried Heller

Prof. Dr. Ralph Eismann

Vorsitzender der Promotionskommission: Prof. Dr. rer. medic. H.-P. Wiesmann 
Dieses Werk wurde unter der Lizenz „Creative Commons Namensnennung 4.0 International“ (CC BY 4.0) veröffentlicht. Den rechtsverbindlichen Lizenzvertrag finden Sie unter https://creativecommons.org/licenses/by/4.0/deed.de 


\section{Danksagung}

Ich bin dankbar, dass ich an der Universität studieren, dort forschen und damit auch diese Dissertation vorlegen konnte. Meine grundlegende Motivation war dabei, gegen Ungerechtigkeiten und damit auch zur Lösung eines der drängendsten Probleme - der Klimakrise - einen kleinen Beitrag leisten zu können.

Ich bin ein Gast auf Erden ${ }^{1}$ und möchte, dass andere ebenso gastfreundlich wie ich empfangen werden. Ich hoffe, dass ich mit dieser Arbeit im kleinen Rahmen daran mitwirken kann, eine lebenswerte und enkeltaugliche Erde weiterzugeben.

Diese Arbeit würde es ohne die Unterstützung vieler lieber Menschen nicht geben:

Ich danke meinen Eltern Iris und Michael Panitz, die das Fundament dafür gelegt haben, dass ich diese Arbeit schreiben konnte. Ich danke meiner lieben Frau Juliane Panitz, die mir stets den Rücken frei gehalten und mich bestärkt hat. Ich danke meinen vier Kindern Clara, Justus, Alfred und Jona, die mir viel Verständnis entgegengebracht haben und die es mir einfacher machten, auch mal auf andere Gedanken zu kommen. Ebenso danke ich meinen Schwiegereltern und meiner Schwägerin für die vielfältige Unterstützung. Almuth Lerche danke ich für das Lektorat.

Ich danke meinem Hauptgutachter Prof. Dr.-Ing. Clemens Felsmann sowie den Gutachtern Prof. Dr. Ralph Eismann und Prof. Dr.-Ing. habil. Winfried Heller für das entgegengebrachte Vertrauen, die angenehme Zusammenarbeit und die Motivation, die Arbeit fertigzustellen.

Prof. Dr. Ralph Eismannn danke ich weiterhin für die nahezu jährliche Ermutigung auf der Solarthermie-Tagung in Kloster Banz. Seine Worte zu meinen Ergebnissen waren wichtige Bestärkung für weitergehende Forschung und diese Dissertation.

Insbesondere danke ich Dr.-Ing. Karin Rühling für die Beantragung des Forschungsprojekts, auf dessen Grundlage diese Arbeit entstehen konnte. Ich danke ihr für die vielen guten Ideen, die kritische und motivierende Begleitung meiner Forschung und die ehrliche und direkte fachliche Diskussion. Ohne ihre feste Überzeugung, dass meine geleistete Arbeit promotionswürdig ist, würde es diese Dissertation nicht geben.

Ich danke Martin Heymann für die tolle Zusammenarbeit in den Projekten, die guten Anregungen, vielseitige Unterstützung und den geteilten Spaß an der Sache. Was haben wir gelacht!

Vera Boß danke ich dafür, dass sie mir in einem anderen Projekt zeitweise viel Arbeit abgenommen hat, sodass ich diese Dissertation zu Ende bringen konnte.

Ich danke dem ZET-Team für die geleistete Unterstützung. Insbesondere danke ich Knut Gietzelt für seine gewissenhafte Arbeit bei Versuchsaufbau und -durchführung und die angenehme Zusammenarbeit!

Ich danke Jens Haupt für die Starthilfe bei der Automatisierung des Versuchsstands. Ich danke den Studenten, die bei der Versuchsdurchführung geholfen haben, namentlich Max

\footnotetext{
${ }^{1}$ Gerhard Schöne (1991) bzw. ursprünglich Psalm 119,19
} 
Bachmann, Toni Rosemann, Haihua Rao. Besonderer Dank gilt Max Pham und Sebastian Unger für ihre Studienarbeiten zum Thema.

Allen Kollegen danke ich für das stets angenehme gemeinsame Arbeiten am Lehrstuhl.

Dr. Bernd Hafner danke ich für gute Ideen bei den Projekttreffen zu Beginn der Dissertation. Besonderer Dank gilt Herrn Dr. Frank Hillerns und der Firma TYFOROP Chemie GmbH für die Bereitstellung der Wasser-Glykol-Gemische und die fachliche Begleitung der Arbeit. Ich danke Dr. Schirutschke vom SGS Institut Fresenius für die unkomplizierte Bereitstellung der Gaschromatographie.

Die Arbeit wäre ebenso ohne die kollegiale Unterstützung durch andere Lehrstühle der TU Dresden nicht möglich gewesen. Ich danke Dr.-Ing. Andreas Jäger von der Professur Technische Thermodynamik für die Diskussionen zur Gemisch- und Mehrphasenthermodynamik, André Kupka von der Arbeitsgruppe Mechanische Verfahrenstechnik sowie Dr. Kathrin Gebauer, Tom Liebschner und Anja Seifert vom Lehrstuhl Energieverfahrenstechnik für die Unterstützung mit Messtechnik. Ebenso danke ich Prof. Dr.-Ing. habil. Hartmut Weise vom Lehrstuhl für Fertigungsmesstechnik und Qualitätssicherung sowie Dr.-Ing. Daniel Haufe vom Lehrstuhl für Mess- und Sensorsystemtechnik für Diskussionen und Ratschläge zum Thema Messunsicherheit. Ebenso sei dem gesamten Werkstatt-Team der Fakultät Maschinenwesen gedankt. Jens Hennig danke ich als Raumverantwortlichem für unkomplizierte Lösungen und Absprachen.

Ich bin dankbar, dass ich aus öffentlichen Geldern finanziert und in großer Freiheit Forschung betreiben konnte, und hoffe, der damit einhergehenden Verantwortung mit Veröffentlichung dieser Arbeit ein stückweit gerecht geworden zu sein.

Rückblickend und als einer, der sich selber oft schwer entscheiden kann, möchte ich, gerichtet an alle, die sich ebenso schwer damit tun, schließen mit Worten von Herman van Veen.

Wenn nichts mehr geht, erst viel zu spät, dann wird man klug;

was fängt man dann mit Klugheit an?

nicht erst dann, irgendwann,

jetzt und hier -

worauf warten wir? 


\section{Inhaltsverzeichnis}

Symbolverzeichnis $\quad$ v

Abkürzungsverzeichnis $\quad$ ix

1 Ausgangspunkt und Motivation $\quad 1$

2 Löslichkeit von Gasen 5

2.1 Theoretische Grundlagen . . . . . . . . . . . . . . . . . . 5

2.1.1 Löslichkeit und Phasengleichgewicht . . . . . . . . . . . . 5

2.1.2 Partialdruck und Daltonsches Gesetz . . . . . . . . . . . . . . 5

2.1.3 Phasengleichgewicht - Allgemeingültige Formulierung . . . . . . . . 6

2.1.4 Approximationen für geringe Drücke und Temperaturen - „Von der

Isofugazität zum vereinfachten Henry-Gesetz" . . . . . . . . . . 10

2.1 .5 Henry-Gesetz . . . . . . . . . . . . . . . . . . . . 13

2.1.6 Technischer Löslichkeitskoeffizient . . . . . . . . . . . . . . . 13

2.1.7 Technischer Löslichkeitskoeffizient bei Berücksichtigung der Poynting-

Korrektur . . . . . . . . . . . . . . . . . . 15

2.1.8 Gebräuchliche Größen in der Praxis . . . . . . . . . . . . . . . 16

2.2 Löslichkeit von Stickstoff und Sauerstoff in Wasser . . . . . . . . . . . . . 19

2.2.1 Technischer Löslichkeitskoeffizient . . . . . . . . . . . . . . . . . 19

2.2.2 Gegenseitige Beeinflussung der Gase . . . . . . . . . . . . . . . . . 19

2.3 Berechnung der Löslichkeit und Diskussion von Vereinfachungen am Beispiel

Stickstoff in Wasser . . . . . . . . . . . . . . . . . . . . . . 20

2.3.1 Motivation . . . . . . . . . . . . . . . . . . 20

2.3.2 Gasphase - Fugazitätskoeffizenten der Komponenten . . . . . . . . . 21

2.3.3 Flüssigphase. . . . . . . . . . . . . . . . . . . . . . 23

2.3.4 Lösung des Gleichungssystems des Phasengleichgewichts für ein binäres Gas-Wasser-Gemisch . . . . . . . . . . . . . . . . 26

2.3.5 Ergebnisse und Fazit . . . . . . . . . . . . . . . . . . . . . 27

2.3.6 Fundamentalgleichungen und andere Approximationen für Stickstoff in Wasser . . . . . . . . . . . . . . . . . . . . 31

2.3.7 Konsequenz für die vereinfachte Bestimmung von Löslichkeitskoeffizienten . . . . . . . . . . . . . . . . . . 32

3 Wasser-Glykol-Gemische 35

3.1 Wasser-Propylenglykol- und Wasser-Ethlylenglykol-Gemische . . . . . . . . 35

3.2 Reale Fertigmischungen . . . . . . . . . . . . . . . . . . . . . 37

3.3 Gaslöslichkeit . . . . . . . . . . . . . . . . . . . . . . . . . . 37

4 Methodik zur experimentellen Bestimmung von Löslichkeitskoeffizienten 39

4.1 Gegenstand der Untersuchung . . . . . . . . . . . . . . . . . . . . . 39

4.2 Einordnung der verwendeten Versuchsapparatur . . . . . . . . . . . . . . . 39

4.3 Beschreibung der Versuchsapparatur . . . . . . . . . . . . . . . . . . . . . 41

4.3.1 Prinzip . . . . . . . . . . . . . . . . . . . . . . . . . . . . 41 
4.3.2 Kalibrierung mit Wasser . . . . . . . . . . . . . . . . . . . . 41

4.3.3 Detailaufbau und Messtechnik . . . . . . . . . . . . . . . . . 42

4.3.4 Automatisierung . . . . . . . . . . . . . . . . . . . . . . 45

4.4 Versuchsablauf . . . . . . . . . . . . . . . . . . . . . . . . . . . . 47

4.4.1 Besonderheiten in der Messmethode . . . . . . . . . . . . . . . . 47

4.4 .2 Versuchsablauf . . . . . . . . . . . . . . . . . . . . . 51

4.4.3 Versuchsrelevante Fragestellungen und Detailprobleme . . . . . . . . 55

4.5 Berechnungsalgorithmus der Löslichkeitskoeffizienten . . . . . . . . . . . . . 58

4.5 .1 Allgemeines . . . . . . . . . . . . . . . . . . . . 58

4.5 .2 Bestimmung der Volumina . . . . . . . . . . . . . . . . . . . . 59

4.5 .3 Gasreservoir . . . . . . . . . . . . . . . . . . . . . . . . . . 61

4.5 .4 Reaktor . . . . . . . . . . . . . . . . . . . . . . . 61

4.5.5 Gasphase des Reaktors . . . . . . . . . . . . . . . . . . . . 62

4.5.6 Flüssigphase des Reaktors . . . . . . . . . . . . . . . . . . . 65

4.5.7 Bestimmung des Löslichkeitskoeffizienten . . . . . . . . . . . . 66

4.5.8 Einordnung und Lösung des aufgestellten Gleichungssystems . . . . . 67

4.6 Kalibrierung der Apparatur . . . . . . . . . . . . . . . . . . . . . . . . 71

4.7 Erläuterungen zu Einflussgrößen und deren Unsicherheit . . . . . . . . . . 72

4.7 .1 Allgemeines . . . . . . . . . . . . . . . . . . . . . 72

4.7.2 Gase im Ausgangzustand und im Phasengleichgewicht 1 . . . . . . 72

4.7.3 Zusammensetzung der Synthetischen Luft $y_{\mathrm{N} 2}^{\mathrm{G}} \ldots \ldots . . . .74$

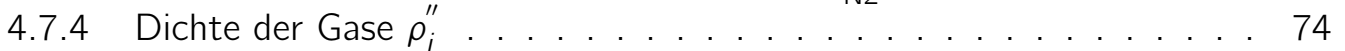

4.7.5 Dichte des Lösungsmitteldampfs $\rho_{\mathrm{LM}}^{\prime \prime} \ldots \ldots \ldots . . \ldots 75$

4.7.6 Dichte des Lösungsmittels $\rho_{\mathrm{LM}}^{\prime} \ldots \ldots \ldots . \ldots . . \ldots 75$

4.7 .7 Dampfdruck $p_{\mathrm{s}} \ldots \ldots \ldots \ldots \ldots . \ldots . \ldots . \ldots . \ldots 78$

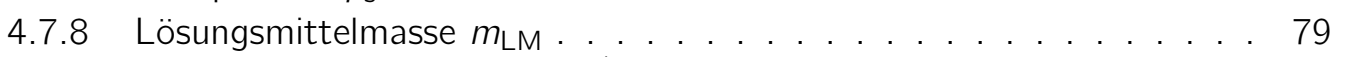

4.7.9 Partielles molares Volumen $v_{\infty, i, \mathrm{LM}}^{\prime}$ der Gase in der Flüssigkeit . . . . 80

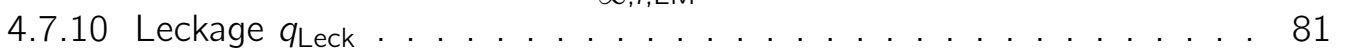

4.7.11 Löslichkeitskoeffizient von Wasser $\lambda_{i, \mathrm{H} 2 \mathrm{O}, \infty, \text { Lit }}$ zur Kalibrierung mit Wasser-Versuchen . . . . . . . . . . . . . . . . . 81

4.7.12 Gleichungsungenauigkeit durch Vereinfachungen . . . . . . . . . . 82

4.7 .13 Weitere Einflüsse . . . . . . . . . . . . . . . . . . . . . . 82

4.8 Bestimmung der Messunsicherheit . . . . . . . . . . . . . . . . . . . . . . . . . 82

4.8 .1 Allgemeines . . . . . . . . . . . . . . . . . . . . . . . . 82

4.8 .2 Grundlagen . . . . . . . . . . . . . . . . . . 83

4.8.3 Messunsicherheitsbudget . . . . . . . . . . . . . . . . 85

4.8.4 Minimierte Messunsicherheit durch optimierte Versuchsparameter . . 88

4.8.5 Messunsicherheit bei der kalibrierten Bestimmung . . . . . . . . . . . . 93

4.9 Messunsicherheitsbeiträge und Gesamt-Messunsicherheit . . . . . . . . . . . 95

4.9.1 Gesamt-Messunsicherheit . . . . . . . . . . . . . . . . . 95

4.9.2 Ermittelte Messunsicherheitsbeiträge . . . . . . . . . . . . . . . . 95

4.10 Wertung und Verbesserungspotential des Versuchsstands . . . . . . . . . . 100

5 Durchgeführte Versuche und deren Auswertung 103

5.1 Übersicht . . . . . . . . . . . . . . . . . . . . . . . . . . 103

5.2 Druckstufen, Gültigkeitsbereich des Löslichkeitskoeffizienten und kalibrierte Ergebnisse . . . . . . . . . . . . . . . . . . . . . . 105

5.3 Stickstoff-Löslichkeitskoeffizienten für Tyfocor ${ }^{\circledR}$ LS . . . . . . . . . . . . 107

5.3.1 Tyfocor ${ }^{\circledR}$ LS und Sauerstoffbindung . . . . . . . . . . . . . . . . 107

5.3.2 Reduktion der Messunsicherheit durch Kalibrierung . . . . . . . . . . 109 
5.3.3 $\mathrm{N}_{2}$-Löslichkeitskoeffizienten für Tyfocor ${ }^{\circledR}$ LS . . . . . . . . . . . 109

5.4 Stickstoff-Löslichkeitskoeffizienten für Wasser-Glykol-Gemische . . . . . . . 110

5.4.1 Temperaturabhängige Messdaten . . . . . . . . . . . . . . . . . 110

5.4.2 Abhängigkeit vom Glykolanteil und Vergleich mit Literaturwerten . . 114

5.4.3 Wirkung von Additiven in Wasser-Glykol-Gemischen . . . . . . . . 116

5.5 Untersuchungen mit Luft . . . . . . . . . . . . . . . . . . . . 117

5.5.1 Stickstoff-Löslichkeitskoeffizienten im Vergleich mit Ergebnissen aus

Stickstoff-Versuchen . . . . . . . . . . . . . . . . . 117

5.5.2 Sauerstoff-Löslichkeitskoeffizienten für Wasser-Glykol-Gemische . . 118

5.5.3 Sauerstoff in Tyfocor ${ }^{\circledR}$ LS . . . . . . . . . . . . . . . . . . 119

5.5.4 Wirkung von Additiven in Wasser-Glykol-Gemischen . . . . . . . . 122

5.6 Zusammenfassung der Ergebnisse . . . . . . . . . . . . . . . . . . 122

6 Löslichkeit in Wasser-Glykol-Gemischen in Theorie und Praxis 125

6.1 Grundlagen . . . . . . . . . . . . . . . . . . . . 125

6.1 .1 Allgemeines . . . . . . . . . . . . . . . . . . . . . . 125

6.1 .2 Henry-Diagramm . . . . . . . . . . . . . . . . . . . 127

6.1.3 Interpretation der Bereiche des Henry-Diagramms, Einordnung des

Henry-Diagramms in reale Zustände des Systems . . . . . . . . . 128

6.2 Stickstoff-Löslichkeitsverhalten der untersuchten Stoffe . . . . . . . . . . . 129

6.2 .1 Allgemeines . . . . . . . . . . . . . . . . . . . . . . . . . 129

6.2.2 Stickstoff-Löslichkeit in Wasser-Glykol-Gemischen . . . . . . . . . 130

6.2.3 Stickstoff-Löslichkeit in Wasser-Glykolen mit Additiven . . . . . . . 134

6.2.4 Unsicherheit der Henry-Diagramme . . . . . . . . . . . . . . . . . 135

6.3 Einfluss von Sauerstoff auf Löslichkeitsgrenzen . . . . . . . . . . . . . . 135

6.3.1 Allgemeines zum Gemisch von Stickstoff und Sauerstoff . . . . . . . 135

6.3 .2 Gleichgewicht mit Luft . . . . . . . . . . . . . . . . . . . 136

6.3.3 Sauerstoff-Anteil nach der Anlagenbefüllung . . . . . . . . . . . 138

6.3.4 Chemische Bindung durch Inhibitor . . . . . . . . . . . . . . . . . 140

6.4 Einige Ableitungen für energietechnische Wasser-Glykol-Kreisläufe und vali-

dierende Beispiele . . . . . . . . . . . . . . . . . . . . . . . . . . 141

6.4.1 Gaslöslichkeit in realen Anlagen . . . . . . . . . . . . . . . . . . . 141

6.4.2 Ergebnisvalidierende Praxis-Beispiele . . . . . . . . . . . . . . . . 148

7 Zusammenfassung und Fazit $\quad 155$

7.1 Versuchsstand und Methodik . . . . . . . . . . . . . . . . . . . 155

7.2 Messtechnische Untersuchungen . . . . . . . . . . . . . . . . 156

7.3 Löslichkeit von Gasen in Theorie und Praxis . . . . . . . . . . . . . . . . . 157

8 Literaturverzeichnis 161

$\begin{array}{ll}\text { A Anhang } & 169\end{array}$

A.1 Tabelle der verbleibenden Isofugazitäts-Gleichungen entsprechend der Vereinfachungen . . . . . . . . . . . . . . . . . . . . . 169

A.2 Herleitung Zusammenhang der Vereinfachungen A-F und A-G . . . . . . 170

A.3 Angaben zum Aufbau . . . . . . . . . . . . . . . . . . . . . . 170

A.4 Charakterisierung der Versuchskonfigurationen . . . . . . . . . . . . . . 173

A.5 Abschätzung des Lösungsmittelmasse-Verlusts bei entgastem Ausgangszustand . . . . . . . . . . . . . . . . . . . . . . . 176

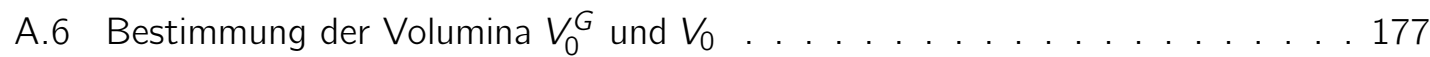

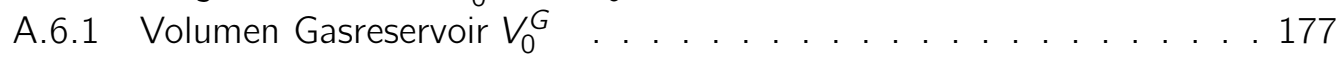




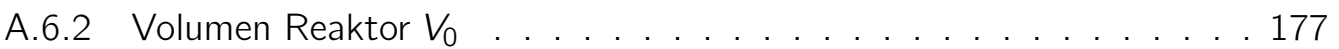

A.6.3 Ermittelte Volumina . . . . . . . . . . . . . . . . . . . . . . . 179

A.7 Kalibrierkurven und deren Unsicherheit - Theorie . . . . . . . . . . . . . . 181

A.7.1 Hintergrund / Einordnung . . . . . . . . . . . . . . . . . . . . . 181

A.7.2 Kalibrierkurve / Korrekturfunktion . . . . . . . . . . . . . . . . . 181

A.7.3 Bestimmung der Koeffizienten der Kalibrierkurve . . . . . . . . . . 182

A.7.4 Unsicherheit der Stützstellen . . . . . . . . . . . . . . . . . . 182

A.7.5 Unsicherheit der Koeffizienten . . . . . . . . . . . . . . . . . 182

A.7.6 Unsicherheit der Kalibrierkurve . . . . . . . . . . . . . . . . . . 183

A.7.7 Integration in Berechnungsformel . . . . . . . . . . . . . . 183

A.8 Herleitung Unsicherheitsfortpflanzung für maximal korrelierte Unsicherheiten . 184

A.9 Unsicherheit der Kalibrierung - Anwendung . . . . . . . . . . . . . 185

A.9.1 Temperaturkalibrierung . . . . . . . . . . . . . . . . . . . . . . 185

A.9.2 Drucksensorkalibrierung . . . . . . . . . . . . . . . . . . . 185

A.10 Herleitung der Kalibrierungsformel . . . . . . . . . . . . . . . . . 186

A.11 Gasphasenzusammensetzung bei gegebener Flüssigphasenzusammensetzung . 190

A.12 Herleitung des Temperaturverhaltens bei statischer Druckhaltung mit MAG . 191

A.13 Auswertung . . . . . . . . . . . . . . . . . . . . . . . . . . . . . . . . 194

A.13.1 Drucksprungdiagramme . . . . . . . . . . . . . . . . . . . . . 194

A.13.2 Ermittelte Löslichkeiten aus den Versuchen . . . . . . . . . . . . . 195

A.14 Dichtemessungen . . . . . . . . . . . . . . . . . . . . . 200

A.15 Henry-Diagramme der untersuchten Gemische mit Additiven . . . . . . . . 202 


\section{Symbolverzeichnis}

\section{Formelzeichen}

\begin{tabular}{|c|c|c|}
\hline Symbol & Bedeutung & Einheit \\
\hline a & Breite der systematischen Unsicherheit, Koeffizient & \\
\hline$A$ & Fläche & $\mathrm{m}^{2} /-$ \\
\hline$B$ & Virialkoeffizient & - \\
\hline$c_{1}, c_{2}, c_{3}$ & $\begin{array}{l}\text { Koeffizienten der Polynom-Regression für die } \\
\text { Lösungsmitteldichte bzw. Korrekturkoeff. der } \\
\text { Messwerte }\end{array}$ & \\
\hline$c_{i}$ & $\begin{array}{l}\text { Konzentration des Gases } i \text { im Lösungsmittel LM } \\
\text { (Flüssigphase) }\end{array}$ & $\frac{m g_{i}}{l}$ \\
\hline$c_{p}$ & spezifische Wärmekapazität & $\frac{\mathrm{kJ}}{\mathrm{kg} \cdot \mathrm{K}}$ \\
\hline$f$ & Fugazität & bar \\
\hline$f_{\mathrm{Z}}$ & $\begin{array}{l}\text { Faktor zur Berücksichtigung von Zusatzvolumina bei } \\
\text { der Druckhaltung }\end{array}$ & \\
\hline$g$ & Erdbeschleunigung & $\frac{\mathrm{kg} \cdot \mathrm{m}}{\mathrm{s}^{2}}$ \\
\hline$\Delta H_{\mathrm{ST}}$ & statische Anlagenhöhe & $\mathrm{m}$ \\
\hline K & $\begin{array}{l}\text { zusammenfassender Faktor in der } \\
\text { Phasengleichgewichtsberechnung }\end{array}$ & - \\
\hline$K_{H}$ & $\begin{array}{l}\text { Henry-Flüchtigkeitskoeffizient; bei Angabe ohne oberen } \\
\text { Index ist } K_{H}^{f x} \text { gemeint. }\end{array}$ & je nach Index \\
\hline$K_{H}^{f x}$ & $\begin{array}{l}\text { Henry-Flüchtigkeitskoeffizient, definiert als } \\
K_{H}^{f x}=\lim _{x_{i} \rightarrow 0} \frac{f_{i}^{\prime}}{x_{i}}\end{array}$ & $\mathrm{MPa}$ \\
\hline$L$ & Ostwald-Koeffizient & - \\
\hline$M_{k}$ & molare Masse der Komponente $k$ & $\frac{\mathrm{kg}}{\mathrm{mol}}$ \\
\hline$m$ & Masse & $\mathrm{kg}$ \\
\hline$m_{\text {Schein }}$ & $\begin{array}{l}\text { scheinbare Masse durch Wägung bei Wirkung des } \\
\text { Auftriebs der Umgebungsluft }\end{array}$ & $\mathrm{kg}$ \\
\hline$n_{k}$ & Stoffmenge der Komponente $k$ & $\mathrm{~mol}$ \\
\hline$p$ & Absolutdruck, Überdruck & bar, bar \\
\hline$p_{k}$ & Partialdruck für Komponente $k$ & bar \\
\hline
\end{tabular}




\begin{tabular}{|c|c|c|}
\hline Symbol & Bedeutung & Einheit \\
\hline$p_{\mathrm{LM}, \mathrm{s}}$ & Sättigungsdampfdruck des reinen Lösungsmittels LM & bar \\
\hline Poyk $_{k}$ & Poynting-Korrektur der Komponente $k$ & - \\
\hline$q_{\text {Leck }}$ & Leckagerate & $\frac{\mathrm{mbar} \cdot \mathrm{I}}{\mathrm{h}}$ \\
\hline$R$ & Universelle Gaskonstante & $\frac{\mathrm{J}}{\mathrm{mol} \cdot \mathrm{K}}$ \\
\hline$R_{i}$ & Spezifische Gaskonstante der Komponente i & $\frac{\mathrm{J}}{\mathrm{kg} \cdot \mathrm{K}}$ \\
\hline$t$ & Temperatur & ${ }^{\circ} \mathrm{C}$ \\
\hline$T$ & Temperatur & K \\
\hline$u$ & Standardmessunsicherheit & \\
\hline$U$ & Erweiterte Messunsicherheit & \\
\hline$v, v_{0, i}$ & molares Volumen & $\mathrm{m}^{3} / \mathrm{mo}$ \\
\hline$v_{i}$ & partielles molares Volumen $v_{i}=\left(\frac{\partial V}{\partial n_{i}}\right)_{t, p, n_{k}, k \neq j}$ & $\mathrm{~m}^{3} / \mathrm{mo}$ \\
\hline$V$ & Volumen & $\mathrm{m}^{3}$ \\
\hline$V_{\mathrm{e}}$ & Ausdehnungsvolumen des Anlagenfluids & $\mathrm{m}^{3}$ \\
\hline$V_{Z}$ & von der Druckhaltung aufzunehmendes Zusatzvolumen & $\mathrm{m}^{3}$ \\
\hline$w_{i}$ & $\begin{array}{l}\text { Gasgehalt des Gases } i \text { in der Flüssigphase (in } \\
\text { Normvolumen des Gases pro Gesamtmasse Flüssigkeit) }\end{array}$ & $\frac{\mathrm{Ncm}^{3}}{\mathrm{~g}}$ \\
\hline$x_{k}$ & $\begin{array}{l}\text { Stoffmengenanteil der Komponente } k \text { in der } \\
\text { Flüssigphase }\end{array}$ & - \\
\hline$y_{k}$ & Stoffmengenanteil der Komponente $k$ in der Gasphase & - \\
\hline
\end{tabular}

\section{Griechische Symbole}

\begin{tabular}{lll}
\hline Symbol & Bedeutung & Einheit \\
\hline$\alpha$ & Längenausdehnungskoeffizient & $\mathrm{K}^{-1}$ \\
$\beta$ & Volumenausdehnungskoeffizient & $\mathrm{K}^{-1}$ \\
$\gamma_{k}$ & Aktivitätskoeffizient der Komponente $k$ & - \\
$\gamma_{k}^{*}$ & rationeller Aktivitätskoeffizient der Komponente $k$ & - \\
$\Delta_{\text {rel } X}$ & relativer Fehler von $x$ & - \\
$\rho$ & Dichte & $\frac{\mathrm{kg}}{\mathrm{m}^{3}}$ \\
$\kappa$ & Kompressibilität & $\mathrm{MPa}^{-1}$
\end{tabular}




\begin{tabular}{lll}
\hline Symbol & Bedeutung & Einheit \\
\hline$\lambda$ & $\begin{array}{l}\text { technischer Löslichkeitskoeffizient; entspricht nach [Sander, } \\
\text { 2015] } H^{w p}\end{array}$ & $\frac{\mathrm{Ncm}^{3}}{\mathrm{gLM} \cdot \mathrm{bar}}$ \\
& chemisches Potential der Komponente $k$ & $\frac{\mathrm{J}}{\mathrm{mol}}$ \\
$\mu_{k}$ & Zeit & $\mathrm{s}$ \\
$\tau$ & Masseanteil der Komponente $k$ (Flüssigphase) & - \\
$\xi_{k}$ & Fugazitätskoeffizient der Komponente $k$ & - \\
$\varphi_{k}$ & Sättigungsgrad für gelöstes Gas & - \\
$\phi_{\text {sat }}$ & Volumenanteil der Komponente $k$ im Gemisch & - \\
$\Phi_{k}^{\vee}$ & &
\end{tabular}

\section{Indizes}

\begin{tabular}{ll}
\hline Indize & Bedeutung \\
\hline$\prime$ & Fluidphase \\
+ & Gasphase \\
0 & Bezugszustand \\
$\infty$ & Reinstoff, Startzustand der Iteration \\
a & unendliche Verdünnung \\
approx & Anfangszustand \\
Ausg & approximiert \\
DS & Ausgangszustand \\
e & Druckstufe/Drucksprung \\
G & Endzustand \\
GGW & hochgestellt Gasreservoir, tiefgestellt Glykol \\
HP & Phasengleichgewicht \\
$i$ & Hochpunkt \\
$i, j, I, m, n$ & Gaskomponente mit $i \in\{$ Gas 1, Gas 2, ... bzw. Laufindex \\
$k$ & Laufvariablen \\
Lit & Komponente mit $k \in\{$ LM, Gas 1, Gas 2, ... $\}$ \\
LM & Literaturwert \\
max & Lösungsmittel \\
mess & Maximum \\
& Messwert
\end{tabular}




\begin{tabular}{ll}
\hline Indize & Bedeutung \\
\hline $\mathrm{N}$ & Normzustand \\
$\mathrm{N} 2$ & Stickstoff \\
$\mathrm{NP}$ & Nullprope \\
$\mathrm{O} 2$ & Sauerstoff \\
$\mathrm{R}$ & Reaktor \\
$\mathrm{R} 0$ & Reaktor ohne Toträume \\
R1 & Reaktor-Totraum 1 \\
R2 & Reaktor-Totraum 2 \\
Rest & Restgase \\
$\mathrm{S}$ & Siedezustand \\
tr & trocken (ohne Flüssigkeit) \\
$U$ & Umgebungszustand \\
ü & Überdruck \\
$z$ & Variable für stationären Zustand \\
\hline
\end{tabular}




\section{Abkürzungsverzeichnis}

\begin{tabular}{ll}
\hline Abkürzung & Erklärung \\
\hline AKA & Absorptionskälteanlage \\
DH & Druckhaltung \\
E & Ethylenglykol \\
25E/ 41E/ & Wasser-Ethylenglykol-Gemisch mit 25/ 41,84/ 75/ 100 \\
$75 E /$ 100E & Gewichtsprozent Glykol \\
EP & Endprobe \\
GM & Gasmaus \\
GUM & Guide of uncertainty of measurements [GUM; DIN V ENV 13005] \\
HDR & Hinterdruckregler \\
LS & Wärmeträgermedium Tyfocor ${ }^{\circledR}$ LS \\
MAG & Membran-Druckausdehnungsgefäß \\
$\mathrm{N}_{2}$ & Stickstoff \\
$\mathrm{NP}$ & Nullprobe \\
$\mathrm{O}_{2}$ & Sauerstoff \\
$\mathrm{P}$ & Propylenglykol / Probenahmezylinder \\
$25 P / 41 P /$ & Wasser-Propylenglykol-Gemisch mit 25/ 41,84/ 75/ 100 \\
$75 P / 100 P$ & Gewichtsprozent Glykol \\
RHzg & Rohrheizung \\
RKW & Rückkühlwerk \\
TUD & Technische Universität Dresden \\
$41 T$ & Wasser-Tyfocor ${ }^{\circledR}$-Gemisch mit 41,84 Gewichtsprozent Tyfocor ${ }^{\circledR}$ \\
V & Ventil \\
VaPu & Vakuumpumpe \\
VE-Wasser & vollentsalztes Wasser \\
\hline & \\
&
\end{tabular}





\section{Ausgangspunkt und Motivation}

Flüssigkeitskreisläufe dienen in energietechnischen Anlagen dem Transport thermischer Energie. Als Wärmeträgermedium kommt aufgrund der physikalischen Eigenschaften, der Verfügbarkeit aber auch wirtschaftlicher und ökologischer Aspekte bevorzugt Wasser zum Einsatz. Ein Zusatz von Frostschutzmitteln - hier Glykolen - ist erforderlich, wenn Medientemperaturen im Bereich des Gefrierpunktes von Wasser auftreten. Ein wichtiger Parameter zur Charakterisierung der Beschaffenheit von Flüssigkeiten ist der Gasgehalt.

Die Flüssigkeiten der Kreisläufe enthalten nahezu immer Gase (z. B. Stickstoff, Sauerstoff) in gelöster Form. Gase können aber auch in freier Form als im Medium mittransportierte Gasblasen oder als Gasansammlung an einem Ort des Kreislaufs in sogenannten Gaspolstern auftreten. Sie gelangen bei Befüll- oder Nachfüllvorgängen durch Lufteinschluss und gelöste Gase im Wärmeträgermedium in den Kreislauf, vgl. [Rühling u. a., 2013b]. Weiterhin werden diese über Diffusionsprozesse durch permeable Bauteile wie Dichtungen, Membranen und Rohrleitungen (z. B. bei Erdsonden aus diffusionsoffenen Kunststoffrohren) ins System transportiert, vgl. [Sittiho, 2011]. Bei falscher Druckhaltung der Anlage oder Fluidverlust kann Unterdruck enstehen. Da Dichtungen nicht auf Unterdruck-Dichtheit gegen Umgebungsdruck ausgelegt sind, wird folglich Umgebungsluft eingesaugt, vergleiche [Panitz und Rühling, 2017]. Ebenso ist interne Gasbildung durch Korrosion oder biologische Prozesse, z. B. Methan und Wasserstoff, möglich (vgl. [Rühling, 2002]). [VDI 2035-2] fokussiert auf den Eintrag von Sauerstoff in Heizungsanlagen und nennt eine ähnliche Sammlung an Gründen.

In der Praxis werden vielfältige Probleme mit Gasen beobachtet. Einige Beispiele sind:

- Verringerung des durchströmten Rohrquerschnitts durch Gaspolster und damit Erhöhung von Druckverlusten [Umbreit, 2014],

- Verringerung des hydraulischen Wirkungsgrads von Pumpen ([Kosmowski und Hergt, 1990]) durch Gasblasen(-bildung),

- Störung der gleichmäßigen Durchströmung hydraulisch parallel verschalteteter Strömungswege durch Luftblasen in Sammler/Verteiler oder einzelnen Strömungswegen (z. B. in Plattenwärmeübertragern oder in bzw. zwischen Solarkollektoren) mit entsprechenden Auswirkungen wie Störung der Wärmeübertragung bzw. partielle Stagnation speziell bei Solarthermieanlagen,

- Fließgeräusche und Erosion durch transportierte Gasblasen,

- lokale Anlagerung von Gasen an zur Kühlung überströmten Oberflächen, lokale Überhitzung und Schädigung des zu kühlenden Bauteils (z. B. bei der Kühlung von Brennstoffzellenmembranen),

- gestörte Messtechnik (Anlagerung von Gasblasen an Temperatursensoren, Störung der Ultraschallsignale von Volumenstromsensoren wie in [Panitz und Rühling, 2017]).

Um die Wirkungen der Gase in Flüssigkeiten auf Systemeffizienz, Inbetriebnahmesowie Instandhaltungsaufwand, Systemfunktionalität, Anlagenverfügbarkeit und Lebensdauer energietechnischer Anlagen quantifizieren zu können, ist die Kenntnis der 
Löslichkeitsgrenze essentiell und deshalb Kernpunkt der vorgelegten Dissertationsschrift.

Das Wissen um das Löslichkeitsverhalten von Gasen im Fluid und Bedingungen für die Überbzw. Unterschreitung der Löslichkeitsgrenzen sind wichtige Grundlagen zur Beschreibung der realen Gasbeladung bzw. des Sättigungsgrads des Gases in der Flüssigkeit, aber auch zur Beschreibung der physikalischen Vorgänge Gastransport, Gasdesorption, Gasabsorption und Gasdiffusion. Damit können oben beschriebene Effekte in Anlagen analysiert und verstanden sowie durch angepasste Konstruktion, Auslegung und Betriebsweise, aber auch durch geeignete Befüllung, Entlüftung und Entgasung der Anlagen, gezielt vermieden werden.

Die stoffspezifische Beschreibung der Gaslöslichkeit geschieht für technische Anwendungen z. B. mit sogenannten technischen Löslichkeitskoeffizienten. Für Wasser sind diese Löslichkeitskoeffizienten für einen weiten Temperaturbereich gut untersucht. Wichtige energietechnische Anlagen, wie bspw. Solarthermieanlagen, Rückkühlwerke und Erdwärmesonden bzw. -kollektoren, aber zum Beispiel auch Motorkühlkreisläufe, nutzen jedoch Frostschutzgemische auf Wasser-Glykol-Basis als Wärmeträger. Für Wasser-Glykol-Gemische sind keine temperaturabhängigen Löslichkeitskoeffizienten verfügbar. Damit bildet die Bestimmung der Gaslöslichkeitskoeffizienten für Wasser-Glykol-Gemische die Grundlage für praktische und theoretische Untersuchungen und die Interpretation des Verhaltens dieser energietechnischen Anlagen unter Einwirkung von Gasen.

Vor dieser Arbeit ist in der ingenieurtechnischen Praxis aufgrund fehlenden Wissens teilweise davon ausgegangen worden, dass Gase in Wasser-Glykol-Gemischen ein ähnliches Verhalten wie in Wasser aufweisen. Entsprechend dieser Annahmen sind z. B. Inbetriebnahmevorschriften und konstruktive Aspekte wie Entlüfter geplant und auch das Betriebsverhalten interpretiert worden.

In Tabelle 1.1 sind die Zusammensetzung der Luft und die Zusammensetzung der gelösten Gase in (luftgesättigtem) Wasser bei Umgebungsbedingungen dargestellt. Neben Stickstoff und Sauerstoff als Hauptbestandteile der Umgebungsluft sind Argon und Kohlendioxid wichtige Bestandteile. Die Zusammensetzung des Gasmix im gelösten Zustand verschiebt sich etwas. Dies liegt an der unterschiedlichen Löslichkeit der einzelnen Gase im Wasser. Vor allem die Löslichkeit von Kohlendioxid ist hier deutlich höher als die der anderen betrachteten Gase. Trotzdem bleiben auch im gelösten Zustand die Anteile von Argon und Kohlendioxid gering. Daher sollen die Betrachtungen hier auf die aus technischer Sicht wesentlichen Gase Stickstoff und Sauerstoff und deren Löslichkeitskoeffizienten beschränkt werden. Weitere Gase, die durch Korrosionsprozesse entstehen, werden nicht betrachtet.

Die Fragestellung soll zu wesentlichen Teilen experimentell mit einem entsprechend zu konfigurierenden Versuchsstand beantwortet werden. Im Sinne eines überschaubaren experimentellen Aufwands wird auf

- Fluidtemperaturen von $10^{\circ} \mathrm{C}$ bis $110^{\circ} \mathrm{C}$ und

- einen Gesamtdruck von 0 bar bis 10 bar

fokussiert. Die Betriebsbereiche vieler energietechnischer Anlagen sind damit abgedeckt.

In der Praxis eingesetzte Wasser-Glykol-Fertiggemische enthalten weitere Bestandteile, die bspw. den Korrosionsschutz garantieren. Wichtig ist demnach auch, den Einfluss dieser Additive auf die physikalische Gaslöslichkeit und etwaige chemische Bindung der Gase zu untersuchen. 
Tabelle 1.1: Zusammensetzung trockener Luft (wesentliche Bestandteile) und gelöste Gasmengen in luftgesättigtem Wasser bei Umgebungsbedingungen $\left(1,01325\right.$ bar und $25^{\circ} \mathrm{C}$ ); gelöste Gasmenge angegeben als Normvolumen reines Gas bei Normzustand 1,01325 bar und $0{ }^{\circ} \mathrm{C}$ )

\begin{tabular}{|c|c|c|c|}
\hline Bestandteil & $\begin{array}{l}\text { Volumenanteil } \\
\text { in tr. Luft }{ }^{a, b}\end{array}$ & $\begin{array}{l}\text { Gelöste Menge } \\
\text { in } 1 \mathrm{~kg} \mathrm{Wasser}{ }^{c, e}\end{array}$ & $\begin{array}{r}\text { Zusammensetzung } \\
\text { gelöster Gase in Wasser } \\
\end{array}$ \\
\hline Stickstoff & $78,08 \%$ & $11,13 \mathrm{Ncm}^{3}$ & $63,35 \%$ \\
\hline Sauerstoff & $20,95 \%$ & $5,86 \mathrm{Ncm}^{3}$ & $33,34 \%$ \\
\hline Argon & $0,93 \%$ & $0,29 \mathrm{Ncm}^{3}$ & $1,63 \%$ \\
\hline Kohlendioxid & $403 \mathrm{ppm}=0,0403 \%$ & $0,30 \mathrm{Ncm}^{3}$ & $1,69 \%$ \\
\hline Sonstiges $\left(\mathrm{Ne}, \mathrm{He}, \mathrm{CH}_{4}, \mathrm{Kr}, \ldots\right)$ & $<30$ ppm & - & - \\
\hline \multicolumn{4}{|c|}{$\begin{array}{l}\text { a nach [Wallace und Hobbs, 2006] } \\
\text { b für } \mathrm{CO}_{2} \text { globaler Mittelwert 2016 (aktuell zusätzlich } 2.3 \mathrm{ppm} \text { pro Jahr) nach [Dlugokencky und Tans, 2017] } \\
\text { c eigene Berechnungen mit Henry-Gesetz, Löslichkeitskoeffizienten nach [IAPWS, 2004]; Partialdruck des Wasserdampfs berücksichtigt } \\
\text { d bezogen auf Normvolumen aller gelösten Gase } \\
\text { e Normvolumen des Gases }\end{array}$} \\
\hline
\end{tabular}

In der vorliegenden Arbeit wird Wasser-Glykol-Gemisch als homogenes Lösungsmittel betrachtet, die Wirkungen der Wasser- bzw. Glykol-Anteile nicht separat betrachtet. Wirkungen der Additive werden über Vergleichsmessungen phänomenologisch ermittelt.

Es wird eine vergleichende Bestimmung der Gaslöslichkeit angestrebt. Als Referenzstoff wird dazu Wasser eingesetzt. Für Wasser sind Löslichkeitskoeffizienten aus der Literatur in guter Genauigkeit bekannt.

Nach Konfiguration und Optimierung eines Versuchsstands, Erstellung eines geeigneten Versuchsablaufs und Berechnungsverfahrens zur Bestimmung der Löslichkeit und der zugehörigen Ergebnisunsicherheit sind für Wasser-Glykole Löslichkeitskoeffizienten in einer Vielzahl an Messungen bestimmt worden. Anschließend sind Regressionsgleichungen für die Temperaturabhängigkeit erstellt worden. Welche Auswirkung das ermittelte Löslichkeitsverhalten in den Anlagen hat und welche Unterschiede sich zu Wasser zeigen, wird anhand von sogenannten Henry-Diagrammen diskutiert. Anhand praktischer Anwendungsbeispiele und beobachteter Phänomene, die mit den ermittelten Daten nun erklärbar sind, wird die Plausibilität und der Nutzen der Daten verdeutlicht. 



\section{Löslichkeit von Gasen}

\subsection{Theoretische Grundlagen}

\subsubsection{Löslichkeit und Phasengleichgewicht}

Gase können in Flüssigkeiten gelöst werden (sorbierter Anteil) oder liegen als freie Gase (nicht sorbierter oder desorbierter Anteil) vor. Bei abgeschlossenen Ausgleichsvorgängen eines Zweiphasengemischs, einem sogenannten Phasengleichgewicht zwischen einem Gasund einem Flüssigkeitsvolumen, kann der Stoffmengenanteil $x_{i}$ des gelösten Gases $i$ mit $i \in\{$ Gas 1, Gas 2, ... $\}$ eindeutig beschrieben werden. Die Menge des im Phasengleichgewicht gelösten Gases ist abhängig von den Druck- und Temperaturbedingungen, der quantitativen Zusammensetzung der Gasphase und der qualitativen Zusammensetzung des Gemischs (beteiligte Gasarten $i$ und Lösungsmittel LM).

Hier sei angenommen, dass die Gasphase so groß ist, dass in dieser der gleiche, von der Oberflächenspannung unbeeinflusste Druck wie in der Flüssigphase herrscht.

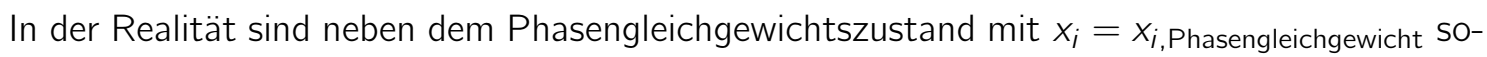
wohl Zustände mit Gasuntersättigung als auch Gasübersättigung in der Flüssigphase möglich, die jedoch Ausgleichsvorgänge in Kraft setzen:

- Im Falle der Gasuntersättigung in der Flüssigkeit treten Absorptionsvorgänge in Kraft.

- Im Falle der Gasübersättigung in der Flüssigkeit wirken Desorptionsvorgänge.

Ist keine Gasphase vorhanden, so können auch beliebige (im Phasengleichgewicht als untersättigt geltende) Zustände stabil sein, dann gilt

$$
x_{i}<x_{i, \text { Phasengleichgewicht }} \text {. }
$$

Sind keine Gasphase und keinerlei Keimstellen vorhanden, können auch übersättigte Zustände stabil sein, vgl. [Heller, 2005].

In realen Anlagen sind jedoch erfahrungsgemäß immer Keimstellen und damit Phasengrenzflächen vorhanden. In der Praxis wird daher der Wert des Phasengleichgewichts

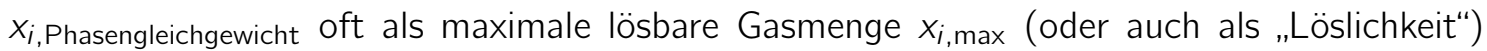
bei gegebenem $(p, t)$-Zustand interpretiert. Dies gilt demnach jedoch nur für Systeme, in denen Ausgleichsvorgänge abgeschlossen sind und genügend Phasengrenzflächen für Desorption existieren.

\subsubsection{Partialdruck und Daltonsches Gesetz}

Vor der Einführung in die Zusammenhänge des Phasengleichgewichts sei hier die Begrifflichkeit des Partialdruckes eingeführt, da dieser anschließend oft benötigt wird. Der Partialdruck $p_{k}$ der Komponente $k$ mit $k \in\{L M$, Gas 1 , Gas $2, \ldots\}$ in einer Gasmischung ist durch den 
Stoffmengenanteil in der Gasphase $y_{k}$, vgl. z. B. mit [Denbigh, 1974, S.90], definiert als

$$
p_{k}=y_{k} \cdot p
$$

Für den Spezialfall einer (idealen) Mischung idealer Gase ist der Partialdruck der Druck, den die Komponente $k$ einnimmt, wenn sie das Volumen $V$ bei gleicher Temperatur $T$ allein ausfüllte.

Für den Gesamtdruck gilt stets

$$
p=\sum_{k} p_{k} .
$$

Dieser Zusammenhang ist bekannt als das Gesetz von Dalton.

\subsubsection{Phasengleichgewicht - Allgemeingültige Formulierung}

Grundlage aller weiteren Betrachtungen ist der Zustand, in dem Gasphase " und Flüssigphase ' koexistieren und Ausgleichsvorgänge abgeschlossen sind: das Phasengleichgewicht. Das thermodynamische Gleichgewicht zeichnet sich durch

- thermisches Gleichgewicht (beide Phasen haben das gleiche thermische Potential, also die gleiche Temperatur, also gilt $t^{\prime \prime}=t^{\prime}$ ),

- mechanisches Gleichgewicht (beide Phasen haben das gleiche mechanische Potential, also den gleichen Druck, also gilt $p^{\prime \prime}=p^{\prime}$ ) und

- stoffliches Gleichgewicht

aus (vgl. z. B. [Pfennig, 2003, S.36]). Das stoffliche Gleichgewicht wird dadurch beschrieben, dass für jede Komponente $k$ das chemische Potential $\mu_{k}$ (das sich als eine Art Triebkraft zum Stoffaustausch vorstellen lässt) in den Phasen gleich groß ist, also

$$
\mu_{k}^{\prime \prime}=\mu_{k}^{\prime}
$$

gilt.

Im Umgang mit Phasengleichgewichten wird das stoffliche Gleichgewicht oft über die Fugazität $f_{k}$ ausgedrückt. Diese ist definiert durch

$$
\mu_{k}=\mu_{k}^{+}\left(p^{+}, T\right)+R \cdot T \cdot \ln \frac{f_{k}}{f_{k}^{+}},
$$

wobei $\mu_{k}^{+}$das chemische Potential im Bezugszustand (bei gleicher Temperatur $T$ ) ist. Die Fugazität $f_{k}$ entspricht bei Idealgasverhalten und idealer Mischung der Gasphase dem Partialdruck $p_{k}$ der Komponente $k$ bzw. stellt im Fall von nicht-idealer Mischung der Gase bzw. Realgasverhalten einen korrigierten Partialdruck dar. Diese Korrektur kann auch über den Fugazitätskoeffizienten $\varphi_{k}$ ausgedrückt werden als

$$
f_{k}=\varphi_{k} \cdot p_{k}
$$




\section{Isofugazitätskriterium}

Das im Phasengleichgewicht geltende stoffliche Gleichgewicht kann dann ebenso ausgedrückt werden durch die sogenannte Isofugazität

$$
f_{k}^{\prime \prime}=f_{k}^{\prime}
$$

und muss für alle Komponenten $k$ gelten. Das Phasengleichgewicht für zwei Komponenten ist in Abbildung 2.1 dargestellt.

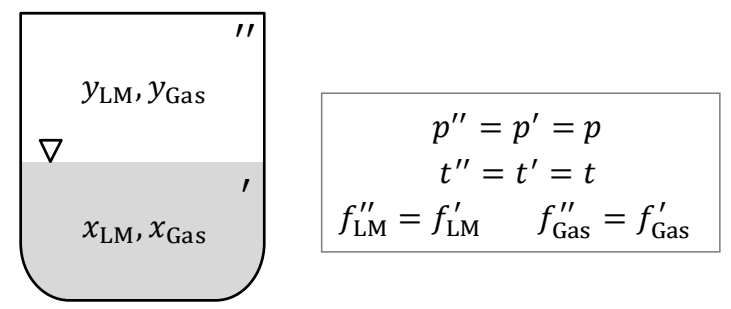

Abbildung 2.1: Phasengleichgewicht für zwei Komponenten (ein Gas und ein Lösungsmittel LM)

Die Fugazität der Gasphase kann mit dem Stoffmengenanteil in der Gasphase $y_{k}$ und dem Gesamtdruck $p$, der mittels Fugazitätskoeffizienten $\varphi_{k}$ korrigiert wird, ausgedrückt werden als

$$
f_{k}^{\prime \prime}=y_{k} \cdot p \cdot \varphi_{k}\left(p, t, y_{k}\right)
$$

Die Fugazität der Flüssigphase kann mit dem Korrekturfaktor Aktivitätskoeffizient $\gamma_{k}$ durch den Stoffmengenanteil in der Flüssigphase $x_{k}$ und der Fugazität in einem Bezugszustand, der sogenannten Standardfugazität $f_{k}^{\prime}{ }^{+}$, bei gleichem Druck ausgedrückt werden als

$$
f_{k}^{\prime}=\gamma_{k}\left(p, t, x_{k}\right) \cdot x_{k} \cdot f_{k}^{\prime+}
$$

mit $\gamma_{k}=1 \mathrm{im}$ Bezugszustand. Als Komponenten $k$ werden im Folgenden das Lösungsmittel mit Index LM und die Gase mit dem Laufindex $i$ unterschieden.

Für das Lösungsmittel ( $k=L M)$ wird für die Flüssigphase als Bezugszustand der Reinstoff (gekennzeichnet durch den Index 0) beim Druck $p$ mit der Standardfugazität $f_{0, L M}^{\prime}$ (Reinstoff-Fugazität) gewählt. Dann kann die Fugazität im Gemisch ausgedrückt werden als

$$
f_{\mathrm{LM}}^{\prime}=\gamma_{\mathrm{LM}}\left(p, t, x_{k}\right) \cdot x_{\mathrm{LM}} \cdot f_{0, \mathrm{LM}}^{\prime}(p, t)
$$

mit dem Aktiviätskoeffizienten $\gamma_{\mathrm{LM}}$, wobei im Bezugszustand $x_{\mathrm{LM}} \rightarrow 1$ der Aktivitätskoeffizient $z u \quad \gamma_{L M} \rightarrow 1$ definiert ist. Es ergibt sich nach weiteren Umformungen für die Standardfugazität, beschrieben z. B. in [Stephan u. a., 2010, S. 264], folgende Gleichung

$$
f_{\mathrm{LM}}^{\prime}=\gamma_{\mathrm{LM}} \cdot x_{\mathrm{LM}} \cdot p_{\mathrm{LM}, \mathrm{s}} \cdot \varphi_{0, \mathrm{LM}, \mathrm{s}} \cdot \exp \int_{p \mathrm{LM}, \mathrm{s}}^{p} \frac{v_{0, \mathrm{LM}}^{\prime}}{R \cdot T} \mathrm{~d} p .
$$


Hier sind der Sättigungsdampfdruck $p_{\mathrm{LM}, \mathrm{s}}$, zugehöriger Fugazitätskoeffizient $\varphi_{0, \mathrm{LM}, \mathrm{s}}$ und das molare Volumen $v_{0, \mathrm{LM}}^{\prime}$ Eigenschaften des reinen Lösungsmittels.

Für die Isofugazität (Gleichung 2.7) ergibt sich nun

$$
y_{\mathrm{LM}} \cdot p \cdot \varphi_{\mathrm{LM}}=\gamma_{\mathrm{LM}} \cdot x_{\mathrm{LM}} \cdot p_{\mathrm{LM}, \mathrm{s}} \cdot \varphi_{0, \mathrm{LM}, \mathrm{s}} \cdot \exp \int_{p L \mathrm{~L}, \mathrm{~s}}^{p} \frac{v_{0, \mathrm{LM}}^{\prime}}{R \cdot T} \mathrm{~d} p .
$$

Für Gase wird üblicherweise für die Flüssigphase als Bezugszustand der Zustand der unendlichen Verdünnung gewählt. Dann ergibt sich nach einigen Umformungen der z. B. in [Stephan u.a., 2010, S. 274] beschriebene Zusammenhang für die Isofugazität

$$
f_{i}^{\prime}=x_{i} \cdot \gamma_{i}^{*} \cdot f_{i, \infty}^{\prime+}\left(p^{+}\right) \cdot \exp \int_{p^{+}}^{p} \frac{v_{\infty, i}^{\prime}}{R \cdot T} \mathrm{~d} p
$$

mit der Standardfugazität der unendlich verdünnten Gaskomponente $f_{i, \infty}^{\prime+}\left(p^{+}\right)$und dem sogenannten rationellen Aktivitätskoeffizienten mit $\gamma_{i}^{*} \rightarrow 1$ für $x_{i} \rightarrow 0$, der die Abweichungen von der Standardfugazität für $x_{i}>0$ beschreibt.

Mit der Definition des Henry-Koeffizienten ${ }^{1}$

$$
K_{\mathrm{H}, i}=\lim _{x_{i} \rightarrow 0} \frac{f_{i}^{\prime}}{x_{i}}
$$

ergibt sich

$$
f_{i}^{\prime}=x_{i} \cdot \gamma_{i}^{*} \cdot K_{\mathrm{H}, i}\left(p^{+}\right) \cdot \exp \int_{p^{+}}^{p} \frac{v_{\infty, i}^{\prime}}{R \cdot T} \mathrm{~d} p
$$

und der Henry-Koeffizient fungiert als Standardfugazität des Gases. Hier sind HenryKoeffizient $K_{\mathrm{H}, i}\left(p^{+}\right)$sowie partielles molares Volumen $v_{\infty, i}^{\prime}$ Eigenschaften des unendlich ver-

\footnotetext{
${ }^{1}$ Insbesondere für den Henry-Koeffizienten existieren eine Vielzahl von Bezeichnungen. Die hier vorgestellte Variante wird in Lehrbüchern gern als H (z. B. [C. Lüdecke und D. Lüdecke, 2000], [Stephan u. a., 2010] oder [Eismann, 2017]) bezeichnet, in Publikationen findet man häufig $k_{H}$ (z. B. [Fernández-Prini u.a., 2003]).

[Sander, 2015] schlägt jedoch eine Systematik zur Bezeichnung vor. Diese ermöglicht es, die Vielzahl an in der Literatur verwendeten Konstanten, die den Zusammenhang zwischen Gas- und Flüssigphase beschreiben, in eindeutiger Weise zu bezeichnen: Es wird unterschieden in Henry-Flüchtigkeitskonstanten $K_{H}$ und Henry-Löslichkeitskonstanten $H$. Henry-Flüchtigkeitskonstanten $K_{H}$ setzen die Zusammensetzung der Gasphase $(G)$ zu der der Flüssigphase $(L)$ ins Verhältnis $\frac{G}{L}$. Henry-Löslichkeitskonstanten $H$ das Reziproke $\frac{L}{G}$. In einem hochgestellten Index werden dann die in Korrelation gestellten Größen der Gas- und Flüssigphase beschrieben.

Die hier gewählte Größe $K_{\mathrm{H}, i}$ müsste also vollständig $K_{H, i}^{f x}$ heißen, wird hier aus Übersichtlichkeitsgründen abgekürzt.
} 
dünnten Gases $i$ im Gemisch. Für die Isofugazität des Gases ergibt sich durch Gleichsetzen der Fugazitäten (Gleichung 2.7) entsprechend

$$
y_{i} \cdot p \cdot \varphi_{i}=x_{i} \cdot \gamma_{i}^{*} \cdot K_{\mathrm{H}, i}\left(p^{+}\right) \cdot \exp \int_{p^{+}}^{p} \frac{v_{\infty, i}^{\prime}}{R \cdot T} \mathrm{~d} p .
$$

Die letzten Faktoren der Gleichungen 2.12 und 2.16 werden auch als Poynting-Korrekturen

$$
\text { PoyLM }=\exp \int_{p L M, s}^{p} \frac{v_{0, L M}^{\prime}}{R \cdot T} \mathrm{~d} p
$$

und

$$
P_{o y}=\exp \int_{p_{0}}^{p} \frac{v_{\infty, i}^{\prime}}{R \cdot T} \mathrm{~d} p
$$

bezeichnet.

\section{Henry-Koeffizient bei Dampfdruck des Lösungsmittels}

Oft ist wie z. B. in [IAPWS, 2004] der Henry-Koeffizient für $p^{+} \rightarrow p_{\mathrm{LM}, \mathrm{s}}$, bezeichnet als $K_{\mathrm{H}, i, \infty}=K_{\mathrm{H}, i}\left(p_{\mathrm{LM}, \mathrm{s}}\right)$, angegeben. Dieser Henry-Koeffizient $K_{\mathrm{H}, i, \infty}$ ist nur noch von der Temperatur abhängig. Dann gilt die Gleichung

$$
y_{i} \cdot p \cdot \varphi_{i}=x_{i} \cdot \gamma_{i}^{*} \cdot K_{\mathrm{H}, i, \infty} \cdot \exp \int_{p\llcorner\mathrm{~L}, \mathrm{~s}}^{p} \frac{v_{\infty, i}^{\prime}}{R \cdot T} \mathrm{~d} p .
$$

\section{Fazit}

Alle Größen, die nun zur exakten Bestimmung des Phasengleichgewichts über obige Isofugazitätsgleichungen benötigt werden, sind in Abbildung 2.2 zusammengefasst.

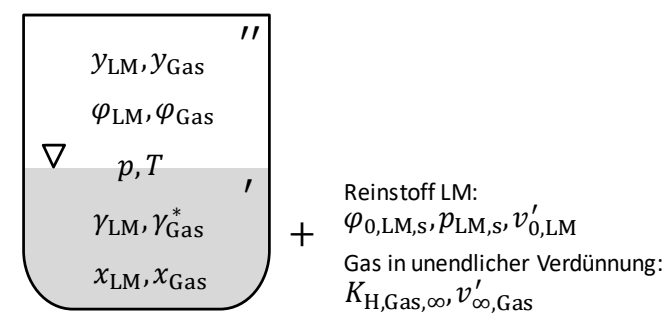

Abbildung 2.2: Notwendige Größen für das Phasengleichgewicht für ein Gas und ein Lösungsmittel (LM) 


\subsubsection{Approximationen für geringe Drücke und Temperaturen - „Von der Isofugazität zum vereinfachten Henry-Gesetz“}

Ausgehend von den oben dargestellten exakten Beziehungen, werden in der angewandten Thermodynamik (insbesondere für geringe Drücke, wie dies für energietechnische Flüssigkeitskreisläufe meist der Fall ist) oft einige wesentliche Vereinfachungen verwendet. Die bekannte Literatur zu Gasen in energietechnischen Flüssigkeitskreisläufen basiert zumeist vollständig auf deutlich idealisierten und vereinfachten Phasengleichgewichts-Beziehungen. Diese sollen für die Betrachtungen die Genauigkeit nur unwesentlich reduzieren, vereinfachen jedoch die Berechnung ungemein bzw. ermöglichen aufgrund oft fehlender vollständiger Beschreibung der Stoffgemische die Berechnung erst. Für das allgemeine Verständnis sollen diese Vereinfachungen hier noch einmal klar dokumentiert werden. Dafür sind alle verwendeten Vereinfachungen als Übersicht in Tabelle 2.1 dargestellt und werden nachfolgend unter Benutzung der Kennung in Spalte 1 erläutert.

Tabelle 2.1: Übersicht möglicher Vereinfachungen

\begin{tabular}{|c|c|c|}
\hline Bez. & Approximationen & Kurzbeschreibung \\
\hline A & $\begin{array}{l}v_{0, \mathrm{LM}}^{\prime}=v_{0, \mathrm{LM}, \mathrm{s}}^{\prime}=f(t) \\
v_{\infty, i}^{\prime}=f(t)\end{array}$ & Partielle molare Volumina druck- bzw. gasgehaltsunabhängig \\
\hline B & $\begin{array}{l}\gamma_{\mathrm{LM}}=1 \\
\gamma_{i}^{*}=1\end{array}$ & Aktivität des LM vernachlässigbar, konstante Aktivität des Gases \\
\hline $\mathrm{C}$ & PoyLM $=1$ & Poynting-Korrektur des Lösungsmittels vernachlässigbar \\
\hline $\mathrm{D}$ & $\begin{array}{l}\varphi_{\mathrm{LM}}=\varphi_{0, \mathrm{LM}, \mathrm{s}}=\text { const } \\
\varphi_{i}=\varphi_{0, i}=\mathrm{const}\end{array}$ & $\begin{array}{l}\text { Ideale Mischung - Fugazitätskoeffizient entspricht dem des } \\
\text { Reinstoffes (bei LM Siedezustand verwendet) }\end{array}$ \\
\hline$E$ & $\begin{array}{l}\varphi_{\mathrm{LM}}=\varphi_{0, \mathrm{LM}, \mathrm{s}}=1 \\
\varphi_{i}=1\end{array}$ & Idealgas - Fugazitätskoeffizient ist vernachlässigbar \\
\hline $\mathrm{F}$ & Poy $_{i}=1$ & Poynting-Korrektur des Gases vernachlässigbar \\
\hline G & $\begin{array}{l}x_{\mathrm{LM}}=1 \text { in } \\
\text { Isofugazitätsglg. }\end{array}$ & $\begin{array}{l}\text { Stoffmengenanteil des Gases } x_{i} \text { hat auf Fugazität der Flüssigphase } \\
\text { des Lösungsmittels näherungsweise keinen Einfluss }\end{array}$ \\
\hline $\mathrm{H}$ & z.B. $x_{i}=\frac{n_{i}^{\prime}}{n_{\text {ges }}^{\prime}} \approx \frac{n_{i}^{\prime}}{n_{\text {LM }}^{\prime}}$ & $\begin{array}{l}\text { Gas-Gehaltsangaben für beliebige Gehaltsgrößen im Fluid lassen } \\
\text { sich wg. } x_{i} \ll x_{\mathrm{LM}} \text { vereinfacht bestimmen. }\end{array}$ \\
\hline
\end{tabular}

\section{A - Vereinfachungen der Poynting-Korrekturen}

Da die hier betrachteten Lösungsmittel Wasser bzw. Wasser-Glykol annähernd inkompressibel sind, kann $v_{0, \mathrm{LM}}^{\prime}$ als druckunabhängig angenommen werden. Nun lässt sich das Integral der Poynting-Korrektur des Lösungsmittels auflösen zu

$$
\text { PoyLM }=\exp \left[\frac{v_{0, \mathrm{LM}, \mathrm{s}}^{\prime} \cdot\left(p-p_{\mathrm{LM}, \mathrm{s}}\right)}{R \cdot T}\right]
$$

mit dem Molvolumen des Lösungsmittels am Siedepunkt $v_{0, \mathrm{LM}, \mathrm{s}}^{\prime}=v_{0, \mathrm{LM}}^{\prime}\left(p_{\mathrm{LM}, \mathrm{s}}\right)$. Damit geht die Gleichung 2.12 über in

$$
y_{\mathrm{LM}} \cdot p \cdot \varphi_{\mathrm{LM}}=\gamma_{\mathrm{LM}} \cdot x_{\mathrm{LM}} \cdot p_{\mathrm{LM}, \mathrm{s}} \cdot \varphi_{0, \mathrm{LM}, \mathrm{s}} \cdot \exp \left[\frac{v_{0, \mathrm{LM}, \mathrm{s}}^{\prime} \cdot\left(p-p_{\mathrm{LM}, \mathrm{s}}\right)}{R \cdot T}\right] \text {. }
$$

Setzt man das partielle Molvolumen $v_{\infty, i}^{\prime}$ des Gases $i$ in der Flüssigkeit ebenso als druckunabhängig voraus, was laut [Stephan u. a., 2010] in einigem Abstand vom kritischen Gebiet 
zulässig ist und auch mit den Messwerten der Tabelle 2.5 bestätigt wird, dann gilt für die Poynting-Korrektur des Gases $i$

$$
\text { Poy }_{i}=\exp \left[\frac{v_{\infty, i}^{\prime} \cdot\left(p-p_{\mathrm{LM}, \mathrm{s}}\right)}{R \cdot T}\right]
$$

und Gleichung 2.16 geht über in

$$
y_{i} \cdot p \cdot \varphi_{i}=x_{i} \cdot \gamma_{i}^{*} \cdot K_{\mathrm{H}, i, \infty} \cdot \exp \left[\frac{v_{\infty, i}^{\prime} \cdot\left(p-p_{\mathrm{LM}, \mathrm{s}}\right)}{R \cdot T}\right] .
$$

\section{B - Aktivitätskoeffizienten}

Der Aktivitätskoeffizient des Gases $\gamma_{i}^{*}$ kann für kleine gelöste Gasmengenanteile, im sogenannten „Gültigkeitsbereich der Henry-Konstante“, vernachlässigt und die Fugazität allein mit dem Henry-Koeffizienten $K_{\mathrm{H}, i, \infty}$ abgeschätzt werden. Der rationelle Aktivitätskoeffizient ist demzufolge in diesem Bereich $\gamma_{i}^{*}=1$ (B2). In der Literatur wird diese Vereinfachung oft als „Henry-Gesetz" bezeichnet, ist jedoch nur eine notwendige Bedingung für die Gültigkeit des Henry-Gesetzes, wie es hier später eingeführt wird.

Da die Aktivitätskoeffizienten der Gemischkomponenten nach der Gibbs-Duhem-Gleichung voneinander abhängig sind, wie bspw. in [C. Lüdecke und D. Lüdecke, 2000] gezeigt, ließe sich die Aktivität des Lösungsmittels $\gamma_{\mathrm{LM}}$ bei bekannten Aktivitätskoeffizienten der Gase $\gamma_{i}^{*}$ berechnen. Aber da gilt $\gamma_{\mathrm{LM}} \rightarrow 1$ für $x_{\mathrm{LM}} \rightarrow 1$, ist der Einfluss des Aktivitätskoeffizienten des Lösungsmittels bei dem nur marginal unter dem Wert 1 liegenden Stoffmengenanteil des Lösungsmittels sehr gering und daher soll näherungsweise $\gamma_{\mathrm{LM}}=1$ gelten (B1).

\section{C/F - Vernachlässigung Poynting-Korrekturen}

Wie zum Beispiel [Stephan u.a., 2010] schreibt, kann für mäßige Drücke und nicht allzu tiefe Temperaturen die Poynting-Korrektur vernachlässigt werden. Dies liegt an den im Vergleich zum Idealgas sehr geringen (partiellen) molaren Volumina in der Flüssigphase.

Die Poynting-Korrektur des Lösungsmittels wird bei (C) vernachlässigt, d.h. $P_{\text {OyLM }}=1$. Die Poynting-Korrektur des Gases wird bei $(F)$ ebenso vernachlässigt, d. h. $\operatorname{Poy}_{i}=1$.

\section{D/E- Fugazitätskoeffizienten}

Für eine ideale Mischung realer Gase ändern sich die Fugazitätskoeffizienten nicht mit dem Stoffmengenanteil in der Gasphase (D2). Es gilt also

$$
\varphi_{i}=\varphi_{0, i}=\text { const. }
$$

und für das Lösungsmittel soll auch der Fugazitätskoeffizient des Reinstoffes gelten (D1), der hier mit dem Zustand Siedepunkt abgeschätzt wird

$$
\varphi_{\mathrm{LM}}=\varphi_{0, \mathrm{LM}, \mathrm{s}}=\text { const. }
$$


Für die Bedingungen des idealen Gases müssen die Fugazitätskoeffizienten $\varphi_{i}=1$ und $\varphi_{\mathrm{LM}}=$ 1 sein $(\mathrm{E} 1+\mathrm{E} 2)$.

\section{G - Stoffmenge des Lösungsmittels}

Ist die Stoffmenge des gelösten Gases klein im Verhältnis zur Stoffmenge des Lösungsmittels, dann ist $x_{\mathrm{LM}}$ nahezu eins und kann in Gleichung 2.12 vernachlässigt werden.

\section{H - Gasgehaltsgrößen}

Gasgehaltsgrößen im Fluid lassen sich vereinfacht bestimmen, da $n_{\mathrm{LM}} \gg n_{i}$. Dadurch gilt z. B., dass die Gehaltsgröße Stoffmengenanteil der Gehaltsgröße Stoffmengenverhältnis entspricht: $x_{i}=\frac{n_{i}^{\prime}}{n_{\text {ges }}^{\prime}} \approx \frac{n_{i}^{\prime}}{n_{\mathrm{LM}}^{\prime}}$.

Gewisse Löslichkeitsgrößen, wie z. B. der Löslichkeitkoeffizient $\lambda$, beschreiben die gelöste Gasmenge bezogen auf die zugehörige reine Lösungsmittelmenge (statt auf die Gesamtheit der Flüssigphase) und stellen damit eher ein Verhältnis der Komponenten und keinen Anteil dar. Die Approximation $\mathrm{H}$ vereinfacht hier die Umrechnung.

\section{Verbleibende Gleichungen nach Anwendung der Approximationen}

Tabelle A.1 im Anhang gibt eine Übersicht über die je nach angewendeten Vereinfachungen verbleibenden Isofugazitäts-Gleichungen. Die nach den Vereinfachungen A-F verbleibende Gleichung für das Phasengleichgewicht des Lösungsmittels ist

$$
y_{\mathrm{LM}} \cdot p=x_{\mathrm{LM}} \cdot p_{\mathrm{LM}, \mathrm{s}}
$$

und entspricht dem sogenannten Raoultschen Gesetz. Mit zusätzlicher Anwendung der Approximation $\mathrm{G}$ gilt

$$
y_{\mathrm{LM}} \cdot p=p_{\mathrm{LM}, \mathrm{s}} .
$$

Die verbleibende Gleichung für das Phasengleichgewicht des Gases i ist

$$
y_{i} \cdot p=x_{i} \cdot K_{H, i, \infty}
$$

und beschreibt das sogenannte Henry-Gesetz.

Diese Gleichungen sind im Gegensatz zu den allgemeinen Isofugazitätsgleichungen von linearer Beschaffenheit und unabhängig voneinander. Mit den Gleichungen $\sum_{k} x_{k}=1$ und $\sum_{k} y_{k}=1$ lässt sich nun z. B. für gegebenen Druck das Phasengleichgewicht, also $x_{\mathrm{LM}}, y_{\mathrm{LM}}, x_{i}, y_{i}$ vollständig beschreiben. ${ }^{2}$

\footnotetext{
${ }^{2}$ Entsprechend der Anzahl der Gase $n_{\text {Gase }}$ im Gemisch ergeben sich: $n_{\text {Gase }}+3$ Gleichungen für $2 \cdot n_{\text {Gase }}+2$ Unbekannte $x_{\mathrm{LM}}, y_{\mathrm{LM}}, x_{i}, y_{i}$. Das heißt, für mehrere Gase im Gemisch $\left(n_{\text {Gase }}>1\right)$ muss $z$. B. noch jeweils ein Mengenverhältnis der Gaskomponenten (üblicherweise in der Gasphase) gegeben sein.
} 
Für ein Binäres Gemisch aus Gas und Lösungsmittel lässt sich die gelöste Gasmenge jetzt einfach bestimmen zu

$$
x_{i}=\frac{1}{K_{\mathrm{H}, i, \infty}} \cdot\left(p-p_{\mathrm{LM}, \mathrm{s}}\right) \text {. }
$$

\subsubsection{Henry-Gesetz}

Die Gleichung 2.28 enthält (nach Anwendung der Näherungen für eine ideale Mischung, ideales Gas und Vernachlässigung der Poynting-Korrektur) als einzig verbleibende Stoffgröße die Henry-Konstante $K_{H, i, \infty}(t)$ und beschreibt für das Gas $i$ den linearen Zusammenhang zwischen den Stoffmengenanteilen in Gasphase und Flüssigphase: das HenryGesetz.

Mit Einführung des Partialdrucks $p_{i}=y_{i} \cdot p$ lässt sich das Henry-Gesetz auch in der ursprünglichen Form basierend auf [Henry, 1803] schreiben

$$
p_{i}=x_{i} \cdot K_{\mathrm{H}, i, \infty}
$$

bzw. umgestellt nach dem gelösten Gasmengenanteil

$$
x_{i}=\frac{1}{K_{\mathrm{H}, i, \infty}(t)} \cdot p_{i} .
$$

Es besagt also, dass der in der Flüssigphase gelöste Stoffmengenanteil des Gases $x_{i}$ proportional zum Partialdruck des Gases in der korrelierenden Gasphase ist. William Henry beschreibt in [Henry, 1803] dieses Phänomen leicht abgewandelt so:

„... water takes up, of gas condensed by one, two, or more additional atmospheres, a quantity which, ordinarily compressed, would be equal to twice, thrice, \&c. the volume absorbed under the common pressure of the atmosphere." 3

Hier sei nochmals daraufhin gewiesen, dass in einigen Quellen bereits für Bereiche der Verdünnung, in denen $\gamma_{i}^{*}=1$ gilt, von der "Gültigkeit des Henry-Gesetz" gesprochen wird. Dies ist für die hier vorgestellte Definition jedoch lediglich eine notwendige und keine hinreichende Bedingung. Der Fall $\gamma_{i}^{*}=1$ wird in dieser Arbeit als "Gültigkeitsbereich der Henry-Konstante" bezeichnet.

Inwiefern diese idealisierten Zusammenhänge Abweichungen vom realen Verhalten des Gemisches zeigen, soll exemplarisch für Stickstoff in Wasser im Kapitel 2.3 gezeigt werden.

\subsubsection{Technischer Löslichkeitskoeffizient}

Oft wird in der Praxis und in Normen (z. B. [VDI 2035-2]) statt des Henry-Koeffizienten die technisch anschaulichere Definition des Löslichkeitskoeffizienten $\lambda_{i, \mathrm{LM}}(t)$ verwendet. Dieser ist u. a. in [C. Lüdecke und D. Lüdecke, 2000] definiert als das Normvolumen des im Pha-

\footnotetext{
${ }^{3}$ Nach William Henry sei also die im reinen Lösungsmittel gelöste Stoffmenge des Gases proportional zum Partialdruck des Gases, also $\frac{n_{i}^{\prime}}{n_{\mathrm{LM}}^{\prime}} \sim p_{i}$. Bei unendlicher Verdünnung des Gases gilt $x_{i}=\frac{n_{i}^{\prime}}{n_{\text {ges }}^{\prime}}=\frac{n_{i}^{\prime}}{n_{\mathrm{LM}}^{\prime}}$ und die Aussage ist somit gleichbedeutend mit obiger, allgemeinerer Gleichung.
} 
sengleichgewicht gelösten Gases ${ }^{4} V_{i, N}^{\prime}$ bezogen auf die Masse $m_{\mathrm{LM}}^{\prime}$ des Lösungsmittels und den Gaspartialdruck $p_{i}$ als

$$
\lambda_{i}:=\frac{V_{i, \mathrm{~N}}^{\prime}}{m_{\mathrm{LM}}^{\prime} \cdot p_{i}} .
$$

[D'Ans und Lax, 1997] beschreibt $\lambda_{i}$ genauer als ,... diejenige Gasmenge in $\mathrm{Ncm}^{3}$, die von $1 \mathrm{~g}$ Lösemittel aufgenommen wird, wenn der Teildruck des Gases 980,67 hPa (1 at) beträgt“. Demzufolge wird dort als Einheit $\frac{\mathrm{Ncm}^{3}}{\mathrm{~g} \cdot \mathrm{at}}$ mit der heute veralteten technischen Atmosphäre (at) sowie der heute nicht mehr zulässigen Schreibweise $\mathrm{Ncm}^{3}$, vergleiche [DIN EN ISO 13443], verwendet. Heutzutage hat sich bewährt, als Einheit $\frac{\mathrm{cm}^{3}}{\mathrm{~g} \cdot \mathrm{bar}} \mathrm{zu}$ verwenden. Diese wird im Folgenden benutzt. Entsprechend Physikalisch technischer Bundesanstalt bzw. [DIN EN ISO 13443] sind Schreibweisen wie $\mathrm{Ncm}^{3}$ oder $\mathrm{cm}^{3} \mathrm{~N}$ und andere Indizierungen von Einheiten eigentlich nicht mehr zulässig. In dieser Arbeit wird jedoch von dieser Regel an Stellen, wo es einer klareren Darstellung im Sinne von Praxisnähe und Lesbarkeit dient, bewusst abgewichen.

In verdünnten Lösungen im fokussierten Druckbereich gilt näherungsweise $m_{\mathrm{LM}}^{\prime} \approx$ $m_{\text {ges }}^{\prime}$. Dann lässt sich der Löslichkeitskoeffizient mit dem Gasgehalt, definiert als

$$
w_{i}=w_{i}^{\prime}:=\frac{V_{\mathrm{i}, \mathrm{N}}^{\prime}}{m_{\mathrm{ges}}^{\prime}},
$$

im Phasengleichgewicht auch einfacher ausdrücken als

$$
\lambda_{i}=\frac{w_{i}}{p_{i}}
$$

Nun soll der Bezug dieser neuen Größe $\lambda_{i}$ zum Henry-Gesetz erläutert werden: Wegen $w_{i}=\frac{V_{i, \mathrm{~N}}^{\prime}}{m_{L M}^{\prime}}=\frac{n_{i}^{\prime}}{n_{L M}^{\prime}} \cdot \frac{v_{0, i, \mathrm{~N}}}{M_{L M}}$ und mit der Approximation $\mathrm{H}$ in stark verdünnten Lösungen $x_{i}=\frac{n_{i}^{\prime}}{n_{\text {ges }}^{\prime}} \approx \frac{n_{i}^{\prime}}{n_{\mathrm{LM}}^{\prime}}$ lässt sich die Definition nach Gleichung 2.32 auch umschreiben zu

$$
\lambda_{i}=\frac{x_{i}}{p_{i}} \cdot \frac{v_{0, i, \mathrm{~N}}}{M_{\mathrm{LM}}}
$$

Bei Gültigkeit des Henry-Gesetzes $p_{i}=x_{i} \cdot K_{H, i, \infty}$ nach Gleichung 2.30 lassen sich der Löslichkeitskoeffizient $\lambda_{i}$ und der Henry-Koeffizient $K_{H, i, \infty}$ ineinander umrechnen:

$$
\lambda_{i}=\frac{M_{i}}{M_{\mathrm{LM}} \cdot \rho_{i, \mathrm{~N}}} \cdot \frac{1}{K_{\mathrm{H}, i, \infty}}=\frac{v_{0, i, \mathrm{~N}}}{M_{\mathrm{LM}}} \cdot \frac{1}{K_{\mathrm{H}, i, \infty}}
$$

bzw.

$$
K_{\mathrm{H}, i, \infty}=\frac{M_{i}}{M_{\mathrm{LM}} \cdot \rho_{i, \mathrm{~N}}} \cdot \frac{1}{\lambda_{i}}=\frac{v_{0, i, \mathrm{~N}}}{M_{\mathrm{LM}}} \cdot \frac{1}{\lambda_{i}}
$$

\footnotetext{
${ }^{4}$ Gemeint ist das Volumen, das die gelöste Gasmenge alternativ als Einzelstoff im physikalischen Normzustand bei 1,01325 bar und $0{ }^{\circ} \mathrm{C}$ (vgl. [DIN 1343]) einnehmen würde. Obwohl $V_{i, \mathrm{~N}}^{\prime}$ ein gasförmiges Volumen beschreibt, ist die gelöste Gasmenge gemeint und wird daher mit dem Index ' gekennzeichnet.
} 
Demzufolge ist der Löslichkeitskoeffizient dann also ebenso eine Konstante wie der HenryKoeffizient. Das Phasengleichgewicht lässt sich also auch über den Löslichkeitskoeffizienten ausdrücken zu

$$
p_{i}=\frac{v_{0, i, \mathrm{~N}}}{M_{\mathrm{LM}}} \cdot \frac{1}{\lambda_{i}} \cdot x_{i}
$$

bzw. mit dem Gasgehalt $w_{i}$ zu

$$
p_{i}=\frac{1}{\lambda_{i}} \cdot w_{i}
$$

Nach der Systematik von [Sander, 2015] müsste der technische Löslichkeitskoeffizient $\lambda$ als Henry-Löslichkeitskonstante $H^{w p}$ bezeichnet werden,

Für das Lösungsmittel Wasser sind für verschiedene Gase die Henry-Koeffizienten oder auch technische Löslichkeitskoeffizienten tabelliert (z. B. [IAPWS, 2004; D'Ans und Lax, 1997]). Analog zum „Gültigkeitsbereich der Henry-Konstante“ wird im Folgenden auch vom "Gültigkeitsbereich des Löslichkeitskoeffizienten" gesprochen.

\subsubsection{Technischer Löslichkeitskoeffizient bei Berücksichtigung der Poynting-Korrektur}

Für die Arbeit wird sich später zeigen, dass das - mit Ausnahme auf die Poynting-Korrektur (Approximation F) - vereinfachte Phasengleichgewicht

$$
y_{i} \cdot p=x_{i} \cdot K_{\mathrm{H}, i, \infty} \cdot \operatorname{Poy}_{i}\left(p_{\mathrm{LM}, \mathrm{s}} ; p\right)
$$

ebenso wichtig ist. Dieser Zusammenhang soll im Folgenden auch als Henry-Gesetz mit Berücksichtigung der Poynting-Korrektur bezeichnet werden.

Jetzt kommt zum Tragen, dass der Löslichkeitskoeffizient, anders als oft angegeben, eben nicht nur von der Temperatur, sondern exakterweise auch vom Druck $p$ abhängig ist. Daher ist die Angabe eines Drucks bzw. Partialdrucks exakterweise zwingend notwendig (wie in [D'Ans und Lax, 1997] die Partialdruckangabe von 1 at). Bestimmt man den HenryKoeffizient $K_{H, i, \infty}$ aus dem Löslichkeitskoeffizienten $\lambda_{i}$, der beim Druck $p_{\lambda}$ bestimmt wurde, gilt

$$
K_{\mathrm{H}, i, \infty}=\frac{v_{0, i, \mathrm{~N}}}{M_{\mathrm{LM}}} \cdot \frac{1}{\lambda_{i}} \cdot \frac{1}{\operatorname{Poy}_{i}\left(p_{\mathrm{LM}, s} ; p_{\lambda}\right)} .
$$

Definiert man nun analog zum Henry-Koeffizienten $K_{\mathrm{H}, i ; \infty}$ einen Löslichkeitskoeffizienten bei unendlicher Verdünnung

$$
\lambda_{i, \infty}=\lambda_{i} \cdot \operatorname{Poy}_{i}\left(p_{\mathrm{LM}, \mathrm{s}} ; p_{\lambda}\right),
$$

dann lässt sich das Phasengleichgewicht für jeden beliebigen Druck $p$ auch schreiben als

$$
y_{i} \cdot p=x_{i} \cdot \frac{v_{0, i, \mathrm{~N}}}{M_{\mathrm{LM}}} \cdot \frac{1}{\lambda_{i, \infty}} \cdot \operatorname{Poy}_{i}\left(p_{\mathrm{LM}, s} ; p\right)
$$

oder auch

$$
y_{i} \cdot p=w_{i} \cdot \frac{1}{\lambda_{i, \infty}} \cdot \operatorname{Poy}_{i}\left(p_{\mathrm{LM}, s} ; p\right) .
$$


Es können also die gleichen Phasengleichgewichts-Gleichungen wie oben (ohne Berücksichtigung der Poyntingkorrektur) benutzt werden, wenn für den Löslichkeitskoeffizienten $\lambda_{i}=\lambda_{i}(p)=\lambda_{i, \infty} \cdot \frac{1}{\operatorname{Poy}_{i}\left(p_{\mathrm{LM}, s} ; p\right)}$ genutzt wird. Poynting-Korrekturen für Stickstoff und Sauerstoff sind bei einem Partialdruck $p_{i}$ von 1 at bzw. 1 bar (und einem Gesamtdruck $p=p_{\mathrm{LM}}+p_{i}$, also ohne weitere Komponenten) sehr klein, also gilt näherungsweise $\lambda_{i, \infty} \approx \lambda_{i}\left(p_{i}=1\right.$ at $) \approx \lambda_{i}\left(p_{i}=1\right.$ bar $)$.

\subsubsection{Gebräuchliche Größen in der Praxis}

Neben dem Stoffmengenanteil $x_{i}$ und dem Gasgehalt $w_{i}$ sind auch die Konzentration $c_{i}$, definiert als Masse des gelösten Gases bezogen auf das Volumen des Lösungsmittels, und der Masseanteil $\xi_{i}$ gebräuchliche Gehaltsgrößen im Umgang mit gelösten Gasen in der Praxis. Übliche Umrechnungen für die vorgestellten Vereinfachungen sind in Tabelle 2.2 zusammengefasst. Weitere notwendige Zusammenhänge sind in Tabelle 2.3 dargestellt.

Wie im Folgenden noch dargestellt wird, stellt die Anwendung der Vereinfachungen aber Berücksichtigung der Poynting-Korrektur des Gases $P o y_{i}\left(p_{\mathrm{LM}, s} ; p\right)$ (d.h. A-E und G-H, also ohne F) für die Arbeit eine ebenso wichtige Approximationskonfiguration dar. Tabelle 2.2 kann ebenso bei Berücksichtigung der Poynting-Korrektur des Gases genutzt werden, wenn $K_{\mathrm{H}, i} \hat{=} K_{\mathrm{H}, i}(p)=K_{\mathrm{H}, i, \infty} \cdot \operatorname{Poy}_{i}\left(p_{\mathrm{LM}, s} ; p\right)$ bzW. $\lambda_{i} \widehat{=} \lambda_{i}(p)=\lambda_{i, \infty} \cdot \frac{1}{\operatorname{Poy}_{i}\left(P_{\mathrm{LM}, s} ; p\right)}$ verwendet wird.

Eine weitere, übliche und anschauliche Kenngröße zur Beschreibung der Löslichkeit ist der Ostwald-Koeffizient. Dieser ist laut [Young u.a., 1987] definiert als das Verhältnis von absorbierter Gasmenge $V_{g}$ zum Volumen des reinen Lösungsmittels $V_{L M}$, also

$$
L_{i}=\frac{V_{\mathrm{g}}}{V_{\mathrm{LM}}}
$$

bei Druck $p$ und Temperatur $t$. Unpräzise ist, dass hier die Gasmenge $V_{\mathrm{g}}$ nicht weiter erläutert wird. Erst über die bei [Young u. a., 1987] angegebene Umrechnungsformel in den Stoffmengenanteil mit $x_{i}=\left(\frac{R \cdot T}{p_{i} \cdot L_{i} \cdot V_{0, L M}}+1\right)^{-1}$ wird indirekt deutlich, dass hier das Volumen gemeint ist, dass das reine Gas bei einem Gesamtdruck einnehmen würde, der dem Partialdruck $p_{i}$ des Gases im Gemisch entspricht. Nach [Hefter und Tomkins, 2003] oder auch [Gamsjäger u. a., 2010] existieren neben dieser Definition aber auch eine Vielzahl anderer Definitionen für den Ostwald-Koeffizienten. Daher wird von der Verwendung des Ostwald-Koeffizienten eher abgeraten.

Der technische Löslichkeitskoeffizient $\lambda_{i}$ bzw. der Gasgehalt $w_{i}$ haben für den Anwender zwei Eigenschaften, die sich als praktischer Vorteil gegenüber anderen Größen erweisen:

- Gegenüber dem Henry-Koeffizienten $K_{\mathrm{H}, i}$ bzw. dem Molanteil $x_{i}$ : Die Löslichkeit kann auch bei unbekannter molarer Zusammensetzung des Lösungsmittels $M_{\mathrm{LM}}$ beschrieben werden. Dies ist auch für Stoffgemische sehr hilfreich. Diesen Vorteil hat auch der Ostwald-Koeffizient.

- Gegenüber dem Ostwald-Koeffizienten $L_{i}$ bzw. der Konzentration $c_{i}$ : Bei gleicher gelöster Gasmenge pro Lösungsmittelmenge bei anderer Temperatur ändert sich der Löslichkeitskoeffizient $\lambda_{i}$ bzw. der Gasgehalt $w_{i}$ nicht, während sich der Ostwald-Koeffizient $L_{i}$ bzw. die Konzentration $c_{i}$ (durch die temperaturabhängige Dichte) ändert. 
Deshalb soll in dieser Arbeit der technische Löslichkeitskoeffizient $\lambda_{i}$ und der Gasgehalt $w_{i}$ genutzt werden, um die Gaslöslichkeit zu beschreiben. 


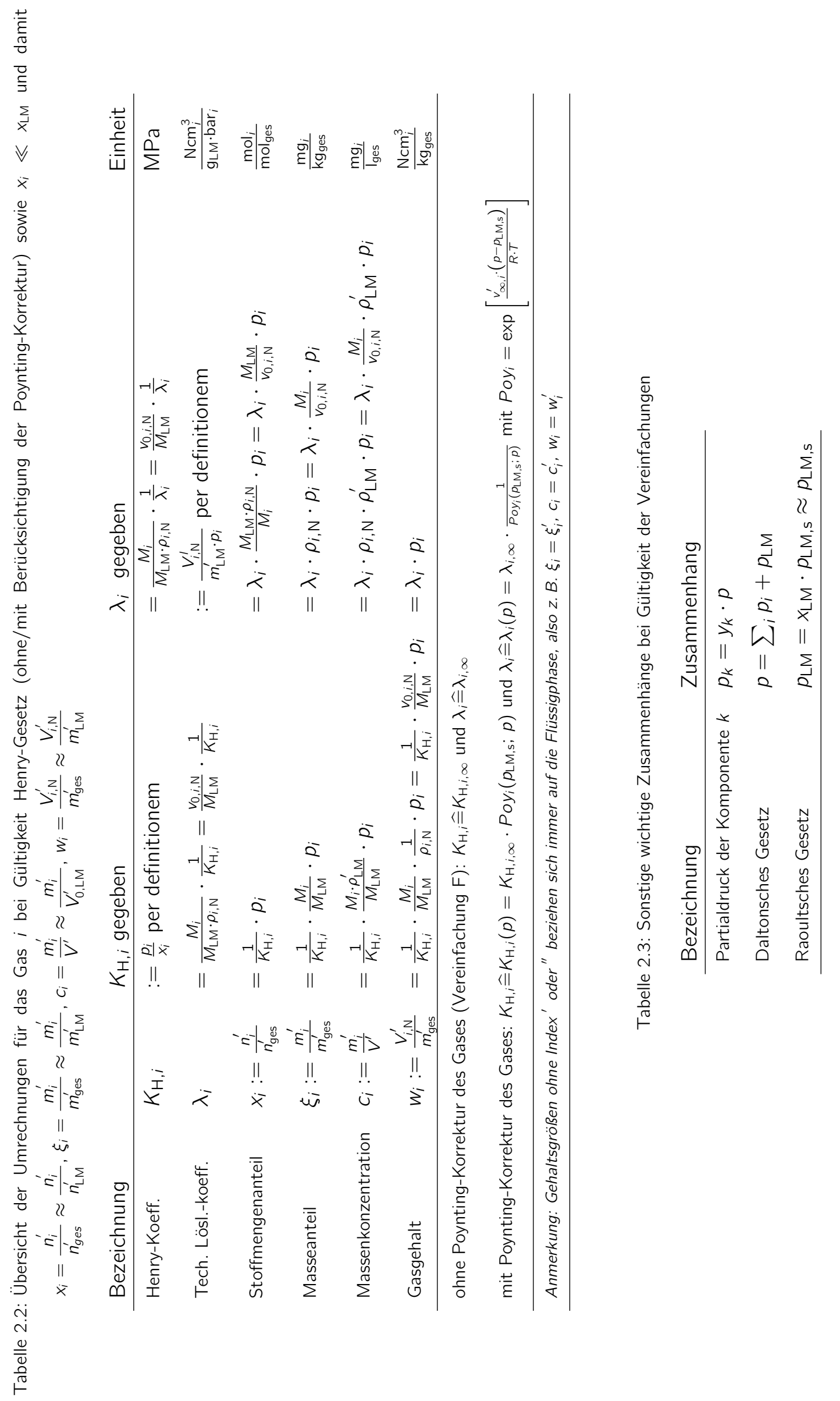




\subsection{Löslichkeit von Stickstoff und Sauerstoff in Wasser}

\subsubsection{Technischer Löslichkeitskoeffizient}

In Abbildung 2.3 sind Löslichkeitskoeffizienten für Stickstoff und Sauerstoff in Wasser dargestellt. Sauerstoff zeigt eine etwa doppelt so hohe Löslichkeit wie Stickstoff. Mit zunehmender Temperatur nimmt für beide Gase die Löslichkeit deutlich ab. Minima erreichen die Löslichkeitskoeffizienten nach dieser Regression bei $82^{\circ} \mathrm{C}$ für Stickstoff und $94,6{ }^{\circ} \mathrm{C}$ für Sauerstoff.

In [IAPWS, 2004] wird eine Vielzahl an Messpunkten verschiedener Literaturquellen genutzt um eine möglichst genaue Regression über einen weiten Temperaturbereich zu erstellen. Für die Berechnung der Löslichkeit werden in der Praxis hingegen oft die tabellierten Löslichkeitskoeffizienten aus [D'Ans und Lax, 1997] verwendet. So verweist auch [VDI 2035-2] auf diese Quelle. In Abbildung 2.3 sind diese mit den Regressionen nach [IAPWS, 2004] verglichen und es zeigt sich eine sehr gute Übereinstimmung der Werte.

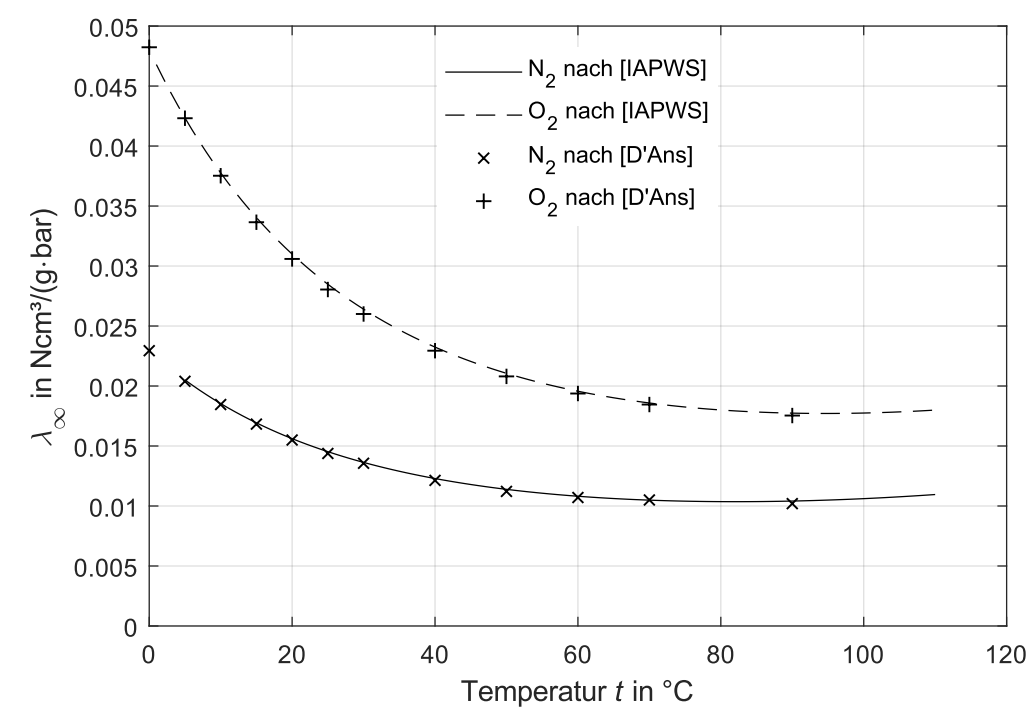

Abbildung 2.3: Vergleich Löslichkeitskoeffizienten $\lambda_{i, \infty}$ von Stickstoff und Sauerstoff nach [IAPWS, 2004] (umgerechnet von Henry-Koeffizienten $K_{H, i, \infty}$ zu $\lambda_{i, \infty}$ ) und nach [D'Ans und Lax, 1997]; Gültigkeitsbereich nach [IAPWS, 2004]: ab $5{ }^{\circ} \mathrm{C}$ für $\mathrm{N}_{2}$, ab $1{ }^{\circ} \mathrm{C}$ für $\mathrm{O}_{2}$

\subsubsection{Gegenseitige Beeinflussung der Gase}

In der Praxis wird eine gegenseitige Beeinflussung von Stickstoff- und Sauerstofflöslichkeit nicht berücksichtigt, da diese als gering eingeschätzt wird. Als Literaturquelle zu dieser Thematik konnte [Wilcock und Battino, 1974] gefunden werden. Dort wurden bei Umgebungsdruck und $25^{\circ} \mathrm{C}$ für Stickstoff sowie Sauerstoff in Wasser und anschließend mit der gleichen Apparatur auch für ein Gemisch aus Sauerstoff (49,5 Mol-\%) und Stickstoff (50,5 Mol-\%) der Anteil der gelösten Gase bestimmt. Die gelöste Menge des Gasgemischs, berechnet aus der Löslichkeit der einzelnen Gase, ist hier nur 3\% geringer als die gemessene Menge bei einer Messunsicherheit der Einzelmessungen von 1\%. Andere Autoren messen deutlich höhere Abweichungen, jedoch vermutet [Wilcock und Battino, 1974], dass dies lediglich an zu hohen Messunsicherheiten der Verfahren liegt. Für sichere Aussagen zur Querbeeinflussung müssten mehr Untersuchungen vorliegen. 


\subsection{Berechnung der Löslichkeit und Diskussion von Vereinfachungen am Beispiel Stickstoff in Wasser}

\subsubsection{Motivation}

Das vereinfachte Henry-Gesetzes lässt sich deutlich einfacher zur Löslichkeitsbestimmung anwenden als die umfangreiche Berechnung der Löslichkeit mittels exakter, nicht vereinfachter Isofugazitätsbetrachtung. Daher stellt sich die Frage, wie stark die Ergebnisse durch Anwendung des vereinfachten Henry-Gesetzes von denen des exakten Verfahrens abweichen.

In [D'Ans und Lax, 1997] findet sich eine Angabe zum Gültigkeitsbereich des Henry'schen Gesetzes $\left(\mathrm{O}_{2}\right.$ in Wasser: $0 \ldots 20$ bar, $\mathrm{N}_{2}$ in Wasser: $0 \ldots 30$ bar $)$, ohne jedoch klar zu definieren, was mit dem Gültigkeitsbereich gemeint ist bzw. was die Gültigkeitskriterien sind. Zu erwarten ist, dass die mit dem Henry-Gesetz vereinfacht bestimmten Löslichkeitskoeffizienten nur mit einer gewissen Abweichung zur realen Löslichkeit bestimmt werden können. Diese Abweichung sollte prinzipiell mit zunehmendem Druck und damit zunehmendem Gasgehalt $x_{i}$ größer werden, da man sich dann vom Zustand unendlicher Verdünnung entfernt.

Eine systematische Darstellung der Auswirkung der einzelnen Näherungen konnte selbst für Wasser in der Literatur nicht gefunden werden, obwohl diese Näherungen in der technischen Praxis nahezu immer verwendet werden. Welche der Einflüsse auf oben beschriebenes, das Phasengleichgewicht exakt beschreibende Gleichungssystem (mit den exakt gültigen, das Isofugazitätskriterium erfüllenden Gleichungen 2.12 für das Lösungsmittel bzw. 2.16 für das Gas) hier wesentlich sind und wie groß der Genauigkeitsverlust der mit dem Henry-Gesetz vereinfacht bestimmten Löslichkeiten wirklich ist, soll daher exemplarisch anhand des gut bekannten binären Gemischs Stickstoff-Wasser analysiert werden. Die verwendeten Vereinfachungen sollen im Folgenden nacheinander diskutiert werden.

Ausgangspunkt der Diskussionen soll eine möglichst detaillierte Berechnung sein, hier Referenz genannt. Diese nutzt die im Folgenden beschriebenen Stoffdaten. Zu beachten ist dabei, dass auch die Referenz bereits die Vereinfachungen A und B aus Tabelle 2.1 enthalten soll, da diese Vereinfachungen im betrachteten Druck- und Temperaturbereich marginale Auswirkung haben und nur wenige Stoffdaten für den Bereich vorliegen. Ergebnisse weiterer akkumulierter Vereinfachungen, wie in Tabelle 2.4 dargestellt, sollen mit den Ergebnissen der Referenz verglichen werden. Vereinfachungen $\mathrm{G}$ und $\mathrm{H}$ werden separat diskutiert.

Tabelle 2.4: Approximationen zur Ermittlung des $\mathrm{N}_{2}$-Wasser-Phasengleichgewichts

\begin{tabular}{|c|c|c|c|c|c|c|c|c|}
\hline Name & A & $\mathrm{B}$ & $\mathrm{C}$ & $\mathrm{D}$ & $\mathrm{E}$ & $\mathrm{F}$ & G & $\mathrm{H}$ \\
\hline „Referenz" & $\sqrt{ }$ & $\sqrt{ }$ & - & - & - & - & - & - \\
\hline "C" & $\sqrt{ }$ & $\sqrt{ }$ & $\sqrt{ }$ & - & - & - & - & - \\
\hline "C-D" & $\sqrt{ }$ & $\sqrt{ }$ & $\sqrt{ }$ & $\sqrt{ }$ & - & - & - & - \\
\hline „C-E“" (Henry-Gesetz mit Berücks. Poynting-Korrektur) & $\sqrt{ }$ & $\sqrt{ }$ & $\sqrt{ }$ & $\sqrt{ }$ & $\sqrt{ }$ & - & - & - \\
\hline "C-F" (Henry-Gesetz) & $\sqrt{ }$ & $\sqrt{ }$ & $\sqrt{ }$ & $\sqrt{ }$ & $\sqrt{ }$ & $\sqrt{ }$ & - & - \\
\hline
\end{tabular}

Die nachfolgenden Betrachtungen sollen zudem für die nicht bzw. wenig-vereinfachte Betrachtung (Referenz) deutlich machen:

- dass eine Vielzahl an zusätzlichen Stoffdaten notwendig ist, 
- wie verstreut die Literatur für diese Stoffdaten vorliegt,

- dass relativ wenig fundierte Literatur für einzelne Aspekte der konkreten GemischBeschreibung vorliegt und

- wie komplex die Anwendung der exakten Beschreibung für den Praktiker wäre.

\subsubsection{Gasphase - Fugazitätskoeffizenten der Komponenten}

Die realen Fugazitätskoeffizienten $\varphi_{k}$ der Komponenten $k$ sollen durch die zweiten Virialkoeffizienten für Stickstoff und Wasser, hier gegeben als Temperaturfunktionen, abgeschätzt werden. Nach [C. Lüdecke und D. Lüdecke, 2000] gilt allgemein für ein binäres System mit zwei Komponenten 1 und 2

$$
\begin{aligned}
& \varphi_{1}=\left[2 \cdot\left(y_{1} \cdot B_{11}+y_{2} \cdot B_{12}\right)-B\right] \cdot \frac{p}{R \cdot T} \\
& \varphi_{2}=\left[2 \cdot\left(y_{2} \cdot B_{22}+y_{1} \cdot B_{12}\right)-B\right] \cdot \frac{p}{R \cdot T}
\end{aligned}
$$

mit

$$
B=y_{1}^{2} \cdot B_{11}+2 \cdot y_{1} \cdot y_{2} \cdot B_{12}+y_{2}^{2} \cdot B_{22} .
$$

Als Virialkoeffizienten der Reinstoffe $B_{\mathrm{N} 2}(T)$ und $B_{\mathrm{H} 2 \mathrm{O}}(T)$ werden die Werte aus [Bell u. a., 2014 ] benutzt. Für den Kreuzvirialkoeffizienten $B_{\mathrm{N} 2, \mathrm{H} 2 \mathrm{O}}(T)$ wird die Beziehung nach [Tulegenov u. a., 2008] benutzt.

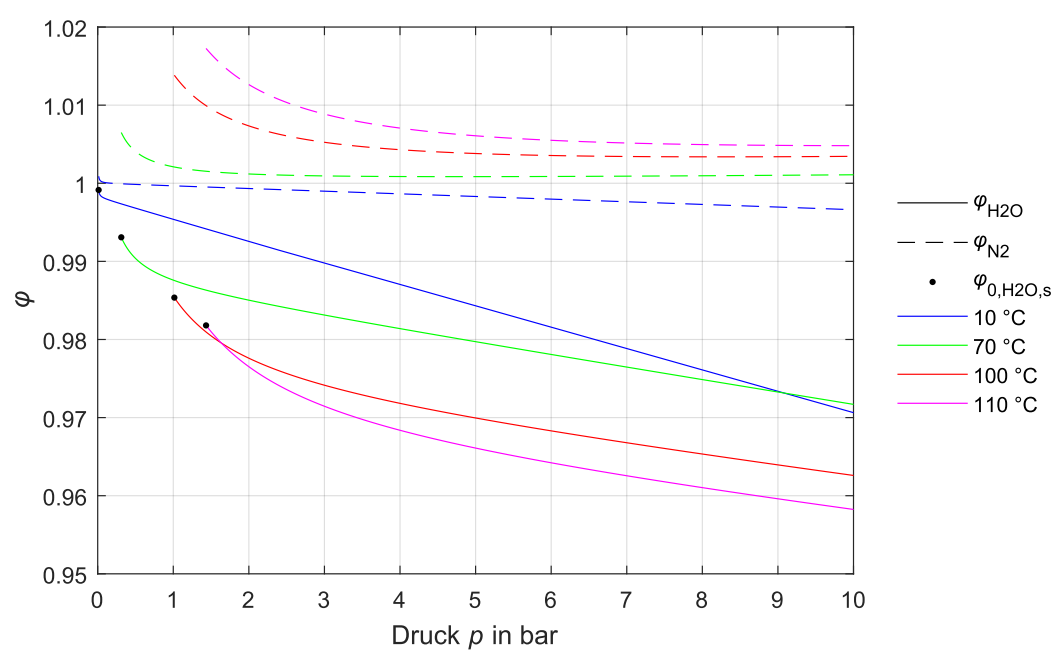

Abbildung 2.4: Fugazitätskoeffizienten der Gasphase für $\mathrm{N}_{2}$-Wasser-Gemisch in Abhängigkeit des Gesamtdrucks (durchgehende Linien für Wasser; gestrichelte Linien für Stickstoff)

Die sich ergebenden Fugazitätskoeffizienten sind in Abhängigkeit des Gesamtdruckes der Gasphase in Abbildung 2.4 dargestellt. ${ }^{5}$ Die abgebildeten Isothermen des Fugazitätskoeffizi-

\footnotetext{
${ }^{5}$ Für die folgenden Darstellungen der Fugazität $\varphi_{k}$ soll es hier ausreichend sein, in der vorgelagerten Berechnung die Konzentration $y_{k}$ direkt aus dem Gesamtdruck abzuschätzen. Unter der Annahme geringer gelöster Gasmengen $\left(x_{\mathrm{N} 2} \ll x_{\mathrm{H}_{2} \mathrm{O}}\right)$ und idealem Gasverhalten gilt, dass sich die Konzentration $y_{\mathrm{H} 2 \mathrm{O}}$ direkt aus dem Gesamtdruck zu $y_{\mathrm{H} 2 \mathrm{O}}=\frac{p_{\mathrm{S}, \mathrm{H} 2 \mathrm{O}}}{p}$ berechnen lässt.
} 
enten beginnen jeweils ab dem entsprechenden Dampfdruck des Wassers, da erst ab diesem Druck das Zweiphasengebiet beginnt (Kondensationslinie). Fugazitätskoeffizienten für Stickstoff (gestrichelte Linien) sind für kleine Drücke (d. h. für $y_{N_{2}} \rightarrow 0$ ) deutlich größer als für höhere Druckbereiche.

In Abbildung 2.5 sind Stickstoff-Fugazitäten $\varphi_{\mathrm{N} 2}$ im Gemisch (gestrichelte Linien) den Fugazitäten des Reinstoffes $\varphi_{0, \mathrm{~N} 2}$ (durchgezogene Linien) in Abhängigkeit des Druckes gegenübergestellt. Erwartungsgemäß nähert sich der Fugazitätskoeffizient von Stickstoff im Gemisch für hohe Drücke, und damit für $y_{\mathrm{N} 2} \rightarrow 1$, dem Fugazitätskoeffizienten des Reinstoffes $\varphi_{0, \mathrm{~N} 2}$ an. Bei geringen Drücken wird mit der Näherung ,ideales Gemisch“ ( $\varphi_{i}=\varphi_{0, i}=$ const) die Fugazität für Stickstoff jedoch deutlich ungenauer abgeschätzt. Für $10{ }^{\circ} \mathrm{C}$ sind die beiden Kurven $\varphi_{\mathrm{N} 2}$ und $\varphi_{0, \mathrm{~N} 2}$ noch wenig voneinander entfernt und nähern sich schnell an. Mit steigender Temperatur steigen jedoch die Unterschiede und damit die Fehler der Näherung.

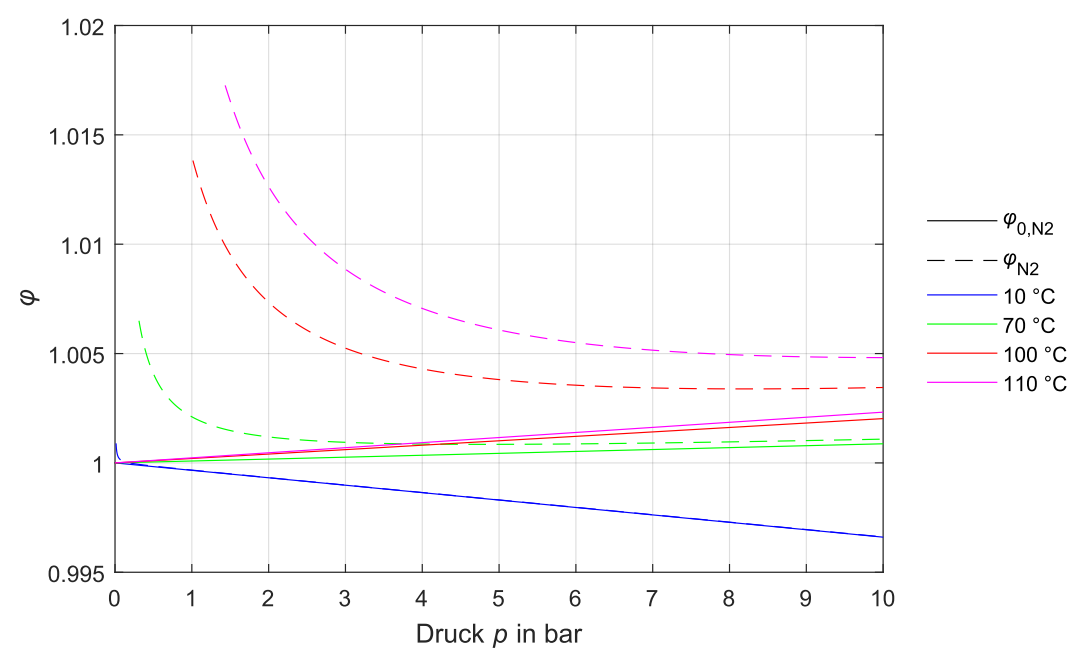

Abbildung 2.5: Fugazitätskoeffizienten von Stickstoff für gesättigtes $N_{2}$-Wasser-Gemisch und für reinen Stickstoff in Abhängigkeit des Drucks (durchgehende Linien für reinen Stickstoff; gestrichelte Linien für Stickstoff im Gemisch)

Für die Annahme des idealen Gases $\left(\varphi_{i}=\right.$ const $\left.=1\right)$ sind für $10^{\circ} \mathrm{C}$ höchste Abweichungen bei hohen Drücken zu sehen. Im Gegensatz dazu zeigen die Kurven für höhere Temperaturen die größten Abweichungen bei niedrigen Drücken.

Fugazitätskoeffizienten gehen zwar allgemein für $p \rightarrow 0$ gegen $\varphi_{i}=1$. Für unendliche Verdünnung können Fugazitätskoeffizienten jedoch deutlicher von 1 abweichen als für höhere Drücke und gehen nicht, wie fälschlicherweise z. B. in [Sander, 1999] angenommen, für unendliche Verdünnung also $p_{i} \rightarrow 0$ gegen $\varphi_{i}=1$.

In Abbildung 2.4 ist auch der Fugazitätskoeffizient von Wasser dargestellt. Dieser weicht mit steigenden Drücken sowohl stärker von $\varphi_{\mathrm{N} 2}=1$ (ideale Gase) als auch stärker vom Fugazitätskoeffizienten des Reinstoffs $\varphi_{0, \mathrm{H} 2 \mathrm{O}, \mathrm{s}}$ (ideales Gemisch) ab. Im Gegensatz zu Stickstoff steigen die Fehler beider Näherungen mit steigendem Druck.

Fugazitäten des Stoffgemisches lassen sich prinzipiell auch direkt über die Fundamentalgleichungen nach [Gernert, 2013] mit [Bell u. a., 2014] bestimmen. Jedoch weist [Gernert, 2013] darauf hin, dass die zweiten Kreuzvirialkoeffizienten durch das Modell ungenügend repräsentiert werden. Er geht zwar nicht direkt auf die Fugazitätskoeffizienten ein, es besteht damit jedoch die Vermutung, dass auch diese hier signifikante Ungenauigkeiten auf- 
weisen. Die Ergebnisse der Fugazitätskoeffizienten zeigen hier deutlich abweichendes Verhalten.

\subsubsection{Flüssigphase}

\subsubsection{Poynting-Korrekturen}

Für die Bestimmung der Poynting-Korrektur des Gases ist die Kenntnis des partiellen molaren Volumens $v_{i, \infty}^{\prime}$ notwendig. Dazu existieren relativ wenig Messungen. In Tabelle 2.5 sind Literaturwerte des partiellen molaren Volumens $v_{i}^{\prime}$ verschiedener Gase in Wasser auch für höhere Drücke dargestellt. Wie oben bereits angenommen, ist auch in der Tabelle zu sehen, dass sich die partiellen molaren Volumina mit dem Druck nur wenig ändern. Auch der Einfluss der Temperatur scheint für Stickstoff vergleichsweise gering.

Tabelle 2.5: Partielles molares Volumen von Gasen in Wasser

\begin{tabular}{|c|c|c|c|c|c|c|c|c|}
\hline & [Zhou und $\mathrm{E}$ & ttino, 2001] & [O'Sulliva & und Smitl & , 1970] & & [Moore & [Hildebrand und \\
\hline & & & & & & & & \\
\hline & $25^{\circ} \mathrm{C}$ & $30^{\circ} \mathrm{C}$ & $1025^{\circ} \mathrm{C}$ & & $125^{\circ} \mathrm{C}$ & & $25^{\circ} \mathrm{C}$ & $25^{\circ} \mathrm{C}$ \\
\hline Gas & $v_{\mathrm{Gas}}^{\prime} /\left(\mathrm{cm}^{3}\right.$. & $\left.\mathrm{ol}^{-1}\right)$ & & & & & & \\
\hline $\mathrm{N}_{2}$ & $33,1 \pm 1,6$ & $32,5 \pm 0,8$ & 37,7 & 37,7 & 43,1 & 38,9 & 35,7 & 40 \\
\hline $\mathrm{O}_{2}$ & $32,1 \pm 0,1$ & $28,6 \pm 1,2$ & & & & & 33,2 & 31 \\
\hline $\mathrm{CH}_{4}$ & $32,0 \pm 2,0$ & $25,3 \pm 1,5$ & 45,9 & 41,1 & 54,4 & 46,6 & 34,5 & 37 \\
\hline $\mathrm{Ar}$ & $32,7 \pm 0,4$ & $28,7 \pm 0,1$ & & & & & 32,6 & \\
\hline $\mathrm{CO}_{2}$ & & & & & & & 33,9 & 33 \\
\hline $\mathrm{H}_{2}$ & & & & & & & & 26 \\
\hline $\mathrm{He}$ & $24,6 \pm 3,0$ & & & & & & & \\
\hline
\end{tabular}

Für die überschlägigen Berechnungen des Poynting-Einflusses für Stickstoff in Wasser wurde im Folgenden vereinfacht ein konstanter Wert von $v_{\mathrm{N} 2, \infty}^{\prime}=33 \mathrm{~cm}^{3} / \mathrm{mol}$, der sich an den aktuellsten Ergebnissen von [Zhou und Battino, 2001] orientiert, verwendet. Die sich für diese Annahme ergebenden Poynting-Korrekturen für Stickstoff sowie die Poynting-Korrekturen für Wasser sind für verschiedene Gase in Wasser in Abbildung 2.6 für verschiedene Temperaturen dargestellt. Diese steigen erwartungsgemäß mit zunehmenden Drücken, liegen jedoch im dargestellten Bereich nahe eins. Für Stoffdaten von Wasser wird die Stoffdatenbibliothek LiblF97 von [Kretzschmar, 2010] verwendet. 


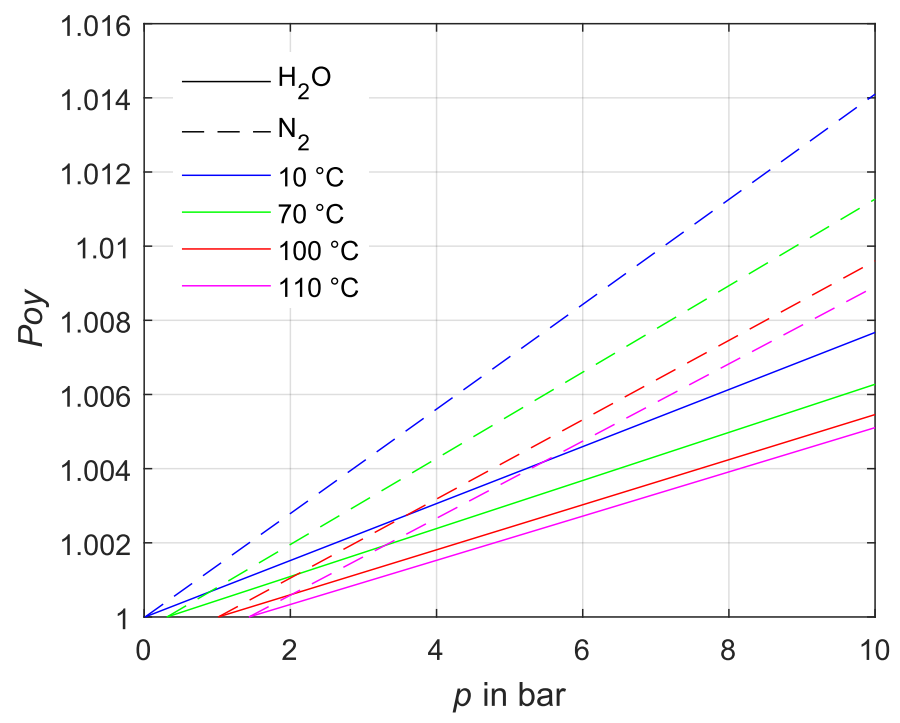

Abbildung 2.6: Poynting-Korrektur-Faktor für $\mathrm{N}_{2}$-Wasser-Gemisch

\subsubsection{Flüssigphase - Henry-Koeffizienten}

Henry-Koeffizienten $K_{\mathrm{H}, i, \infty}$ für unendliche Verdünnung sind für alle Atmosphärengase in Wasser als Regressionsgleichungen basierend auf der Arbeit von [Fernández-Prini u.a., 2003] aufbereitet in [IAPWS, 2004] gegeben. Aufgrund der großen Anzahl der für diese Regression verwendeten Messpunkte $\left(\mathrm{N}_{2}\right.$ : 67 Messpunkte aus 7 Literaturquellen, 278,12..636,46 K; $\mathrm{O}_{2}$ : 52 Messpunkte aus 3 Literaturquellen, 274,15...616,52 K) wird angenommen, dass die Unsicherheit der so bestimmten Koeffizienten klein ist. Wie der Vergleich zwischen Regression und Messwerten in Abbildung 2.7 zeigt, kann der temperaturabhängige Verlauf sehr gut abgebildet werden. Eine Analyse der Abweichung zwischen Regressionsgleichung und exemplarisch gewählten Messpunkten der Literatur von [Alvarez und Fernandez-Prini, 1991; Rettich u. a., 1984; Morrison und Billett, 1952] in Abbildung 2.8 zeigt jedoch, dass einzelne Abweichungen auch im für diese Arbeit fokussierten Temperaturbereich nicht marginal sind und daher die Unsicherheit der Regressionsgleichung des Henry-Koeffizienten durchaus nicht marginal sein muss.

Da in Anlagen verwendetes Wasser stark unterschiedliche Qualität hat, soll auch dies hier kurz beleuchtet werden. Angaben zur Reinheit, z. B. der elektrischen Leitfähigkeit, des verwendeten Wassers werden in den Originalquellen nicht oder nur wenig konkret angegeben. So sprechen die Quellen allgemein von „deionisiertem“ (vgl. [Chapoy u. a., 2004]) bzw. „destilliertem" Wasser (vgl. [Murray u. a., 1969] ,[Murray und Riley, 1969], [Saddington und Krase, 1934] ${ }^{6}$ ), während andere Quellen (vgl. [Alvarez u. a., 1988], [Alvarez und Fernandez-Prini, 1991] ${ }^{6}$, [Morrison und Billett, 1952]) keine konkreten Angaben zur Reinheit des Wassers machen. Hingegen wird bei [Rettich u. a., 1984] ${ }^{6}$ konkret ein mittels „Umkehrosmose, lonenAustauscher und Destillation" erreichter Reinigungsgrad vom mehr als $5 \cdot 10^{5} \Omega \mathrm{m}$ angegeben.

Es gibt auch Löslichkeitsuntersuchungen für wässrige Elektrolytlösungen. So wird beispielsweise in [Sittiho, 2011] für eine Carbonathärte von $8,4^{\circ} \mathrm{dH}$ (Annahme: sonst keine anderen Elektrolyte) ein gegenüber nichtelektrolytischem Wasser um 0,13\% erhöhter Henry-Koeffizient von Sauerstoff ermittelt, was einem um 0,133\% reduzierten Löslich-

\footnotetext{
${ }^{6}$ Diese Literaturquellen wurden u. a. für Regressionen nach [IAPWS, 2004] verwendet
} 
keitskoeffizienten entspricht. Damit bleibt der Einfluss der gelösten Ionen im Wasser gering.

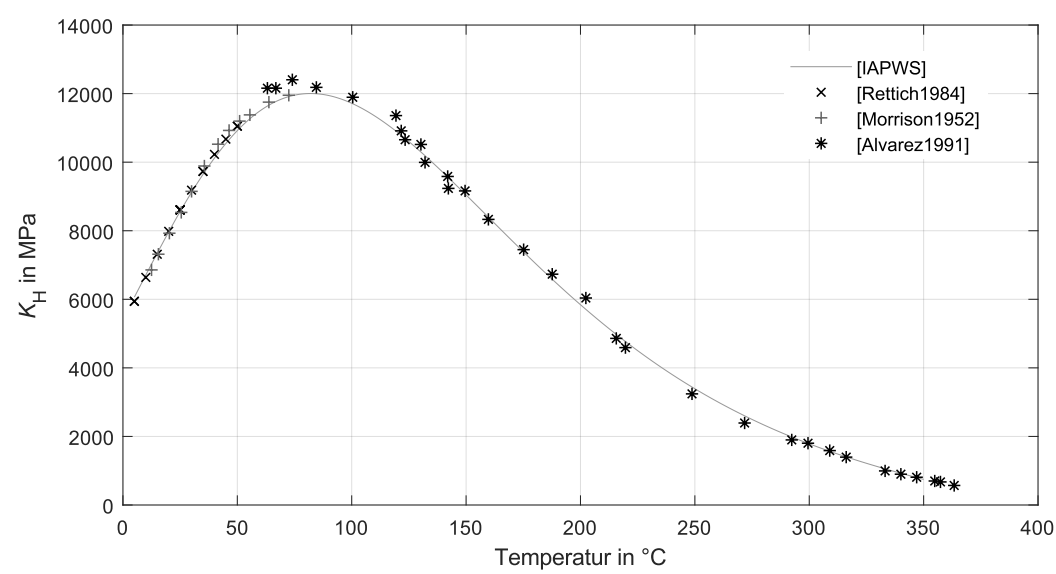

Abbildung 2.7: Henry-Koeffizient für $\mathrm{N}_{2}$-Wasser-Gemisch

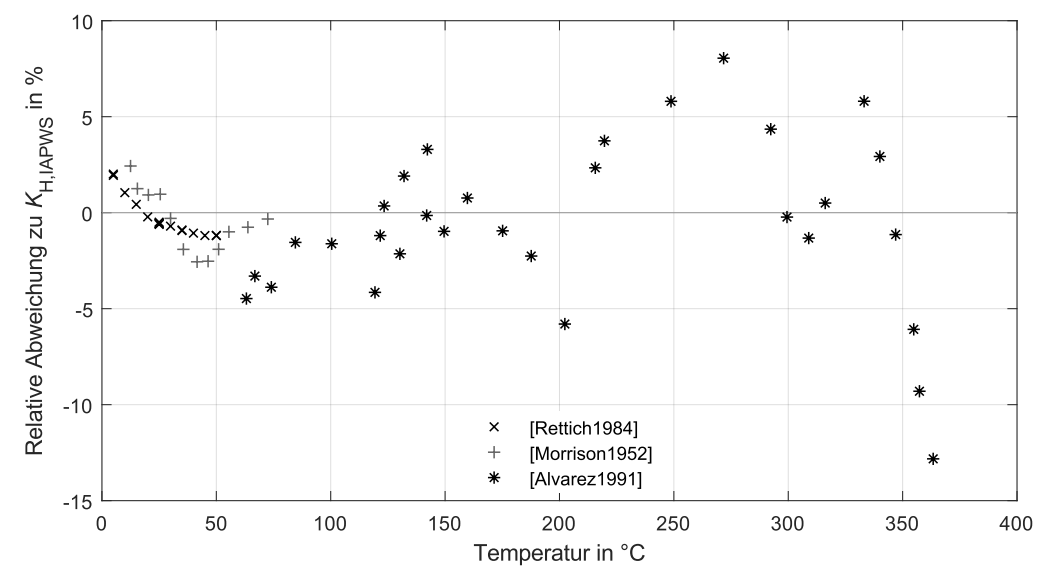

Abbildung 2.8: Henry-Koeffizient für $\mathrm{N}_{2}$-Wasser-Gemisch

\subsubsection{Flüssigphase - Aktivitätskoeffizienten}

Der rationelle Aktivitätskoeffizient des Gases $\gamma^{*}\left(t, x_{i}\right)$ wird in [Alvarez und Fernandez-Prini, 1991] für Stickstoff in Wasser für verschiedene $(p, T)$-Werte bestimmt. Es zeigt sich ein mit der Temperatur steigender Einfluss des rationellen Aktivitätskoeffizienten, der sich dadurch äußert, dass bei steigenden Temperaturen und bei gleichem Partialdruck dieser weiter nach unten von Eins abweicht. In [Alvarez und Fernandez-Prini, 1991] wird der rationelle Aktivitätskoeffizient $\gamma_{i}^{*}$ bspw. auch noch bei einem Partialdruck von ca. $p_{\mathrm{N} 2}=19$ bar und einer Temperatur von $142,25^{\circ} \mathrm{C}(415,4 \mathrm{~K})$ abgeschätzt zu $\gamma_{\mathrm{N} 2}^{*}=0,998$. Dies entspräche einer Abweichung der ermittelten Löslichkeit von 0,2 \%. Eine einfach zu implementierende Korrelation des rationellen Aktivitätskoeffizienten des Gases für den fokussierten Druck- und Temperaturbereich liegt nicht vor. Entsprechend des hier fokussierten Druck- und Temperaturbereichs kann aber der rationelle Aktivitätskoeffizient von Stickstoff $\gamma_{\mathrm{N} 2}^{*}$ nur noch weniger von Eins abweichen und kann daher für technische Anwendungen vernachlässigt werden, also $\gamma_{\mathrm{N} 2}^{*}=1$. 
Wie oben beschrieben, soll auch hier die Aktivität des Lösungsmittels vernachlässigt werden.

\subsubsection{Lösung des Gleichungssystems des Phasengleichgewichts für ein binäres Gas-Wasser-Gemisch}

Hier soll der Fall betrachtet werden, dass die Gaskonzentration $x_{\mathrm{N} 2}$ und $y_{\mathrm{N} 2}$ gesucht sind, während $p$ und $T$ gegeben sind. Zusätzlich zu den Isofugazitätsgleichungen sind (für die Bestimmung der vier Unbekannten) die Gleichungen für die Stoffmengenbilanzen in Flüssigund Gasphase notwendig:

$$
x_{\mathrm{N} 2}+x_{\mathrm{LM}}=1
$$

$$
y_{\mathrm{N} 2}+y_{\mathrm{LM}}=1
$$

Für die Lösung dieses nichtlinearen Gleichungssystems, in der Literatur oft als „Flashcalculation" bezeichnet, hat sich entsprechend der gesuchten Größen die in Abb. 2.9 dargestellte Iteration hier als sinnvoll erwiesen. Für die jeweiligen Vereinfachungen C-F aus Tabelle 2.1 vereinfacht sich die Berechnung entsprechend. 


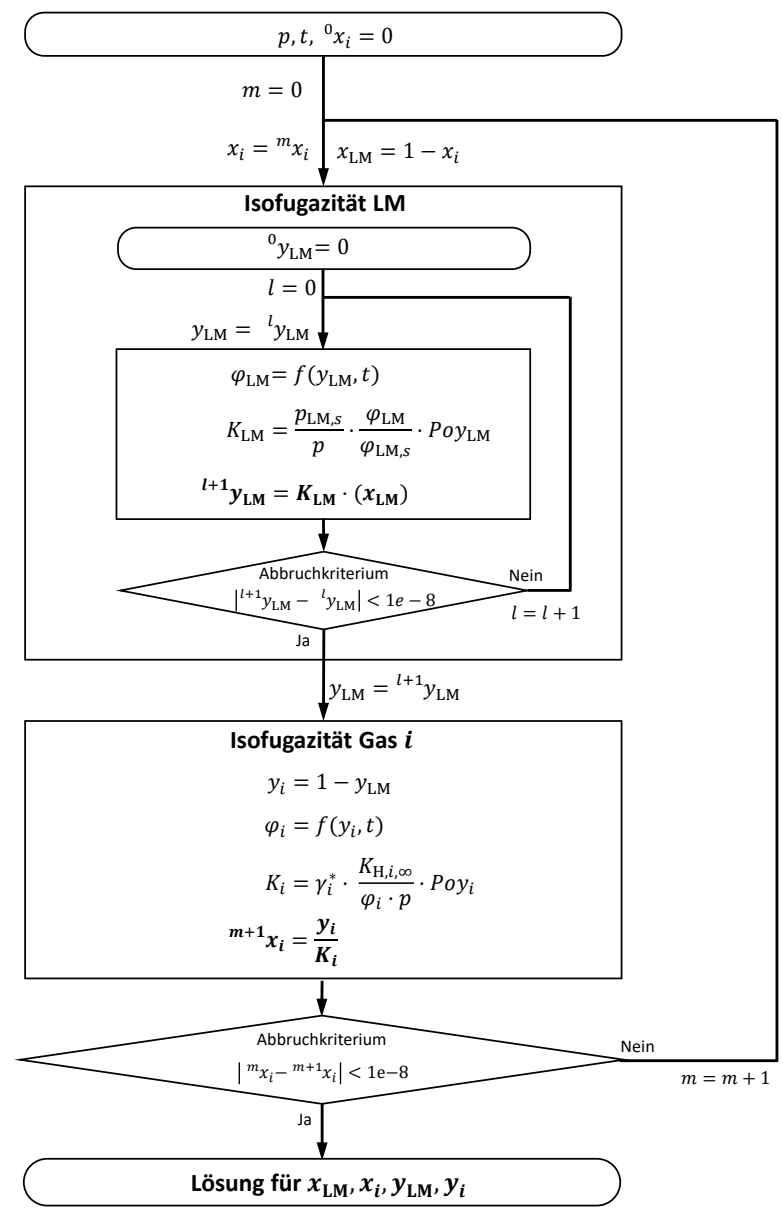

Abbildung 2.9: Iterative Bestimmung der gelösten Stoffmenge des Gases i=N2 für ein binäres Gemisch, Zählvariablen der Iteration: I, $m$

Ebenso ist eine rein mathematische Lösung des nichtlinearen Gleichungssystems in der umgestellten Form $F(x)=0$ möglich, wie es [Wolf, 2018] mit dem Trust-Region-Dogleg-Verfahren in $M A T L A B^{\circledR}$ durchführt. Eigene Untersuchungen zeigen, dass die Wahl der Startbedingungen $x_{0}$ aufgrund der Beschaffenheit des Gleichungssystems bereits wesentlichen Einfluss auf die Lösbarkeit des Gleichungssystems innerhalb der vorgegebenen Iterationsgrenzen hat. AuBerdem waren Rechenzeiten etwa drei- bis zehnmal höher als beim angepassten iterativen Algorithmus.

\subsubsection{Ergebnisse und Fazit}

\section{Vereinfachungen $\mathrm{C}-\mathrm{F}$}

Im Folgenden wurden Einzelrechnungen des Phasengleichgewichts bei konstanter Temperatur und unterschiedlichen Drücken im Bereich $p_{\mathrm{H} 2 \mathrm{O}, \mathrm{s}} \ldots 20$ bar (in Stufen von 10 mbar) durchgeführt. Daraus lassen sich Isothermen-Kurven der Löslichkeit generieren. Berechnet wurde jeweils das Phasengleichgewicht für die Referenz und für die zusätzlichen Vereinfachungen C, C-D, C-E, C-F.

In Abbildung 2.10 sind sich ergebende Löslichkeiten $x_{\mathrm{N} 2}$ im Phasengleichgewicht für jeweils konstante Temperatur in Abhängigkeit des Gesamtdruckes für die Referenz-Berechnungen 


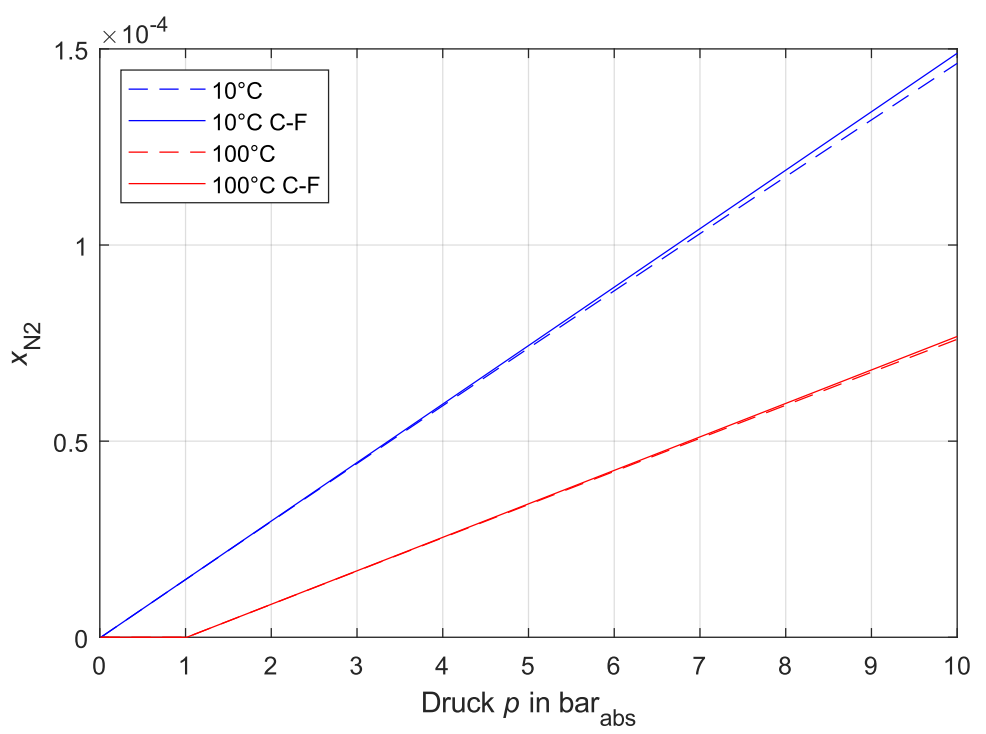

Abbildung 2.10: Löslichkeit von Stickstoff in Wasser in Abhängigkeit des Absolutdrucks für die ReferenzRechnung und mit den zusätzlichen Vereinfachungen C-F

und für die Berechnungen mit den Vereinfachungen C-F (Henry-Gesetz) dargestellt. Die Berechnung mit Vereinfachungen $\mathrm{C}-\mathrm{F}$ zeigt die zu erwartende lineare Abhängigkeit vom Druck. Die Referenz weicht davon kaum sichtbar ab. Die zugehörige relative Abweichung ist in Abbildung 2.11 dargestellt. Die Abweichungen der Ergebnisse zwischen der Rechnung mit den Näherungen $\mathrm{C}-\mathrm{F}$ und der Rechnung ohne diese Näherungen liegen im fokussierten Temperaturund Druckbereich $\left(10-110{ }^{\circ} \mathrm{C}, 0-10\right.$ bar $)$ stets unter $+2 \%$. Damit ist die vereinfachte Berechnung (Idealgas, ohne Poynting-Korrekturen) für technische Belange in den meisten Fällen wohl ausreichend. ${ }^{7}$

Welche der Vereinfachungen welchen Einfluss auf die Abweichung der berechneten gelösten Gasmenge gegenüber den Ergebnissen der Referenz-Rechnung haben, ist detailliert in Abbildung 2.12 dargestellt. Hier zeigt sich, dass für höhere Drücke insbesondere die PoyntingKorrektur des Gases Poy 2 den größten Abweichungsbetrag verursacht. Berücksichtigt man die Poynting-Korrektur des Gases Poy ${ }_{\mathrm{N} 2}$ (Variante C-E in Abbildung 2.12), dann können die Fehler (für den Maximalfehler-Fall 10 bar, $10^{\circ} \mathrm{C}$ ) bereits von $1,75 \%$ auf etwa $0,3 \%$ gesenkt werden.

Höchste Abweichungen der kumulierten Vereinfachungen C-F werden im gesamten Temperaturbereich bei maximalem Druck erreicht. Diese Abweichungen sind in Abbildung 2.13 nochmals dargestellt.

Extrapoliert man für höhere Drücke, zeigt sich, dass die in [D'Ans und Lax, 1997] festgelegten "Gültigkeitsbereiche" für technische Löslichkeitskoeffizienten (was implizit vermutlich den Gültigkeitsbereich für die vereinfachte Berechnung nach dem Henry-Gesetz meint) mit 30 bar für $\mathrm{N}_{2}$ (und 20 bar für $\mathrm{O}_{2}$ ) einen recht hohen Fehler akzeptieren.

\footnotetext{
${ }^{7} \mathrm{Zu}$ beachten ist hier nochmals, dass lediglich die Genauigkeit zweier Berechnungverfahren vergleichend gegenübergestellt wurden. Auch die Referenz-Berechnung beinhaltet hier die o.g. Vereinfachungen $A$ und B sowie die nicht weiter betrachteten Unsicherheiten der verwendeten Stoffwerte und Regressionen. Insbesondere die Unsicherheit der Henry-Koeffizienten-Regression sei hier beachtet.
} 


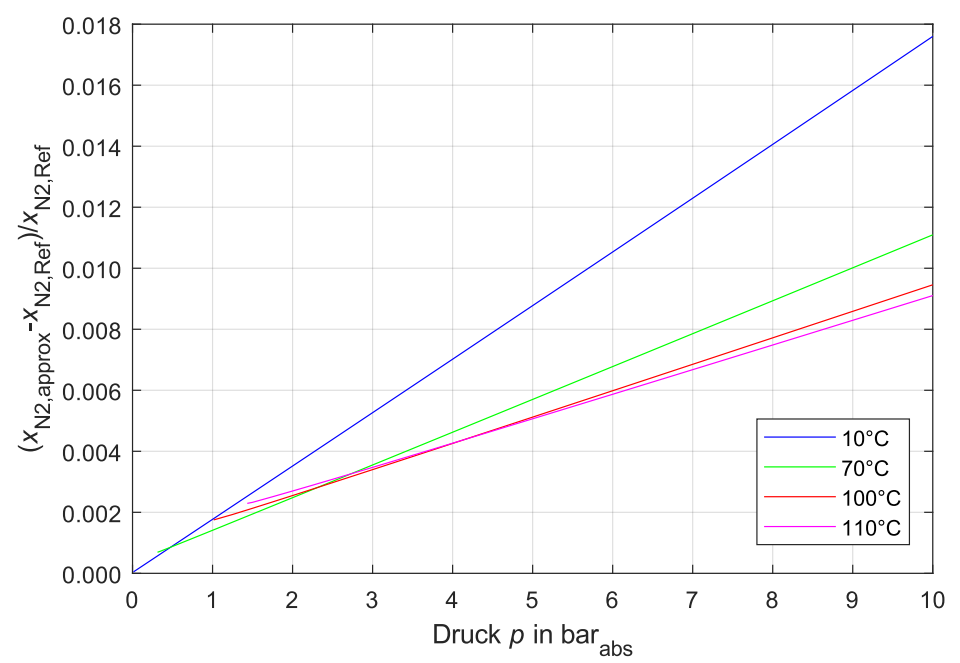

Abbildung 2.11: Relativer Fehler der Approximationen C-F gegenüber der Referenz für alle vier untersuchten Temperaturen

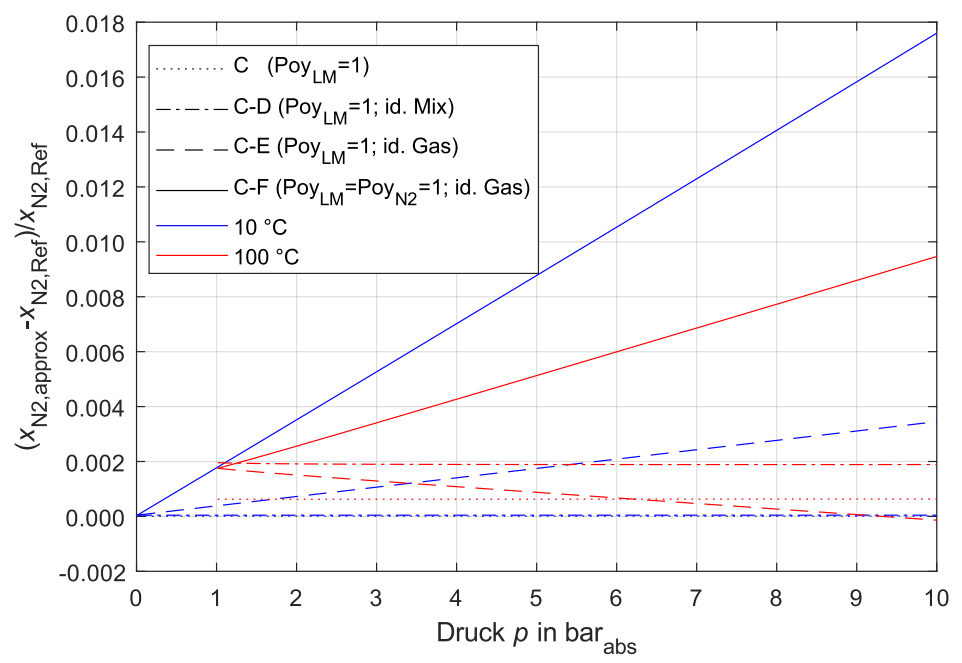

Abbildung 2.12: Relativer Fehler der Approximationen gegenüber der Referenz für $10^{\circ} \mathrm{C}$ und $100^{\circ} \mathrm{C}$

\section{Vereinfachungen G}

Die gelöste Gasmenge Stickstoff für Vereinfachungen A-G kann man auch mit der gelösten Gasmenge nach Vereinfachungen A-F $x_{i, A-F}$ ausdrücken als

$$
x_{i, \mathrm{~A}-\mathrm{G}}=\left(1-\frac{p_{\mathrm{LM}, \mathrm{s}}}{K_{\mathrm{H}, i, \infty}}\right) \cdot x_{i, \mathrm{~A}-\mathrm{F}}
$$

ist also um einen Anteil $\frac{P_{L M, s}}{K_{H, i, \infty}}$ kleiner als der Wert für A-F (Herleitung in Anhang A.2). Dieser Anteil ist druckunabhängig und, wie in Abbildung 2.14 zu sehen, sehr klein. Vereinfachung $G$ verursacht eine Unterschätzung der gelösten Gasmenge um $<0,002 \%$ und ist damit eine sinnvolle Vereinfachung. 


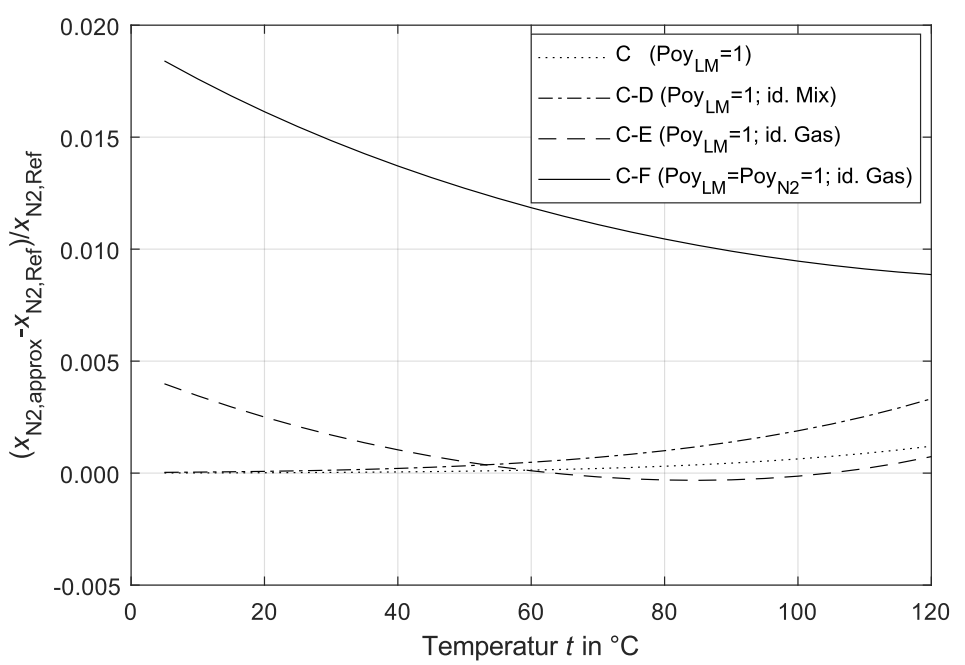

Abbildung 2.13: Relativer Fehler der kumulierten Approximationen gegenüber der Referenz für 10 bar

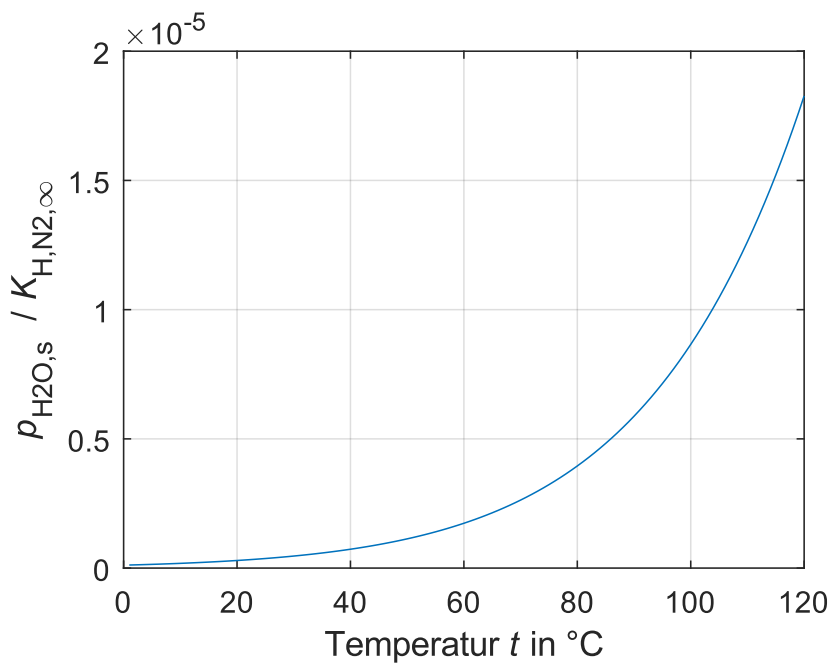

Abbildung 2.14: Relative Abweichung durch Anwendung von Vereinfachung G

\section{Vereinfachungen $\mathbf{H}$}

Fehler der Vereinfachung $\mathrm{H}$ sind umso höher, je höher der Stoffmengenanteil des gelösten Gases ist. Daher wurden die Betrachtungen hier für den Stoffmengenanteil von $x_{\mathrm{N} 2}=1,5 \cdot 10^{-4}$, der als Obergrenze im gesuchten Bereich gelten kann, durchgeführt. Tabelle 2.6 stellt die Abweichungen für die üblichen Gehaltsgrößen gegenüber. ${ }^{8}$ Unterschiede sind, wie in Tabelle 2.6 zu sehen, marginal. ${ }^{9}$

${ }^{8}$ Für die Konzentration $c_{i}$ wird folgende Gleichung für das molare Volumen der Flüssigphase genutzt $v^{\prime}=$ $v_{\mathrm{N} 2, \infty}^{\prime} x_{\mathrm{N} 2}+v_{\mathrm{H} 2 \mathrm{O}, 0} \cdot\left(1-x_{\mathrm{N} 2}\right)$

${ }^{9}$ Da die molare Masse des Stickstoffs größer ist als die des Wassers, sind Abweichungen für den Masseanteil höher als für den Stoffmengenanteil 
Tabelle 2.6: Wirkung der Vereinfachung $\mathrm{H}$ einer gegebenen Menge $x_{\mathrm{N} 2}=1,5 \cdot 10^{-4}$ bei $10^{\circ} \mathrm{C}$ gegenüber dem Wert ohne Vereinfachung (Ref)

\begin{tabular}{cccc}
\hline Ref & Wert & $\mathrm{H}$ & relative Abweichung $(\mathrm{H}$ - Ref) / Ref \\
\hline \hline$x_{\mathrm{N} 2}$ & $1,5 \cdot 10^{-4}$ & $\frac{n_{i}}{n_{\mathrm{H} 2}}$ & $0,015 \%$ \\
\hline$\xi_{i}$ & $2,33 \cdot 10^{-4}$ & $\frac{m_{i}}{m_{\mathrm{H} 20}}$ & $0,0233 \%$ \\
\hline$c_{i}$ & $233 \mathrm{mg} / \mathrm{I}\left(10^{\circ} \mathrm{C}\right)$ & $\frac{m_{i}}{V_{\mathrm{H} 2 \mathrm{O}}^{\prime}}$ & $0,0275 \%$ \\
\hline$w_{i}$ & $0,186 \frac{\mathrm{cm}^{3}}{\mathrm{~g}}$ & $\frac{m_{i}}{m_{\mathrm{H} 2 \mathrm{O}}^{\prime}}$ & $0,0233 \%$ \\
\hline
\end{tabular}

\section{Fazit}

Trotz der Anwendung der Vereinfachung A-H lassen sich für das Beispiel Stickstoff in Wasser im gewählten Druck- und Temperaturbereich (0-10 bar, $10-110^{\circ} \mathrm{C}$ ) für technische Belange sehr akzeptable Fehler der Berechnung der gelösten Stoffmenge von maximal ca. 1,8\% ggü. der Referenz-Berechnung (und je nach $(p, t)$ meist deutlich darunter) erreichen. Gleichzeitig reduziert sich der Berechnungsaufwand von einem komplexen, nichtlinearen Gleichungssystem mit vielen Stoffgrößen auf ein einfaches lineares Gleichungsystem bzw. schlussendlich eine lineare Gleichung für die gelöste Stoffmenge. Dabei verbleiben nur zwei und nur temperaturabhängige Stoffgrößen: die Flüchtigkeit des Gases $K_{\mathrm{H}, i, \infty}$ sowie der Dampfdruck des Lösungmittels $p_{\mathrm{LM}, \mathrm{s}}$.

Möchte man die Löslichkeit insbesondere bei höheren Drücken genauer ermitteln, empfiehlt sich die Vereinfachung F nicht anzuwenden, d. h. die (vereinfachte) Poynting-Korrektur des Gases zu berücksichtigen. Hier lassen sich Fehler von maximal 0,3\% erreichen, jedoch erhöht sich der Berechnungsaufwand leicht und es kommt mit $v_{i, \infty}^{\prime}$ eine weitere Stoffgröße hinzu, die oft nicht sonderlich gut bekannt ist. Es ist zu beachten, dass für den Praktiker durch die höhere Komplexität eine zusätzliche Hemmschwelle der Anwendung entstünde, die durch den Genauigkeitsgewinn vermutlich nur selten zu rechtfertigen wäre. Außerdem erhöht sich mit der Komplexität die Gefahr von Berechnungsfehlern.

\subsubsection{Fundamentalgleichungen und andere Approximationen für Stickstoff in Wasser}

Die Verwendung von Fundamentalgleichungen zur Bestimmung von Gaslöslichkeit ist theoretisch völlig gleichwertig möglich und aufgrund von zur Verfügung stehenden Berechnungsprogrammen wie z. B. CoolProp von [Bell u. a., 2014] oder TREND von [Span u. a., 2016] leicht und ohne Aufwand anzuwenden. Allerdings sind die Ansatzfunktionen der Fundamentalgleichungen anscheinend selten mit dem Fokus auf die Gaslöslichkeit bei geringen Drücken ermittelt und parametriert worden, sondern an eine Vielzahl experimentell ermittelter thermodynamischer Messwerte der Gemische in weiten Druck- und Temperaturbereichen angepasst worden. Die Ergebnisse der gelösten Gasmengen $x_{N 2}$ aus eigenen, oben beschriebenen Referenz-Berechnungen (ohne Vereinfachungen) sind denen mit der Anwendung Fundamentalgleichung nach [Gernert, 2013] in Abbildung 2.15 gegenübergestellt. Bei $100{ }^{\circ} \mathrm{C}$ liegen ermittelte gelöste Gasmengen mit der Fundamentalgleichung nach [Gernert, 2013] bereits mehr als $25 \%$ über den Werten der eigenen Berechnungen. Dies ist umso verwunderlicher, da [Gernert, 2013] sogar auch Löslichkeitsdaten zur Anpassung seiner Regression nutzt und die Regressionsgüte anschließend graphisch überprüft. Bei genauerer Analyse fällt auf, dass die logarithmische Skalierung der Achsen seiner Graphen die Regression fälschlicherweise genau erscheinen lässt. 
In der Literatur finden sich auch direkte Korrelationen für Stickstoff-Wasser-Gemische, gesammelt in [Fogg, 1991], zur Bestimmung von $x_{N 2}$ in Abhängigkeit der Temperatur (Korrelation von Battino: 1 bar 273-350 K, Korrelation von Clever und Han: 1 bar, 350..600 K) bzw. in Abhängigkeit von Temperatur und Druck (Korrelation von Battino et. al. , ab $350 \mathrm{~K}$, 25..1000 bar, hier extrapoliert) vor. Diese validieren, dargestellt in Abbildung 2.16, hier auch die absoluten Ergebnisse der eigenen Berechnungen und bestätigen den signifikanten Fehler der Ergebnisse mit der Fundamentalgleichung von [Gernert, 2013] bei hohen Temperaturen.

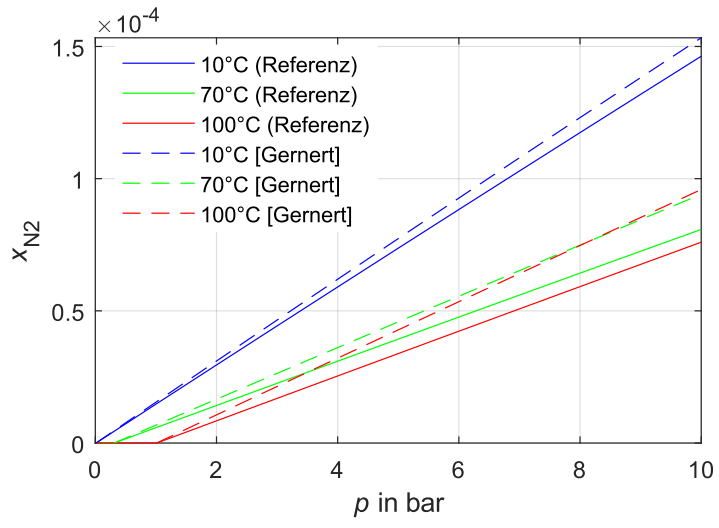

Abbildung 2.15: Löslichkeit $N_{2}$ in Wasser nach [Gernert, 2013], berechnet mit CoolProp von [Bell u. a., 2014]

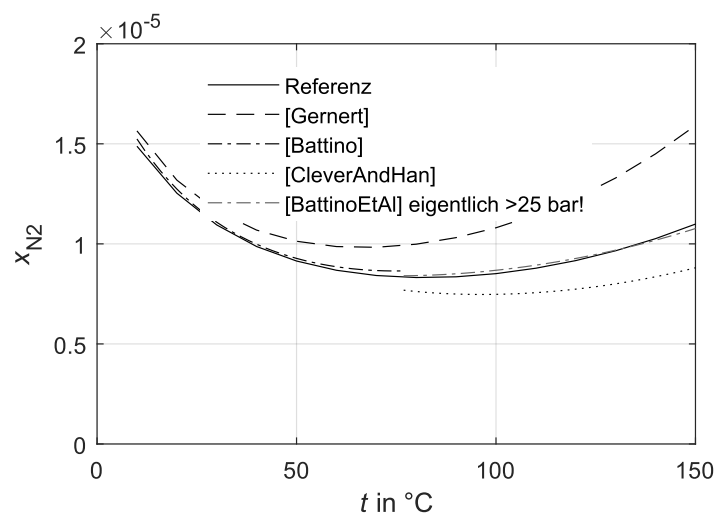

Abbildung 2.16: Löslichkeit $\mathrm{N}_{2}$ in Wasser bei $p=$ $p_{\mathrm{LM}, \mathrm{s}}(t)+1$ bar für verschiedene Modelle

Auch die Fundamentalgleichungen, die den Anspruch erheben, Stoffgemische in einem weiten Temperatur- und Druckbereich thermodynamisch gut zu beschreiben und per se alle angesprochenen Einflüsse (Poynting-Korrekturen, Fugazitätskoeffizienten) berücksichtigen, sind bei der Anwendung für den Spezialfall der Gaslöslichkeit, also bei vergleichsweise geringen Drücken, teilweise ungeeignet. Daher müssen diese zumindest mit großer Vorsicht auf die Anwendbarkeit im gewählten Temperatur- und Druckbereich getestet werden.

So lassen sich paradoxerweise selbst bei Verwendung der vorgestellten stark vereinfachten Zusammenhänge entsprechend des Henry-Gesetzes deutlich präzisere Ergebnisse erzielen.

\subsubsection{Konsequenz für die vereinfachte Bestimmung von Löslichkeitskoeffizienten}

Für die vereinfachte Bestimmung des Gasgehaltes mit Approximation A-H , also entsprechend des Henry-Gesetzes, verbleiben als Gleichungen

$$
x_{i, \text { approx }}=\frac{p_{i}}{K_{\mathrm{H}, i ; \infty}}
$$

oder auch

$$
x_{i, \text { approx }}=\lambda_{i} \cdot \frac{M_{\mathrm{LM}}}{v_{0, i, \mathrm{~N}}} \cdot p_{i}
$$

mit dem Partialdruck bei einer Gaskomponente

$$
p_{i}=p-p_{\mathrm{LM}, \mathrm{s}} .
$$


Oben ist der relative Approximationsfehler der gelösten Gasmenge $\Delta_{\mathrm{rel}} x_{i}=\frac{x_{i, \text { approx }}-x_{i}}{x_{i}}$ ermittelt worden. In der Arbeit ist hingegen nun statt des Stoffmengenanteils $x_{i}$ der Löslichkeitskoeffizient $\lambda_{i}$ gesucht. Dieser lässt sich mit den Vereinfachungen (Approximationen A-H) nach Gleichung 2.35 bestimmen zu

$$
\lambda_{i, \text { approx }}=\frac{v_{0, i, \mathrm{~N}}}{M_{\mathrm{LM}}} \cdot \frac{x_{i}}{p_{i}} .
$$

Mit Gleichung 2.53 ergibt sich

$$
\lambda_{i, \text { approx }}=\lambda_{i} \cdot \frac{x_{i}}{x_{i, \text { approx }}}
$$

und die Abweichung zum exakten Wert $\lambda_{i}$ lässt sich abschätzen zu

$$
\lambda_{i, \text { approx }}=\lambda_{i} \cdot \frac{1}{1+\Delta_{\mathrm{rel}} x_{i}}
$$

Der Approximationsfehler $\Delta_{\text {rel }} \lambda_{i}=\frac{\lambda_{i, \text { approx }}-\lambda_{i}}{\lambda_{i}}$ ergibt damit zu

$$
\Delta_{\mathrm{rel}} \lambda_{i}=\frac{\frac{1}{1+\Delta_{\mathrm{rel}} x_{i}} \cdot \lambda_{i}-\lambda_{i}}{\lambda_{i}}=-\frac{\Delta_{\mathrm{rel}} x_{i}}{1+\Delta_{\mathrm{rel}} x_{i}} .
$$

An einem Beispiel soll das Ergebnis erklärt werden: Für Stickstoff-Wasser bei einem Druck von 5,5 bar und einer Temperatur von $10^{\circ} \mathrm{C}$ beträgt der oben abgeschätzte Fehler der Approximation $\Delta_{\text {rel }} x_{i} \approx 1 \%$. Demzufolge gilt im Umkehrschluss für den bei bekannter Zusammensetzung bestimmten Löslichkeitskoeffizienten $\lambda_{i \text {,approx }}$ ein Fehler von $\Delta_{\text {rel }} \lambda_{i}=-\frac{0,01}{1+0,01} \approx-0,99 \%$. Es ergeben sich also Approximationsfehler in gleicher Größenordnung wie bei der Bestimmung der gelösten Gasmenge $x_{i}$, nur mit anderem Vorzeichen.

Wird die Poynting-Korrektur des Gases doch berücksichtigt (keine Vereinfachung F), also das Henry-Gesetz mit Berücksichtigung der Poynting-Korrektur angewendet, dann gilt

$$
\lambda_{i, \infty, \text { approx }}=\frac{v_{0, i, \mathrm{~N}}}{M_{\mathrm{LM}}} \cdot \frac{x_{i}}{p_{i}} \cdot \operatorname{Poy}\left(p ; p_{\mathrm{LM}, \mathrm{s}}\right)
$$

Für das Beispiel ergibt sich ein Fehler von $\Delta_{\text {rel }} \lambda_{i}=-\frac{0,002}{1+0,002} \approx-0,2 \%$. Für die Bestimmung der Löslichkeitskoeffizienten hat die Berücksichtigung der Poynting-Korrektur also doch einen deutlichen Einfluss auf die Genauigkeit des Ergebnisses. Daher soll die PoyntingKorrektur für die Bestimmung der Löslichkeitskoeffizienten formal berücksichtigt werden und Vereinfachung $F$ also nicht genutzt werden. Auch für die Bestimmung der Löslichkeitskoeffizienten ist es zulässig, die vereinfachten Zusammenhänge nach Tabelle 2.2 zu verwenden.

Die für eine noch exaktere Bestimmung der Löslichkeitskoeffizienten weiteren benötigten Stoffdaten, wie z. B. Fugazitätskoeffizienten der Gasphase für Nutzung ohne Vereinfachung $D / E$, sind für viele Stoffgemische, wenn überhaupt vorhanden, teilweise mit hohen Unsicherheiten belegt. Daher würde die Unsicherheit bei Verwendung der prinzipiell exakten Gleichung sowieso nur eingeschränkt sinken. Da für die zu untersuchenden Stoffgemische auf WasserGlykol-Basis diese Einflussgrößen sowieso gänzlich unbekannt sind, ist diese Näherung hier zusätzlich sinnvoll und notwendig. 
Für Sauerstoff werden vom Autor (aufgrund ähnlicher physikalischer Beschaffenheit und ähnlicher Größenordnung gelöster Gasmengen) ähnlich geringe Abweichungen der vereinfachten Gleichung (Approximationen A-F) wie bei Stickstoff in Wasser erwartet.

Es wird ebenso angenommen, dass der vereinfachte Zusammenhang auch noch bei mehreren Gasen gilt, wo der Partialdruck sich zu

$$
p_{i}=p-p_{\mathrm{LM}, s}-\sum_{j} p_{j \neq i}
$$

bestimmen lässt. 


\section{Wasser-Glykol-Gemische}

\subsection{Wasser-Propylenglykol- und Wasser-Ethlylenglykol-Gemische}

Hauptgrund für den Einsatz von Wasser-Glykol-Gemischen ist die Herabsetzung des Gefrierpunkts. In der Praxis werden Propylenglykol (1,2-Propandiol / $\mathrm{C}_{3} \mathrm{H}_{8} \mathrm{O}_{2}$ ) und Ethylenglykol (1,2-Ethandiol / $\left.\mathrm{C}_{2} \mathrm{H}_{6} \mathrm{O}_{2}\right)$, im Folgenden auch als $\mathrm{P}$ bzw. $E$ bezeichnet, eingesetzt. Im Gegensatz zu Wasser hat Wasser-Glykol zusätzlich den Vorteil, dass dieses bei Unterschreitung des Gefrierpunkts nicht unmittelbar mit einer fast zehnprozentigen Volumenzunahme vollständig erstarrt, sondern allmählich Eis ausflockt und erst bei noch geringeren Temperaturen vollständig erstarrt, vgl. [Stichel, 1998]. Der sich für Wasser-GlykolGemische ergebende Eisflockenpunkt, dessen Bestimmung in [ASTM D1177-17] festgelegt ist, ist in Abbildung 3.1 in Abhängigkeit des Glykolanteils $\xi_{\mathrm{G}}$ dargestellt und zeigt ähnliche Verläufe für Wasser-Propylenglykol und -Ethylenglykol-Gemische, wobei Ethylenglykol bei gleichem Masseanteil eine höhere Gefrierpunktabsenkung zeigt. Fertiggemische, hier das später noch eingeführte Gemisch Tyfocor ${ }^{\circledR}$ LS auf Propylenglykol-Basis als Beispiel, können aufgrund von zusätzlichen Additiven einen leicht verschobenen Eisflockenpunkt aufweisen.

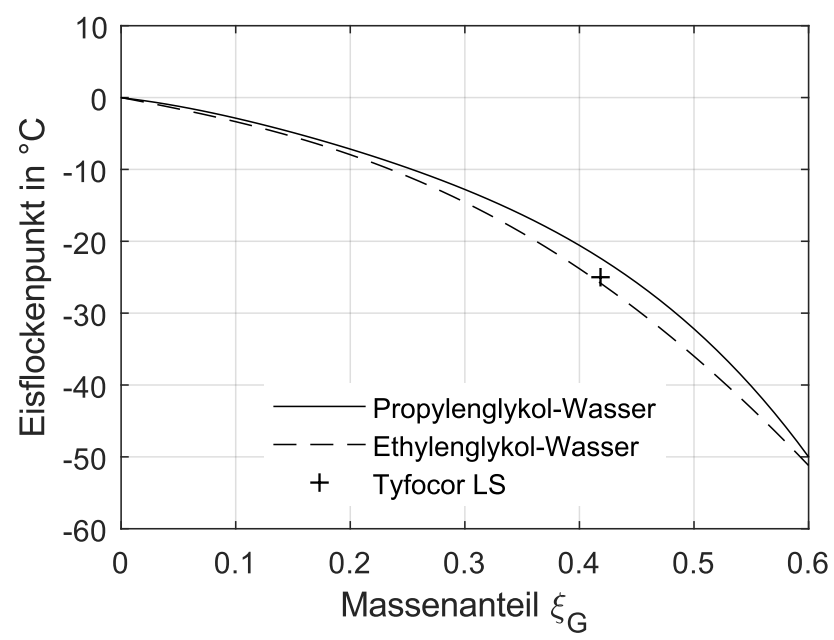

Abbildung 3.1: Eisflockenpunkt von Wasser-Glykol-Gemischen in Abhängigkeit des Masseanteils Glykol $\xi_{\mathrm{G}}$ nach [Melinder, 2010] und Eisflockenpunkt des Fertiggemischs Tyfocor ${ }^{\circledR}$ LS nach Sicherheitsdatenblatt [LS-SDB]

Neben der notwendigen Bedingung des Frostschutzes muss ein Wärmeträgerfluid eine hohe Energietransporteffizienz und guten Wärmeübergang bereitstellen. Dazu sind eine hohe spezifische Wärmekapazität, Wärmeleitfähigkeit und Dichte sowie eine geringe Viskosität notwendig. Weitere Anforderungen sind u. a. nach [Melinder, 2010; F. Hillerns, 2002; Holfeld, 2008]:

- Umweltverträglichkeit, geringe Toxizität, biologische Abbaubarkeit,

- Sicherheits- und Arbeitsschutzaspekte, wie z. B. Entflammbarkeit, 
- chemische Beständigkeit unter Betriebsbedingungen, Alterungsbeständigkeit, geringe Korrosionswirkungen sowie -schäden,

- Kompatibilität mit Dichtungsmaterialien,

- Verhinderung von Ablagerungen und Schaumbildung

- und nicht zuletzt geringe Herstellkosten.

Unter diesen Aspekten haben sich die Wasser-Glykol-Gemische mit entsprechenden Additiven als Wärmeträger etabliert, auch wenn diese nicht alle Kriterien in gleichem Maße wie reines Wasser erfüllen. Beispielsweise müssen eine höhere Viskosität und eine geringere Speicherdichte in Kauf genommen werden (vgl. die Eigenschaften der reinen Wasser-Glykol-Gemische in Abbildung 3.2).

Aus wirtschaftlichen Gründen, der etwas besseren thermischen Beständigkeit, aber auch wegen der wärmetechnischen Vorteile wird vornehmlich Ethylenglykol genutzt, vergleiche [Duchardt u. a., 2016; Stichel, 1998]. Propylenglykol wird aufgrund seiner toxikologischen Unbedenklichkeit dort eingesetzt, wo im Fall von Leckagen die Kontamination von Trinkwasser oder Nahrungsmitteln zu befürchten ist, also z. B. in Solarthermieanlagen und der Nahrungsmittelindustrie (vgl. [Stichel, 1998]). In Solarthermieanlagen eingesetzte Wärmeträger werden in der Praxis auch oft als Solarflüssigkeiten bezeichnet.
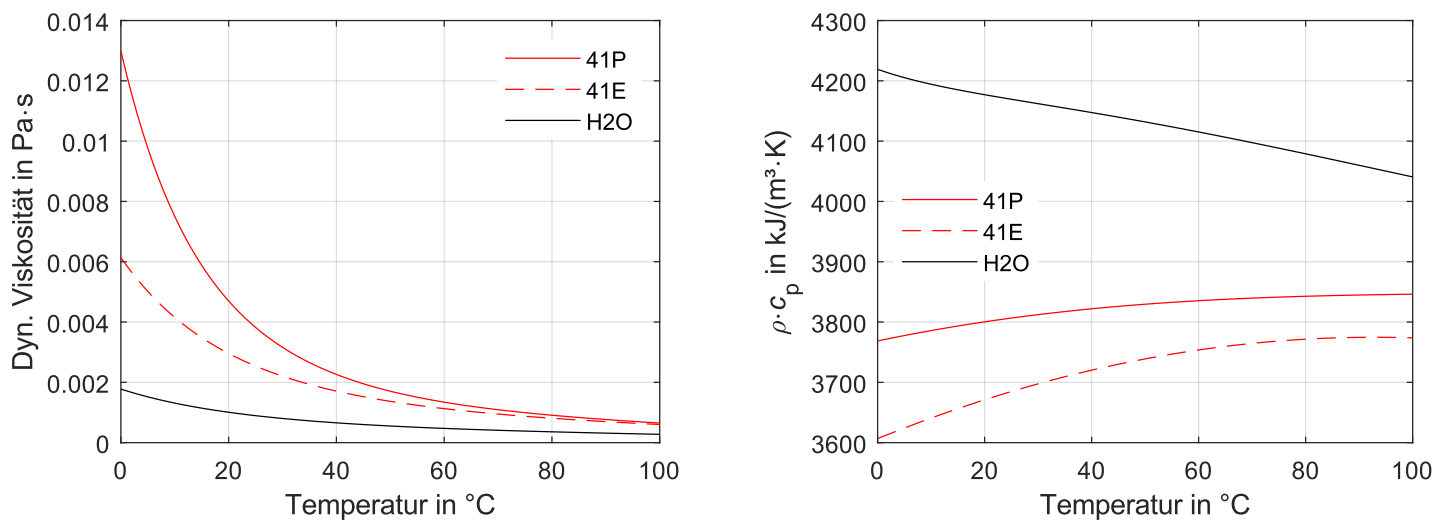

Abbildung 3.2: Viskosität (links) und Speicherdichte $\rho \cdot c_{p}$ (rechts) für Wasser-Glykol-Gemische mit 41,84 Gewichtsprozent Propylen- (41P) bzw. Ethylenglykol (41E) nach [Melinder, 2010] im Vergleich zu Wasser

[Melinder, 2010] stellt eine umfangreiche Stoffdatensammlung für Wasser-Propylenglykol und -Ethylenglykol-Gemische für einen eingeschränkten Temperatur- $\left(\xi_{G} \cdot 100{ }^{\circ} \mathrm{C}<\right.$ $t<100^{\circ} \mathrm{C}$ ) und Mischungsbereich $\left(0<\xi_{\mathrm{G}}<60 \%\right)$ zur Verfügung. Diese Daten sind teilweise in der Stoffdaten-Software CoolProp [Bell u.a., 2014] implementiert.

Für die Bestimmung der Gaslöslichkeit sind vor allem die Dichte und der Dampfdruck der Flüssigkeit von Bedeutung. Die temperaturabhängige Dichte der eingesetzten Stoffgemische wird in hoher Genauigkeit benötigt und daher in Vorversuchen experimentell ermittelt. Zum Dampfdruck der Wasser-Glykole finden sich in der Stoffdatensammlung von [Melinder, 2010] keine Regressionskurven. Diese werden daher vereinfacht mittels Raoultschem Gesetz aus den Siededrücken der Reinstoffe bestimmt. Wie in Abschnitt 4.7.7 noch detailliert ausgeführt wird, zeigen Literaturquellen, dass die Aktivität der Stoffe im Gemisch gering ist und der Dampfdruck des Gemisches in guter Genauigkeit mit dem Raoultschen Gesetz abgeschätzt werden kann. Der Dampfdruck der Wasser-Glykol-Gemische ist deutlich geringer als 


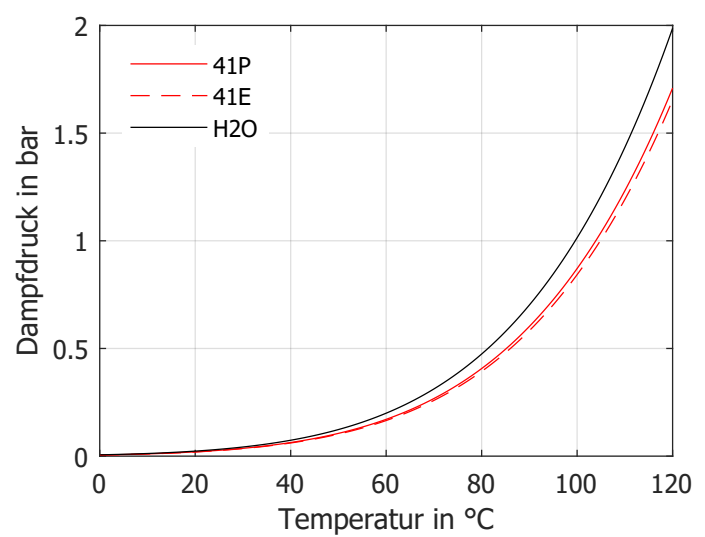

Abbildung 3.3: Dampfdruck für Wasser-Glykol-Gemische mit 41,84 Gewichtsprozent Propylen- (41P) bzw. Ethylenglykol (41E) (nach [Rane u. a., 2016] für P bzw. [Ambrose und Hall, 1981] für E sowie Raoultschem Gesetz) und im Vergleich zu Wasser

der von Wasser (vgl. Abschnitt 3.3) mit den entsprechenden Auswirkungen auf die lösbare Gasmenge.

\subsection{Reale Fertigmischungen}

In der Praxis eingesetzte Wasser-Glykol-Gemische besitzen zusätzlich auf die verwendeten Werkstoffe und den Temperatur-Einsatzbereich abgestimmte Korrosionsschutz-Inhibitoren und Bestandteile zur Vorhaltung einer Reservealkalität sowie zur Sicherstellung weiterer Sekundäreigenschaften (z. B. Reduzierung der Schaumbildung). Die genaue Zusammensetzung der Additive der Anbieter wird aus Wettbewerbsgründen nicht veröffentlicht.

Als Fertigmischungen wurden die Vertreter Tyfocor ${ }^{\circledR}$ LS auf Propylenglykol-Basis und Tyfocor $^{\circledR}$ auf Ethylenglykol-Basis ausgewählt.

Tyfocor $^{\circledR}$ LS wird als Fertigmischung angeliefert und enthält 41,84 Gew.-\% Propylenglykol sowie 1-2 Gew.-\% Additive. Wichtigster Inhibitor ist laut Angaben des Herstellers Isopropanolamin. Aufgrund der gezielten Entwicklung des Tyfocor ${ }^{\circledR}$ LS für Solarthermiekollektoren mit hohen Stagnationstemperaturen (und entsprechend siedendem Wärmeträger im Stagnationsfall) wurden hier ausschließlich flüssige Additive verwendet. Einsatzgebiete sind laut Hersteller „thermisch hochbelastete Solaranlagen“, vgl. [TyfocorLS].

Tyfocor ${ }^{\circledR}$ wird als Konzentrat angeliefert und wurde in den Versuchen mit einer Gewichtskonzentration von 41,84 Gew.-\% Tyfocor ${ }^{\circledR}-$ Konzentrat verwendet. Einsatzgebiete sind laut Hersteller Kühl- und Heizsysteme, Klima- und Wärmepumpenanlagen sowie Flächenheizungen (vgl. [Tyfocor]).

Eine Übersicht von weiteren eingesetzten Fertigmischungen und deren Stoffdaten findet sich in [Holfeld, 2008].

\subsection{Gaslöslichkeit}

In [Yamamoto u. a., 1994] und [Yamamoto und Tokunaga, 1994] wurden für 101, 325 kPa und 298, 15 K Ostwald-Koeffizienten für Stickstoff und Sauerstoff in Propylenglykol-Wasser bzw. 
Ethylenglykol-Wasser bei unterschiedlichen Gykol-Masseanteilen $\xi_{\mathrm{G}}$ bestimmt. Diese Ergebnisse sind, umgerechnet auf technische Löslichkeitskoeffizienten, in Abbildung 3.4 und Abbildung 3.5 dargestellt. ${ }^{1}$ Die jeweils auf der y-Achse liegende Löslichkeit für Wasser $\left(\xi_{\mathrm{P}}=0 \mathrm{bzw}\right.$. $\left.\xi_{\mathrm{E}}=0\right)$ unterscheidet sich teilweise deutlich von der Löslichkeit der Glykol-Wassergemische im technisch relevanten Konzentrationsbereich.

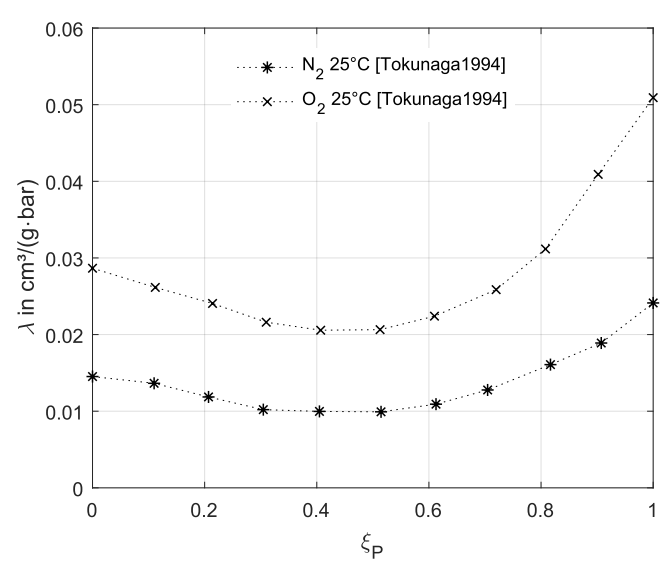

Abbildung 3.4: Löslichkeitskoeffizienten für $\mathrm{N}_{2}$ und $\mathrm{O}_{2}$ in Propylenglykol-Wasser nach [Yamamoto u. a., 1994]

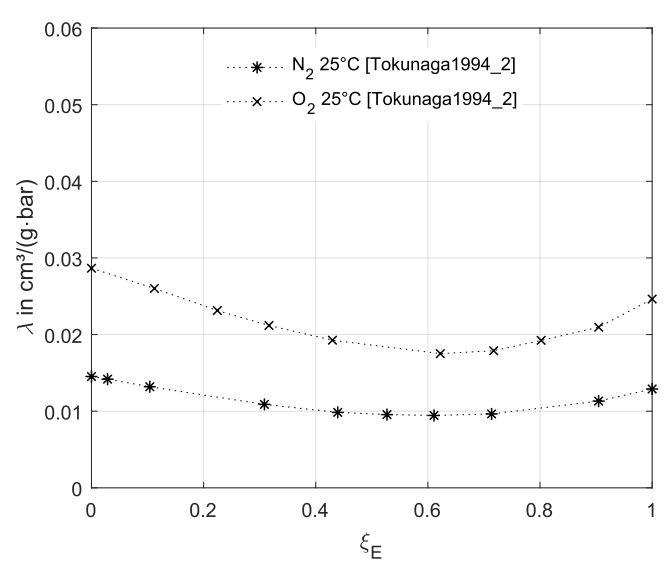

Abbildung 3.5: Löslichkeitskoeffizienten für $\mathrm{N}_{2}$ und $\mathrm{O}_{2}$ in Ethylenglykol-Wasser nach [Yamamoto und Tokunaga, 1994]

In [Abdi u.a., 2007] werden Untersuchungen für Stickstoff in 40 bzw. 60 gew.-\% Ethylenglykol-Wasser bei $10{ }^{\circ} \mathrm{C}, 0^{\circ} \mathrm{C}$ und $-10^{\circ} \mathrm{C}$ durchgeführt. Messergebnisse liegen hier jedoch nur für hohe Drücke von $15 \mathrm{bzw}$. $20 \mathrm{MPa}$ vor. Die Ergebnisse zeigen jedoch von $10^{\circ} \mathrm{C}$ bis $-10^{\circ} \mathrm{C}$ eine Zunahme der Löslichkeit um über $30 \%$. Ob dies auch für Bereiche mit geringen Drücken bzw. für die Henry-Koeffizienten gilt oder aber die bei hohen Drücken zunehmend an Einfluss gewinnenden Faktoren Realgasverhalten und Poyntingkorrekturen dafür verantwortlich sind, kann nicht gesagt werden.

Untersuchungen zu höheren Temperaturen und zur Druckabhängigkeit der Löslichkeit bzw. zum Druck-Gültigkeitsbereich von Löslichkeitskoeffizienten konnten nicht gefunden werden und sind daher ein Hauptanliegen dieser Arbeit.

${ }^{1}$ Die Quellen [Yamamoto u. a., 1994] und [Yamamoto und Tokunaga, 1994] nutzen für die Definition des Ostwald-Koeffizienten nach 2.45, also $V_{g}$ das Volumen des reinen gelösten Gases ist, das sich bei einem Gesamtdruck einstellt, der dem Partialdruck des Gases im Gemisch entspricht ( $p_{i}=1,01325$ bar). Die Umrechnung erfolgte mit $\lambda_{i, \infty}=\frac{\rho_{i}\left(1,01325 \mathrm{bar}, 25^{\circ} \mathrm{C}\right)}{\rho_{i, \mathrm{~N}} \cdot \rho_{\mathrm{LM}}\left(25^{\circ} \mathrm{C}\right) \cdot 1,01325 \mathrm{bar}} \cdot L_{i}$. Für die Dichte des Lösungsmittels $\rho \mathrm{LM}$ wurden Messwerte aus diesen Literaturquellen genutzt. Die Poynting-Korrektur wird hier aufgrund des geringen Drucks nicht berücksichtigt. 


\section{Methodik zur experimentellen Bestimmung von Löslichkeitskoeffizienten}

\subsection{Gegenstand der Untersuchung}

Es sollen Untersuchungen zur Löslichkeit der Gase Stickstoff und Sauerstoff durchgeführt werden. Die Untersuchung der Löslichkeit von Sauerstoff wurde jedoch aufgrund des deutlich geringeren Anteils gegenüber Stickstoff in der Luft und zusätzlichen Anforderungen an die Sicherheit des Versuchsstands nicht direkt durchgeführt. Ausschlaggebend war hier insbesondere die mögliche Gefährdung bei erhöhten Sauerstoffpartialdrücken (z. B. Gefahr der Bildung selbstentzündender Gemische). Dafür erfolgten aber Untersuchungen zur Löslichkeit von synthetischer Luft bestehend aus Stickstoff und Sauerstoff. Dies ist der für technische Belange interessantere Fall, da in realen Anlagen nie reiner Sauerstoff eingeschlossen ist, sondern dieser stets in einem Gemisch von Stickstoff und Sauerstoff vorliegt.

Mit dem Versuchstand sollen typische Vertreter von Propylenglykol- bzw. EthylenglykolWasser-Fertiggemischen unbekannter Additiv-Zusammensetzung untersucht werden. Ebenso sollen reine entsprechende Glykol-Wasser-Gemische untersucht werden, um im Vergleich zu den Fertiggemischen den Einfluss der Inhibitoren zu identifizieren. Insbesondere in Solarthermieanlagen eingesetzte Flüssigkeiten erfahren durch starke thermische Beanspruchung und Korrosion eine Änderung der chemischen Zusammensetzung. Daher soll es möglich sein, mit der Apparatur auch gealterte Gemische mit unbekannter Zusammensetzung und möglicherweise stark flüchtigen Komponenten zu untersuchen.

Grundsätzlich sollen Untersuchungen bei 0-100\% Glykolanteil möglich sein. Bei Ermittlung ausreichend vieler Werte unterschiedlicher Konzentration erhofft man sich, Löslichkeit bei beliebigem Frostschutzniveau und damit Glykolanteil abschätzen zu können.

In dieser Arbeit wird entsprechend üblicher Einsatzbereiche der Wasser-Glykol-Kreisläufe und unter Berücksichtigung des Funktionsbereichs des Versuchsstandes folgender Parameterbereich fokussiert:

- Drücke im Bereich 0...10 bar

- Temperaturen $10 \ldots 110^{\circ} \mathrm{C}$

\subsection{Einordnung der verwendeten Versuchsapparatur}

In dieser Arbeit konnte auf die von [Wagner, 2010] konstruierte Apparatur zurückgegriffen werden. Erste Test-Versuchsreihen von [Wagner, 2010] mit Wasser lieferten jedoch noch keine plausiblen Messergebnisse. Basierend auf einem geänderten Berechnungsalgorithmus mit ausführlicher Messunsicherheitsbetrachtung wurden im Rahmen dieser Arbeit am Versuchsstand und insbesondere an der Versuchsdurchführung wesentliche Änderungen vorgenommen, um die gewünschten Löslichkeitskoeffizienten mit für technische Belange ausreichender Messunsicherheit ermitteln zu können. 
In der Literatur sind viele Varianten und Apparaturen zur Messung von Phasengleichgewichten zu finden. Übersichten dazu können z. B. in [Wagner, 2010] oder [Fogg, 1991] entnommen werden. Die Methoden erfassen entweder vom gasfreien zum gasgesättigten Zustand absorbierte bzw. vom gasgesättigten zum gasfreien Zustand desorbierte Gasmengen oder ermitteln direkt im gelösten Zustand chemisch-analytisch bzw. gravimetisch die Menge gelöster Gase.

Die im Rahmen der Arbeit verwendete Apparatur basiert in großen Teilen auf der in [Melzer, 1990] beschriebenen Lösung und ist für eine synthetisch-statische Bestimmung mittels volumetrisch-manometrischer Methode mit konstanten Volumina (isochore Durchführung) konstruiert worden. Die der Gleichgewichtszelle zugeführten Komponentenmengen müssen für die synthetische Methode vorgelagert ermittelt werden. Die Lösungsmittelmenge wird mittels Wägung, die Gasmenge mittels Zustand im Gasvorratsbehälter und bekanntem p$V$ - $T$-Verhalten ermittelt. Für die Ermittlung der absorbierten Gasmenge und des eingestellten Phasengleichgewichts verbleiben als einzig notwendige Messgrößen während des Versuchs Druck und Temperatur. Dies war entsprechend [Wagner, 2010] u.a. ausschlaggebend für die Wahl des Verfahrens, da für das Messen dieser beiden Größen umfangreiche Erfahrungen vorlagen und Materialkosten vergleichsweise gering sind. Darüber hinaus mussten andere Verfahren aufgrund der Anforderungen an Messunsicherheit, konstruktiven Unwägbarkeiten z. B. hinsichtlich Leckage sowie aufgrund des geforderten Druck- und Temperatur-Bereichs ausgeschlossen werden. Die hier verwendete Apparatur ist prinzipiell für einen Einsatzbereich von 0 bar bis 10 bar und Temperaturen von $-10^{\circ} \mathrm{C}$ bis $150^{\circ} \mathrm{C}$ ausgelegt.

Bereits die Voruntersuchungen in [Wagner, 2010] hatten gezeigt, dass Apparaturen zur Bestimmung der technischen Löslichkeitskoeffizienten $\lambda_{i}(t)$ für Wasser-Glykol-Gemische nur dann mit einem vergleichsweise geringen Finanzbudget umsetzbar sind, wenn eine Kalibrierung der Apparatur unter Zuhilfenahme der bekannten Löslichkeitskoeffizienten für Wasser gelingt.

Weitere Aspekte, die in die gewählte Bestimmungsmethode und Konstruktion einflossen, waren:

- Untersuchung von Mehrkomponentensystemen unbekannter Zusammensetzung.

- Vermeidung der Veränderung der Gemischzusammensetzung mit möglicherweise stark flüchtigen Komponenten. Dementsprechend sind Verdunstungsvorgänge beim Probenhandling zu minimieren.

- Werkstoffverträglichkeit der Bauteile mit den zu untersuchenden Stoffen.

- Gute Reinigbarkeit der Oberflächen der Apparatur und der Sensorik auf Grund der nicht auszuschließenden Schutzschichtbildung durch inhibierende Bestandteile der Flüssigkeiten.

- Optische Kontrollmöglichkeit des Gemisches während der Absorption für ggf. auftretende chemische Prozesse, z. B. Farbveränderungen, Ausfällungen.

Weitere Erläuterungen dazu finden sich in [Wagner, 2010] und [Rühling u.a., 2013b]. 


\subsection{Beschreibung der Versuchsapparatur}

\subsubsection{Prinzip}

Der Versuchsaufbau (Abbildung 4.1) besteht aus zwei festen Messzellen: Gasreservoir und Reaktorgefäß. Diese werden durch den Thermostat temperiert und sind mit Temperaturund Drucksensorik ausgestattet. Im Gasreservoir ist das zu untersuchende Gas in Reinform bevorratet, im Reaktorgefäß (oft auch als Gleichgewichtszelle bezeichnet) befinden sich eine durch vorhergehende Wägung bestimmte Menge Flüssigkeit und eine Gasphase. Bei konstanter Temperatur werden durch definierte Übergabe von reinem Gas aus dem Gasreservoir über das Ventil V2 in das Reaktorgefäß Gleichgewichtszustände im Reaktorgefäß hergestellt. Aus der zugegebenen Menge an Gas und der Druckdifferenz zwischen zwei gesättigten, stationären Zuständen kann auf die in Lösung gegangene Menge des Gases geschlossen werden. Aufgrund der im Vergleich zur Flüssigkeitsmenge geringen Grenzfläche gasförmig/flüssig muss die Einstellung des Gleichgewichtszustandes durch einen Rührprozess unterstützt werden. Zur Berechnung der Löslichkeitskoeffizienten sind mindestens zwei Gleichgewichtszustände bei einer definierten Temperatur $t$ erforderlich.

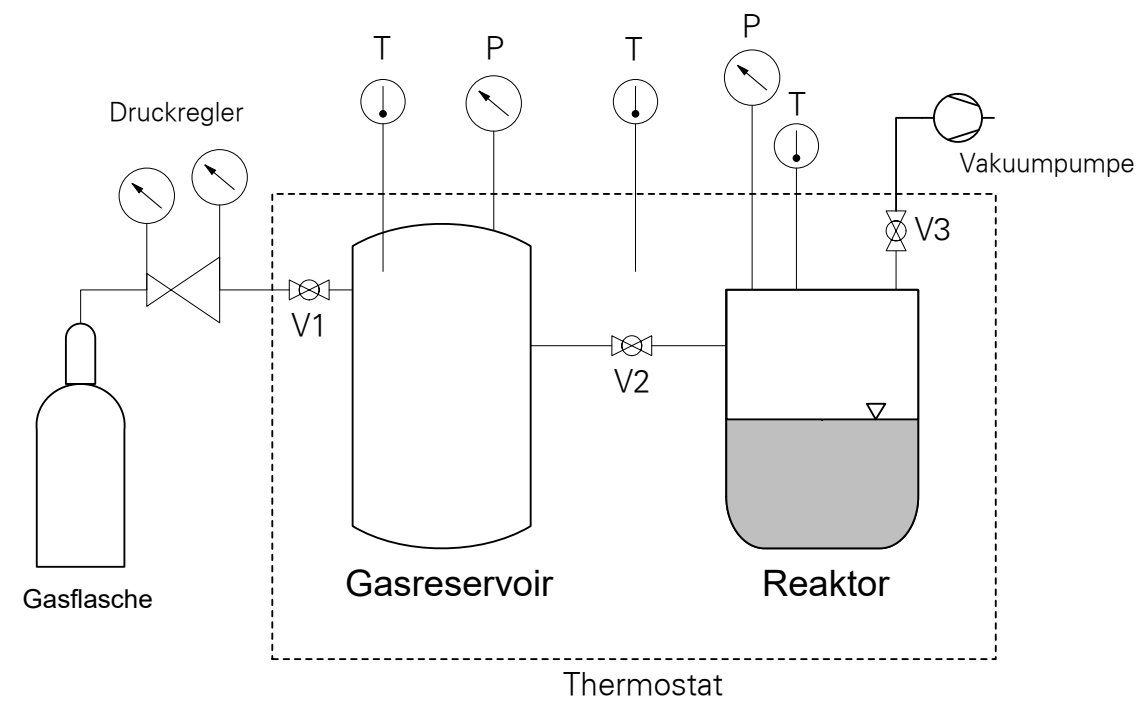

Abbildung 4.1: Prinzipieller Aufbau der Apparatur nach [Wagner, 2010]

\subsubsection{Kalibrierung mit Wasser}

Aufgrund der (entsprechend der Literaturwerte) zu erwartenden geringen Gaslöslichkeiten für Stickstoff und Sauerstoff ist eine Messapparatur mit hohen Güteanforderungen hinsichtlich isothermer, nahezu stoffdichter Systemgrenze, sehr präziser Messtechnik und einer festgelegten, stets gleichen Vorgehensweise bei der Versuchsdurchführung notwendig. Da mit Wasser eine Flüssigkeit zur Verfügung steht, die Gemischbestandteil der zu untersuchenden Wasser-Glykole ist, ähnliche Stoffeigenschaften und auch vermutlich „ähnliche“ Löslichkeitseigenschaften (ausgehend von den in der Literatur gegebenen Löslichkeitskoeffizienten für $25^{\circ} \mathrm{C}$ ) aufweist und für Stickstoff und Sauerstoff in Wasser die Löslichkeitskoeffizienten mit guter Genauigkeit bekannt sind, sollen Referenzversuche mit Wasser durchgeführt werden. Eine Quantifizierung der Abweichungen zu tabellierten Löslichkeitskoeffizienten für Wasser 
erlaubt dann eine Kalibrierung der Apparatur. Für jeden durchgeführten Versuch gibt es also einen zugehörigen Referenzversuch mit Wasser. ${ }^{1}$

Damit wird es möglich, Löslichkeitskoeffizienten für Wasser-Glykol-Gemische mit relativ hoher Genauigkeit mit einer vergleichsweise einfachen Apparatur zu bestimmen.

\subsubsection{Detailaufbau und Messtechnik}

\subsubsection{R\&I-Schema}

In Abbildung 4.2 und in Tabelle 4.1 ist die konstruktive und messtechnische Umsetzung dargestellt, die in den folgenden Unterpunkten erläutert wird.

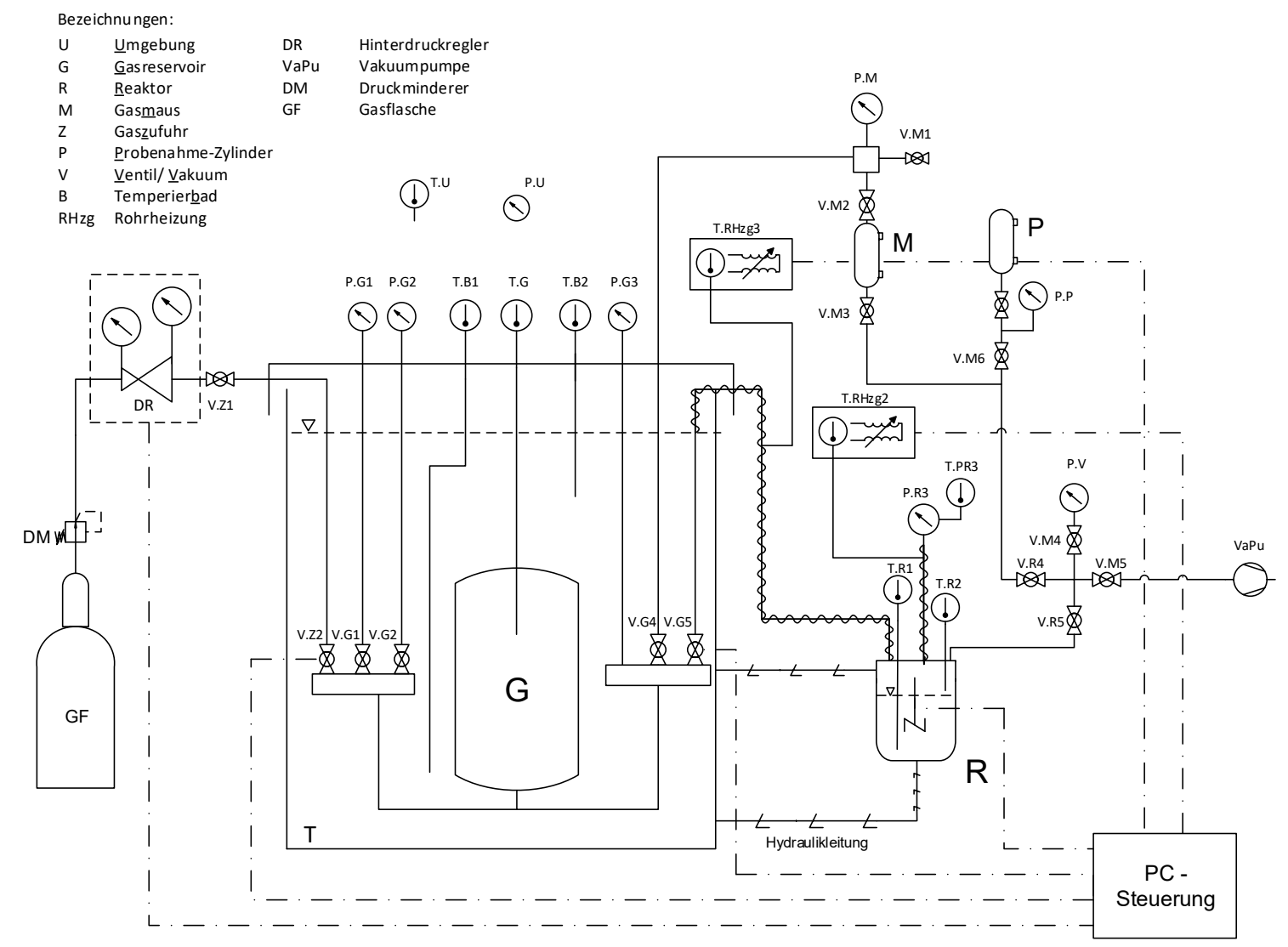

Abbildung 4.2: Detailaufbau der Apparatur (Konfiguration D, andere Konfigurationen im Anhang A.4)

\footnotetext{
${ }^{1}$ Insofern keine Umbauten/Reparaturen vorgenommen wurden, Versuche gleicher Temperatur unmittelbar anschlossen und keine größeren Pausen zwischen den Versuchen lagen, wurde teilweise ein Referenzversuch für mehrere Versuche verwendet.
} 
Tabelle 4.1: Aufbau der Apparatur

\begin{tabular}{llll}
\hline Bezeichnung & Funktion / Messbereich & Bezeichnung & Funktion / Messbereich \\
\hline (P.G1) & Druck Gasreservoir 0..2 bar & V.Z1/Z2 & Ventil \\
(P.G2) & Druck Gasreservoir 0..6 bar & V.G1/G2/G5 & Ventil \\
P.G3 & Druck Gasreservoir 0...16 bar & V.R4/R5(/R1/R2) & Ventil \\
(P.R1) & Druck Reaktor 0...2 bar & V.M1-M6 & Ventil \\
(P.R2) & Druck Reaktor 0...6 bar & & \\
P.R3 & Druck Reaktor 0...16 bar & B & Temperierbad \\
P.M & Druck Gasmaus 0...3 bar & G & Gasreservoir \\
P.V & Druck bei Evakuierung 0...200 mbar & GF & Gasflasche \\
P.P & Druck Probe 0...10 bar & DM & Druckminderer \\
P.U & Druck Umgebung 0...3 bar & DR & Digitaler Hinterdruckregler \\
T.G & Temperatur Gasreservoir & M & Gasmaus \\
T.R1 & Temperatur Fluid im Reaktor & P & Probezylinder \\
T.R2 & Temperatur Gasphase im Reaktor & R & Reaktorgefäß \\
T.B1 & Öltemperatur im Temperierbad oben & VaPu & Vakuumpumpe \\
T.B2 & Öltemperatur im Temperierbad unten & W & Waage 0...7000g \\
(T.RHzg1) & Temperatur 1 Totvolumen $V^{\text {R1 }}$ & & \\
T.RHzg2 & Temperatur 2 Totvolumen $V^{\text {R1 }}$ & & \\
T.RHzg3 & Temperatur Totvolumen $V^{\text {R2 }}$ & & \\
T.U & Temperatur Umgebung & & \\
\hline
\end{tabular}

\subsubsection{Gasreservoir G und Gaszufuhr}

Als Gasreservoir wird ein Probenentnahmezylinder (handelsüblicher Zylinder aus nichtrostendem Stahl) genutzt, der komplett in das Temperierbad eingelassen ist. Dieser ist verbunden mit der Gasflasche GF zur Gaszuführung, mit dem Reaktorgefäß R zur Übergabe des Probegases und mit der Vakuumpumpe VaPu zum Spülen des Gefäßes. Der Zylinder besitzt ein Fassungsvermögen von ca. $1 \mathrm{dm}^{3}$.

Die Gaszufuhr erfolgt aus der Gasflasche über einen Druckminderer DM und eine diffusionsdichte Leitung bis zum Gasreservoir. Zur automatischen und definierten Druckbeladung des Gasreservoirs wird ein Hinterdruckregler DR eingesetzt. Die gasdichte Absperrung erfolgt über Kugelhähne (V.Z1, V.Z2).

\subsubsection{Reaktorgefäß R}

Der Reaktor R stellt das Kernstück der Gesamtapparatur dar. Fokus bei der Wahl des Reaktorgefäßes lag auf der optischen Kontrolle der Reaktionsprodukte sowie der Beobachtung des Mischungsvorgangs der Phasen. Daher besteht das etwa $1 \mathrm{dm}^{3}$ fassende Reaktorgefäß aus doppelwandigem Glas. Der Zwischenraum, auch als Heizmantel bezeichnet, wird mit transparentem Thermoöl durchströmt und dient der Temperierung des Gefäßes. Der Deckelflansch aus nichtrostendem Stahl wird ebenso mit Thermoöl durchströmt und dadurch temperiert. Zur Unterstützung der Absorption für ein zügiges Einstellen eines stationären Zustands wurde eine magnetisch angetriebene Begasungsrührwelle genutzt. 


\subsubsection{Temperierung}

Ein Temperierbad übernimmt die Temperierung des Gasreservoirs. Das gesamte Volumen des Gasreservoirs soll im Sinne einer isothermen Systemgrenze temperiert sein. Deshalb sind auch jegliche Ventile im Temperierbad platziert und nur deren Hebel durch Verlängerungen aus dem Temperierbad herausgeführt worden. Mittels Hydraulikleitungen wird Temperieröl aus dem Temperierbad in die zur Temperierung durchströmten Bauteile des Reaktors gepumpt.

Die zum Reaktorgefäßvolumen zugehörigen Anschlussleitungen und Drucksensorzuführungen werden mittels elektrischer Heizschnüre auf Solltemperatur temperiert. Die Beheizung der Toträume soll große, schwer abschätzbare Temperaturgradienten im Volumen, eigenständige Schwerkraftströmungen und innere Kondensation vermeiden.

Zur gleichmäßigen Temperatureinstellung, Eingrenzung des Wärmeverlusts und weitgehenden Unabhängigkeit von Außentemperaturschwankungen wurde das Reaktorgefäß nach außen vollständig gedämmt. Dies war aufgrund der äußeren Temperierung von Reaktorbehälter und -deckel vorerst nicht angedacht, jedoch wurden anhand der vorhandenen Temperatursensoren erhebliche Temperaturunterschiede im Reaktorvolumen festgestellt. Diese werden durch unvollkommene Temperierung (ungleichmäßige Durchströmung des Heizmantels des Reaktors und nicht ausschleusbare Luft im Heizmantel, starke Wärmeableitung in Anbauteile und Halterung des Reaktors) verursacht.

\subsubsection{Messtechnik}

\section{Temperaturmessung}

Die Temperaturmessung erfolgt mit Pt-100 Mantelwiderstandsthermometern. Um im Temperierbad eine gute Durchmischung überprüfen zu können, sind zwei Thermometer (T.B1, T.B2) im Thermoöl angebracht. Um wirklich stationäre Bedingungen im Gasreservoir erreichen und kontrollieren zu können, wird zusätzlich direkt die Temperatur des Gases im Gasreservoir (T.G) gemessen. Im Reaktor werden sowohl die Temperatur im Fluid (T.R1) als auch die im Gas (T.R2) gemessen.

Die Temperaturen in den elektrisch beheizten Toträumen werden (zur Heizleistungseinstellung und Auswertung) getrennt benötigt und mittels Anlegefühler erfasst (T.RHzg1/2/3).

Nachträglich wurden weiterhin der Sensor T.PR3 (und T.PR2 in einer früheren Konfiguration) zur Messung der Drucksensortemperatur angebracht, um die Einstellung stationärer Zustände auch an den Drucksensoren überprüfen zu können. Weiterhin besteht damit die Möglichkeit einer temperaturabhängigen Kalibrierung der Drucksensoren.

\section{Druckmessung}

In Anlehnung an die Druckmessanordnung in der Arbeit von [Meyer, 2002] hat [Wagner, 2010] die Apparatur ursprünglich mit absperrbaren Absolutdrucksensoren in Abstufungen der Messbereiche $0 . .2$ bar (P.G1 und P.R1), $0 . .6$ bar (P.G2 und P.R2) und 0..16 bar (P.G3 und P.R3) ausgestattet. Dadurch sollten je nach Messpunkt die entsprechenden Sensoren zugeschaltet und gleichzeitig der Messfehler minimiert werden. Die Drucksensoren wurden entsprechend der Anforderungen und mit Fokus auf hohe Genauigkeit, frontbündige Membran und Resistenz gegen korrosive und inhibierende Medien ausgewählt. 
Es konnte festgestellt werden, dass sich die Unsicherheiten durch Nutzung unterschiedlicher Drucksensoren je nach Druckstufe und folglich auch unterschiedlicher Volumina in einem Versuch deutlich erhöhen und nicht zielführend sind. Weiterhin zeigten die verwendeten Drucksensoren $0 \ldots 2$ bar und $0 \ldots 6$ bar starke Temperatursensitivität. Für alle Versuche dieser Arbeit sind nur die Drucksensoren P.G3 und P.R3 mit einem Wertebereich bis 16 bar verwendet worden.

\subsubsection{Sonstige Bauteile}

Die Vakuumpumpe VaPu wird genutzt, um Gasreservoir und Reaktorgefäß annähernd luftund feuchtefrei zu machen. Bei späteren Versuchen wird die Vakuumpumpe auch genutzt, um die Flüssigkeit vor Versuchsbeginn zu entgasen.

Bei der Gasmaus M handelt es sich um einen beidseitigen Entnahmezylinder mit $75 \mathrm{ml}$ Fassungsvermögen. Diese wurde für diverse Hilfestellungen genutzt. Beispielsweise wurde die Gasmaus als Referenzvolumen für das Auslitern von Gasreservoir und Reaktor genutzt. Weiterhin diente sie für spätere Versuche als Hilfsvolumen zur definierten sukzessiven Entgasung der Flüssigkeit.

Für die Untersuchung der Gaszusammensetzung der Gasphase im Reaktorgefäß wurde ein diffusionsdichter Probenahmezylinder $\mathrm{P}$ installiert.

\subsubsection{Arbeitsgas}

Das für die Löslichkeitsmessungen benötigte hochreine Gas, im Folgenden auch Arbeitsgas genannt, wird von der Firma Air Liquide bezogen. Die entsprechende Zusammensetzung ist in Tabelle 4.2 dargestellt. Für die Versuche mit Luft als Arbeitsgas wird synthetische Luft verwendet. Es wurde erst nach den Versuchen festgestellt, dass in dem verwendeten Produkt das Verhältnis von Sauerstoff und Stickstoff, wie in Tabelle 4.3 zu sehen, nur mit relativ hoher Unsicherheit von 0,5\% eingestellt wird. Zukünftig sollte man hier ein deutlich präziser eingestelltes Produkt verwenden. Auf das Messergebnis hat dies jedoch durch die Kalibrierung der Versuche durch Referenzversuche mit Wasser nur geringen Einfluss.

Tabelle 4.2: Zusammensetzung des verwendeten Stickstoff (Alphagaz 2) nach [Air Liquide]

\begin{tabular}{c|cccccc}
\hline Stickstoff & \multicolumn{6}{|c}{ Fremdanteil (ppm-mol) } \\
$\mathrm{N}_{2}($ Vol- $\%)$ & $\mathrm{H}_{2} \mathrm{O}$ & $\mathrm{O}_{2}$ & $\mathrm{C}_{\mathrm{n}} \mathrm{H}_{\mathrm{m}}$ & $\mathrm{CO}$ & $\mathrm{CO}_{2}$ & $\mathrm{H}_{2}$ \\
\hline 99,9999 & $<0,5$ & $<0,1$ & $<0,1$ & $<0,1$ & $<0,1$ & $<0,1$ \\
\hline
\end{tabular}

Tabelle 4.3: Zusammensetzung Synthetische Luft KW-frei nach [Air Liquide 2]

\begin{tabular}{|c|c|c|c|c|c|}
\hline Stickstoff & Sauerstoff & \multicolumn{4}{|c|}{ Fremdanteil (ppm-mol) } \\
\hline $\mathrm{N}_{2}(\mathrm{Vol}-\%)$ & $\mathrm{O}_{2}(\mathrm{Vol}-\%)$ & $\mathrm{H}_{2} \mathrm{O}$ & $\mathrm{C}_{n} \mathrm{H}_{\mathrm{m}}$ & $\mathrm{CO}_{2}$ & $\mathrm{NO}_{x}$ \\
\hline $79,5 \pm 0,5$ & $20,5 \pm 0,5$ & $<0,5$ & $<0,1$ & $<0,5$ & $<0,1$ \\
\hline
\end{tabular}

\subsubsection{Automatisierung}

Der Versuchsstand wird weitestgehend automatisiert betrieben. Folgende Elemente werden gesteuert bzw. geregelt: Thermostat, Hinterdruckregler (HDR), Ventile V.Z2 und V.G5, Rührer und Temperatur der Begleitheizbänder. 
Architektur und verwendete Schnittstellen der Steuerung sind im Schema in Abbildung 4.3 dargestellt. Neben manuellen Messungen (Wägung) und manuellen Stellhandlungen in Vor- oder Nachbereitung, die in einem separaten Protokoll dokumentiert werden (,protokoll.xls“), wird für den automatisierten Versuchsablaufs ein definierter Ablauf („automationRegime.csv") eingeladen und der zeitliche Ablauf entsprechend protokolliert („automationProtokoll.csv").

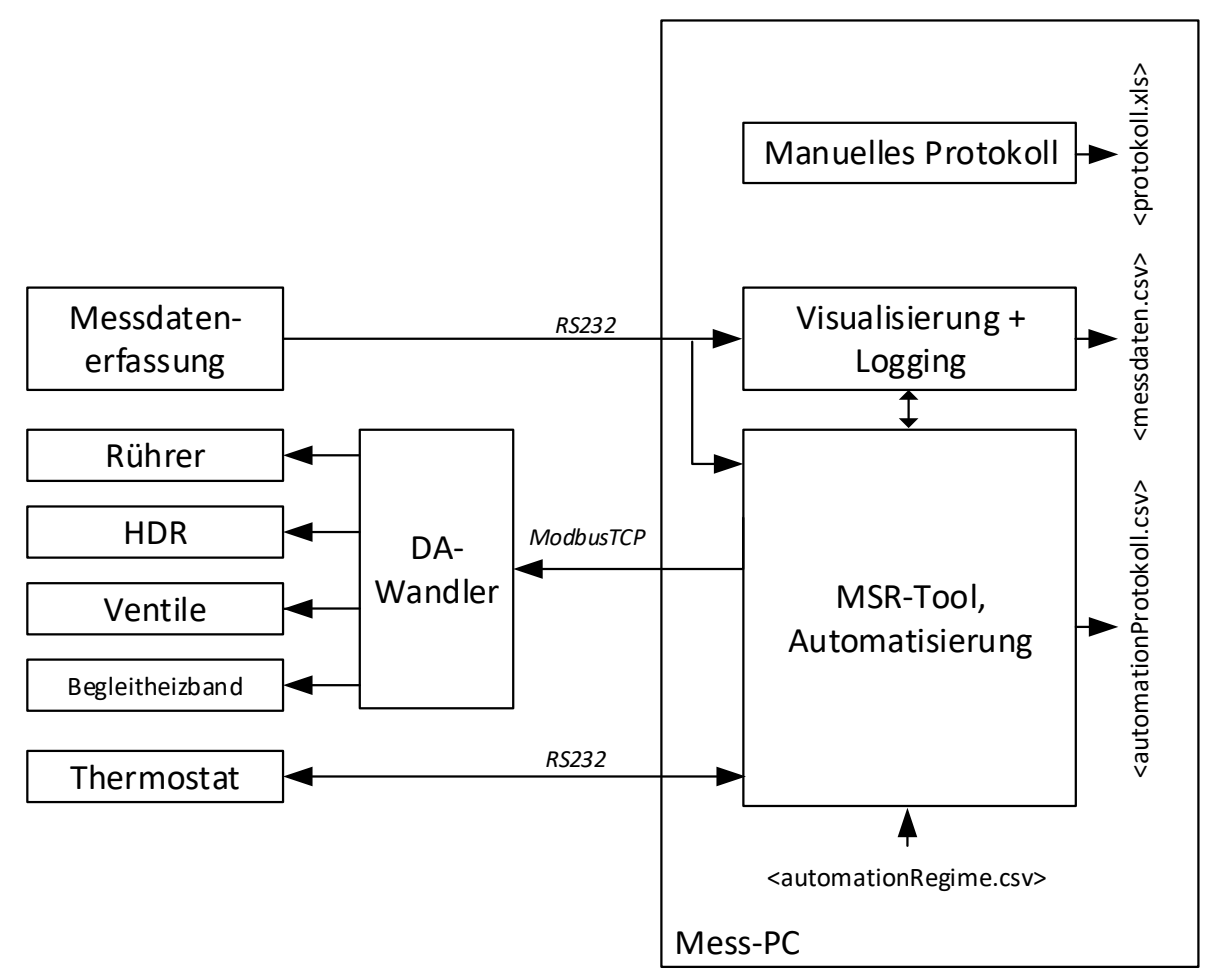

Abbildung 4.3: MSR-Konzept

Die zeitliche Umsetzung des Versuchsablaufs wird über Timer (z. B. „120 Sekunden warten“) und Events (z.B. „stationärer Zustand erreicht") realisiert. Die Zeitpunkte der Eingriffe und Events werden gespeichert (in Datei automationProtokoll.csv). Zusätzlich sind sowohl programmtechnisch Sicherheitsfunktionen als auch programmunabhängige autarke Temperaturwächter für einen sicheren, vollständig automatisierten Betrieb eingebaut.

Durch die Automatisierung werden vor allem Bedienungsfehler vermieden und ein annähernd gleicher Ablauf der Versuche sichergestellt. Durch die automatische Speicherung der Zeitpunkte zu Aktionen und Events werden Protokollierungsfehler und daraus folgende Auswertefehler minimiert. Die Automatisierung ist insbesondere für die Kalibrierung der Versuche durch vorgelagerte, exakt gleich ablaufende Versuche mit Wasser hilfreich und ermöglicht die reproduzierbare Durchführung von langwierigen Versuchen.

Die ebenso automatisierte rechentechnische Auswertung nach Abschluss der Versuche, d. h. Berechnung der Löslichkeitskoeffizienten, erfolgt auf Grundlage der archivierten versuchsspezifischen Daten:

- manuelles Protokoll (<protokoll.xls $>$ ) mit Angaben u. a. zu Solltemperatur, untersuchtem Stoff, Gasart, eingefüllter Masse,

- Messdaten (<messdaten.csv $>$ ) und 
- gespeicherte Zeitpunkte der Eingriffe und Events (<automationProtokoll.csv>).

\subsection{Versuchsablauf}

\subsubsection{Besonderheiten in der Messmethode}

\subsubsection{Ausgangszustand des Lösungsmittels}

Für den Ausgangszustand des Reaktors und demnach auch des Lösungsmittels für den Versuch wird unterschieden in die Varianten

- luftgesättigt und

- entgast.

Nach dem Anbau des befüllten Reaktorgefäßes befindet sich über der Flüssigkeit Umgebungsluft als Gasphase. ${ }^{2}$ Zur Bestimmung der Löslichkeit des Arbeitsgases im Lösungsmittel sollten sich idealerweise nur Arbeitsgas und Lösungsmittel im Reaktorgemisch befinden und der Ausgangszustand das reine, entgaste Lösungsmittel beim Dampfdruck des Lösungmittels sein. Jedoch wurde für erste Stickstoff-Versuche als Ausgangzustand im Reaktor der stoffliche Zustand nach dem Anbau des Reaktorgefäßes verwendet. Folgende Gründe sind dafür zu nennen:

- Ein einfacher und schneller Versuchsstart ohne aufwendiges Entgasen der Flüssigkeit ist möglich.

- Ein Austrag von Lösungsmitteldampf während der Entgasung und damit ein Verfälschen der gemessenen eingefüllten Lösungsmittelmasse wird verhindert.

- Bei der Entgasung findet möglicherweise ein unproportionaler Austrag der Lösungsmittelkomponenten statt. Dies würde insbesondere bei stark flüchtigen Lösungsmittelkomponenten zu einer Änderung der Zusammensetzung der Flüssigkeit führen. Damit ist die Variante auch für gealterte Fertiggemische unbekannter Zusammensetzung mit möglicherweise stark flüchtigen Komponenten geeignet.

Diese Variante hat jedoch folgende Nachteile:

- Messung der Löslichkeit kann für das Arbeitsgas Stickstoff nicht bei Partialdruck $p_{\mathrm{N} 2}=$ 0 bar beginnen.

- Die aus der Luft verbleibenden Störgase (Sauerstoff, Argon, Kohlendioxid, Neon etc.) könnten mit dem zu untersuchenden Gas und dessen Löslichkeit wechselwirken.

- Chemische Reaktionen des Restgases mit dem Lösungsmittel sind möglich (Sauerstoffabbau).

- Die genaue Zusammensetzung der Gasphase und der Partialdruck des Arbeitsgases $p_{\mathrm{N} 2}$ sind (ohne zusätzliche Messungen) nur abschätzbar, aber nicht bekannt.

- Desorption/Absorption/Reaktion der Störgase im Versuchsverlauf sind nicht ausgeschlossen.

\footnotetext{
${ }^{2}$ Es wird davon ausgegangen, dass die Lösungsmittel beim Einfüllen bereits näherungsweise für Umgebungsdruck luftgesättigt sind, da die Lösungsmittel in diffusionsoffenen Behältern aufbewahrt werden. Daher sollten auch gelöste Gase nur Atmosphärengase sein.
} 
Für die in dieser Arbeit angestrebte Messgenauigkeit soll dieser Ansatz ausreichend sein. Dieser Ausgangszustand wird vereinfacht als luftgesättigt bezeichnet.

Im Verlauf der Stickstoff-Versuche hat sich gezeigt, dass teilweise chemische Reaktionen des Sauerstoffs stattfinden und diese die Auswertung der Stickstoff-Versuche entsprechend erschweren. Als zusätzlich erkennbar war, dass keine gealterten Wasser-Glykol-Gemische mit möglicherweise stark flüchtigen Komponenten im Rahmen dieser Arbeit zur Verfügung stehen würden, ist für die nachfolgenden Versuche ein stark entgaster Ausgangszustand im Reaktor und der darin befindlichen Flüssigkeit hergestellt worden. Die Gase im Reaktorgefäß werden dazu in ein evakuiertes Volumen (Gasmaus) abgesaugt. Die Desorption der in der Flüssigphase gelösten Gase wird durch Rühren unterstützt. Dieser Vorgang wird wiederholt, bis die Druckänderung im Reaktorgefäß in den letzten drei Zyklen jeweils deutlich unter $1 \mathrm{mbar}$ liegt und also der Dampfdruck nahezu erreicht ist.

Eine vollständige Entgasung wird hier jedoch ausdrücklich nicht erreicht, da der Fokus auf der Begrenzung des Lösungsmittelsaustrags und der Zusammensetzungsänderung lag. Jedoch wird die Partialdrucksumme der eingeschlossenen Gase, basierend auf dem asymptotischen Verlauf des Drucks während des iterativen Entgasungsablaufs, stets als deutlich unter 10 mbar liegend abgeschätzt. Dadurch ergeben sich einige Vorteile gegenüber der Variante mit luftgesättigtem Ausgangszustand:

- Es liegt ein nahezu reines Lösungsmittel bzw. im weiteren Versuchsverlauf reines Arbeitsgas-Lösungsmittel-Gemisch vor. Auswirkungen von möglichen Wechselwirkungen und Reaktionen der Störgase sind dadurch marginalisiert.

- Die Messung der Löslichkeit der Arbeitsgase $i$ kann bei Partialdruck nahe $p_{i}=0$ bar beginnen.

Der mit der Entgasung stattfindende zusätzliche Lösungsmittelaustrag wird entsprechend berücksichtigt.

\subsubsection{Ableitung von Messmethode und Löslichkeitskoeffizienten}

\section{Hintergrund}

In der Literatur werden Henry-Koeffizienten bzw. technische Löslichkeitskoeffizienten oft bestimmt, indem vollständig entgastes Fluid im zweiten Schritt mit Gas $i$ beladen wird, bis ein Partialdruck $p_{i}=1$ bar erreicht ist. Kennt man die in Lösung gegangene Menge Gas, lässt sich der Stoffmengenanteil $x_{i}$ und schließlich der Löslichkeitskoeffizient unter Anwendung der Approximationen leicht bestimmen nach Gleichung 2.42

$$
\lambda_{i, \infty}=\frac{n_{i} \cdot v_{0, i, \mathrm{~N}} \cdot \operatorname{Poy}\left(p ; p_{\mathrm{LM}, \mathrm{s}}\right)}{m_{\mathrm{LM}} \cdot p_{i}}
$$

bzw. mit dem absoluten Gasgehalt $w_{i}$ nach Tabelle $2.2 \mathrm{zu}$

$$
\lambda_{i, \infty}=\frac{w_{i}}{p_{i}} \cdot \operatorname{Poy}_{i}\left(p_{\mathrm{LM}, \mathrm{s}} ; p\right) \text {. }
$$

Dies kann hier nicht angewendet werden, da für die genutzte Methodik sowohl für den als „luftgesättigt" als auch für den als „entgast" bezeichneten Ausgangszustand wegen genannter Gründe kein komplett gasfreies Phasengleichgewicht mit $p_{i, 1}=0$ bzw. $w_{i, 1}=0$ vorliegt. Daraus folgt insbesondere für den Ausgangszustand luftgesättigt, in geringerem Maß aber auch für den Ausgangszustand entgast: 
- Der absolute Gasgehalt $w_{i}$ der gesättigten Lösungen kann bei dem verwendeten Verfahren nicht bestimmt bzw. nur mit hoher Unsicherheit abgeschätzt werden. Die Änderung zwischen zwei Zuständen $\Delta w_{i, 1 \rightarrow 2}$ durch Zugabe von Arbeitsgas aus dem Gasreservoir ist hingegen bestimmbar.

- Ebenso kann der absolute Partialdruck $p_{i}$ des Arbeitsgases $i$ auch nicht bestimmt werden bzw. nur mit hoher Unsicherheit abgeschätzt werden. Die Änderung $\triangle p_{i, 1 \rightarrow 2}$ zwischen zwei Zuständen, die nur durch die Zugabe des Arbeitsgases hervorgerufen wird, ist hingegen bestimmbar.

\section{Alternative Bestimmung im Versuch}

Vernachlässigt man die Poynting-Korrektur, kann der Löslichkeitskoeffizient $\lambda_{i}$ für eine Temperatur $t$ auch als Anstieg der linearen Funktion $w_{i}\left(p_{i}\right)=\lambda_{i} \cdot p_{i}$, wie in Abbildung 4.4 links dargestellt, interpretiert werden. Mittlere Anstiege können messtechnisch bestimmt werden: Dazu werden $\triangle w_{i}$ und $\triangle p_{i}$ in den Versuchen durch Auswertung der Differenzen zwischen jeweils zwei Phasengleichgewichten (GGW) 1 und 2 bestimmt. Der Löslichkeitskoeffizient ergäbe sich dann zu

$$
\left.\lambda_{i}\right|_{\left[p_{i, 1} ; p_{i, 2}\right]}=\frac{w_{i, 2}-w_{i, 1}}{p_{i, 2}-p_{i, 1}}=\frac{\triangle w_{i}}{\triangle p_{i}} .
$$

Berücksichtigt man die Poynting-Korrektur, gilt $w_{i}\left(p_{i}\right)=\lambda_{i, \infty} \cdot p_{i}^{*}$ mit $p_{i}^{*}=\frac{p_{i}}{\operatorname{Poy}\left(\left(p_{\llcorner\mathrm{M}, \mathrm{s}} ; p\right)\right.}$, und es ergibt sich

$$
\left.\lambda_{i, \infty}\right|_{\left[p_{i, 1} ; p_{i, 2}\right]}=\frac{w_{i, 2}-w_{i, 1}}{p_{i, 2}^{*}-p_{i, 1}^{*}}=\frac{\triangle w_{i}}{\Delta p_{i}^{*}}=\frac{\Delta w_{i}}{\frac{p_{i, 2}}{\operatorname{Poy}\left(p_{2}, p_{\llcorner\mathrm{M}, s}\right)}-\frac{p_{i, 1}}{\operatorname{Poy}\left(p_{1}, p_{\llcorner\mathrm{M}, \mathrm{s}}\right)}} .
$$

In der Gleichung ist nun leider nicht mehr die Partialdruckdifferenz $\Delta p_{i}$, sondern sind doch die Partialdrücke $p_{i, 1}$ und $p_{i, 2}$ notwendig. Die Gleichung lässt sich aber auch schreiben als

$$
\left.\lambda_{i, \infty}\right|_{\left[p_{i, 1} ; p_{i, 2}\right]}=\frac{\Delta w_{i}}{\Delta p_{i}+p_{i, 2} \cdot\left(\frac{1}{\operatorname{Poy}\left(p_{2}, p_{\mathrm{LM}, \mathrm{s}}\right)}-1\right)-p_{i, 1} \cdot\left(\frac{1}{\operatorname{Poy}\left(p_{1}, p_{\mathrm{LM}, \mathrm{s}}\right)}-1\right)} .
$$

Da $\frac{1}{P o y}$ nahe eins ist, zeigt sich jetzt, dass sich der Löslichkeitskoeffizient (bei ausreichend großen Partialdrucksprüngen $\Delta p_{i}$ ) im Wesentlichen doch wieder durch die Partialdruckdifferenz $\Delta p_{i}$ bestimmen lässt. Der zweite und dritte Term im Nenner hat nur schwachen Einfluss auf das Messergebnis. Dafür reichen geschätzte absolute Partialdrücke $p_{i, 2}$ und $p_{i, 1}$ aus.

Damit steht eine Berechnungsmöglichkeit des Löslichkeitskoeffizienten zur Verfügung, die nicht auf genaue absolute Partialdrücke angewiesen ist.

\section{„Gültigkeitsbereich der Henry-Konstante“}

Für die untersuchten Flüssigkeiten ist jedoch noch nicht bekannt, ob man sich im Gültigkeitsbereich der Henry-Konstante (Erklärung im Abschnitt 2.1.5) und damit Gültigkeitsbereich des Löslichkeitskoeffizienten befindet, also der Anstieg der Funktion $w_{i}\left(p_{i}^{*}\right)$

$$
\frac{\mathrm{d} w_{i}}{\mathrm{~d} p_{i}^{*}}=\lambda_{i, \infty}=\text { const }
$$


für beliebige $p_{i}^{*}$ im zu untersuchenden Druckbereich überhaupt gilt. Dies kann zumindest stichprobenartig überprüft werden durch die Ermittlung von mittleren Anstiegen für verschiedene Druckbereiche/Drucksprünge (DS) $\left[p_{i, 1} ; p_{i, 2}\right]^{3}$

Im Gültigkeitsbereich des Löslichkeitskoeffizienten muss gelten

$$
\left(\frac{\triangle w_{i}}{\triangle p_{i}^{*}}\right)^{D S 1}=\left(\frac{\triangle w_{i}}{\triangle p_{i}^{*}}\right)^{D S 2}=\ldots=\left(\frac{\triangle w_{i}}{\triangle p_{i}^{*}}\right)^{D S n}
$$

und nur dann kann eigentlich von einem Löslichkeitskoeffizienten mit

$$
\lambda_{i, \infty}=\text { const }=\left(\frac{\triangle w_{i}}{\triangle p_{i}^{*}}\right)
$$

gesprochen werden.

Befindet man sich nicht im Gültigkeitsbereich des Löslichkeitskoeffizienten, so ergibt sich für jeden Druckbereich $\left[p_{i, 1} ; p_{i, 2}\right]$ ein anderer mittlerer Gasgehaltsanstieg $\frac{\triangle w_{i}}{\Delta p_{i}^{*}}$, wie in Abbildung 4.4 schematisch dargestellt. Wird nur ein Drucksprung $\left[p_{i, 1} ; p_{i, 2}\right]$ untersucht, dann kann der Löslichkeitskoeffizient nur unter dem Vorbehalt, dass man sich im Gültigkeitsbereich des Löslichkeitskoeffizienten befindet, angegeben werden.

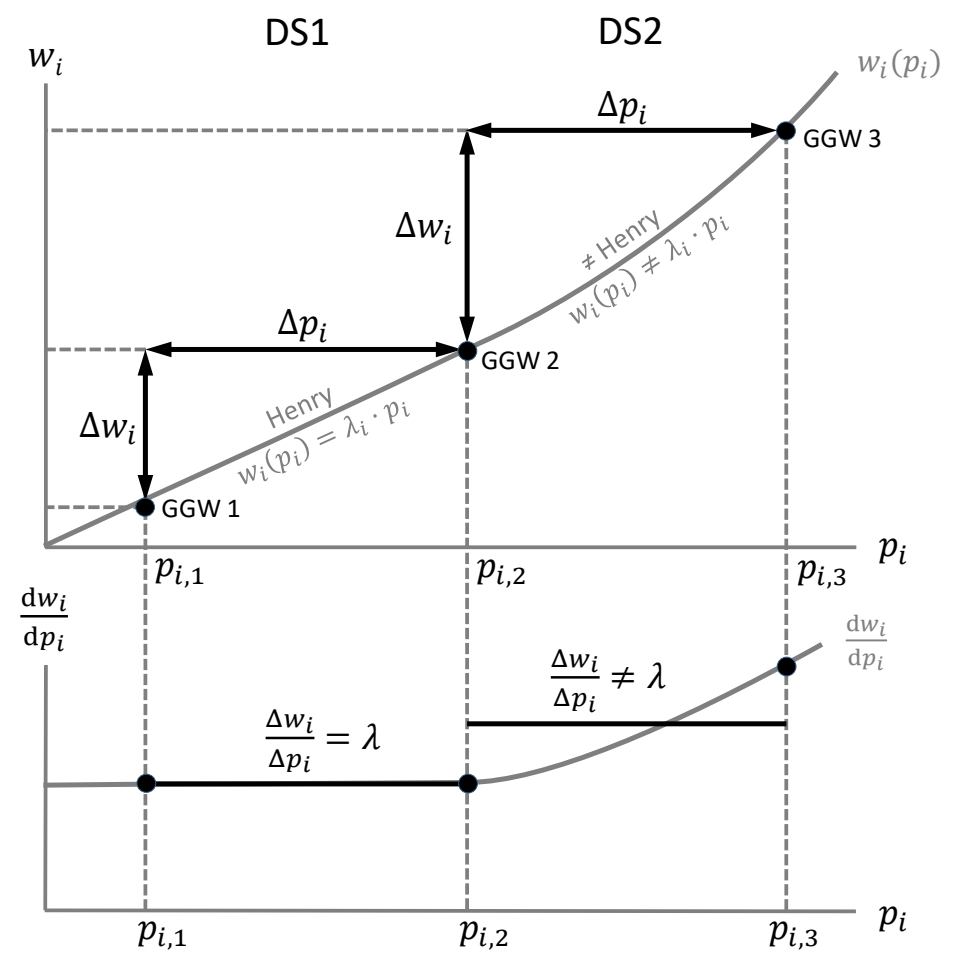

Abbildung 4.4: Schematischer Gasgehaltsverlauf mit zwei Drucksprüngen (DS) bei konst. Temperatur (bei Berücksichigung der Poyntingkorrektur muss $p_{i}$ durch $p_{i}^{*}$ ersetzt werden)

DS1: Verlauf im Gültigkeitsbereich des Löslichkeitskoeffizienten,

DS2: fiktiver Verlauf außerhalb des Gültigkeitsbereichs des Löslichkeitskoeff.

${ }^{3} \mathrm{Im}$ Folgenden wird $\left(\frac{\Delta w_{i}}{\Delta p_{i}^{*}}\right)$ immer als „mittlerer Gasgehaltsanstieg“ im definierten Druckbereich $\left[p_{i, 1} ; p_{i, 2}\right]$ bezeichnet. Für die Bestimmung der Poynting-Korrekturen werden jedoch immer auch die Gesamtdrücke $\left[p_{1} ; p_{2}\right]$ benötigt. 


\section{Realisierung im Versuch}

Für den Nachweis des Gültigkeitsbereich des Henry-Koeffizienten bzw. Löslichkeitskoeffizienten für Stickstoff werden Gasgehaltszunahmen durch Partialdruckzunahmen in unterschiedlichen Druckniveaus untersucht (Abbildung 4.5). Der erste Drucksprung soll die Löslichkeit im Druckbereich bis 6 bar charakterisieren, der zweite Drucksprung im Druckbereich bis 10 bar. Realisiert wird dies durch die Einstellung von drei Phasengleichgewichten sowie durch die Auswertung der sich ergebenden Drucksprünge $1 \rightarrow 2$ bzw. $2 \rightarrow 3$.

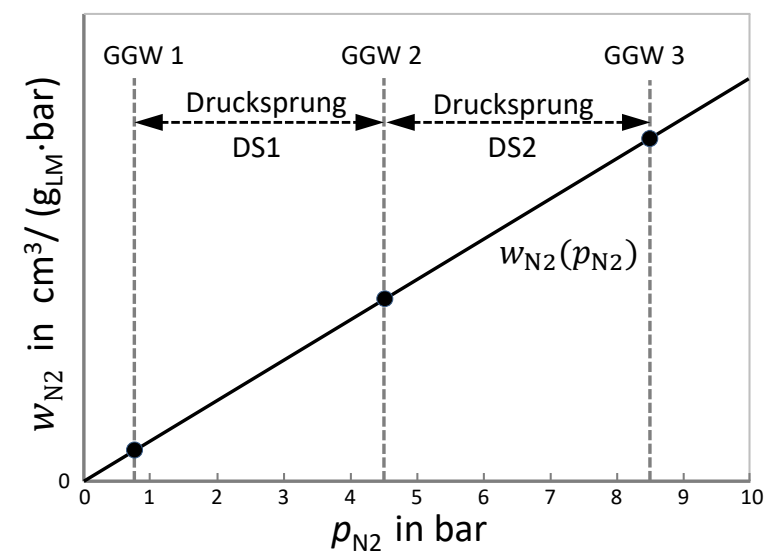

Abbildung 4.5: Richtwerte der Partialdruckniveaus der Phasengleichgewichtsszustände (GGW) im Kontext des Gasgehaltsverlaufs (hier Gültigkeitsbereich des Löslichkeitskoeffizienten angenommen)

\subsubsection{Versuchsablauf}

\subsubsection{Stickstoff als Arbeitsgas}

In Abbildung 4.6 ist für die Drücke in Reaktorgefäß und Gasreservoir (P.R3 bzw. P.G3) und die Temperaturen T.G und T.R2 der Verlauf am Beispiel eines konkreten Stickstoff-Versuchs mit Ausgangszustand luftgesättigt dargestellt. Ein analoger Versuchsablauf für einen Versuch mit entgastem Fluid im Ausgangszustand findet sich in Abbildung 4.7. Die einzelnen Versuchsschritte werden im Folgenden erklärt:

\subsection{Vorspülung Gasreservoir und Reaktorraum}

Das Gasreservoir wird wiederholt evakuiert und mit dem Arbeitsgas Stickstoff gefüllt, um Fremdstoffe beim Versuch im Gasreservoir möglichst ausschließen zu können. Vor Beginn des eigentlichen Versuches wird das getrocknete, leere Reaktorgefäß montiert und mit Luft (bzw. in den Versuchen mit Ausgangszustand entgast mit Arbeitsgas) gespült, um eine definierte, immer gleiche Gasphase beim Zeitpunkt der Montage sicherzustellen (nähere Erläuterung in Abschnitt 4.4.1.1).

1.2 Wägung Lösungsmittel und Befüllen

Nach der Wägung des Lösungsmittels (LM) wird dieses in das geöffnete Reaktorgefäß eingefüllt und der Reaktorraum abgeschlossen. Damit befindet sich das Lösungsmittel (mit unbekannter Ausgangsgasbeladung) und Luft im Reaktorgefäß. Durch ein erstes Rühren und die Kontrolle des Drucks im Reaktorgefäß besteht hier die Möglichkeit festzustellen, ob und in welchem Maße das Lösungsmittel bereits luftgesättigt ist.

1.3 Entgasung des Reaktorgefäßes (nur Versuche mit Ausgangszustand entgast) 


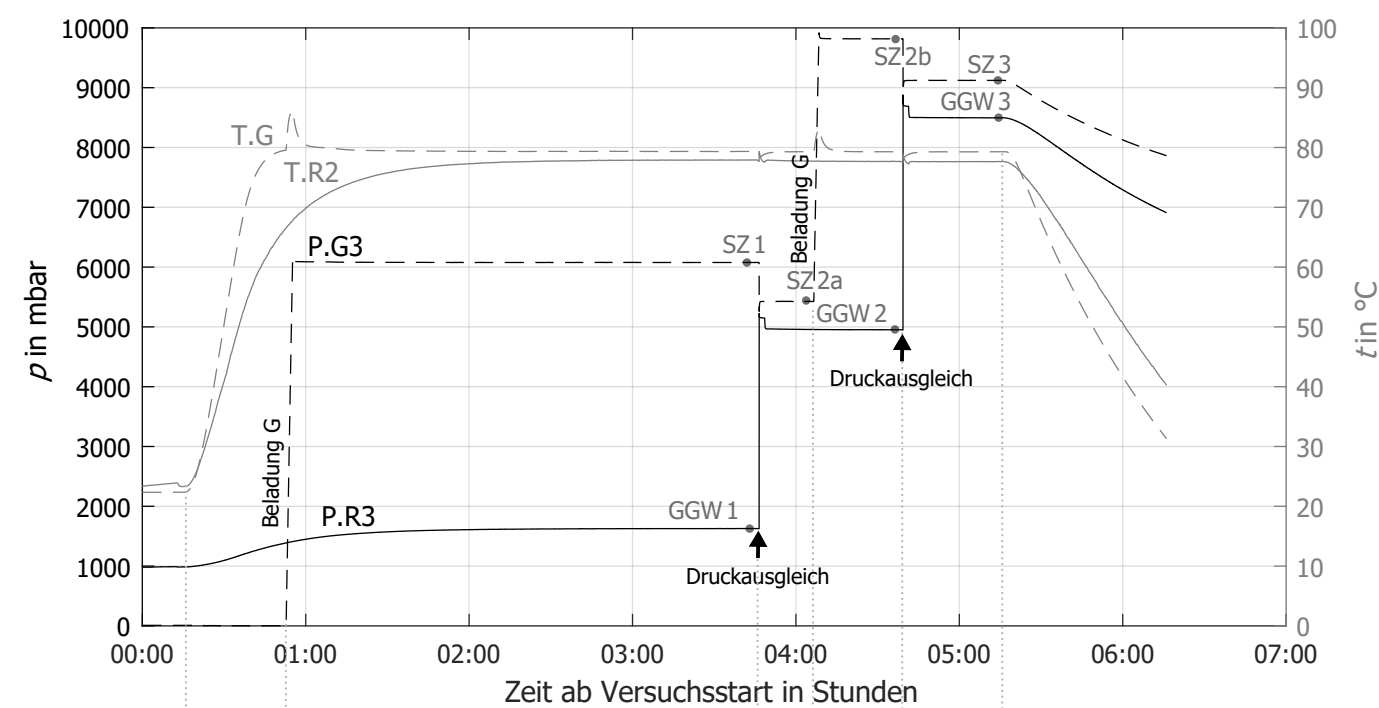

Versuchsschritte:

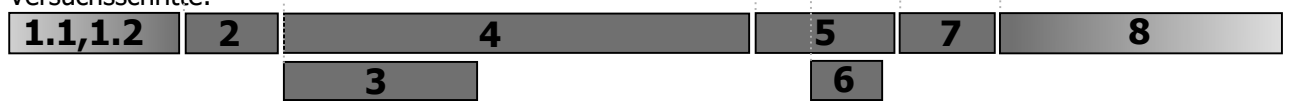

Abbildung 4.6: Versuchsablauf mit Schrittnummern, Ausgangszustand luftgesättigt (hier: 41 gew.-\% Propylenglykol-Wasser-Gemisch bei $80^{\circ} \mathrm{C}$ )

Durch sukzessive Druckabsenkung im Reaktorgefäß über ein wiederholt evakuiertes Hilfsvolumen (Gasmaus) wird das Druckniveau bis nahezu auf Dampfdruck reduziert und dabei durch Unterstützung des Rührers die Flüssigkeit entgast.

2. Temperieren der Anlage auf die gewählte Zieltemperatur.

3. Erste Beladung Gasreservoir und Herstellung stationärer Zustand 1 (SZ 1)

Nach Beginn der Temperierung wird das Gasreservoir mit Arbeitsgas aus der Gasflasche gespeist (auf ca. 6 bar). Dafür werden die Ventile V.Z1 und V.Z2 kurzzeitig geöffnet. Anschließend wird gewartet bis im Gasreservoir ein stationärer Zustand 1 hergestellt ist.

4. Herstellung Phasengleichgewicht 1 (GGW 1)

Nach abgeschlossener Temperierung stellt sich unter stetigem Rühren im Reaktor ein Phasengleichgewicht ein. Alle im Reaktor befindlichen Gase liegen jetzt in der Gasphase und auch mit entsprechender gelöster Menge in der Flüssigphase vor.

5. Druckausgleich und Herstellung Phasengleichgewicht 2 (GGW 2)

Durch Druckausgleich zwischen Gasreservoir und Reaktorgefäß (durch Öffnen des Ventils V.G5 für ca. $1 \mathrm{~s}$ ) und nachfolgendem Rühren entsteht im Reaktor ein neuer gesättigter Zustand auf höherem Partialdruckniveau. Im Gasreservoir stellt sich ein neuer stationärer Zustand 2a (SZ 2a) auf niedrigerem Druckniveau ein.

6. Zweite Beladung Gasreservoir (auf ca. 10 bar)

Der stationäre Zustand 2a im Gasreservoir wird zügig erreicht, während die Einstellung des Phasengleichgewichts 2 deutlich länger dauert. Um Versuchszeit zu sparen, wird parallel zur Einstellung des Phasengleichgewichts 2 im Reaktor - eine erneute Beladung des Gasreservoirs durchgeführt und bis zur Erreichung des stationären Zustands 2b (SZ 2b) im Gasreservoir gewartet. 

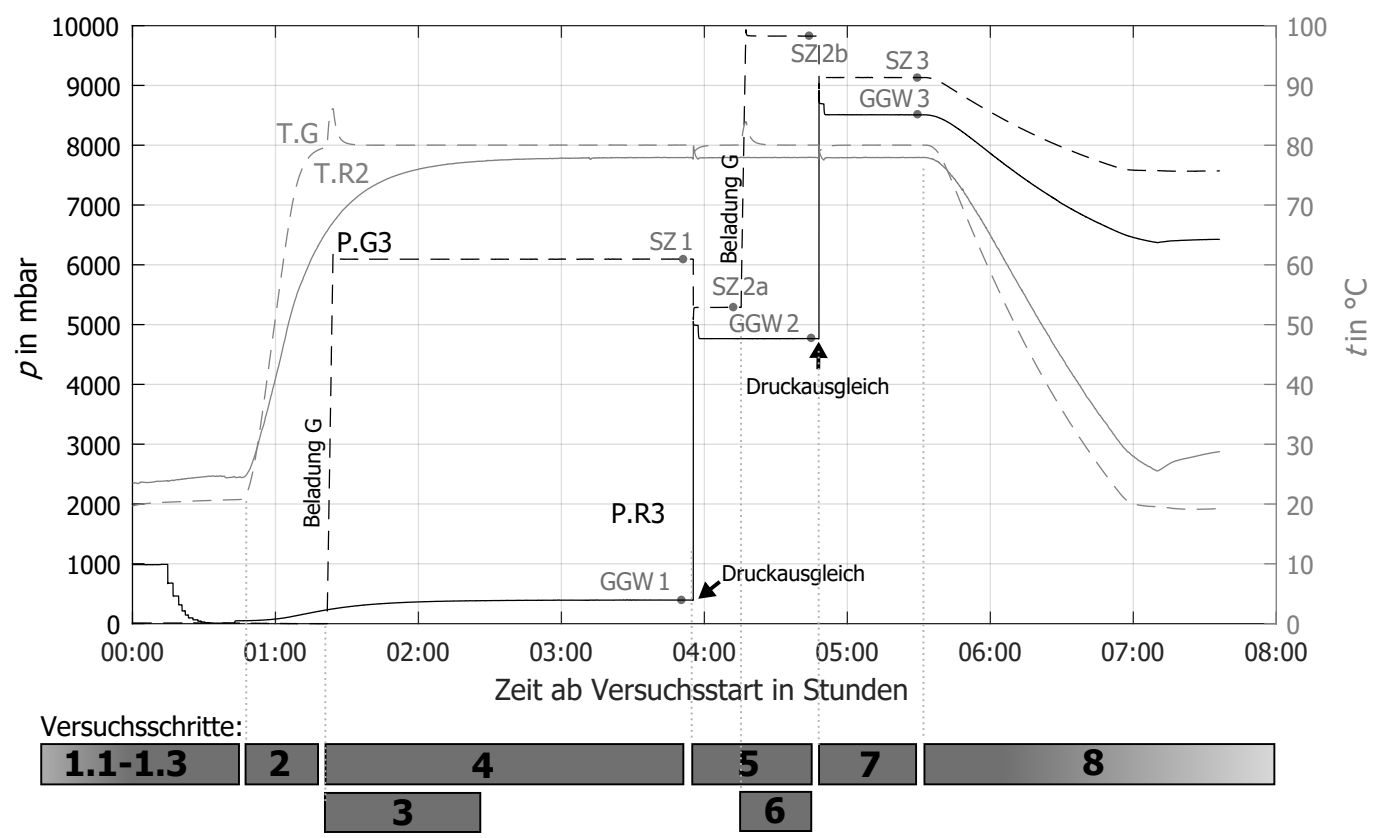

Abbildung 4.7: Versuchsablauf mit Schrittnummern, Ausgangszustand entgast (hier: 41,84 gew.- $\%$ Ethylenglykol-Wasser-Gemisch bei $80^{\circ} \mathrm{C}$ )

7. Druckausgleich und Herstellung Phasengleichgewicht 3 (GGW 3) im Reaktor sowie stationärer Zustand 3 (SZ3) im Gasreserovir

Nachdem sowohl Phasengleichgewicht 2 im Reaktor als auch der stationäre Zustand 2b im Gasreservoir erreicht sind, erfolgt ein erneuter Druckausgleich zwischen Gasreservoir und Reaktorgefäß (durch erneutes Öffnen des Ventils V.G5 für ca. $1 \mathrm{~s}$ ). Durch nachfolgendes Rühren entsteht ein neuer gesättigter Zustand auf höherem Partialdruckniveau (Phasengleichgewicht 3). Im Gasreservoir wird abgewartet, bis der stationäre Zustand 3 erreicht ist.

8. Temperieren, Entleerung und Trocknung

Nach dem Temperieren auf Raumtemperatur und Druckausgleich mit der Umgebung muss die Anlage entleert, gereinigt und getrocknet werden.

Der Versuchsablauf stellt den in mehreren Schritten gefundenen optimierten Ablauf dar, dem die in Abschnitt 4.4.3 erläuterten Überlegungungen und Versuche zugrunde liegen. Es erfolgten im Rahmen der Messreihen dann nur noch leichte Anpassungen des Versuchsablaufs. ${ }^{4}$

Zur Auswertung der zwei Drucksprünge stehen nun jeweils zwei definierte Zustände in Gasreservoir und Reaktor zur Verfügung

- 1. Drucksprung - stationärer Zustand 1 und 2a im Gasreservoir, Phasengleichgewicht 1 und Phasengleichgewicht 2 im Reaktor

\footnotetext{
${ }^{4}$ In den ersten Versuchen mit Ausgangszustand luftgesättigt (Versuche mit Tyfocor ${ }^{\circledR}$ LS) wurde das erste Phasengleichgewicht zusätzlich auch durch Absorption angefahren. Dazu wird das Gasreservoir nach Schritt 1.1 zusätzlich mit $\mathrm{N}_{2}$ so beladen, dass P.G3 nach der Temperierung ca. 0, 5 bar über dem Druck im gefüllten, verschlossen und temperierten Reaktorgefäß P.R3 liegt. Nach einem zusätzlichen vor Beladung des Gasreservoirs (Schritt 3) erfolgten Druckausgleich stellte sich hier durch eine geringe Absorption das Phasengleichgewicht 1 ein. Da jedoch keine Ergebnisunterschiede zu Versuchen ohne diesen Zusatzaufwand festgestellt werden konnten, wurde darauf bei nachfolgenden Versuchen verzichtet. Entsprechende Versuche werden durch „luftgesättigt $+0,5$ bar" bezeichnet.
} 
- 2. Drucksprung - stationärer Zustand 2b und 3 im Gasreservoir, Phasengleichgewicht 2 und Phasengleichgewicht 3 im Reaktor

\subsubsection{Synthetische Luft als Arbeitsgas}

Versuche mit Synthetischer Luft als Arbeitsgas (bestehend aus Stickstoff und Sauerstoff) werden im Folgenden auch als Luft-Versuche bezeichnet. Durch die unterschiedliche Löslichkeit von Stickstoff und Sauerstoff ändert sich nach der Luft-Zugabe im Phasengleichgewicht 2 die Zusammensetzung der Gasphase gegenüber dem Ausgangsgemisch. Um die Zusammensetzung und damit die Partialdrücke zu bestimmen, erfolgte eine Probenahme der Gasphase des Reaktors. Dazu wurde eine Probemenge in den vorher evakuierten Probenahmezylinder gesaugt und diese anschließend gaschromatographisch ausgewertet. Da die entfernte Lösungsmittelmenge (Dampf und v.a. mitgerissene Tropfen) nicht exakt quantifizierbar ist, ist bei diesen Versuchen die Fortführung bis zum Phasengleichgewicht 3 nicht mehr sinnvoll. Hier hätten für einen zweiten Drucksprung zusätzliche Versuche stattfinden müssen. Da hier jedoch v. a. die Überlagerung von physikalischer Löslichkeit und chemischer Bindung im Fokus standen, wurde hier auf eine Überprüfung, ob man sich im Gültigkeitsbereich des Löslichkeitskoeffizienten befindet, durch Auswertung eines zweiten Drucksprungs verzichtet.

Um mit den Luft-Versuchen auch die chemische Bindung des Sauerstoffs zu untersuchen, wurde der Schritt 5 erweitert. Für rein physikalische Bindung stellt sich das Phasengleichgewicht zügig (in weniger als einer Stunde) ein. Anschließend wurde jedoch mindestens 5 Stunden gewartet, da bei allmählicher chemischer Bindung sich ein weiterer Druckabfall zeigt, der das Phasengleichgewicht entsprechend stetig verschiebt. Der Zeitpunkt des für die Berechnung/Bilanzierung notwendigen Phasengleichgewichts 2 liegt hier kurz vor der Probenahme. Die zwischen den Zuständen Phasengleichgewicht 1 und 2 formal stets berechenbaren Löslichkeitskoeffizienten bringen jedoch bei Lösungsmitteln mit vorhandener chemischer Bindung keine Aussage. Eine stattfindende chemische Bindung kann entsprechend des Druckverlaufs erkannt werden.

Ein exemplarischer Druck- und Temperaturverlauf (für einen Versuch ohne chemische Bindung) findet sich in Abbildung 4.8. In Tabelle 4.4 ist der Versuchsablauf im Vergleich zum Versuchsablauf mit Stickstoff dargestellt. 


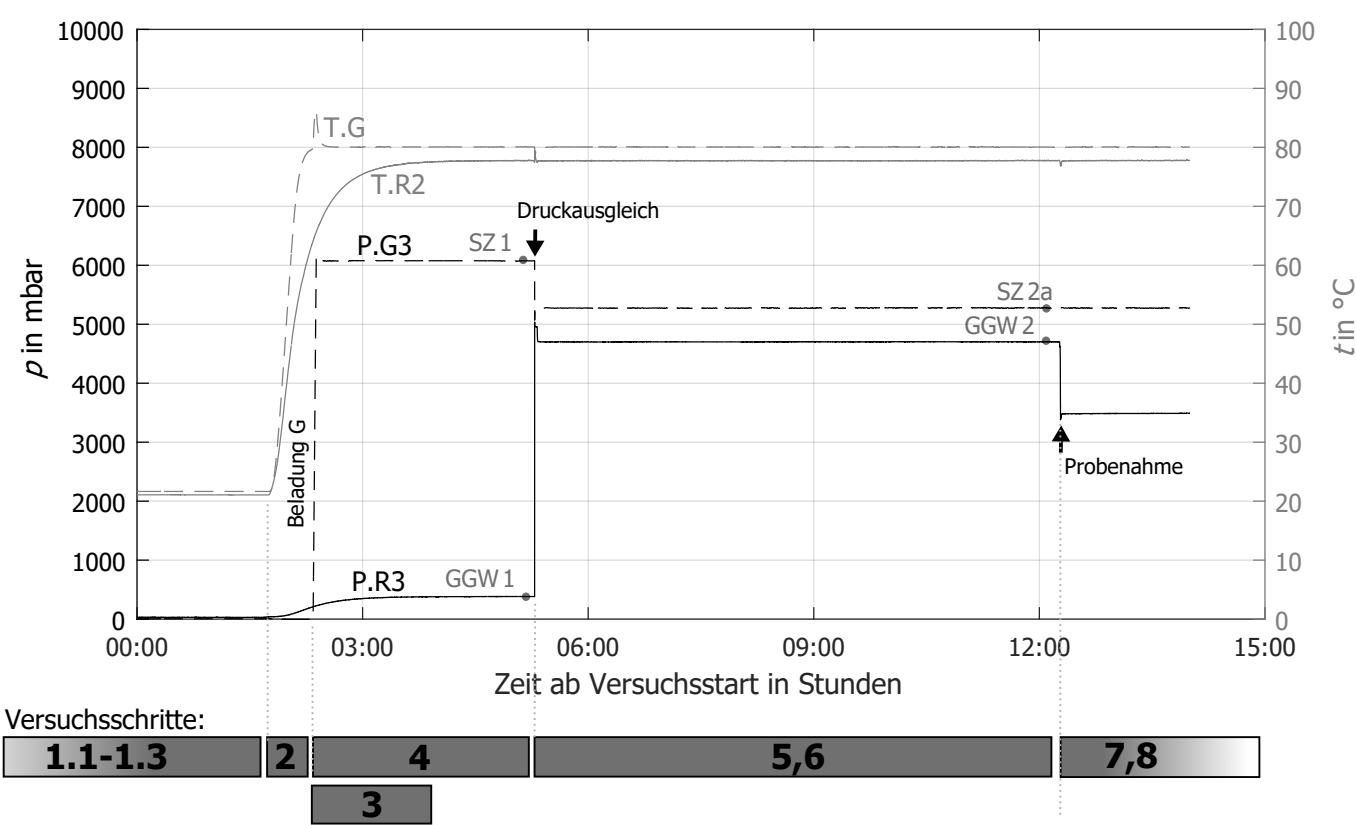

Abbildung 4.8: Versuchsablauf für Luftversuche mit Schrittnummern (hier: 41,84 gew.-\% EthylenglykolWasser-Gemisch bei $80^{\circ} \mathrm{C}$, ohne Effekte der chemischen Bindung)

Tabelle 4.4: Versuchsablauf für die Stickstoff- und Luft-Versuche

\begin{tabular}{|c|c|c|}
\hline $\begin{array}{l}\text { Arbeitsgas Stickstoff } \\
\text { Ausgangszustand: luftgesättigt }\end{array}$ & $\begin{array}{l}\text { Arbeitsgas Stickstoff } \\
\text { Ausgangszustand: entgast }\end{array}$ & $\begin{array}{l}\text { Arbeitsgas Luft } \\
\text { Ausgangszustand: entgast }\end{array}$ \\
\hline $\begin{array}{l}1.1 \text { Vorspülung Gasreservoir mit } \mathrm{N}_{2} \text { und } \\
\text { Reaktor mit Luft }\end{array}$ & \multicolumn{2}{|c|}{ 1.1 Vorspülung Gasreservoir und Reaktorraum mit Arbeitsgas } \\
\hline \multicolumn{3}{|l|}{ 1.2 Wägung Lösungsmittel und Befüllen } \\
\hline- & \multicolumn{2}{|c|}{ 1.3 Entgasung des Reaktorgefäßes } \\
\hline \multicolumn{3}{|c|}{ 2. Temperieren der Anlage auf Zieltemperatur } \\
\hline \multicolumn{3}{|c|}{ 3. Beladung Gasreservoir (auf ca. 6 bar) und Herstellung stationärer Zustand 1 in G } \\
\hline \multicolumn{3}{|l|}{ 4. Herstellung Phasengleichgewicht 1} \\
\hline \multicolumn{3}{|c|}{ 5. Druckausgleich und Herstellung Phasengleichgewicht 2 und stationärer Zustand 2a in G } \\
\hline \multicolumn{2}{|c|}{$\begin{array}{l}\text { 6. Zweite Beladung Gasreservoir (auf ca. } 10 \text { bar) und Herstellung stat. } \\
\text { Zustand } 2 \text { b in G }\end{array}$} & $\begin{array}{l}\text { (6. Mindestwartezeit } 5 \text { Std. } \\
\text { nach Druckausgleich) }\end{array}$ \\
\hline \multicolumn{2}{|c|}{$\begin{array}{l}\text { 7. Druckausgleich und Herstellung Phasengleichgewicht } 3 \text { und stat. } \\
\text { Zustand } 3 \text { in G }\end{array}$} & 7. Gasphasen-Probenahme \\
\hline
\end{tabular}

\subsubsection{Versuchsrelevante Fragestellungen und Detailprobleme}

\subsubsection{Rührer im Reaktorgefäß}

Aufgrund der im Vergleich zur Flüssigkeitsmenge geringen Grenzfläche gasförmig/flüssig muss die Einstellung des Gleichgewichtszustandes durch einen Rührprozess unterstützt werden. Dazu wird eine Begasungsrührwelle eingesetzt, die Gas aus dem oberen Bereich des Gefäßes ansaugt und an den Rührschaufeln ins Fluid abgibt. 
Aus Vorversuchen konnte optisch festgestellt werden, dass eine vollständige Durchmischung und Begasung der Flüssigkeit bei richtiger Stellung des Verwirbelers ab einer Rührerdrehzahl von $1000 \mathrm{~min}^{-1}$ sichergestellt ist. Bei $100 \%$ Propylenglykol ist für geringe Temperaturen aufgrund der hohen Zähigkeit eine Mindestrührerdrehzahl von $1100 \mathrm{~min}^{-1}$ notwendig, um den Begasungseffekt der Rührwelle zu aktivieren.

\subsubsection{Phasengleichgewicht}

Für die Messung des Phasengleichgewichts im Reaktorgefäß wird der Rührerbetrieb nicht unterbrochen. Dies hat hauptsächlich zwei Gründe:

- Da der Rührer selbst eine nicht unerhebliche Leistung im Reaktor umsetzt, fällt trotz äußerer Temperierung des Reaktorgefäßes die Temperatur nach Ausschalten des Rührers leicht ab. Entsprechend leicht geänderter Löslichkeit und geändertem Dampfdruck würde man damit das mit Rührer erreichte Phasengleichgewicht wieder verlassen.

- Aufgrund der unterschiedlichen Wärmeübergänge und Wandtemperaturen im nicht perfekt temperierten Reaktormantel bildet sich nach Ausschalten des Rührers ein örtlich stark unterschiedliches Temperaturbild aus, das messtechnisch mit den zwei vorliegenden T-Sensoren nicht erfasst werden kann. Dies lässt sich zwar durch zusätzliche Dämmung am Reaktormantel und -flansch verringern, jedoch nicht vermeiden.

Das Rühren verursacht die Ausbildung eines Druckfeldes. Der gemessene Druck $p$ im Reaktorgefäß gibt einen zeitlich gemittelten Druck eines Punktes des Druckfeldes wieder. Folgendes wird angenommen:

- Die durch den Rührerbetrieb erzeugte Veränderung der gemessenen Absolutdrücke werden wg. der differentiellen Bestimmung der Löslichkeit vernachlässigt, da ein gleichmäBiger Offset von ca. $1 . .4$ mbar bei allen Druckniveaus beobachtet wird.

- Lokal unterschiedliche Drücke im Reaktorgefäß bedeuten, dass an Stellen geringeren Drucks Gas desorbiert und an Stellen höheren Drucks Gas wieder absorbiert wird. Da die Desorption i. A. schneller abläuft als die Absorption, ist zu vermuten, dass das Fluid für den gemessenen Druck also nicht vollständig gesättigt ist. Da dieser Effekt eher klein sein wird und in allen Phasengleichgewichten der unterschiedlichen Druckniveaus gleichmäßig auftritt, sollte er auf die Druckdifferenz keinen Einfluss haben.

\subsubsection{Gasphasen-Probenahme bei Luft-Versuchen}

Das Volumen des Probenahmezylinders von $75 \mathrm{ml}$ ist so gewählt, dass der Lösungsmittelaustrag aus dem Reaktor und die damit einhergehende Druckabsenkung gering bleiben, sodass der Einfluss der einsetzenden Desorption aus der Flüssigkeit auf die entnommene Gasphasenprobe gering bleibt und gleichzeitig die Probemenge und der notwendige ProbenÜberdruck ausreicht, um die anschließende Gasanalyse durchzuführen. Damit nur geringe Gasdesorption während der Probenahme stattfindet, wird der Rührer unmittelbar vor der Probenahme ausgeschaltet. Der evakuierte Probenahmezylinder wird anschließend auf 1 bar $_{u}$, wie auch in [Pham, 2015] beschrieben, mit Gasphasengemisch des Reaktors befüllt.

Die Gasprobe konnte gaschromatographisch bei der SGS Institut Fresenius GmbH analysiert und das Verhältnis von Sauerstoff und Stickstoff ermittelt werden. Dafür konnte auf das vom Institut für Energietechnik der TU Dresden gemeinsam mit SGS Institut Fresenius 
GmbH entwickelte und in einer Vielzahl von Messungen validierte Verfahren (u. a. in [Rühling und Uhlmann, 1998] und [Rühling, 2002]) und auf den zugehörigen Gaschromatographen zurückgegriffen werden. ${ }^{5}$

\subsubsection{Lösungsmittelmasse in Versuch und Referenzversuch}

Allgemein ist zu beachten, dass für den Versuch mit Probeflüssigkeit und den Referenzversuch mit Wasser annähernd gleiche Versuchsbedingungen gelten sollen (siehe auch Abschnitt 4.6).

Für die Wasserversuche wurde eine Masse von $700 \mathrm{~g}$ eingefüllt. Für die Probeflüssigkeiten würde eine massegleiche Einfüllung ein verändertes Flüssigvolumen $V^{\prime}$ und damit auch ein deutlich verändertes verbleibendes Gasvolumen $V^{\prime \prime}$ im Reaktor im Gegensatz zum WasserReferenzversuch bedeuten $\left(+13 \%\right.$ bei $t_{\text {soll }}=10^{\circ} \mathrm{C}$ bei Tyfocor ${ }^{\circledR}$ LS $)$. Dies könnte zum Beispiel

- ein anderes Rühr- und Begasungsverhalten durch veränderten Füllstand,

- andere Temperaturgradienten im Reaktorgefäß sowie

- ein verändertes Druckniveau im Reaktor nach Druckausgleich mit dem Gasreservoir

zur Folge haben. Daher wurde die volumengleiche Füllung bei Solltemperatur entsprechend nachstehender Gleichung angewendet.

$$
m_{\mathrm{LM}, \mathrm{soll}}=\rho_{\mathrm{LM}}\left(t_{\mathrm{soll}}\right) \cdot V_{\mathrm{soll}}^{\prime}=\rho_{\mathrm{LM}}\left(t_{\mathrm{soll}}\right) \cdot \frac{m_{\mathrm{H} 2 \mathrm{O}}}{\rho_{\mathrm{H} 2 \mathrm{O}}\left(t_{\mathrm{soll}}\right)}
$$

\subsubsection{Temperierung der Toträume}

Für die Temperierung der Toträume mittels elektrischer Begleitheizung ist vor allem zu beachten, dass innere Kondensation verhindert wird, da diese Lösungsmittelanteile nur teilweise an der Absorption teilnehmen und außerdem eine geänderte Gemischzusammensetzung nicht ausgeschlossen werden kann. Darüber hinaus ist der Reinigungs-/Trocknungsaufwand bei starker Kondensation in Toträumen höher. Folglich ist für die Anlage zu beachten, dass bei der Aufheizung und dann auch während des Versuchs die Temperatur der Toträume immer über der Temperatur im Reaktorgefäß liegt (ca. +2..3 K).

\subsubsection{Temperiergüte des Reaktors}

Die real gemessenen Temperaturen des Fluids im Reaktor unterscheiden sich trotz aufwändiger Dämmung signifkant von den eingestellten Solltemperaturen, die im Gasreservoir hingegen sehr gut erreicht werden. Dies liegt daran, dass es sich als günstiger erwiesen hat, nach der Temperierbadtemperatur zu temperieren und eine hohe Regelgüte für das Gasreservoir zu erreichen. Das Reaktorgefäß wird von der temperierten Flüssigkeit, wie beschrieben, in Mantel und Deckel umflossen. Da jedoch die Dämmung des Reaktors nicht perfekt ist und

\footnotetext{
${ }^{5}$ Als Trägergas wurde Helium eingesetzt. Im Gaschromatographen kann nicht zwischen Sauerstoff und Argon unterschieden werden, sodass der summarische Anteil von Sauerstoff und Argon erfasst wird. Da alle Luft-Versuche jedoch mit dem Ausgangszustand entgast starten, sind Argon-Anteile in der Gasphase des Reaktors zu vernachlässigen. Weiterhin wird im Gaschromatographen auch der Methan-Anteil erfasst. Hier konnten in den Versuchen erwartungsgemäß keine Anteile detektiert werden.
} 
auch der Rührer einen Wärmeeintrag generiert, unterscheidet sich die Temperatur des Fluids im Reaktor von der eingestellten Thermostattemperatur.

Die realen Fluidtemperaturen variieren zwischen den Versuchen gleicher Solltemperatur um $\pm 0,75 \mathrm{~K}$. Ursache dafür sind vor allem

- die vorhandene Abhängigkeit von der Raumtemperatur und der

- unterschiedlicher Wärmeeintrag durch den Rührer (konstante Rührerdrehzahl, jedoch deutlich höhere Viskosität bei höherem Glykolanteil).

Für die Berechnung und Darstellung der Ergebnisse werden stets die real gemessenen Temperaturen im Reaktorgefäß und nicht die Solltemperaturen verwendet.

\subsubsection{Trocknung des Reaktorgefäßes}

Vor Beginn eines neuen Versuches ist die vollständige Trocknung des Reaktorgefäßes notwendig. Für Wasser und Tyfocor ${ }^{\circledR}$ LS konnte ausreichende Trocknung durch längeren konstanten Betrieb der Vakuumpumpe erreicht werden. Zusätzlich werden die elektrisch beheizten Toträume auf $110^{\circ} \mathrm{C}$ erhitzt, um einen leichteren Lösungsmittelaustrag aus diesen zu erreichen.

Aufgrund des geringen Dampfdrucks von Glykol dauerte dieser Vorgang bei Wasser-GlykolGemischen mit hohem Glykolanteil deutlich zu lange. Daher wurde das Verfahren abgeändert und zusätzlich zur genannten Beheizung der Toträume auch das Haupt-Reaktorvolumen auf $80^{\circ} \mathrm{C}$ aufgeheizt, um den Dampfdruck des Lösungsmittels und damit dessen potentielle Anreicherung im gasförmigen Zustand zu erhöhen. Dann wird das Reaktorgefäß evakuiert und damit Luft und gasförmige Lösungsmittelbestandteile entfernt. Anschließend wird wieder mit Umgebungsluft befüllt, die sich entsprechend der Temperierung zügig erwärmt und dabei Lösungsmittel aufnimmt. Zusätzlich wird die Verdunstung der Lösungsmittelbestandteile durch die Aktivierung des Rührers begünstigt. Dieser Vorgang wird wiederholt, bis der Druck im evakuierten Zustand sich nicht mehr änderte. Es muss dabei verhindert werden, dass das warme, abzusaugende verdampfte Lösungsmittel nicht in einer kalten Verbindungsleitung zur Vakuumpumpe kondensiert und wieder in den Reaktorraum zurückfließt. Um diesen Effekt zu vermeiden, wurde eine zusätzliche direkte Abführung am Reaktor mit kurzer senkrechter Strecke angebracht.

\subsection{Berechnungsalgorithmus der Löslichkeitskoeffizienten}

\subsubsection{Allgemeines}

In Abbildung 4.9 sind der schematische Ablauf eines Versuchs und die MessgröBen (gemessene Masse $m_{\text {schein }}$ sowie Druck- und Temperaturverhältnisse) dargestellt. Auch wichtige Störeffekte sind bereits gekennzeichnet. Auf diese wird später eingegangen.

Die folgenden Ausführungen unterscheiden sowohl für Gasreservoir als auch Reaktorgefäß nur zwischen einem Zustand $z=1$ vor dem Druckausgleich bzw. $z=2$ nach dem Druckausgleich. Die Berechnungen sind jedoch für beide Drucksprünge gleichermaßen anwendbar. In Tabelle 4.5 sind die zugehörigen, im Versuchsablauf genannten Zustände zugeordnet. 


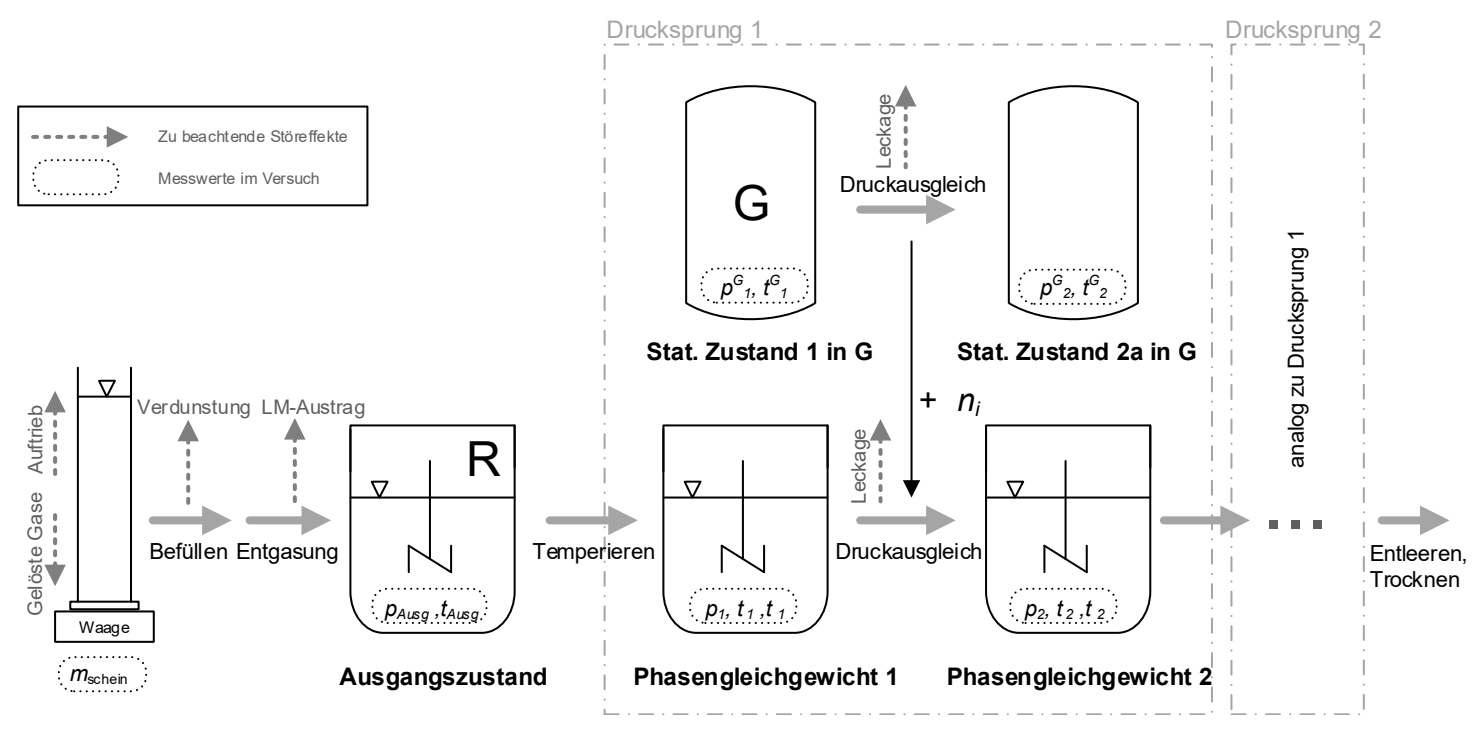

Abbildung 4.9: Schematischer Versuchsablauf eines Stickstoff-Versuchs mit Ausgangszustand entgast

Es wird davon ausgegangen, dass in den Zuständen $z$

- im Gasreservoir ein stationärer Zustand vorliegt und

- im Reaktorgefäß Stoffausgleichsvorgänge für alle vorhandenen Komponenten abgeschlossen sind, also ein Phasengleichgewicht vorliegt.

Tabelle 4.5: Zustand $z$ der Berechnung und Zuordnung zu Phasengleichgewichten des Versuchablaufs

\begin{tabular}{llll}
\hline & & Gasreservoir & Reaktor \\
\hline \hline \multirow{2}{*}{ Drucksprung 1 } & Zustand $z=1$ & stat. Zustand 1 & Phasengleichgewicht 1 \\
& Zustand $z=2$ & stat. Zustand 2a & Phasengleichgewicht 2 \\
\hline \multirow{2}{*}{ Drucksprung 2 } & Zustand $z=1$ & stat. Zustand 2b & Phasengleichgewicht 2 \\
& Zustand $z=2$ & stat. Zustand 3 & Phasengleichgewicht 3 \\
\hline
\end{tabular}

\subsubsection{Bestimmung der Volumina}

Das Volumen der Gasmaus $V_{0}^{\mathrm{M}}$ wurde von [Wagner, 2010] bereits mit Wasser ausgelitert. Die stark verzweigten Volumina Reaktorgefäß $V_{0}$ und Gasreservoir $V_{0}^{G}$ sind aufgrund fehlender Präzisionswägetechnik in diesem Massebereich nicht geeignet für ein Auslitern mit Wasser. ${ }^{6}$ Hier wurde das Gasmausvolumen als Referenz genutzt. Dieses Volumen wird mit $\mathrm{N}_{2}$ druckbeaufschlagt und im zweiten Schritt in das andere Volumen entspannt. Aus gemessenen Drücken und Temperaturen der Volumina kann mit Hilfe des bekannten $p-V$ - $T$-Verhaltens von Stickstoff auf das gesuchte Volumen rückgeschlossen werden. Da insbesondere der Fehlereinfluss des Reaktorvolumens $V_{0}$ auf die Löslichkeitsbestimmung sehr groß ist, wie im später folgenden Abschnitt 4.9 gezeigt, wurde beim Reaktorgefäß eine definierte Wassermenge zugegeben, um nur den verbliebenen Gasraum zu vermessen und damit die Genauigkeit der Volumen-Messung zu erhöhen. Die Erläuterung der Vorgehensweise zur Bestimmung der Volumina $V_{0}^{G}$ und $V_{0}$ erfolgt in Anlage A.6. Eine Übersicht der ermittelten Volumina

\footnotetext{
${ }^{6}$ Für das Gesamtvolumen des Reaktorgefäßes $V_{0}$ wird aus Gründen der Übersichtlichkeit der Vielzahl noch folgender Gleichungen auf einen Index R verzichtet.
} 
inklusive Messunsicherheit und angewandtem Verfahren ist im Anhang in Tabelle A.3 gegeben.

Die Volumina von Reaktorgefäß R und Gasreservoir $\mathrm{G}$ sind aufgrund der volumetrischen thermischen Ausdehnungskoeffizienten $\beta$ der Hüllwerkstoffe nicht als konstant zu betrachten. ${ }^{7}$ Die experimentelle Bestimmung des Volumens $V^{G}$ erfolgte bei einer Bezugstemperatur $t_{0}^{\mathrm{G}}$ mit der Bezeichnung $V_{0}^{G}=V^{G}\left(t=t_{0}^{G}\right)$. Für das Gasreservoir ergibt sich allgemein auch für von $t_{0}^{\mathrm{G}}$ abweichende Temperaturen $t^{\mathrm{G}}$

$$
V^{G}=\left(1+\beta^{G} \cdot\left(t^{G}-t_{0}^{G}\right)\right) \cdot V_{0}^{G} .
$$

Der Volumenausdehnungskoeffizient $\beta$ ergibt sich aus dem aus Literaturwerten bekannten Längenausdehnungskoeffizient $\alpha$ näherungsweise wie folgt

$$
\beta=(1+\alpha)^{3}-1 \approx 3 \cdot \alpha
$$

Relevante Ausdehnungskoeffizienten sind in Tabelle A.4 im Anhang dargestellt.

Das Reaktorgefäßes $V$ setzt sich aus drei Volumina zusammen:

- dem mit Thermoöl temperierten Reaktorgefäßvolumen $V^{\mathrm{RO}}$ mit der Temperatur $t^{\mathrm{RO}}$,

- der elektrisch temperierten Verbindungsleitung zwischen Reaktorgefäß und Gasreservoir $V^{\mathrm{R} 1}$ mit der Temperatur $t^{\mathrm{R} 1}$ und

- dem elektrisch temperierten Leitungsvolumen zwischen Reaktorgefäß und Drucksensoren (bzw. Ventil V.R4) $V^{R 2}$ mit der Temperatur $t^{R 2}$.

Die experimentelle Bestimmung des Volumens $V$ erfolgte bei einer Bezugstemperatur in allen Teilvolumina $t^{\mathrm{R} 0}=t^{\mathrm{R} 1}=t^{\mathrm{R} 2}=t_{0}^{\mathrm{R}}$ mit der Bezeichnung $V_{0}$. Als Temperatur des Totvolumens $t^{\mathrm{R} 1}$ wird je nach Anlagenkonfiguration die Messstelle T.RHzg1 oder das arithmetische Mittel aus T.RHzg1 und T.RHzg2 verwendet. Für $t^{\mathrm{R} 2}$ wird die Messstelle T.RHzg3 verwendet. Für die Berechnung des Reaktorvolumens (ohne Toträume) $V^{\text {R0 }}$ wird die gemessene Flüssigphasentemperatur verwendet, weil die Flüssigphase den größten Anteil einnimmt.

Da die elektrisch temperierten Bereiche im Gegensatz zu $V^{R 0}$ mit Edelstahl umgrenzt sind und eine andere Temperatur erreichen, werden diese Bereiche in der Rechnung getrennt bilanziert. Daher ist auch die Kenntnis dieser Teilvolumina notwendig. Die Totvolumina $V_{0}^{R 1}$ und $V_{0}^{R 2}$ werden anhand der geometrischen Abmessungen berechnet. Das Volumen ohne Toträume bei Bezugstemperatur $V_{0}^{R 0}$ wird dann mit dem bekannten Gesamtvolumen $V_{0}$ bestimmt zu

$$
V_{0}^{\mathrm{R} 0}=V_{0}-V_{0}^{\mathrm{R} 1}-V_{0}^{\mathrm{R} 2}
$$

Für das Reaktorgefäß gilt nun bei beliebiger Temperatur

$$
V=V^{\mathrm{R} 0}+V^{\mathrm{R} 1}+V^{\mathrm{R} 2}
$$

mit der Temperaturabhängigkeit für $m=\mathrm{R} 0, \mathrm{R} 1, \mathrm{R} 2$

$$
V^{m}=\left(1+\beta^{m} \cdot\left(t^{m}-t_{0}^{R}\right)\right) \cdot V_{0}^{m}
$$

${ }^{7}$ Die mechanische Ausdehnung durch Innendruck kann aufgrund der deutlich geringeren Größenordnung gegenüber der thermischen Ausdehnung vernachlässigt werden. 


\subsubsection{Gasreservoir}

Gesucht ist die an das Reaktorgefäß abgegebene Stoffmenge der Gaskomponenten $i$ von Zustand 1 nach 2

$$
\triangle n_{i, 1 \rightarrow 2}=n_{i, 1}^{G}-n_{i, 2}^{G} .
$$

Folgende Annahmen werden getroffen:

- Aufgrund der beschriebenen Reinheit des eingesetzten Arbeitsgases (vgl. Tabelle 4.2 und 4.3) und der Vorspülung befindet sich nur das trockene Arbeitsgas im Gasreservoir.

- Die Gasreservoir-Temperatur $t^{\mathrm{G}}$ ist konstant über das gesamte, von Temperierflüssigkeit umgebene Gasvolumen $V^{G}$.

Die Stoffmenge für das Gas $i$ im stationären Zustand $z$ bestimmt sich $z u$

$$
n_{i, z}^{\mathrm{G}}=\frac{V_{z}^{\mathrm{G}}}{M_{i}} \rho_{i, z}^{\mathrm{G}}\left(p_{z}^{\mathrm{G}} ; t_{z}^{\mathrm{G}} ; y^{\mathrm{G}}\right)
$$

wobei $\rho_{i, z}^{\mathrm{G}}$ aus Realgasgleichungen von Stickstoff bzw. Stickstoff-Sauerstoff-Gemisch über FluidLAB von [Kretzschmar, 2010] bestimmt wird. Für das Zweikomponenten-Gasgemisch Synthetische Luft ( $i=\mathrm{N}_{2}, \mathrm{O}_{2}$ ) muss dafür zusätzlich zu Druck und Temperatur auch der Molanteil $y_{\mathrm{N} 2}^{G}$ vorliegen. Angelehnt an FluidLAB wird hierfür vereinfacht mit einem idealen Gemisch realer Gase gerechnet.

\subsubsection{Reaktor}

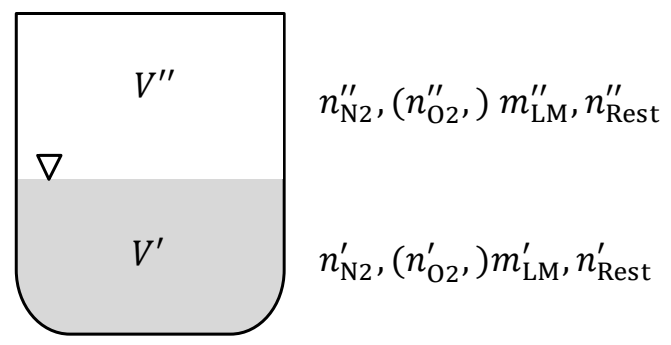

Abbildung 4.10: Physikalische Größen für Phasengleichgewicht im Reaktor

Im Reaktor befindet sich die Lösungsmittelmasse $m_{\mathrm{LM}}$, das Arbeitsgas und je nach Ausgangszustand die im Folgenden als Restgase bezeichneten Atmosphärengase $\mathrm{CO}_{2}$, Ar etc. (sowie $\mathrm{O}_{2}$ bei Stickstoff-Versuchen) und möglicherweise auch zusätzliche Gase, die herstellbedingt im gelieferten Fluid gelöst sind. Bei entgastem Ausgangszustand ist der Anteil der Restgase sehr gering.

Folgende Annahmen werden getroffen:

- Der Druck p' wird am Deckel des Gefäßes gemessen. Es wird angenommen, dass der Druck im gesamten Reaktorvolumen gilt, also $p_{z}=p_{z}^{\prime}=p_{z}^{\prime \prime}$. Hydrostatischer Druck wird vernachlässigt.

- Die Temperatur $t_{z}^{\prime}$ bzw. $t_{z}^{\prime \prime}$ ist repräsentativ für das jeweilig zugehörige Volumen $V_{z}^{\prime}$ bzw. $V_{z}^{\prime \prime}$. 
- Die geringen bzw. minimalen Anteile an Restgasen haben keinen Quereinfluss auf die Löslichkeit des Arbeitsgases $i$.

\subsubsection{Gasphase des Reaktors}

\subsubsection{Beschreibung des Gasgemischs}

Als effektive Temperatur in der Gasphase $t^{\prime \prime}$ wird näherungsweise der volumengewichtete Mittelwert der Temperatur des Sensors T.R2 $\left(t_{z}^{R 0}\right)$ und der Temperaturen der elektrisch temperierten Rohrleitungen ( $t_{z}^{\mathrm{R} 1}$ und $t_{z}^{\mathrm{R} 2}$ ) verwendet.

Das Gemisch soll idealisiert über Partialdrücke beschrieben werden können, die das Gesetz nach Dalton erfüllen. Für die Stickstoffversuche ergibt sich der Druck ausgedrückt über Partialdrücke im Zustand $z$ zu

$$
p_{z}=p_{\mathrm{N} 2, \mathrm{z}}+p_{\mathrm{Rest}, \mathrm{z}}+p_{\mathrm{LM}, z}
$$

bzw. für Luftversuche zu

$$
p_{z}=p_{\mathrm{N} 2+\mathrm{O} 2, z}+p_{\mathrm{Rest}, \mathrm{z}}+p_{\mathrm{LM}, z} .
$$

Die Arbeitsgase Stickstoff und synthetische Luft sollen durch die Partialdrücke $p_{\mathrm{N}_{2}}$ bzW. $p_{\mathrm{N}_{2}+\mathrm{O}_{2}}$ beschrieben werden können. Die restlichen Luftbestandteile Argon, $\mathrm{CO}_{2}$ und möglicherweise unbekannte, im Produktionsprozess gelöste Gase sowie Sauerstoff (bei StickstoffVersuchen) sollen durch den Partialdruck $p_{\text {Rest }}$ beschrieben werden. Der Partialdruck $p_{\text {Rest, } z}$ beträgt beispielsweise bei einem Stickstoff-Versuch mit luftgesättigtem Ausgangszustand (bei Temperierung auf $20^{\circ} \mathrm{C}$ ) nur ca. 0,22 bar, beim Versuch mit entgastem Ausgangszustand sogar deutlich unter 10 mbar. $^{8}$

Die Bestimmung des Drucks $p_{\text {Rest,z }}$ wird später detailliert erläutert und bei der Gesamtunsicherheitsbetrachtung mit einer großen Unsicherheit berücksichtigt.

Weiterhin wird angenommen, dass nach Raoult der Dampfdruck des Lösungsmittels $p_{\mathrm{LM}}$ durch die geringe Gaskonzentration annähernd dem Dampfdruck des reinen Lösungsmittels $p_{\mathrm{LM}, \mathrm{s}}$ entspricht. ${ }^{9}$

$$
p_{\mathrm{LM}, z}=x_{\mathrm{LM}, \mathrm{z}} \cdot p_{\mathrm{LM}, \mathrm{s}}\left(t_{z}^{\prime \prime}\right) \approx p_{\mathrm{LM}, \mathrm{s}}\left(t_{z}^{\prime \prime}\right) .
$$

Für Stickstoffversuche ergibt sich daher der Partialdruck des Arbeitsgases zu

$$
p_{\mathrm{N}_{2}, z}=p_{z}-p_{\mathrm{Rest}, z}-p_{\mathrm{LM}, \mathrm{s}}
$$

\footnotetext{
${ }^{8}$ Es wird durch das Rechnen mit Partialdrücken also angenommen, dass auch das Restgas das Verhalten der sonstigen Gase im Gasraum nicht beeinflusst. Diese Annahmen scheinen plausibel, da die Restgase selbst für Versuche mit dem Ausgangszustand „luftgesättigt“ und um so mehr bei Ausgangszustand „entgast“ nur einen kleinen Beitrag einnehmen. Damit hat das abweichende Realverhalten (Einfluss auf das Gasphasengemisch) der Restgase nur einen vernachlässigbaren Einfluss auf die zu ermittelnden Stoffmengen von $\mathrm{N}_{2}$ (und $\mathrm{O}_{2}$ bei Luft-Versuchen).

${ }^{9}$ Die Abweichung beträgt z. B. für Stickstoff in Wasser bei $110{ }^{\circ} \mathrm{C}$ und 10 bar $\left(x_{\mathrm{N} 2} \approx 0,0001, p_{\mathrm{s}, \mathrm{H} 2 \mathrm{O}} \approx\right.$ 1, 4 bar) etwa 0,14 mbar und liegt damit deutlich unter den Messunsicherheiten der Drucksensorik.
} 
Für die Luftversuche ergibt sich der Partialdruck des interessierenden Gasgemisches $\mathrm{N}_{2}$ und $\mathrm{O}_{2} \mathrm{zu}$

$$
p_{\mathrm{N}_{2}+\mathrm{O}_{2}, z}=p_{z}-p_{\text {Rest }, \mathrm{z}}-p_{\mathrm{LM}, \mathrm{s}} .
$$

Die Stoffmengen von LM und Arbeitsgas im Gasraum werden nun entsprechend der einzelnen $(p, V, T)$-Beziehungen unter Verwendung der Partialdrücke bestimmt. Für einen Stickstoff-Versuch ist die Partialdruck-Vereinfachung schematisch in Abbildung 4.11 dargestellt. Wichtig für hohe Genauigkeit ist, dass trotz der idealisierten Verwendung von Partialdrücken für die Bestimmung der Stoffmengen des Arbeitsgases nun jedoch reale $(p, V, T)$ Beziehungen der Reinstoffe (Realgas) verwendet werden. Diese Vereinfachung nutzt u.a. [Kretzschmar, 2010] für die recht genaue Bestimmung des Luft-Wasser-Gemisch und wird auch in [Fischer und Wilken, 2001] für recht genaue Löslichkeitsuntersuchungen angewendet.

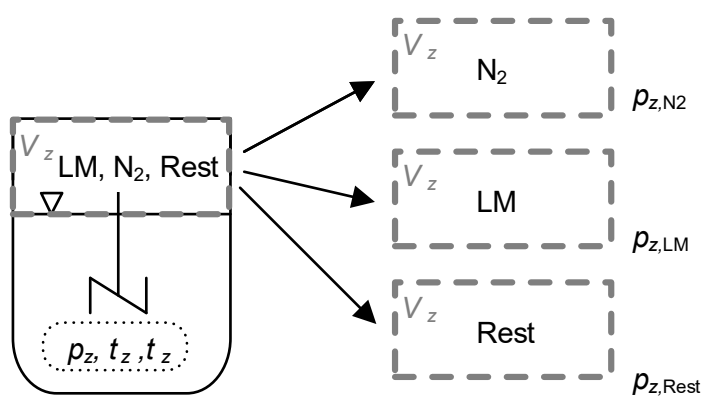

Abbildung 4.11: Partialdruckannahme für Gasphase am Beispiel des Stickstoff-Versuchs

Ist das Volumen der Fluidphase bekannt, lässt sich das Volumen der Gasphase für den stationären Zustand $z$ berechnen über

$$
V_{z}^{\prime \prime}=V_{z}-V_{z}^{\prime}
$$

\subsubsection{Masse des Lösungsmittels in der Gasphase}

Wegen des angenommenen Idealgasverhaltens des Lösungsmitteldampfes gilt für das Gasvolumen

$$
V_{z}^{\prime \prime}=n_{\mathrm{LM}, z}^{\prime \prime} \cdot v_{0, \mathrm{LM}}^{\prime \prime}\left(p_{\mathrm{LM}, z}, t\right) .
$$

Da die molare Zusammensetzung des Lösungsmittels teilweise unbekannt ist, ist es notwendig, statt mit Stoffmenge $n_{\mathrm{LM}}^{\prime \prime}$ mit der Masse $m_{\mathrm{LM}}^{\prime \prime}$ zu rechnen. Die Masse des Lösungsmittels in der Gasphase $m_{\mathrm{LM}}^{\prime \prime}$ kann mit der Dichte des reinen LM-Dampfes des Lösungsmittels abgeschätzt werden zu

$$
m_{\mathrm{LM}, z}^{\prime \prime}=V_{z}^{\prime \prime} \cdot \rho_{\mathrm{LM}, z}^{\prime \prime}
$$

mit $\rho_{\mathrm{LM}, z}^{\prime \prime} \approx \rho_{\mathrm{LM}, \mathrm{s}}^{\prime \prime}\left(t_{z}^{\prime \prime}\right)$. Da Werte für Wasser-Glykol-Gemische nicht bekannt sind, werden diese in Abschnitt 4.7 plausibel abgeschätzt. 


\subsubsection{Stoffmenge des Arbeitsgases für Stickstoff-Versuche}

Für die Stickstoff-Versuche lässt sich die Stoffmenge $n_{\mathrm{N} 2}$ in der Gasphase bestimmen über

$$
n_{\mathrm{N} 2, z}^{\prime \prime}=\frac{V_{z}^{\prime \prime}}{v_{0, \mathrm{~N} 2}^{\prime \prime}\left(p_{\mathrm{N} 2, z}, t_{z}^{\prime \prime}\right)}
$$

mit $v_{0, \mathrm{~N} 2, z}^{\prime \prime}\left(p_{\mathrm{N} 2, z}, t_{z}^{\prime \prime}\right)=\frac{M_{i}}{\rho_{\mathrm{N} 2}\left(p_{\mathrm{N} 2, z}, t_{z}^{\prime \prime}\right)}$. Die Dichte $\rho_{\mathrm{N} 2}^{\prime \prime}\left(p_{\mathrm{N} 2, z}\right)$ wird mit Realgasgleichungen aus FluidLAB von [Kretzschmar, 2010] bestimmt.

\subsubsection{Stoffmenge der Arbeitsgase für Luft-Versuche}

Das Problem wurde durch Vereinfachungen reduziert auf ein Gemisch der Gase $\mathrm{N}_{2}$ und $\mathrm{O}_{2}$ beim Druck $p_{\mathrm{N} 2+\mathrm{O} 2, z}$. Da Stickstoff und Sauerstoff auch den größten Beitrag in der Gasphase ausmachen und v. a. deren Mengen sich zwischen den Zuständen wesentlich ändern, werden diese nicht getrennt idealisiert, sondern als Gemisch realer Gase betrachtet. Stoffdaten dafür sind vorhanden. Ein ideales Gemisch realer Gase (wie es auch in FluidLAB angenommen wird) soll hier jedoch ausreichen. Dann gilt folglich, dass die partiellen molaren Volumina der Gasphase konzentrationsunabhängig sind und also dem molaren Volumen der Reinstoffe entsprechen, also $v_{i}^{\prime \prime}=v_{0, i}^{\prime \prime}=$ konst. gilt.

Es verbleibt die Gleichung zur Bestimmung des Gesamtvolumens, in der die gesuchten Stoffmengen für Stickstoff und Sauerstoff eingehen

$$
V_{z}^{\prime \prime}=n_{\mathrm{N} 2, z}^{\prime \prime} \cdot v_{0, \mathrm{~N} 2}^{\prime \prime}\left(p_{\mathrm{N}_{2}+\mathrm{O}_{2}, \mathrm{z}}\right)+n_{\mathrm{O} 2, \mathrm{z}}^{\prime \prime} \cdot v_{0, \mathrm{O} 2}^{\prime \prime}\left(p_{\mathrm{N}_{2}+\mathrm{O}_{2}, \mathrm{z}}\right)
$$

bzw. bei bekannter Zusammensetzung $y_{\mathrm{O} 2}$ in $\mathrm{N} 2+\mathrm{O} 2, z$

$$
V_{z}^{\prime \prime}=n_{\mathrm{N} 2+\mathrm{O} 2, z}^{\prime \prime} \cdot\left[\left(1-y_{\mathrm{O} 2 \text { in } \mathrm{N} 2+\mathrm{O} 2, z}\right) \cdot v_{0, \mathrm{~N} 2}^{\prime \prime}\left(p_{\mathrm{N}_{2}+\mathrm{O}_{2}, z}\right)+y_{\mathrm{O} 2 \text { in } \mathrm{N} 2+\mathrm{O} 2, z} \cdot v_{0, \mathrm{O} 2}^{\prime \prime}\left(p_{\mathrm{N}_{2}+\mathrm{O}_{2}, z}\right)\right] \text {. }
$$

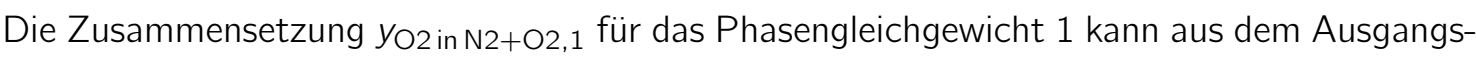
zustand nach der Befüllung abgeschätzt werden. Der Stoffmengenanteil $y_{\mathrm{O} 2}$ in $\mathrm{N} 2+\mathrm{O} 2,2 \mathrm{im}$ Phasengleichgewicht 2 wird durch Probenahme über einen Gaschromatographen bestimmt. Darauf wird später eingegangen.

Nun kann die Stoffmenge Stickstoff und Sauerstoff ermittelt werden $\mathrm{zu}$

$$
n_{\mathrm{N} 2+\mathrm{O} 2, z}^{\prime \prime}=\frac{v_{z}^{\prime \prime}}{\left(1-y_{\mathrm{O} 2 \text { in } \mathrm{N} 2+\mathrm{O} 2, z}\right) \cdot v_{0, \mathrm{~N} 2}^{\prime \prime}\left(p_{\mathrm{N}_{2}+\mathrm{O}_{2}, \mathrm{z}}, t_{z}^{\prime \prime}\right)+y_{\mathrm{O} 2 \text { in N2} 2+\mathrm{O} 2, z} \cdot v_{0, \mathrm{O} 2}^{\prime \prime}\left(p_{\mathrm{N}_{2}+\mathrm{O}_{2}, z}, t_{z}^{\prime \prime}\right)}
$$

wobei die molaren Volumina wiederum aus der bekannten Dichte der Reinstoffe zu $v_{0, i}^{\prime \prime}=\frac{M_{i}}{\rho_{i}^{\prime \prime}}$ bestimmt werden.

Anschließend lassen sich nun die Einzel-Stoffmengen von Stickstoff bzw. Sauerstoff bestimmen zu

$$
n_{i, z}^{\prime \prime}=y_{i \text { in } \mathrm{N} 2+\mathrm{O} 2, z} \cdot n_{\mathrm{N} 2+\mathrm{O} 2, z}^{\prime \prime} \text {. }
$$


Die Temperaturen $t_{1}^{\prime \prime}$ und $t_{2}^{\prime \prime}$ sind ungefähr gleich groß, werden jedoch für die exakte Berechnung beide genutzt.

4.5.5.5 Stoffmengenzunahme der Gasphase $\triangle n_{i, 1 \rightarrow 2}^{\prime \prime}$

Die Stoffmengenzunahme in der Gasphase für das Gas $i$ kann nun bestimmt werden zu

$$
\triangle n_{i, 1 \rightarrow 2}^{\prime \prime}=n_{i, 2}^{\prime \prime}-n_{i, 1}^{\prime \prime}
$$

\subsubsection{Flüssigphase des Reaktors}

\subsubsection{Bestimmung der gelösten Stoffmengen}

Die durch den Druckanstieg im Fluid gelöste Gasmenge ergibt sich aus der verbleibenden eingeschobenen Gasmenge zu

$$
\triangle n_{i, 1 \rightarrow 2}^{\prime}=\triangle n_{i, 1 \rightarrow 2}^{G}-\triangle n_{i, 1 \rightarrow 2}^{\prime \prime} .
$$

Da in die Bestimmung von $\triangle n_{i, 1 \rightarrow 2}^{\prime \prime}$ wiederum das Volumen der Flüssigphase $V^{\prime}$ eingeht, ist dieses, wie folgt, zu bestimmen.

\subsubsection{Abschätzung des Volumens der Flüssigphase}

Die in der Flüssigphase befindliche Lösungmittel-Masse berechnet sich mit der über Wägung bestimmten Masse $m_{\mathrm{LM}} \mathrm{zu}$

$$
m_{\mathrm{LM}, z}^{\prime}=m_{\mathrm{LM}}-m_{\mathrm{LM}, z}^{\prime \prime} .
$$

Das Volumen der Flüssigphase $V_{z}^{\prime}$ kann unter Vernachlässigung der Restbestandteile ${ }^{10}$ aber mit Berücksichtigung des gelösten Arbeitsgases näherungsweise bestimmt werden. Mit der Reinstoffdichte für Wasser $\rho_{0, \mathrm{LM}, z}^{\prime}$ und den partiellen molaren Volumina für Stickstoff $v_{\infty, \mathrm{N} 2}^{\prime}$ und Sauerstoff $v_{\infty, \mathrm{O} 2}^{\prime}$ bei „unendlicher Verdünnung" ergibt sich für Stickstoffversuche

$$
v_{z}^{\prime}=v_{0, \mathrm{LM}, z}^{\prime} \cdot n_{\mathrm{LM}, z}^{\prime}+v_{\infty, \mathrm{N} 2}^{\prime} \cdot n_{\mathrm{N} 2, z}^{\prime}=\frac{m_{\mathrm{LM}, z}^{\prime}}{\rho_{0, \mathrm{LM}, z}^{\prime}}+v_{\infty, \mathrm{N} 2}^{\prime} \cdot n_{\mathrm{N} 2, z}^{\prime}
$$

und Luftversuche

$$
v_{z}^{\prime}=\frac{m_{\mathrm{LM}, \mathrm{z}}^{\prime}}{\rho_{0, \mathrm{LM}, \mathrm{z}}^{\prime}}+v_{\infty, \mathrm{N} 2}^{\prime} \cdot n_{\mathrm{N} 2, \mathrm{z}}^{\prime}+v_{\infty, \mathrm{O} 2}^{\prime} \cdot n_{\mathrm{O} 2, \mathrm{z}}^{\prime} .
$$

Wichtig ist hier, dass die Kompressibilität des Lösungmittels, also $\rho_{0, \mathrm{LM}, z}^{\prime}=f\left(p_{z}, t_{z}^{\prime}\right)$, berücksichtigt wird. Verwendete Werte für $\rho_{0, \mathrm{LM}, z}^{\prime}$ und $v_{\infty, i}$ werden später in Abschnitt 4.7 diskutiert.

${ }^{10}$ Der Einfluss gelöster Restgase auf das Volumen $V^{\prime}$ kann selbst bei Ausgangszustand luftgesättigt als deutlich kleiner als andere Unsicherheiten wie die der Masse abgeschätzt werden. 
Für eine präzise Bestimmung des Volumens werden also auch die jeweils gelösten Stoffmengen der Gase $n_{\mathrm{N} 2, z}^{\prime}$ (und für Luftversuche auch $n_{\mathrm{O} 2, z}^{\prime}$ ) im Zustand $z=1$ und $z=2$ benötigt. ${ }^{11}$

Bei bekanntem Löslichkeitskoeffizienten $\lambda_{i}$ lässt sich die gelöste Gasmenge im Zustand 1 über

$$
n_{i, 1}^{\prime}=\lambda_{i, \infty} \cdot \frac{m_{\mathrm{LM}, 1}^{\prime}}{v_{0, i, \mathrm{~N}}} \cdot \frac{p_{i, 1}}{\operatorname{Poy}\left(p_{1}, p_{\mathrm{LM}, \mathrm{s}}\right)}
$$

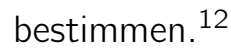

Da der Löslichkeitskoeffizient $\lambda_{i}$ selbst gesucht ist, wird ein iteratives Verfahren benötigt. $^{13}$

Für Zustand 2 lässt sich die gelöste Gasmenge bestimmen über

$$
n_{i, 2}^{\prime}=n_{i, 1}^{\prime}+\triangle n_{i, 1 \rightarrow 2}^{\prime}
$$

\subsubsection{Bestimmung des Löslichkeitskoeffizienten}

Die Änderung des Gasgehalts in der Flüssigkeit zwischen den zwei Phasengleichgewichten bestimmt sich zu

$$
\Delta w_{i, 1 \rightarrow 2}=w_{i, 2}-w_{i, 1}=\left[\frac{n_{i, 2}^{\prime}}{m_{\mathrm{LM}, 2}^{\prime}}-\frac{n_{i, 1}^{\prime}}{m_{\mathrm{LM}, 1}^{\prime}}\right] \cdot v_{0, i, \mathrm{~N}}
$$

Als Partialdruck in den Stickstoff-Versuchen wird

$$
p_{\mathrm{N} 2, z}=p_{z}-p_{\text {Rest }}-p_{\mathrm{LM}, \mathrm{s}}\left(t_{z}^{\prime \prime}\right)
$$

verwendet. Für die Luft-Versuche werden die Partialdrücke mit

$$
p_{\mathrm{N} 2, z}=y_{\mathrm{N} 2 \text { in } \mathrm{N} 2+\mathrm{O} 2, z} \cdot p_{\mathrm{N} 2+\mathrm{O} 2, z}=y_{\mathrm{N} 2 \text { in } \mathrm{N} 2+\mathrm{O} 2, z} \cdot\left(p_{z}-p_{\mathrm{Rest}}-p_{\mathrm{LM}}\left(t_{z}^{\prime \prime}\right)\right)
$$

und

$$
p_{\mathrm{O} 2, z}=y_{\mathrm{O} 2 \text { in } \mathrm{N} 2+\mathrm{O} 2, z} \cdot p_{\mathrm{N} 2+\mathrm{O} 2, z}=y_{\mathrm{O} 2 \text { in } \mathrm{N} 2+\mathrm{O} 2, z} \cdot\left(p_{z}-p_{\mathrm{Rest}}-p_{\mathrm{LM}}\left(t_{z}^{\prime \prime}\right)\right)
$$

bestimmt. ${ }^{14}$

\footnotetext{
${ }^{11}$ Für den Ausgangszustand „entgast“ sind im Phasengleichgewicht 1 (Drucksprung 1) gelöste Gasanteile und damit auch deren Einfluss auf das Flüssigvolumen marginal. Diese werden jedoch im Sinne eines einheitlichen Berechnungsalgorithmus ebenso berechnet.

${ }^{12}$ Der Partialdruck $p_{i, 1}$ in der Gleichung wird im nächsten Abschnitt behandelt.

${ }^{13}$ Für den Drucksprung 2 könnte auch auf den aus Drucksprung 1 berechneten Löslichkeitkoeffizienten zurückgegriffen werden. Im Sinne unabhängiger Auswertungen wird davon jedoch abgesehen.

${ }^{14}$ Während für die Bestimmung der Stoffmengen in der Gasphase für Luft-Versuche aus Genauigkeitsgründen nicht mit Partialdrücken der Einzelgase, sondern $p_{\mathrm{N} 2+\mathrm{O} 2}$ gerechnet worden ist, soll jetzt für die Bestimmung der Löslichkeit sehr wohl mit der idealisierten Hilfsgröße der Partialdrücke der Einzelgase gerechnet werden.
} 
Damit ergibt sich der (partialdruckabhängige) Anstieg des Gasgehalts nun zu

$$
\frac{\triangle W_{i, 1 \rightarrow 2}}{\triangle p_{i, 1 \rightarrow 2}^{*}}=\frac{\left[\frac{n_{i, 2}^{\prime}}{m_{\mathrm{LM}, 2}^{\prime}}-\frac{n_{i, 1}^{\prime}}{m_{\mathrm{LM}, 1}^{\prime}}\right] \cdot v_{0, i, \mathrm{~N}}}{\left(\frac{p_{i, 2}}{\operatorname{Poy}\left(p_{2} ; p_{\mathrm{LM}, \mathrm{s}}\right)}-\frac{p_{i, 1}}{\operatorname{Poy}\left(p_{1} ; p_{\mathrm{LM}, \mathrm{s}}\right)}\right)} .
$$

Wenn davon ausgegangen werden kann, dass

$$
\frac{\mathrm{d} w_{i}}{\mathrm{~d} p_{i}^{*}} \approx \text { konst. }
$$

im Bereich $p_{i}=0$.. $p_{i, 2}$ (Gültigkeitsbereich des Löslichkeitskoeffizienten) gilt, dann entspricht dies dem Löslichkeitskoeffizienten, wie bereits in Abschnitt 4.4.1.2 diskutiert. Damit gilt

$$
\lambda_{i, \infty}=\frac{\triangle w_{i, 1 \rightarrow 2}}{\triangle p_{i, 1 \rightarrow 2}^{*}}
$$

Als zum berechneten Löslichkeitskoeffizienten $\lambda_{i, \text { LM }}$ zugehörige Temperatur wird formal die Temperatur $t_{2}^{\prime}$ verwendet. Die Temperatur im Zustand $1 t_{1}^{\prime}$ weicht in den meisten Versuchen um weniger als $0,1 \mathrm{~K}$ ab. ${ }^{15}$

\subsubsection{Einordnung und Lösung des aufgestellten Gleichungssystems}

\subsubsection{Beschreibung des Phasengleichgewichts am Beispiel Stickstoff}

Zur systematischen Einordnung der obigen Gleichungen in die allgemeine Bestimmung eines Phasengleichgewichts soll Abbildung 4.12 helfen. Auf der linken Seite ist das verwendete Gleichungssystem zur Bestimmung des Phasengleichgewichts in üblicher, allgemeiner Form dargestellt. Alle eingegebenen Stoffmengen $n_{k}$ sind dort direkt bekannt. Auch alle Zusammenhänge zwischen Gas- und Flüssigphase $y_{k}=f\left(x_{k}\right)$ sind bekannt, jedoch nicht für das gesuchte Gas $j$. Da auch das Volumen $V$ bekannt ist, lässt sich das Phasengleichgewicht vollständig bestimmen. Das Gleichungssystem ist nichtlinear. Dies macht eine iterative Bestimmung für jedes Phasengleichgewicht notwendig.

Durch oben beschriebene Vereinfachungen und Anpassungen an die Spezifika des Versuchsstands und der Berechnung lässt sich das Gleichungssystem auf die auf der rechten Seite in Abbildung 4.12 dargestellten Gleichungen reduzieren. Während in einem Standard-Verfahren die eingegebenen Stoffmengen $n_{i}$ bekannt sind, sollen für die Restgase hier stattdessen die Partialdrücke bekannt sein. Das Gleichungssystem bleibt auch im vereinfachten Zustand (rechts) nichtlinear und muss weiterhin iterativ berechnet werden. Iterative Berechnungsalgorithmen für ähnliche Bedingungen finden sich z. B. in [Pfennig, 2003, S. 164] oder [Fischer und Wilken, 2001].

${ }^{15}$ In wenigen Versuchen liegt die Temperatur $t_{1}^{\prime}$ bis zu 0,5 K unter $t_{2}^{\prime}$. Dies waren Versuche bei hohen Temperaturen. Es wird vermutet, dass die starke Entgasung doch zu einem deutlich anderen Rührverhalten führt und entsprechend die eingetragene Rührerleistung sowie die gleichmäßige Temperierung des Fluids beeinflusst. Dass die hohen Temperaturunterschiede nur beim ersten Drucksprung auftreten, stärkt diese Vermutung. 


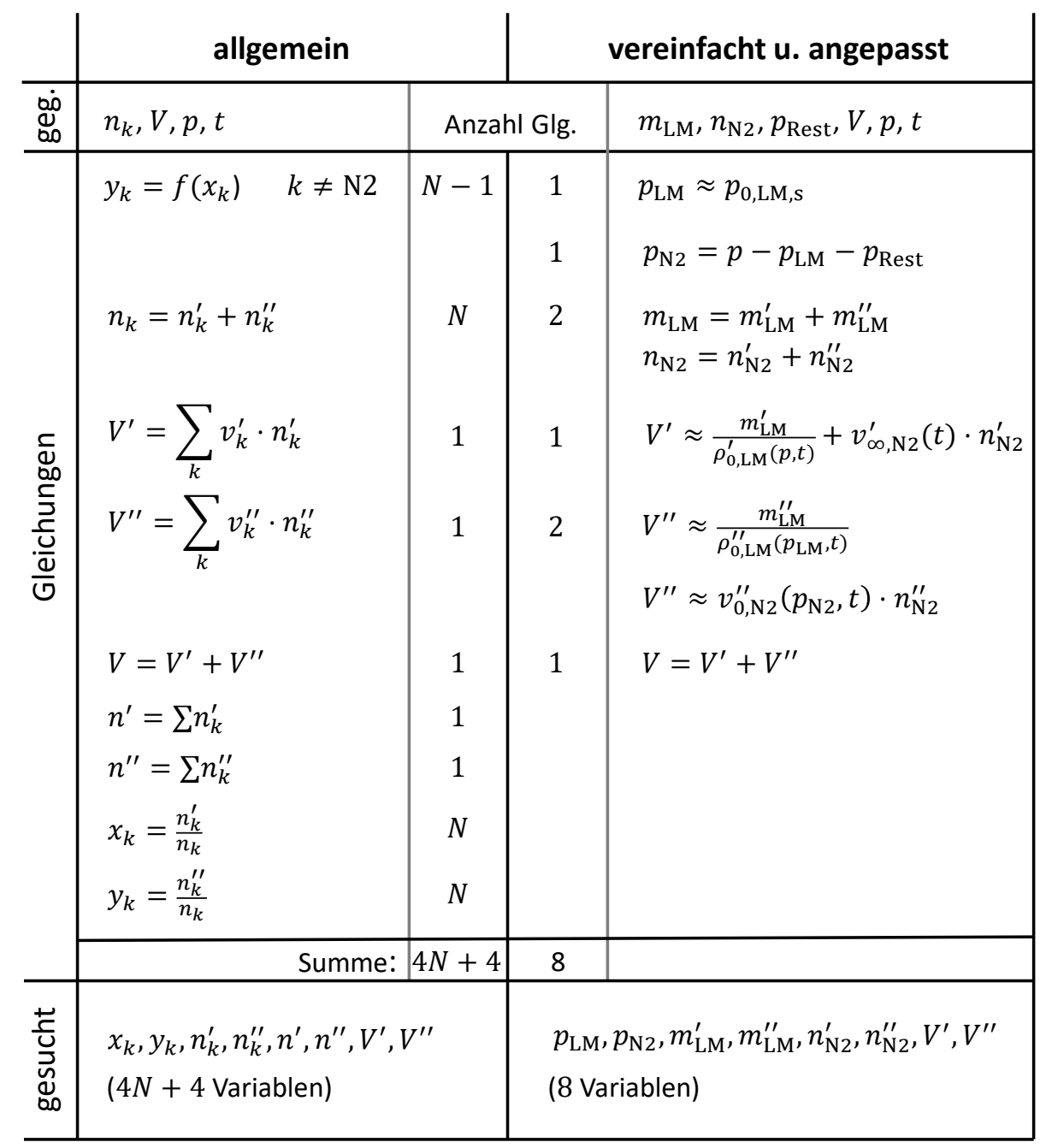

Abbildung 4.12: Gleichungen für ein Phasengleichgewicht bei unbekanntem Löslichkeitsverhalten von Stickstoff. Links: allgemein für Komponenten $k=1 . . N$. Rechts: am Beispiel der StickstoffVersuche

\subsubsection{Bestimmung des Löslichkeitskoeffizienten - Besonderheit des Versuchsablaufs}

Der technische Löslichkeitskoeffizient ließe sich nun entsprechend Gleichung 4.1 einfach bestimmen zu

$$
\lambda_{\mathrm{N} 2, \infty}=\frac{n_{\mathrm{N} 2,2}^{\prime} \cdot v_{0, \mathrm{~N} 2, \mathrm{~N}} \cdot \operatorname{Poy}\left(p ; p_{0, \mathrm{LM}, \mathrm{s}}\right)}{m_{\mathrm{LM}}^{\prime \prime} \cdot p_{\mathrm{N} 2,2}} .
$$

Nun soll aber die im Reaktor befindliche Stoffmenge Gas $n_{N 2,1}$ und damit auch $n_{N 2,2}$ nicht direkt bekannt sein, da eine gewisse nur grob abschätzbare Menge Stickstoff bereits im Phasengleichgewicht 1 eingeschlossen ist. Stattdessen soll der Löslichkeitskoeffizient $\lambda_{i, \infty}$ aus zwei Phasengleichgewichten generiert werden und gleichzeitig aus dem bestimmten Löslichkeitsverhalten $\lambda_{i, \infty}$ die gelöste Gasmenge $n_{i, 1}^{\prime}$ im Zustand 1 abgeschätzt werden. Damit können beide Phasengleichgewichte und der Löslichkeitskoeffizient, wie in Abbildung 4.13 dargestellt, nur gemeinsam ermittelt werden. 


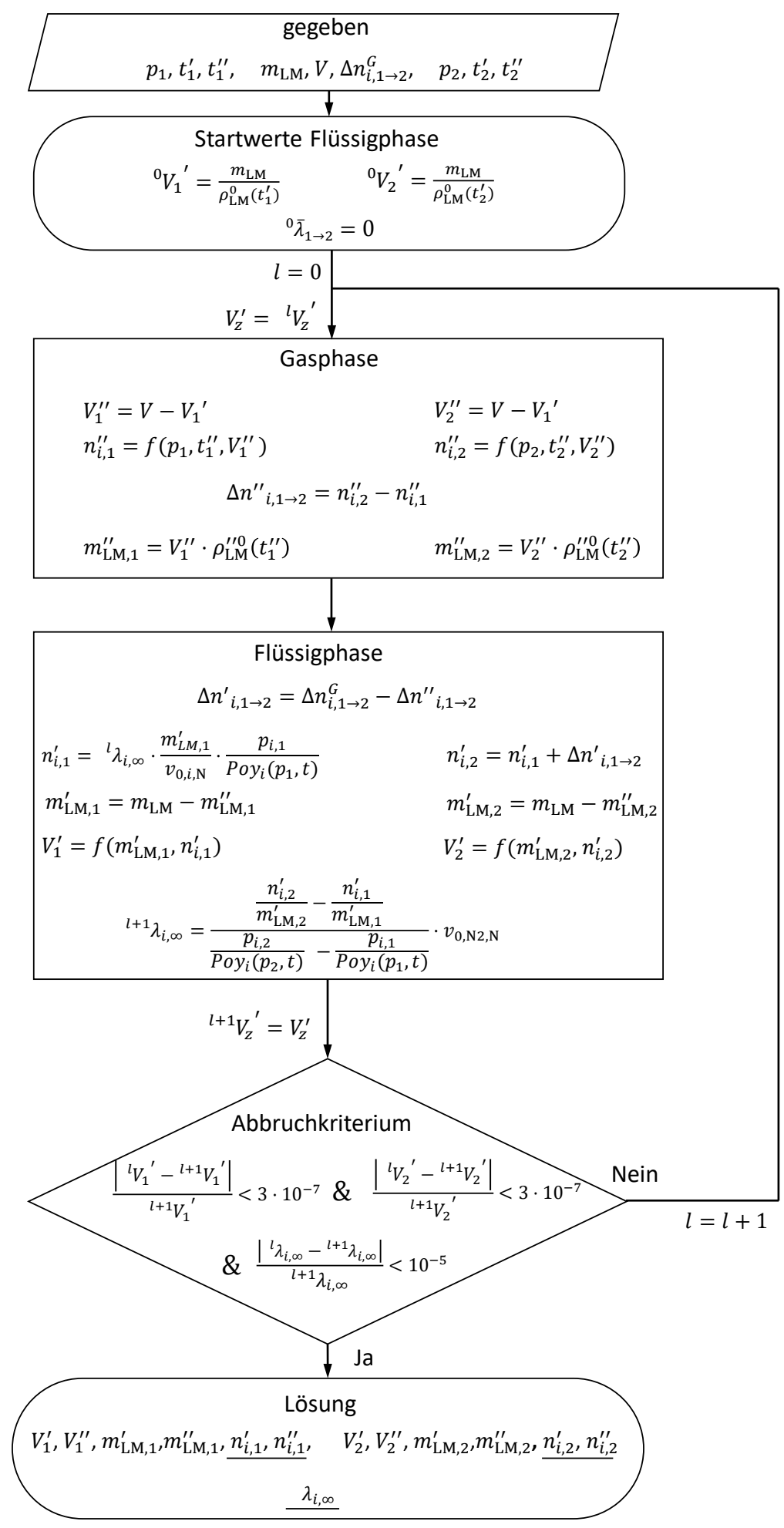

Abbildung 4.14: Berechnungsalgorithmus für das Phasengleichgewicht für Stickstoff-Versuche; $i=\mathrm{N}_{2}$

Abbruchkriterien sind so festgelegt, dass die verbleibende Iterations-Unsicherheit nicht nur für das Ergebnis, sondern auch für die einzelnen Messunsicherheitsbeiträge der anschließenden Messunsicherheitsbetrachtung marginal sind. Die Versuchsauswertungen zeigen, dass 
für die durchgeführten Versuche bis $110^{\circ} \mathrm{C}$ hier bereits in der Regel bei $/=3$ oder $I=4$ das Abbruchkriterium ${ }^{16}$ erreicht ist und somit die Rechengeschwindigkeit akzeptabel bleibt.

\subsection{Kalibrierung der Apparatur}

Die Bestimmung der Löslichkeit $\lambda_{i, L M}, \infty$,mess für das gelöste Gas $i$ ist stark mit Messabweichungen behaftet. Da Löslichkeiten für Wasser bekannt sind, werden die gemessenen Löslichkeiten für Wasser und der zu untersuchenden Flüssigkeit (bei sonst gleichen Bedingungen wie z. B. Temperaturen, Drücke, Versuchsablauf, Zeiten) vergleichend betrachtet.

Mit folgender Formel kann durch die Kalibriermessungen mit Wasser ein Abweichungsoffset der Messung der unbekannten Löslichkeit im Lösungsmittel LM entfernt werden:

$$
\lambda_{i, \mathrm{LM}, \infty, \text { kalibriert }}=\lambda_{i, \mathrm{LM}, \infty, \text { mess }}-\left(\lambda_{i, \mathrm{H} 2 \mathrm{O}, \infty, \text { mess }}-\lambda_{i, \mathrm{H} 2 \mathrm{O}, \infty, \mathrm{Lit}}\right) .
$$

Die ausführliche mathematische Begründung für dieses Vorgehen und warum es genau zu dieser Formel kommt, wird im Anhang A.10 auf Seite 186 geliefert. Der korrigierte Messwert wird im folgenden als kalibrierter Messwert im Gegensatz zum unkalibrierten Messwert bezeichnet.

Mit dieser Variante der Kalibrierung wird nicht nur der Wert des Messergebnisses näher an den wahren Wert gebracht, sondern es kann auch der Messunsicherheitsbereich deutlich verkleinert werden. Der Einfluss bekannter Messunsicherheiten und auch unbekannter bzw. schlecht quantifizierbarer Messunsicherheiten wird minimiert.

Die Löslichkeiten von LM (Versuch I) und Wasser (Versuch II) werden in getrennten Versuchen bestimmt. Stellt man diese Versuche gegenüber, so gibt es in den Berechnungen

- sich unterscheidende, variable Eingangsgrößen (z. B. zufällige Messunsicherheiten der Druckanzeige, Messunsicherheit Dichte),

- gleiche, konstante Eingangsgrößen mit gleicher (unbekannter) Messabweichung (z. B. Volumina $V_{0}^{G}$ und $V_{0}^{R j}$, Kalibrierkurven der Sensoren).

Der Einfluss der Messabweichung der gleichen/konstanten Eingangsgrößen auf das Messergebnis kann durch Kenntnis der präzisen tabellierten Löslichkeit eines Referenzversuchs (Versuch II) verringert werden. Über sich unterscheidende Eingangsgrößen kann keine Information aus Referenzversuchen gewonnen werden. Daher „addieren“ sich hier sogar die Messunsicherheiten aus Versuch I und II bei der kalibrierten Berechnung. Im günstigen Fall haben die gleichen Eingangsgrößen einen deutlich höheren Anteil an der Gesamtunsicherheit

${ }^{16}$ Das sich aus der eingefüllten Masse $m_{\mathrm{LM}}$ ergebende Volumen hat etwa die gleiche Sensitivität auf das Berechnungsergebnis wie das Fluid- oder Gasvolumen. Daher kann das Abbruchkriterium an der relativen (systematischen) Messunsicherheit der Masse orientiert werden. Die Festlegung auf ca. ein Zehntel der systematischen Unsicherheit der Masse sollte demnach reichen, damit die verbleibende IterationsUngenauigkeit für das Gesamtergebnis marginal ist. Jedoch wird zur Berechnung und Analyse der einzelnen Unsicherheitsbeiträge ein deutlich schärferes Kriterium benötigt, damit die Iterations-Ungenauigkeit nur einen marginalen Einfluss auf die einzelnen Messunsicherheitsbeiträge besitzt. Dieses wird daher festgelegt zu einem Hundertstel der systematischen Unsicherheit der Masse zu $\frac{1}{100} \cdot \frac{0,02 \mathrm{~g}}{700 \mathrm{~g}} \approx 3 \cdot 10^{-7}$. Zusätzlich wird der technische Löslichkeitskoeffizient auf eine nur noch marginale Änderung von $10^{-5}$ eingestellt. 
als die sich unterscheidenden Eingangsgrößen. Dann ist die Verwendung der kalibrierten Berechnung sinnvoll, weil sich die Gesamt-Messunsicherheit reduzieren lässt. Dies ist bei den Versuchen der Fall. ${ }^{17}$

Zusätzlich werden Abweichungen, auch möglicherweise unbekannte, die in Referenzversuch und Versuch absolut gleich sind, durch die Kalibrierung gänzlich eliminiert. Daher sind Versuche so „ähnlich“ wie möglich durchzuführen.

\subsection{Erläuterungen zu Einflussgrößen und deren Unsicherheit}

\subsubsection{Allgemeines}

Für die Ermittlung der Löslichkeitskoeffizienten aus den vorgestellten Versuchen sind einige Stoffwerte zu beachten. Ebenso ist eine Reihe versuchsspezifischer Einflussgrößen zu berücksichtigen. In der Tabelle 4.6 sind die demnach verbleibenden relevanten Größen und deren für die Rechnung verwendete Unsicherheit dargestellt. Diese werden im Folgenden einzeln vorgestellt.

Das molare Normvolumen $v_{0, i, N}$ taucht nicht separat auf, wird aber über

$$
v_{0, i, \mathrm{~N}}=\frac{\rho_{i, \mathrm{~N}}}{M_{i}}=\frac{\rho_{i}^{\prime \prime}\left(1,01325 \text { bar; } 0^{\circ} \mathrm{C}\right)}{M_{i}}
$$

bestimmt. Molare Massen $M_{i}$ werden genügend genau aus FluidLab von [Kretzschmar, 2010] (und daher ohne Berücksichtigung von Unsicherheiten) verwendet.

\subsubsection{Gase im Ausgangzustand und im Phasengleichgewicht 1}

Die Stoffzusammensetzung im Ausgangszustand (abgeschlossener Reaktor vor der Temperierung) lässt sich nur abschätzen. Der Partialdruck der Restgase lässt sich bestimmen über

$$
p_{\text {Rest,Ausg }}=y_{\mathrm{tr}, \text { Rest,Ausg }} \cdot p_{\mathrm{tr}, \text { Ausg }} \text {, }
$$

wobei $p_{\text {tr,Ausg }}=p_{\text {Ausg }}-p_{\mathrm{LM}, \mathrm{s}}$ den Gesamtpartialdruck aller Gase ohne Lösungsmitteldampf im Ausgangszustand nach der Befüllung darstellt.

Die Zusammensetzung der Gasphase und daher auch die angenommenen Anteile $y_{\text {tr, Rest,Ausg }}$ sind nicht gemessen worden. Für die Berechnung müssen diese daher mit großem Unsicherheitsbereich eingeordnet werden:

Stickstoff-Versuche wurden mit „luftgesättigtem“ und „entgastem“ Ausgangszustand durchgeführt. Vermutet wurde, dass im Ausgangszustand "luftgesättigt" nach dem Verschließen des Reaktors die Luftbestandteile bereits im Gleichgewicht mit der Lösung stehen, da die Lösungsmittel längere Zeit in nicht diffusionsdichten Kunststoffbehältern gelagert

\footnotetext{
${ }^{17}$ Für Referenzversuche mit Wasser hätte man den Berechnungsalgorithmus hinsichtlich Isofugazität weiter präzisieren können, da hier gute thermodynamische Beschreibungen für das Mehrkomponentengemisch Wasser mit Atmosphärengasen existieren. Dies hätte eine geringfügig genauere Bestimmung der Löslichkeit für die Referenzversuche geliefert. Jedoch entfällt dann der Vorteil, dass durch vereinfachte Berechnung verursachte Abweichungen sich durch obige Kalibrierung teilweise gegenseitig aufheben.
} 
Tabelle 4.6: Hilfsgrößen, Konstanten und Stoffwerte und deren angenommene Unsicherheit

\begin{tabular}{|c|c|c|c|c|}
\hline Größe & Anwendungsbereich & Wert & Unsicherheit & Quelle der Daten \\
\hline$V_{0}^{\mathrm{G}}, V_{0}^{\mathrm{R} 0}, V_{0}^{\mathrm{R} 1}, V_{0}^{\mathrm{R} 2}$ & - & siehe Tabelle A.3 & & eigene Messungen \\
\hline$\alpha$ für Volumina & - & siehe Tabelle A.4 & & Schätzung \\
\hline \multirow[t]{2}{*}{$y_{\text {tr, Rest, Ausg }}$} & Stickstoff-Versuche & $0,22\left(\right.$ mit $\left.\mathrm{O}_{2}\right)$ & 0,2 & $\begin{array}{l}\text { Schätzung basierend auf Luftzusammen- } \\
\text { setzung nach [Wallace und Hobbs, 2006] }\end{array}$ \\
\hline & Luft-Versuche & 0,1 (ohne $\mathrm{O}_{2}$ ) & 0,1 & $\begin{array}{l}\text { Schätzung basierend auf Luftzusammen- } \\
\text { setzung und nichtproportionaler Entgasung }\end{array}$ \\
\hline$y_{\mathrm{N} 2}^{\mathrm{G}}$ & nur Luft-Versuche & 0,795 & 0,005 & Synthetische Luft KW-frei, [Air Liquide 2] \\
\hline$y_{\mathrm{O} 2}$ in $\mathrm{N} 2+\mathrm{O} 2,1$ & nur Luft-Versuche & $\frac{0,209}{0,78+0,209} \approx 0,21$ & 0,21 & $\begin{array}{l}\text { Schätzung basierend auf Luftzusammen- } \\
\text { setzung nach [Wallace und Hobbs, 2006] } \\
\text { und nichtproportionaler Entgasung }\end{array}$ \\
\hline \multirow[t]{2}{*}{$\overline{\rho_{i}^{\prime \prime}}$} & $\begin{array}{l}\text { Stickstoff-Versuche, } \\
i=\mathrm{N}_{2}\end{array}$ & $f\left(p_{\mathrm{N} 2}, t\right)$ & - & FluidLAB von [Kretzschmar, 2010] \\
\hline & $\begin{array}{l}\text { Luft-Versuche, } \\
i=\mathrm{N}_{2}, \mathrm{O}_{2}\end{array}$ & $f\left(p_{\mathrm{N} 2+\mathrm{O} 2}, t\right)$ & - & FluidLAB von [Kretzschmar, 2010] \\
\hline \multirow[t]{2}{*}{$\rho_{\mathrm{LM}, \mathrm{s}}^{\prime \prime}$} & Wasser & $f(t)$ & $0,003 \%$ & [IAPWS IF-97, 2007] \\
\hline & $\begin{array}{l}\text { Wasser-Glykol } \\
\text { (mit/ohne Additive) }\end{array}$ & $\rho_{\mathrm{H} 2 \mathrm{O}, \mathrm{s}}^{\prime \prime}(t)$ & $100 \%$ & grobe Schätzung \\
\hline \multirow[t]{2}{*}{$\overline{\rho_{\mathrm{LM}}^{\prime}}$} & Wasser & $f(p, t)$ & $0,003 \%$ & [IAPWS IF-97, 2007] \\
\hline & $\begin{array}{l}\text { Wasser-Glykol } \\
\text { (mit/ohne Additive) }\end{array}$ & $f(1$ bar,$t)$ & $0,1 \%$ & Biegeschwingermessungen \\
\hline \multirow[t]{6}{*}{$p_{\mathrm{s}}$} & Wasser & $f(t)$ & $0,03 \%$ & [IAPWS IF-97, 2007] \\
\hline & Tyfocor LS & $f(t)$ & $5 \%$ & Datenblatt Tyfocor ${ }^{\circledR}$ LS \\
\hline & Tyfocor & $f(t)$ & $20 \%$ & Datenblatt Tyfocor ${ }^{\circledR}$ \\
\hline & $(41,84$ gew.- $\%)$ & & & \\
\hline & reines Wasser-Glykol & $f(t)$ & $10 \%$ & [Ambrose und Hall, 1981]/ \\
\hline & & & & [Rane u. a., 2016] u. Raoultsches Gesetz \\
\hline \multirow[t]{2}{*}{$\overline{\phi_{\text {sat }, i}}$} & Wasser & 1 & 0,15 & Schätzung \\
\hline & $\begin{array}{l}\text { Wasser-Glykol } \\
\text { (mit/ohne Additive) }\end{array}$ & 0,5 & 0,5 & Schätzung \\
\hline \multirow[t]{2}{*}{$v_{\infty, \mathrm{N} 2 / \mathrm{O} 2}^{\prime}$} & Wasser & $33 \mathrm{~cm}^{3} / \mathrm{mol}$ & $15 \mathrm{~cm}^{3} / \mathrm{mol}$ & grobe Einordnung nach Tab. 2.5 \\
\hline & $\begin{array}{l}\text { Wasser-Glykol } \\
\text { (mit/ohne Additive) }\end{array}$ & $30 \mathrm{~cm}^{3} / \mathrm{mol}$ & $30 \mathrm{~cm}^{3} / \mathrm{mol}$ & grobe Einordnung nach Tab. 2.5 \\
\hline \multirow[t]{3}{*}{$\kappa_{t}$} & Wasser & indirekt über $\rho_{\mathrm{H} 2 \mathrm{O}}^{\prime}$ & $=f(p, t)$ & [IAPWS IF-97, 2007] \\
\hline & Wasser-Glykol & $4 \cdot 10^{-4} \mathrm{MPa}^{-1}$ & $3 \cdot 10^{-4} \mathrm{MPa}^{-1}$ & Schätzung basierend auf \\
\hline & (mit/ohne Additive) & & & [Guignon u. a., 2010] \\
\hline \multirow[t]{2}{*}{$q_{\text {Leck }}$} & Gasreservoir & $0,12 \mathrm{mbar} / \mathrm{h}$ & $0,12 \mathrm{mbar} / \mathrm{h}$ & Leckagemessungen \\
\hline & Reaktor & $0,25 \mathrm{mbar} / \mathrm{h}$ & $0,25 \mathrm{mbar} / \mathrm{h}$ & Leckagemessungen \\
\hline \multirow[t]{3}{*}{$\lambda_{i, \mathrm{H} 2 \mathrm{O}, \infty, \mathrm{Lit}}$} & Kalibrierung der & $f(t)$ & $2 \%$ & [IAPWS, 2004] \\
\hline & Glykol-Wasser- & & & \\
\hline & Versuche & & & \\
\hline
\end{tabular}

Wasser-Stoffdaten aus [IAPWS IF-97, 2007] werden über FluidLAB von [Kretzschmar, 2010] eingebunden. 
wurden. Jedoch zeigt sich beim ersten Rühren bei dem untersuchten Stoff Tyfocor ${ }^{\circledR}$ LS eine leichte Absorption. Es ist nicht klar, ob dies Sauerstoff und Stickstoff gleichermaßen betrifft. Für Ausgangszustand „entgast" ist durch das Evakuieren insbesondere aufgrund unterschiedlicher Henry-Koeffizienten für Sauerstoff und Stickstoff, und folglich unterschiedlichen ausgasenden Mengen, mit einer veränderten Gasphase zu rechnen. Der Anteil $y_{t r, R e s t, A u s g}$ für Stickstoff-Versuche wird orientierend an der Luftzusammensetzung (vgl. [Wallace und Hobbs, 2006]) aber für alle Versuche relativ unsicher abgeschätzt zu $y_{\text {tr, Rest,Ausg }}=(22 \pm 20) \%$.

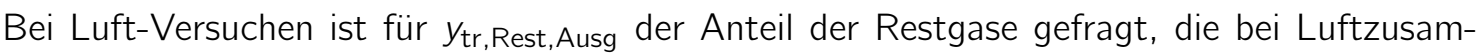
mensetzung ca. $1 \%\left(\mathrm{CO}_{2}\right.$, Ar etc.) einnehmen. Luft-Versuche beginnen alle mit einem entgasten Ausgangszustand. Um die möglicherweise stattfindende deutliche Anteilserhöhung durch nichtproportionale Entgasung der Restgase zu berücksichtigen, wird hier sicherheitshalber ein deutlich größerer Anteil als bei Luft mit $y_{\mathrm{tr} \text {,Rest,Ausg }}=0 . .20 \%$ angenommen. Zur Angabe eines symmetrischen Unsicherheitsbereichs des Ergebnisses wird zur Berechnung $y_{\text {tr,Rest,Ausg }}=(10 \pm 10) \%$ verwendet. In der später folgenden Messunsicherheitsbetrachtung kann man sehen, dass die Unsicherheit dieser Werte nur geringe Auswirkung auf die Messergebnisse haben.

Es wird davon ausgegangen, dass $\frac{p_{\text {Rest.z }}}{T_{z}^{\prime \prime}}=$ konst. für den Ausgangzsustand (mit 293,15 K angenommen ${ }^{18}$ ) und alle Phasengleichgewichte $z$ des Versuchsablaufs näherungsweise gilt. Der Partialdruck $p_{\text {Rest, } z}$ in den Phasengleichgewichten mit dem Temperaturniveau $t_{z}^{\prime \prime}$ ergibt sich dann zu

$$
p_{\text {Rest }, z}=\frac{T_{z}^{\prime \prime}}{293,15 \mathrm{~K}} \cdot p_{\text {Rest,Ausg }}
$$

19. Diese vernachlässigt, dass sich die gelöste Menge an Restgasen durch temperaturabhängige Löslichkeit und Veränderung von $V^{\prime \prime}$ und damit der Partialdruck $p_{\text {Rest }}$ leicht ändern wird. Es wird angenommen, dass diese Effekte marginal sind.

Für Luft-Versuche ist zusätzlich der Anteil an Sauerstoff im Phasengleichgewicht 1 $y_{\mathrm{O} 2}$ in N2+O2,1 gesucht. Dieser wird auch vereinfacht grob abgeschätzt wie Tabelle 4.6 angegeben.

\subsubsection{Zusammensetzung der Synthetischen Luft $y_{\mathrm{N} 2}^{G}$}

Die Unsicherheit in der Zusammensetzung der synthetischen Luft orientiert sich an den Vorgaben des Herstellers. Eigene gaschromatographische Messungen bestätigen die Werte.

\subsubsection{Dichte der Gase $\rho_{i}^{\prime \prime}$}

Für die druck- und temperaturabhängige Dichte der reinen Gase $\mathrm{N}_{2}$ bei Stickstoff-Versuchen bzw. $\mathrm{N}_{2}$ und $\mathrm{O}_{2}$ bei Luft-Versuchen wird die Realgasbeziehung aus FluidLAB von [Kretzschmar, 2010] verwendet.

\footnotetext{
${ }^{18}$ Dies soll für die Abschätzung ausreichen. Die Temperatur des Ausgangszustands der Versuche variierte zwischen $18^{\circ} \mathrm{C} .22^{\circ} \mathrm{C}$.

${ }^{19}$ Temperatur und Druck $p_{\text {tr,Ausg }}$ des Ausgangszustands werden nicht mit Messunsicherheit beaufschlagt. Dies soll durch die reichliche Unsicherheit von $y_{\text {Rest,Ausg }}$ abgegolten sein.
} 


\subsubsection{Dichte des Lösungsmitteldampfs $\rho_{\mathrm{LM}}^{\prime \prime}$}

Für die Dichte des reinen Dampfes werden bei dem Lösungmittel Wasser entsprechend Tabelle 4.6 Werte aus FluidLAB von [Kretzschmar, 2010] verwendet. Für die Dichte des reinen Dampfes der zu untersuchenden Lösungsmittel sind keine Werte bekannt. Die Dampfdichte der Wasser-Glykol-Gemische wird daher sehr grob mit der Dampfdichte des Wasser abgeschätzt. Die später folgende Messunsicherheitsbetrachtung wird zeigen, dass die Ergebnisse trotz der starken Unsicherheit keinen großen Einfluss haben. ${ }^{20}$

\subsubsection{Dichte des Lösungsmittels $\rho_{\mathrm{LM}}^{\prime}$}

\subsubsection{Einfluss des Dichtemesswerte oder Näherungsgleichungen}

Bei zulässigen Dichtetoleranzen in der Qualitätssicherung des Herstellers (Tyforop Chemie $\mathrm{GmbH}$ ) von bspw. $\rho_{\text {TyfLS, } 20^{\circ} \mathrm{C}}=1,032 . .1,035 \frac{\mathrm{g}}{\mathrm{cm}^{3}}$ des Endprodukts ist eine direkte Dichtebestimmung der im Versuch verwendeten Produktchargen notwendig. Die Dichtemessungen konnte mit dem zur Verfügung stehenden Messgerät bei Umgebungsdruck und nur bis $90{ }^{\circ} \mathrm{C}$ erfolgen. Werte und entsprechende Vergleichswerte aus der Literatur sind in den Tabellen A.20, A.21 und A.22 dokumentiert.

Die gemessenen Dichten von 25 gew.- $\%$ und 41,84 gew.- \% Propylenglykol-Wasser- bzw. Ethylenglykol-Wasser-Gemischen konnten mit den von [Melinder, 2010] vorgeschlagenen Regressionsgleichungen mit einem Gültigkeitsbereich bis $60 \mathrm{Gew} . \%$ verglichen werden. Die maximale Abweichung zwischen [Melinder, 2010] und den eigenen Messungen betrug für Temperaturen bis $80^{\circ} \mathrm{C}$ deutlich unter $0,05 \%$. Jedoch konnte für höhere Temperaturen eine zunehmende Abweichung festgestellt werden. Da sich die eigenen Werte jedoch zusätzlich mit den extern in Auftrag gegebenen Referenzmessungen decken, wird hier eher vermutet, dass [Melinder, 2010] hier tendenziell weniger genau ist.

Um für beliebige gemessene Temperaturen die Dichte zu bestimmen, wurden aus den gemessenen Dichte-Werten für jedes zu untersuchende Gemisch Regressionsgleichungen, wie im Anhang in Abbildungen A.16 und A.16 dargestellt, gebildet. Unter der Annahme, dass der gewählte quadratische Polynomansatz $\rho_{\mathrm{LM}, 1 \text { bar }}(t)=c_{0, \mathrm{LM}}+c_{1, \mathrm{LM}} \cdot t+c_{2, \mathrm{LM}} \cdot t^{2}$ für den relevanten Temperaturbereich eine sehr gute Beschreibung ermöglicht, sollten die Regressionsgleichungen (im gemessenen Temperaturbereich) per se eine geringere Unsicherheit als die Einzel-Messwerte aufweisen.

Für die Versuche mit einer Solltemperatur von $110^{\circ} \mathrm{C}$ musste die Dichte durch Extrapolation bestimmt werden. Auch hierfür wurden die ermittelten Regressionsgleichungen statt der Regression von [Melinder, 2010] verwendet, zumal in [Melinder, 2010] die Gültigkeit auch nur bis $100^{\circ} \mathrm{C}$ angegeben wird. Der Unsicherheiten der Dichtemessungen bzw. der verwendeten Näherungsgleichungen der Literatur (z. B. für Wasser) sind in Tabelle 4.6 dargestellt.

\footnotetext{
${ }^{20}$ Schätzt man für reines Glykol die Dampfdichte $\rho_{\mathrm{LM}}^{\prime \prime}$ stark vereinfacht mit Idealgasverhalten nach$$
\rho_{\mathrm{LM}}^{\prime \prime} \leq M_{\mathrm{LM}} \cdot \frac{p_{\mathrm{LM}, \mathrm{s}}}{R \cdot T}
$$

ab, ergibt sich eine Dichte, die im fokussierten Temperaturbereich $10-110^{\circ} \mathrm{C}$ stets deutlich unter der Dichte von reinem Wasserdampf liegt. Somit ist zu vermuten, dass der gewählte Messunsicherheitsbereich völlig ausreichend ist. Für Inhibitoren wird angenommen, dass deren Einfluss auf die Dichte marginal ist, da diese laut Hersteller noch geringere Dampfdrücke als Glykol besitzen. 


\subsubsection{Einfluss des gelösten Gases auf die Dichtemessung}

Für die Dichtemessungen werden die Flüssigkeiten bei Umgebungstemperatur bis auf einen Phasengleichgewichts-Gesamtdruck von ca. 0,1 bar entgast, damit während der Temperierung im Messgerät bei keiner angefahrenen Temperatur Gasdesorption stattfindet. Damit befinden sich während der Messung gelöste Gase in der Flüssigkeit, die die gemessene Dichte des reinen Lösungsmittels $\rho_{\mathrm{LM}}$ verfälschen. Wie groß der Einfluss der gelösten Gase ist, soll exemplarisch für Wasser abgeschätzt werden. Für die zu untersuchenden Wasser-Glykole wird eine ähnliche Größenordnung erwartet.

Die Dichte $\rho$ bzw. das spezifische Volumen $\frac{1}{\rho}$ des Lösungsmittels inklusive gelöster Gase bestimmt sich massengewichtet zu

$$
\frac{1}{\rho}=\xi_{\mathrm{LM}} \cdot \frac{1}{\rho_{\mathrm{LM}}}+\sum_{i} \xi_{i} \cdot \frac{v_{\infty, i}}{M_{i}}
$$

Das partielle molare Volumen der Lufthauptbestandteile Stickstoff bzw. Sauerstoff in Wasser $v_{\infty, \mathrm{N} 2}=33,1 \mathrm{~cm}^{3} / \mathrm{mol}$ (entspricht $846 \mathrm{~kg} / \mathrm{m}^{3}$ ) und $v_{\infty, \mathrm{O} 2}=32,1 \mathrm{~cm}^{3} / \mathrm{mol}$ aus Tabelle 2.5 entspricht einem partiellen spezifischen Volumen von $\frac{V_{\infty}, \mathrm{N} 2}{M_{\mathrm{N} 2}}=1,181 \frac{\mathrm{l}}{\mathrm{kg}} \mathrm{bzw}$. $\frac{v_{\infty, 02}}{M_{\mathrm{O} 2}}=1,0031 \frac{\mathrm{l}}{\mathrm{kg}}$. Diese Werte weichen nur gering vom Wasserwert $\frac{1}{\rho_{\mathrm{H} 2 \mathrm{O}}}=1,00296 \frac{\mathrm{l}}{\mathrm{kg}}$ ab. Damit sind die sich ergebenden Auswirkungen auf das spezifische Volumen bzw. die Dichte des gasbeladenen Lösungsmittels per se beschränkt (während sich hingegen das absolute Volumen durch die gelösten Gase sehr wohl ändert). Bei einem Luftdruck von 1 bar kann die Abweichung zur reinen Lösungsmittel-Dichte bei $10{ }^{\circ} \mathrm{C}$ zu maximal $-0,00037 \%$ abgeschätzt werden. Dieser Wert deckt sich mit [Harvey u. a., 2005] oder auch der in [Tanaka u. a., 2001] empirisch erhaltenen Gleichung des Einflusses gelöster Luft auf die Wasserdichte (bei $101,325 \mathrm{kPa}$ ) $\Delta \rho=-0,004612 \frac{\mathrm{kg}}{\mathrm{m}^{3}}+0,000106 \frac{\mathrm{kg}}{\mathrm{m}^{3} \cdot{ }^{\circ} \mathrm{C}} \cdot t$ gegenüber reinem Wasser im Bereich $t=0 \ldots 25^{\circ} \mathrm{C}$.

Durch die zusätzliche Entgasung der Flüssigkeit vor Beginn der Dichtemessung ist mit einem noch geringeren Einfluss zu rechnen. Da die Messunsicherheit der Dichtemessung eine vielfach höhere Unsicherheit besitzt als die beschriebene Abweichung durch die gelösten Gase, wird letztere im Folgenden vernachlässigt. Die gemessene Dichte ist also näherungsweise die des reinen Lösungsmittels $\rho_{\mathrm{LM}}$.

\subsubsection{Dichteänderung durch Verdampfung}

Während der Versuche nimmt die Gasphase Lösungsmitteldampf auf, der der Flüssigphase entzogen wird. Die Verdunstung größerer Mengen Wasser als Glykol entsprechend der sich stark unterscheidenden Dampfdrücke hat Einfluss auf die Stoffmengenzusammensetzung in der Flüssigkeit, also auf $x_{\mathrm{H} 2 \mathrm{O}}$ und $x_{\mathrm{Glykol}}$ und damit auf $\rho_{\mathrm{LM}}^{\prime}$.

Dieser Einfluss fällt aufgrund der geringen verdunsteten Masse und der ähnlichen Einzeldichten (oder exakter aufgrund der erstens sehr ähnlichen und zweitens über den ganzen Bereich $x_{\mathrm{H} 2 \mathrm{O}}$ nicht stark schwankenden partiellen molaren Volumina $\left.v_{\mathrm{H}_{2} \mathrm{O}}^{\prime}\left(x_{\mathrm{H}_{2} \mathrm{O}}\right), v_{\mathrm{Glykol}}^{\prime}\left(x_{\mathrm{H} 2 \mathrm{O}}\right)\right)$ der Komponenten sehr gering aus und wird hier vernachlässigt.

\subsubsection{Kompressibilität}

Da während der Versuche die Flüssigkeit mit bis zu 10 bar beaufschlagt wird, soll die Kompressibilität der Lösungsmittel beachtet werden. Diese hat wiederum Einfluss auf die jeweils zu verwendende Dichte des Lösungsmittels $\rho_{\mathrm{LM}}$. 
Für Wasser wird auf die Werte $\rho_{\mathrm{H} 2 \mathrm{O}}=f(p, t)$ aus [IAPWS IF-97, 2007] zurückgegriffen. Für alle Glykol-Wasser-Gemische existieren keine druckabhängigen Dichtewerte. Diese sollen daher vereinfacht über die isotherme Kompressibilität $\kappa_{t, L M}$, definiert als

$$
\kappa_{t, \mathrm{LM}}=-\frac{1}{V}\left(\frac{\partial V}{\partial p}\right)_{t}
$$

abgeschätzt werden. Ist $\kappa_{t, \mathrm{LM}}$ im interessierenden Druckbereich konstant, lässt sich die Dichte wie folgt bestimmen

$$
\rho_{\mathrm{LM}}(p, t)=\frac{\rho_{\mathrm{LM}}\left(p_{0}, t\right)}{\left(1-\kappa_{t, \mathrm{LM}} \cdot\left(p-p_{0}\right)\right)},
$$

wobei $\rho_{\mathrm{LM}}\left(p_{0}\right)$ die gemessene Dichte bei Umgebungsdruck $p_{0}=1$ bar darstellt.

In [Guignon u. a., 2010] finden sich sowohl für Propylenglykol (P) als auch Ethylenglykol (E) und je ein Glykol-Wasser-Gemisch (mit prozentualer Angabe des Glykolanteils, jedoch leider ohne Definition des verwendeten Konzentrations-Maßes) Untersuchungen zur Kompressibiliät $\kappa_{t}$. Wie in Abbildung 4.15 dargestellt, ist die Druckabhängigkeit der Kompressibilität im hier interessierenden Druckbereich marginal und soll vernachlässigt werden. Da die in [Guignon u. a., 2010] vorgestellten Werte jedoch nicht annähernd den gesamten interessierenden Temperatur- und Konzentrations-Bereich abdecken und die Gemische bekanntermaßen deutlich nichtideales Verhalten zeigen, wird die Kompressibilität basierend auf Abbildung 4.15 im relevanten Temperatur- und Druckbereich grob, aber gleichzeitig als druckunabhängig abgeschätzt zu

$$
\kappa_{t, \mathrm{G}-\mathrm{H} 2 \mathrm{O}}=\kappa_{\mathrm{G}-\mathrm{H} 2 \mathrm{O}}=(4 \pm 3) \cdot 10^{-4} \mathrm{MPa}^{-1} .
$$

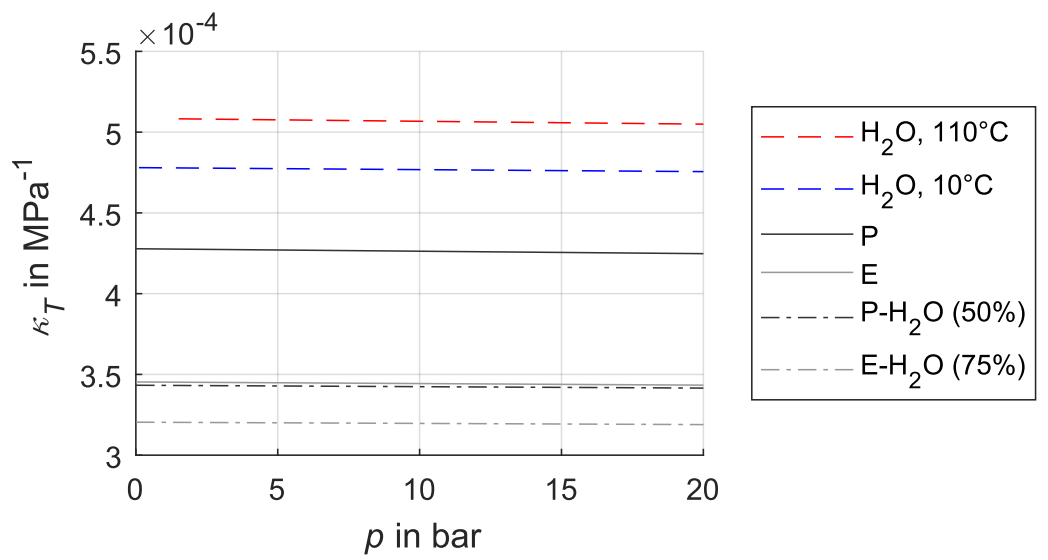

Abbildung 4.15: Druckabhängigkeit der Kompressibilität $\kappa_{t}$ für Wasser $\left(10^{\circ} \mathrm{C}\right.$ und $\left.110^{\circ} \mathrm{C}\right)$ nach [IAPWS IF97,2007 ] und Glykole P/E bzw. Glykol-Wasser-Gemische $\mathrm{P}-\mathrm{H}_{2} \mathrm{O} / \mathrm{E}-\mathrm{H}_{2} \mathrm{O}$ (Gültigkeitsbereich $0{ }^{\circ} \mathrm{C}$ bis $40^{\circ} \mathrm{C}$ ) nach [Guignon u. a., 2010] (leider ohne Angabe des verwendeten Konzentrationsmaßes für die Gemische) 


\subsubsection{Dampfdruck $p_{\mathrm{s}}$}

Für reines Propylenglykol bzw. Ethylenglykol liegen Dampfdruckkurven vor ([Rane u. a., 2016] bzw. [Ambrose und Hall, 1981]).

Für Wasser-Glykol-Gemische wurden in [Fendu und Oprea, 2014] für Propylenglykol (für $44-104^{\circ} \mathrm{C}$ ) und in [Chiavone-Filho u. a., 1993] für Ethylenglykol (für $70^{\circ} \mathrm{C}$ bzw. $90^{\circ} \mathrm{C}$ ) Messungen zum Zweiphasengleichgewicht bei unterschiedlichem Glykolgehalt durchgeführt und Aktivitätskoeffizienten bestimmt. Wendet man die angegebenen Korrelationen der Aktivitätskoeffizienten an, so ergeben sich die in Abb. 4.16 oben dargestellten Dampfdrücke. Die in Abb. 4.16 unten dargestellten Abweichungen zum vereinfacht mit dem Raoultschen Gesetzes bestimmten Dampfdruck

$$
p_{\mathrm{S}}=x_{\mathrm{G}} \cdot p_{\mathrm{s}, \mathrm{G}}+x_{\mathrm{H} 2 \mathrm{O}} \cdot p_{\mathrm{s}, \mathrm{H} 2 \mathrm{O}}
$$

sind gering. Daher soll hier diese Näherung genügen.
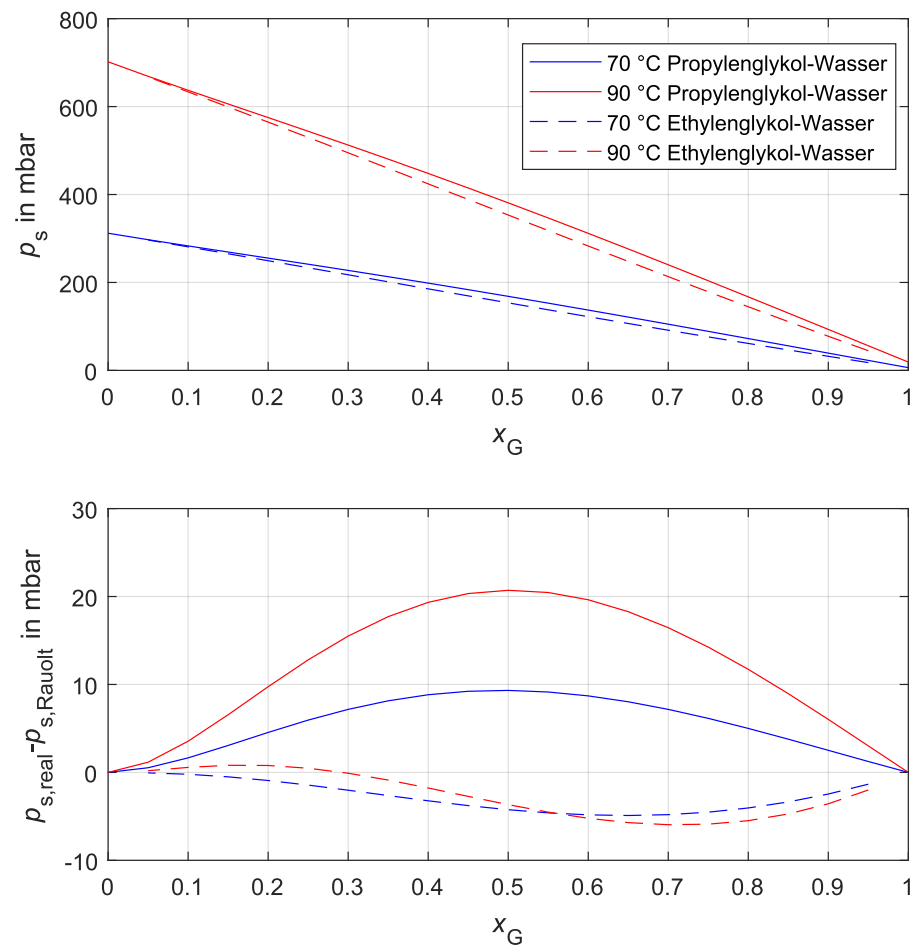

Abbildung 4.16: Realer Dampfdruckverlauf (oben) und Abweichung bei Dampfdruckbestimmung mittels Raoult (unten) in Abhängigkeit des Glykolanteils

Für Dampfdrücke der Fertigmischungen werden Regressionsgleichungen auf Basis der Angaben in den Datenblättern gebildet (vgl. Tabelle 4.7).

Die angenommenen Unsicherheiten der Dampfdrücke werden hier sehr reichlich abgeschätzt. In der Unsicherheitsberechnung wird sich zeigen, dass der Einfluss auf Unsicherheit der Löslichkeitskoeffizienten trotzdem gering ist. 
Tabelle 4.7: Ermittelte Antoine-Dampfdruck-Regressionsgleichungen $\log _{10} \frac{p_{\mathrm{LM}, \mathrm{s}}}{\mathrm{bar}}=\left(A-\frac{B}{t /{ }^{\circ} \mathrm{C}+C}\right)$ der Fertiggemische

\begin{tabular}{lcccl} 
Gemisch & $A$ & $B$ & $C$ & basierend auf Quelle \\
\hline LS & 4.20236 & 1152.431 & 172.437 & [TyfocorLS] \\
\hline 41T & 4,23526 & 1191,062 & 176,168 & [Tyfocor] \\
\hline
\end{tabular}

\subsubsection{Lösungsmittelmasse $m_{\mathrm{LM}}$}

\subsubsection{Auftrieb bei Wägung}

Bei Wägung der Lösungsmittel (LM) mit Präzisionswaage in Umgebungsluft wird die scheinbare Masse $m_{\text {schein }}$ ermittelt. Aufgrund des Luftauftriebs muss zur Bestimmung der wahren Masse $m$ noch die verdrängte Luftmasse addiert werden

$$
\begin{aligned}
& m \cdot g=m_{\text {schein }} \cdot g+m_{\text {Luft }} \cdot g \\
& m=m_{\text {schein }}+m_{\text {Luft }}=m_{\text {schein }}+\rho_{\text {Luft }} \cdot V_{\text {LM }}=m_{\text {schein }}+\rho_{\text {Luft }} \cdot \frac{m}{\rho_{\text {LM }}} \\
& m=\frac{m_{\text {schein }}}{1-\frac{\rho_{\text {Luft }}}{\rho_{\text {LM }}}} .
\end{aligned}
$$

Die Dichte wurde bei den Wäge-Randbedingungen $\left(p=1013,25 \pm 40 \mathrm{hPa} ; t=20 \pm 5^{\circ} \mathrm{C}\right.$; $\left.\varphi_{\mathrm{r}}=50 \pm 20 \%\right) \mathrm{zu} \rho_{\text {Luft }}=1,993 \mathrm{~kg} / \mathrm{m}^{3} \pm 1,63 \%$ bestimmt, vergleiche EURAMET. Damit ergibt sich bei Wägung von $700 \mathrm{~g}$ Wasser eine maximale Masseabweichung der Rechnung von $11 \mathrm{mg}$, bei Nichtbeachtung des Auftriebs jedoch ein systematische Abweichung von bis zu $1,4 \mathrm{~g}$.

\subsubsection{Gelöste Gase bei Wägung}

Die ermittelte Masse $m$ enthält nun noch Anteile der unter Umgebungsbedingungen bzw. Anlieferbedingungen gelösten Gase, die vom Massewert abgezogen werden müssen. Dies ist notwendig, da die Größenordnung dieser Korrektur für manche der untersuchten Gemische durchaus in gleicher Größenordnung wie die Messgenauigkeit der Waage liegen kann. Es ist jedoch ausreichend, dass für die Abschätzung nur die Gase Stickstoff und Sauerstoff betrachtet werden:

$$
m_{\mathrm{LM}}=m-m_{\mathrm{N} 2}-m_{\mathrm{O} 2} .
$$

Die sich unter Umgebungsbedingungen einstellenden gelösten Stoffmengen können abgeschätzt werden mit

$$
\begin{aligned}
& m_{\mathrm{N} 2}=\phi_{\mathrm{sat}, i} \cdot \lambda_{\mathrm{N} 2, \infty} \cdot \rho_{\mathrm{N} 2, \mathrm{~N}} \cdot 0,78 \mathrm{bar} \cdot m, \\
& m_{\mathrm{O} 2}=\phi_{\mathrm{sat}, i} \cdot \lambda_{\mathrm{O} 2, \infty} \cdot \rho_{\mathrm{O} 2, \mathrm{~N}} \cdot 0,21 \mathrm{bar} \cdot m,
\end{aligned}
$$


wobei $\phi_{\text {sat }}$ den Sättigungsgrad gegenüber Umgebungsdruck angeben soll. Für die Berechnung und Messunsicherheitsbetrachtung für Wasser wird $\phi_{\text {sat, } i}=1$ mit max. $15 \% \mathrm{Ab}$ weichung angenommen, da hier wirklich von Luftsättigung ausgegangen werden kann. Für die Glykole wurde teilweise untersättigter Anlieferungszustand beobachtet, daher wird dort $\phi_{\text {sat }, i}=0,5 \pm 0,5$ angenommen. Für die Löslichkeitskoeffizienten $\lambda_{i, \infty}$ werden für diese Abschätzung die bereits vorgestellten Literaturwerte bei $25^{\circ} \mathrm{C}$ für Wasser aus [IAPWS, 2004] bzw. für Wasser-Glykole aus [Yamamoto u. a., 1994] und [Yamamoto und Tokunaga, 1994] (in Abhängigkeit des Glykolgehalts) bei $25{ }^{\circ} \mathrm{C}$ verwendet. Inhibitoren werden hier nicht berücksichtigt.

Für bei Umgebungsdruck luftgesättigtem Wasser $700 \mathrm{~g}$ beträgt die zusätzliche Masse durch gelöste Gase ca. $+0.02 \mathrm{~g}$ und liegt damit bereits in der Größenordnung der Messunsicherheit der Wägung selbst. Insbesondere für reine Glykole können entsprechend der höheren Löslichkeit auch deutlich höhere Werte erreicht werden.

\subsubsection{Verdunstung beim Befüllen}

Nach dem Wägen der Flüssigkeit folgt der Befüllprozess. Dabei wird die Flüssigkeit aus dem Messzylinder in das Reaktorgefäß gegeben. Bis zum luftdichten Abschluss des Messraums $V$ (Einfüllen, Anschrauben des Reaktorgefäßes) bzw. bis zum erneuten Messen des Messzylinders vergehen 5-10 min.

Nimmt man für Wasser an, dass die komplette Luft bis Reaktorgefäßrand (ca. $200 \mathrm{ml}$ ) als auch ca. $800 \mathrm{ml}$ Luft im Messzylinder für die Wägung vollständig von $50 \%$ Ausgangsluftfeuchte auf $100 \%$ Luftfeuchte gebracht wurde, dann wären etwa 0,01 g Verdunstungsverluste zu verzeichnen. Ein Nachstellen des Wäge- und Befüllprozesses mit Massekontrolle vorher-nachher bestätigt diese Größenordnung. Außerdem ist zu beachten, dass ein Großteil des Verdunstungsfehlers bei Versuch und Referenzversuch gleichartig sein wird.

Damit liegt der Verdunstungsfehler (und insbesondere der zwischen Versuch und Referenzversuch variierende Anteil) unter der Messunsicherheit der Waage und wird hier vernachlässigt.

\subsubsection{Zusätzlicher Lösungsmittelaustrag beim Ausgangszustand „entgast“}

Anhand der Anzahl der durchgeführten Zyklen der sukzessiven Druckabsenkung durch Druckausgleich in das evakuierte Hilfsvolumen, dem bekannten Hilfsvolumen und der Medientemperatur lässt sich die maximal mit dem Gas ausgetretene Lösungsmittelmenge bestimmen. Für die untersuchten Lösungsmittel kann der Lösungsmittelaustrag und die Zusammensetzungsänderung als marginal abgeschätzt werden (vgl. Anlage A.5), da die Entgasung bei Umgebungstemperatur erfolgte und die Dampfdrücke der Komponenten gering sind.

\subsubsection{Partielles molares Volumen $v_{\infty, i, \mathrm{LM}}^{\prime}$ der Gase in der Flüssigkeit}

Die partiellen molaren Volumina der Gase in Wasser wurden basierend auf den vorgestellten Literatur-Werten in Tabelle 2.5 für den interessierenden Temperaturbereich grob abgeschätzt. Für die Wasser-Glykole konnten keine Werte in der Literatur gefunden werden. Daher konnten diese auch nur basierend auf den Werten in Wasser, jedoch mit deutlich 
größerer Unsicherheit abgeschätzt werden. Die Annahmen finden sich in Tabelle 4.6 und dienen vordergründig der Berücksichtigung in der umfassenden Messunsicherheitsbetrachtung.

\subsubsection{Leckage $q_{\text {Leck }}$}

Aufgrund der relativ hohen Anzahl an Verbindungsstellen (metallisch dichtende SwagelokVerbindungen und flach- bzw. gewindedichtende Teflondichtungen) muss die Anlage auf Leckage überprüft werden. Dazu wurde ein Helium Detektor der Firma Balzers Typ HLT 160 mit Schnüffelsensor zur lokalen Lecksuche eingesetzt. Nach Identifizierung und Behebung von Leckstellen erfolgten anschließend globale Dichtheitstest von Reaktorgefäß und Gasreservoir unter versuchsähnlichen Bedingungen. Daraus wurden maximale Leckageraten $q_{\text {Leck,max }}$ für Gasreservoir $(0,25 \mathrm{mbar} / \mathrm{h})$ und befüllten Reaktor $(0,5 \mathrm{mbar} / \mathrm{h})$ ermittelt.

Da Gasreservoir und Reaktorgefäß immer nur differenziell zwischen stationärem Zustand 1 und 2 bilanziert werden, ist nur die Leckage, die zwischen diesen Zuständen stattfindet, maßgebend. Diese beeinflusst also auch nur $p_{2}$ und $p_{2}^{G}$. Als maximale Zeitdauer zwischen den stationären Zuständen soll eine Stunde angenommen werden. Der Druck $p$, der sich ohne Leckage einstellen würde, wird grob abgeschätzt mit

$$
p=p_{\text {Mess }}+q_{\text {Leck }} \cdot 1 \mathrm{~h}
$$

mit $q_{\text {Leck }}=0,5 \cdot q_{\text {Leck,max }} \pm 0,5 \cdot q_{\text {Leck,max }}$. Die Druckabhängigkeit der Leckage soll hier nicht beachtet werden, sondern es wird vereinfacht immer der maximale Wert verwendet. $^{21}$

Bei Versuch und Referenzversuch wird ein Teil der Leckage gleich sein und sich in der kalibrierten Berechnung aufheben. Diese teilweise Einflussaufhebung des Konstantanteils der Leckage kann genutzt werden, wenn möglichst gleiche Druckniveaus und gleiche Versuchszeiten eingestellt werden.

\subsubsection{Löslichkeitskoeffizient von Wasser $\lambda_{i, \mathrm{H} 2 \mathrm{O}, \infty, \mathrm{Lit}}$ zur Kalibrierung mit Wasser-Versuchen}

Die bereits im Theorieteil vorgestellten in der Literatur gegebenen Regressionen für Wasser in Stickstoff bzw. Sauerstoff werden hier für $\lambda_{i, \mathrm{H} 2 \mathrm{O}, \infty, \mathrm{Lit}}(t)$ verwendet und mit einer Unsicherheit von $2 \%$ abgeschätzt.

\footnotetext{
${ }^{21}$ Luft-Versuche dauern deutlich länger. Auf die Bilanz im Gasreservoir hat dies keinen Einfluss, da auch hier, sobald ein stationärer Zustand erreicht ist, dieser Zeitpunkt als Zustand nach dem Druckausgleich für die Bilanzierung gewählt wird. Für das Reaktorgefäß wird allerdings ein Zustand nach Ablauf der Mindestwartezeit von 5 Stunden nach Druckausgleich gewählt, während in der Zwischenzeit Leckage stattfinden kann. Diese Versuche wurden jedoch mit besonderem Fokus auf geringe Leckageraten durchgeführt. AuBerdem konnte die Leckage in gewissem Maße auch während des Versuchs überprüft werden, da über die lange Mindestwartezeit bereits Beobachtungen eines Druckabfalls möglich sind. Weiterhin ist das Druckniveau im Reaktor bei Luft-Versuchen aufgrund nur eines Druckausgleiches auf ca. 5 bar beschränkt. Daher wird hier trotz der längeren Wartezeiten von ähnlichen absoluten Maximal-Leckagen wie bei StickstoffVersuchen (0,5 mbar) ausgegangen. Da die Leckage nur einen geringen Einfluss auf die Gesamtunsicherheit des Ergebnisses hat, soll diese Vereinfachung genügen.
} 


\subsubsection{Gleichungsungenauigkeit durch Vereinfachungen}

Die im Kapitel 2 exemplarisch für Stickstoff in Wasser beschriebenen maximalen Abweichungen bei Verwendung des Henry-Gesetzes (Vereinfachungen A-F) statt der thermodynamisch exakten Gleichungen sind im Vergleich zur verbleibenden Gesamtmessunsicherheit des Ergebnisses klein (1\%). Durch die zusätzliche Berücksichtigung der Poynting-Korrektur (ohne Vereinfachung F) des Gases wird hier sogar die Gleichungsungenauigkeit zur Ermittlung des Löslichkeitskoeffizienten zu 0,2 \% reduziert.

Wenn man annimmt, dass die untersuchten Stoffgemische eine ähnliche Charakteristik wie das Stoffgemisch Stickstoff-Wasser zeigen, so kann die Ungenauigkeit für beide Drucksprünge also als deutlich unter 0,2\% eingeschätzt werden. Da Abschätzungen zu unbekannten Stoffdaten der untersuchten Stoffgemische schwierig sind und die sich ergebende Unsicherheit keine experimentelle Unsicherheit ist, soll dieser Unsicherheitsbeitrag formal nicht in die Bestimmung der Gesamtunsicherheit eingehen, zumal er klein gegenüber anderen Unsicherheiten ist.

\subsubsection{Weitere Einflüsse}

Folgende Einflüsse wurden nicht beachtet bzw. abgeschätzt:

- Totvolumen im Kugelventil V.G5;

- Ungenauigkeit der Realgasgleichungen;

- Inhomogenitäten in der Gaszusammensetzung über das Volumen $V^{\prime \prime}$ insbesondere durch die Totvolumina $V^{R 1}, V^{R 2}$ und damit verbundene mögliche Partialdruckverschiebung;

- Nichtideale Mischung von Restgasen, Arbeitsgas und Lösungsmitteldampf.

Es wird angenommen, dass diese Aspekte nur geringen Einfluss auf die Messergebnisse haben. Außerdem kann davon ausgegangen werden, dass einige der Effekte in Versuch und Referenzversuch "gleichmäßig" auftreten und sich dadurch zumindest bei der Ermittlung der kalibrierten Löslichkeiten teilweise aufheben.

\subsection{Bestimmung der Messunsicherheit}

\subsubsection{Allgemeines}

Das Messverfahren und das zugehörige Berechnungsverfahren zur Bestimmung von Löslichkeiten (Sauerstoff, Stickstoff) ist sehr sensitiv bezüglich verschiedener Eingangsgrößen, während andere Eingangsgrößen hingegen nur sehr geringen Einfluss haben. Diese Sensitiviäten sind nicht unbedingt intuitiv und aufgrund der Komplexität der Berechnung nicht mehr hinreichend gut überschaubar. Daher ist eine ausführliche und exakte Messunsicherheitsbetrachtung notwendig, die mögliche Trugschlüsse in der Deutung von Messergebnissen unterbindet.

Mit Hilfe der ausführlichen Messunsicherheitsbetrachtung konnte der Versuchsablauf sukzessive so geändert werden, dass eine Minimierung der Gesamtunsicherheit des Messergebnisses möglich war. Sowohl praktisch als auch durch die folgende Messunsicherheitsbetrachtung wurde erkannt, dass eine geringe Änderung an gewissen Parametern nicht nur zur Vergrößerung der Unsicherheitsbandbreiten, sondern sogar zur Unverwertbarkeit von Versuchen und Ergebnissen führt. 
Bei den folgenden Betrachtungen sind vor allem zwei methodische Aspekte hervorzuheben, da diese deutlich über eine allgemein übliche Messunsicherheitsbetrachtung hinausgehen, deren Berücksichtigung sich hier aber trotzdem als notwendig erweist:

1. Gehen mehrere Messwerte eines Sensors in die Berechnung, z. B. die Druckmessungen $p_{1}$ und $p_{2}$, ein, so führt eine einfache, separate Unsicherheitsbehandlung der Messwerte schnell zu stark überschätzenden Unsicherheitsbandbreiten des Ergebnisses. Stattdessen müssen die systematischen Unsicherheiten gesamtheitlich für den Sensor betrachtet werden.

2. Die Messergebnisse der Versuche sollen durch gleichartige Referenzversuche mit Wasser kalibriert werden, um die Messunsicherheit des Ergebnisses zu reduzieren. Dazu ist eine geeignete Formel zur Ermittlung eines kalibrierten Ergebnisses anzuwenden als auch die zugehörige Unsicherheit des kalibrierten Ergebnisses zu ermitteln.

\subsubsection{Grundlagen}

\subsubsection{Verfahren}

Die Messunsicherheitsbetrachtung folgt im Wesentlichen den Vorgaben des GUM. GUM steht für "Guide to the Expression of Uncertainty in Measurement" [GUM]. Diese stellt die Methoden für eine ganzheitliche Messunsicherheitsbetrachtung zur Verfügung und wird als verbindliche Grundlage auch von zertifizierten Kalibrierlaboratorien (z. B. Deutscher Kalibrierdienst) genutzt. Wichtige Eigenschaften sind:

- Im GUM werden auch nichtnormalverteilte Eingangsgrößen betrachtet. So können z. B. zufällige Messunsicherheiten, die auf eine Anzeigeungenauigkeit (letzte Ziffer) zurückgehen, erfasst werden. Diese Messunsicherheiten entsprechen zumeist einer Rechteckverteilung.

- Systematische Messunsicherheiten werden als quasi-zufällige Messunsicherheiten mit Rechteckverteilung betrachtet, d.h. jeder Wert im abgeschätzten Wertebereich $\left[x_{j}-a_{j} ; x_{j}+a_{j}\right]$ wird als gleich wahrscheinlich angenommen. ${ }^{22}$

- Eine wesentliche Rolle für die Berechnung einer Gesamtmessunsicherheit aus unterschiedlich verteilten Zufallsgrößen spielt der zentrale Grenzwertsatz. Aus diesem ergibt sich, dass trotz teilweise stark von der Normalverteilung abweichende Verteilungen die Unsicherheit des Messergebnisses gut mit Hilfe des Gauss 'schen Fehlerfortpflanzungsgesetzes abgeschätzt werden kann [DIN V ENV 13005, S.69].

Allgemein ergibt sich die kombinierte erweiterte Messunsicherheit für eine Funktion $f\left(x_{1}, x_{2}, \ldots, x_{n}\right)$ für beliebige, unkorrelierte Eingangsgrößen zu

$$
U_{c}=\sqrt{\left(\frac{\partial f}{\partial x_{1}} U_{1}\right)^{2}+\left(\frac{\partial f}{\partial x_{2}} U_{2}\right)^{2}+\ldots+\left(\frac{\partial f}{\partial x_{n}} U_{n}\right)^{2}},
$$

wobei sich die erweiterte Unsicherheit der Eingangsgrößen $U_{j}$ wiederum aus mehreren Unsicherheiten zusammen setzen können, im einfachsten Fall aus einer zufälligen Stan-

\footnotetext{
${ }^{22}$ Die Standardunsicherheit der Rechteckverteilung beträgt $u=\frac{a}{\sqrt{3}}$ mit der Rechteckbreite $2 \cdot a$
} 
dardmessunsicherheit $u_{\mathrm{zuf}, j}$ und einer systematischen maximalen Abweichung $a_{\mathrm{sys}, j} \mathrm{zu}$

$$
U_{j}=k \cdot \sqrt{\left(\frac{\partial f}{\partial x_{j}} u_{\text {zuf }, j}\right)^{2}+\left(\frac{\partial f}{\partial x_{j}} \frac{a_{\text {sys }, j}}{\sqrt{3}}\right)^{2}} .
$$

Die so ermittelte Unsicherheitsfortpflanzung ist aufgrund der linearen Näherung abschätzender Natur. Für $u_{j}$ wird die Standardmessunsicherheit eingesetzt. Mit dem Erweiterungsfaktor $k$ wird die Standardmessunsicherheit $u$ auf die sogenannte erweiterte Messunsicherheit $U$ übertragen. Hier wurde dieser auf $k=2$ und damit die Unsicherheitsabschätzung auf ein Konfidenzintervall von $95 \%$ (Vertrauensbereich) festgelegt.

Das wahre Messergebnis liegt also mit einer Wahrscheinlichkeit von 95\% in einem Bereich um das Messergebnis y derart

$$
y-U_{c} \leq y \leq y+U_{c} .
$$

\subsubsection{Partielle Ableitung $\frac{\partial f}{\partial x_{j}}$}

Die partiellen Ableitungen werden in den Berechnungen gemäß [GUM, S. 5.1.3] durch den Differenzenquotienten

$$
\frac{\partial f}{\partial x_{j}} \approx \frac{f\left(x_{1}, \ldots, x_{j}+u_{j}, \ldots, x_{n}\right)-f\left(x_{1}, \ldots, x_{j}-u_{j}, \ldots, x_{n}\right)}{2 \cdot u_{j}}
$$

ersetzt.

\subsubsection{Bewertung des Verfahrens}

Der GUM steht u. a. in der Kritik, systematische und zufällige Aspekte der Messabweichung zu vermischen, siehe [Grabe, 2000]. Der Ansatz, die systematischen Messunsicherheiten als quasi-zufällig zu betrachten, liefert eine wahrscheinliche Abschätzung für das Messergebnis, jedoch keine sichere Eingrenzung. Der so eingegrenzte Unsicherheitsbereich kann bei korrelierten systematischen Messabweichungen in Realität deutlich überschritten werden. Insbesondere bei vielen gleichartigen Messstellen oder Messgeräten ist eine gleichartige Abweichung wahrscheinlich und daher der GUM nicht direkt anwendbar. Nichtsdestotrotz steht mit dem GUM ein robustes und etabliertes einheitliches Verfahren zur Verfügung, das in den meisten Fällen ein sehr gutes Werkzeug darstellt.

Für die Bestimmung des Löslichkeitskoeffizienten liegt eine Vielzahl an systematischen Unsicherheiten von verschiedensten Messgrößen, Konstanten und Stoffwerten vor, die außerdem größtenteils als nicht korreliert angesehen werden können. Das macht den GUM (Verfahren II) mit guter Genauigkeit anwendbar. Das klassische Verfahren I mit worst-caseAbschätzung der systematischen Unsicherheiten würde das Unsicherheitsband deutlich größer schätzen.

\subsubsection{Messunsicherheitsbeiträge}

Um den Einfluss einzelner Größen $x_{j}$ auf die Gesamtmessunsicherheit $U_{c}$ vergleichen und bewerten zu können, werden hier sogenannte Messunsicherheitsbeiträge der einzelnen Größen 
$x_{j}$ verwendet. Da sich die Gesamtunsicherheit $U_{c}$ nicht durch Addition einzelner Beiträge ergibt, muss eine andere Definitionen des Messunsicherheitsbeitrags verwendet werden: Hier soll der Messunsicherheitsbeitrag die Messunsicherheit der Zielgröße beschreiben, wenn nur die Größe $x_{j}$ unsicherheitsbehaftet wäre.

Der Messunsicherheitsbeitrag einer zufälligen Messabweichung ist dann gekennzeichnet durch die Unsicherheit $U_{z u f, j}$ auf das Messergebnis mit

$$
\left|\frac{\partial f}{\partial x_{j}}\right| \cdot U_{\text {zuf }, j}
$$

definiert.

Ein systematischer Messunsicherheitsbeitrag sei (durch die worst-case-Abschätzung) durch

$$
\left|\frac{\partial f}{\partial x_{j}}\right| \cdot a_{\mathrm{sys}, j}
$$

definiert.

\subsubsection{Messunsicherheitsbudget}

\subsubsection{Unsicherheit der Konstanten und Stoffwerte}

Unsicherheiten für Hilfsgrößen, Stoffwerte u. ä. sind in der bereits vorgestellten Tabelle 4.6 zusammengefasst. Diese sind systematische Unsicherheiten.

\subsubsection{Unsicherheit der Messwerte}

\section{Allgemeines}

Für die Messunsicherheitenrechnung des Versuchsstandes muss bei den genutzten Messwerten unterschieden werden in:

- zufällige Messunsicherheiten - hier tritt die Messabweichung bei jedem Messwert in Betrag und Richtung unterschiedlich auf;

- systematische Messunsicherheiten, bei der die unbekannte Messabweichung für jeden anderen Messwert eines Sensors in Betrag und Richtung unterschiedlich auftreten können - „messwertabhängige Messabweichungen“;

- systematische Messunsicherheiten, bei der die unbekannte Messabweichung für jeden anderen Messwert eines Sensors in Betrag und Richtung gleich auftreten - „sensorabhängige Messabweichungen".

Für die Unsicherheitsbetrachtung der Messwerte werden diese in eine messwertbezogene Unsicherheit und sensorbezogene Unsicherheit aufgeteilt. Dies ist für Berechnung der realen kombinierten Messunsicherheit des Ergebnisses bei Beachtung o.g. Aspekte 1. Verwendung mehrerer Messewerte eines Sensors sowie 2. Kalibrierung durch vorgelagerte Wasser-Versuche wichtig. Dazu wird der Messwert $x_{j}$ als Funktion mehrerer Eingabegrößen dargestellt. Diese sind jeweils der Anzeigewert $x_{j, \text { Anz }}$ und weitere Eingabegrößen, die sensorabhängig sind. In Tabelle 4.8 ist die Zusammensetzung der in der Berechnung verwendeten Messwerte dargestellt: 
- Die Masse ergibt sich aus Anzeigewert der Wägung $m_{\llcorner M}, A n z$ und einem Korrekturfaktor $c^{m}$ (ermittelt mit Referenzgewichten).

- Für Druck- und Temperaturmessungen wird der wahre Wert durch den Anzeigewert plus sogenannte Korrekturen (Differenz zwischen wahrem Wert und Anzeigewert) geschätzt. Als Korrekturfunktionen werden quadratische Polynome verwendet. Zur Ermittlung der Korrekturen (d.h. Kalibrierung) der Drucksensoren und Temperatursensoren und der Methodik zur Bestimmung der Messunsicherheit wird auf den Anhang A.9 verwiesen.

- Die Temperaturen in den Toträumen T.RHzg1/2/3 ergeben sich aus einem Anzeigewert und den Korrekturen $c^{T . R H z g}$. Die Korrekturen stellen die Abweichung zwischen gemessener Temperatur und der effektiv im Gasvolumen vorliegenden Temperatur dar. Als Wert der Korrektur wird $c^{T}$ RHzg $=0$ angenommen. Dieser hat also rechnerisch keine Auswirkung auf das Messergebnis, aber ist wichtig für die Unsicherheitsbetrachtung. Eine Kalibrierung der T.RHzg1/2/3 ist nicht notwendig, da die Unsicherheitsbandbreite aufgrund der zu erwartenden Abweichung zur realen Gastemperatur ausreichend hoch gewählt ist.

- Der gaschromatographisch bestimmte Sauerstoffanteil $y_{\mathrm{O} 2}$ in N2+O2,2 setzt sich aus Messwert und einem konstanten, messwertunabhängigen Unsicherheitsbeitrag $c_{y} \mathrm{O} 2,2$ zusammen. Auch dieser ist $c_{\mathrm{yO}, 2}=0$ und hat also keine rechnerische Auswirkung auf das Messergebnis, sehr wohl aber auf die zugehörige Unsicherheit.

Tabelle 4.8: Zusammensetzung der Messwerte

\begin{tabular}{|c|c|c|}
\hline Größe & Zusammensetzung & Messstelle in Schema \\
\hline$m_{\llcorner\mathrm{M}}$ & $m_{\mathrm{LM}, \mathrm{Anz}} \cdot c^{m}$ & - \\
\hline$t_{z}^{\mathrm{G}}$ & $t_{A n z, z}^{G}+K^{\top \cdot G}\left(t_{A n z, z}^{G}\right)=t_{A n z, z}^{G}+c_{1}^{\top \cdot G}+c_{2}^{\top \cdot G} \cdot t_{A n z, z}^{G}+c_{3}^{\top \cdot G} \cdot\left(t_{A n z, z}^{G}\right)^{2}$ & T.G \\
\hline$t_{z}^{\prime}$ & $t_{\mathrm{Anz}, z}^{\prime}+K^{\top \cdot R 1}\left(t_{\mathrm{Anz}, \mathrm{z}}^{\prime}\right)=t_{\mathrm{Anz}, z}^{\prime}+c_{1}^{\top \cdot R 1}+c_{2}^{\top \cdot R 1} \cdot t_{\mathrm{Anz}, z}^{\prime}+c_{3}^{\top \cdot R 1} \cdot\left(t_{\mathrm{Anz}, \mathrm{z}}^{\prime}\right)^{2}$ & T.R1 \\
\hline$t_{z}^{\mathrm{RO}}$ & $t_{\mathrm{Anz}, z}^{\mathrm{R} 0}+K^{\top \cdot \mathrm{R} 2}\left(t_{\mathrm{Anz}, \mathrm{z}}^{\mathrm{R} 0}\right)=t_{\mathrm{Anz}, z}^{\mathrm{R} 0}+c_{1}^{\top \cdot R 2}+c_{2}^{\top \cdot R 2} \cdot t_{\mathrm{Anz}, z}^{\mathrm{R} 0}+c_{3}^{\top} \cdot \mathrm{R} 2 \cdot\left(t_{\mathrm{Anz}, z}^{R 0}\right)^{2}$ & T.R2 \\
\hline$p_{z}^{\mathrm{G}}$ & $p_{A n z, z}^{G 3}+K^{P . G}\left(p_{A n z, z}^{G}\right)=p_{A n z, z}^{G 3}+c_{1}^{P . G 3}+c_{2}^{P . G 3} \cdot p_{A n z, z}^{G}+c_{3}^{P . G 3} \cdot\left(p_{A n z, z}^{G}\right)^{2}$ & P.G3 \\
\hline$p_{z}$ & $p_{\mathrm{Anz}, z}^{\mathrm{R} 3}=p_{\mathrm{Anz}, \mathrm{z}}^{\mathrm{R} 3}+c_{1}^{\mathrm{P} \cdot \mathrm{R} 3}+c_{2}^{\mathrm{P} \cdot \mathrm{R} 3} \cdot p_{\mathrm{Anz}, \mathrm{z}}+c_{3}^{\mathrm{P} \cdot \mathrm{R} 3} \cdot\left(p_{\mathrm{Anz}, \mathrm{z}}\right)^{2}$ & P.R3 \\
\hline$t_{z}^{\mathrm{RHzg} 1}$ & $t_{\mathrm{Anz}, \mathrm{z}}^{\mathrm{RHzg} 1}+c^{\mathrm{T} \cdot \mathrm{RHzg} 1}$ & T.RHzg1 \\
\hline$t_{z}^{\mathrm{RHzg} 2}$ & $t_{\mathrm{Anz}, \mathrm{z}}^{\mathrm{RHzg} 2}+c^{\mathrm{T} \cdot \mathrm{RHzg} 2}$ & T.RHzg2 \\
\hline$t_{z}^{\mathrm{RHzg} 3}$ & $t_{\mathrm{Anz}, \mathrm{z}}^{\mathrm{RHzg}}+c^{\mathrm{T} \cdot \mathrm{RHzg} 3}$ & T.RHzg3 \\
\hline $\mathrm{yO}_{\mathrm{O}}$ in $\mathrm{N} 2+\mathrm{O} 2,2$ & $\mathrm{y}_{\mathrm{O} 2 \text { in N2+O2,2, Mess }}+c^{y \mathrm{O} 2,2}$ & - \\
\hline
\end{tabular}

\section{Systematische Unsicherheit der Messwerte}

Tabelle 4.9 zeigt die Zusammenstellung der systematischen Unsicherheiten:

- Bei der Massewägung wird nicht der Kalibrierfaktor $c^{m}$ abgeschätzt, sondern direkt der Fehler von $m_{\mathrm{LM}}$. Damit werden die systematischen Abweichungen der Wägungen in den Versuchen unabhängig voneinander betrachtet.

- Die systematische Unsicherheit der $p$ - und t-Messungen wird über die Unsicherheiten der Korrekturfunktionen erfasst. Die Unsicherheiten der Koeffizienten $c=\left(c^{k}\right)$ sind für jeden Messwert des Sensors $k$ gleich groß und damit sensorabhängige systematische Unsicherheiten.

- Die effektiven Gastemperaturen in den Totvolumina können ggf. deutlich verschoben gegenüber den Werten der Anlegefühler $t^{\mathrm{RHzg1/2} / 3}$ sein (v. a. durch ungleichmäßige 
Wicklung und Kontaktfläche des Heizbandes, ungleichmäßige Dämmung). Bei konstanter Versuchs-Solltemperatur bleibt diese Abweichung jedoch (in Versuch und Referenzversuch) annähernd konstant. Dies drückt der Wert $c^{T . R H z g 1 / 2 / 3}=0 \pm 10 \mathrm{~K}$ aus.

- Der gemessene Sauerstoffgehalt $y_{\mathrm{O} 2}$ in N2+O2,2 wird ebenso mit einem konstanten, sensorabhängigen und messwertunabhängigen Unsicherheitsbeitrag $c_{y} \mathrm{O}, 2$ beaufschlagt. Es wird für Versuch und Referenzversuch also die gleiche systematische Messabweichung angenommen. Dies ist zulässig, da die Messwerte von Versuch- und Referenzversuch hier meist in gleicher Größenordnung $\left(y_{\mathrm{O} 2}\right.$ in N2+O2,2 $\left.=0,19 \ldots 0,195\right)$ lagen. Eine separate, bei Versuch und Referenzversuch nicht gleiche systematische Messabweichung wird folglich deutlich niedriger eingeschätzt. Beide Unsicherheitsbeiträge sollen sowohl die Messabweichung des Gaschromatographen als auch die Unsicherheiten bei der Probenentnahme selbst berücksichtigen.

Tabelle 4.9: Systematische Unsicherheit

\begin{tabular}{|c|c|c|c|}
\hline Größe & Art & sys. Unsicherheit $a_{\text {sys }}$ & Messgröße \\
\hline$m_{\llcorner\mathrm{M}}$ & Unsicherheit Kalibrierkurve & $0,02 \mathrm{~g}$ & $\mathrm{~m}$ \\
\hline$c^{\top \cdot G}$ & Unsicherheit Kalibrierkurve & siehe Kalibrierung & T.G \\
\hline$c^{T \cdot R 1}$ & Unsicherheit Kalibrierkurve & siehe Kalibrierung & T.R1 \\
\hline$c^{T \cdot R 2}$ & Unsicherheit Kalibrierkurve & siehe Kalibrierung & T.R2 \\
\hline$c^{\mathrm{P} . \mathrm{G} 3}$ & Unsicherheit Kalibrierkurve & siehe Kalibrierung & P.G3 \\
\hline$c^{P . R 3}$ & Unsicherheit Kalibrierkurve & siehe Kalibrierung & P.R3 \\
\hline$c^{T \cdot R H z g 1 / 2 / 3}$ & Verschiebung gegenüber Gastemperatur & $10 \mathrm{~K}$ & $\mathrm{~T} . \mathrm{RHzg} 1 / 2 / 3$ \\
\hline$c^{y \mathrm{O} 2,2}$ & verfahrensbedingt (nur Luftversuche) & $0,5 \%$ & \\
\hline$y_{\mathrm{O}} 2$ in $\mathrm{N} 2+\mathrm{O} 2,2, \mathrm{Mess}$ & verfahrensbedingt (nur Luftversuche) & $0,1 \%$ & \\
\hline
\end{tabular}

\section{Zufällige Unsicherheit der Messwerte}

In Tabelle 4.10 sind die zufälligen Unsicherheiten dargestellt. Aufgrund des hochwertigen Thermostatbades und vorhandener thermischer Trägheiten treten keine Abweichungen in Form von Streuung von Messwerten auf. Damit beschränkt sich die zufällige Unsicherheit auf die Auflösung der Messgeräte. Nur für die Messung der Temperaturen $t^{\mathrm{RHzg} 1}, t^{\mathrm{RHzg} 2}$, $t^{\mathrm{RHzg} 3}$, der Totvolumina treten Schwankungen der Temperatur bedingt durch die Regelgüte auf. Diese werden hier aufgrund der Erfahrung mit dem Versuchsstand auf 0,3 K abgeschätzt. Die Auflösung der Messwerte ist hier vernachlässigbar.

Für den gemessenen Sauerstoffgehalt $y_{\mathrm{O} 2}$ in $\mathrm{N} 2+\mathrm{O} 2,2$ der Luft-Versuche wird eine zufällige Unsicherheit angenommen, die auf Test-Messungen von [Pham, 2015] basiert.

\subsubsection{Grobe Fehler}

Als grobe Fehler werden Abweichungen vom wahren Wert bezeichnet, die durch ein Versehen oder durch äußere Einflüsse das Ergebnis deutlich in eine Richtung verzerren. Als Beispiele sind hier

- Ablesefehler,

- Ventilbedienfehler, 
Tabelle 4.10: Zufällige Unsicherheiten der Messgrößen

\begin{tabular}{|c|c|c|c|c|c|}
\hline Größe & Art & Auflösung & Intervall & Verteilung & Messgröße \\
\hline$m_{\mathrm{LM}, \mathrm{Anz}}$ & $\begin{array}{c}\text { Standardabweichung laut } \\
\text { Datenblatt }\end{array}$ & - & $\pm 0,01 \mathrm{~g}$ & Normal & $\mathrm{m}$ \\
\hline$t_{\text {Anz }}^{G}$ & Auflösungsgenauigkeit & $0,01 \mathrm{~K}$ & $\pm 0,005 \mathrm{~K}$ & Rechteck & T.G \\
\hline$t_{\mathrm{Anz}}^{\prime}$ & Auflösungsgenauigkeit & $0,01 \mathrm{~K}$ & $\pm 0,005 \mathrm{~K}$ & Rechteck & T.R1 \\
\hline$t_{\mathrm{Anz}}^{R 0}$ & Auflösungsgenauigkeit & $0,01 \mathrm{~K}$ & $\pm 0,005 \mathrm{~K}$ & Rechteck & T.R2 \\
\hline$p_{\text {Anz }}^{G}$ & $\begin{array}{l}\text { Auflösungsgenauigkeit, } \\
\text { Wiederholpräzision }\end{array}$ & 0,001 bar & $\pm 0,001$ bar & Rechteck & P.G3 \\
\hline$p_{\text {Anz }}$ & $\begin{array}{c}\text { Auflösungsgenauigkeit, } \\
\text { Wiederholpräzision }\end{array}$ & 0,001 bar & $\pm 0,001$ bar & Rechteck & P.R3 \\
\hline$t_{\mathrm{Anz}}^{\mathrm{RHzg} 1}$ & Temperaturschwankung & - & $\pm 0,3 \mathrm{~K}$ & Rechteck & T.RHzg1 \\
\hline$t_{\mathrm{Anz}}^{\mathrm{RHzg} 2}$ & Temperaturschwankung & - & $\pm 0,3 \mathrm{~K}$ & Rechteck & T.RHzg2 \\
\hline$t_{\mathrm{Anz}}^{\mathrm{RHzg} 3}$ & Temperaturschwankung & - & $\pm 0,3 \mathrm{~K}$ & Rechteck & T.RHzg3 \\
\hline$y_{\mathrm{O}} 2$ in $\mathrm{N} 2+\mathrm{O} 2,2, \mathrm{Mess}$ & $\begin{array}{l}\text { Wiederholpräzision } \\
\text { (Gaschromatograph- und } \\
\text { Probenahmeunsicherheit) }\end{array}$ & - & $\pm 0,1 \%$ & Normal & \\
\hline
\end{tabular}

- unvollständige Reinigung bzw. Trocknung oder auch

- Flüssigkeitsverlust bei unkorrektem Einfüllen (Spritzer)

zu nennen. Selbstverständlich sind auch bisher nicht erkannte bzw. beachtete Einflüsse als grobe Fehler möglich. Die Erfahrung hat gezeigt, dass aufgrund der Vielzahl der Versuchsschritte, nach einer relativ zeitintensiven Einarbeitung des Protokollanten, die Anzahl grober Fehler deutlich verringert werden konnte. Jedoch kann das Auftreten grober Fehler gerade aufgrund der Sensibilität der Messergebnisse zu starken Abweichungen führen.

Viele grobe Fehler können durch Mehrfachmessung eindeutig ausgeschlossen werden. Wiederholungsmessungen konnten innerhalb der bisherigen Arbeit aufgrund des enormen Zeitaufwands pro Messpunkt jedoch nicht durchgeführt werden. Zur Minimierung bzw. Entdeckung der groben Fehler tragen jedoch das ausführliche Protokoll jeder Messung. Plausibilitätskontrollen beim Experiment sowie bei der Auswertung (zeitlicher Messgrößenverlauf) und die druckgestufte Löslichkeitsmessung bei. Weiterhin werden die Löslichkeiten bei verschiedenen Temperaturen gemessen. Ausreißer können also auch über den temperaturabhängigen Verlauf der Löslichkeit identifiziert werden.

\subsubsection{Minimierte Messunsicherheit durch optimierte Versuchsparameter}

\subsubsection{Allgemeines}

Für die bestehende Apparatur besteht nun weiterhin die Möglichkeit, Versuchsparameter im Nachhinein so anzupassen, dass die Gesamtmessunsicherheit relativ gering ist. Dazu gehören im Wesentlichen nur

- die Druckdifferenz und

- die Lösungmittelmasse. 
Durch eine hohe Druckdifferenz steigt die zu bilanzierende Gasmenge und damit (bei o. g. Annahmen des idealisiertem Löslichkeitsverhalten im Druckbereich) sinken die Messunsicherheitsbeiträge. Jedoch ist man hier beschränkt durch die Grenzen der Apparatur, den aufgrund der technischen Relevanz bewusst gewählten Druckbereich, aber eben auch durch die Annahme idealisierten Löslichkeitsverhaltens, die nur bei geringen Drücken gültig ist.

Einen wesentlicher Faktor, der gut beeinflusst werden kann, stellt hier die die eingefüllte Lösungsmittelmenge $m_{\mathrm{LM}}$ dar. Erste Versuche mit der Apparatur von [Wagner, 2010] mit Wasser als Lösungsmittel führten nicht zum Erfolg. Es wird vermutet, dass hier der Einfluss der Lösungsmittelmenge nicht ausreichend berücksichtigt wurde und die Lösungsmittelmengen ungünstig gewählt waren, sodass die Gesamtmessunsicherheit das Messergebnis deutlich überstieg.

\subsubsection{Konstruktion eines fiktiven Versuchs}

Zur Analyse der Messunsicherheit in Abhängigkeit gewisser Versuchsparameter müssen Versuchsdaten vorhanden sein, um die Messunsicherheit bestimmen zu können. Für die Vorbetrachtung sollten jedoch keine realen Versuche durchgeführt werden, sondern fiktive Versuche bei beliebigen Versuchsparametern analysiert werden. Bei gegebenen Stoffdaten des Lösungsmittels lassen sich die Drücke abschätzen, die sich für eine angenommene Löslichkeit $\lambda_{\text {soll }}$ nach dem Druckausgleich theoretisch einstellen müssten. Ausgehend von den fiktiven Messwerten lässt sich dann die Messunsicherheit abschätzen.

Für die Voruntersuchungen wurde angenommen, dass alle Temperatursensoren die gleiche Temperatur zeigen:

$$
t^{\mathrm{G}}=t^{\prime}=t^{\prime \prime}=t^{\mathrm{RHzg} 1}=t^{\mathrm{RHzg} 2}=t^{\mathrm{RHzg} 3}=t .
$$

Die Druckwerte im stationären Zustand $1 p_{1}^{\mathrm{G}}$ und $p_{1}$ wurden vereinfacht vorgegeben. Unbekannt sind die Drücke für den Zustand 2 und diese stellen sich in Abhängigkeit der Löslichkeit ein.

\section{Realistischer Druck $p_{2}^{G}$}

Für die fiktive Versuchsdurchführung wurde angenommen, dass es einen stationären Zustand 2a direkt nach dem Druckausgleich gibt, bei dem die Drücke in Gasreservoir und Reaktorgefäß gleich groß sind, jedoch noch kein Gas in Lösung gegangen ist. Nun wird das Ventil geschlossen und der Druck im Gasreservoir bleibt, also $p_{2}^{G}=$ $p_{2 a}^{G}{ }^{23}$

Für die Bestimmung von $p_{2}^{G}$ wurde der Berechnungsalgorithmus zur Bestimmung von $\lambda_{\infty}$ genutzt. Gesucht ist für den Zustand 2 genau der Druck $p_{2}^{G}$, der sich in Gasreservoir und Reaktor einstellt, wenn kein Gas in Lösung geht, also der Druck für den gilt

$$
\lambda_{\infty}\left(p_{2}^{\mathrm{G}} ; p_{2}\right) \stackrel{!}{=} 0
$$

${ }^{23}$ Für einen solchen Zwischenzustand spricht, dass während des Druckausgleichs der Rührer ausgeschaltet ist und die über die Phasengrenze stattfindende Absorption während der Zeit des Druckausgleichs nur einen vernachlässigbar geringen Gasanteil löst. Real tritt dieser stationäre Zustand 2a jedoch nicht auf, da beispielsweise während des Druckausgleichs dynamische und nicht-isotherme Vorgänge der Gasentspannung bzw. Kompression stattfinden oder auch die Dampfsättigung im Gasraum des Reaktors nicht sofort wieder gewährleistet ist. 
mit der Nebenbedingung

$$
p_{2}=p_{2}^{\mathrm{G}} .
$$

Durch numerische Berechnung erhält man $p_{2}^{G}$.

\section{Realistischer Druck $p_{2}$}

Nun ist noch die Variable $p_{2}$ für Zustand 2 gesucht. Diese muss so gewählt werden, dass die gemessene Löslichkeit gleich der angenommenen Löslichkeit $\lambda_{\text {soll, } \infty}$ ist, also

$$
\left.\lambda\right|_{p_{2}^{G}, \infty}\left(p_{2}\right) \stackrel{!}{=} \lambda_{\text {soll, } \infty}
$$

gilt. Auch diese nichtlineare Gleichung wird numerisch gelöst, um $p_{2}$ zu ermitteln.

Mit den ermittelten Messwerten $p_{2}^{G}$ und $p_{2}$ können nun, wie bei experimentell ermittelten Messwerten, die Löslichkeit (die hier nur der Kontrolle dient) und die zugehörigen Messunsicherheiten bestimmt werden. ${ }^{24}$

\subsubsection{Unsicherheitsanalyse in Abhängigkeit der eingefüllten Lösungsmittelmasse}

Da die Löslichkeitseigenschaften der Glykole unbekannt sind, aber vermutet wird, dass diese in ähnlicher Größenordnung wie für das Lösungsmittel Wasser liegen, wird für die Messunsicherheitsuntersuchungen angenommen, dass der gesuchte Löslichkeitskoeffizient gleich dem von Wasser bei gleicher Temperatur ist. Alle anderen Stoffwerte und insbesondere die zugehörigen Unsicherheiten werden jedoch von der zu untersuchenden Flüssigkeit Tyfocor ${ }^{\circledR}$ LS verwendet, da diese doch teilweise deutlich höhere Unsicherheiten als entsprechende Daten für Wasser aufweisen wie z. B. für Dampfdruck und Dichte der Fall. In den Ausführungen wird sich hier auf Stickstoff als gelöstes Gas beschränkt.

In Abbildung 4.17 ist für die gewählten Versuchsparameter dargestellt, welche Druckwerte sich, berechnet nach beschriebener Methodik, im Zustand 2 in Abhängigkeit der eingefüllten Lösungsmittelmasse einstellen. Ein Füllungsgrad von eins entspräche einem Volumen von $868 \mathrm{ml}$ bzw. bei der gewählten Temperatur einer Lösungsmittelmasse von 854,4 g. Eine geringe eingefüllte Lösungmittelmenge bedeutet ein großes Gasvolumen $V^{\prime \prime}$ im Reaktor, das bei Druckausgleich eine große Gasmenge aufnimmt. Daher sinkt der Druck im Gasreservoir relativ stark. Anschließend wird das Gas gelöst und es stellt sich im Reaktor der Druck $p_{2}^{\mathrm{R}}$ ein. Für eine geringe Lösungsmittelmenge ist im Vergleich zur Gasmenge in $V^{\prime \prime}$ die in Lösung gehende Gasmenge sehr klein, so dass sich der Druck nur wenig vom Druck direkt nach dem Druckausgleich, also von $p_{2}^{\mathrm{G}}$, unterscheidet.

Mit steigendem Füllungsgrad sinkt das Gasvolumen $V^{\prime \prime}$, damit verringert sich die beim Druckausgleich übertragene Gasmenge und folglich sinkt die Druckdifferenz im Gasreservoir. Bei vollständiger Füllung des Reaktorgefäßes mit Lösungsmittel findet kein Gasaustausch und damit auch keine Druckabsenkung im Gasreservoir statt, wie in der Abbildung 4.17 durch Erreichen von $p_{2}^{R}=5,5$ bar zu sehen. Das durch die Gasabsorption bedingte Absinken des Reaktordruckes nach dem Druckausgleich wird mit steigendem Füllungsgrad größer, da die in Lösung gehende Gasmenge im Vergleich zur Gasmenge in $V^{\prime \prime}$ steigt

\footnotetext{
${ }^{24}$ Hier wird der Sonderfall betrachtet, dass durch die fiktiven Messwerte gerade die wahre Löslichkeit als
} Ergebnis erhalten wird. 


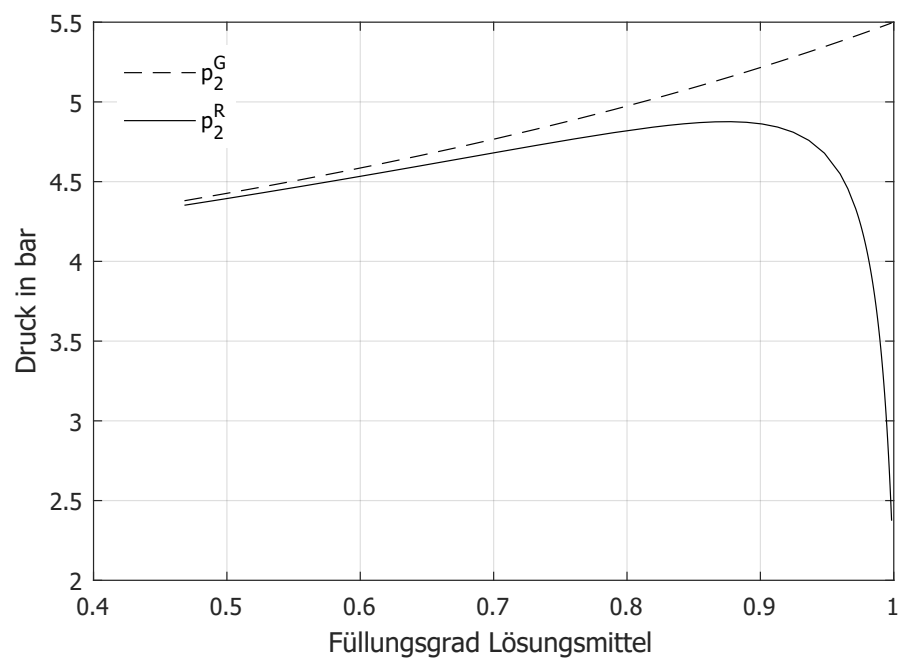

Abbildung 4.17: Drücke im stationären Zustand 2 in Abhängigkeit des Lösungsmittel-Füllungsgrads im Reaktor bei (fiktiver) wasserähnlicher Löslichkeit von Tyfocor ${ }^{\circledR}$ LS, $t_{\text {soll }}=90^{\circ} \mathrm{C} ; \lambda_{\text {soll, N2, } \infty}=$ $0,0104 \frac{\mathrm{cm}^{3}}{\mathrm{gLM} \cdot \mathrm{bar}}$, Druckausgleich bei $p_{1}^{\mathrm{G}}=5,5$ bar und $p_{1}^{\mathrm{R}}=2$ bar

und durch den höheren Partialdrucksprung die in Lösung gehende Gasmenge auch absolut steigt.

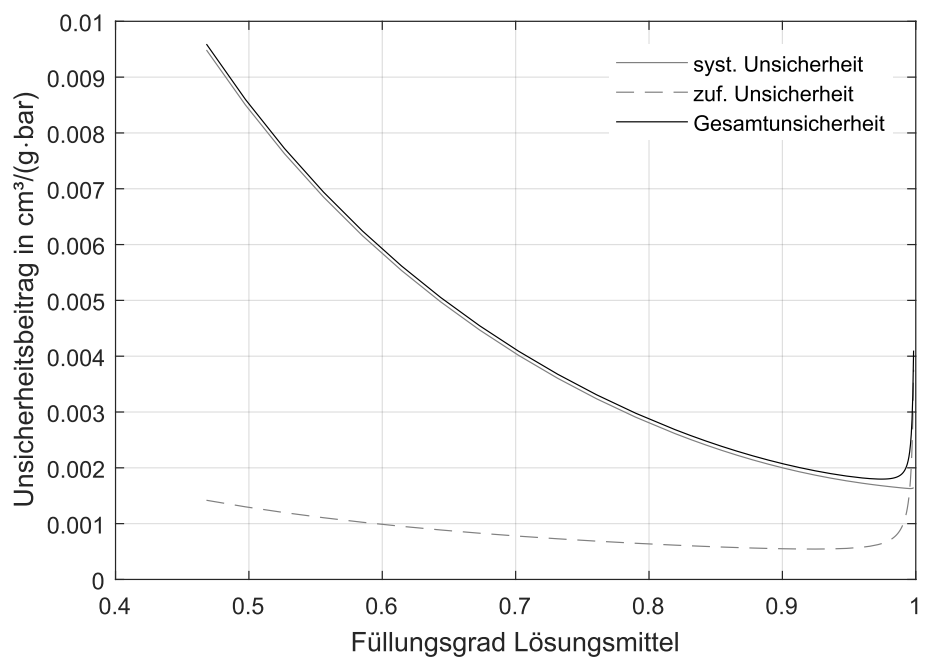

Abbildung 4.18: Messunsicherheit in Abhängigkeit der Masse an Lösungsmittel, Randbedingungen wie in Abbildung 4.17

In Abbildung 4.18 ist nun der die Messunsicherheit (zufällig und systematisch nach GUM) für den fiktiven Versuch mit den angenommenen Unsicherheitsbudgets dargestellt. Interessant ist, dass bei einem Füllungsgrad von 0,5 die Messunsicherheit bereits etwa so groß ist wie das Messergebnis selbst. Durch die Erhöhung der Lösungsmittelmenge $m_{\mathrm{LM}}$ kann die Messunsicherheit der Apparatur deutlich gesenkt werden. Dies liegt im Wesentlichen darin begründet, dass der Gasraum im Reaktorgefäß deutlich kleiner ist und die gasförmige Gasmenge $\triangle n_{i, 1 \rightarrow 2}^{\prime \prime}$ daher genauer (absolut) abgeschätzt werden kann (Unsicherheit der Messung P.R2 bzw P.R3 hat deutlich geringeren Einfluss). Dadurch und durch die höhere Menge an gelöstem Gas $\triangle n_{i, 1 \rightarrow 2}^{\prime}$ lässt sich diese genauer bestimmen. 


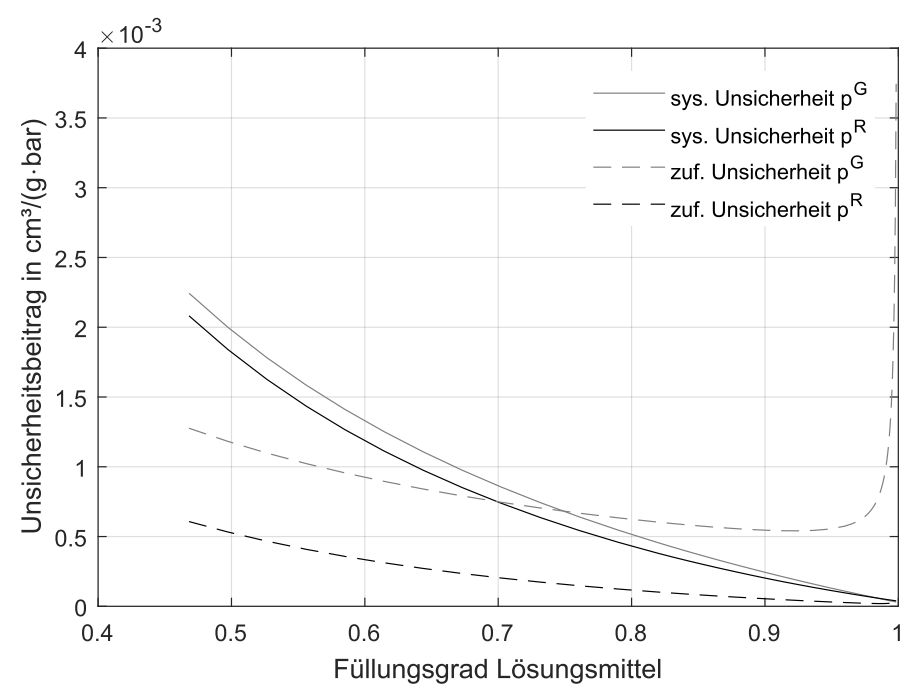

Abbildung 4.19: Zufällige und systematische Messunsicherheitsbeiträge der Drücke $p^{G}$ und $p$

Bei einer sehr großen Lösungsmittelmenge sinkt die zufällige Messunsicherheit nicht weiter, sondern steigt sogar wieder an. Dies passiert in so starkem Maße, dass auch die Gesamtmessunsicherheit ansteigt. Dieser Effekt beruht im Wesentlichen auf der geringeren vom Gasreservoir überführten Gasmenge, die sich in einer geringeren Druckdifferenz im Gasreservoir, wie in Abbildung 4.17 dargestellt, äußert. Durch die geringere Druckdifferenz steigt der Einfluss der zufälligen Messunsicherheit der gemessenen Druckwerte, wie die in Abbildung 4.19 dargestellten Messunsicherheitsbeiträge zeigen. Einen weiterer Grund für den Messunsicherheitsanstieg sind die in Temperatur und Volumen nur ungenau definierten Toträume in $V^{\prime \prime}$. Denn mit steigender Lösungsmittelmenge nimmt das Gasvolumen im Reaktor ab und dadurch steigt der Anteil der Totvolumina am Gesamtvolumen $V^{\prime \prime}$.

Aufgrund der obigen Überlegungen liegt es nahe, die einzufüllende Lösungsmittelmenge am Minimum der Gesamtunsicherheit und damit bei einem Füllgrad von 0.9 zu orientieren. Gegen eine so hohe Lösungsmittelmenge im Reaktorgefäß sprechen hingegen die in der Unsicherheitsbetrachtung nicht beachteten, weil schwer quantifizierbaren Gründe wie

- schwieriges Handling, erhöhte Gefahr des (möglicherweise unerkannten) Flüssigkeitsverlustes (Schwäppern) bei Montage des Gefäßes,

- unperfekte Gasmischung in Toträumen, die dann einen hohen Anteil an $V^{\prime \prime}$ einnehmen würden,

- Spritzen/Transport von Flüssigkeit in Totvolumina durch Rühren und Festsetzung dort,

- Funktion der Begasungsrührwelle bei zu hohem Füllstand nicht mehr sicher.

Die Lösungsmittelmenge wurde aufgrund der o.g. Einflüsse auf einen Füllgrad von etwa $0.8 \mathrm{bzw}$. ein Volumen von $700 \mathrm{ml}$ festgelegt. Durch die vorgelagerten Dichteversuche kann jeweils auf die einzufüllende Masse $m_{\mathrm{LM}}$ geschlossen werden. Die entsprechende Gesamtmessunsicherheit liegt nur wenig über dem -rein rechnerisch- optimalen Wert in Abbildung 4.18. 


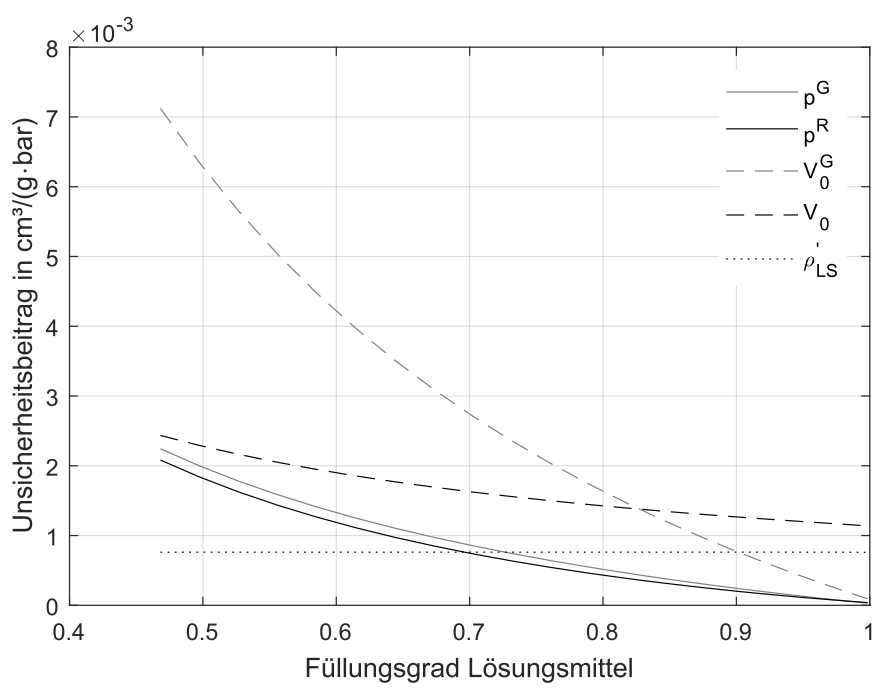

Abbildung 4.20: Größte systematische Unsicherheitsbeiträge im Überblick: Volumina (VG_0, V_0), Dichte (arho_LS), Kalibrierung des Drucksensors (ap,apG)

\subsubsection{Messunsicherheitsbeitrag der Dichte}

In Abb 4.20 sind wichtige systematische Messunsicherheitsbeiträge dargestellt. Dort zeigt sich die Besonderheit, dass der Unsicherheitsbeitrag der Dichte des Lösungsmittels (arho_LS, hier Annahme 0,1\% Dichteunsicherheit) einen wesentlichen Beitrag darstellt und im Gegensatz zu den anderen Beiträgen durch Erhöhung der Lösungmittelmenge im Versuch nicht verringert werden kann. Auf eine Herleitung wird hier verzichtet. Erste Vorversuche wurden noch auf Basis von Dichtemessungen mit Pyknometer (0,2\% Dichteunsicherheit) durchgeführt. Für folgende Versuche wurden dann diverse Biegeschwingmessgeräte mit deutlich höherer Genauigkeit (laut Datenblatt 0,01\% Unsicherheit) eingesetzt, um den Unsicherheitsbeitrag gering zu halten.

\subsubsection{Messunsicherheit bei der kalibrierten Bestimmung}

Alle Eingangsgrößen $\vec{x}$ in die Berechnung von $\lambda_{i, \mathrm{LM}, \infty \text {,mess }}\left(\vec{x}_{\mathrm{LM}}\right)$ des Versuchs und $\lambda_{i, \mathrm{H} 2 \mathrm{O}, \infty \text {, mess }}\left(\vec{x}_{\mathrm{H} 2 \mathrm{O}}\right)$ des Referenzversuchs setzen sich zusammen aus

- unterschiedlichen Eingangsgrößen $\vec{x}_{\mathrm{var}}$ und

- gleichen Eingangsgrößen $\vec{x}_{\text {con }}$,

Für den Versuch ergibt sich der Vektor an Eingangsgrößen zu

$$
\vec{x}_{\mathrm{LM}}=\left(\begin{array}{c}
\vec{x}_{\mathrm{LM}, \mathrm{var}} \\
\vec{x}_{\mathrm{Con}}
\end{array}\right)
$$

und für den Referenzversuch mit Wasser zu

$$
\vec{x}_{\mathrm{H} 2 \mathrm{O}}=\left(\begin{array}{c}
\vec{x}_{\mathrm{H} 2 \mathrm{O}, \mathrm{var}} \\
\vec{x}_{\mathrm{con}}
\end{array}\right) \text {. }
$$

Unterschiedliche Eingangsgrößen sind 
- stoffspezifische Größen wie Dampfdrücke $p_{\mathrm{LM}, \mathrm{s}}$ und $p_{\mathrm{H} 2 \mathrm{O}, \mathrm{s}}$ mit jeweils zugehöriger systematischer Unsicherheit

- Messwerte des jeweiligen Versuchs, wie z. B. $p_{\mathrm{Anz}, z}$

Eine gleiche Eingangsgröße ist z. B. das Volumen des Reaktorgefäßes V. Gleiche Eingangsgrößen weisen immer den gleichen Wert und damit die gleiche Messabweichung für jede anschließende Nutzung in den $\lambda$-Berechnungen auf. Zusätzlich können auch unterschiedliche Eingangsgrößen, wie z. B. $y_{\mathrm{O} 2}$ in $\mathrm{N} 2+\mathrm{O} 2,2$ in den Versuchen gleiche Messabweichungen enthalten. Dann wird die Größe aufgeteilt in erstens eine in den Versuchen unterschiedliche und zweitens eine in den Versuchen gleiche Größe, im Beispiel zu

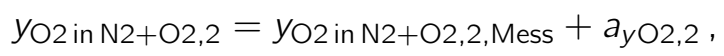

wobei

- der Messwert $y_{\mathrm{O} 2}$ in N2+O2,2,Mess eine in den Versuchen unterschiedliche Eingangsgröße und

- die Abweichung $a_{y} \mathrm{O}, 2$ eine in den Versuchen gleiche Eingangsgröße (mit Wert null und entsprechender Unsicherheit)

darstellt.

\section{Effekt der kalibrierten Bestimmung}

Die kombinierte Messunsicherheit der kalibrierten Bestimmung von $\lambda_{i, \mathrm{LM}, \infty}$, kalibriert nach Gleichung 4.46 in Abschnitt 4.6 lässt sich nun mit den Unsicherheiten der unterschiedlichen Eingangsgrößen $U_{\mathrm{LM}, \mathrm{var}, j_{1}}$ bzw. $U_{\mathrm{H} 2 \mathrm{O}, \mathrm{var}, j_{2}}$ und den Unsicherheiten der gleichen Eingangsgrößen $U_{\text {con, } m}$ sowie der Unsicherheit des Literaturwertes $U_{\lambda_{\text {Lit }}}$ nach dem Unsicherheitsfortpflanzungsgesetz ausdrücken als

$$
\begin{aligned}
U_{\lambda, \text { kalibriert }}^{2} & =\sum_{j_{1}}\left(\frac{\partial \lambda_{i, \mathrm{LM}, \infty, \text { mess }}}{\partial x_{\mathrm{LM}, \mathrm{var}, j_{1}}}\right)^{2} U_{\mathrm{LM}, \mathrm{var}, j_{1}}^{2}+\sum_{j_{2}}\left(\frac{\partial \lambda_{i, \mathrm{H} 2 \mathrm{O}, \infty, \mathrm{mess}}}{\partial x_{\mathrm{H} 2 \mathrm{O}, \mathrm{var}, j_{2}}}\right)^{2} U_{\mathrm{H} 2 \mathrm{O}, \mathrm{var}, j_{2}}^{2} \\
& +\sum_{m}\left(\frac{\partial \lambda_{i, \mathrm{LM}, \infty, \text { mess }}}{\partial x_{\mathrm{con}, m}}-\frac{\partial \lambda_{i, \mathrm{H} 2 \mathrm{O}, \infty, \text { mess }}}{\partial x_{\mathrm{con}, m}}\right)^{2} U_{\mathrm{con}, m}^{2}+U_{\lambda_{\mathrm{Lit}}}^{2}
\end{aligned}
$$

im Gegensatz zur Unsicherheit der Einzelmessung

$$
U_{\lambda}^{2}=\sum_{j}\left(\frac{\partial \lambda_{i, \mathrm{LM}, \infty, \mathrm{mess}}}{\partial x_{\mathrm{LM}, \mathrm{var}, j}}\right)^{2} U_{\mathrm{LM}, \mathrm{var}, j}^{2}+\sum_{m}\left(\frac{\partial \lambda_{i, \mathrm{LM}, \infty, \mathrm{mess}}}{\partial x_{\mathrm{con}, m}}\right)^{2} U_{\mathrm{con}, m}^{2} .
$$

Im Vergleich der Gleichung 4.76 für die kalibrierte Bestimmung mit der Gleichung 4.77 für die unkalibrierte Bestimmung ist zu erkennen:

- Sind die Sensitiväten $\frac{\partial \lambda_{i, L M}, \infty, \text { mess }}{\partial x_{c o n, m}}$ und $\frac{\partial \lambda_{i, H 2 O, \infty, \text { mess }}}{\partial x_{c o n, m}}$ einer Größe $x_{m}$ ähnlich, so lässt sich durch die kalibrierte Berechnung der Beitrag der Unsicherheit $U_{\text {con, } m}$ der Einflussgröße $x_{c o n, m}$ deutlich reduzieren, im Idealfall sogar gänzlich eliminieren.

- Die Sensitivitäten sind umso ähnlicher, je ähnlicher sich die Versuche und die Stoffe sind. Daher wurde Wasser ausgewählt und auf annähernd gleiche Versuchsdurchführung geachtet. 
- Bei der kalibrierten Berechnung $\lambda_{i, \mathrm{LM}, \infty}$, kalibriert kommen aber auch zusätzlich Beiträge durch die Unsicherheiten $U_{\mathrm{H} 2 \mathrm{O}, v a r, j_{2}}$ wie zufällige Unsicherheiten, StoffwertUnsicherheiten (in den jeweiligen Sensitivitäten) hinzu. Außerdem kommt direkt die Unsicherheit des Literaturwertes $U_{\lambda_{\text {Lit }}}$ hinzu. Deshalb ist darauf zu achten, dass diese zusätzlichen Unsicherheiten den Vorteil der kalibrierten Bestimmung nicht aufheben. Hier ist das jedoch nicht der Fall.

\subsection{Messunsicherheitsbeiträge und Gesamt-Messunsicherheit}

\subsubsection{Gesamt-Messunsicherheit}

In Abbildung 4.21 sind für einen Versuch mit Tyfocor ${ }^{\circledR}$ LS bei $80^{\circ} \mathrm{C}$ (LS80) die Ergebnisse des Referenzversuchs, der unkalibrierte Wert und der kalibrierte Wert dargestellt. Die Messunsicherheit des Referenzversuchs ist recht hoch. Eine ähnlich hohe Messunsicherheit ergibt sich für den unkalibrierten Versuch. Das Ergebnis des kalibrierten Versuchs ist um den Offset zwischen Ergebnis des Wasserversuchs und Literaturwert verschoben und die Gesamtmessunsicherheit ist deutlich reduziert.

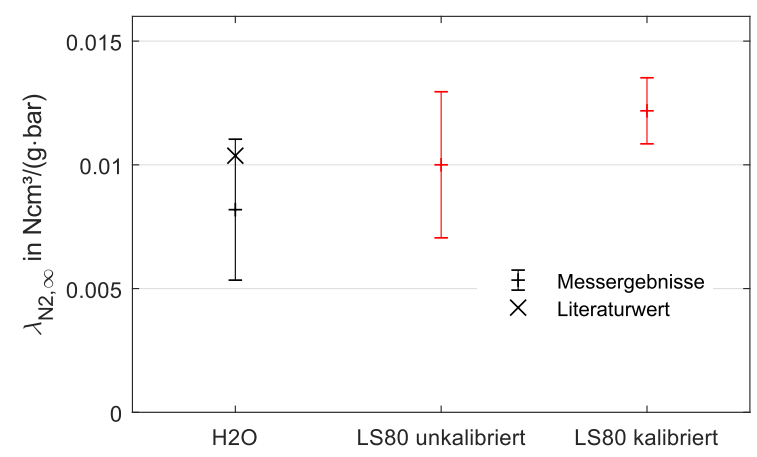

Abbildung 4.21: Ergebnisse eines LS80-Versuchs: H2O-Referenzversuch, unkalibriertes Ergebnis, kalibriertes Ergebnis

In Abbildung 4.22 sind unterschiedliche kalibrierte Messergebnisse für das Lösungsmittel 41,84 gew.- $\%$ Propylenglykol-Wasser bei $80{ }^{\circ} \mathrm{C}$ (41P80) dargestellt. Es ist zu sehen, dass das Messergebniss $\lambda_{\mathrm{N} 2, \infty}$ im Luft-Versuch gut mit dem ermittelten Wert $\lambda_{\mathrm{N} 2, \infty}$ des StickstoffVersuchs übereinstimmt und die Gesamtmessunsicherheit nur leicht höher ist. Ergebnisse für Sauerstoff $\lambda_{\mathrm{O}, \infty}$ zeigen etwas höhere Unsicherheiten.

\subsubsection{Ermittelte Messunsicherheitsbeiträge}

\subsubsection{Stickstoff-Versuche}

In den folgenden Abbildungen 4.23 (für unkalibrierte Berechnung) und 4.25 (für kalibrierte Berechnung) ist die Auswirkung einzelner Messunsicherheiten auf das Messergebnis, hier für einen Tyfocor ${ }^{\circledR}$ LS-Versuch, prozentual dargestellt. ${ }^{25}$

\footnotetext{
${ }^{25}$ Bemerkung: Es ist zu beachten, dass sich in den Berechnungen „absolut“ und „,kalibriert" nicht nur andere Messunsicherheiten, sondern auch andere Messergebnisse ergeben. Zur besseren Darstellung wurde hier für beide Berechnungen der Unsicherheitsbeitrag jeweils prozentual auf das Messergebnis des kalibrierten Messergebnisses bezogen. Außerdem ist zu beachten, dass bei Anwendung von GUM der gesamte, syste-
} 


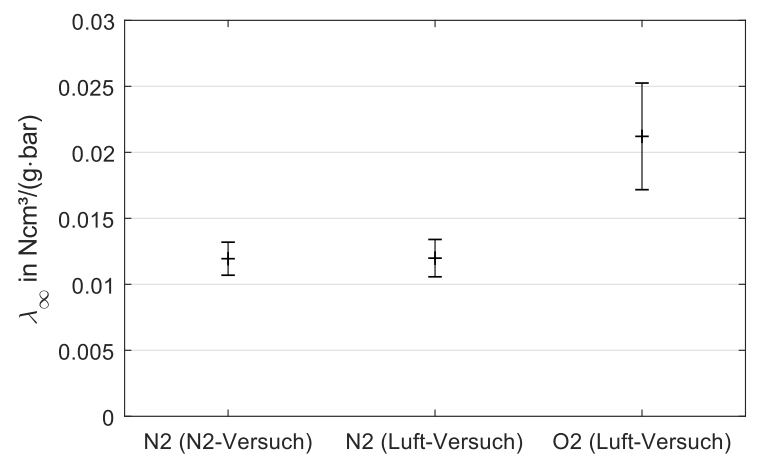

Abbildung 4.22: Ergebnisse kalibrierter 41P80-Versuche

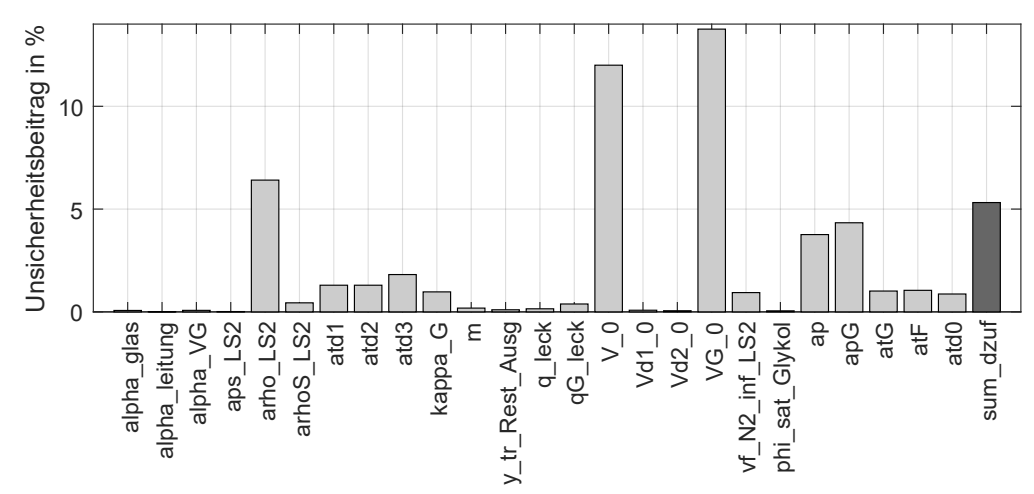

Abbildung 4.23: Systematische Unsicherheitsbeiträge und gesamter zufälliger Unsicherheitsbeitrag für die unkalibrierte Berechnung (LS80_675g_10bar_2011-12-16; Drucksprung 1); Legende in Tabelle 4.11

Bei der direkten Bestimmung der Löslichkeit (Abbildung 4.23) zeigt sich, dass trotz aufwändigem Ausliterns die Unsicherheiten der Volumina des Gasreservoirs $\Delta V^{G}$ und des Reaktors $\Delta V$ deutlichen Einfluss auf das Messergebnis haben. Außerdem sind auch die Einflüsse der Drucksensor-Unsicherheiten für Gasreservoir und Reaktorgefäß relativ groß. Die Unsicherheit der Dichte zeigt ebenfalls deutlichen Einfluss. Vernachlässigbar sind hingegen Messunsicherheiten durch Ausdehnungskoeffizienten, Dampfdruck. Zufällige Unsicherheiten haben einen relevanten Einfluss. Wie in Abbildung 4.24 zu sehen, haben auch hier die Druckmessungen den größten Anteil.

matische Unsicherheitsbeitrag kleiner als die Summe der einzelnen systematischen Unsicherheitsbeiträge ist. Die zufällige Unsicherheit ist bereits nach GUM abgeschätzt.

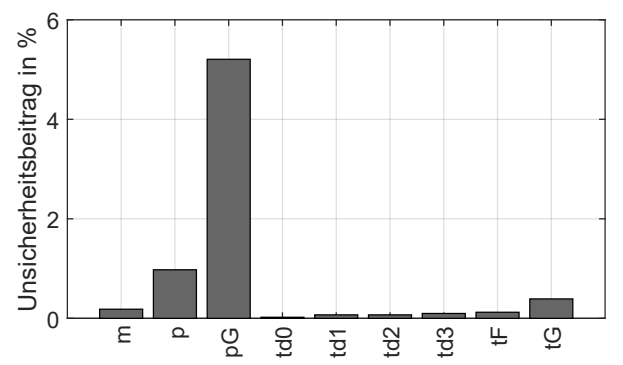

Abbildung 4.24: Zufällige Unsicherheitsbeiträge für die unkalibrierte Berechnung (LS80_675g_10bar_201112-16; Drucksprung 1); Legende in Tabelle 4.11 
Tabelle 4.11: Legende zu Abbildungen

\begin{tabular}{ll}
\hline Abkürzung & Einflussgröße \\
\hline alpha_leitung & $\begin{array}{l}\text { Ausdehungskoeff. } \alpha \text { der } \\
\text { Totraumvolumina }\end{array}$ \\
alpha_glas & Ausdehnungskoeff. $\alpha$ des Reaktors \\
alpha_VG & Ausdehnungskoeff. $\alpha$ des \\
& Gasreservoirs \\
aps_H2O & Dampfdruck Wasser \\
aps_LS & Dampfdruck Tyfocor LS \\
arho_H2O & Dichte Wasser \\
arho_LS & DichteTyfocor LS \\
arhoS_H2O & Dampfdichte Wasser \\
arhoS_LS2 & Dampfdichte Tyfocor LS \\
atd1 & T.RHzg1 gegenüber Gastemperatur \\
atd2 & T.RHzg2 gegenüber Gastemperatur \\
atd3 & T.RHzg3 gegenüber Gastemperatur \\
kappa_G & Kompressibilität Glykol $\kappa$ \\
$\mathrm{m}$ & Wägung $m$ \\
y_tr_Rest_Ausg & Anteil Restgase ytr,Rest,Ausg \\
q_leck & Leckage Reaktor \\
qG_leck & Leckage Gasreservoir \\
V_0 & Volumen Vo \\
\hline
\end{tabular}

\begin{tabular}{|c|c|}
\hline Abkürzung & Einflussgröße \\
\hline Vd1_o & Volumen Totraum \\
\hline Vd2_0 & Volumen Totraum \\
\hline VG_0 & Volumen $V_{0}^{G}$ \\
\hline vf_N2_inf_H2O & $v_{\mathrm{N} 2, \mathrm{H} 2 \mathrm{O}, \infty}^{\prime}$ \\
\hline $\mathrm{vf}_{-} \mathrm{N} 2{ }_{-}{ }^{\text {inf_LS2 }}$ & $v_{\mathrm{N} 2, \mathrm{LS}, \infty}^{\prime}$ \\
\hline phi_sat_H2O & $\phi_{\text {sat }, \mathrm{H} 2 \mathrm{O}}$ \\
\hline phi_sat_Glykol & $\phi_{\text {sat, Glykol }}$ \\
\hline ap & Kalibrierung Drucksensor P.R2 \\
\hline apG & Kalibrierung Drucksensor P.G2 \\
\hline at $\mathrm{G}$ & Kalibrierung T.G \\
\hline atF & Kalibrierung T.R1 \\
\hline atd0 & Kalibrierung T.R2 \\
\hline sum_dzuf & gesamter zuf. Unsicherheitsbeitrag \\
\hline$y_{-} \mathrm{O} 2 \mathrm{ofN} 2 \mathrm{O} 2 \_1 / 2$ & Zusammensetzung Gasphase $\mathrm{O} 2+\mathrm{N} 2$ \\
\hline ay_O2ofN2O2_2 & Zusammensetzung Gasphase $\mathrm{O} 2+\mathrm{N} 2$ \\
\hline$y G_{-} N 2$ & Zusammensetzung Arbeitsgas \\
\hline vf_O2_inf_H2O & $v_{\mathrm{O} 2, \mathrm{H} 2 \mathrm{O}, \infty}^{\prime}$ \\
\hline$\underline{\mathrm{vf} \_\mathrm{O} 2 \_ \text {inf_LS2 }}$ & $v_{\mathrm{O} 2, \mathrm{LS}, \infty}^{\prime}$ \\
\hline
\end{tabular}

Bei der kalibrierten Bestimmung der Löslichkeit kommen weitere Einflussgrößen des Referenzversuchs hinzu (u. a. mit "ref " bezeichnet) sowie die Unsicherheit des verwendeten Literaturwerts $\lambda_{i, \mathrm{H} 2 \mathrm{O} \text {, Lit }}$ (a_Lambda_H2O_Lit). Auch die zufällige Unsicherheit wird durch die erhöhte Anzahl an Messwerten erhöht. Durch die Kalibrierung können jedoch insbesondere die systematischen Unsicherheitsbeiträge der Volumina, der Temperatursensoren und der Drucksensoren deutlich reduziert werden. Als größte systematische Unsicherheitsbeiträge verbleiben die Unsicherheit der Dichte, die Unsicherheit tabellierter Wasserwerte, die unsichere Kompressibilität des Wasser-Glykols sowie die Unsicherheit der partiellen molaren Volumina von Stickstoff im Lösungsmittel. Zufällige Unsicherheitsbeiträge machen einen höheren Beitrag aus und sind, wie in Abbildung 4.26 dargestellt, dominiert durch den Einfluss der Druckmessung für beide Versuche.

\subsubsection{Luft-Versuche}

In Abbildungen 4.27 und 4.28 sind analog die Messunsicherheitsbereiche eines exemplarischen Luft-Versuchs dargestellt. Durch die Kalibrierung reduziert sich die Unsicherheit der gaschromatographischen Bestimmung des Sauerstoffanteils deutlich. Ebenso hat die Unsicherheit der Zusammensetzung des Arbeitsgases keinen Einfluss mehr. 


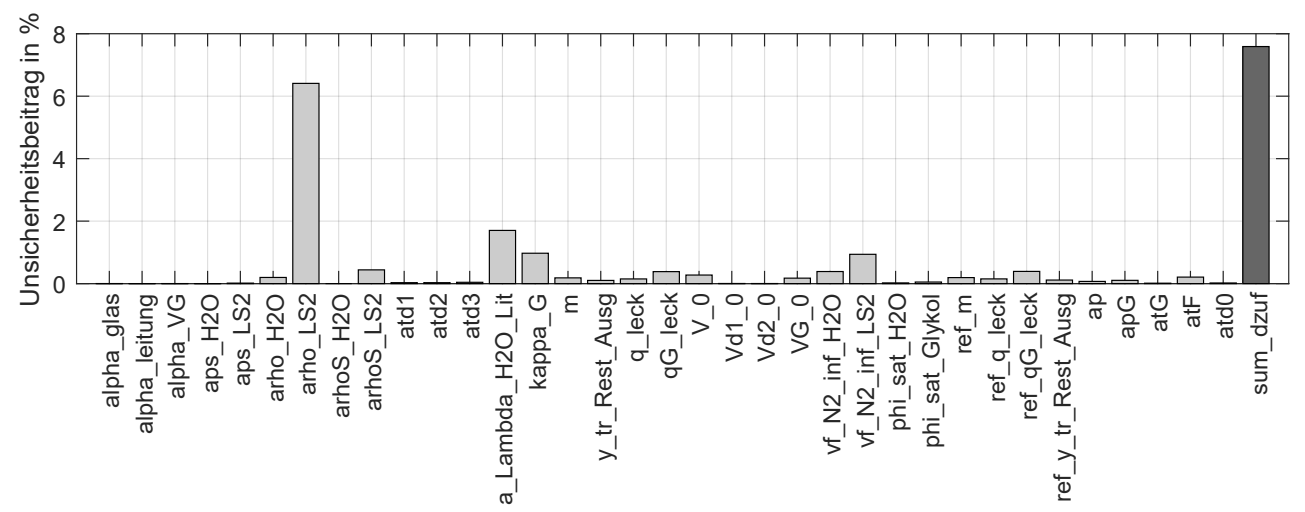

Abbildung 4.25: Systematische Unsicherheitsbeiträge und gesamter zufälliger Unsicherheitsbeitrag für die kalibrierte Berechnung (LS80_675g_10bar_2011-12-16; Drucksprung 1); bezogen auf das Ergebnis der kalibrierten Berechnung; Legende in Tabelle 4.11

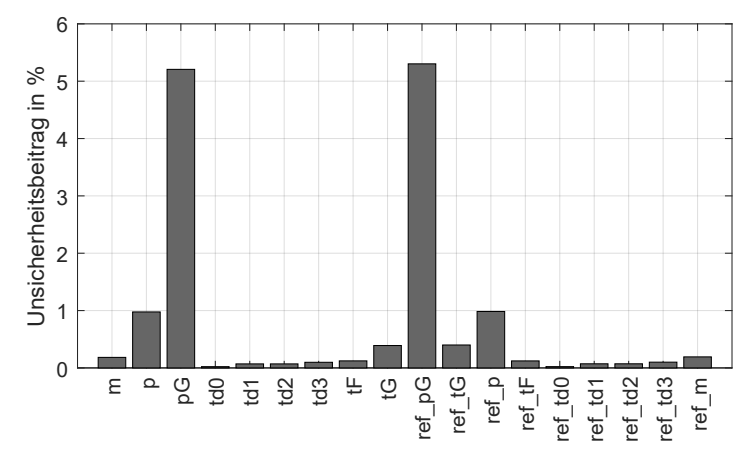

Abbildung 4.26: Zufällige Unsicherheitsbeiträge für die kalibrierte Berechnung (LS80_675g_10bar_2011-1216; Drucksprung 1); Legende in Tabelle 4.11

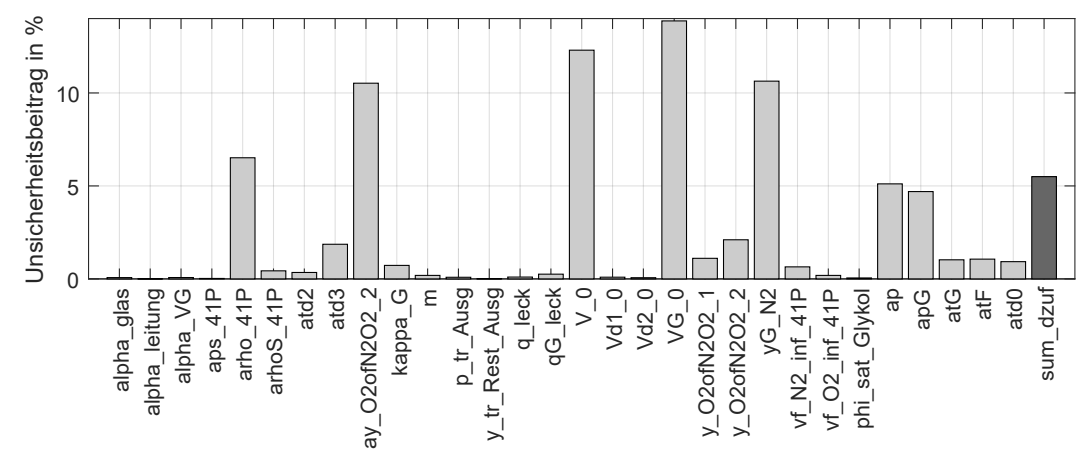

Abbildung 4.27: Systematische Unsicherheitsbeiträge und gesamter zufälliger Unsicherheitsbeitrag für die unkalibrierte Berechnung für O2 (Versuch 41P80); Legende in Tabelle 4.11 


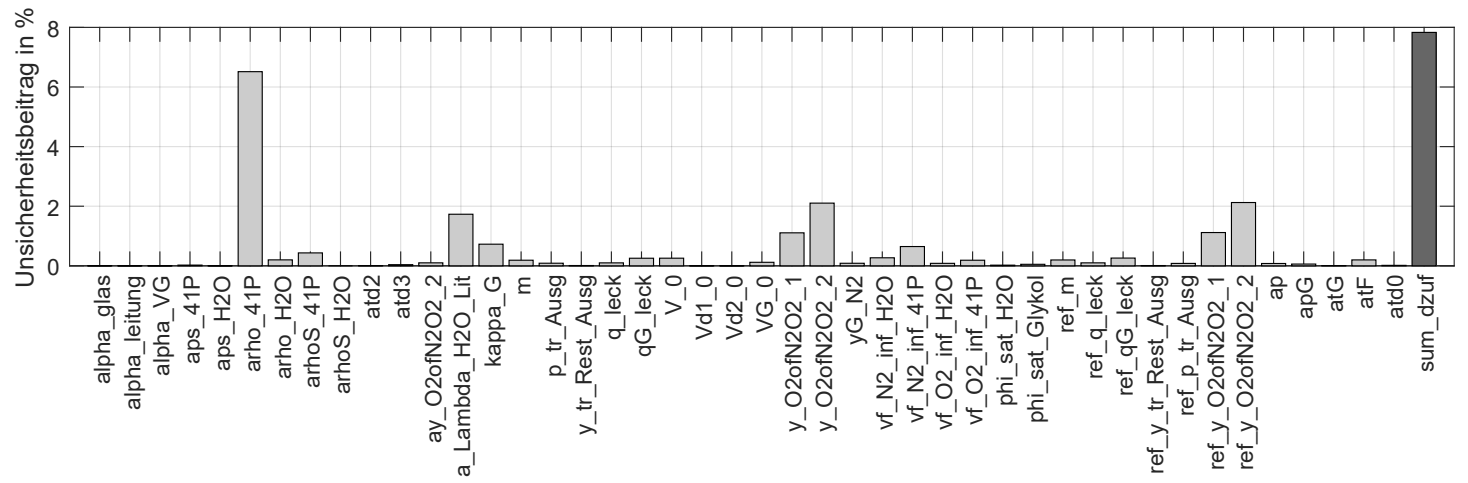

Abbildung 4.28: Systematische Unsicherheitsbeiträge und gesamter zufälliger Unsicherheitsbeitrag für die kalibrierte Berechnung für O2 (Versuch 41P80); Legende in Tabelle 4.11 


\subsection{Wertung und Verbesserungspotential des Versuchsstands}

Für die anspruchsvollen Anforderungen (unbekannte, möglicherweise reaktive Lösungsmittel mit ggf. flüchtigen Substanzen, großer Temperaturbereich, geringer Finanzumfang) konnten eine solide Apparatur und ein robuster Versuchsablauf erarbeitet werden. Die Automatisierung des Versuchsstands hat sich bewährt. Löslichkeitskoeffizienten können mit für technische Anwendungen akzeptablen Messunsicherheiten ermittelt werden.

Insgesamt bietet die Apparatur auf Grundlage der vorliegenden Erfahrungen dennoch Optimierungspotential hinsichtlich Zeitaufwand, Genauigkeit und Messaufwand. Dieser liegt vor allem in konstruktiven Abänderungen (Minimierung der Verbindungsstellen und Ventile, Einsatz von Ventilen höherer Güte, Verkleinerung und optimale Abstimmung der Volumina für den speziellen Versuchsablauf, Integration Reaktorgefäß in Temperierbad etc.). Der Aufwand, eine gasdichte und gut temperierte Apparatur mit den gegebenen Druck- und Temperaturparametern zu erstellen, lag deutlich über dem veranschlagten Aufwand. Für Justierung und Optimierung von Versuchsanlage und Ablauf (Optimierung elektrische Heizung, Auslitern, Leckage, Rührerstellung, Dämmwirkung, Testversuche, Optimierung Abfolge und Zeiten, Kalibrierung etc.) wurden über 80 Versuchstage benötigt, um schließlich zu reproduzierbaren Versuchen zu kommen.

Das System besitzt eine hohe thermische Trägheit. Dadurch werden jeweils Zeiträume von mehreren Stunden bis zum Erreichen des stationären Zustands benötigt. Dies hat entsprechend Auswirkungen auf den Zeitaufwand der Versuche und Leckagemengen. Ein wichtiger Nachteil des Verfahrens ist, dass die Dichte des Lösungsmittels mit hoher Genauigkeit bekannt sein muss. Dafür sind vorgelagerte Dichtemessungen der Lösungsmittel notwendig.

Eine Möglichkeit, den Versuchsaufwand stark zu minimieren, besteht darin, den sich einstellenden Druck im Phasengleichgewicht nicht nur bei einer Temperatur zu messen, sondern immer für eine ganze Temperaturreihe abzufahren. Anschließend wird ein Drucksprung realisiert und die gleiche Temperaturreihe auch für das zweite Phasengleichgewicht abgefahren. Damit ergeben sich aus einer Reaktorbefüllung Ergebnisse für beliebig viele Temperaturen. ${ }^{26}$ Es wurden ein entsprechender Automatisierungsablauf erstellt und einige Testversuche nach einem solchen Regime mit fünf Temperaturstufen durchgeführt. Ergebnisse zeigen, dass die Leckagen aufgrund der langwierigen Temperiervorgänge für die Apparatur hier einfach zu hoch sind, um solide Messergebnisse zu generieren.

Für eine weitere Verbesserung des Versuchsstands sollte auch die Gleichgewichtszelle in das Temperierbad eingetaucht werden. Damit ist eine gleichmäßige Temperierung gegeben. Separat zu temperierende Totvolumina würden entfallen. Die thermische Trägheit des Systems wird kleiner. Diese kann weiter reduziert werden, wenn die Größe der Gleichgewichtszelle reduziert wird. Eine exakte Bestimmung des Dampfdrucks wird durch die sehr gleichmäßige Temperierung möglich. Dies ist beim aktuellen Reaktorgefäß nicht möglich. Anschließend sollte die Größe des Gasreservoirs in Abhängigkeit der zu untersuchenden Stoffe hinsichtlich geringer Gesamtmessunsicherheit optimiert ausgewählt werden. Alternativ ist die Verwendung von (temperierten) Kolben-Injektoren wie beispielsweise in [Fischer und Gmehling, 1994; Fischer und Wilken, 2001] für den definierten und sehr gut messbaren Gas- bzw. Lösungsmitteleintrag in die Gleichgewichtszelle in Betracht zu ziehen. Zur Untersuchung der Druckabhängigkeit der Löslichkeit ist eine mehrfache, automatisierte und dosierte Zugabe von Gas bei nur einer Gasbeladung von Gasreservoir oder Kolben-Injektor im Sinne geringer Messunsicherheiten und vieler Messpunkte wünschenswert. Ein einfaches Handling der

\footnotetext{
${ }^{26}$ Dieses Vorgehen funktioniert nicht, wenn das Gas chemische Bindung eingeht.
} 
Befüllung und Entleerung durch Einsaugen und Absaugen des Fluids ohne Öffnen des Behälters kann den Versuchsaufwand und die Fehleranfälligkeit reduzieren. Drucksensoren sollten nicht mittemperiert sondern durch dünne Verbindungsleitungen vom Temperierbad getrennt werden. Dann sind auch am Markt verfügbare (aber im Temperaturbereich eingeschränkte) Drucksensoren mit höherer Genauigkeit nutzbar. 



\section{Durchgeführte Versuche und deren Auswertung}

\section{1 Übersicht}
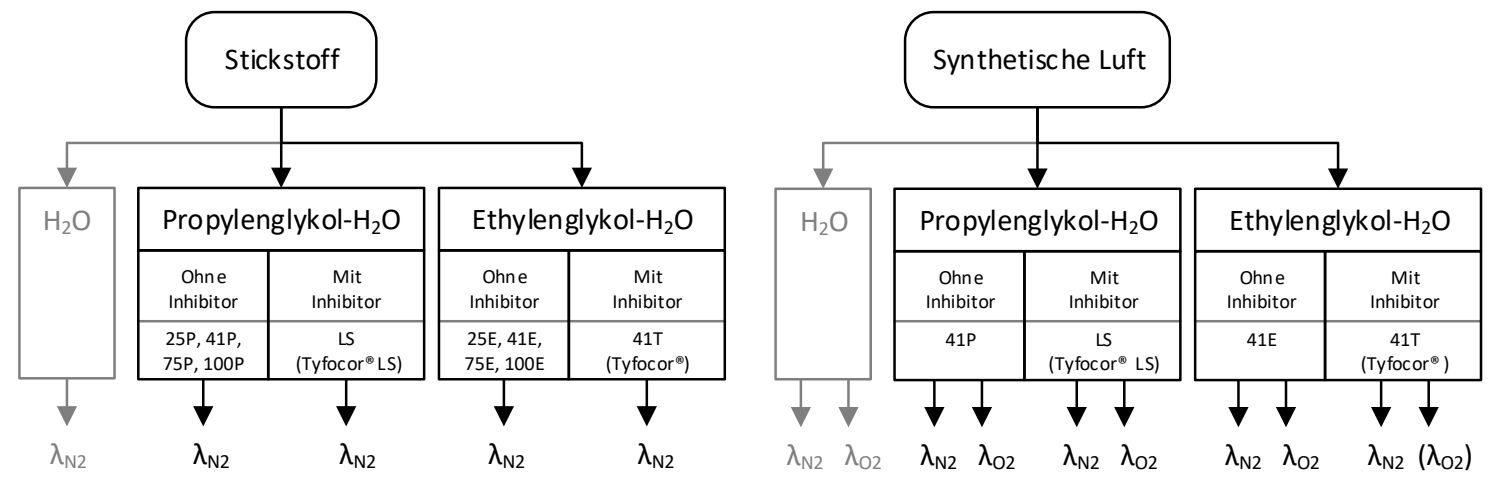

Abbildung 5.1: Übersicht der durchgeführten Versuche zur Bestimmung technischer Löslichkeitskoeffizienten

Abbildung 5.1 zeigt schematisch die nach intensiver Versuchs- und Anlagenoptimierung durchgeführten Versuche für Stickstoff und Synthetische Luft. Während aus StickstoffVersuchen Löslichkeitskoeffizienten für Stickstoff abgeleitet werden können, können aus LuftVersuchen sowohl Löslichkeitskoeffizienten für Stickstoff als auch Sauerstoff abgeleitet werden.

Erste Stickstoff-Versuche fanden für das Fertiggemisch Tyfocor ${ }^{\circledR}$ LS (Kurzbezeichnung LS) mit einem Propylenglykolgehalt laut Hersteller von 41,84 Gew.- $\%$ statt. Um den Einfluss der Additive auf die Löslichkeit zu analysieren, wurde diesem ein reines Wasser-PropylenglykolGemisch mit gleichem Glykolanteil gegenübergestellt (Kurzbezeichnung 41P). Um Aussagen auch für andere Mischungsverhältnisse abzuleiten, wurden außerdem reine WasserPropylenglykol-Mischungen für 25, 75 und $100 \mathrm{Gew} .-\%$ (mit Kurzbezeichnung 25P, 75P, 100P) untersucht.

Analoge Stickstoff-Versuche fanden für reine Wasser-Glykol-Gemische auf EthylenglykolBasis statt (mit Kurzbezeichnungen 25E, 41E, 75E und 100E). Als Vertreter für eine Fertigmischung mit Additiven wurde hier ein Tyfocor ${ }^{\circledR}$-Wasser-Gemisch (Kurzbezeichnung 41T) verwendet. Dabei wurde aus Vergleichsgründen das gleiche Mischungsverhältnis (mit 41,84 Gew.-\% Tyfocor ${ }^{\circledR}$-Gehalt) wie für Tyfocor ${ }^{\circledR}$ LS gewählt. ${ }^{1}$ Die Fertiggemische erreichen ähnliche Gefrierpunkte $\left(-25^{\circ} \mathrm{C}\right.$ für $\mathrm{LS}$, ca. $-24,3^{\circ} \mathrm{C}$ für 41T).

Die untersuchten Glykolgehalte $75 \mathrm{Gew} .-\%$ und $100 \mathrm{Gew} .-\%$ mit einem Eisflockenpunkt deutlich unter $-50^{\circ} \mathrm{C}$ haben hinsichtlich des in dieser Arbeit fokussierten technischen Einsatzgebiets keine Relevanz. Die $75 \mathrm{gew} .-\%$ Gemische sollten jedoch allgemein den Trend für Gemische mit höherem Glykolanteil zeigen. Die Untersuchungen der reinen Glykole erfolgten eher der Vollständigkeit halber und um möglicherweise erste allgemeine Ansätze zur

\footnotetext{
${ }^{1}$ Da dem Autor der genaue Glykol-Gehalt in Tyfocor ${ }^{\circledR}$ nicht bekannt war, wurde hier zwar die gleiche Konzentration wie bei Tyfocor LS, jedoch hier inklusive Additiven, hergestellt.
} 
Tabelle 5.1: Untersuchte Flüssigkeiten; Reinheitsangaben nach [Hillerns, 2013]

\begin{tabular}{|c|c|c|c|}
\hline Kurzbez. & Bezeichnung & Zusammensetzung & Hersteller \\
\hline W, $\mathrm{H} 2 \mathrm{O}$ & Wasser, Wasser als Komponente & $\begin{array}{l}\text { entionisiertes Leitungswasser mit } \\
0,055 . .0,06^{-} \mathrm{S} / \mathrm{cm} ; \\
\text { (TKA Smart2Pure 08.2060) }\end{array}$ & TU Dresden \\
\hline W, $\mathrm{H} 2 \mathrm{O}$ & Wasser in Wasser-Propylenglykol & VE-Wasser mit $5^{-} \mathrm{S} / \mathrm{cm}$ & Tyforop Chemie AG \\
\hline 100P, P, & Propylenglykol, Propandiol, P & 1,2-Propandiol, Reinheit > 99, $5 \%$ & Tyforop Chemie AG \\
\hline 100E, E & Ethylenglykol, Ethandiol, E & 1,2-Ethandiol, Reinheit > 99, $5 \%$ & Tyforop Chemie AG \\
\hline $25 \mathrm{P}, 41 \mathrm{P}, 75 \mathrm{P}$ & Wasser-Propylenglykol-Gemisch & $\begin{array}{l}25 ; 41,84 ; 75 \text { Gew.-\% } \\
\text { Propylenglykol-Anteil* }^{*}\end{array}$ & Tyforop Chemie AG \\
\hline $25 \mathrm{E}, 41 \mathrm{E}, 75 \mathrm{E}$ & Wasser-Ethylenglykol-Gemisch & $\begin{array}{l}25 ; 41,84 ; 75 \text { Gew.-\% } \\
\text { Ethylenglykol-Anteil* }\end{array}$ & TU Dresden \\
\hline LS & Tyfocor ${ }^{\circledR}$ LS & $\begin{array}{l}\text { Fertigmischung mit Inhibitoren mit } \\
41,84 \text { Gew.- } \% \text { Glykol-Anteil }\end{array}$ & Tyforop Chemie AG \\
\hline $\mathrm{T}$ & Tyfocor ${ }^{\circledR}$-Konzentrat & $\begin{array}{l}\text { Ethylenglykol-Konzentrat mit } \\
\text { Inhibitoren }\end{array}$ & Tyforop Chemie AG \\
\hline $41 \top$ & Tyfocor $^{\circledR}$-Wasser-Gemisch & $\begin{array}{l}\text { Fertigmischung mit 41,84 } \\
\text { Gew.-\%-Tyfocor }{ }^{\circledR} \text {-Anteil* }\end{array}$ & TU Dresden \\
\hline
\end{tabular}

*Unsicherheit des angegebenen Mischungsverhältnisses 0, $01 \%$

glykolgehaltsabhängigen Löslichkeit basierend auf den Reinstoff-Löslichkeiten zu generieren.

Versuche mit Synthetischer Luft beschränken sich aus Kapazitätsgründen auf die WasserGlykol-Gemische mit 41 Gewichtsprozent Glykol mit und ohne Additiven.

Alle Versuche wurden mit unbenutzten Gemischen oder Einzelkomponenten durchgeführt. Flüssigkeiten wurden auch in den Versuchen nicht wiederverwendet. Ursprünglich war auch geplant, mit gealterten Flüssigkeiten zu experimentieren, da mögliche Änderungen des Löslichkeitsverhaltens der Flüssigkeiten auch Auswirkungen auf den Langzeitbetrieb der Anlage hätten. Im Rahmen der Arbeit konnte jedoch keine repräsentativ gealterte Solarflüssigkeit in den für die Untersuchung benötigten Mengen zur Verfügung gestellt werden. Gealterte Solarflüssigkeit aus einer untersuchten Feldanlage zeigte einen deutlich zu hohen Wasseranteil ( $77 \mathrm{Gew} .-\%$ statt $57 \mathrm{Gew} .-\%$ ), gealterte Solarflüssigkeit aus einer eigenen Anlage deutlich zu hohe Eisenwerte, jedoch keine nennenswerte thermische Alterung.

Bezeichnung, Zusammensetzung, Herkunft und Reinheit der untersuchten Stoffe sind in Tabelle 5.1 zusammengestellt. Wasser-Propylenglykol-Gemische wurden bereits in den entsprechenden Mischungsverhältnissen geliefert. Wasser-Ethylenglykol-Gemische wurde selbst hergestellt aus Wasser und reinem Ethylenglykol (100E) bzw. TyfocorKonzentrat $(T)$.

Details zu den einzelnen durchgeführten Versuchen finden sich in Abbildungen 5.2 und 5.3. Die zu jedem Versuch zugeordnete Versuchskonfiguration hat sich entsprechend der Reihenfolge der durchgeführten Versuche ergeben. Details zu den Versuchskonfigurationen finden sich im Anhang A.4. Aus technischen Gründen sind dabei nicht alle Versuche eines Stoffes mit der gleichen Versuchskonfiguration durchgeführt worden. Die jeweils zugehörigen WasserReferenzversuche sind aber selbstverständlich jeweils mit der gleichen Versuchskonfiguration durchgeführt worden. Dabei wurde darauf geachtet, dass zwischen Versuch und Referenzversuch jeweils keine Umbauten/Reparaturen vorgenommen wurden, keine weiteren Versuche oder nur wenige gleichartige Versuche bei gleicher Temperatur (aber z. B. anderem Lösungs- 
mittel) stattfanden und zeitliche Abstände zwischen Versuch und Referenzversuch gering blieben.

Versuche fanden für Solltemperaturen von $10{ }^{\circ} \mathrm{C}$ bis $110^{\circ} \mathrm{C}$ im Raster von $10 \mathrm{~K}$ bzw. $15 \mathrm{~K}$ statt. Für Stickstoff-Versuche mit Ethylenglykol-Wasser-Gemischen und reinem Ethylenglykol sowie für die Luft-Versuche wurde das Temperaturraster aus Gründen der Zeitrationalität leicht reduziert. Die realen Temperaturen in den Versuchen weichen leicht von den Solltemperaturen ab (vgl. Begründung in Abschnitt 4.4.3.6).

Tabelle 5.2: Übersicht der durchgeführten Stickstoff-Versuche und Versuchskonfiguration A-D nach Anlage A.4

\begin{tabular}{l|l|llllllll} 
& \multirow{2}{*}{ Ausgangszustand } & \multicolumn{7}{|c}{ Thermattemperatur in ${ }^{\circ} \mathrm{C}$} \\
& & 10 & 20 & 35 & 50 & 65 & 80 & 95 & 110 \\
\hline LS2 & luftgesättigt + & A & A & A & A & A & A & A & (A) \\
& $\begin{array}{l}\text { 0,5 bar } \\
\text { luftgesättigt }\end{array}$ & A & A & B & B & A & A & A & A \\
$41 P$ & luftgesättigt & A & A & A & A & A & A & A & A \\
$75 P$ & luftgesättigt & B & B & C & B & C & B & C & C \\
$100 P$ & luftgesättigt & B & A & B & B & A & B & B & B \\
\hline $41 T$ & entgast & D & D & D & D & & D & & D \\
$25 E$ & entgast & D & D & D & D & & D & & D \\
$41 E$ & entgast & D & D & D & D & & D & & D \\
$75 E$ & entgast & D & D & D & D & & D & & D \\
$100 E$ & entgast & D & D & D & D & & D & & D \\
\hline
\end{tabular}

Tabelle 5.3: Übersicht der durchgeführten Luft-Versuche und Versuchkonfiguration nach Anlage A.4

\begin{tabular}{l|l|cccccccc} 
& \multirow{2}{*}{ Ausgangszustand } & \multicolumn{7}{|c}{ Thermostattemperatur in ${ }^{\circ} \mathrm{C}$} \\
& & 10 & 20 & 35 & 50 & 65 & 80 & 95 & 110 \\
\hline LS2 & entgast & D & D & D & D & & D & D & (D) \\
$41 \mathrm{P}$ & entgast & D & D & D & D & D & D & D \\
\hline $41 T$ & entgast & D & D & D & D & D & & D \\
$41 \mathrm{E}$ & entgast & D & D & D & D & D & & D \\
\hline
\end{tabular}

\subsection{Druckstufen, Gültigkeitsbereich des Löslichkeitskoeffizienten und kalibrierte Ergebnisse}

In Abbildung 5.2 und Abbildung 5.3 sind sich ergebende Löslichkeitskoeffizienten $\lambda_{\mathrm{N} 2, \infty}$ für verschiedenene Temperaturen exemplarisch für das Lösungsmittel Tyfocor ${ }^{\circledR}$ LS und zugehörige Wasser-Referenzversuche über dem Partialdruck von Stickstoff $p_{\mathrm{N} 2}$ dargestellt. ${ }^{2}$

Eine waagrechte Linie stellt jeweils den Löslichkeitskoeffizienten, ermittelt als Anstieg des Gasgehalts $\lambda_{\mathrm{N} 2, \infty}=\frac{\triangle w_{\mathrm{N} 2}}{\triangle p_{\mathrm{N} 2}^{*}}$ zwischen den beiden Druckpunkten $\left[p_{\mathrm{N} 2,1} ; p_{\mathrm{N} 2,2}\right]$ der Phasengleichgewichte eines Drucksprungs (vor und nach dem Druckanstieg), dar. Der erste Drucksprung (DS1) findet ungefähr von 1 bar auf 4 bar statt, der zweite Drucksprung (DS2) ungefähr von 4 bar auf 8 bar.

\footnotetext{
${ }^{2}$ Der in den Diagrammen dargestellte Partialdruck $p_{\mathrm{N} 2}=p-p_{\mathrm{H} 2 \mathrm{O}}-p_{\text {Rest }}$ wird, wie beschrieben, nicht direkt gemessen und kann aufgrund des bereits beschriebenen unsicheren Anteils $p_{\text {Rest }}$ nur abgeschätzt werden.
} 
Im Gültigkeitsbereich des Löslichkeitskoeffizienten sollten sich, wie in Abschnitt 4.4.1.2 beschrieben, bei konstanter Temperatur für beliebige Paare $\left[p_{N 2,1} ; p_{N 2,2}\right]$, also auch für die zwei untersuchten Drucksprünge, gleiche Werte ergeben, also

$$
\lambda_{\infty, \mathrm{DS} 1}(t) \approx \lambda_{\infty, \mathrm{DS} 2}(t) .
$$

Dies sollte hier also zumindest für Wasser gelten und wird in Abbildung 5.2 unter Berücksichtigung der Unsicherheit der Messergebnisse auch erreicht.

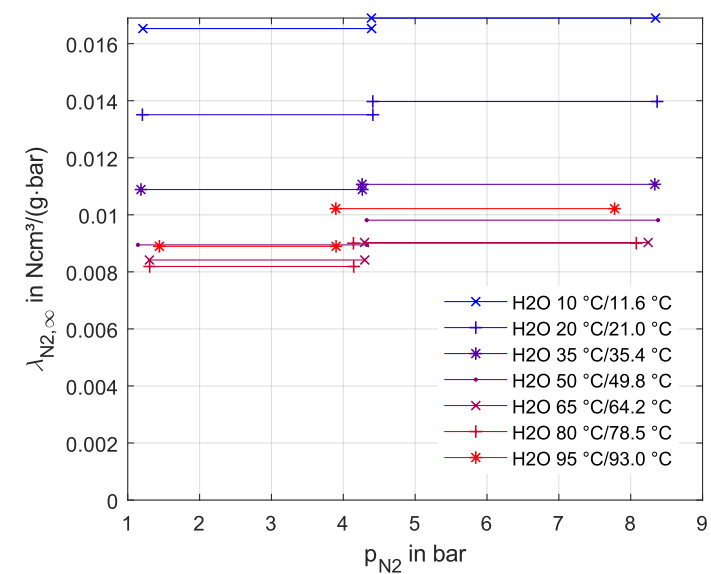

Abbildung 5.2: Löslichkeitskoeffizienten Druckstufen für $\mathrm{N}_{2}$ in Wasser: Soll-/Ist-Temperatur in Legende

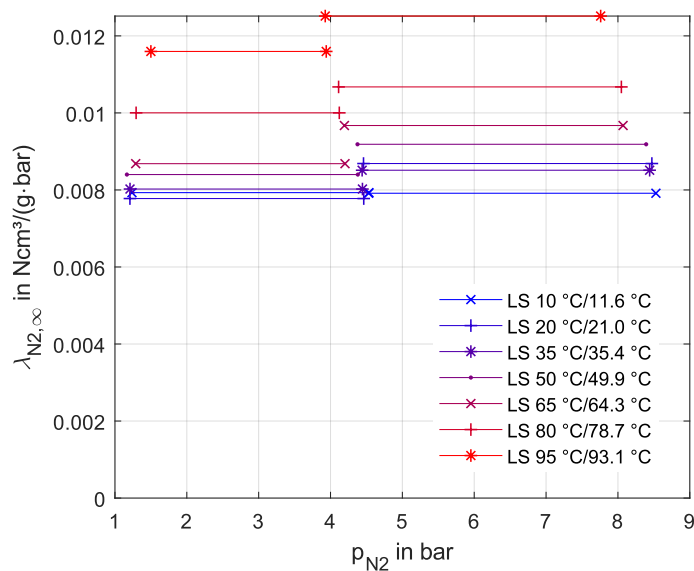

Abbildung 5.3: Löslichkeitskoeffizienten beider Druckstufen für $\mathrm{N}_{2}$ in Tyfocor $^{\circledR}$ LS (unkalibriert); Soll-/Ist-Temperatur in Legende

Wie zu sehen, konnten im Sinne der "Ähnlichkeit des Versuchsablaufs" in Versuch und Referenzversuch für die jeweilige Temperatur bezüglich des Druckes in etwa gleiche Druckstufen hergestellt werden. Bei höheren Temperaturen sind Anfangsdrücke des Phasengleichgewichts dabei aufgrund des höheren Dampfdrucks nach dem Temperieren etwas höher.

Der zweite Drucksprung $4 \rightarrow 8$ bar zeigt für alle Versuche tendenziell etwas höhere ermittelte Löslichkeitskoeffizienten im Vergleich zu den niedrigen Druckstufen. Die Ergebnis-Unterschiede zwischen den Druckstufen sind insbesondere für den jeweiligen Versuch und Referenzversuch relativ gleichmäßig. Ursachen dafür könnten z. B.

- die aufgrund höheren Druckniveaus höheren Leckageraten (werden als in Lösung gegangene Gasmengen interpretiert) und

- die in Betrag und Vorzeichen möglicherweise andere Messabweichung der Drucksensoren (P.G3 und P.R3) im höheren Druckbereich und damit andere Abweichung der Löslichkeitskoeffizienten

sein. 


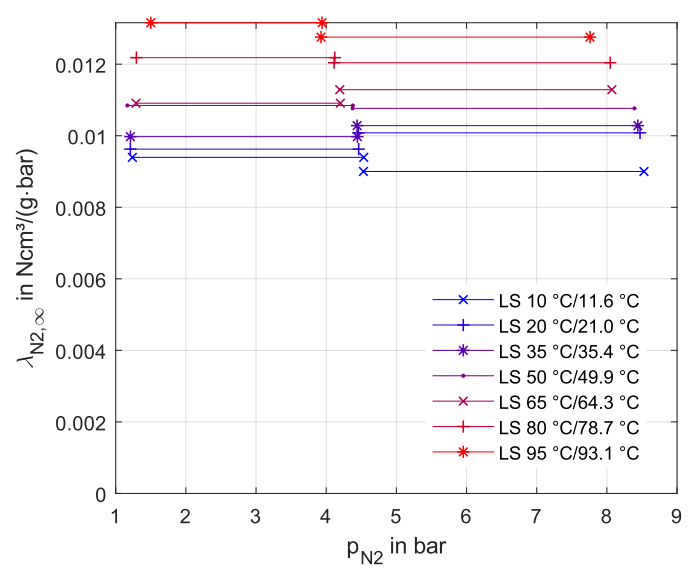

Abbildung 5.4: Löslichkeitskoeffizienten beider Druckstufen für kalibrierte Tyfocor ${ }^{\circledR}$ LS-Ergebnisse; Soll-/IstTemperatur in Legende

In Abbildung 5.4 sind nun die ermittelten Löslichkeitskoeffizienten für Gas $i=N_{2}$ in Tyfocor $^{\circledR}$ LS nach Kalibrierung unter Nutzung der Referenzversuche mit Wasser nach Gleichung 4.46 aufgetragen. Hier zeigt sich, dass die bestimmten Werte für die gemessenen Druckbereiche im Rahmen der Messunsicherheit sehr gute Übereinstimmung zeigen und damit als druckunabhängig bezeichnet werden können. Damit kann davon ausgegangen werden, dass man sich für den untersuchten Druckbereich im „Gültigkeitsbereich des Löslichkeitskoeffizienten" (zumindest nachweislich im Sinne technisch notwendiger Genauigkeit) befindet.

Weitere Diagramme der Druckstufen für alle durchgeführten Stickstoff-Versuchsreihen finden sich in Anhang A.13.1. Für jedes Lösungsmittel- $\mathrm{N}_{2}$-Gemisch wird zuerst in diesen Diagrammen geprüft, ob für die untersuchten Drucksprünge im Rahmen der Messgenauigkeit in etwa gleiche ermittelte Löslichkeitskoeffizienten $\lambda_{\infty}$ für die Druckbereiche gefunden werden konnten. Wenn dies der Fall ist, kann angenommen werden, dass keine Versuchsunregelmäßigkeiten vorliegen und man sich im „Gültigkeitsbereich des Löslichkeitskoeffizienten" befindet. Exakterweise kann erst dann von Henry-Koeffizienten bzw. technischen Löslichkeitskoeffizienten gesprochen werden. Für alle untersuchten Lösungsmittel war dies der Fall.

Für die weiteren Darstellungen der Löslichkeitskoeffizienten und Regressionskurven werden immer die Ergebnisse des ersten Drucksprungs verwendet. Diese werden aufgrund der geringeren Leckage als sicherer bewertet.

\subsection{Stickstoff-Löslichkeitskoeffizienten für $\operatorname{Tyfocor}^{\circledR}$ LS}

\subsubsection{Tyfocor $^{\circledR}$ LS und Sauerstoffbindung}

In Versuchen mit Tyfocor ${ }^{\circledR}$ LS mit dem Ausgangszustand „luftgesättigt" wurde erstmals am Versuchsstand beobachtet, dass sich kein konstanter Druck einstellt, sondern eine leichte Druckniveau-unabhängige aber temperaturabhängige Druckabnahme stattfindet, wie in Abbildung 5.5 zu sehen. Ursache ist die allmähliche chemische Bindung des Sauerstoffs, der bei „luftgesättigten“ Versuchen in größeren Mengen eingeschlossen ist. Diesem Effekt wird sich später in Abschnitt 5.5.3 noch ausführlich gewidmet. Zur Bestimmung der StickstoffLöslichkeit ist dieser Effekt störend. 


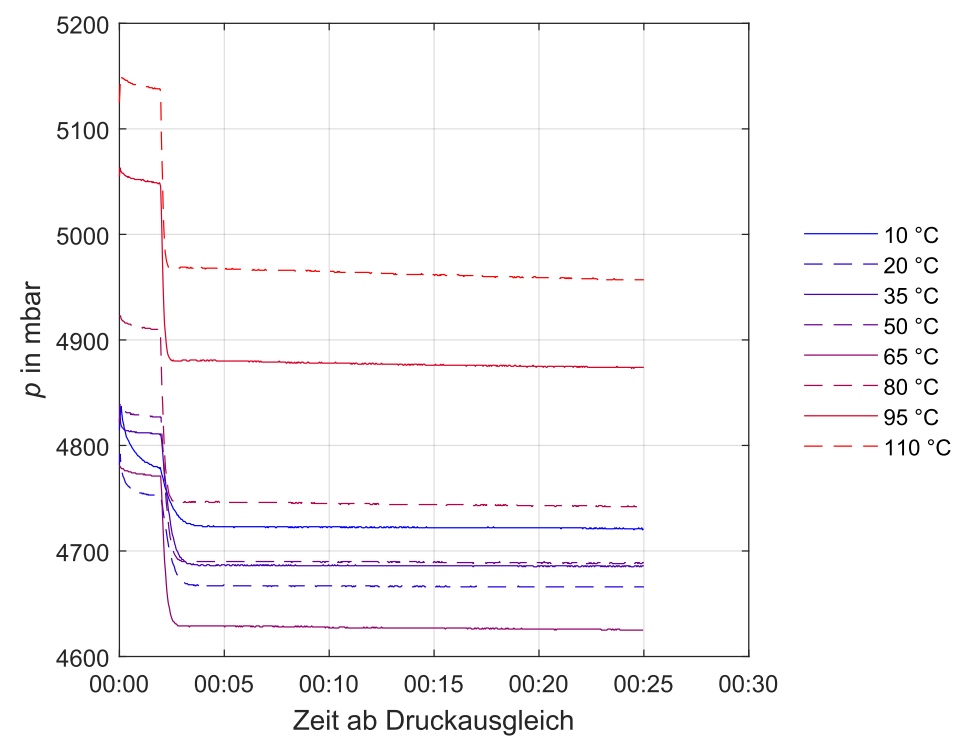

Abbildung 5.5: Druck im Reaktorgefäß nach Druckausgleich für verschiedene Temperaturen für Tyfocor ${ }^{\circledR}$ LS; Zeit in hh:mm

Um Auswirkungen auf die Genauigkeit der Messungen der Stickstoff-Löslichkeit gering zu halten, wurde der stationäre Zustand im Reaktorgefäß nach dem Druckausgleich abweichend vom Vorgehen bei anderen Flüssigkeiten so gewählt, dass

- die Absorption und Einstellung der stationären Temperaturen möglichst abgeschlossen ist,

- jedoch wenig Zeit seit dem Druckausgleich vergangen ist, um möglichst den Einfluss der $\mathrm{O}_{2}$-Bindung gering zu halten.

Für diesen Kompromiss wurde für die Stickstoff-Versuche mit Tyfocor ${ }^{\circledR}$ LS (und die zugehörigen Referenzversuche mit Wasser) der stationäre Zustand auf 5 min nach Einschalten des Rührers festgelegt. Für den $110{ }^{\circ} \mathrm{C}$-Versuch ist jedoch die ablaufende chemische Bindung zu stark, um sicher auf den Stickstoff-Löslichkeitskoeffizienten zu schließen. Daher wird dieser Versuch nicht weiter betrachtet. 


\subsubsection{Reduktion der Messunsicherheit durch Kalibrierung}

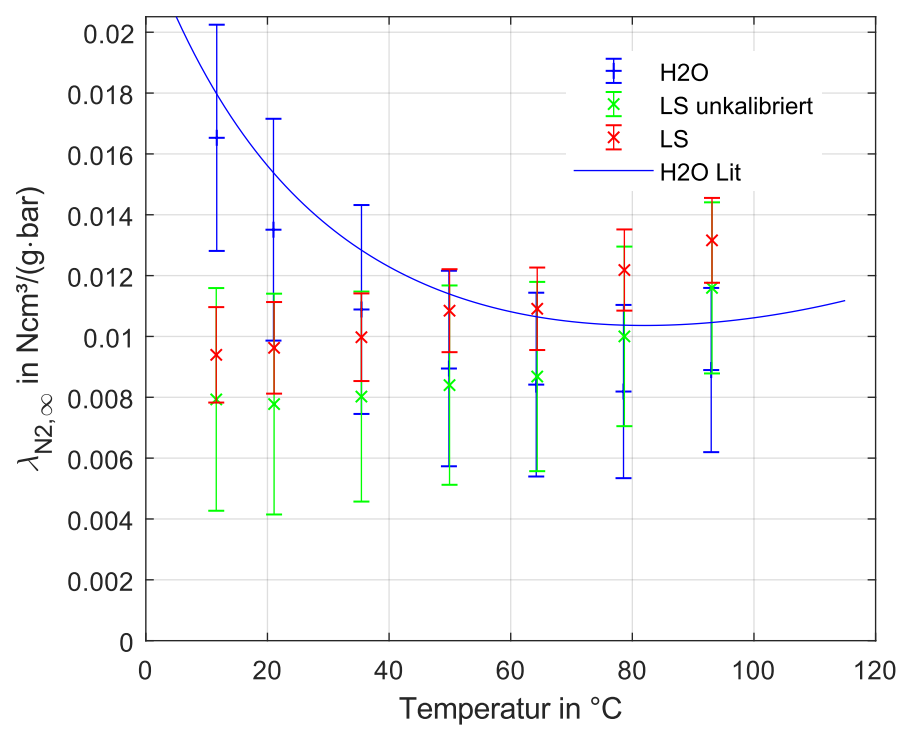

Abbildung 5.6: Unkalibrierte und kalibrierte Werte von Tyfocor $^{\circledR}$ LS

In Abbildung 5.6 sind nun die sich ergebenden Löslichkeitskoeffizienten für den 1. Drucksprung für Stickstoff in Wasser und Stickstoff in Tyfocor ${ }^{\circledR}$ LS

- für die unkalibrierten Werte sowie

- für die mit den Wasser-Versuchen kalibrierten Werte

eingezeichnet. Messunsicherheitsbereiche der unkalibrierten Werte sind ähnlich hoch wie für Wasser. Es ist grafisch zu erkennen, dass - um die kalibrierten Ergebnisse zu erhalten - die unkalibrierten Ergebnisse für Tyfocor ${ }^{\circledR}$ LS exakt um die Differenz $\left(\lambda_{i, \mathrm{H} 2 \mathrm{O}, \infty \text {, mess }}-\lambda_{i, \mathrm{H} 2 \mathrm{O}, \infty, \mathrm{Lit}}\right)$ nach oben verschoben wurden. Für die kalibrierten Werte ergeben sich damit deutlich geringere Messunsicherheitsbereiche. In den folgenden Ausführungen und Darstellungen werden, wenn nicht gesondert bezeichnet, nun nur noch die kalibrierten Messergebnisse verwendet.

\subsection{3 $\mathrm{N}_{2}$-Löslichkeitskoeffizienten für Tyfocor ${ }^{\circledR}$ LS}

Abbildung 5.7 zeigt nun die kalibrierten Messergebnisse für Tyfocor $^{\circledR}$ LS und die zugehörigen Referenzversuche mit Wasser jeweils mit zugehörigen Messunsicherheitsbudgets sowie eine ermittelte Regressionskurve für Tyfocor ${ }^{\circledR}$ LS-Versuche.

Es zeigt sich, dass Tyfocor $^{\circledR}$ LS ein grundsätzlich anderes Löslichkeitsverhalten als Wasser besitzt. ${ }^{3}$ Während für Wasser der Löslichkeitskoeffizient entsprechend der Regression nach [IAPWS, 2004] bis zum Wendepunkt bei ca. $80^{\circ} \mathrm{C}$ fällt und dann bis $110^{\circ} \mathrm{C}$ nur schwach ansteigt, zeigt der Löslichkeitskoeffzient von Tyfocor ${ }^{\circledR} \operatorname{LS} \lambda_{\mathrm{N} 2, \mathrm{LS} 2, \infty}(t)$ (im gesamten fokussierten Temperaturbereich) ein mit zunehmender Temperatur deutlich steigendes Verhalten. Der Löslichkeitskoeffizient von Tyfocor ${ }^{\circledR}$ LS ist bei Temperaturen von $10^{\circ} \mathrm{C}$ etwa nur halb

\footnotetext{
${ }^{3}$ Ergebnisse des jeweiligen Arbeitsstands wurden vom Autor u.a. in [Rühling u.a., 2011a], [Rühling u. a., 2011b], [Rühling u. a., 2012a], [Rühling u. a., 2013a] und [Rühling u. a., 2012b] veröffentlicht.
} 


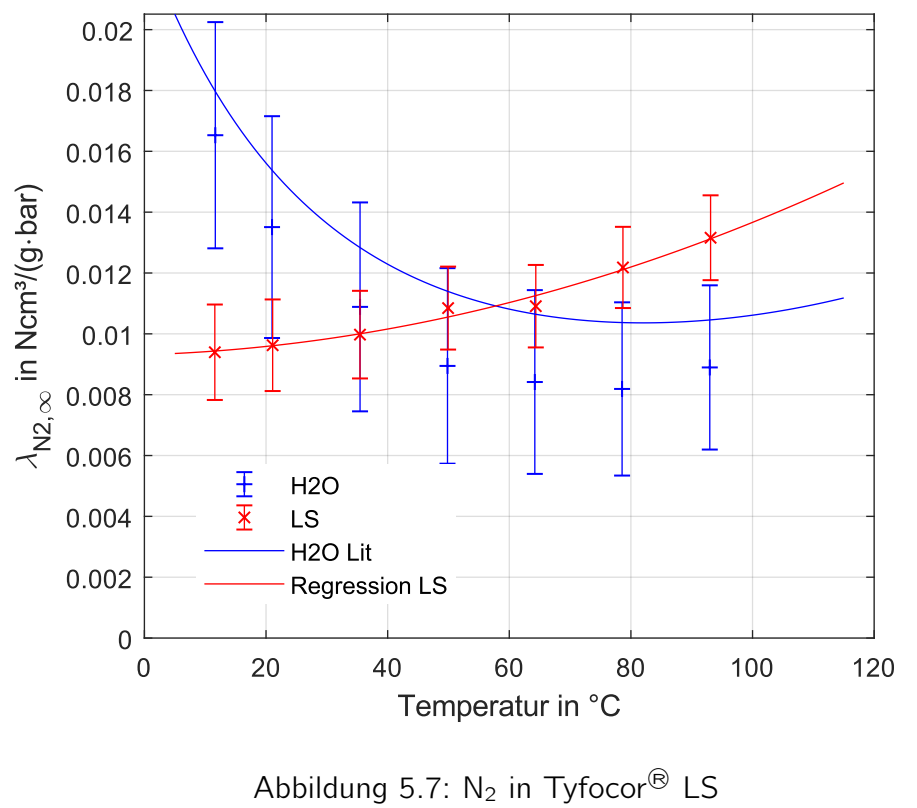

so groß wie der Löslichkeitskoeffizient von Wasser. Bei $55^{\circ} \mathrm{C}$ nehmen die Löslichkeitskoeffizienten in etwa gleiche Werte an. Bei hohen Temperaturen liegen Löslichkeitskoeffizienten von Tyfocor ${ }^{\circledR}$ LS signifikant über den Werten von Wasser.

Als Regressionsgleichung, die die Temperaturabhängigkeit des Löslichkeitskoeffizienten beschreibt, wird generell ein Polynom zweiten Grades nach Gleichung 5.2 verwendet:

$$
\lambda_{\infty}(t)=a_{0}+a_{1} \cdot t+a_{2} \cdot t^{2} .
$$

Koeffizienten für Tyfocor ${ }^{\circledR}$ LS finden sich in Tabelle 5.4. Diese Regression kann als Grundlage für weitergehende Untersuchungen $u$. a. von Solaranlagen im entsprechenden Temperaturbereich dienen und fand bereits Eingang in das Fachbuch „Thermohydraulische Dimensionierung von Solaranlagen" von [Eismann, 2017].

Tabelle 5.4: Regressionskoeffizienten für Löslichkeitskoeffizienten $N_{2}$ in Tyfocor ${ }^{\circledR}$ LS

\begin{tabular}{cccc}
\hline Medium & $\begin{array}{c}a_{0} \\
\mathrm{~cm}^{3} / \mathrm{g} \cdot \text { bar }\end{array}$ & $\begin{array}{c}a_{1} \\
\mathrm{~cm}^{3} / \mathrm{g} \cdot \mathrm{bar} \cdot{ }^{\circ} \mathrm{C}\end{array}$ & $\begin{array}{c}\mathrm{a}_{2} \\
\mathrm{~cm}^{3} / \mathrm{g} \cdot \mathrm{bar} \cdot{ }^{\circ} \mathrm{C}^{2}\end{array}$ \\
\hline Tyfocor ${ }^{\circledR}$ LS & 0,0093 & $6,2 \cdot 10^{-6}$ & $3,7 \cdot 10^{-7}$ \\
\hline
\end{tabular}

\subsection{Stickstoff-Löslichkeitskoeffizienten für Wasser-Glykol-Gemische}

\subsubsection{Temperaturabhängige Messdaten}

Analog zum oben beschriebenen Ablauf für Tyfocor ${ }^{\circledR}$ LS erfolgten weitere Versuche für andere Stoffgemische und zugehörige Auswertungen. Im Gegensatz zu Tyfocor ${ }^{\circledR}$ LS zeigten jedoch alle anderen Stoffe im untersuchten Temperatur- und Druckbereich keine chemische Sauerstoff-Bindung, so dass hier kein Kompromiss zwischen vollständiger Absorption und dem 


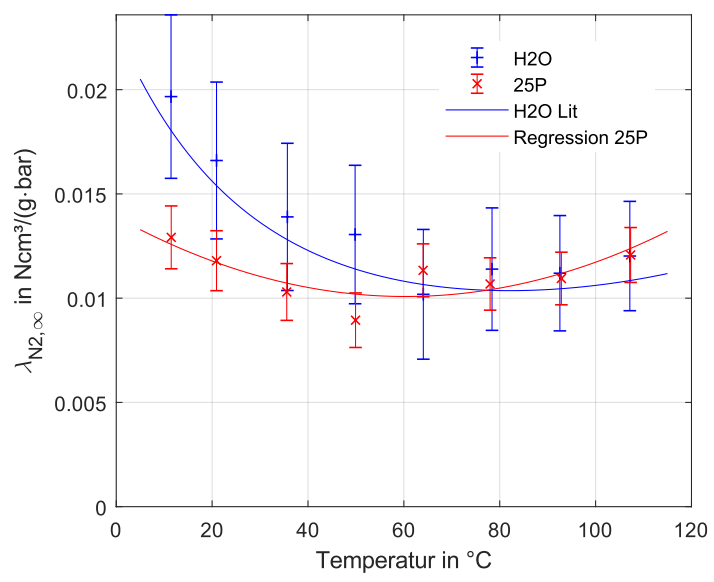

Abbildung 5.8: $\mathrm{N}_{2}$-Löslichkeitskoeffzienten für Wasser und $25 \mathrm{Gew} .-\%$ WasserPropylenglykol-Gemisch (25P)

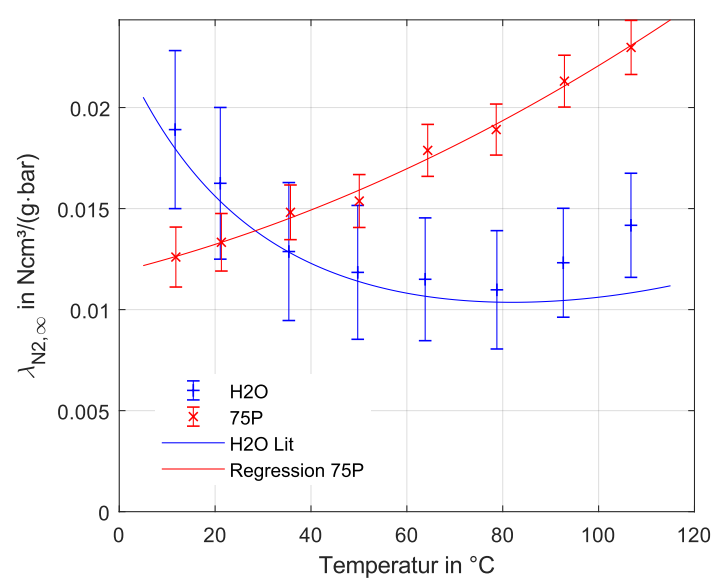

Abbildung 5.10: $\mathrm{N}_{2}$-Löslichkeitskoeffizienten für Wasser und $75 \mathrm{Gew} .-\%$ WasserPropylenglykol-Gemisch (75P)

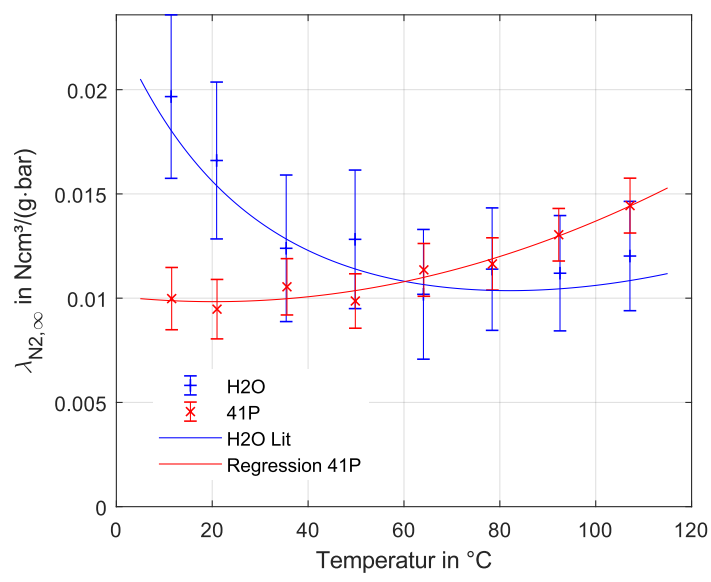

Abbildung 5.9: $\mathrm{N}_{2}$-Löslichkeitskoeffizienten für Wasser und 41,84 Gew.-\% WasserPropylenglykol-Gemisch

(41P)

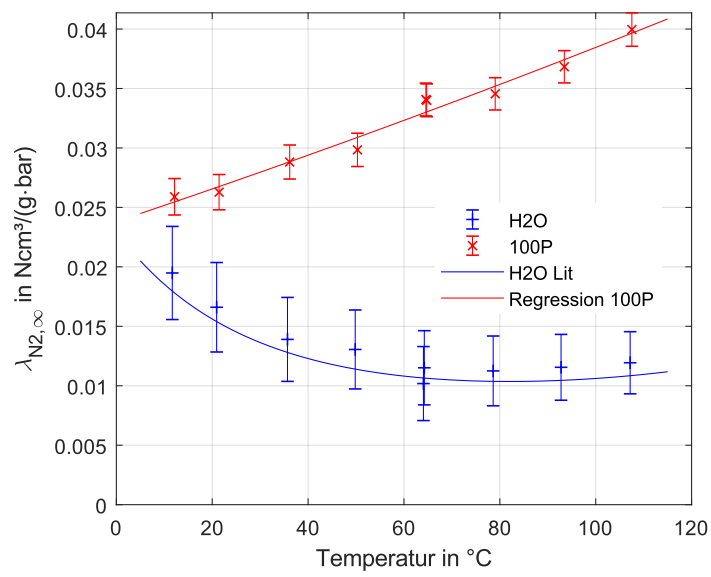

Abbildung 5.11: $\mathrm{N}_{2}$-Löslichkeitskoeffizienten für Wasser und $100 \mathrm{Gew} .-\%$ Propylenglykol (100P)

Einstellen eines stationären Zustands einerseits und einem möglichst kurz gehaltenen Zeitraum nach dem Druckausgleich zwecks geringer $\mathrm{O}_{2}$-Bindung andererseits gefunden werden musste. Somit konnten ausreichend lange Wartezeiten für eine sichere vollständige Absorption und für Stationärität eingestellt werden.

Die entsprechenden Ergebnisse finden sich für die untersuchten Propylenglykol-WasserGemische und für Propylenglykol in den Abbildungen 5.8 bis 5.11 sowie für die untersuchten Ethlyenglykol-Wasser-Gemische und Ethylenglykol in den Abbildungen 5.12 bis $5.15 .{ }^{4}$ Die Diagramme für die Druckstufen finden sich in Anhang A.13.1. Diese zeigen annähnernd gleiche Werte für die zwei untersuchten Druckstufen, so dass auch hier von annähernd druckunabhängigen Löslichkeitskoeffizienten im untersuchten Druckbereich ausgegangen werden kann.

In den Abbildungen 5.16 bzw. 5.17 sind die Ergebnisse für alle Propylenglykol- bzw. Ethylenglykol-basierten Gemische zusammengefasst. Während Wasser ein Minimum der Lös-

\footnotetext{
${ }^{4}$ Ergebnisse des jeweiligen Bearbeitungsstands wurden vom Autor bereits in [Rühling u. a., 2013a] und [Heymann u. a., 2014] veröffentlicht.
} 


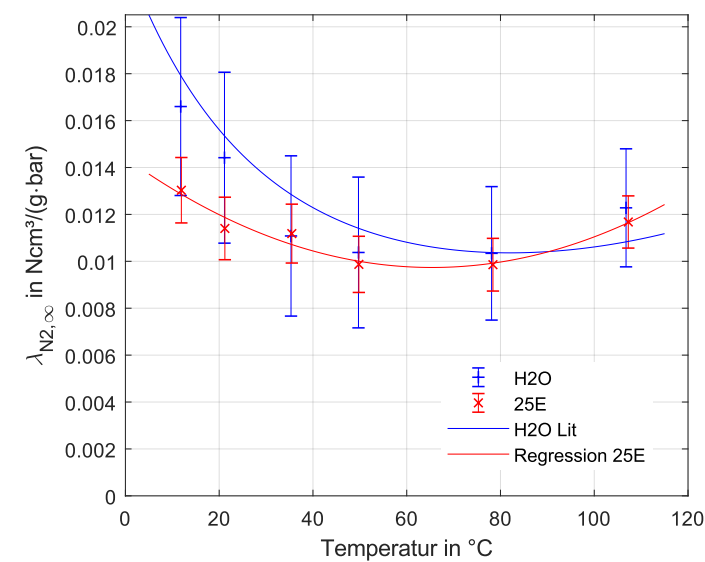

Abbildung 5.12: $\mathrm{N}_{2}$-Löslichkeitskoeffzienten für Wasser und $25 \mathrm{Gew} .-\%$ WasserEthylenglykol-Gemisch (25E)

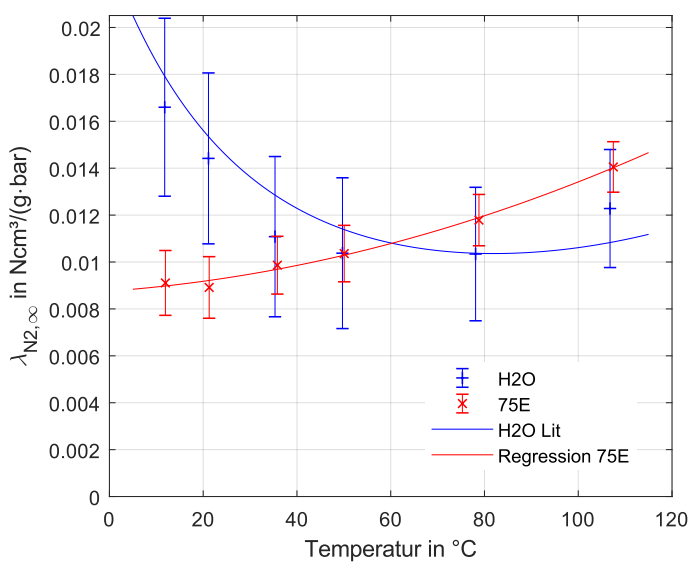

Abbildung 5.14: $\mathrm{N}_{2}$-Löslichkeitskoeffizienten für Wasser und $75 \mathrm{Gew} \%$ WasserEthylenglykol-Gemisch (75E)

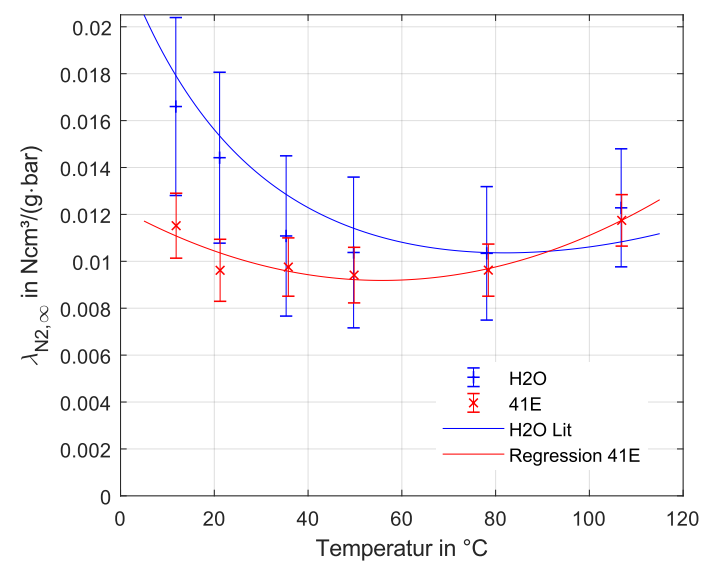

Abbildung 5.13: $N_{2}$-Löslichkeitskoeffizienten für Wasser und 41,84 Gew.\% WasserEthylenglykol-Gemisch

(41E)

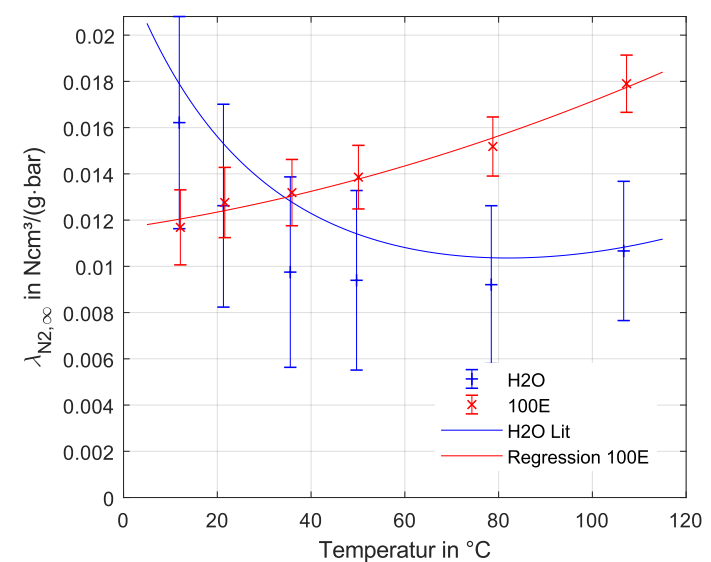

Abbildung 5.15: $\mathrm{N}_{2}$-Löslichkeitskoeffizienten für Wasser und $100 \mathrm{Gew} .-\%$ Ethylenglykol (100E)

lichkeit zeigt und diese bis dahin mit steigender Temperatur stark sinkt, zeigen beide reinen Glykole mit zunehmender Temperatur steigende Löslichkeitskoeffizienten. Für reines Propylenglykol werden mit ca. 0,025...0,04 $\frac{\mathrm{cm}^{3}}{\mathrm{~g} \cdot \mathrm{bar}}$ deutlich höhere Werte als bei den untersuchten Wasser-Glykol-Gemischen, aber auch deutlich höhere Werte als bei reinem Ethylenglykol (100E), das Werte im Bereich 0,012 ..0,018 $\frac{\mathrm{cm}^{3}}{\mathrm{~g} \cdot \mathrm{bar}}$ erreicht, gemessen. Die Löslichkeit in 100P liegt mit ca. $50 \%$ höheren Werten bei $10{ }^{\circ} \mathrm{C}$ und ca. $300 \%$ höheren Werten bei $110^{\circ} \mathrm{C}$ auch deutlich über der Löslichkeit von Wasser.

In Mischungen steigt mit zunehmendem Glykolanteil die Abweichung des temperaturabhängigen Verlaufs der Löslichkeitskoeffizienten von der Wasser-Charakteristik. Eine Abweichung gegenüber Wasser ist bereits bei 25 Gew.-\% Glykolanteil deutlich erkennbar. Für geringe Temperaturen sinkt die Löslichkeit deutlich gegenüber Wasser, für Temperaturen über $90^{\circ} \mathrm{C}$ wird hingegen die Löslichkeit von Wasser überschritten. Löslichkeitskoeffizienten sind sowohl für 25P als auch 25E im untersuchten Temperaturbereich noch nicht mit zunehmender Temperatur stetig steigend, sondern es ist ein Minimum der Löslichkeit bei ca. $55-65^{\circ} \mathrm{C}$ erkennbar. Für Propylenglykol-Wasser-Gemische tritt ein stetiger Anstieg bei 41,48 Gew.-\% Glykol auf, bei Ethylenglykol-Wassergemischen erst bei $75 \mathrm{Gew} .-\%$. 


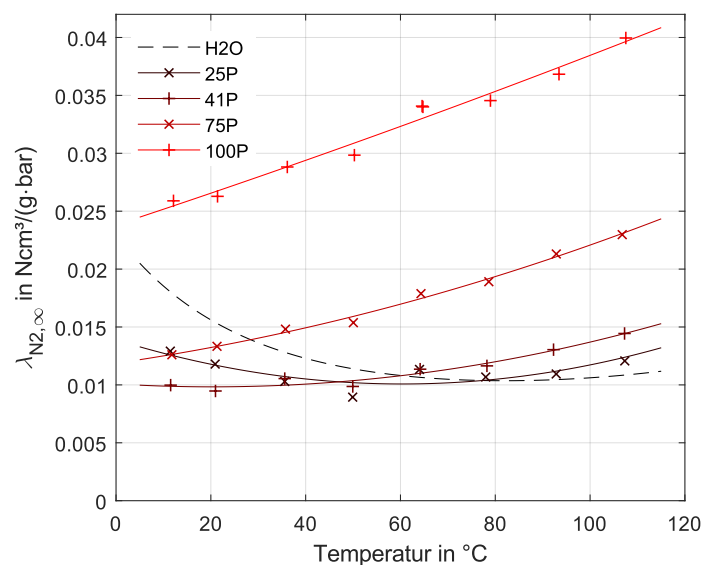

Abbildung 5.16: $\mathrm{N}_{2}$-Löslichkeitskoeffizienten Wasser-Propylenglykol-Gemisch 25 Gew. \% (25P), 41,84 Gew.\% (41P), 75 Gew. $\%$ (75P) und $100 \%(100 \mathrm{P})$

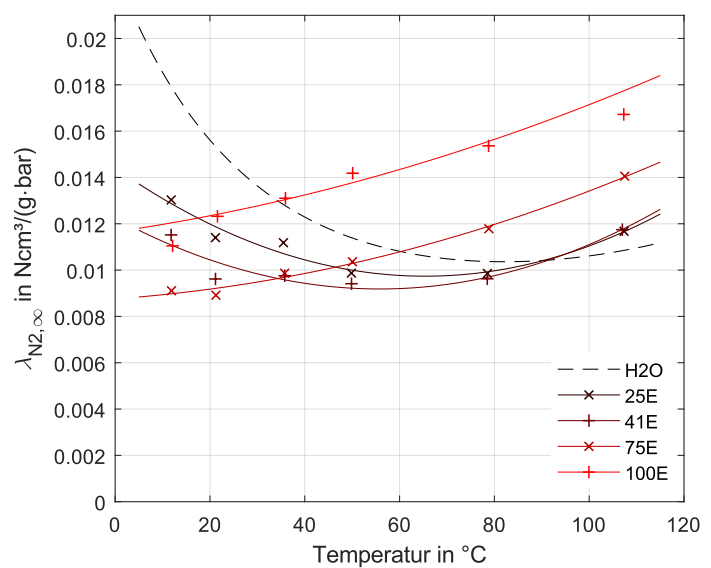

Abbildung 5.17: $\mathrm{N}_{2}$-Löslichkeitskoeffizienten Wasser-Ethylenglykol-Gemisch 25 Gew. \% (25E), 41,84 Gew.\% (41E), 75 Gew- $\%$ (75E) und $100 \%$ $(100 \mathrm{P})$

Vergleicht man Wasser-Referenzversuche (unterschiedlicher Lösungsmittel), die bei gleicher Temperatur durchgeführt worden sind, ist festzustellen, dass einige Referenzversuche trotz gleicher Temperatur deutlich unterschiedliche Ergebnisse liefern. Hier seien exemplarisch einige Gründe kurz genannt:

- Änderungen im Versuchsablauf bspw. bezüglich Ausgangszustand, Trocknungsprozess.

- Änderungen der Versuchskonfiguration.

- Messstellendrift über die lange Nutzungszeit des Versuchsstands (mehrere Jahre) führen zu anderen Ergebnissen.

- Austausch der im Reaktorraum liegenden Gleitlager des Rührers führen zu leicht verändertem Volumen des Inhalts.

- Je nach versuchsdurchführender Person trotz Drehmomentschlüssel mögliches subjektiv unterschiedliches Anziehen der Schrauben, die den Pressdruck auf die Dichtung bestimmen (0,01 mm Unterschied entsprechen ca. 0,1 ml Volumenänderung). Das Anziehen ist ebenso abhängig vom Zustand der Gewindeschmierung.

- Der Versuchsstand zeigt starke Sensibiliät gegenüber der Dämmung. Anbringung und Alterung der Dämmung führt möglicherweise zu unterschiedlichem Temperaturfeld im Reaktor.

- Versuchsvorbereitungszeit und damit potentielle Zeit von Lösungsmittelverlust durch Verdunstung hängt stark von der Routiniertheit des jeweiligen Durchführenden ab.

- Exaktheit des eingestellten Vakuums trotz festgelegter Kriterien je nach Durchführendem eventuell unterschiedlich.

Es wurde intensiv darauf geachtet, dass sowohl Versuch als auch Referenzversuch so gleichartig wie möglich stattfanden (gleicher Durchführender, geringe Zeiträume zwischen Versuch und Referenzversuch, gleiche Versuchskonfiguration, gleicher Versuchsablauf, gleiche Füllvolumina etc.). Da wirklich zu jedem Versuch ein gleichartiger Referenzversuch existiert, hat hier die Methode der Kalibrierung der Versuche durch die Referenzversuche den Charme, dass genannte, schwer einzugrenzende Messunsicherheitsbeiträge bei den kalibrierten Versuchen entsprechend rausfallen oder zumindest stark reduziert werden. 
In Tabelle 5.4 sind die in den vorigen Abbildungen dargestellten Regressionskurven der GlykolWasser-Gemische zusammengestellt. Mit diesen kann erstmals die Löslichkeit für WasserGlykol-Gemische für beliebige Temperaturen im untersuchten Temperaturbereich ermittelt werden.

Tabelle 5.5: Regressionskoeffizienten für Löslichkeitskoeffizienten $\mathrm{N}_{2}$ in Wasser-Glykol-Mischungen

\begin{tabular}{rrrrrr}
\hline Medium & $\begin{array}{r}\xi_{\text {Glykol }} \\
-\end{array}$ & $\begin{array}{r}a_{0} \\
\mathrm{~cm}^{3} / \mathrm{g} \cdot \mathrm{bar}\end{array}$ & $\mathrm{cm}^{3} / \mathrm{g} \cdot \mathrm{bar} \cdot{ }^{\circ} \mathrm{C}$ & $\mathrm{cm}^{3} / \mathrm{g} \cdot \mathrm{bar}^{\circ}{ }^{\circ} \mathrm{C}^{2}$ \\
\hline Propylenglykol-Wasser & 0,25 & 0,0139 & $-1,27 \cdot 10^{-4}$ & $1,04 \cdot 10^{-6}$ \\
Propylenglykol-Wasser & 0,4184 & 0,0101 & $-2,5 \cdot 10^{-5}$ & $6,1 \cdot 10^{-7}$ \\
Propylenglykol-Wasser & 0,75 & 0,0119 & $6,0 \cdot 10^{-5}$ & $4,3 \cdot 10^{-7}$ \\
Propylenglykol & 1 & 0,0238 & $1,35 \cdot 10^{-4}$ & $1,1 \cdot 10^{-7}$ \\
\hline Ethylenglykol-Wasser & 0,25 & 0,0144 & $-14,3 \cdot 10^{-5}$ & $1,09 \cdot 10^{-6}$ \\
Ethylenglykol-Wasser & 0,4184 & 0,0122 & $-11,0 \cdot 10^{-5}$ & $9,8 \cdot 10^{-7}$ \\
Ethylenglykol-Wasser & 0,75 & 0,0088 & $1,5 \cdot 10^{-5}$ & $3,2 \cdot 10^{-7}$ \\
Ethylenglykol & 1 & 0,0116 & $3,0 \cdot 10^{-5}$ & $2,5 \cdot 10^{-7}$ \\
\hline
\end{tabular}

\subsubsection{Abhängigkeit vom Glykolanteil und Vergleich mit Literaturwerten}

Im Folgenden soll nun noch die Abhängigkeit der Löslichkeitskoeffizienten vom Glykolanteil diskutiert werden.
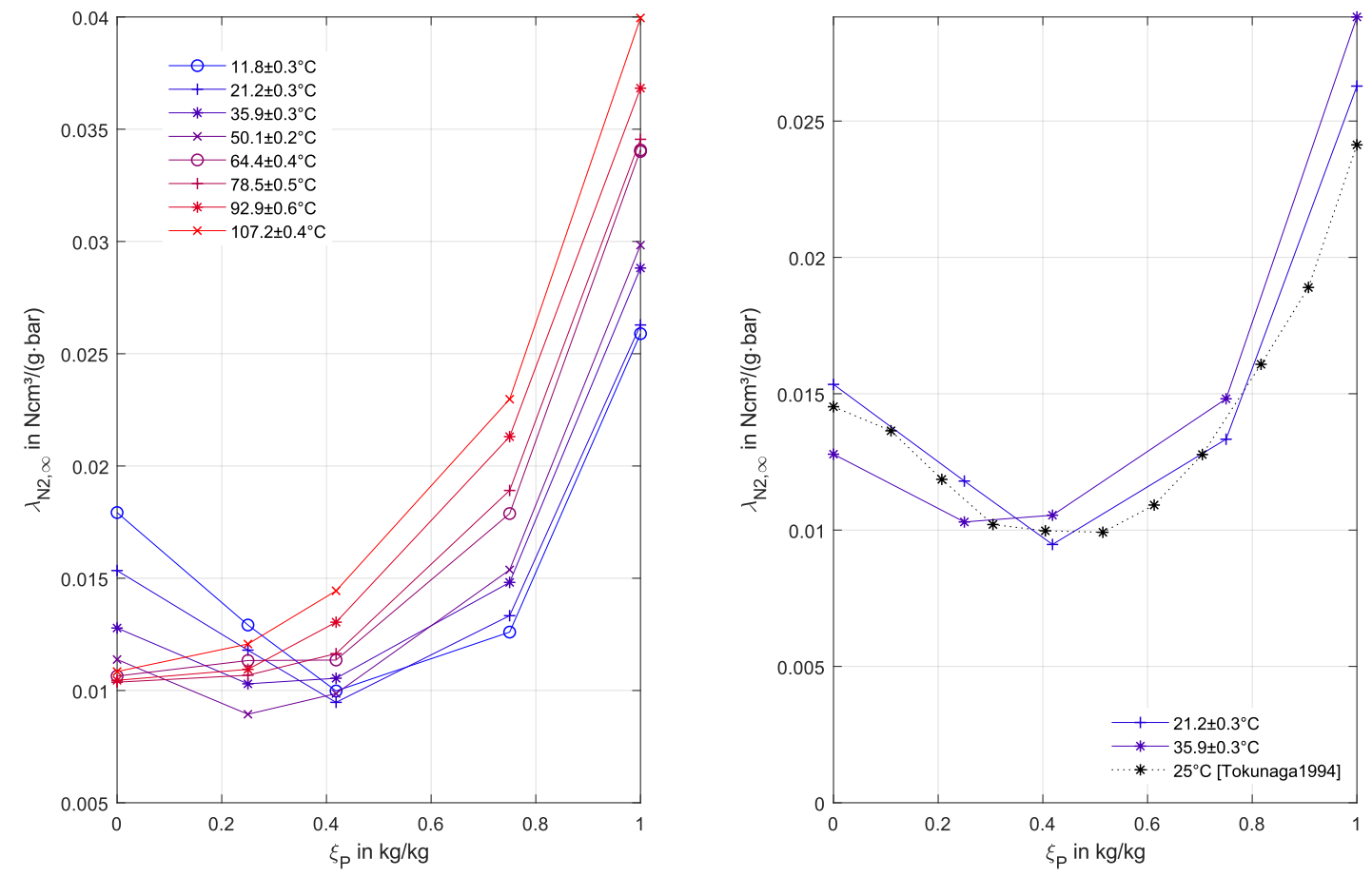

Abbildung 5.18: Konzentrationsabhängige $\mathrm{N}_{2}$-Löslichkeit in Wasser-Propylenglykol-Mischungen; links: gesamter untersuchter Temperaturbereich; rechts: Vergleich mit Literaturwerten von $25^{\circ} \mathrm{C}$ 

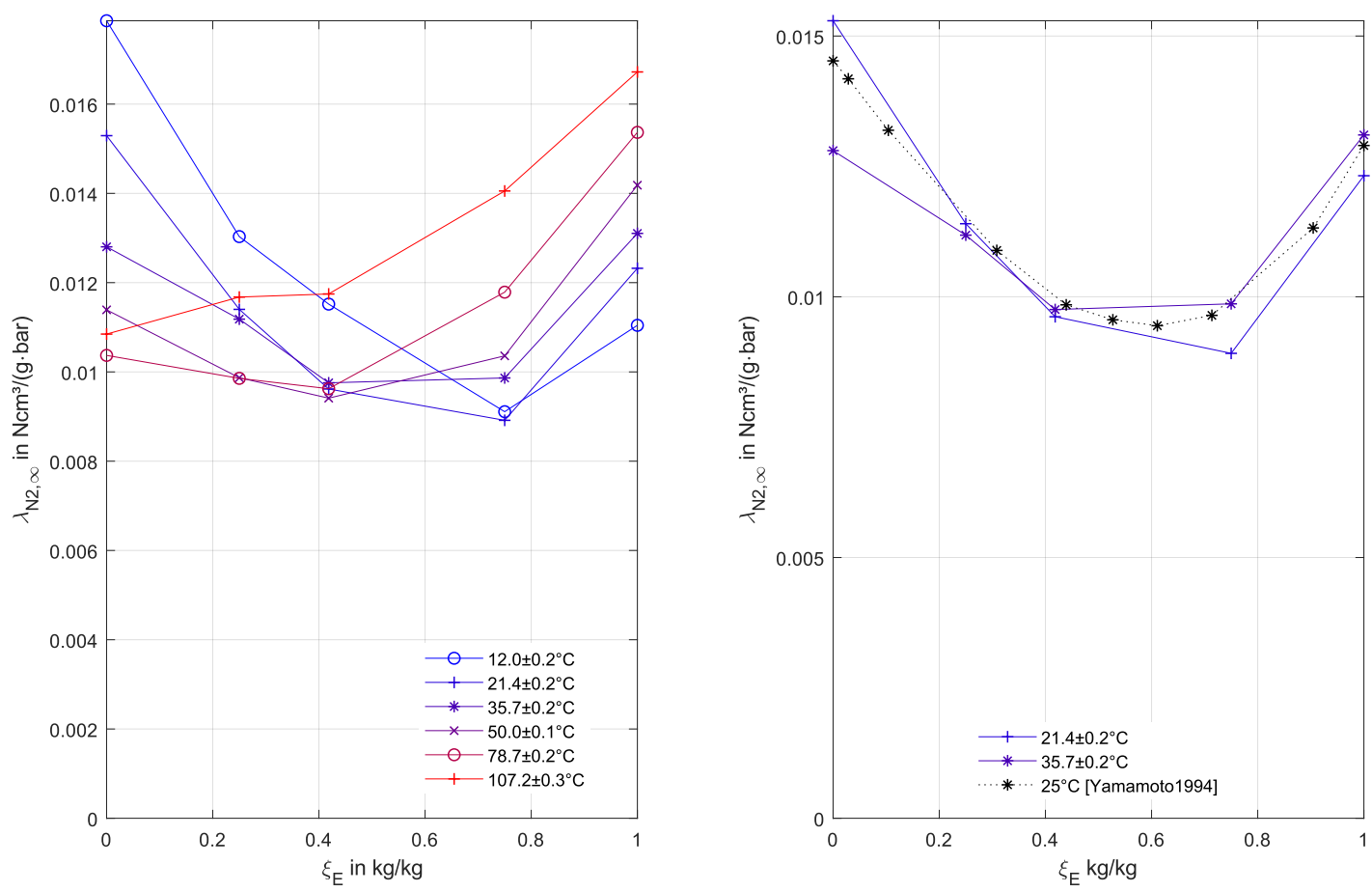

Abbildung 5.19: Konzentrationsabhängige $\mathrm{N}_{2}$-Löslichkeit in Wasser-Ethylenglykol-Mischungen; links: gesamter untersuchter Temperaturbereich; rechts: Vergleich mit Literaturwerten von $25^{\circ} \mathrm{C}$

Abbildung 5.18 zeigt die gemessenen Löslichkeitskoeffizienten der Propylenglykol-WasserGemische in Abhängigkeit des Glykolanteils für die unterschiedlichen Temperaturniveaus der Versuche. In der Legende ist dabei immer der Bereich der in den Versuchen real gemessenen Fluidtemperatur dargestellt und um den Wert von Wasser (0\% Glykolanteil) nach [IAPWS, 2004] ergänzt.

Für Temperaturen von $50^{\circ} \mathrm{C}$ und darunter fällt auf, dass die Löslichkeit der untersuchten Gemische sogar unter der Löslichkeit der einzelnen Komponenten Wasser und Glykol liegt. Das Minimum der Löslichkeit befindet sich für $25^{\circ} \mathrm{C}$ laut [Yamamoto u. a., 1994] etwa zwischen $40 \mathrm{Gew} .-\%$ und $50 \mathrm{Gew} .-\%$ und damit genau im Bereich üblicher Solarflüssigkeiten. Für Temperaturen von $64,4^{\circ} \mathrm{C}$ steigt die Löslichkeit mit zunehmendem Glykolanteil stetig an. Wie bereits in [Yamamoto u. a., 1994] beschrieben, zeigen auch die Messwerte von 25 Gew.-\% Glykol und $41.84 \mathrm{Gew} . \%$ Glykol, dass eine lineare Interpolation der Löslichkeit zwischen den Reinstoffen $\xi_{\mathrm{Glykol}}=0$ (Wasser) und $\xi_{\mathrm{Glykol}}=1$ (reines Glykol) nicht zielführend ist (siehe Abbildung 5.18).

Die Messergebnisse zeigen allgemein gute Übereinstimmung mit den von [Yamamoto u. a., 1994] bei $25^{\circ} \mathrm{C}$ ermittelten Werten (Abbildung 5.18 rechts).

Die Ergebnisse für Ethylenglykol-Wasser-Gemische sind in Abbildung 5.19 dargestellt. Auch hier werden für kältere Temperaturen Löslichkeitskoeffizienten erreicht, die signifikant niedriger als die der Reinstoffe sind. Die Spannweite der Löslichkeitskoeffizienten ist hier deutlich geringer als bei Propylenglykol-Wasser-Gemischen. 


\subsubsection{Wirkung von Additiven in Wasser-Glykol-Gemischen}

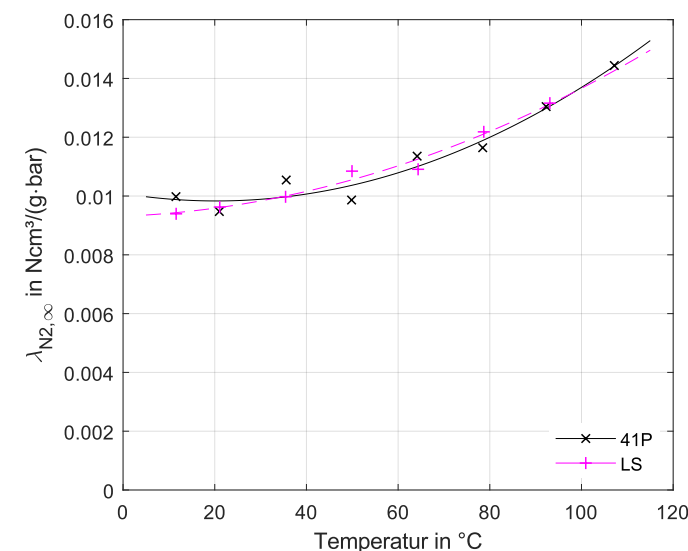

Abbildung 5.20: $\mathrm{N}_{2}$-Löslichkeitskoeffizienten für $\quad 41,84 \mathrm{Gew} .-\% \quad$ WasserPropylenglykol (41P) und konzentrationsgleiche Fertigmischung Tyfocor $^{\circledR}$ LS (LS)

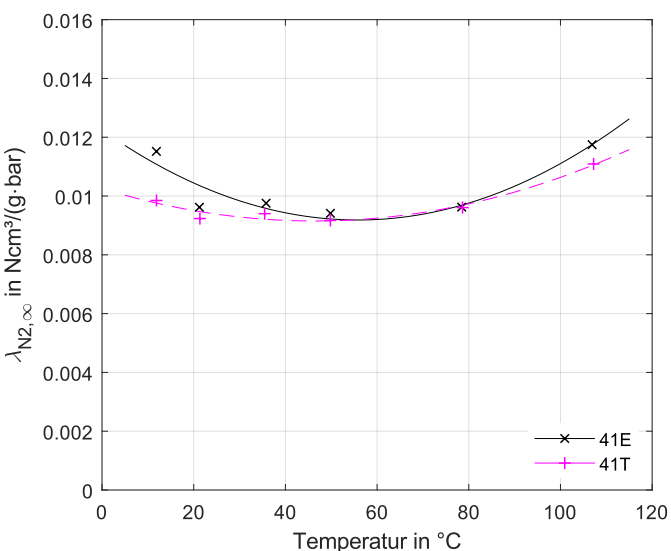

Abbildung 5.21: $\mathrm{N}_{2}$-Löslichkeitskoeffizienten für 41,84 Gew.-\% Wasser-Ethylenglykol (41E) und konzentrationsgleiche Fertigmischung Tyfocor ${ }^{\circledR}$ (41T)

Um den Einfluss der Additive in Tyfocor ${ }^{\circledR}$ LS und Tyfocor ${ }^{\circledR}$ auf die Löslichkeit zu untersuchen, werden die experimentell bestimmten Löslichkeitskoeffzienten mit denen der reinen Wasser-Glykol-Mischung mit gleichem Propylenglykol- bzw. Ethylenglykolanteil in Abbildung 5.21 bzw. Abbildung 5.20 verglichen. ${ }^{5}$ Die Messwerte zeigen nur geringe Abweichungen, die im Rahmen der Messunsicherheit liegen. Der Einfluss der Additive auf die Löslichkeit von Stickstoff kann daher als gering eingeschätzt werden ${ }^{6}$.

Es kann daher für technische Berechnungen bezüglich des Gases Stickstoff mit genügender Genauigkeit davon ausgegangen werden, dass für Fertigmischungen mit Additiven auf Propylenglykol- und Ethylenglykol-Basis mit unterschiedlichen Inhibierungsbestandteilen die Löslichkeitskurven des reinen Glykol-Wassergemisches für Analysen zum Löslichkeitsverhalten genutzt werden können. Dies kann vermutlich auch auf andere Glykol-WasserKonzentrationsverhältnisse übertragen werden.

${ }^{5}$ Erste Ergebnisse für Tyfocor $^{\circledR}$ LS wurden vom Autor auch in [Rühling u. a., 2013a] und [Heymann u. a., 2014] veröffentlicht.

${ }^{6} \mathrm{Ob}$ der deutliche Unterschied beim $10^{\circ} \mathrm{C}$-Wert für $41 \mathrm{~T}$ und $41 \mathrm{E}$ ein realer Unterschied ist oder eher auf Messabweichungen zurückzuführen ist, konnte im Rahmen der durchgeführten Messreihen nicht ermittelt werden. 


\subsection{Untersuchungen mit Luft}

\subsubsection{Stickstoff-Löslichkeitskoeffizienten im Vergleich mit Ergebnissen aus Stickstoff-Versuchen}

Aus den Luftversuchen lassen sich auch die Löslichkeiten von Stickstoff bestimmen. Dazu sind in Abbildung 5.22 und Abbildung 5.23 die Ergebnisse der Messungen dargestellt. Eine erneute Regressionskurve wurde nicht bestimmt. Hier ist zum Vergleich die bereits bestimmte Regressionskurve für die Stickstoff-Versuche des jeweiligen Stoffgemisches eingezeichnet. Es zeigt sich eine sehr gute Übereinstimmung mit den Ergebnissen der Stickstoff-Versuche. Relevante Quereinflüsse von Sauerstoff auf die Löslichkeit von Stickstoff können hier demnach nicht beobachtet werden.

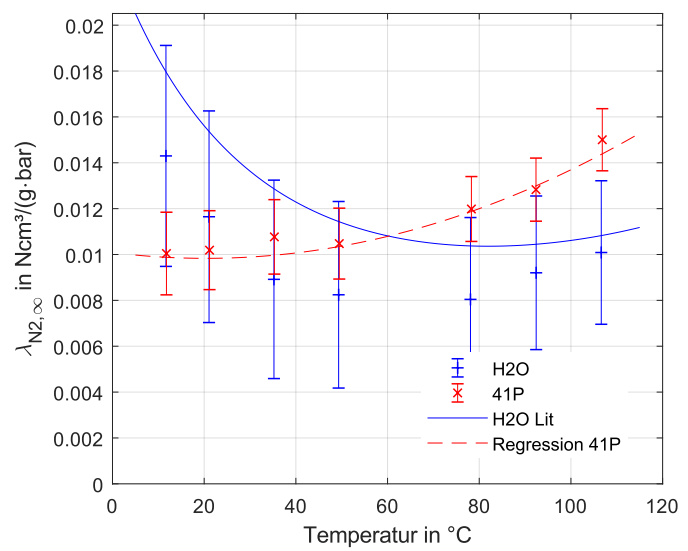

Abbildung 5.22: $\mathrm{N}_{2}$-Löslichkeitskoeffzienten aus LuftVersuchen für 41P und Wasser; Regressionspolynom 41P aus $\mathrm{N}_{2}$ Versuchen

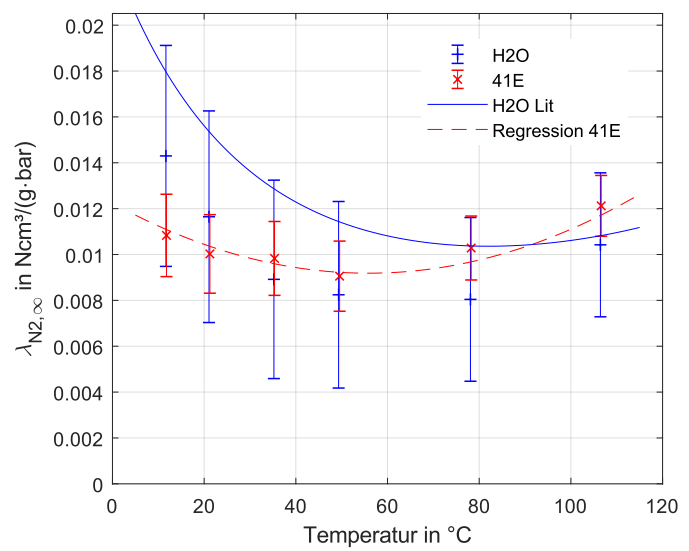

Abbildung 5.23: $\mathrm{N}_{2}$-Löslichkeitskoeffizienten aus Luft-Versuchen für $41 \mathrm{E}$ und Wasser; Regressionspolynom 41E aus $\mathrm{N}_{2}$-Versuchen

Luft-Versuche zeigen für Wasser als auch Wasser-Glykol, wie in Abschnitt 4.9 ausführlich dargelegt, eine etwas höhere ermittelte Messunsicherheit als bei den StickstoffVersuchen.

Auch für die Fertigmischungen Tyfocor ${ }^{\circledR}$ LS (Abbildung 5.24) und Tyfocor ${ }^{\circledR}$ (Abbildung 5.25) zeigt sich eine gute Übereinstimmung mit den bereits in den Stickstoff-Versuchen bestimmten Löslichkeitskoeffizienten. Lediglich für hohe Temperaturen von Tyfocor ${ }^{\circledR}$ LS zeigen sich etwas erhöhte errechnete Stickstoff-Löslichkeiten. Hier wird vermutet, dass die einsetzende chemische Bindung des $\mathrm{O}_{2}$ die Genauigkeit der $\mathrm{N}_{2}$-Ergebnisse einschränken, da bspw. dann für die gaschromatographische Bestimmung andere Messwerte in anderen Messbereichen als beim Referenzversuch mit Wasser relevant sind und damit nicht mehr von gleichen systematischen Messabweichungen ausgegangen werden kann. Auch nicht berücksichtigte, möglicherweise stattfindende Dichteänderungen durch die chemische Bindung könnten das Ergebnis hier verfälschen. Angegebene Messunsicherheitsbereiche können bei diesen Versuchen in der Realität ggf. überschritten werden. Es kann jedoch mit den Versuchen auch nicht ausgeschlossen werden, dass die starke chemische Bindung des $\mathrm{O}_{2}$ aus dem Arbeitsgas hier doch zu einer Veränderung der $N_{2}$-Löslichkeit führt. Für die Tyfocor ${ }^{\circledR}$-Versuche in Abbildung 5.25 sind keine solchen Auffälligkeiten vorhanden. 


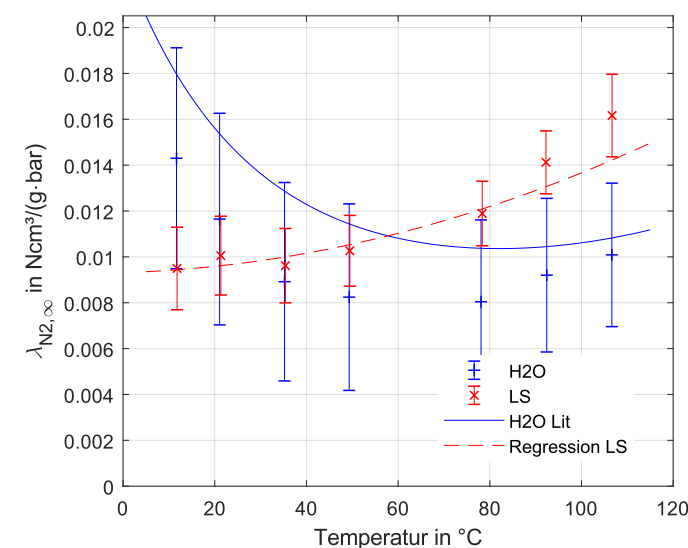

Abbildung 5.24: $\mathrm{N}_{2}$-Löslichkeitskoeffzienten aus LuftVersuchen für Tyfocor $^{\circledR}$ LS (LS) und Wasser; Regressionspolynom LS2 aus $\mathrm{N}_{2}$-Versuchen

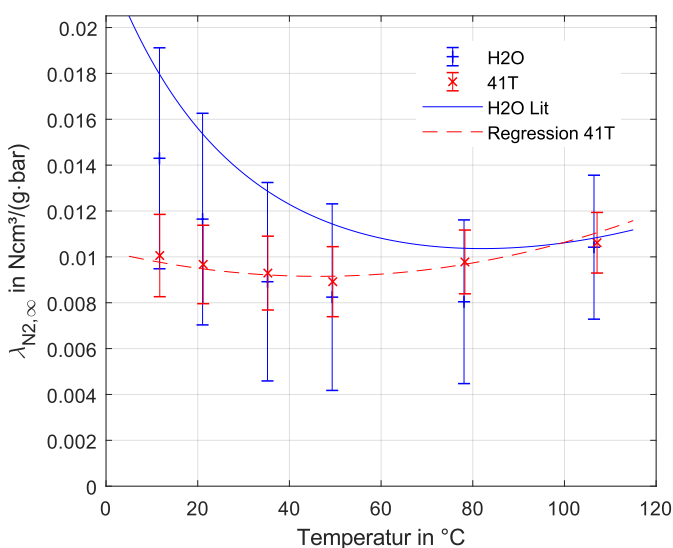

Abbildung 5.25: $\mathrm{N}_{2}$-Löslichkeitskoeffizienten aus Luft-Versuchen für $41 T$ und Wasser; Regressionspolynom $41 T$ aus $\mathrm{N}_{2}$-Versuchen

\subsubsection{Sauerstoff-Löslichkeitskoeffizienten für Wasser-Glykol-Gemische}

Wie im vorherigen Abschnitt gezeigt, konnte kein Quereinfluss von Sauerstoff auf die Löslichkeit von Stickstoff festgestellt werden. Da keine Versuche mit reinem Sauerstoff vorliegen, sondern nur mit synthetischer Luft, kann im Folgenden nur angenommen werden, dass auch Stickstoff keinen wesentlichen Quereinfluss auf die Löslichkeitskoeffizienten von Sauerstoff zeigt und die berechneten Werte auch für reine Sauerstoff-Wasser-Glykol-Gemische gelten.

Die ermittelten $\mathrm{O}_{2}$-Löslichkeitskoeffizienten für die untersuchten reinen Wasser-GlykolGemische in Abbildungen 5.26 und 5.27 zeigen in der Abweichung von den Löslichkeiten von Wasser eine ähnliche temperaturabhängige Charakteristik wie bei Stickstoff, jedoch bei deutlich höheren Werten. Bei beiden 41,84 Gew.-\% Glykol-Wasser-Gemischen ist auch für die Löslichkeit von Sauerstoff ein Anstieg für höhere Temperaturen erkennbar. Die ermittelten Regressionskoeffizienten sind in Tabelle 5.6 zusammengefasst.

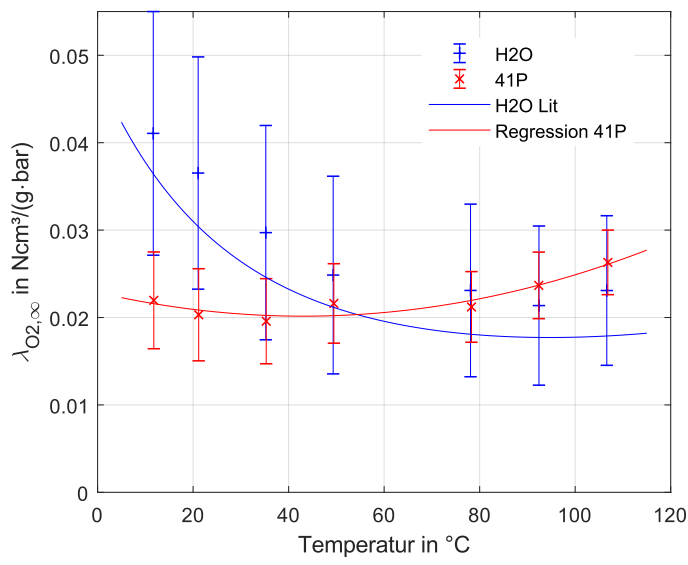

Abbildung 5.26: $\mathrm{O}_{2}$-Löslichkeitskoeffzienten für Wasser und 41,84 Gew.-\% Propylenglykol-Wasser (41P)

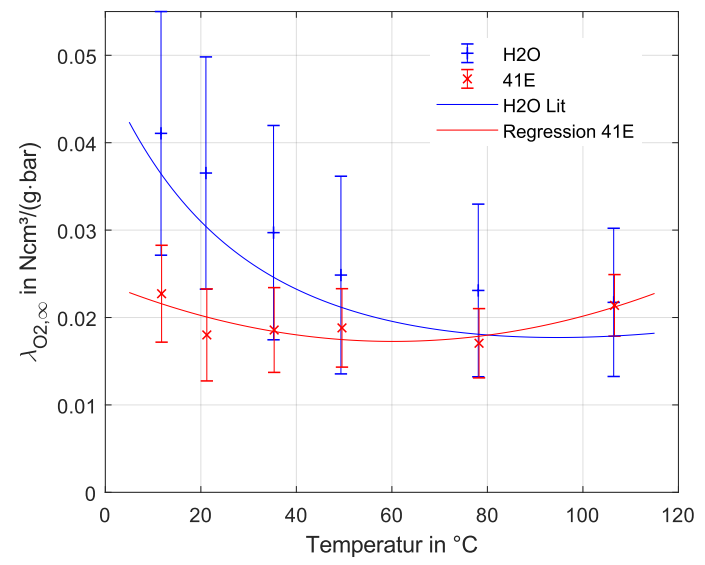

Abbildung 5.27: $\mathrm{O}_{2}$-Löslichkeitskoeffizienten für Wasser und 41,84 Gew.- $\%$ Ethylenglykol-Wasser (41E) 
Tabelle 5.6: Regressionskoeffizienten für $\mathrm{O}_{2}$ in Wasser-Glykol

\begin{tabular}{rrrrr}
\hline Medium & $\begin{array}{r}\xi_{\text {Glykol }} \\
-\end{array}$ & $\mathrm{cm}^{3} / \mathrm{g} \cdot \mathrm{bar}$ & $\mathrm{cm}^{3} / \mathrm{g} \cdot \mathrm{bar} \cdot{ }^{\circ} \mathrm{C}_{1}$ & $\mathrm{~cm}^{3} / \mathrm{g} \cdot \mathrm{bar} \cdot{ }^{\circ} \mathrm{C}^{2}$ \\
\hline Propylenglykol-Wasser & 0,4184 & 0,0229 & $-1,26 \cdot 10^{-4}$ & $1,46 \cdot 10^{-6}$ \\
\hline Ethylenglykol-Wasser & 0,4184 & 0,0239 & $-2,21 \cdot 10^{-4}$ & $1,83 \cdot 10^{-6}$ \\
\hline
\end{tabular}

Auch wenn Sauerstoff-Löslichkeitskoeffizienten nur für 41,84 Gew.-\% Glykolanteil vorhanden sind, seien der Vollständigkeit halber auch hier die Werte in Abhängigkeit vom Glykolanteil dargestellt (vgl. 5.28). Der Vergleich mit den Literaturwerten für $25^{\circ} \mathrm{C}$ zeigt im Rahmen der Messunsicherheit plausible Ergebnisse.
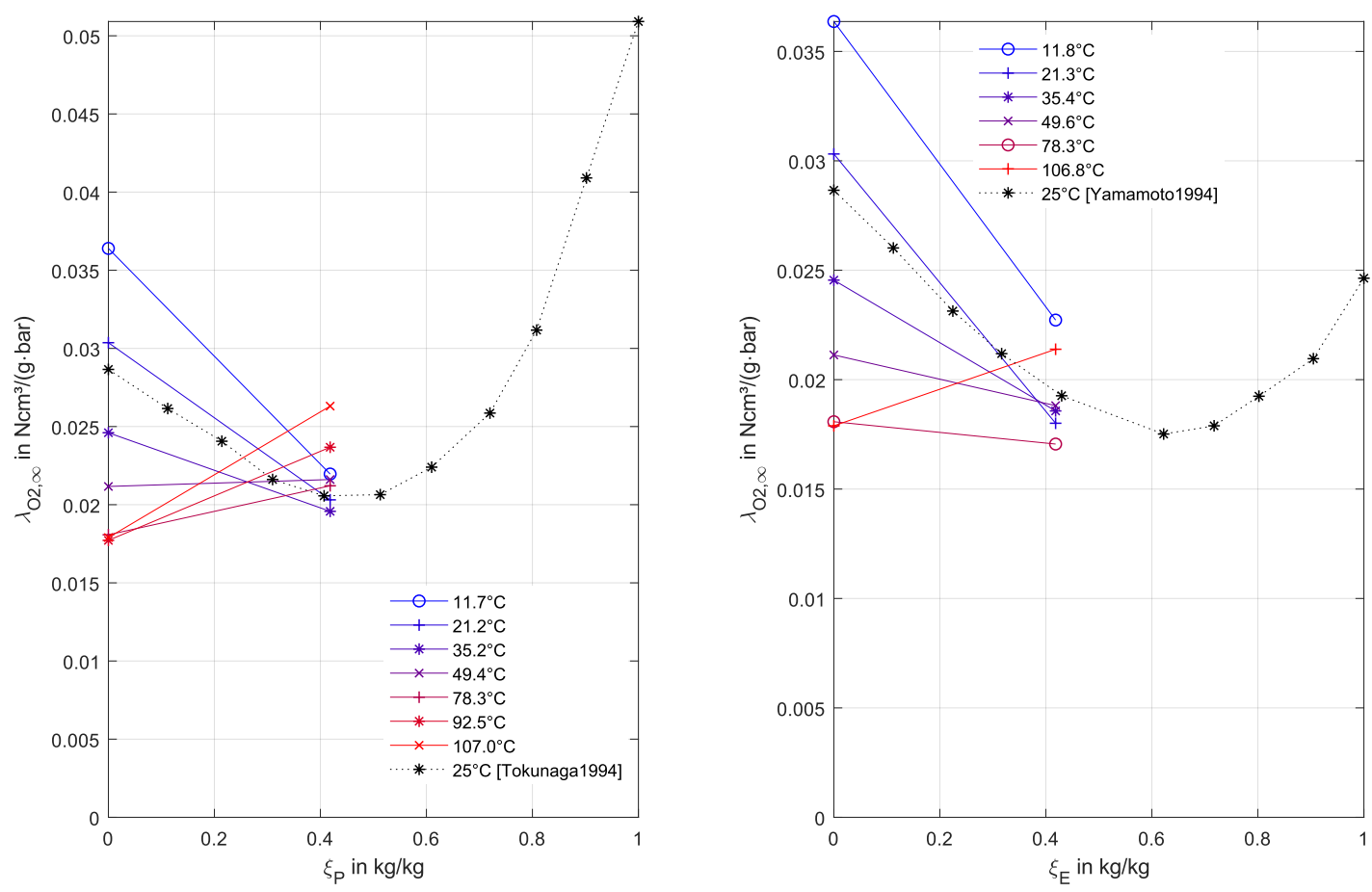

Abbildung 5.28: Konzentrationsabhängige Löslichkeit von $\mathrm{O}_{2}$; links: Propylenglykol-Wasser-Gemisch; rechts: Ethylenglykol-Wassergemisch

\subsubsection{Sauerstoff in Tyfocor ${ }^{\circledR}$ LS}

Wie bereits in [Panitz und Rühling, 2016] basierend auf dem Entwurf dieser Dissertation veröffentlicht, zeigt Tyfocor ${ }^{\circledR}$ LS ein signifikant anderes Löslichkeitsverhalten von Sauerstoff. Sauerstoff wird in dem üblichen Solarfluid (Tyfocor ${ }^{\circledR}$ LS) nicht nur physikalisch, sondern auch chemisch gebunden.

In Abbildung 5.29 und Abbildung 5.30 sind die Druckverläufe der Luft-Versuche, die sich in der Solltemperatur unterscheiden, für Wasser und 41P im temperierten, mit Fluid befüllten Behälter nach dem Zuführen von Luft (Druckausgleich zwischen Gasreservoir und Reaktor) zu sehen. Bereits nach einem wenige Minuten dauernden, durch Rühren unterstützten physikalischen Lösungsvorgang von $\mathrm{N}_{2}$ und $\mathrm{O}_{2}$, bei dem der Druck deutlich sinkt, wird 


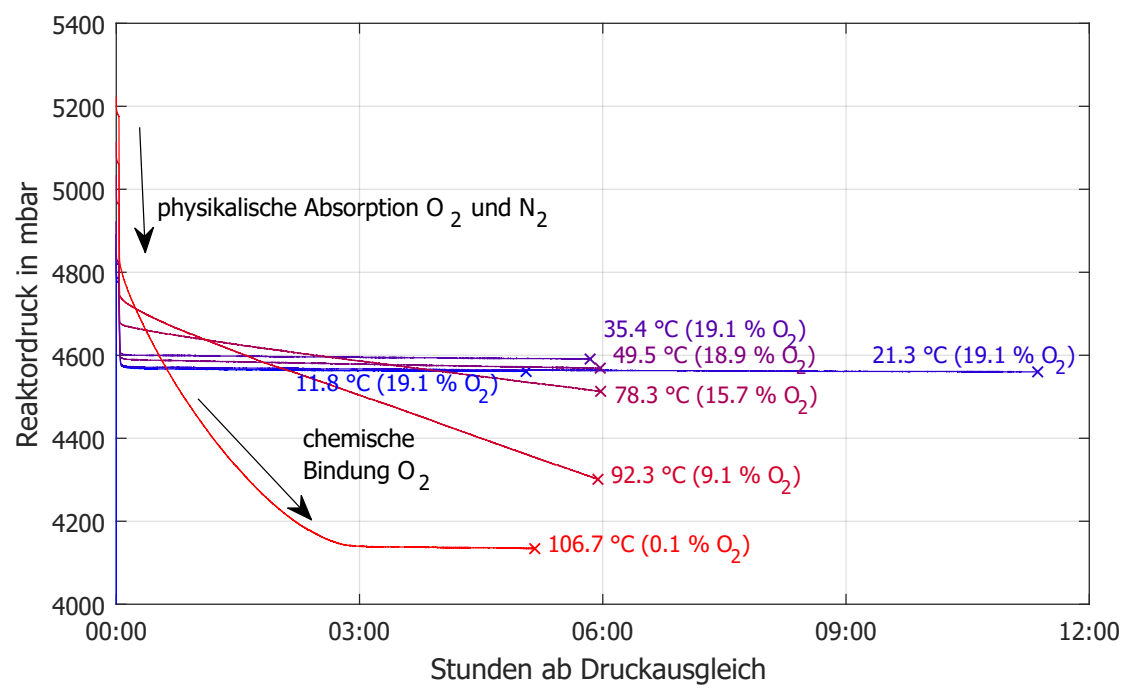

Abbildung 5.31: Druckverlauf nach Zugabe von Luft in Tyfocor ${ }^{\circledR}$ LS in umgewälztem Reaktorgefäß (ca. $859,5 \mathrm{ml}$, davon ca. $156,5 \mathrm{ml}$ Gasphase); mit $\mathrm{O}_{2}$-Gehalt der Gasphase $y_{\mathrm{O} 2}$ in N2+O2,2 am Versuchsende

bei Wasser und auch beim Propylenglykol-Wasser-Gemisch 41P ohne Inhibierung ein stationärer Zustand erreicht. Am Ende der Beharrungszeit wird die Gasprobe entnommen. Über den Gaschromatographen gemessene Sauerstoffgehalte $y_{\mathrm{O}} 2$ in N2+O2,2, die für die Berechnung verwendet wurden, sind in den Diagrammen dargestellt. Aufgrund des relativ großen Volumens der Gasphase ändert sich der Anteil des Sauerstoffs in der Gasphase gegenüber dem Ausgangszustand nur gering. Entsprechend der höheren Löslichkeitskoeffizienten von Sauerstoff im Vergleich zu Stickstoff ist der sich ergebende Sauerstoffgehalt $y_{\mathrm{O} 2}$ in N2+O2,2 stets kleiner als der Sauerstoffgehalt der aus dem Gasreservoir zugegebenen synthetischen Luft mit $y_{\mathrm{O} 2}^{G}=20,5 \%$.

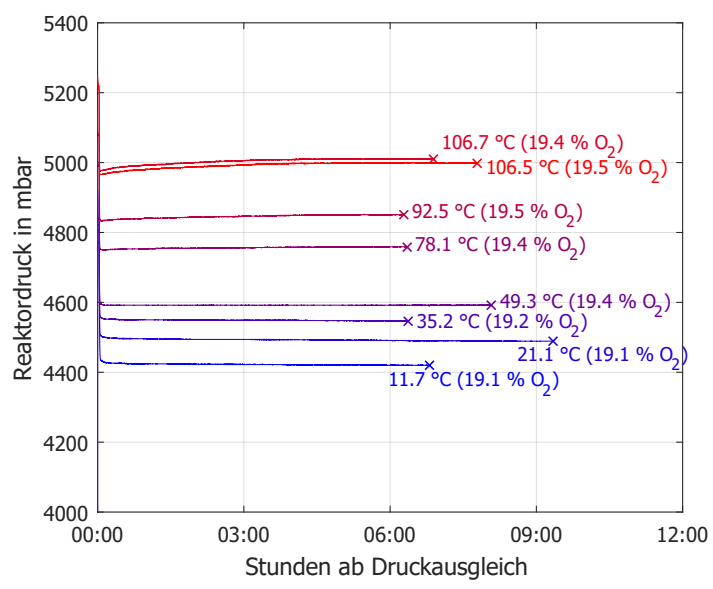

Abbildung 5.29: Druckverlauf nach Zugabe von Luft für Wasser; mit $\mathrm{O}_{2}$-Gehalt der Gasphase $y_{0} 2$ in $\mathrm{N} 2+\mathrm{O} 2,2$ am Versuchsende

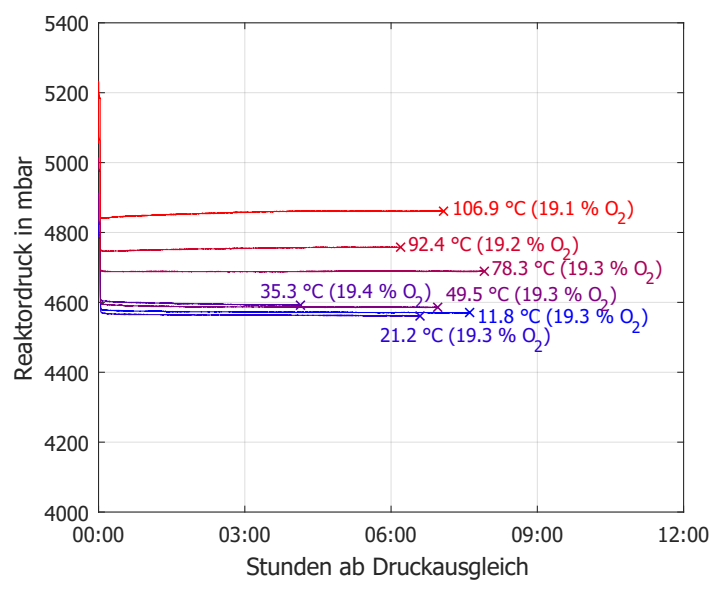

Abbildung 5.30: Druckverlauf nach Zugabe von Luft für 41P; mit $\mathrm{O}_{2}$-Gehalt der Gasphase $y_{\mathrm{O} 2}$ in $\mathrm{N} 2+\mathrm{O} 2,2$ am Versuchsende

Bei Tyfocor ${ }^{\circledR}$ LS - einem Gemisch mit Inhibierung -, dargestellt in Abbildung 5.31, trifft dies nur bis zu ca. $50^{\circ} \mathrm{C}$ zu. Bei höheren Temperaturen (hier untersucht ab $78,3^{\circ} \mathrm{C}$ ) ist ein weiterer 
allmählicher Druckabfall zu beobachten. Dieser Effekt kann durch die chemische Bindung des Sauerstoffs über die Inhibitorwirkung erklärt werden. Die chemische Reaktionsgeschwindigkeit steigt mit höheren Temperaturen deutlich. Anhand des bestimmten $\mathrm{O}_{2}$-Gehalts der Gasphase lässt sich schlussfolgern, dass bei $107^{\circ} \mathrm{C}$ innerhalb von drei Stunden nahezu jeglicher im Reaktor vorhandene Sauerstoff chemisch gebunden wird. Im Reaktorgefäß wurden bei diesem Versuch ca. $94 \mathrm{~cm}^{3} \mathrm{~N}$ Stickstoff gebunden, dies entspricht einer chemischen Bindung von Sauerstoff im Tyfocor ${ }^{\circledR}$ LS von $w_{\text {chem }}=138 \frac{\mathrm{cm}_{\mathrm{N}}^{3}}{\mathrm{~kg}}$.

Nachgelagert wurde ein separater Versuch bei $107^{\circ} \mathrm{C}$ durchgeführt, um die Stabilität der chemischen Bindung nach Abkühlung zu bestätigen. Der Sauerstoffgehalt der Probenahme betrug hier vor Abkühlung 0,2\% und lieferte bei erneuter Messung durch Probenahme nach Abkühlung des Gemischs von $107^{\circ} \mathrm{C}$ auf $25^{\circ} \mathrm{C}$ und nach dem Einstellen stationärer Verhältnisse einen Wert von 0,4\%. Damit kann davon ausgegangen werden, dass der Sauerstoff im Wesentlichen dauerhaft chemisch gebunden ist.

Damit unterscheidet sich Tyfocor ${ }^{\circledR}$ LS deutlich von den anderen untersuchten Gemischen. Auch bei dem Gemisch Ethylenglykol-Wasser (41E) und dem konzentrationsgleichen Gemisch mit dem mit Inhibitoren versetzten $\operatorname{Tyfocor}^{\circledR}$ (41T) findet sich kein Hinweis auf ein solches Phänomen, wie die äquivalenten Darstellungen in Abbildung 5.32 und Abbildung 5.33 zeigen.

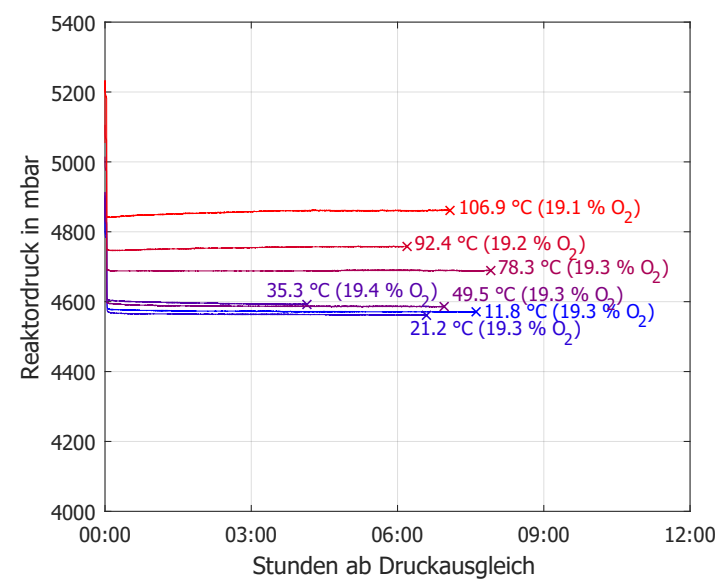

Abbildung 5.32: Druckverlauf nach Zugabe von Luft für 41E; mit $\mathrm{O}_{2}$-Gehalt der Gasphase $y_{\mathrm{O} 2}$ in $\mathrm{N} 2+\mathrm{O} 2,2$ am Versuchsende

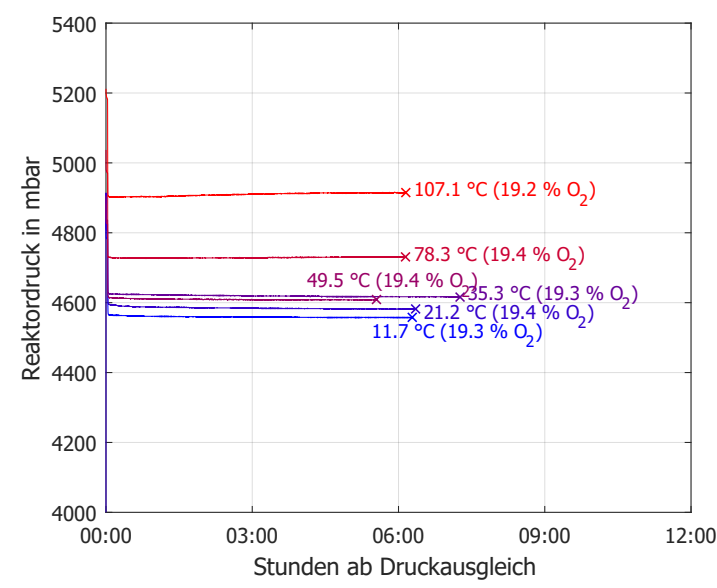

Abbildung 5.33: Druckverlauf nach Zugabe von Luft für 41T; mit $\mathrm{O}_{2}$-Gehalt der Gasphase $y_{\mathrm{O} 2}$ in $\mathrm{N} 2+\mathrm{O} 2,2$ am Versuchsende

Das spezielle Verhalten von Tyfocor ${ }^{\circledR}$ LS kann nur auf die eingesetzten Inhibitoren zurückgeführt werden. Es kann abgeleitet werden, dass der speziell für Tyfocor ${ }^{\circledR}$ LS entwickelte flüssige Inhibitor-Mix mit dem wesentlichen Bestandteil Isopropanolamin für die chemische Bindung des $\mathrm{O}_{2}$ verantwortlich ist. Somit tritt hier ein positiver Nebeneffekt auf. Der Inhibitor, der eigentlich für die Bereitstellung der Reservealkalität vorhanden ist, kann hier bereits den Sauerstoff vorher direkt binden und damit die Säurebildung des Propylenglykols nicht nur kompensieren sondern, vermutlich sogar direkt verhindern. Dies ist ein positiver Nebeneffekt des speziellen Inhibitors. Jedoch muss im Weiteren untersucht werden, wie sich der Sauerstoff hier chemisch auswirkt und den Inhibitor möglicherweise „verbraucht“. Mit Problemen wie Aufsalzung und Bildung aggressiver lonen wie bei früher eingesetzten $\mathrm{O}_{2}$-Bindern wie Thiosulfat ist jedoch nicht zu rechnen. 


\subsubsection{Wirkung von Additiven in Wasser-Glykol-Gemischen}

Das Propylenglykol-Wasser-Gemisch mit Additiven (Tyfocor ${ }^{\circledR}$ LS) zeigt in Abbildung 5.34 bis $35^{\circ} \mathrm{C}$ leicht erhöhte Löslichkeits-Werte gegenüber dem reinen Propylenglykol-WasserGemisch (41P). Ab $50{ }^{\circ} \mathrm{C}$ setzt hier definitiv eine chemische Bindung ein. Die entsprechend des Formalismus berechneten Löslichkeitskoeffizienten zeigen einen starken Anstieg mit steigender Temperatur, da die beschriebene chemische Bindung wie eine hohe Löslichkeit erscheint. Diese Werte sind jedoch keine Löslichkeitskoeffizienten im eigentlichen Sinne mehr. $\mathrm{Ob}$ die leicht erhöhten Löslichkeitskoeffizienten des Gemischs mit Inhibitoren unter $50^{\circ} \mathrm{C}$ ebenso auf Anteile chemischer Bindung zurückzuführen sind, kann anhand der Versuche nicht näher ermittelt werden.

Tyfocor $^{\circledR}$ (41T) hingegen zeigt, wie in Abbildung 5.35 zu sehen, ein sehr ähnliches Verhalten wie das entsprechende reine Ethylenglykol-Wasser-Gemisch (41E). Teilweise beziehen sich die kalibrierten Versuche der Lösungsmittel mit und ohne Inhibitor bei gleicher Temperatur auf den gleichen Referenz-Wasserversuch. Daher sind bei den kalibrierten Versuchen Messabweichungsbeiträge, die aus dem eingehenden Referenz-Wasser-Versuch stammen, gleich. Dies erklärt die gleichen Unstetigkeiten z. B. bei $20^{\circ} \mathrm{C}$.

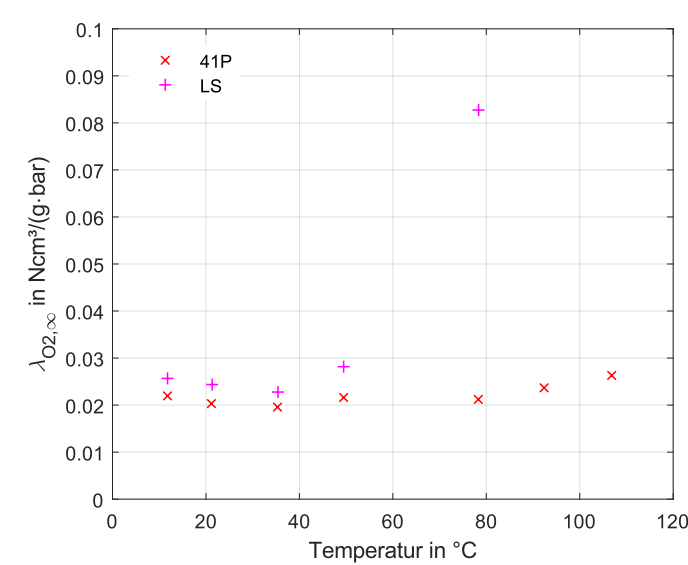

Abbildung 5.34: $\mathrm{O}_{2}$-Löslichkeitskoeffizienten für $\quad 41,84 \mathrm{Gew} .-\%$ WasserPropylenglykol (41P) und konzentrationsgleiche Fertigmischung Tyfocor $^{\circledR}$ LS (LS)

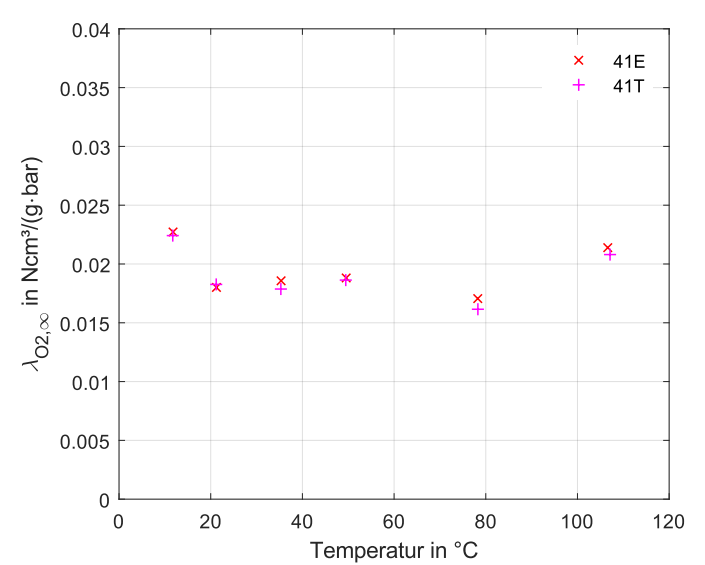

Abbildung 5.35: $\mathrm{O}_{2}$-Löslichkeitskoeffizienten für 41,84 Gew.- $\%$ Wasser-Ethylenglykol (41E) und konzentrationsgleiche Fertigmischung Tyfocor ${ }^{\circledR}$ (41T)

\subsection{Zusammenfassung der Ergebnisse}

Die Ergebnisse sollen wie folgt zusammengefasst werden:

- Es konnten Stickstoff-Löslichkeitskoeffizienten für Wasser-Propylenglykol- bzw. Wasser-Ethylenglykol-Mischungen mit unterschiedlichem Glykolgehalt sowie für reines Glykol bestimmt werden. Ebenso wurden aus anschließenden Luft-Versuchen für Wasser-Glykol-Gemische mit einem Glykolgehalt von 41,84 Gew.-\% vergleichende Stickstoff-Löslichkeitskoeffizienten sowie Sauerstoff-Löslichkeitskoeffizienten ermittelt.

- Vorhandene Literaturwerte für Wasser-Glykole bei $25^{\circ} \mathrm{C}$ liegen im Messunsicherheitsbereich der eigenen Ergebnisse. 
- Es zeigt sich -verglichen mit Wasser- eine grundlegend andere Temperaturabhängigkeit der Stickstoff- und Sauerstoff-Löslichkeit von Glykol-Wasser-Gemischen, wie in Abbildung 5.36 exemplarisch für die zwei untersuchten Stoffe 41P und 41E gegenübergestellt. Ähnlich wie für reines Wasser sind für den untersuchten Temperaturbereich Sauerstoff-Löslichkeiten etwa doppelt so hoch wie Stickstoff-Löslichkeiten bei gleicher Temperatur. Wie bei Wasser ist damit unter atmosphärischen Bedingungen der Anteil von Sauerstoff an der Gesamtmenge gelöster Gase deutlich höher als der Sauerstoffanteil in der Gasphase.

- Die bei unterschiedlichen Drucksprüngen bestimmten StickstoffLöslichkeitskoeffizienten zeigen im Rahmen der Messunsicherheit gute Übereinstimmung. Damit liegt der untersuchte Druckbereich bis ca. 9 bar im „Gültigkeitsbereich des Löslichkeitskoeffizienten".
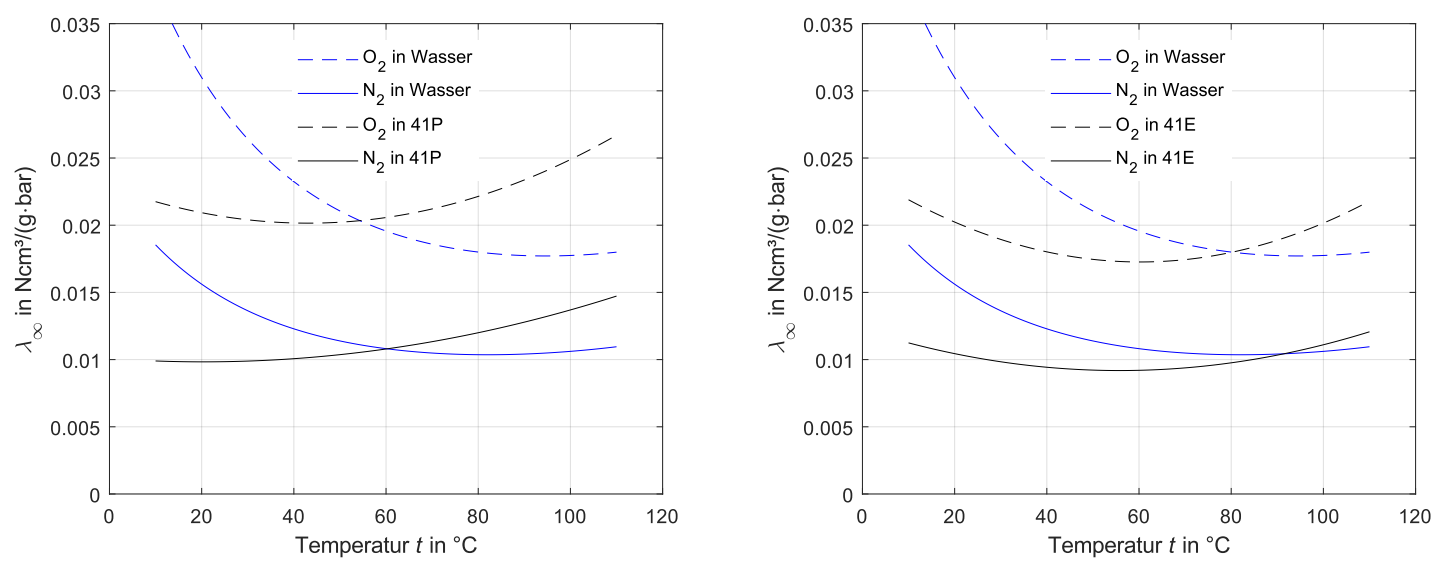

Abbildung 5.36: Löslichkeit von $\mathrm{O}_{2}$ und $\mathrm{N}_{2}$ für 41,84 Gew.-\% Glykol-Wasser-Gemisch und Wasser; links: Propylenglykol-Wasser-Gemisch; rechts: Ethylenglykol-Wasser-Gemisch

- Der Einfluss von in geringen Mengen vorliegenden Inhibitoren auf die Löslichkeit kann als gering eingeschätzt werden. Bei Tyfocor ${ }^{\circledR}$ LS jedoch führt der Inhibitor zumindest bei hohen Temperaturen zur chemischen Bindung des Sauerstoffs.

- Durch vorhandenen Sauerstoff kann - zumindest in atmosphärischen Konzentrationsverhältnissen von Stickstoff und Sauerstoff - keine Beeinflussung der Stickstofflöslichkeit festgestellt werden. Der Vergleich der ermittelten SauerstoffLöslichkeitskoeffizienten mit den wenigen Literaturwerten legt nahe, dass dies andersherum für den Einfluss von Stickstoff auf die Sauerstofflöslichkeit ebenso der Fall ist.

- Die Löslichkeit von Stickstoff im Wasser-Glykol-Gemisch ist stark abhängig vom Glykolgehalt.

- Löslichkeitsbestimmungen für Wasser-Glykol-Gemische für weitere Glykolanteile sollten durchgeführt werden, um mit einer dann erstellbaren Regressionskurve $\lambda\left(t, \xi_{\mathrm{G}}\right)$ für beliebige Temperaturen $t$ und Gemischanteile $\xi_{\mathrm{G}}$ zu interpolieren. 



\section{Löslichkeit von Stickstoff und Sauerstoff in Wasser-Glykol-Gemischen in Theorie und Praxis (Anwendung auf Anlagen)}

\subsection{Grundlagen}

\subsubsection{Allgemeines}

In Kapitel 5 wurden die ermittelten Löslichkeitskoeffizienten $\lambda_{i, \infty}$ vorgestellt und diskutiert. Diese liefern jedoch noch keine vollständige Aussagen über die im Phasengleichgewicht gelösten Gase für einen gewissen $(p, t)$-Zustand für WasserGlykole.

Entsprechend den bereits vorgestellten Beziehungen ergibt sich bei Anwendbarkeit des HenryGesetzes und für ein Gemisch mit einer Gaskomponente i, analog Gleichung 2.29 für $x_{i}$, für den Gasgehalt $w_{i}$ im Phasengleichgewicht bei $(p, t)$

$$
w_{i}(p, t)=\lambda_{i, \infty}(t) \cdot\left(p-p_{\mathrm{LM}, \mathrm{s}}(t)\right)
$$

Für die gelöste Gasmenge im Phasengleichgewicht ist also ebenso der stoffspezifische Dampfdruckverlauf $p_{\mathrm{LM}, \mathrm{s}}(t)$ eine wichtige Einflussgröße.

In Abschnitt 2.3.5 wurde bereits exemplarisch für Stickstoff in Wasser gezeigt, dass dieser vereinfachte Zusammenhang für technische Belange ausreichende Genauigkeiten liefert. Für Wasser-Glykole wird aufgrund der wasserähnlichen Stoffeigenschaften von ähnlichen Genauigkeiten bei Anwendung der Vereinfachungen ausgegangen. Im Gegensatz zum vorhergehenden Kapitel sei also hier in der Anwendung auf technische Anlagen auch die Poynting-Korrektur des Gases nicht mehr berücksichtigt. Da die Poynting-Korrektur vernachlässigt wird, wird als Bezeichnung verkürzt $\lambda_{i}$ statt $\lambda_{i, \infty}$ verwendet. ${ }^{1}$ Demnach können also auch Löslichkeitswerte, die für den Partialdruck von 1 bar tabelliert sind, z. B. die in [D'Ans und Lax, 1997], eingesetzt werden. Diese unterscheiden sich nur marginal, d. h.

$$
\lambda_{i, \infty}(t) \approx \lambda_{i, 1 \mathrm{bar}}(t) .
$$

In der technischen Praxis wird als Gehaltsgröße zumeist die Konzentration $c_{i}$ in der Einheit mg/l genannt (vgl. [VDI 2035-2], [FW 510] und [FW 442]).

Entsprechend Tabelle 2.2 gilt für die im Phasengleichgewicht gelöste Gasmenge bei $(p, t)$ für den vereinfachten Zusammenhang und für ein Gemisch mit einer Gaskomponente $i$

$$
c_{i}(p, t)=\lambda_{i}(t) \cdot \rho_{0, i, \mathrm{~N}} \cdot \rho_{\mathrm{LM}}(t) \cdot\left(p-p_{\mathrm{LM}, \mathrm{s}}(t)\right) .
$$

${ }^{1}$ Will man die Poynting-Korrektur doch berücksichtigen, so ist in den folgenden Gleichungen analog Tabelle $2.2 \lambda_{i}$ stattdessen wie folgt zu bestimmen $\lambda_{i}=\lambda_{i}(p)=\lambda_{i, \infty} \cdot \frac{1}{\operatorname{Poy}_{i}\left(p_{\mathrm{LM}, \mathrm{s}} ;\right)} \operatorname{mit} P O y_{i}=\exp \left[\frac{v_{\infty, i}^{\prime} \cdot\left(p-p_{\mathrm{LM}, \mathrm{s}}\right)}{R \cdot T}\right]$ 
Die Dichte des Lösungsmittels $\rho_{\mathrm{LM}}$ kann vereinfacht als druckunabhängig angenommen werden. Die Dichte unter Normbedingungen lässt sich ersetzen $\rho_{i, N}=\frac{M_{i}}{v_{0, i, N}}$ und mit $v_{0, i, N} \approx$ $22,4 \mathrm{l} / \mathrm{mol}$ unabhängig vom Gas, vgl. Tabelle 6.1, ergibt sich

$$
c_{i}(p, t)=\lambda_{i}(t) \cdot \frac{M_{i}}{22,4 \mathrm{I} / \mathrm{mol}} \cdot \rho_{\mathrm{LM}}(t) \cdot\left(p-p_{\mathrm{LM}, \mathrm{s}}(t)\right) .
$$

Tabelle 6.1: Molares Normvolumen $v_{0, i, \mathrm{~N}}=\frac{M_{i}}{\rho_{i, \mathrm{~N}}}$ der Gase

\begin{tabular}{cc} 
Gas $i$ & Molares Normvolumen $v_{0, i, \mathrm{~N}}$ in $\mathrm{I} / \mathrm{mol}$ \\
\hline Stickstoff & 22,4038 \\
\hline Sauerstoff & 22,3923 \\
\hline
\end{tabular}

Nachteile einer Darstellung als $c_{i}$ gegenüber $w_{i}$ sind:

1. Gas in der Einheit Milligramm ist im Gegensatz zum Normkubikzentimeter praktisch schwer vorstellbar.

2. Bei Gasgemischen ist ein summarisches Normvolumen anschaulicher als eine summarische Masse der gelösten Gase.

3. Die Bezugsgröße Volumen ändert sich mit der Temperatur. Damit ändert sich trotz konstanter gelöster Gasmenge in einer Flüssigkeitsmenge die Konzentration $c_{i}$, der Gasgehalt $w_{i}$ ändert sich hingegen nicht. Hier können schnell Fehler entstehen, z. B. wenn man fälschlicherweise die gemessene Konzentration einer abgekühlten Probe mit der Konzentration im deutlich heißeren Kreislauf gleichsetzt. Auswirkungen sind bei Wasser-Glykolen noch deutlicher, da hier die Dichte im Vergleich zu Wasser stärker von der Temperatur abhängt, vgl. Abbildung 6.1.

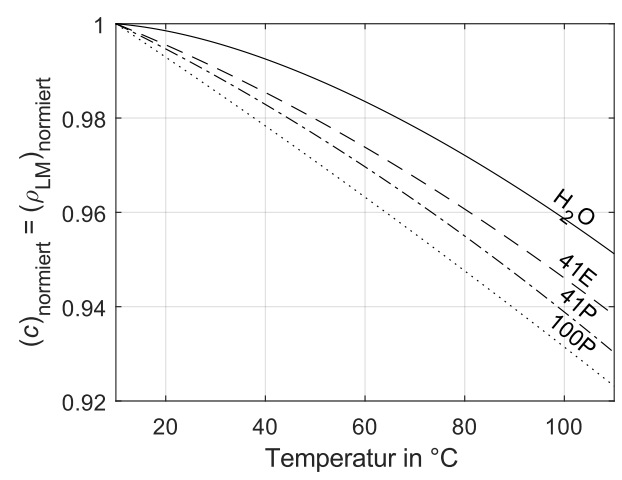

Abbildung 6.1: Änderung der Konzentration ggü. $10^{\circ} \mathrm{C}$ bei gleichbleibendem Gasgehalt des Fluids $w=$ $w\left(10^{\circ} \mathrm{C}\right)=$ const.

4. Zur Bestimmung der Gleichgewichtskonzentration aus Löslichkeitskoeffizienten $\lambda_{i}$ ist mit $\rho_{\mathrm{LM}}$ eine zusätzliche Stoffgröße notwendig. Außerdem geht die Normdichte des Gases $\rho_{0, i, \mathrm{~N}}$ ein.

5. Der Zusammenhang zwischen Stoffgröße $\lambda_{i}$ und $w_{i}$ ist denkbar einfach wg. $w_{i}=\lambda_{i} \cdot p_{i}$. Für die Konzentration $c_{i}$ sollte dann konsequenterweise in der Praxis eine andere, einfachere Stoffgröße zur Beschreibung der Löslichkeit genutzt und entsprechend tabelliert werden, z. B. abgeleitet entsprechend der Notation von [Sander, 2015] eine Größe $H^{c P}$ in $\frac{\mathrm{mg}_{i}}{1 \cdot \text { bar }}$, für die dann der einfache Zusammenhang $c_{i}=H^{C P} \cdot p_{i}$ angewendet werden kann (d. h. $H^{C P}(t):=$ $\left.\lambda_{i}(t) \cdot \rho_{0, i, N} \cdot \rho_{\mathrm{LM}}(t)\right)$. 
Für die ebenso gern verwendete Größe Masseanteil ergibt sich nach Tabelle 6.1

$$
\xi_{i}(p, t)=\lambda_{i}(t) \cdot \frac{M_{i}}{22,4 \mathrm{I} / \mathrm{mol}} \cdot\left(p-p_{\mathrm{LM}, \mathrm{s}}(t)\right) .
$$

Diese hat zumindest in der Anschaulichkeit ähnliche Nachteile wie die Konzentration $c_{i}$ und könnte außerdem einfacher mit einer Stoffgröße $H^{\xi p}$ in $\frac{\mathrm{mg}_{i}}{\mathrm{~kg} \cdot \mathrm{bar}}$, für die gilt $\xi_{i}=H^{\xi p} \cdot p_{i}$, beschrieben werden. Ein wesentlicher Vorteil von $\xi_{i}(p, t)$ ist, dass die Masse des Gases als Größe im Nenner im Gegensatz zum Volumen nicht zustandsabhängig ist und damit die Definition des Normzustandes nicht benötigt.

Der Autor verwendet in Abwägung der genannten Aspekte $w_{i}$ als GehaltsgröBe.

\subsubsection{Henry-Diagramm}

Der Zusammenhang des Gasgehalts im Phasengleichgewicht $f(p, t)$ wird häufig in Form von Isothermen in Abhängigkeit des Gesamtdrucks $p$ in sogenannten Henry-Diagrammen abgebildet.
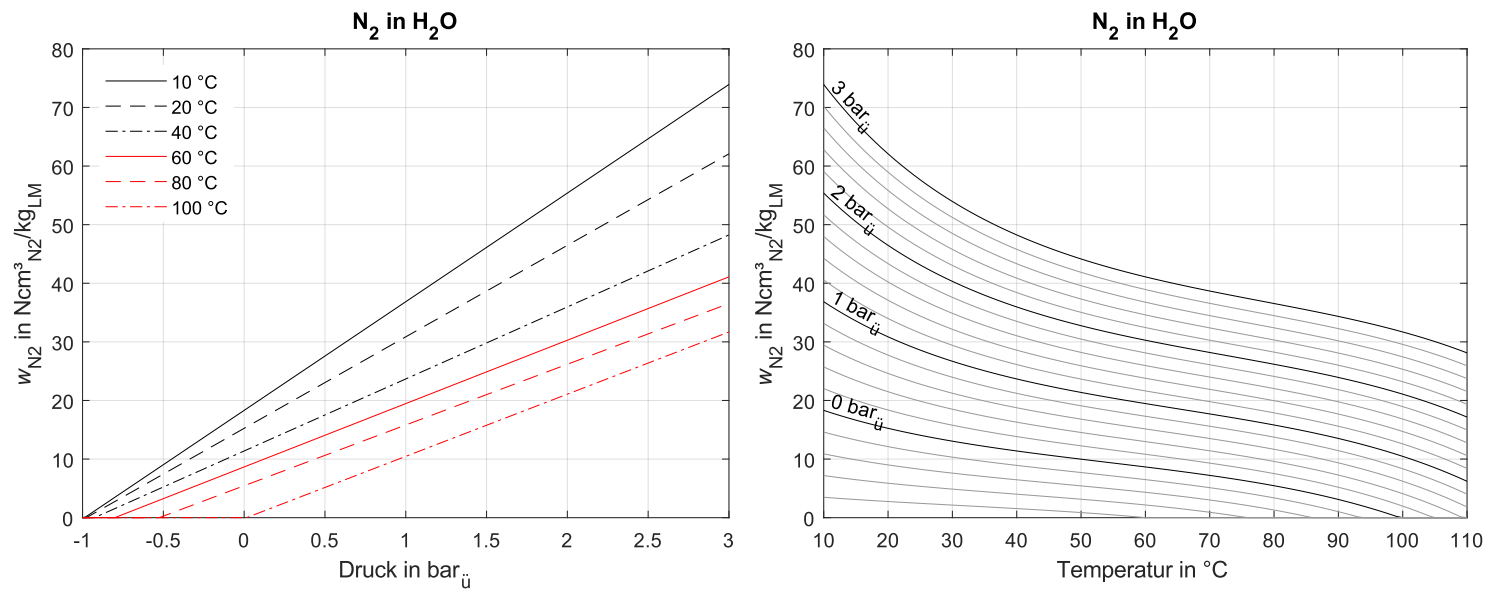

Abbildung 6.2: Henry-Diagramm für Stickstoff in Wasser; links: Isothermen-, rechts: Isobaren-Darstellung

Dies ist am Beispiel Stickstoff in Wasser in Abbildung 6.2 links dargestellt. Die Isothermen sind (bei Gültigkeit von Gleichung 6.1) Geraden, die die x-Achse beim jeweiligen Dampfdruck des Lösungsmittels schneiden. Der Anstieg der Geraden spiegelt die Löslichkeitskoeffizienten wider. Hier lässt sich für einen $(p, T)$-Zustand die im Phasengleichgewicht gelöste Gasmenge schnell ablesen und leicht ermitteln, welchen Einfluss Temperatur- und Druckänderungen auf die Phasengleichgewichts-Gaskonzentration in der Flüssigkeit haben. Außerdem lassen sich Messwerte des Gasgehalts hinsichtlich des Sättigungsgrades der Flüssigkeit schnell einordnen - bezüglich des $(p, T)$-Zustands am Gasgehalts-Messpunkt, aber auch eines beliebigen anderen im Kreislauf auftretenden $(p, T)$-Zustands.

Eine alternative Darstellungsart, die - aus der der gesichteten Literatur mutmaßend - in Normen und in der Praxis bisher keine Anwendung findet, ist die Isobaren-Darstellung, siehe Abbildung 6.2 rechts. Vorteile dieser Darstellung sind:

- In der Isothermen-Darstellung wie in Abbildung 6.2 links kann man zwar für beliebigen Druck (über die $\mathrm{x}$-Achse) die Löslichkeitsgrenze ablesen, jedoch nicht für Temperaturen 
zwischen den dargestellten Isothermen. In der Isobaren-Darstellung hingegen kann man erstens für beliebige Temperatur (über die x-Achse) ablesen. Bei Gültigkeit des HenryGesetzes nach Gleichung 6.1 und der damit gültigen Partialdruck-Proportionalität des Gasgehalts $w_{N 2}$ lässt sich zweitens ebenso für beliebigen Druck zwischen den Isobaren ablesen: Für konstante Temperatur ist die Druckabhängigkeit jeweils linear skaliert (äquidistante Abstände) und startet mit dem Dampfdruck auf der x-Achse. Das zeigen auch die isobaren Hilfslinien, die in den Abbildungen mit einem Abstand von 0,2 bar eingezeichnet sind.

- Sich kreuzende Linien sind im Gegensatz zur Isothermen-Darstellung in dieser Darstellung per se nicht möglich. Dieser Vorteil wird in den folgenden Henry-Diagrammen der untersuchten Glykole noch deutlich.

Henry-Diagramme für Stickstoff als einziges Gas (also ohne Sauerstoff) haben in Praxisanlagen eine große Bedeutung. Denn in den Anlagen wird der Sauerstoff mit der Zeit chemisch abgebaut und bei korrekter Druckhaltung und Verwendung diffusionsdichter Bauteile und Dichtungen ist der Eintrag von neuem Sauerstoff gering, sodass der Anteil von Sauerstoff in der Anlage marginal ist.

Dies wird u.a. durch Feldmessungen in [Rühling u. a., 2013b] von vier Solarthermie-Anlagen mit MAG-Druckhaltung (mit Wärmeträger auf Propylenglykol-Basis) bestätigt. Diese zeigen vernachlässigbare Masseanteile des gelösten Sauerstoffs an der Gesamtmenge gelösten Stickstoffs und Sauerstoffs von $\frac{m_{\mathrm{O} 2}^{\prime}}{m_{\mathrm{N} 2}^{\prime}+m_{\mathrm{O} 2}^{\prime}}=0,14 \ldots 0,34 \%$. In [Panitz und Rühling, 2017] wurde auch ein Rückkühlkreis auf Ethylenglykol-Basis untersucht und ein Masseanteil gelösten Sauerstoffs von $\frac{m_{\mathrm{O} 2}^{\prime}}{m_{\mathrm{N} 2}^{\prime}+m_{\mathrm{O} 2}^{\prime}}=3 \%$ ermittelt. In [Rühling u. a., 2013b] wurden auch drei Solarthermie-Anlagen mit Kompressordruckhaltung untersucht. Hier wurden bereits deutlich höhere Sauerstoffanteile festgestellt, die vermutlich auf Eintrag über Gasdiffusion aus der Umgebungsluft durch die Membran zurückzuführen sind. Diese liegen aber immer noch in Bereichen $\frac{m_{\mathrm{O} 2}^{\prime}}{m_{\mathrm{N} 2}^{\prime}+m_{\mathrm{O} 2}^{\prime}}=8 \ldots 16 \%$, die deutlich unter dem Sauerstoff-Anteil liegen, der sich für Luft-Atmosphäre ergeben würde. Auch Korrisionsprodukte (wie z. B. Methan) sind in korrekt betriebenen Anlagen kaum oder gar nicht vorhanden. Für viele Anwendungsfälle können daher reine Stickstoff-Henry-Diagramme verwendet werden.

\subsubsection{Interpretation der Bereiche des Henry-Diagramms, Einordnung des Henry-Diagramms in reale Zustände des Systems}

In Abbildung 6.3 links sind die im Phasengleichgewicht gelöste Gasmenge $w_{\text {N2}}$ und die sich ergebenden Diagramm-Bereiche am Beispiel Wasser für eine Temperatur von $t=$ $100{ }^{\circ} \mathrm{C}$ dargestellt. Abbildung 6.3 rechts zeigt analog die Diagrammbereiche für $p=$ 1 bar $_{\text {abs. }}$.

Folgende Bereiche können ausgemacht werden:

- Linie I: Flüssigkeit befindet sich im Phasengleichgewicht mit der Gasphase. Die Flüssigkeit ist "gasgesättigt".

- Punkt la: Am Siedepunkt der reinen Flüssigkeit sind keine Gasanteile mehr gelöst.

- Bereich II: Die Flüssigkeit ist gasuntersättigt. Existiert eine Gasphase, so ist der Zustand instationär, da Gas aus der Gasphase bis zum Erreichen des Phasengleichgewichts (Linie I) durch Absorption in die Flüssigphase übergeht. Ein hinsichtlich Stofftransport stationärer Zustand ist in diesem Bereich möglich, wenn keine Gasphase existiert. 

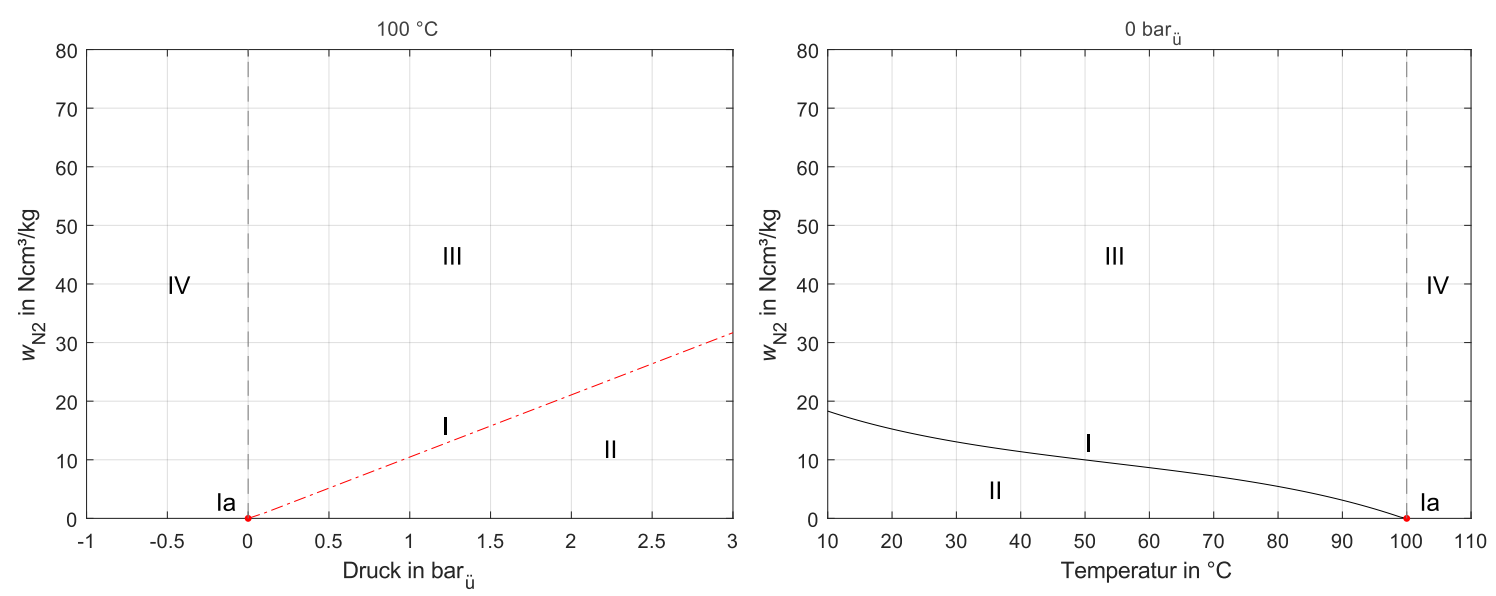

Abbildung 6.3: Bereiche im Henry-Diagramm am Beispiel Stickstoff in Wasser; links: für $100^{\circ} \mathrm{C}$ rechts: für 0 barü

- Bereich III: Die Flüssigkeit ist gasübersättigt. Dieser Zustand ist in der Praxis instationär, da durch das Vorhandensein von Phasengrenzflächen Gas desorbiert. Je nach Beschaffenheit der Phasengrenzflächen können auch leicht über der Kurve I liegende Zustände stationär sein: Sind z.B. nur kleinere Gasblasen vorhanden, dann ist der Innendruck der Gasblasen und damit der für das lokale Phasengleichgewicht relevante Druck aufgrund der Oberflächenspannung höher als der nach außen gemessen Druck des Fluids. Dadurch erhöht sich die lösbare Gasmenge.

- Bereich IV: Bei Gesamtdrücken $p<p_{\mathrm{LM}, \mathrm{s}}$ für die gegebene Temperatur (Abbildung 6.3 links) bzw. Temperaturen $t>t_{\mathrm{LM}, \mathrm{s}}$ für den gegebenen Druck (Abbildung 6.3 links) ist keine Flüssigphase vorhanden, eine gelöste Gasmenge demnach hier nicht existent. Nach [Heller, 2005] kann eine Flüssigkeit hier sehr wohl existieren, aber nur in dem praxisfernen Ausnahmefall, dass keine Phasengrenzflächen vorhanden sind.

Aus der Sichtweise stationärer Bedingungen stellt also die gelöste Gasmenge im Phasengleichgewicht (Linie I) lediglich die maximal lösbare Gasmenge dar, die real gelöste Gasmenge kann darunter, in Ausnahmefällen auch darüber liegen. Der im Henry-Diagramm dargestellte im Phasengleichgewicht (GGW) gelöste Gasgehalt $w_{i}=w_{i, G G W}$ wird daher in der Praxis oft auch als Maximaler Gasgehalt oder Löslichkeitsgrenze $w_{i \text {,max }}$ bezeichnet.

\subsection{Stickstoff-Löslichkeitsverhalten der untersuchten Stoffe}

\subsubsection{Allgemeines}

Um Henry-Diagramme für den Gasgehalt $w_{i}(p, t)$ nach Gleichung 6.1 zu erstellen, werden für die untersuchten Wasser-Glykole folgende Stoffdaten genutzt:

- Löslichkeitskoeffizienten $\lambda_{i}(t)$ entsprechend der in Kapitel 5 vorgestellten Regressionen.

- Dampfdruck $p_{\mathrm{LM}, \mathrm{s}}$ entsprechend den Quellen und Annahmen, die bereits für die Bestimmung der Löslichkeitskoeffizienten verwendet worden sind (siehe Abschnitt 4.7).

Die Unterschiedlichkeit des Verlaufs des Gasgehalts $w_{i}(p, t)$ im Phasengleichgewicht der untersuchten Flüssigkeiten im Vergleich zu Wasser resultiert dabei zu einem großen 
Teil aus der Tatsache, dass der Dampfdruck des Lösungsmittels mit zunehmenden Glykolanteil deutlich unter dem Dampfdruck von Wasser liegt, vergleiche Abbildung 6.4 .

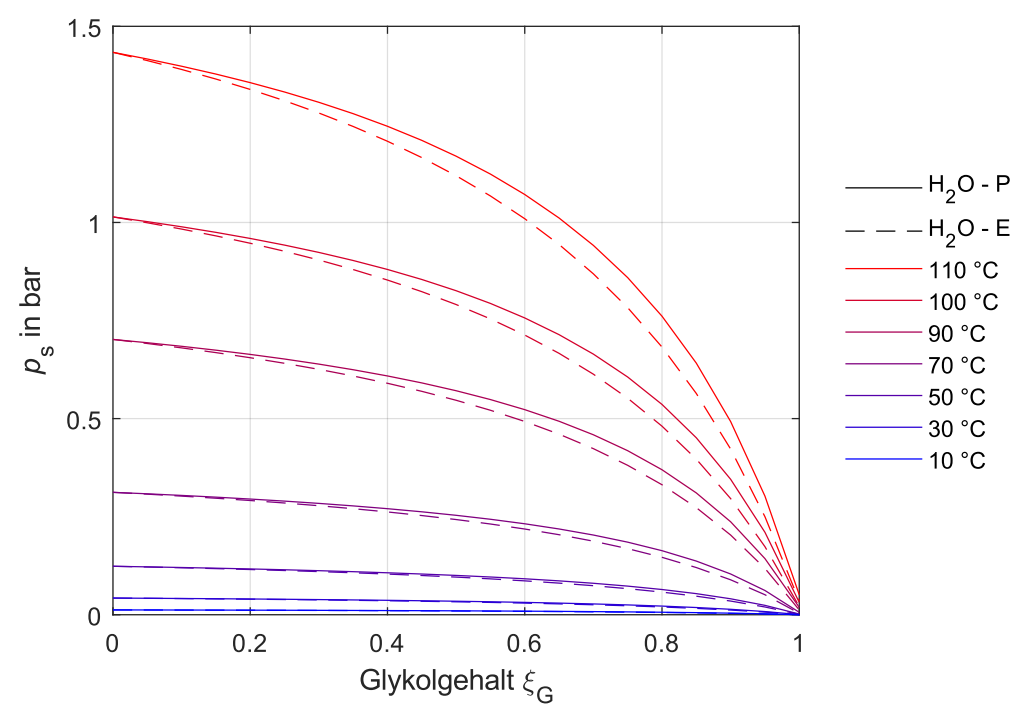

Abbildung 6.4: Dampfdruckverlauf der Wasser-Glykole in Abhängigkeit des Glykolgehalts (Anwendung Raoult 'sches Gesetz); durchgezogene Linie Wasser-Propylenglykol-Gemisch $\left(\mathrm{H}_{2} \mathrm{O}-\mathrm{P}\right)$, gestrichelte Linie Wasser-Ethylenglykol-Gemisch $\left(\mathrm{H}_{2} \mathrm{O}-\mathrm{E}\right)$

\subsubsection{Stickstoff-Löslichkeit in Wasser-Glykol-Gemischen}

In Abbildung 6.5 links ist das Stickstoff-Henry-Diagramm (in Isothermen-Darstellung) für das untersuchte Wasser-Propylenglykol-Gemisch 41P dargestellt. Die absolute Löslichkeit von Stickstoff in $20^{\circ} \mathrm{C}$ kaltem $41 \mathrm{P}$ ist im dargestellten Druckbereich ca. $35 \%$ geringer als die in Wasser. Wie bereits vorgegriffen wurde (als Nachteil der Isothermen-Darstellung), schneiden sich für 41P im Gegensatz zu Wasser die Isothermen, wie in Abbildung 6.5 links zu sehen. Die Löslichkeit von Stickstoff kann also bei konstantem Druck mit steigender Temperatur (trotz zunehmendem Dampfdruck und damit geringerem Partialdruck Stickstoff) sogar noch steigen. Dies liegt an den ermittelten, mit steigender Temperatur stark ansteigenden Löslichkeitskoeffizienten. Noch deutlicher wird dies in der Isobaren-Darstellung in Abbildung 6.5 rechts sichtbar. Je höher der Druck, desto stärker der Effekt.

Im Gegensatz zu Wasser ist ein Herabsetzen der Löslichkeit durch isobare Erwärmung von kalter Flüssigkeit (z. B. $20^{\circ} \mathrm{C}$ ) bereits bei 1 bar ü bis zu einer Temperatur von $75^{\circ} \mathrm{C}$ nicht möglich. Erst über $90^{\circ} \mathrm{C}$ unterschreitet die Löslichkeit die Werte von $20^{\circ} \mathrm{C}$ signifikant. Damit ist eine Gasdesorption wie bei Wasser erst bei viel höheren Temperaturen möglich. Dieses Verhalten entspricht somit nicht dem bisher angenommenen Verhalten von Wasser-Propylenglykol und hat entsprechende Folgen für Inbetriebnahme, Betrieb und Konstruktion entsprechender Anlagen.

In Abbildung 6.6 ist der Verlauf für das entsprechende Wasser-Ethylenglykol-Gemisch (41E) dargestellt. Hier zeigt sich für den dargestellten Druckbereich ein deutlich wasserähnlicherer, mit steigender Temperatur und konstantem Druck stetig fallender Verlauf.

Abbildung 6.7 verdeutlicht die Unterschiedlichkeit der Verläufe im Vergleich zu Wasser am

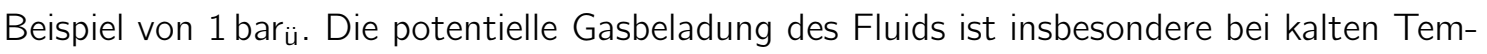



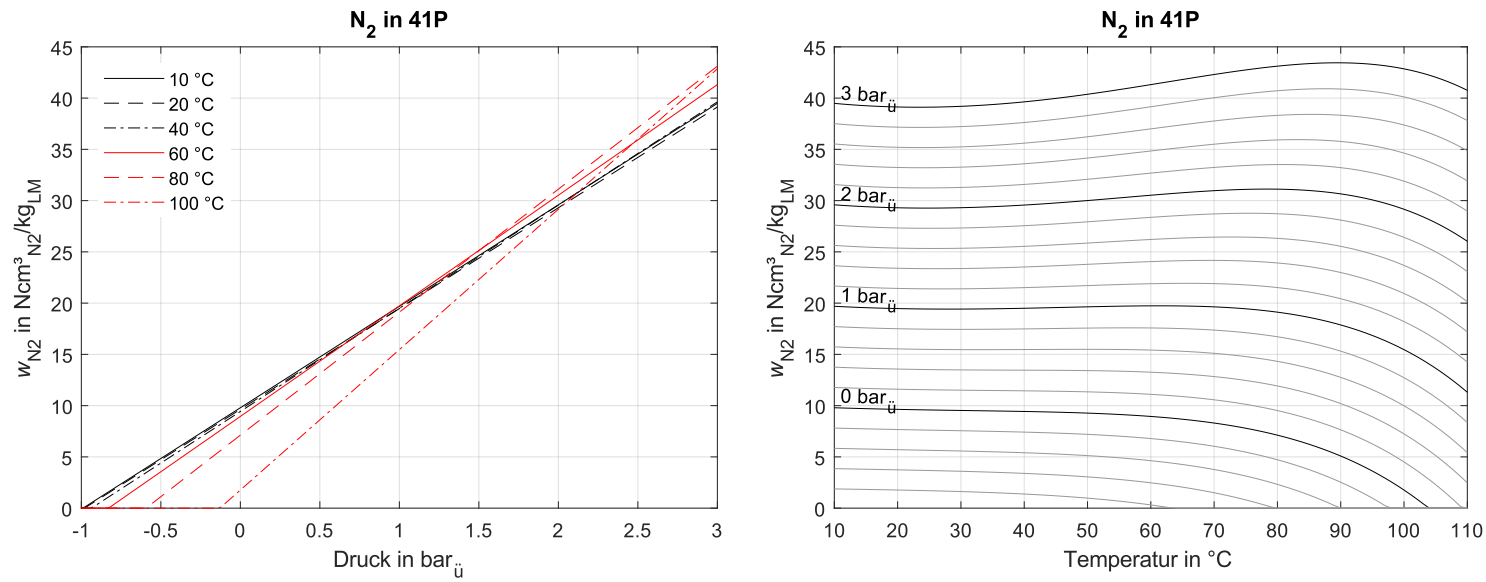

Abbildung 6.5: Löslichkeit von Stickstoff in 41P; links: Isothermen-, rechts: Isobaren-Darstellung
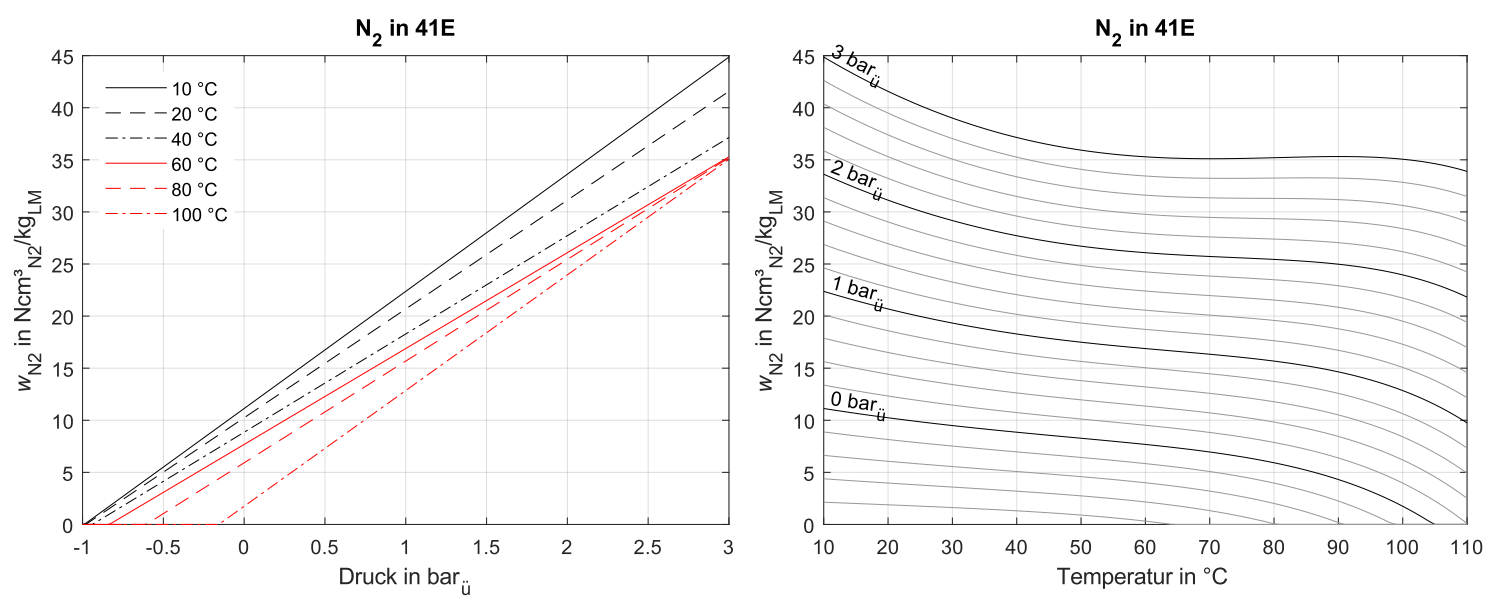

Abbildung 6.6: Löslichkeit von Stickstoff in 41P; links: Isothermen-, rechts: Isobaren-Darstellung

peraturen sowohl bei 41P als auch 41E deutlich geringer als bei Wasser. Bezogen auf einen Liter des Fluids bei 1 bar Überdruck und $20^{\circ} \mathrm{C}$ werden - im Gegensatz zu 30,8 Ncm ${ }^{3}$ Stickstoff in Wasser - nur $20,1 \mathrm{Ncm}^{3}$ bzw. $21,8 \mathrm{Ncm}^{3}$ Stickstoff in 41P bzw. $41 \mathrm{E}$ gelöst. In Abbildung 6.7 ist zum Vergleich auch noch einmal die Konzentration $c_{\mathrm{N} 2}$ dargestellt. Die prinzipiellen Verläufe unterscheiden sich nur gering. Bei 41P fällt jedoch auf, dass es zwar einen Bereich mit bei zunehmender Temperatur leicht steigendem Gasgehalt w gibt, dies jedoch für die Konzentration $c_{\mathrm{N} 2}$ aufgrund der mit Temperatur abnehmenden Dichte kaum der Fall ist.

Ergebnisse für weitere Konzentrationen seien hier nur gekürzt wiedergegeben. Eine Übersicht der Ergebnisse für 1 bar $_{\ddot{u}}$ findet sich in Abbildung 6.8. Isobaren-Darstellungen der einzelnen Gemische finden sich in Abbildung 6.9. Für das 25 gew.-\% Wasser-Propylenglykol-Gemisch (25P) ergibt sich im Gegensatz zu 41P ein wasserähnlicherer, stetig fallender Verlauf. Gleiches gilt für 25E. Bei 75P steigen Löslichkeiten mit zunehmender Temperatur stark an. Auch 75E zeigt nun im Gegensatz zu 41E eine deutlich von Wasser abweichende, mit der Temperatur ansteigende Löslichkeit. Beide reinen Gemische zeigen im dargestellten Bereich eine mit steigender Temperatur stetig steigende Löslichkeit der Isobaren. Die Löslichkeit von reinem Propylenglykol ist signifikant höher als von reinem Ethylenglykol. 

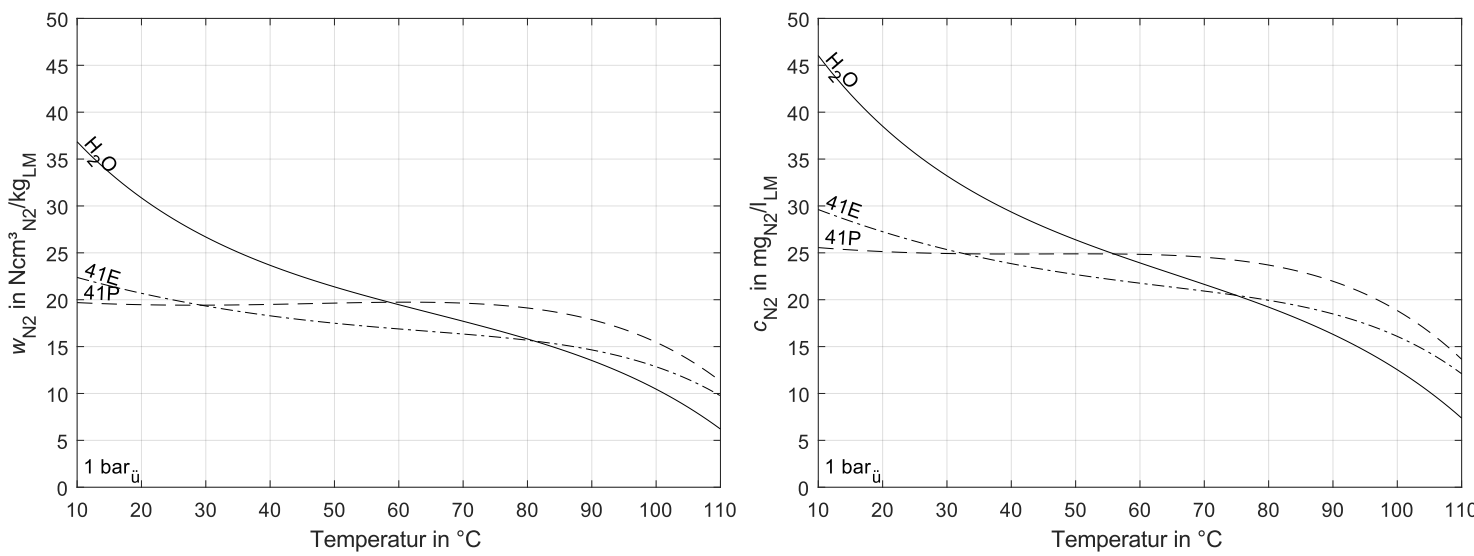

Abbildung 6.7: Löslichkeit von Stickstoff in 41P und 41E im Vergleich zu Wasser bei 1 barü; links: Gasgehalt $w$, rechts: Konzentration $c$
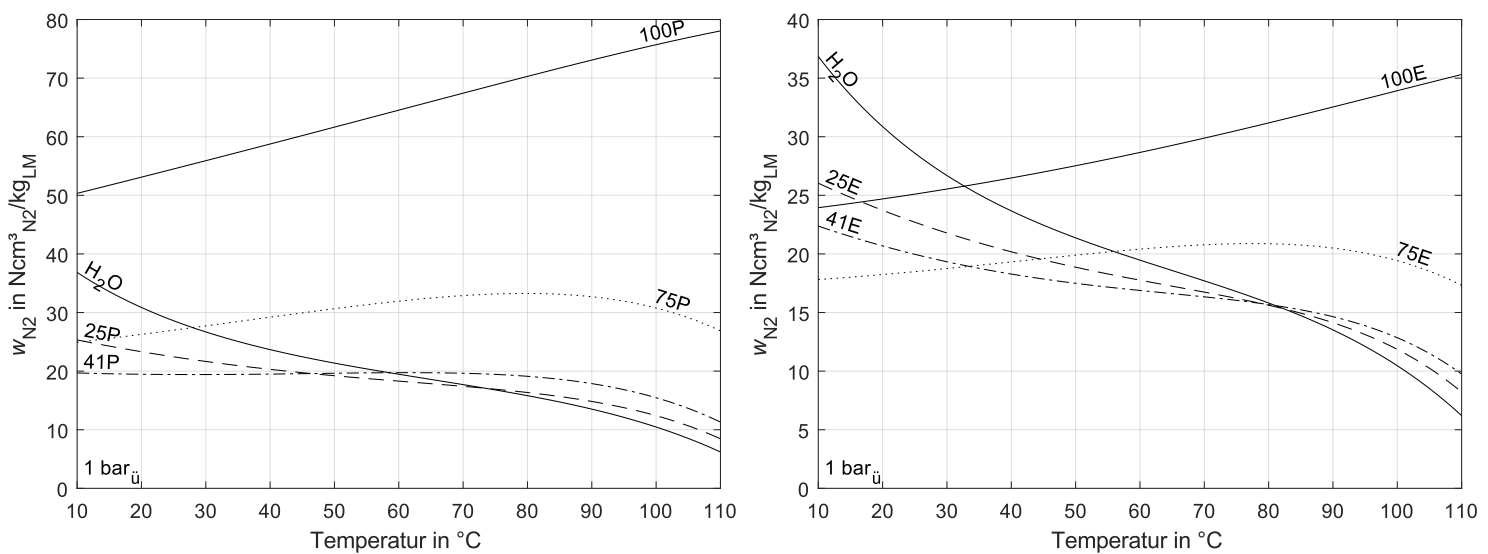

Abbildung 6.8: Löslichkeit von Stickstoff in Abhängigkeit des Glykolgehalts bei 1 bar ü; links: Propylenglykol, rechts: Ethylenglykol 

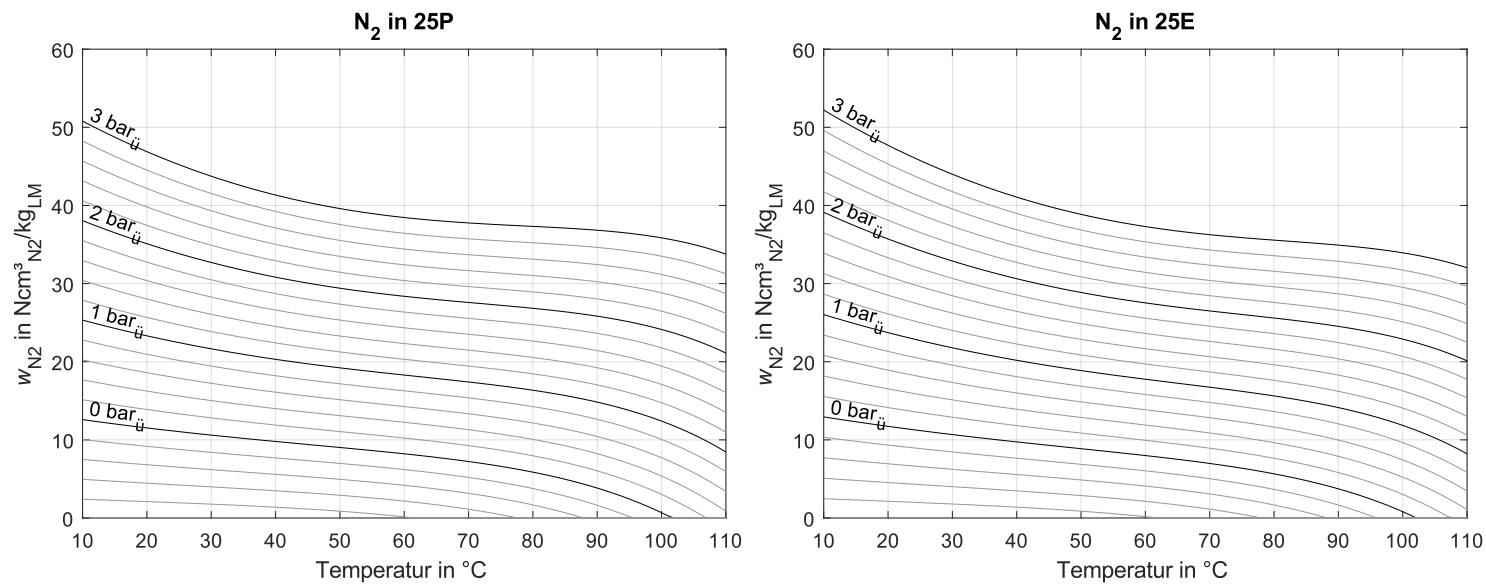

$\mathrm{N}_{2}$ in $75 \mathrm{P}$
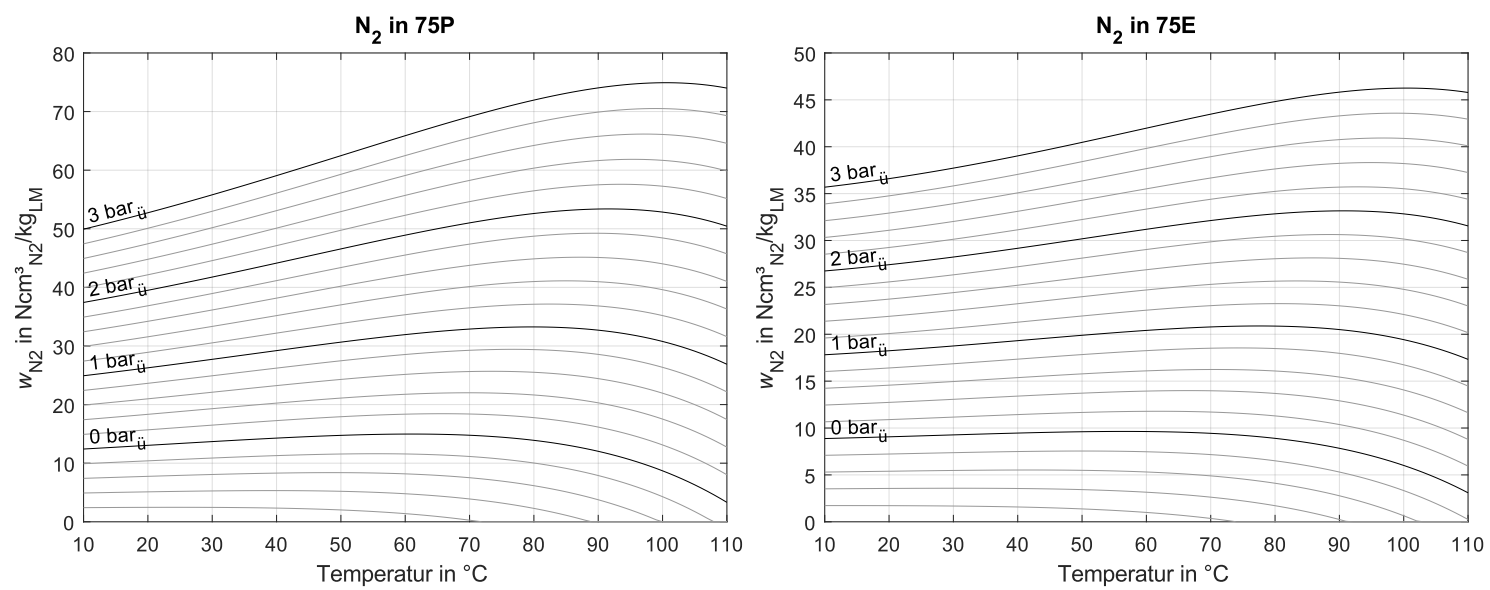

$\mathrm{N}_{2}$ in $100 \mathrm{P}$
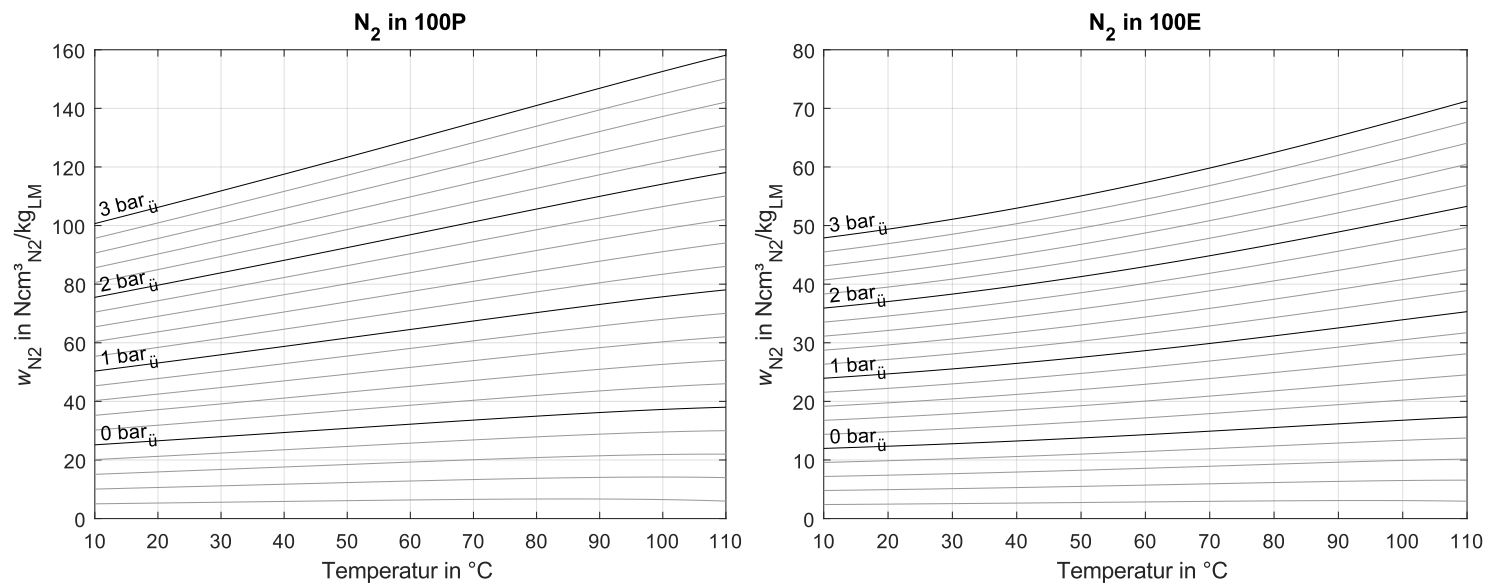

Abbildung 6.9: Löslichkeit von Stickstoff in Wasser-Glykolen mit 25, 75 und 100 gew.- \% Glykol; Isobarendarstellung 


\subsubsection{Stickstoff-Löslichkeit in Wasser-Glykolen mit Additiven}

Aufgrund der in Abschnitt 5.4 .3 beschriebenen starken Ähnlichkeit der StickstoffLöslichkeitskoeffizienten Tyfocor ${ }^{\circledR}$ LS und 41P bzw. 41T und 41E und der sich wenig unterscheidenden Dampfdrücke und Dichten (vgl. Abbildung 6.10 und Abbildung 6.11) ergeben sich sehr ähnliche Löslichkeitsverläufe im Henry-Diagramm, wie für 1 bar $_{\ddot{u}}$ in Abbildung 6.12 vergleichend dargestellt. Im Rahmen der Unsicherheit der ermittelten Regressionskurven der Löslichkeitskoeffizienten können hier keine signifikanten Unterschiede ausgemacht werden. Lediglich die Löslichkeiten bei geringen Temperaturen zeigen für die Gemische mit Additiven leicht niedrigere Werte. Die vollständigen Henry-Diagramme (Isothermen- und Isobarendarstellung) der untersuchten Gemische mit Additiven sind im Anhang A.15 (Abbildungen A.18 und A.19) ebenso dokumentiert.

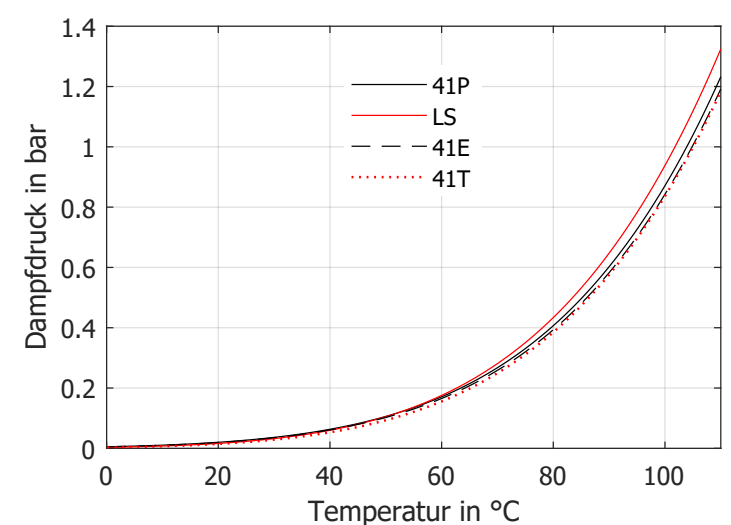

Abbildung 6.10: Vergleich der Dampfdrücke mit/ohne Additive

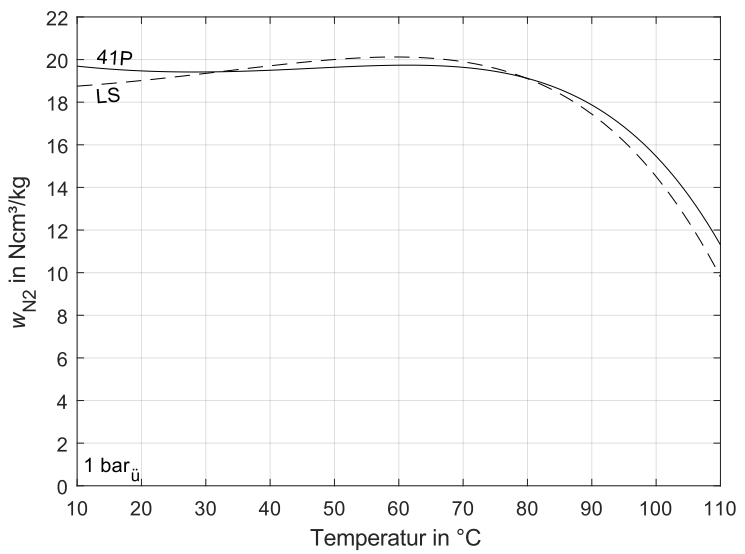

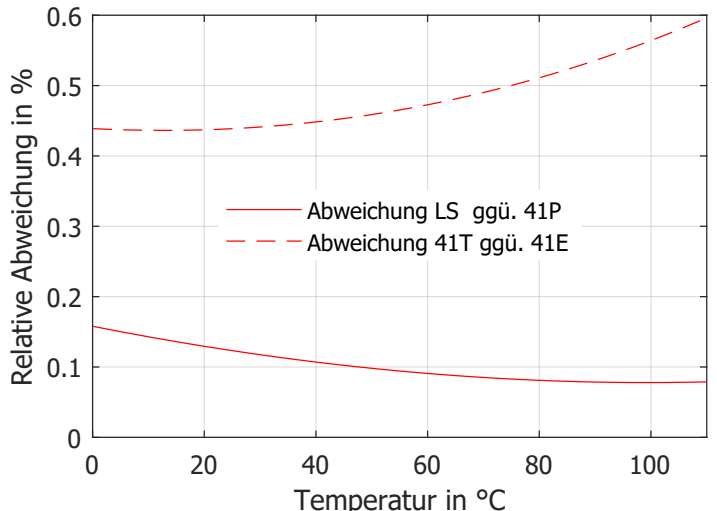

Abbildung 6.11: Abweichungen der Dichte $\frac{\rho_{L S}-\rho_{41 P}}{\rho_{41 \mathrm{P}}}$ bzW. $\frac{\rho_{41 T}-\rho_{41 \mathrm{E}}}{\rho_{41 \mathrm{E}}}$

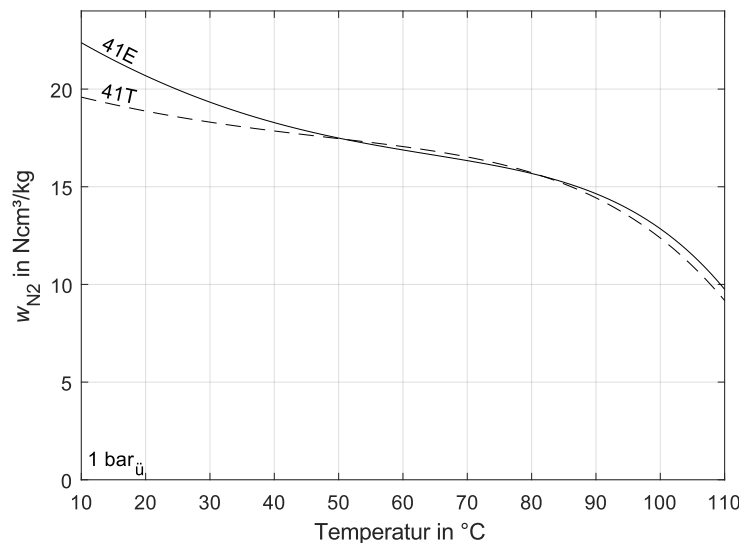

Abbildung 6.12: Löslichkeit von Stickstoff in Gemischen mit/ohne Additiven bei 1 barü; links: PropylenglykolWasser, rechts: Ethylenglykol-Wasser 


\subsubsection{Unsicherheit der Henry-Diagramme}

Die absolute Unsicherheit der in den Henry-Diagrammen dargestellten $w_{i}$ im Phasengleichgewicht ergibt sich (bei Annahme maximaler Korrelation) zu

$$
U\left(w_{i}\right)=\left(p-p_{\mathrm{LM}, \mathrm{s}}\right) \cdot U\left(\lambda_{i}\right)+\lambda \cdot U\left(p_{\mathrm{LM}, \mathrm{s}}\right)
$$

bzw. die relative Unsicherheit zu

$$
\frac{U\left(w_{i}\right)}{w_{i}}=\frac{U\left(\lambda_{i}\right)}{\lambda_{i}}+\frac{p_{\mathrm{LM}, \mathrm{s}}}{p-p_{\mathrm{LM}, \mathrm{s}}} \cdot \frac{U\left(p_{\mathrm{LM}, \mathrm{s}}\right)}{p_{\mathrm{LM}, \mathrm{s}}}=\frac{U\left(\lambda_{i}\right)}{\lambda_{i}}+\frac{p_{\mathrm{LM}, \mathrm{s}}}{p_{i}} \cdot \frac{U\left(p_{\mathrm{LM}, \mathrm{s}}\right)}{p_{\mathrm{LM}, \mathrm{s}}} .
$$

Es addieren sich also die relative Unsicherheit des Löslichkeitskoeffizienten und, mit einem Vorfaktor von $\frac{p_{L M}, s}{p_{i}}$, die relative Unsicherheit des Dampfdrucks. Insbesondere für kleine Partialdrücke im Verhältnis zum Dampfdruck kann die Unsicherheit des Dampfdruckes also eine wesentliche Rolle spielen. Die Dampfdruck-Unsicherheit von reinem Wasser-GlykolGemisch wird jedoch aufgrund der Genauigkeit der Daten der Reinstoffe (erweitere Unsicherheit: 0,03\% für Wasser in [IAPWS IF-97, 2007]; 4\% für Propylenglykol in [Rane u. a., 2016]; $5 \%$ für Ethylenglykol in [Ambrose und Hall, 1981]) und der guten Genauigkeit der Abschätzung des Gemisch-Dampfdrucks mit der Raoult-Regression als gering eingeschätzt. Interessant sind in der Praxis aber oft hohe Partialdrücke. Dort dominiert dann die Unsicherheit des Stoffwertes $\lambda$ entsprechend der Ergebnisse des vorhergehenden Kapitels 5 .

Für die Fertiggemische sind keine Genauigkeitsangaben der Dampfdruck-Messungen verfügbar. Ggf. führen insbesondere herstellungsbedingte Mischungstoleranzen hier zusätzlich zu leichten Abweichungen des Dampfdrucks, z. B. bei angenommenen Schwankungen von $\xi_{\mathrm{G}}=41,84 \pm 1,5 \%$ für $41 \mathrm{P}$ zu ca. $\pm 0,9 \%$ (im Bereich $10 \ldots 110^{\circ} \mathrm{C}$ ). In der Praxis kommen ggf. weitere Unsicherheiten in $p$ und $t$, aber auch Unsicherheiten im konkreten Gemisch (z. B. Glykol-Gehalt) mit entsprechendem Einfluss auf die Unsicherheit auf $\lambda_{i}$ und $p_{\mathrm{LM}, \mathrm{s}}$ und damit $w_{i}$ im Phasengleichgewicht hinzu.

\subsection{Einfluss von Sauerstoff auf Löslichkeitsgrenzen}

\subsubsection{Allgemeines zum Gemisch von Stickstoff und Sauerstoff}

Wie bereits in Abschnitt 6.1 .2 beschrieben, ist bei korrektem Anlagenbetrieb zumeist nur Stickstoff als Gas in nennenswerten Mengen vorhanden. Für Befüllvorgänge von Anlagen, aber auch bei atmosphärisch offenen Systemen wie z. B. einigen Drain-Back-Systemen sind jedoch Sauerstoffanteile nicht zu vernachlässigen und beeinflussen signifikant die Löslichkeitsgrenzen. Ebenso können fehlerhaft betriebene Anlagen (v. a. bei mangelhafter Druckhaltung) oder Anlagen mit diffusionsoffenen Leitungen signifikante Anteile an Sauerstoff aufweisen.

Bei $n$ Gasen lassen sich die Löslichkeitsgrenzen $w_{i}$ nur mit dem Wissen um die Mengenverhältnisse der Gase bestimmen. Neben $p, t$ müssen weitere $n-1$ Größen bekannt sein. Das können Mengenverhältnisse (a) in der Gasphase oder (b) in der Flüssigphase sein. 
Für die Gasphase kann das z. B. der Partialdruck $p_{i}$ direkt sein oder der Stoffmengenanteil, gern in der Form $y_{i, \text { tr }}:=\frac{n_{i}}{\sum_{k \neq \mathrm{LM}} n_{k}}$ verwendet. Dann kann die Löslichkeit leicht über

$$
w_{i}(p, t)=\lambda_{i}(t) \cdot\left(p-p_{\mathrm{LM}, \mathrm{s}}\right) \cdot y_{i, \mathrm{tr}}
$$

bestimmt werden.

In der Praxis ist durch Gasprobenahme oft die Zusammensetzung der Gase in der Flüssigkeit bekannt, z. B. in Form von $x_{i}$ oder $w_{i}$ oder auch $c_{i}$. Auch dort lässt sich (unter der Annahme, dass im Phasengleichgewicht die gleiche Gaszusammensetzung herrscht) die Löslichkeitsgrenze für jedes Gas bestimmen. Hier muss $y_{i, \text { tr }}$ erst ermittelt werden. Entsprechend der Herleitung im Anhang A.11 lässt sich die Zusammensetzung der im Phasengleichgewicht korrespondierenden Gasphase dann wie folgt beschreiben

$$
y_{i, \mathrm{tr}}=\frac{1}{1+\sum_{j \neq i} \frac{\lambda_{i}}{\lambda_{j}} \cdot \frac{n_{j}^{\prime}}{n_{i}^{\prime}}},
$$

wobei für die Stoffmengenverhältnisse der gelösten Gase $\frac{n_{j}^{\prime}}{n_{i}^{\prime}}$ auch $\frac{n_{j}^{\prime}}{n_{i}^{\prime}}=\frac{x_{j}}{x_{i}}=\frac{x_{j, t r}}{x_{i, t r}}$ und (durch die Vereinfachung $v_{0, \mathrm{~N} 2, \mathrm{~N}}=v_{0, \mathrm{O} 2, \mathrm{~N}}$ ) auch $\frac{n_{j}^{\prime}}{n_{i}^{\prime}}=\frac{w_{j}}{w_{i}}=\frac{w_{j, \text { tr }}}{w_{i, \mathrm{tr}}}$ eingesetzt werden kann.

Eine Übersicht der Umrechnungen für die in dieser Arbeit genannten Gehaltsgrößen findet sich in Tabelle 6.2.

Tabelle 6.2: Bestimmung der Gaszusammensetzung in der Gasphase $y_{i, \text { tr }}$

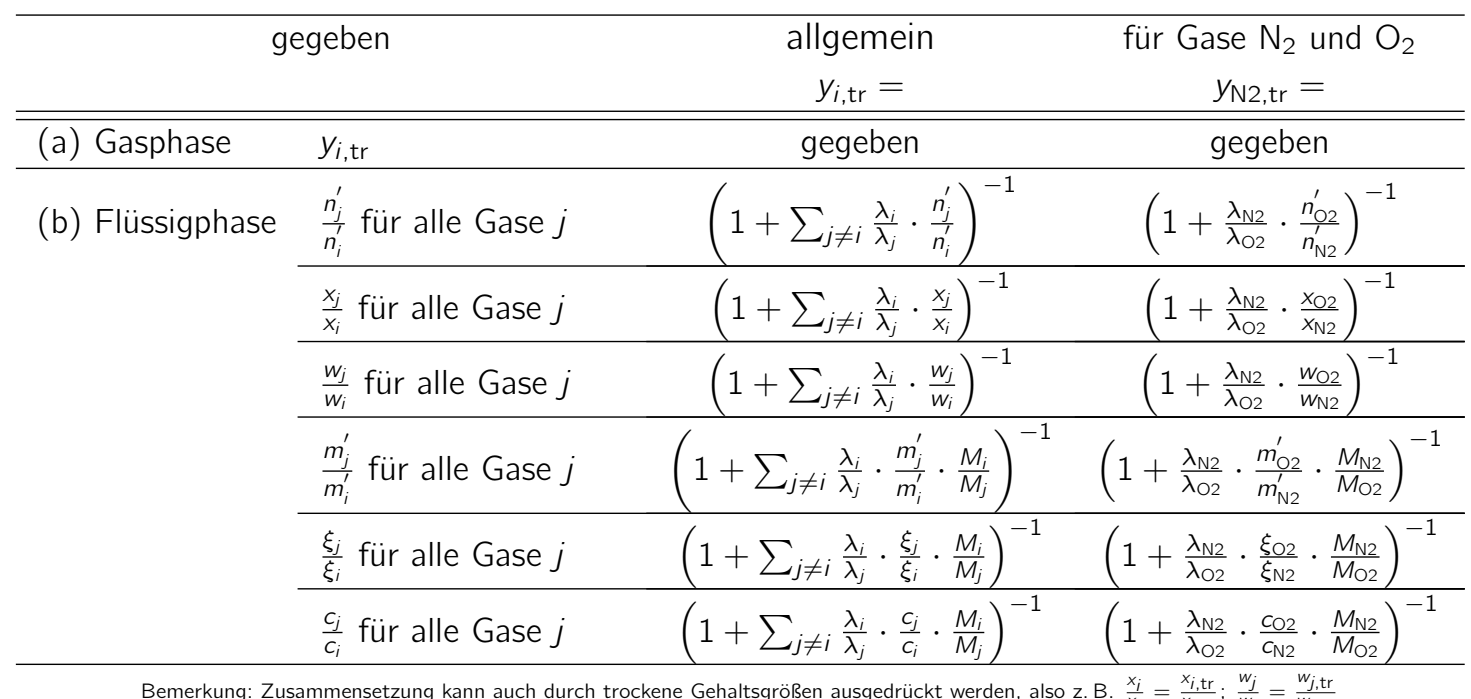

\subsubsection{Gleichgewicht mit Luft}

Für den Fall, dass das Medium direkt mit Umgebungsluft im Gleichgewicht steht, müssen die Stoffmengenanteile $y_{i, t r}$ der Luft berücksichtigt werden. Die Luftzusammensetzung ist bereits in Tabelle 1.1 vorgestellt worden. Oft wird die Luftzusammensetzung, wie auch hier nach [Wallace und Hobbs, 2006], nur in Volumenanteilen $\Phi_{i, \text { tr }}^{V}$ angegeben. Jedoch zeigt die Tabelle 6.3, dass Stoffmengenanteile $y_{i, \text { tr }}$ davon kaum abweichen. 
Tabelle 6.3: Zusammensetzung trockener Luft (wesentliche Bestandteile) - exakt und vereinfacht

\begin{tabular}{|c|c|c|c|}
\hline Bestandteil & $\begin{array}{l}\text { Volumenanteil } \\
\text { in tr. Luft }{ }^{a}\end{array}$ & $\begin{array}{l}\text { Entspr. Molanteil } \\
\text { in tr. Luft }{ }^{b, c}\end{array}$ & $\begin{array}{l}\text { Vereinfachung } \\
\text { Binäres Gemisch }\end{array}$ \\
\hline & $\Phi_{i, \operatorname{tr}}^{\bigvee}$ & $y_{i, \mathrm{tr}}$ & $y_{i, \mathrm{tr}}$ \\
\hline Stickstoff & $78,08 \%$ & $78,072 \%$ & $79 \%$ \\
\hline Sauerstoff & $20,95 \%$ & $20,957 \%$ & $21 \%$ \\
\hline Argon & $0,93 \%$ & $0,930 \%$ & - \\
\hline Kohlendioxid & $403 \mathrm{ppm}=0,0403 \%$ & $0,04049 \%$ & - \\
\hline $\mathrm{Ne}, \mathrm{He}, \mathrm{CH}_{4}, \mathrm{Kr}, \ldots$ & $<30$ ppm & - & - \\
\hline $\begin{array}{l}{ }^{a} \text { aus Tabelle } 1.1 \\
{ }^{b} \text { Berechnung aus Volumen } \\
{ }^{c} \text { Berechnung mit stoffspez }\end{array}$ & $\begin{array}{l}\text { V bei Annahme Standardbedi } \\
\text { molaren Volumina } v_{i, 0} \text { aus }[K\end{array}$ & $\begin{array}{l}\text { ungen }\left(25^{\circ} \mathrm{C} ; 1,01325 \text { bar }\right) \\
\text { tzschmar, 2010] mit } y_{i, t r}=\end{array}$ & \\
\hline
\end{tabular}

Zur Beschreibung der Löslichkeit kann in den meisten Fällen mit für technische Belange ausreichender Genauigkeit auf die Berücksichtigung weiterer Gasbestandteile verzichtet werden (Annahme Binäres Gemisch). Dazu sollen die in der Tabelle 6.3 angegebenen, gerundeten Anteile für Sauerstoff und Stickstoff genutzt werden. In der nachfolgenden Tabelle 6.4 sind die sich ergebenden Gasgehalte unter Umgebungsbedingungen dargestellt. Hier zeigt sich, dass sich durch die Vereinfachung Binäres Gemisch nur geringe Änderungen auf das ermittelte (Gesamt)-Volumen der gelösten Gase ergeben. Etwas höhere Unterschiede zwischen vereinfachter und detaillierter Betrachtung ergeben sich für die Gesamtsumme. Für Wasser beträgt hier die Abweichung durch vereinfachte Betrachtung als binäres Gemisch trotzdem lediglich $2,5 \%$.

Tabelle 6.4: Gelöste Gase (angegeben als Volumen im Normzustand 1,01325 bar und $0{ }^{\circ} \mathrm{C}$ ) für Gleichgewicht mit Luftatmosphäre bzw. Stickstoff für 1 Liter Wärmeträgermedium bei $25^{\circ} \mathrm{C}$ und 1,01325 bar

\begin{tabular}{|c|c|c|c|c|c|c|c|}
\hline \multirow{2}{*}{$\begin{array}{l}\text { Angaben in } \mathrm{cm}^{3} \text { (Normvolumen) } \\
\text { Wärmeträger }\end{array}$} & \multirow{2}{*}{$\frac{\text { Luft detailliert }}{\mathrm{H}_{2} \mathrm{O}}$} & \multicolumn{3}{|c|}{ Luft vereinfacht (Binäres Gemisch) } & \multicolumn{3}{|c|}{ Stickstoff } \\
\hline & & $\mathrm{H}_{2} \mathrm{O}$ & $41 \mathrm{P}$ & $41 \mathrm{E}$ & $\mathrm{H}_{2} \mathrm{O}$ & $41 \mathrm{P}$ & $41 \mathrm{E}$ \\
\hline \multirow[t]{2}{*}{ Stickstoff } & 11,17 & 11,30 & 7,41 & 7,46 & 14,30 & 9,38 & 9,44 \\
\hline & $(63,4 \%)$ & $(65,7 \%)$ & $(64,2 \%)$ & $(66,1 \%)$ & & & \\
\hline \multirow[t]{2}{*}{ Sauerstoff } & 5,88 & 5,89 & 4,13 & 3,83 & - & - & - \\
\hline & $(33,3 \%)$ & $(34,3 \%)$ & $(35,8 \%)$ & $(33,9 \%)$ & & & \\
\hline \multirow[t]{2}{*}{ Argon } & 0,29 & - & - & - & - & - & - \\
\hline & $(1,6 \%)$ & & & & & & \\
\hline \multirow[t]{2}{*}{ Kohlendioxid } & 0,30 & - & - & - & - & - & - \\
\hline & $(1,7 \%)$ & & & & & & \\
\hline SUMME & 17,64 & 17,19 & 11,54 & 11,29 & 14,30 & 9,38 & 9,44 \\
\hline
\end{tabular}

Ähnlich wie bei Wasser erhöht sich durch die signifikant höhere Löslichkeit von Sauerstoff die gelöste Gasmenge deutlich gegenüber reinem Stickstoff. Dabei nimmt Sauerstoff trotz des Gaspartialdruckanteils von $21 \%$ bei Wasser bereits 34,3\% des gelösten Gasnormvolumens, bei den Wasser-Glykolen mit 35,8\% (41P) bzw. 33,9\% (41E) sehr ähnliche Anteile ein.

Dieses Verhältnis ändert sich auch bei hohen Temperaturen nur wenig, da das Verhältnis der Löslichkeitskoeffizienten $\frac{\lambda_{\mathrm{O} 2}}{\lambda_{\mathrm{N} 2}}$ sich nur wenig ändert, vgl. Abbildung 6.13. 


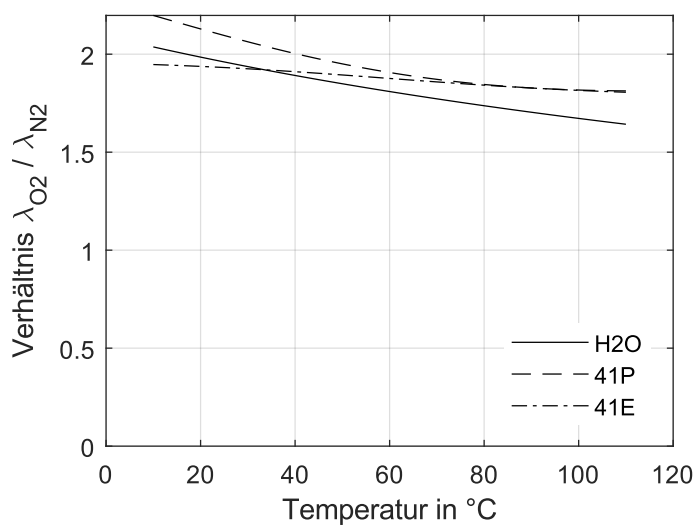

Abbildung 6.13: Verhältnis der Löslichkeitskoeffizienten Sauerstoff und Stickstoff

Im folgenden wurden Henry-Diagramme für die gesamte gelöste Gasmenge $w_{\text {ges }}$ erstellt. In der Darstellung des summarischen Gasgehalts von Sauerstoff und Stickstoff stellt sich einer der genannten Vorteile der Größe w gegenüber der Konzentration c heraus: Das summarische gelöste Normvolumen von Stickstoff und Sauerstoff ist anschaulich, da die Thematik der gelösten Gase immer auf die Menge desorbierbarer Gase fokussiert. Die summarische gelöste Masse der Gases ist hingegen wenig anschaulich, da die Masse das Vermögen, freie Gase zu bilden, eben für Sauerstoff und Stickstoff nicht gleichwertig ausdrückt.
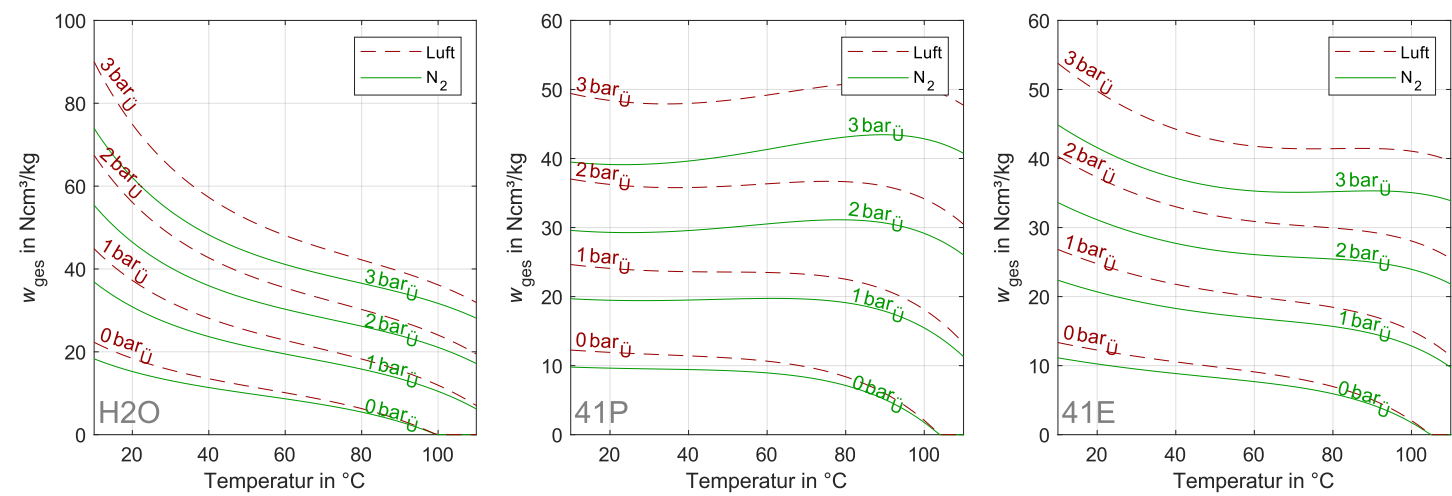

Abbildung 6.14: Gesamtgasgehalte w bei Gleichgewicht von Wasser, 41P bzw. 41E mit Stickstoff bzw. Luftatmosphäre

\subsubsection{Sauerstoff-Anteil nach der Anlagenbefüllung}

Da Sauerstoff allmählich aus dem System durch chemische Bindung entfernt wird, ist Sauerstoff in Anlagen, wenn, dann während und unmittelbar nach der Befüllung der Anlage zu beachten. Hier soll nun die reale Größenordnung des zu berücksichtigenden Sauerstoffanteils untersucht werden.

Da bei Absorption Sauerstoff (zumindest bei quasistationärer Betrachtung des Vorgangs) überproportional gelöst wird, ändert sich das Sauerstoff-Stickstoff-Verhältnis in der korrespondierenden Gasphase. Dies hat schlussendlich Auswirkungen auf die Zusammensetzung Sauerstoff-Stickstoff in der Flüssigphase. Aufgrund der physikalischen Komplexi- 
tät des Befüllprozesses können hier jedoch keine analytischen Aussagen getroffen werden.

In [Heymann und Rühling, 2013] wird der Befüllvorgang und die Entlüfterwirkung von Solarthermieanlagen ausführlich experimentell untersucht. Dabei wird auch die Gaskonzentration in der Solarflüssigkeit ermittelt.

Hier sollen die Ergebnisse der Gaskonzentrationsmessungen unmittelbar nach Befüllung der Anlagen (Nullprobe NP) näher untersucht werden. Dazu werden alle Befüllversuche für die sogenannte Technikumsanlage (bei unterschiedlichen Konfigurationen der Entlüfter und verschiedenen statischen Höhen der Anlage) ausgewertet. In allen Versuchen wird die Anlage mittels Druckbefüllung mit einer Befüllstation befüllt, entlüftet und umgewälzt. Versuche wurden mit Wasser und anschließend mit Tyfocor ${ }^{\circledR}$ LS als Wärmeträgermedium durchgeführt. Die absoluten gelösten Gasmengen der Nullprobe hängen stark von dem jeweiligen Befüllprozess und den statischen Höhen ab. Dies soll hier nicht weiter vertieft werden.

In Abbildung 6.15 sind jedoch die in [Heymann und Rühling, 2013] gemessenen SauerstoffAnteile (zum Zeitpunkt nach Befüllung der Anlage) für die Versuche mit Wasser (links) bzw. Tyfocor ${ }^{\circledR}$ LS (rechts) als Histogramm dargestellt. Der dargestellte Sauerstoffgehalt wurde in den Versuchen von [Heymann und Rühling, 2013] durch Probenahme mit anschließender gaschromatographischer Analyse ermittelt. Zusätzlich sind eingezeichnet: Der Anteil an Sauerstoff für Gleichgewicht mit Luft-Atmosphäre, also $y_{\mathrm{O} 2, \mathrm{tr}}=0,21$, und der Anteil von Sauerstoff bei einem Verhältnis in der Flüssigkeit von $x_{O 2, t r}=0,21$. Letzteres ist der Fall, wenn in einem geschlossenen System die Komponenten gasfreies Fluid und Luft gemischt und so verdichtet werden, bis die Gasphase vollständig verschwindet. Dann entspricht die Zusammensetzung Stickstoff-Sauerstoff der Flüssigphase genau der Zusammensetzung in der Luft. Dies kann also ebenso als gewisse hypothetische Untergrenze gedeutet werden, auch wenn der Befüllprozess durch lokal ablaufende Desorptions- und Absorptionsprozesse und Stoffaustausch deutlich komplexer ist und zumindest rein theoretisch auch ähnlich dem Prozess der Destillation Aufkonzentrationsprozesse, die über die Grenzen hinaus gehen, stattfinden könnten.
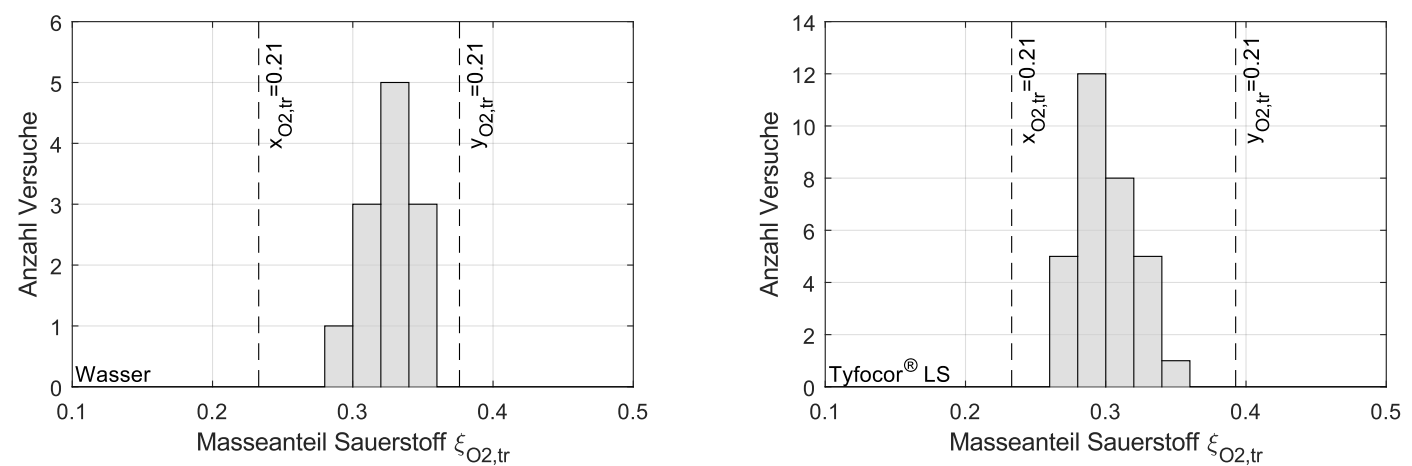

Abbildung 6.15: Anteil gelöster Sauerstoff der Nullproben als Histogramm für die Versuche; links: Versuche mit Wasser, rechts: Versuche mit Tyfocor ${ }^{\circledR}$ LS; Eigene Auswertungen auf Basis von Daten aus [Heymann und Rühling, 2013]. Zur Bestimmung der Obergrenze bei $y_{02, t r}=$ 0,205 wurden Löslichkeitskoeffizienten bei $20^{\circ} \mathrm{C}$ verwendet. Aufgrund des fehlenden $\mathrm{O}_{2-}$ Löslichkeitskoeffizienten für Tyfocor ${ }^{\circledR}$ LS wurden vereinfacht Löslichkeitskoeffizienten für 41P verwendet.

Bei den Versuchen mit Tyfocor ${ }^{\circledR}$ LS wurden hier nur die ersten 31 der in [Heymann und Rühling, 2013] durchgeführten Versuche verwendet. Bei anschließenden weiteren Befüllversuchen sind zusätzliche Versuche mit Stagnation der Solarthermieanlage eingeschoben. Diese 
verändern die Gas-Ausgangskonzentration des Tyfocor $^{\circledR}$ LS für den sich anschließenden Befüllversuch so stark, dass die Ausgangsgasbeladung dieser Versuche hier als nicht repräsentativ für einen klassischen Befüllvorgang gesehen wird.

Zusammenfassend kann festgehalten werden, dass der Sauerstoffanteil $\xi_{\mathrm{O} 2 \text {,tr }}$ nach der Befüllung sowohl für Wasser als auch Tyfocor $^{\circledR}$ LS einen wesentlichen Teil zum Gasgehalt beiträgt und sich im Bereich zwischen den genannten theoretischen Grenzen einordnet. Damit ist für die Analyse von Befüllversuchen bzw. unmittelbar an die Befüllung anschließenden Prozessen der Sauerstoff in den Löslichkeitsbetrachtungen zwingend zu berücksichtigen.

Um jedoch allgemeingültige Aussagen zum Sauerstoffgehalt nach Befüllung abzuleiten und konkrete Aussagen zu treffen, welcher Sauerstoffanteil sich je nach Art der Anlagenbefüllung und des Anlagenaufbaus einstellt, wären weitere Versuche unter Sicherstellung einer gleichen und für die Praxis repräsentativen Ausgangsbeladung des Fluids notwendig.

\subsubsection{Chemische Bindung durch Inhibitor}

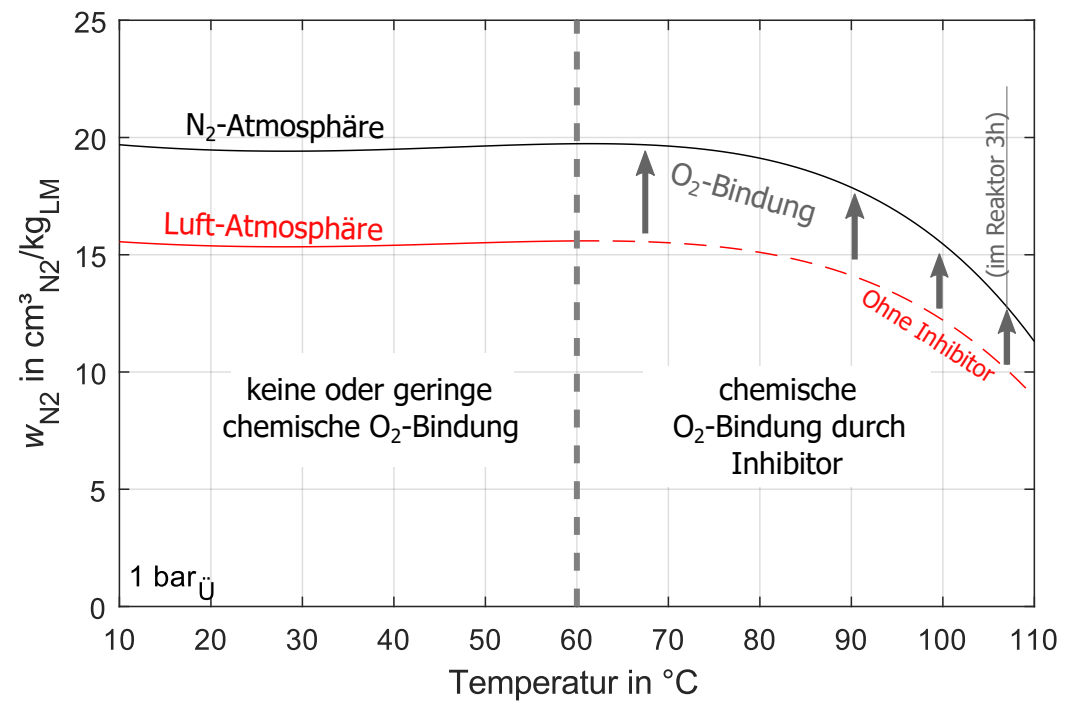

Abbildung 6.16: Wirkungen des Inhibitors: Löslichkeitsgrenzen von Stickstoff in 41 gewichtsprozentigem Wasser-Glykol-Gemisch bei $100 \%$ Stickstoff oder Luft (hier nur Stickstoff-SauerstoffGemisch); Angaben für 1 bar Überdruck.

Im Folgenden soll nun die Wirkung der Inhibitoren, wie sie in Tyfocor ${ }^{\circledR}$ LS enthalten sind, auf die Löslichkeit am Beispiel eines Wasser-Glykol-Gemischs mit 41 Gewichtsprozent Glykol diskutiert werden. Dazu sollen auch die im vorhergehenden Abschnitt gewonnenen Erkenntnisse zum Sauerstoffanteil nach Befüllung der Anlage genutzt werden.

In Abbildung 6.16 ist dazu als obere Kurve die experimentell bestimmte Löslichkeitsgrenze für

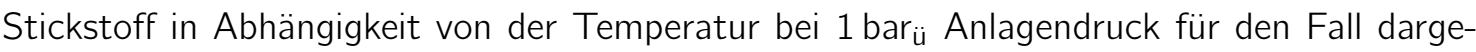
stellt, dass nur Stickstoff vorhanden ist. Für den Fall, dass die Komponenten Sauerstoff und 
Stickstoff vorhanden sind (Verhältnis von $\mathrm{O}_{2}$ und $\mathrm{N}_{2}$ der Gasphase entspricht der trockenen Luft), gelte die untere, rote Kurve. ${ }^{2}$

Den Zustand nach der Befüllung bildet demnach tendenziell eher die untere Kurve ab, da beide Gase durch den Luft-Kontakt des Gemischs gelöst werden. Entsprechend des vorhergehenden Abschnitts stellt die untere Kurve eine Grenzbetrachtung dar (maximal möglicher Sauerstoffanteil).

Aufgrund des geringeren Partialdrucks an Stickstoff bei Luft-Atmosphäre ist auch die Löslichkeit des Stickstoffs hier signifikant geringer gegenüber reiner StickstoffAtmosphäre.

Wird das Fluid nun entsprechend dieser Kurve für Luft-Atmosphäre erwärmt (vereinfachend sei hier der Druck konstant), so sinkt die Löslichkeit von Stickstoff im Gegensatz zum Lösungsmittel Wasser wie bereits beschrieben ab ca. $70 \ldots 80^{\circ} \mathrm{C}$. Hinzu kommt aber nun, dass beim Erwärmen von Tyfocor ${ }^{\circledR}$ LS der Sauerstoff zügig chemisch gebunden wird (vgl. Abschnitt 5.5.3). In der Abbildung 6.16 ist exemplarisch für $107^{\circ} \mathrm{C}$ auch die ermittelte Zeit von 3 Stunden für die nahezu vollständige chemische Bindung von Sauerstoff unter Versuchsbedingungen eingetragen. Je nach Zeitdauer sinkt also der physikalisch gelöste Sauerstoff. Es kann demnach mehr Stickstoff gelöst werden: Die Löslichkeitgrenze von Stickstoff wandert also von der unteren Linie in Abbildung 6.16 (Luft-Atmosphäre) zur oberen Linie (StickstoffAtmosphäre). Dies ist ein weiterer Grund, warum beim Aufheizen einer Solaranlage theoretisch keine zusätzliche Gasdesorption zum Ziele der Anlagenentlüftung bewirkt werden kann. Stattdessen wird beim Aufheizen durch die chemische Bindung der gelöste und freie Sauerstoff allmählich entfernt und das Fluid kann mehr Stickstoff aufnehmen und damit das Volumen ggf. vorhandener Stickstoffblasen reduzieren.

Auch bei allen anderen Medien reduziert sich durch langsam ablaufende Korrosionsprozesse (bei korrekter Druckhaltung und Anlagendichtheit) langfristig der gelöste Sauerstoffgehalt stark. Es stellt sich damit demnach bezüglich der Stickstoff-Löslichkeit langfristig der gleiche Effekt wie bei der schneller ablaufenden chemischen Bindung von Tyfocor ${ }^{\circledR}$ LS ein.

\subsection{Einige Ableitungen für energietechnische Wasser-Glykol-Kreisläufe und validierende Beispiele}

\subsubsection{Gaslöslichkeit in realen Anlagen}

Die Auswirkungen der ermittelten Löslichkeitskoeffizienten auf das Löslichkeitsverhalten in realen Anlagen soll hier aufgrund der Komplexität der physikalischen Prozesse nur auf wenige Einflüsse reduziert und exemplarisch analysiert werden. Eine sehr wichtige Einflussgröße auf die Löslichkeit in energietechnischen Anlagen ist das Verhalten der Druckhaltung. In den folgenden Beispielen wird die statische Druckhaltung mittels Membranausdehnungsgefäßen (MAG), wie es in kleineren und mittleren Anlagen üblich ist, betrachtet.

Für die Betrachtung sollen Strömungsdruckverluste nicht berücksichtigt werden. Ebenso wird die Temperaturverteilung bzw. Temperaturspreizung des Mediums in der Anlage vernachlässigt. Das Medium sei also auf einer einheitlichen Temperatur. Weiterhin werden freie

\footnotetext{
${ }^{2}$ Da die Kurve für Luft-Atmosphäre nicht für LS sondern nur für 41P aus den Messdaten ermittelt werden kann, wird aus Konsistenzgründen auch die Stickstoff-Kurve für 41P (und nicht LS) dargestellt. Diese unterscheiden sich aber nur marginal.
} 
Gasblasen, die ebenso Einfluss auf die Druckhaltung haben, ausgeschlossen. Dynamische Effekte in der Anlage wie Absorption, Desorption, Diffusion und Gastransport sollen nicht untersucht werden. Lediglich der Anlagenschlechtpunkt im Sinne der Gaslöslichkeit wird herausgegriffen und soll unter unterschiedlichen Betriebsbedingungen betrachtet werden. Selbst aus dieser sehr reduzierten, aber eben auch einfachen Betrachtung zum Verhalten der Gase lassen sich jedoch schon sehr wichtige Erkenntnisse zum Verhalten der Anlage gewinnen.

Die Druckänderung in Abhängigkeit der mittleren Medientemperatur $t_{x}$ lässt sich bei Gültigkeit von $p \cdot V=$ const für das Gasvolumen des MAG in einem solchen System leicht ermitteln. Für bekannten Anfangsdruck der Anlage $p_{a}$, definiert als Anlagendruck bei minimaler Medientemperatur $t_{\min }$, und Enddruck $p_{\mathrm{e}}$, definiert als Anlagendruck bei maximaler Medientemperatur $t_{\max }$, kann man für beliebige Zwischenzustände mit der Temperatur $t_{x}$ den Druck der Druckhaltung bestimmen ${ }^{3}$

$$
p\left(t_{x}\right)=\frac{p_{\mathrm{a}}}{1-\frac{\frac{\rho\left(t_{\min }\right)}{\rho\left(x_{)}\right)}-1}{f_{\mathrm{Z}} \cdot\left(\frac{\rho\left(t_{\min }\right)}{\rho\left(t_{\max }\right)}-1\right)} \cdot\left(1-\frac{p_{\mathrm{a}}}{p_{\mathrm{e}}}\right)} .
$$

Die Betrachtungen sind demnach unabhängig von der Größe der Anlage. ${ }^{4}$ Die Herleitung findet sich in Anhang A.12. Diese Gleichung findet sich für den Spezialfall $p_{\mathrm{e}}=p_{\mathrm{e}, \max }$ und vereinfacht für $f_{Z}=1$ auch in [VDI 4708] Anhang E. Der Faktor $f_{Z}$ ist hier definiert als

$$
f_{\mathrm{Z}}:=\frac{V_{\mathrm{Z}}+V_{\mathrm{e}}}{V_{\mathrm{e}}}
$$

und berücksichtigt die größeren Ausdehnungsvolumina der Anlage, wenn zusätzlich zum Wärmedehnungsvolumen $V_{\mathrm{e}}$ des Mediums prozessbedingte Zusatzvolumina $V_{Z}$ wie das Verdampfungsvolumen bei der Solarthermie aufgenommen werden müssen. Die Gleichung ist nur bis zu Temperaturen $t_{x}$ gültig, bis Verdampfungsvolumina auftreten. Für Anlagen ohne Zusatzvolumina, wie z. B. Rückkühlwerken, ist $f_{\mathrm{Z}}=1$.

Als Anfangsdruck am Hochpunkt wird in Anlehnung an [VDI 4708] vereinfacht $p_{a, H P}=$ 0,5 bar $_{\ddot{u}}$ angenommen. ${ }^{5}$ Der Anfangsdruck am MAG berechnet sich mit der Höhendifferenz $H_{\text {st }}$ zwischen Hochpunkt und Position der Druckhaltung (vgl. Abbildung 6.17) zu

$$
p_{\mathrm{a}}=p_{\mathrm{a}, \mathrm{HP}}+\rho\left(t_{\min }\right) \cdot g \cdot H_{\mathrm{st}}
$$

Bei gegebenem Druckspiel im Betriebsbereich $\Delta p_{\mathrm{e}-\mathrm{a}}=p_{\mathrm{e}}-p_{\mathrm{a}}$ ergibt sich

$$
p_{\mathrm{e}}=p_{\mathrm{a}}+\Delta p_{\mathrm{e}-\mathrm{a}}
$$

\footnotetext{
${ }^{3}$ Beachte, dass die in [VDI 4708] in Gleichung 13 angegebene Gleichung für $p_{a}$ lediglich einen Zwischenschritt in der VDI-Berechnung beschreibt. Diese wird dort nur als eine Hilfsgröße verwendet, hat aber mit dem schlussendlich realen $p_{\mathrm{a}}$ nichts zu tun.

${ }^{4}$ Auch die Größe des MAG ist bei gegebenem Enddruck pe keine Eingangsgröße in dieser Berechnung, sondern ergibt sich als Wert.

${ }^{5}$ Für Anlagen mit Nachdruckhaltung kommt zusätzlich der Pumpendifferenzdruck hinzu.
} 


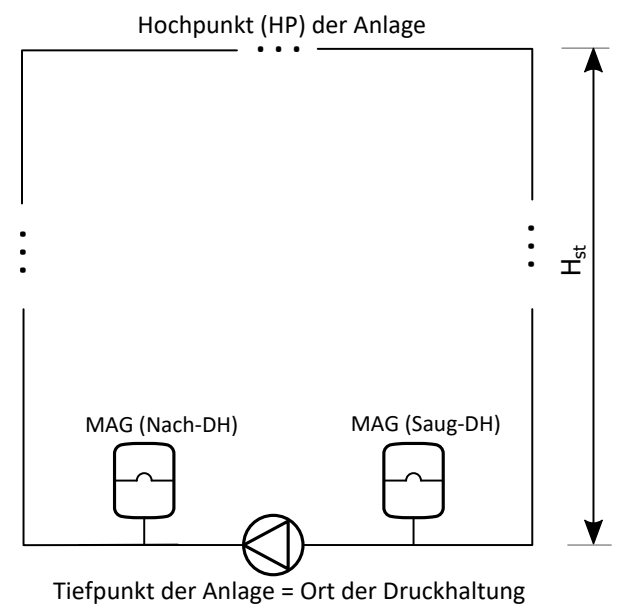

Abbildung 6.17: Prinzipskizze des Fluidkreislaufs der Anlage mit MAG-Position je nach Art der Druckhaltung $(\mathrm{DH})$

Für die Betrachtungen zur Löslichkeitsgrenze ist der Hochpunkt der Anlage der signifikante Punkt. Der Druckverlauf in diesem Punkt ergibt sich mit dem Druck der Druckhaltung $p\left(t_{x}\right)$ aus Gleichung $6.10 \mathrm{zu}$

$$
p_{x, \mathrm{HP}}=p_{x}-\rho\left(t_{x}\right) \cdot g \cdot H_{\mathrm{st}} .
$$

Die Druckänderung am Hochpunkt ergibt sich im Wesentlichen aus dem durch Temperaturdehnung geänderten Druck der Druckhaltung, nur zu einem geringen Teil aus der veränderten hydrostatischen Druckauflastung bis zur Druckhaltung. ${ }^{6}$

Der Verlauf der sich dadurch je nach $\left(p_{x}, t_{x}\right)$ ergebenden Löslichkeitsgrenze

$$
W_{H P, \max }\left(p_{x}, t_{x}\right)
$$

ist am Beispiel eines Rückkühlkreises mit 41-gewichtsprozentigem Ethylenglykol-WasserGemisch (41E) und Stickstoff als einziges relevantes Gas, angenommenen Temperaturgrenzen der Anlage von $-15^{\circ} \mathrm{C}$ und $+45^{\circ} \mathrm{C}$ und verschiedenen praxisnahen Annahmen, siehe auch Tabelle 6.5, in Abbildung 6.18 dargestellt. Alle Ergebnisse beziehen sich auf Stickstoff als alleinige Gaskomponente. ${ }^{7}$ Dargestellt sei hier nur der Bereich ab $10^{\circ} \mathrm{C}$, für den auch Löslichkeitskoeffizienten bestimmt wurden. ${ }^{8}$ Verschiedene statische Höhen $H_{\text {st }}$ sowie Druckspiele $\Delta p_{\mathrm{e}-\mathrm{a}}$ und damit unterschiedliche Anfangs- und Enddrücke sollen die Bandbreite möglichen Verhaltens der Löslichkeit von realen Anlagen aufzeigen.

Aufgrund der Darstellung als Henry-Diagramm mit eingezeichneten Isobaren, lässt sich auch der Druckverlauf am Hochpunkt der Anlage in Abhängigkeit der Temperatur $t_{x}$ des Mediums ebenso nachvollziehen.

\footnotetext{
${ }^{6}$ Bei einer statischen Höhe von 10 Metern ändert sich im Bereich von 10 bis $110^{\circ} \mathrm{C}$ die hydrostatische Druckauflastung bei Tyfocor $^{\circledR}$ LS um ca. 0,072 bar, bei Wasser um 0,048 bar.

${ }^{7}$ Ergebnisse für Stickstoff-Sauerstoff Gemische sind jedoch aufgrund gleichartiger Löslichkeitsverläufe gleichartiger Natur.

${ }^{8}$ Weitere Annahme: Aufgrund der beschränkten Gültigkeitsbereiche der Dichte-Gleichungen für 41P und 41E: Für $t \leq 10^{\circ} \mathrm{C}$ wurden Korrelationen aus [Melinder, 2010] genutzt, für $t>10^{\circ} \mathrm{C}$ hingegen die Regressionsgleichungen, die auf eigenen Dichtemessungen basieren (siehe Anhang A.14).
} 
Tabelle 6.5: Übersicht der betrachteten Kreisläufe; Temperaturwerte in Anlehnung an [VDI 4708]

\begin{tabular}{lrrrrr}
\hline & Kühlkreis 1 & Kühlkreis 2 & Heizung & Solarthermie 1 & Solarthermie 2 \\
\hline Medium & $41 \mathrm{E}$ & $\mathrm{H} 2 \mathrm{O}$ & $\mathrm{H} 2 \mathrm{O}$ & $41 \mathrm{P}$ & $\mathrm{H} 2 \mathrm{O}$ \\
$t_{\min }$ & $-15^{\circ} \mathrm{C}$ & $+5{ }^{\circ} \mathrm{C}$ & $+10{ }^{\circ} \mathrm{C}$ & $-20{ }^{\circ} \mathrm{C}$ & $+1{ }^{\circ} \mathrm{C}$ \\
$t_{\max }$ & $+45^{\circ} \mathrm{C}$ & $+45^{\circ} \mathrm{C}$ & $+90{ }^{\circ} \mathrm{C}$ & $+100{ }^{\circ} \mathrm{C}$ & $+100^{\circ} \mathrm{C}$ \\
$t_{Z}$ & 1 & 1 & 1 & 5 & $9,1 \mathrm{wg} . V_{Z, H 2 O}=V_{Z, 41 \mathrm{P}}$ \\
Druckhaltung (DH) & Saug-DH & Saug-DH & Saug-DH & Nach-DH $\left(\Delta p_{\text {Pu,Auslegung }}=0,5\right.$ bar $)$ \\
\hline
\end{tabular}

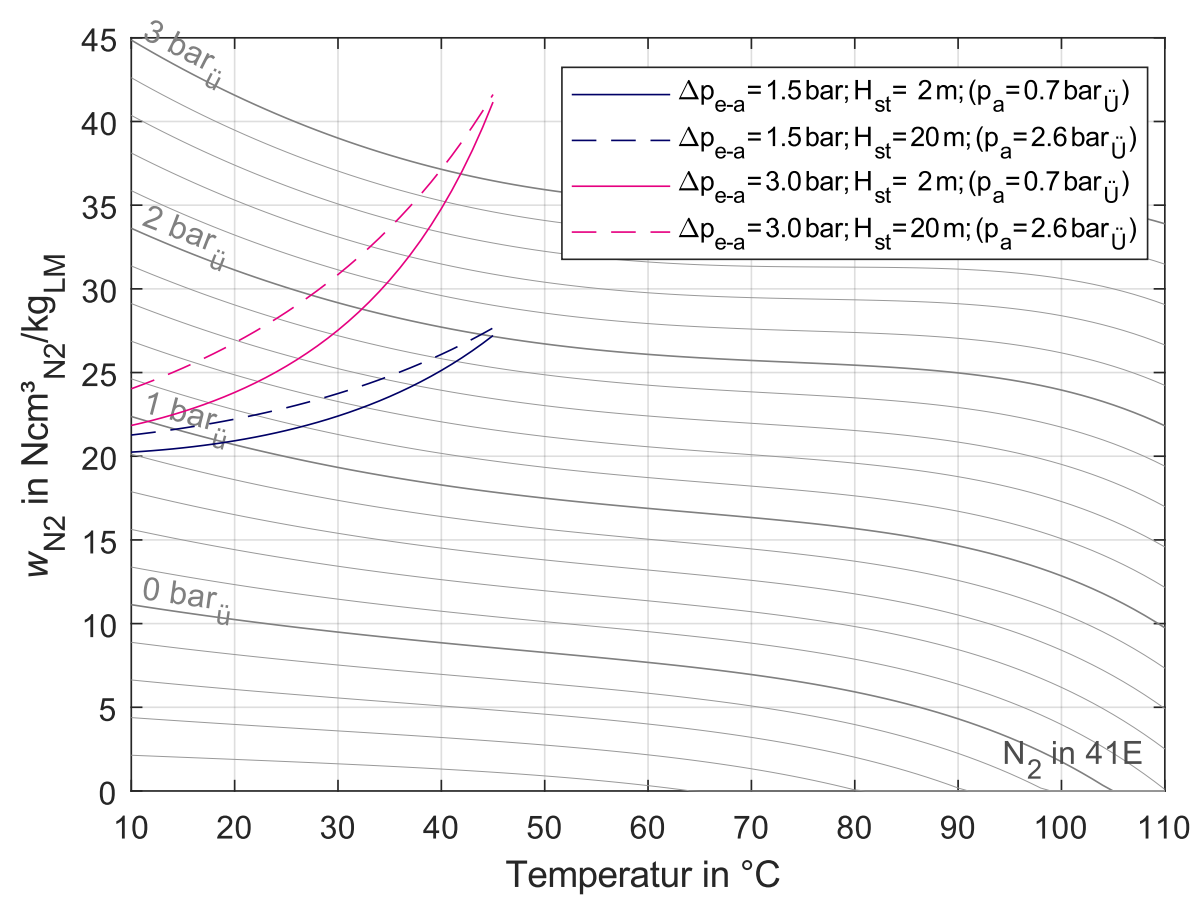

Abbildung 6.18: Löslichkeitsverlauf (am HP) eines Rückkühlkreises mit 41E

Folgende Erkenntnisse können für die spezifischen Rückkühlanlagen aus dem Diagramm in Abbildung 6.18 gewonnen werden:

- Steigende Temperaturen führen stets zu steigenden Drücken entsprechend der dargestellten Verläufe. Je nach Druckspiel $\Delta p_{\mathrm{e}-\mathrm{a}}$ führt dies zu mehr oder weniger starken Abweichungen von den Isobaren-Verläufen.

- Alle Kurven starten bei $t_{\min }=-15^{\circ} \mathrm{C}$ beim gleichen Anfangsdruck $p_{\mathrm{a}, \mathrm{HP}}=0,5$ bar ü am Hochpunkt bzw. bei gleicher Löslichkeitsgrenze $w_{a, H P, \max }$. Aufgrund fehlender Daten für $\lambda$ bei $t<10{ }^{\circ} \mathrm{C}$ ist dieser Teil jedoch nicht dargestellt und dieser Bereich des Verlaufs auch nicht bekannt.

- Drücke steigen mit zunehmender Temperatur jeweils so stark, dass diese entsprechend des Löslichkeitsverhaltens $\lambda_{\mathrm{N} 2}(p, t)$ von Stickstoff in $41 \mathrm{E}$ zu mit der Temperatur stets steigenden Löslichkeiten führt. Dies steht im Gegensatz zu den Isobaren-Verläufen. Das heißt: Trotz mit zunehmender Temperatur deutlich sinkender Löslichkeit der Isobaren kann sich die Löslichkeit in der Anlage entgegengesetzt verhalten.

- Je höher die gewählte Druckdifferenz $\Delta p_{\mathrm{e}-\mathrm{a}}$, desto größer der Enddruck auch am Hochpunkt. Je geringer $\Delta p_{\mathrm{e}-\mathrm{a}}$ (bedeutet in der Praxis größeres MAG), desto mehr nähert sich der Verlauf dem Isobaren-Verlauf an. 
- Bei gleichem $\Delta p_{\mathrm{e}-\mathrm{a}}$, aber bei unterschiedlicher statischer Höhe $H_{\mathrm{st}}$ unterscheiden sich die Verläufe zwischen Anfangs- und Endzustand leicht.

- Dabei ist $p_{\mathrm{e}, \mathrm{HP}}$ für gleiche Druckspiele $p_{\mathrm{e}-\mathrm{a}}$ nahezu, aber nicht vollständig gleich. Das liegt am Einfluss des hydrostatischen Druckes: Je größer die statische Höhe, desto höher ist bei steigender Temperatur die Druckerhöhung durch sich reduzierende hydrostatische Druckauflastung.

- Eine Entgasungswirkung durch Erwärmung ist in den dargestellten Anlagen nicht möglich. Stattdessen können bei höheren Betriebstemperaturen ggf. vorhandene freie Gase durch Absorption noch gebunden werden.

- Eine Entlüftung der Anlagen wäre aus dem hier betrachteten Gesichtspunkt demnach eher bei kalten Temperaturen sinnvoll. Aufgrund der stark vereinfachten Betrachtung (Vernachlässigung Strömungsdruckverluste, temperaturabhängiges Gasblasenund Entlüftungsverhalten u.v. m.) ist diese Aussage jedoch unsicher.

Im Gegensatz dazu ist das Verhalten für einen Rückkühlkreis, der mit Wasser betrieben wird, in Abbildung 6.19 dargestellt. Einziger Unterschied in den Randbedingungen ist die minimale Temperatur. Entsprechend des Gefrierpunktes von Wasser ist hier von einer minimalen Temperatur von $t_{\min }=5^{\circ} \mathrm{C}$ auszugehen. Hier ist deutlich zu sehen, dass es gegenüber $10^{\circ} \mathrm{C}$ bis zu einer Temperatur von etwa 20 bis $25^{\circ} \mathrm{C}$ zu einem leichten, stetigen Absinken der Löslichkeit kommt.

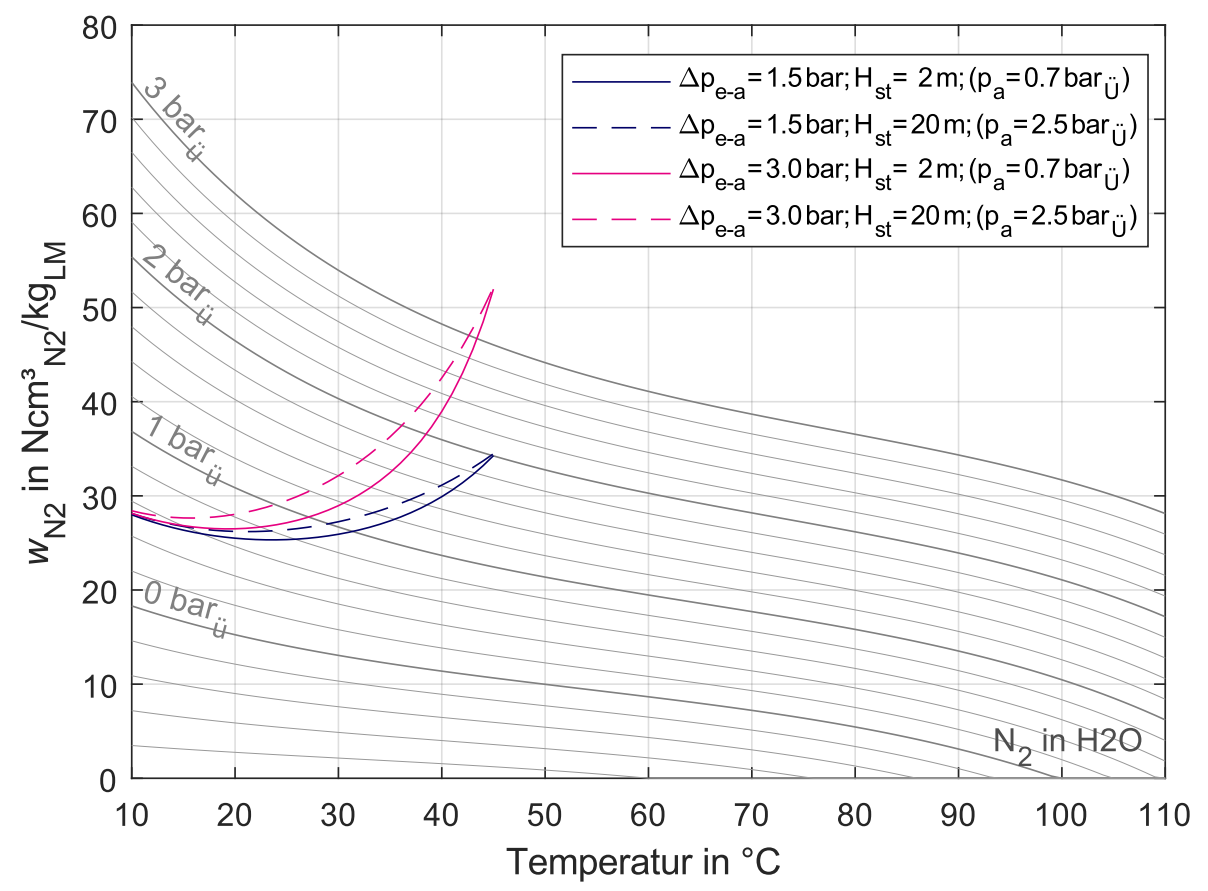

Abbildung 6.19: Löslichkeitsverlauf (am HP) eines Rückkühlkreises mit Wasser

Dieser Effekt ist hinlänglich bekannt, insbesondere aus der Heizungstechnik. Der Vollständigkeit halber sei also auch der Verlauf für einen typischen Heizkreis nochmal dargestellt (vgl. Abbildung 6.20). Hier ist deutlich erkennbar, dass die Löslichkeit in der Anlage gegenüber der Löslichkeit bei Befülltemperatur $\left(10 \ldots 20^{\circ} \mathrm{C}\right)$ deutlich reduziert werden kann. Bei stärkerem Aufheizen steigt insbesondere bei relativ großem Druckspiel $\Delta p_{\mathrm{e}-\mathrm{a}}$ die Löslichkeit jedoch wieder. 


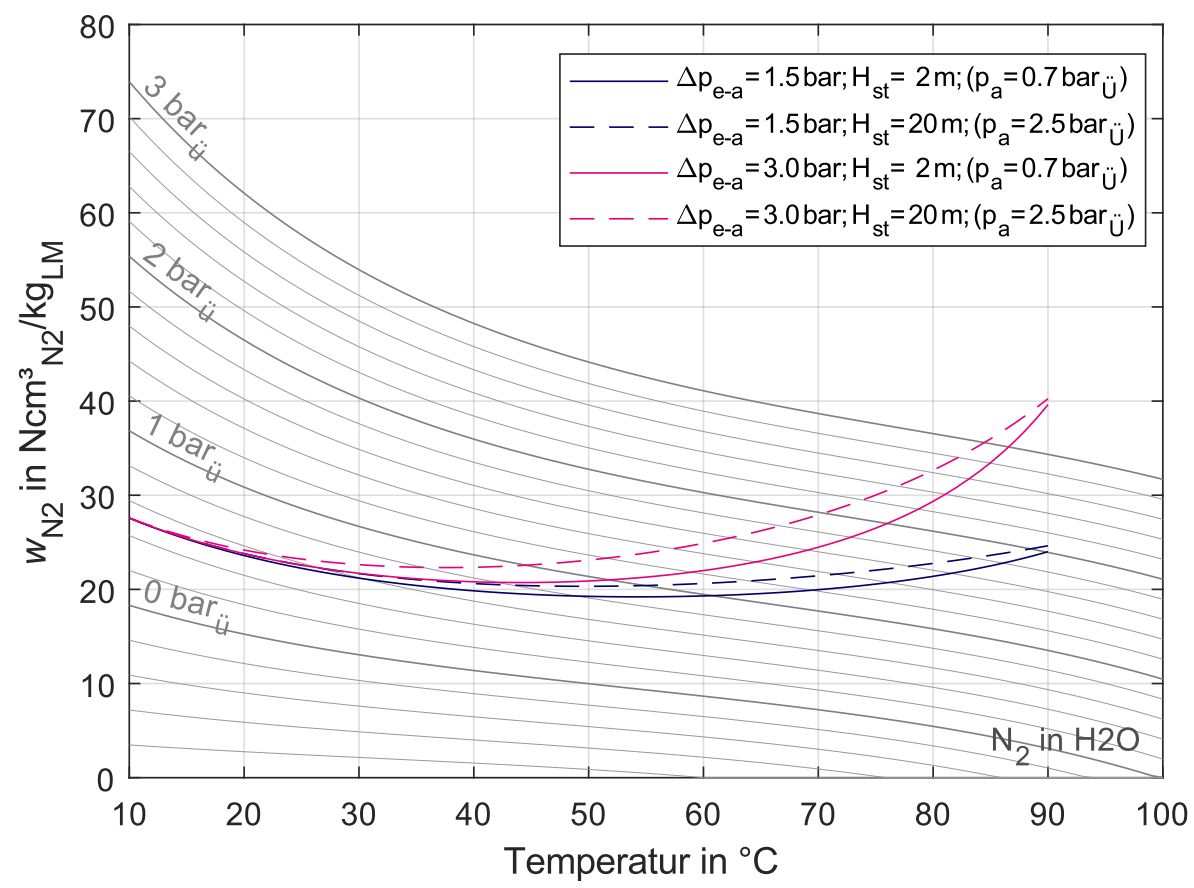

Abbildung 6.20: Löslichkeitsverlauf (am HP) einer Heizung mit Wasser

In Abbildung 6.21 sind die Ergebnisse für die Solarthermieanlagen mit dem Wärmeträgermedium 41P dargestellt. Das Verdampfungsvolumen $V_{Z}$ der Solarthermieanlage wird hier mit einem Faktor von $f_{Z}=5$ berücksichtigt. Dieses kann je nach Kollektortyp, Anlagenaufbau und Rohrleitungslängen deutlich variieren. In [Eismann, 2017] beträgt dieses für eine Beispielanlage mit 10 Flachkollektoren 6,9; in einem Beispiel in [Viess08] für eine Anlage mit 2 Flachkollektoren nur 2,9.

Aufgrund der Nachdruckhaltung ist der Druck $p_{a, m i n}$ (für die hiesige Betrachtung ohne Strömungsdruckverluste) um die angenommene Pumpendruckdifferenz $\Delta p_{\mathrm{Pu}}=0,5$ bar erhöht gegenüber den Anlagen mit Saugdruckhaltung und beträgt $p_{\mathrm{a}, \mathrm{HP}}=1$ bar ü. Es ist deutlich zu sehen, dass das Verdampfungsvolumen bzw. der Faktor $f_{Z}$ dazu führt, dass sich im Bereich der reinen Temperaturdehnung nur geringe Druckerhöhungen ergeben. Daher weichen die Verläufe nur sehr wenig vom Verlauf der Isobaren ab. Da die Isobaren für 41P jedoch im Gegensatz zu Wasser keine starke Löslichkeitsabnahme mit steigender Temperatur hervorrufen, bleiben die Löslichkeiten in der Anlage nahezu gleich über den gesamten betrachteten Temperaturbereich. Dies ist dahingehend interessant, dass also im gesamten Betriebsbereich etwa die gleichen Mengen an gelösten Gasen bzw. das gleiche Gasaufnahmevermögen vorhanden sind. Je höher der Anteil des Dampfvolumens $V_{Z}$ und damit je größer $f_{Z}$ ist, umso mehr verschiebt sich der Verlauf in Richtung Isobare.

Vergleichend sei nun noch eine Solarthermie-Anlage mit Wasser vorgestellt, vgl. Abbildung 6.22. Diese wurde so dimensioniert, dass sie vereinfacht das gleiche Verdampfungsvolumen besitzt. ${ }^{9}$ Hier ergeben sich sehr hohe Löslichkeitsabsenkungen mit steigender Temperatur. Dieses Wissen für Wasser-Anlagen ist lange Zeit auch auf glykolbasierte Solarthermieanlagen, die eigentlich dem Verhalten in Abbildung 6.21 folgen, gedanklich übertragen worden, wie dies bereits in der Einleitung dieser Arbeit beschrieben wurde: „Entsprechend dieser An-

${ }^{9}$ Entsprechend der geringeren Wärmedehnung des Wassers und der höheren Mindesttemperatur ergibt sich ein deutlich höherer Faktor $f_{\mathrm{Z}}=9,1$. 


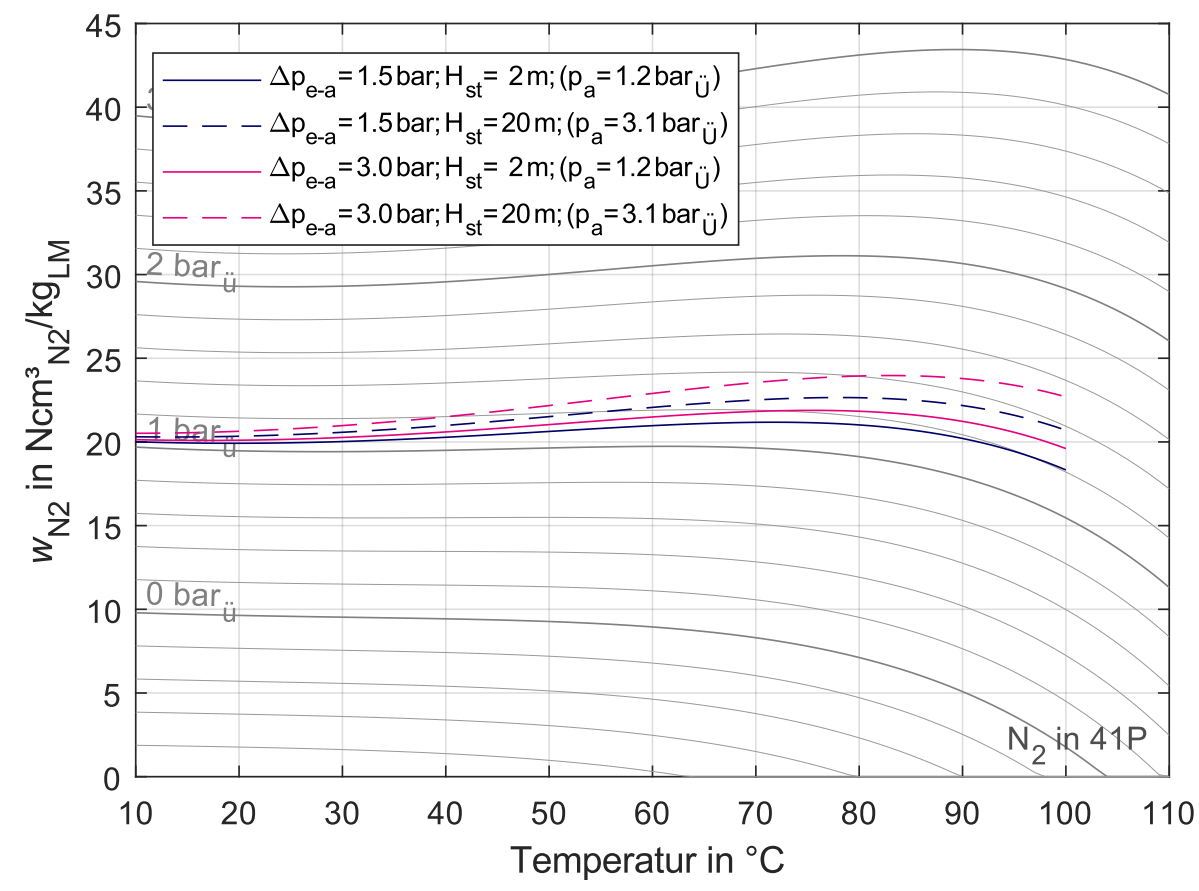

Abbildung 6.21: Löslichkeitsverlauf (am HP) einer Solarthermieanlage mit 41P

nahmen sind z. B. Inbetriebnahmevorschriften und konstruktive Aspekte wie Entlüfter geplant und ist auch das Betriebsverhalten interpretiert worden."

Eine zusätzliche Entlüftung einer Wasser-Glykol-Solarthermieanlage im warmen Zustand ist demnach weder möglich noch nötig. Allein ein fiktiv besserer Gasblasentransport zum und Ausschub aus dem Entlüfter bei hohen Temperaturen könnte den Effekt leicht höherer Löslichkeiten bei hohen Temperaturen überkompensieren. 


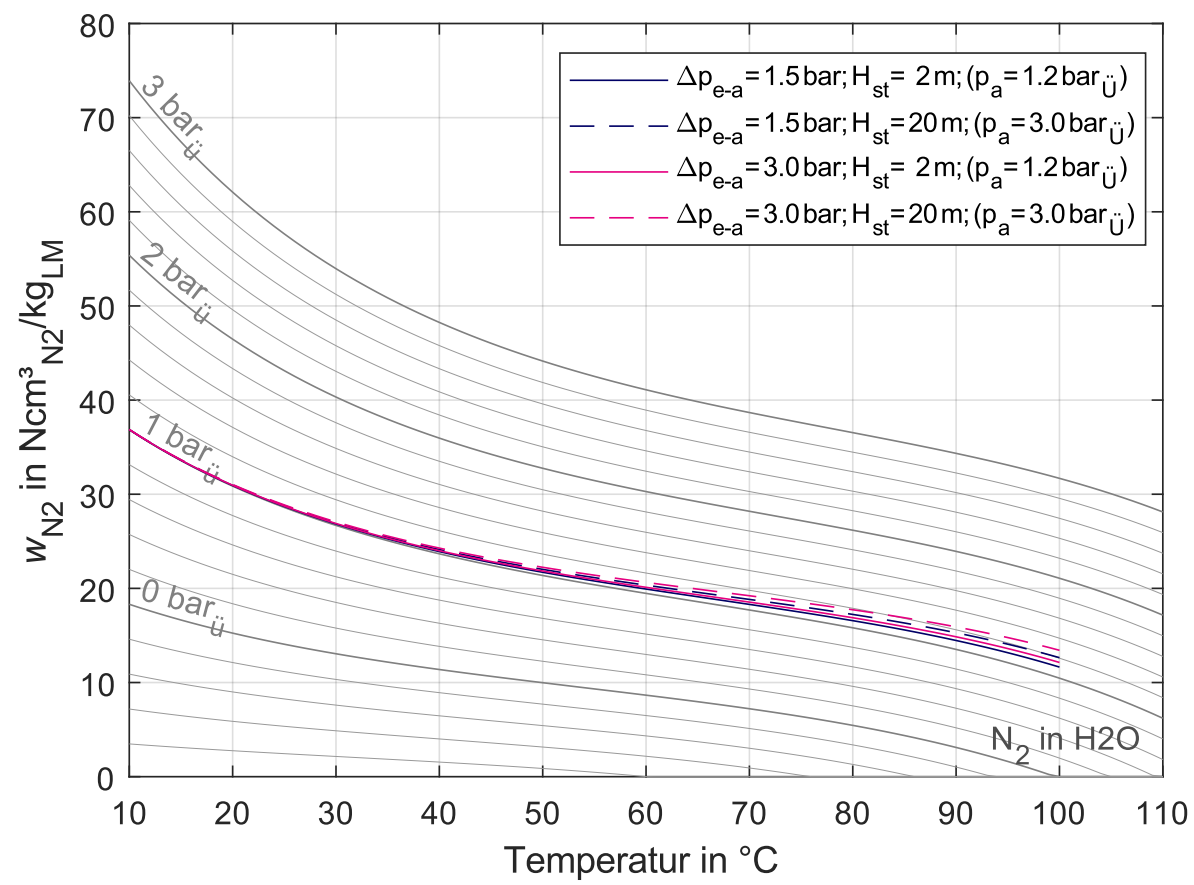

Abbildung 6.22: Löslichkeitsverlauf (am HP) einer Solarthermieanlage mit Wasser (Annahme: gleiches Dampfvolumen $V_{Z}$ wie bei Anlage mit 41P)

\subsubsection{Ergebnisvalidierende Praxis-Beispiele}

\subsubsection{Allgemeines}

Es gibt nur wenige Untersuchungen zu Gasen in energietechnischen Anlagen mit glykolhaltigen Wärmeträgermedien. Daher können die obigen Ergebnisse nur stichprobenartig mit Praxisuntersuchungen verglichen werden. Für Propylenglykol-Wasser sollen dafür die Untersuchungen an Solarthermieanlagen, die in [Heymann und Rühling, 2013] durchgeführt wurden, genutzt werden. Für Ethylenglykol-Wasser werden die Ergebnisse einer Untersuchung eines Kältemaschinen-Rückkühlkreislaufes, die im Rahmen dieser Arbeit entstanden sind (vgl. [Panitz und Rühling, 2017]), genutzt.

\subsubsection{Praxisbeispiel Propylenglykol-Wasser (Solarthermieanlage)}

Bestätigt werden die aus den Messwerten der Löslichkeitsuntersuchungen abgeleiteten, in diesem Kapitel oben dargestellten Schlussfolgerungen durch Messungen der gelösten Gasmenge einer Solaranlage im Technikumsversuchsstand in [Heymann und Rühling, 2013] für Entlüfterversuche bei verschiedenen statischen Höhen der Anlage. Nach der Befüllung der Solaranlage mit Tyfocor ${ }^{\circledR}$ LS wird eine Nullprope (NP) genommen. Anschließend wird die Anlage vier Stunden bei $90^{\circ} \mathrm{C}$ betrieben, anschließend abgekühlt und erneut eine Probe (Endprope EP_90) genommen, siehe Abbildung 6.23.

Vergleicht man die Proben NP und EP_90, so sinkt die gelöste Sauerstoffmenge in den meisten Versuchen um 40-60 \%, während sich die gelöste Stickstoffmenge um 5-30 \% erhöht (Nachweis in über 30 Versuchen, siehe Abbildung 6.24). Gleichartig durchgeführte Versuche mit Wasser zeigen nach vierstündigem Anlagenbetrieb bei $90{ }^{\circ} \mathrm{C}$ nur eine leichte Reduzierung 


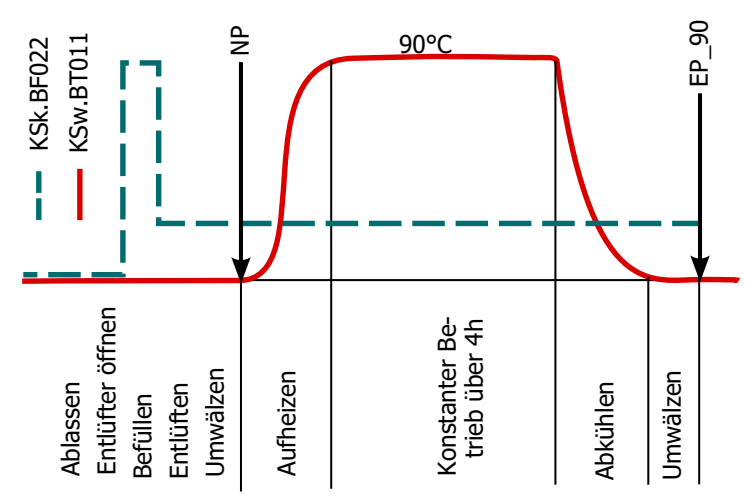

Abbildung 6.23: Schema des Versuchsablaufs mit den Probenahmen NP und EP 90 aus [Heymann und Rühling, 2013]

des Sauerstoffgehalts, vergleiche Abbildung 6.25. Im Gegensatz zum Propylenglykol-WasserGemisch mit Inhibitoren sinkt bei gleichartigen Versuchen mit Wasser als Wärmeträgermedium die Menge des gelösten Stickstoffs im Fluid sogar leicht.

Die Technikumsversuche bestätigen also die theoretischen Schlussfolgerungen aus den Löslichkeitsuntersuchungen, dass eine Aufwärmung des Kreislaufes bei Propylenglykol-Wasser und insbesondere bei dem Gemisch mit Inhibitoren (Tyfocor ${ }^{\circledR}$ LS) zu keiner Desorption aus der Flüssigkeit (Entgasung) führen kann und damit vermutlich auch eine Wirkung der Entlüfter ausbleibt. ${ }^{10}$

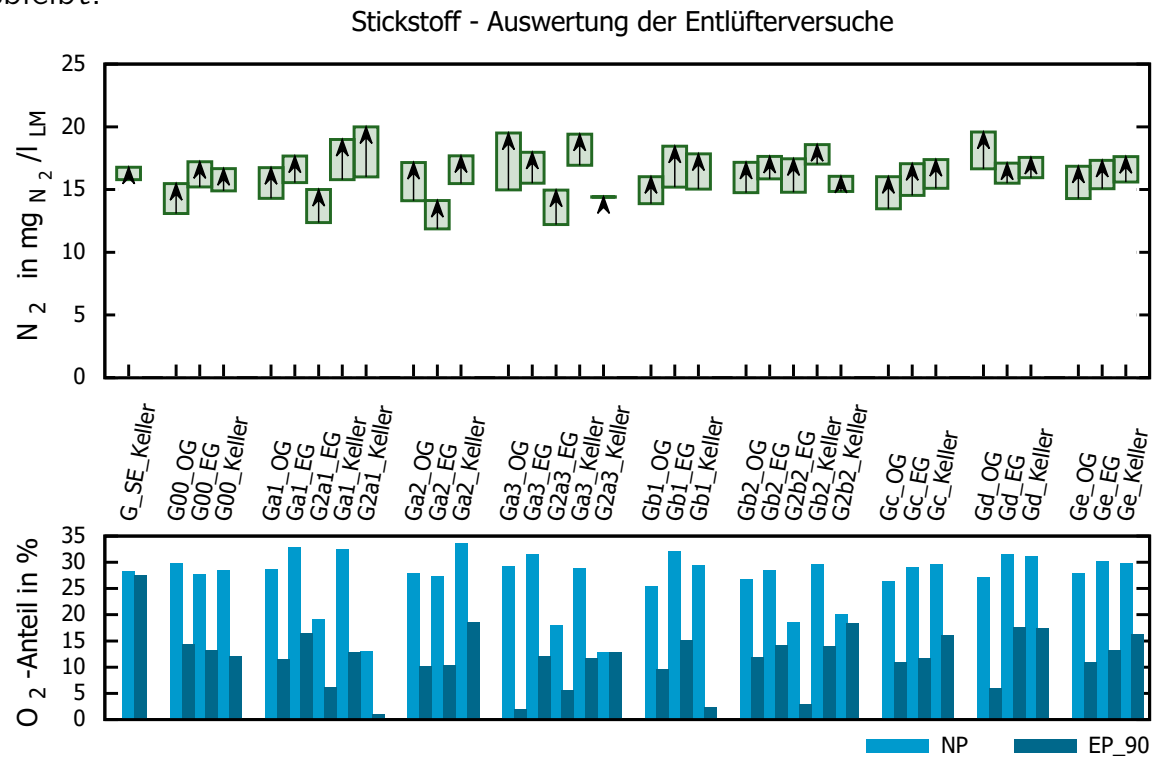

Abbildung 6.24: Ergebnisse für Befüllversuche mit Tyfocor $^{\circledR}$ LS aus [Heymann und Rühling, 2013]

Interessant sind auch die Ergebnisse aus [Heymann und Rühling, 2013] bei höheren Temperaturen. Hier wurde, wie in Abbildung 6.26 dargestellt, gezielt Stagnation, d. h. Pumpenstillstand bei anhaltender Zufuhr von solarer Strahlungsenergie, bei Temperaturen von ca. $140{ }^{\circ} \mathrm{C}$ herbeigeführt. Es werden in der zweiten Versuchsphase zwei weitere Proben (ZP und EP_S) vor bzw. nach der Stagnationsphase entnommen. Die Ergebnisse bestätigen

\footnotetext{
${ }^{10}$ Aufgrund des in [Rühling u.a., 2013b] erst später entwickelten Messverfahrens zu Ermittlung der freien Gasblasen, können zur Entlüftungswirkung dieser Versuche leider keine vollständigen Aussagen gemacht werden.
} 

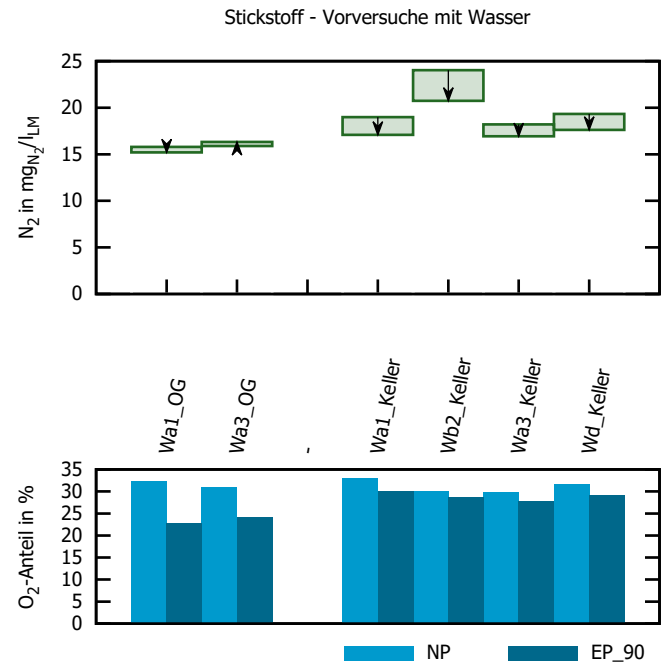

Abbildung 6.25: Ergebnisse für Befüllversuche mit Wasser aus [Heymann und Rühling, 2013]

die theoretischen Überlegungen, dass mit hohen Temperaturen die Sauerstoffkonzentration weiter abfällt wie in Abbildung 6.27 zu sehen. Interessant ist, dass auch nach Abkühlung zwischen Endprobe EP 90 und Zwischenprobe ein Sauerstoffabfall zu beobachten ist. Diese Situation wurde am Löslichkeitsversuchsstand nicht nachgestellt. Da die Zeitspannen zwischen den Proben nicht gesondert ausgewertet wurden, kann nicht abschließend geklärt werden, wie die realen Reaktionsgeschwindigkeiten in den Bereichen zwischen den Proben aussehen.

Die Autoren bemerken, dass gegebenenfalls die Vorlaufzeiten für den Zustand EP_S nicht ausreichen, um durch den Siedevorgang desorbiertes Gas wieder vollständig ins Gas-FlüssigGleichgewicht zu bringen. Da jedoch der Stickstoffanteil der Versuche in allen der vier Proben eines Versuchs immer auf einem hohen Niveau bleibt, ist damit zu rechnen, dass dieser Einfluss auf den Sauerstoffgehalt eher gering sein wird.

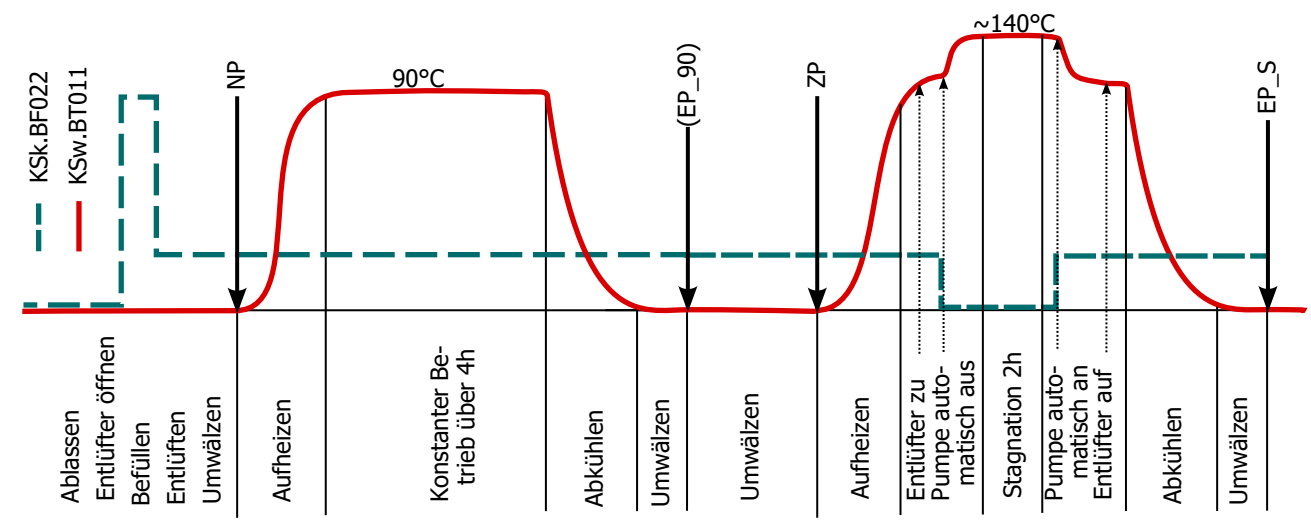

Abbildung 6.26: Schema des Versuchsablaufs mit Stagnation mit den Zeitpunkten der Probenahmen NP, EP_90, ZP und EP_S; aus [Heymann und Rühling, 2013] 


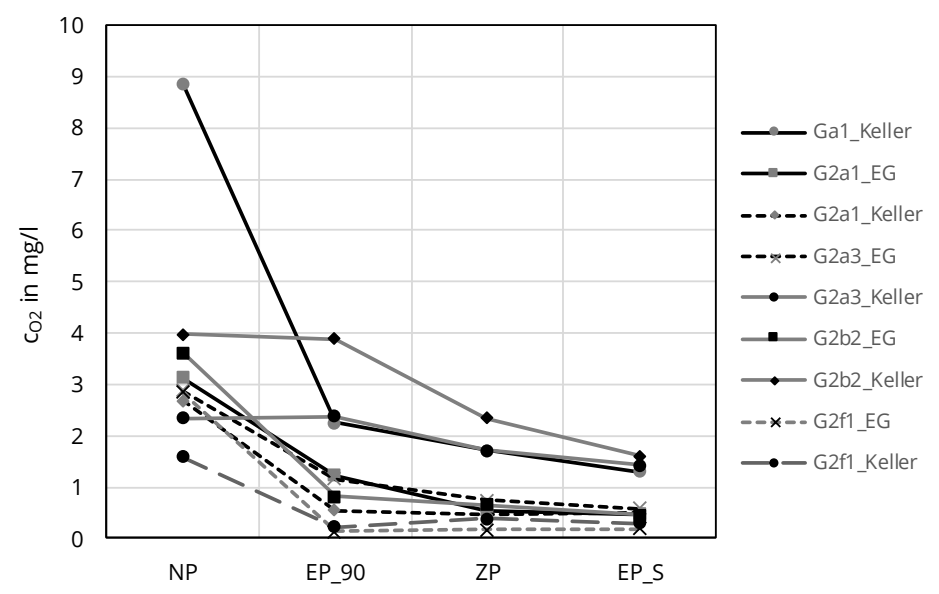

Abbildung 6.27: Verlauf des Sauerstoffgehalts bei Versuchen mit Stagnation. Datenbasis und Versuchsbezeichnungen aus [Heymann und Rühling, 2013]

\subsubsection{Praxisbeispiel Ethylenglykol-Wasser (Rückkühlkreislauf) ${ }^{11}$}

Im Rückkühlkreis einer Absorptionskälteanlage (AKA), vgl. Abbildung 6.28 und Tabelle 6.6, werden Wärmemengenzähler zur Erfassung der Volumenströme mittels Ultraschallverfahren genutzt. Diese werden u. a. zur Regelung der AKA genutzt. Im Rückkühlkreis mit einem Nasskühlturm als Rückkühlwerk (RKW) in geschlossener Bauweise traten bei hohen Volumenströmen Probleme bei der Realisierung eines konstanten Volumenstromsignals auf. Als Ursache für die Signalprobleme wurden Gasblasen im Kreislaufmedium vermutet. Daher wurde der Kreislauf umfänglich auf im Fluid gelöste Gase und vorhandene Gasblasen („freie“ Gase) untersucht. Das verwendete Wärmeträgermedium ist Antifrogen N, ein Gemisch auf Basis von Ethylenglykol, hier mit ca. 21 Gew.-\% Glykolgehalt. Für die Bestimmung der geIösten und freien Gase wurden die an der TU Dresden entwickelten Messmethoden genutzt (vgl. [Rühling u. a., 2013b] und [Rühling u. a., 2013a]).

Tabelle 6.6: Parameter des Rückkühlkreises

Wärmeträger

Anlagenvolumen

Max. Höhendifferenz

Rückkühlwerkstyp

Wärmeträgerinhalt RKW

Wärmeträgerinhalt $A K A$

Erstinbetriebnahme

MAG

Öffnungsdruck Sicherheitsventil

Rohrlänge der Anlage
Antifrogen N, ca. 21 Gew.-\% Ethylenglykol

ca. 1500 Liter

ca. $4,5 \mathrm{~m}$

Gohl, Typ VK2 / 45 / 7+P, Baujahr 1999

970 Liter

200 Liter

September 2014

80 Liter, Vordruck 1,1 bar (Ist) / 1,15 bar (Soll)

5 bar

ca. $30 \mathrm{~m}(\mathrm{DN} 150)$

Die Messung der Ausgangsbeladung des Kreislaufes ergab

- 31,1 Normliter gelöste Gase, aber auch

- 24,2 Normliter freie Gase.

\footnotetext{
${ }^{11}$ Ergebnisse sind vom Autor bereits in Form eines Berichts in [Panitz und Rühling, 2017] veröffentlicht.
} 


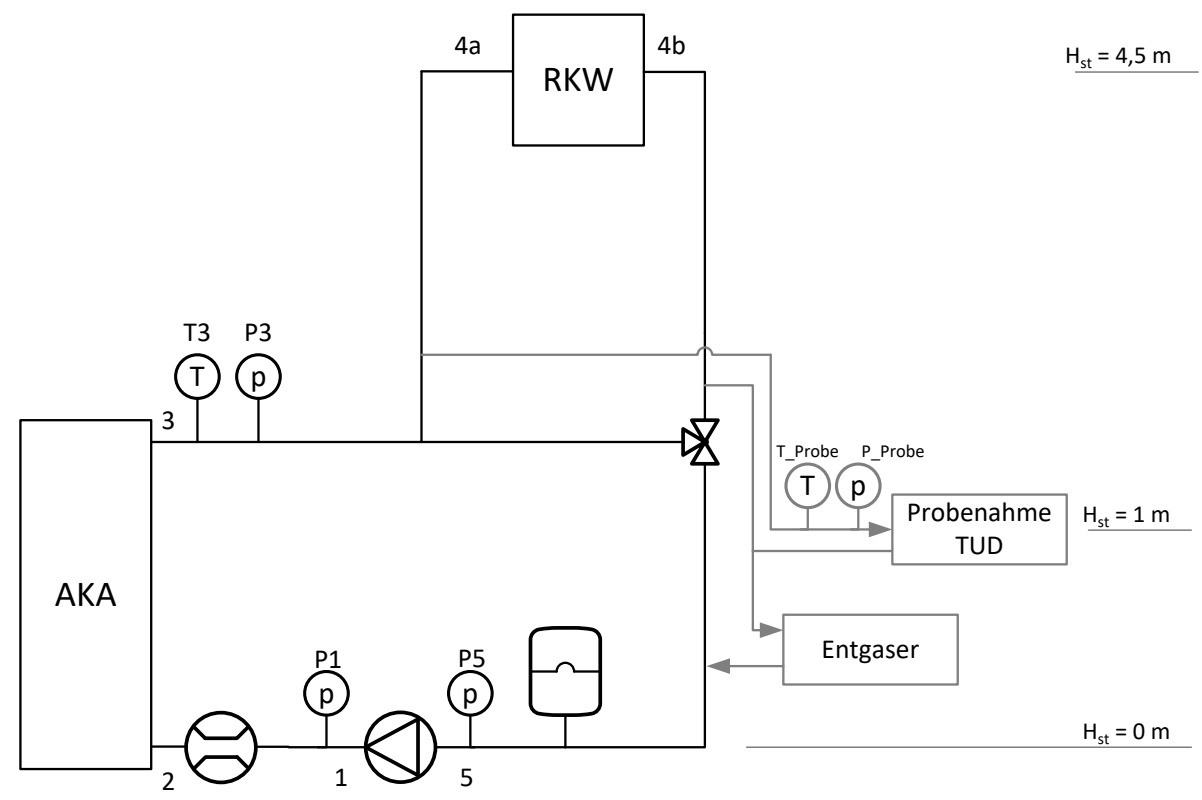

Abbildung 6.28: Prinzipskizze des Kühlkreislaufes mit Beprobungseinheit der TU Dresden; charakteristische Anlagenpunkte 1-5

Der Anteil von gelöstem Sauerstoff in der Fluidprobe betrug $x_{\mathrm{tr}, \mathrm{O} 2}=$ $2,6 \%$.

Im Henry-Diagramm in Abbildung 6.29 sind die Löslichkeitsgrenzen von Stickstoff als Isobaren in Abhängigkeit der Temperatur dargestellt. Aufgrund der in der Probe festgestellten, sehr geringen Menge an Sauerstoff und Methan wurde in der Rechnung vereinfacht von reinem Stickstoff-Lösungsmittel-Gemisch ausgegangen. ${ }^{12}$

Zusätzlich sind die Löslichkeitsgrenzen für charakteristische Punkte 1-5 der Anlage (vgl. Abbildung 6.28) eingezeichnet. Diese wurden aus bekannten bzw. vereinfacht berechneten Druck- und Temperaturverläufen des Betriebszustands der mit einem Volumenstrom von $33 \mathrm{~m}^{3} / \mathrm{h}$ laufenden Anlage ermittelt. Hieraus geht deutlich hervor, dass die minimale Löslichkeit am Hochpunkt 4b (Kühlwasseraustritt aus dem Rückkühlwerk) zu erwarten ist und dieser Punkt als Kriterium für das Vorliegen freier Gase bzw. für den Untersättigungsgrad des Kreislaufs gesehen werden muss. Dabei ist anzumerken, dass bei anderem Druckverlust und anderer Temperaturspreizung im Rückkühlwerk ebenso denkbar ist, dass der Punkt 4a vor dem Rückkühlwerk die minimale Löslichkeitsgrenze bildet, da zwar der höhere Druck, aber eben auch die höhere Temperatur vorliegt.

Weiterhin ist die mittels Entnahme einer Flüssigkeitsprobe und gaschromatographischer Auswertung gemessene Konzentration an gelöstem Stickstoff (bei ca. $20^{\circ} \mathrm{C}$ ) $\mathrm{C}_{\mathrm{N} 2 \text {, Mess }}$ eingezeichnet. Diese ist als Isolinie gleichen Gasgehalts (d.h $w_{\mathrm{N} 2}=$ const. und $x_{\mathrm{N} 2}=$ const.) eingezeichnet. Da das Henry-Diagramm hier einmal für die physikalische Größe Konzentration $c$ dargestellt ist, sind diese Isolinien - wie bereits in Abschnitt 6.1.1 beschrieben - eben keine waagerechten Linien.

${ }^{12}$ Für die Ermittlung von Löslichkeitskoeffizienten wurde hier vereinfacht zwischen Werten von Wasser und $25 \mathrm{E}$ linear (über den Gewichtsanteil $\xi_{\mathrm{E}}$ ) interpoliert. 
Wie zu sehen ist, entspricht der gemessene Gasgehalt nahezu dem theoretischen Wert der Löslichkeit des Punktes 4b. Dies spricht also für die Plausibilität der gewonnenen Löslichkeitskoeffizienten und Anwendbarkeit in der Praxis.

Abschließend sei bemerkt, dass in der Versuchsanordnung anschließend ein Entgaser installiert wurde und damit die Gaskonzentration innerhalb von 2 Wochen Entgaserbetrieb auf $c_{\mathrm{N} 2 \text {, Mess, teilentgast ( }} \mathrm{vgl}$. Abbildung 6.29) reduziert werden konnte und damit im gesamten Kreislauf untersättigtes Fluid vorlag. Die Gasbeladung des Kreislaufs konnte damit reduziert werden auf 25,6 Normliter gelöstes Gas und 9,4 Liter freies Gas. ${ }^{13}$

Durch die vorliegende Untersättigung ist von einer allmählichen Absorption der verbleibenden Gaspolster bis zum Erreichen der Löslichkeitsgrenze am Hochpunkt auszugehen.

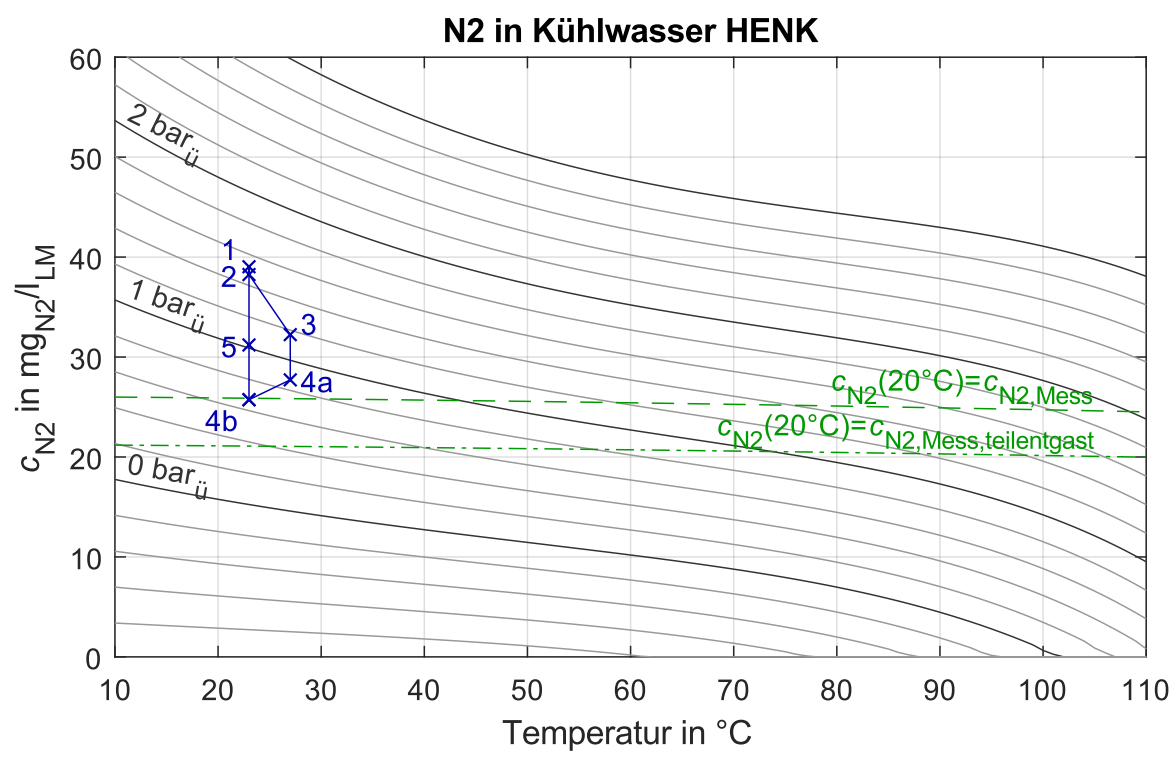

Abbildung 6.29: Maximale Stickstofflöslichkeit an den Anlagenpunkten in Abbildung 6.28 und gemessene gelöste Gasmengen; nur Stickstoff als Gas

Abbildung 6.30 zeigt nach 6 bzw. 9 Tagen Entgasung das Volumenstromsignal des Ultraschalldurchflussmessers bei unterschiedlichen Volumenströmen. Während nach 6 Tagen Entgasung insbesondere über $45 \mathrm{~m}^{3} / \mathrm{h}$ noch deutliche Signalstörungen auftreten, sind nach 9 Tagen Entgasung (und damit weiterer Reduzierung gelöster und freier Gase) die Störungen des Volumenstromsignals im Wesentlichen verschwunden. Als Ursache der Störung sind mit der Strömung mitgerissene Mikroblasen naheliegend, die das Ultraschallsignal des Volumenstrommessers stören. Je größer die Strömungsgeschwindigkeit, desto höher der Anteil mitgerissener und umgewälzter Gasblasen, die sich im Ruhezustand oder unterhalb einer kritischen Strömungsgeschwindigkeit an Hochpunkten absetzen. Bei DN 150 entspricht der Volumenstrom von $45 \mathrm{~m}^{3} / \mathrm{h}$ einer mittleren Strömungsgeschwindigkeit von $0,7 \mathrm{~m} / \mathrm{s}$. Die Untersuchungen zur Selbstentlüftungsgeschwindigkeit für Wasser nach [Walther und Günthert, 1998] im senkrechten Rohr bestätigen diese Größenordnung. Dort wird bei Nennweite DN 150 die Geschwindigkeit auf ca 1,1 m/s abgeschätzt.

\footnotetext{
${ }^{13}$ Aufgrund einer eingeschränkten Funktionalität des eingesetzten Entgasers konnte die Anlage nur teilentgast werden. Ansonsten wären nach der Betriebszeit des Entgasers deutlich niedrigere Beladungen zu erwarten gewesen.
} 

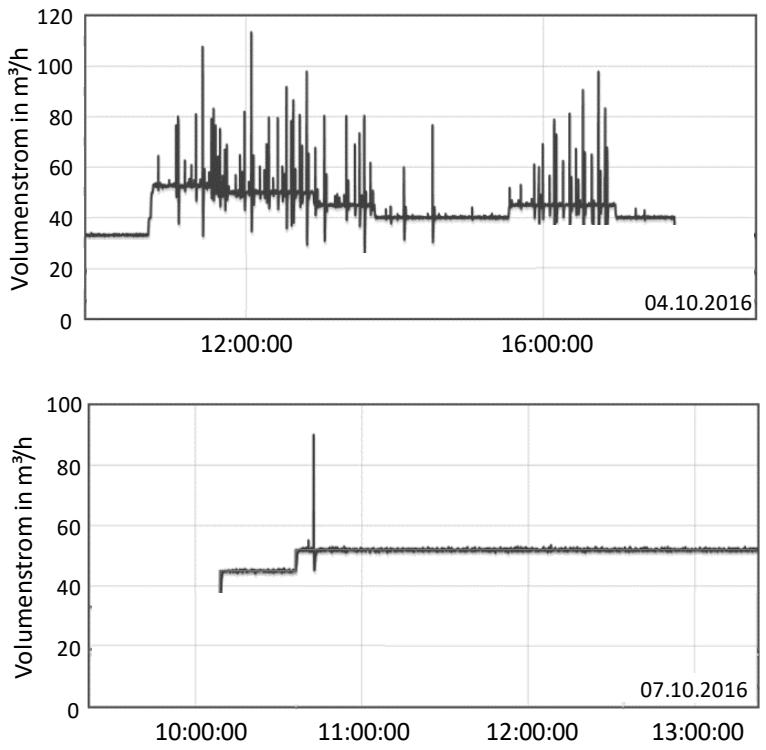

Abbildung 6.30: Volumenstromsignal 6 Tage nach Beginn der Entgasung (oben) bzw. 9 Tage nach Beginn der Entgasung (unten); Samplingrate jeweils 5 s; Daten von [Hunt, 2016] 


\section{Zusammenfassung und Fazit}

\subsection{Versuchsstand und Methodik}

Wichtige Herausforderungen an das Verfahren zur Bestimmung technischer Löslichkeitskoeffizienten und dessen apparative Umsetzung waren die unbekannte Zusammensetzung des Lösungsmittels mit möglicherweise stark flüchtigen Komponenten. Hinzu kam ein begrenztes Finanzbudget, das Kompromisse hinsichtlich Messgüte, Dichtheit, Temperiergüte und Konstruktion nach sich zog, die u. a. durch aufwändige Messunsicherheitsbetrachtungen zu berücksichtigen und zu minimieren waren. Auf Erfahrungen bzw. Veröffentlichungen zu Löslichkeitskoeffizienten von Wasser-Glykolen bei höheren Temperaturen als $25^{\circ} \mathrm{C}$ und bei Wasser-Glykol-Gemischen mit unbekannten Additiven konnte trotz intensiver Literaturrecherche nicht zurückgegriffen werden.

Es ist gelungen ein Verfahren zur Bestimmung von Löslichkeiten umzusetzen, das zu plausiblen Messergebnissen für die gegebenen Anforderungen führt und gleichzeitig eine praktikable und effiziente Versuchsdurchführung und Auswertung ermöglicht. Die Versuchsapparatur, die nach dem volumetrisch-manometrischen Messprinzip arbeitet, ermöglicht die Identifizierung von Löslichkeitskoeffizienten für Stickstoff und Sauerstoff in Glykol-Wasser-Gemischen im Temperaturbereich $10 \ldots 110^{\circ} \mathrm{C}$.

Löslichkeitskoeffizienten für Stickstoff wurden direkt bestimmt. Die für Sauerstoff konnten indirekt aus Versuchen mit technischer Luft statt dem reinen Gas Sauerstoff gewonnen werden. Neben Gründen des Arbeitsschutzes waren die Praxisnähe der Gaszusammensetzung für diese Entscheidung ausschlaggebend. Schließlich tritt auch in Anlagen Sauerstoff immer in Kombination mit Stickstoff auf. Der Versuch wurde dafür um die gaschromatographische Auswertung der Gasphase des Gas-Lösungsmittel-Gemischs erweitert.

Die vergleichende Bestimmung der Löslichkeit durch vorgelagerte Versuche mit Wasser, für das aus der Literatur technische Löslichkeitskoeffizienten bekannt sind, und Kalibrierung der Löslichkeitskoeffizienten der eigentlichen Messungen an diesen Werten konnte erfolgreich durchgeführt werden. Es wurden entsprechende Messunsicherheitsalgorithmen entwickelt, die auch mathematisch zeigen, dass vorhandene Messunsicherheiten durch die Kalibrierung stark reduziert werden können.

Nach der Literatur Pfennig [2003, S. 162] ist für das gewählte synthetisch-statische Verfahren ,... peinlich darauf zu achten, dass in den dosierten Flüssigkeiten keine Fremdgase wie z. B. Luft gelöst sind, da diese die Messung deutlich verfälschen würden." Für einen hohen Entgasungsgrad des Lösungsmittels wird in der Literatur entsprechend hoher Aufwand betrieben. Die hier vorgestellte Methodik zeigt hingegen, dass gewisse Mengen an Störgasen bzw. eine gewisse, bereits im Ausgangszustand bestehende Beladung der Flüssigkeit mit Arbeitsgas bei der hier entwickelten differentiellen Ermittlung der Löslichkeitskoeffizienten zwischen zwei Phasengleichgewichten keinen relevanten Einfluss haben. Der Versuchsaufwand lässt sich damit erheblich reduzieren und ermöglicht auch die Bestimmung der Gaslöslichkeit in einem Flüssigkeitsgemisch mit stark flüchtigen Komponenten.

Die Spezifika des Versuchsstands und -ablaufs wie nicht bzw. nicht vollständig entgaste Lösungsmittel, differentielle Bestimmung des Löslichkeitskoeffizienten aus zwei Phasen- 
gleichgewichtsmessungen, unbekannte molare Zusammensetzung des Lösungsmittels, Kalibrierung mit vorgelagerten Wasserversuchen u.v.a.m. haben einen deutlich komplexeren Berechnungsalgorithmus als für in der Literatur beschriebene Versuchsstände zur Folge.

Versuchsstand und Methodik haben sich in insgesamt mehr als 180 Versuchen bewährt. Dabei wurde im gesamten Temperaturbereich von $10^{\circ} \mathrm{C}$ bis $110^{\circ} \mathrm{C}$ gearbeitet. Erfolgreich durchgeführte Versuche für eine Vielzahl von Stoffgemischen mit stark unterschiedlichen physikalischen Eigenschaften (v. a. Viskosität und Dampfdruck) sowie teilweise besonderen chemischen Eigenschaften durch Additive bei noch dazu signifkant unterschiedlichen Löslichkeiten bescheinigen dem Versuchsstand und dem Verfahren ein hohes Maß an Robustheit und Flexibilität.

Bestehende Schwachstellen von Methodik und Versuchsstand wurden in der Arbeit offen und klar benannt und Möglichkeiten der weiteren Optimierung aufgezeigt.

\subsection{Messtechnische Untersuchungen}

Für die Beschreibung der Löslichkeit wurde die Größe Technischer Löslichkeitskoeffizient $\lambda$ gewählt. Diese wird in der Praxis oft verwendet, ist anschaulich und hat gegenüber anderen Beschreibungen den Vorteil, dass man auch für Flüssigkeiten mit unbekannter Zusammensetzung die Löslichkeit angeben kann.

Im Rahmen der Arbeit wurden Propylenglykol und Ethylenglykol als reiner Stoff sowie im Gemisch mit Wasser untersucht. Die Messunsicherheiten der ermittelten Löslichkeitskoeffizienten liegen in einer für die praktische Anwendung akzeptablen Größe. Für zukünftige Messungen können jedoch mit beschriebenen Abänderungen aufgrund der gewonnenen Erkenntnisse etwas geringere Messunsicherheiten und ein geringerer Versuchsaufwand erwartet werden.

Für Stickstoff sind Löslichkeitskoeffizienten für drei Wasser-Glykol-Gemische (25; 41,84 und 75 Gewichtsprozent Glykol) sowie reines Glykol bei Temperaturen im Bereich $10 \ldots 110^{\circ} \mathrm{C}$ ermittelt worden. Löslichkeitskoeffizienten für Sauerstoff beschränken sich auf Wasser-Glykole mit 41,84 Gewichtsprozent Glykol. Da diese aus Versuchen mit technischer Luft ermittelt wurden, geben diese genau genommen nur die unter gleichzeitiger Gegenwart von Stickstoff bei einem $\mathrm{O}_{2}$-Anteil in der (trockenen) Gasphase von ca. $y_{\mathrm{O} 2}$ in $\mathrm{N} 2+\mathrm{O} 2,2=19 \%$ wirkende Löslichkeit an. Die Versuche haben aber gezeigt, dass zumindest ein Einfluss von Sauerstoff auf Stickstoff-Löslichkeitskoeffizienten nicht erkennbar ist. Daher wird vermutet, dass dies andersherum auch für den Stickstoff-Einfluss auf die Sauerstoff-Löslichkeitskoeffizienten gilt, also die ermittelten $\mathrm{O}_{2}$-Löslichkeitskoeffizienten auch für reinen Sauerstoff gelten. Der Vergleich der Ergebnisse mit den wenigen vorhanden Literaturwerten für Sauerstoff-Löslichkeit stützt diese These.

Der temperaturabhängige Verlauf der ermittelten Löslichkeitskoeffizienten unterscheidet sich für reines Glykol, aber auch für die Wasser-Glykole deutlich von reinem Wasser. Im Vergleich mit Wasser zeigen beispielsweise die Löslichkeitskoeffizienten von einem WasserPropylenglykol-Gemisch mit 41,84 gewichtsprozentigem Glykolgehalt für $\mathrm{N}_{2}$ und auch $\mathrm{O}_{2}$ eine deutlich niedrigere Löslichkeit bei geringen Temperaturen und dann mit steigender Temperatur steigende Löslichkeitskoeffizienten. Durch die Bereitstellung von Regressionskurven $\lambda(t)$ sind die neuen Erkenntnisse den Anwendern einfach zugänglich. Bis zur Angabe einer 
allgemeinen Regression $\lambda\left(\xi_{\mathrm{G}}, t\right)$ für beliebigen Glykolanteil $\xi_{\mathrm{G}}$ sollten aber noch weitere Messpunkte bei anderen Mischungsverhältnissen ermittelt werden, da das Verhalten $\lambda=f\left(\xi_{\mathrm{G}}\right)$ recht nichtlinear ist.

Eine Druckabhängigkeit der Löslichkeitskoeffizienten ist für Stickstoff im untersuchten Druckbereich bis ca. 9 bar nicht erkennbar.

Die untersuchten Fertiggemische Tyfocor $^{\circledR}$ LS sowie Wasser-Tyfocor ${ }^{\circledR}$, die neben dem Wasser-Glykol-Gemisch verschiedene Additive enthalten, zeigen für Stickstoff ein sehr ähnliches Verhalten wie die Wasser-Glykol-Gemische bei gleichem Glykolgehalt.

Nach Beobachtungen mit Tyfocor ${ }^{\circledR}$ LS in der Praxis und vermuteter Überlagerung der physikalischen und chemischen Bindung von Sauerstoff galt es, dies genauer zu untersuchen. Für Tyfocor ${ }^{\circledR}$ LS konnte in den Versuchen gezeigt werden, dass hier Sauerstoff allmählich zusätzlich chemisch gebunden wird. Die chemische Bindung ist bei einem Versuch bei $49,5{ }^{\circ} \mathrm{C}$ noch nicht beobachtbar, bei einem Versuch bei $78^{\circ} \mathrm{C}$ jedoch deutlich sichtbar. Die Reaktionsgeschwindigkeit steigt mit zunehmender Temperatur. Bei einem Versuch bei $107^{\circ} \mathrm{C}$ wird innerhalb von etwa 3 Stunden jeglicher dem Reaktor zugegebene Sauerstoff chemisch gebunden. Nachweislich können 138 Normkubikzentimeter Sauerstoff pro Kilogramm Tyfocor ${ }^{\circledR}$ LS, vermutlich aber deutlich darüber hinaus, chemisch gebunden werden. Wieviel Sauerstoff im Fertiggemisch maximal chemisch gebunden werden kann, sollte in zukünftigen Arbeiten genauer untersucht werden, ebenso die Reaktionsgeschwindigkeit und deren Abhängigkeit von der bereits gebundenen Sauerstoffmenge. Durch die Überlagerung von physikalischer und chemischer Bindung im Versuchsstand konnten für Tyfocor ${ }^{\circledR}$ LS keine Sauerstoff-Löslichkeitskoeffizienten ermittelt werden.

Bei dem Tyfocor ${ }^{\circledR}$-Wasser-Gemisch sowie beiden reinen Wasser-Glykol-Gemischen bei 41,84 gewichtsprozentigem Glykolgehalt ist hingegen keine chemische Bindung beobachtbar. Für das Tyfocor ${ }^{\circledR}$-Wasser-Gemisch gleichen die $\mathrm{O}_{2}$-Löslichkeitskoeffizienten ebenso wie bei $\mathrm{N}_{2}$ nahezu denen des entsprechenden reinen Wasser-Glykol-Gemischs bei gleichem Glykolgehalt.

Für eine vollständige Beschreibung der Vorgänge in energietechnischen Anlagen sind weitere Löslichkeitsbestimmungen bei niedrigeren Temperaturen (z. B. für das Verhalten von Solarthermie-Anlagen im Winter oder Wärmesonden bzw. -kollektoren mit starker Auskühlung) sowie ggf. auch bei höheren Temperaturen (z. B. für Stagnation Solarthermie) notwendig. Ebenso sind Untersuchungen für weitere Gase (v. a. Umgebungsgase für Erdwärmesonden wie Kohlendioxid, aber auch Gase aus Korrosionsprozessen wie Methan und Wasserstoff) hilfreich.

\subsection{Löslichkeit von Gasen in Theorie und Praxis}

Oft wird in der Literatur darauf verwiesen, dass „für ein in geringer Verdünnung gelöstes Gas bzw. für geringe Drücke" das vollständig das Phasengleichgewicht beschreibende Gleichungssystem "in guter Genauigkeit" auf das Henry-Gesetz, hier in der Form $w_{i}=\lambda_{i} \cdot p_{i}$ für das Gas $i$ genutzt, reduziert werden kann und einige weitere Vereinfachungen gelten. Insbesondere in Praxis-Anwendungen werden diese Vereinfachungen nahezu immer angewendet. In der Literatur konnte jedoch auch für das gut bekannte Stickstoff-Wasser- bzw. SauerstoffWasser-Gemisch keine systematische Analyse bzw. Quantifizierung des Fehlers gefunden werden, zu welchem die Anwendung „der für geringe Verdünnung zulässigen“ Vereinfachungen führt. 
Am Beispiel Stickstoff-Wasser wurde daher mit Hilfe der bestmöglichen dem Autor zugänglichen Stoffdaten im interessierenden Druck- und Temperaturbereich analysiert, welche Vereinfachungen welche Abweichung $\Delta w_{i}$ bewirken. Alle betrachteten Vereinfachungen bewirken im untersuchten Bereich summarisch eine Abweichung von maximal 1,8\%. Diese Vereinfachungen werden gerade für praktische Untersuchungen als ausreichend angesehen. Ein deutlicher Genauigkeitsgewinn mit einer resultierenden Abweichung von maximal 0,3\% lässt sich erreichen, wenn man die (vereinfachte) Poynting-Korrektur des Gases berücksichtigt. Daher ist für die Bestimmung der Löslichkeitskoeffizienten diese berücksichtigt worden. Die höhere Komplexität bei Berücksichtigung dieser Poynting-Korrektur ist eine Hemmschwelle in der Anwendung der Gleichungen und spricht gegen die Nutzung in der praktischen Anwendung.

Aus den ermittelten Regressionen der Löslichkeitskoeffizienten ist die sich ergebende Löslichkeit im Phasengleichgewicht $w_{\max }(p, t)$ in sogenannten Henry-Diagrammen dargestellt worden. Als Gehaltsgröße für das gelöste Gas favorisiert der Autor die Größe w als geeignete Größe u. a. wegen der guten Anschaulichkeit und der - bei Gültigkeit des Henry-Gesetzes - denkbar einfachen Beschreibung mittels des technischen Löslichkeitskoeffizienten $\lambda$. Diese bestätigen, dass sich nicht nur die Löslichkeitskoeffizienten $\lambda(t)$, sondern auch die Löslichkeitsgrenzen $w_{\max }$ zwischen Wasser und den Wasser-Glykolen, aber auch den zwischen unterschiedlichen Wasser-Glykol-Gemischen deutlich unterscheiden.

In der Solartechnik wurde vor dieser Arbeit für Belange der Befüllung, Entlüftung, Entgasung und Stagnation aufgrund fehlenden besseren Wissens von einem dem Wasser ähnelnden temperaturabhängigen Löslichkeitsverhalten ausgegangen. Ggf. betraf dies genauso auch die Bereiche Vakuumentgaser-Entwicklung sowie Wasser-Glykole in Kühl- und Sondenkreisläufen. In der Arbeit konnte jedoch gezeigt werden, dass ein Wasser-Glykol-Gemisch ein völlig anderes Löslichkeitsverhalten zeigt (Löslichkeitskoeffizienten, Henry-Diagramm und Löslichkeitsgrenzen in realen Anlagen). Dementsprechend kann die Annahme von wasserähnlichem Verhalten zu völlig falschen Ansätzen in Konstruktion und Betrieb von WasserGlykol-Kreisläufen sowie Fehlinterpretationen des beobachteten Anlagenverhaltens in der Praxis, aber auch in Forschung und Entwicklung führen. Mit den auf dieser Arbeit basierenden Veröffentlichungen konnte v. a. die Solarbranche diesbezüglich sensibilisiert werden. Die Arbeit stellt mit den ermittelten Löslichkeitskoeffizienten, zugehörigen Regressionsgleichungen und Henry-Diagrammen die Grundlagen für die Nutzung der Erkenntnisse in der Praxis bereit.

Vereinfachte theoretische Untersuchungen zur Gaslöslichkeit in einigen energietechnischen Anlagen unter Verwendung von statischer Druckhaltung mittels Membranausdehnungsgefäß und weiteren praxisnahen Randbedingungen zeigen die deutlichen Unterschiede im Verhalten zwischen Wasser und Wasser-Glykol, insbesondere bei der Solarthermie.

Für einige in der Literatur durchgeführte Untersuchungen und beobachtete Phänomene zu Solarthermie-Anlagen mit Fokus Gasbeladung liefern die Erkenntnisse dieser Arbeit zu Wasser-Glykol-Gemischen auf Propylenglykol-Basis plausible Erklärungen. Auch eine durchgeführte umfassende Gasanalyse eines realen Rückkühlkreislaufes lässt sich mit den ermittelten Löslichkeitskoeffizienten für Wasser-Glykole auf Ethylenglykol-Basis logisch darstellen; aufgetretene Phänomene lassen sich schlüssig erklären. Beide Untersuchungen können also als ergebnisvalidierende Praxisbeispiele gelten.

Von dem hier vorgestellten Gesichtspunkt der Löslichkeit sollte eine Wasser-Glykol basierte Solarthermieanlage weniger Gasblasenprobleme aufweisen als eine Anlage mit Wasser. In der Praxis treten jedoch auch bei Wasser-Glykol-Anlagen Probleme mit Gasen auf. Dort 
sind folglich andere Mechanismen, die die Vorgänge bei Befüllung, Entlüftung und Betrieb bestimmen, für die Probleme mit freien Gasen verantwortlich. Das Löslichkeitsverhalten kann aber als Ursache weitgehend ausgeschlossen werden.

Das Verständnis der ablaufenden Prozesse in energietechnischen Anlagen sollte durch detaillierte Anlagensimulationen, die alle wesentlichen Effekte in einer Anlage dynamisch abbilden, erweitert werden. Mit den Erkenntnissen könnten vorhandene Untersuchungen bezüglich des zeitlichen Verlaufs von Gastransport und Gaspermeation in Heizungsanlagen unter Berücksichtigung der Druckhaltung, vgl. [Sittiho, 2011], jetzt auf Wasser-Glykol-Kreisläufe, also Solaranlagen, Kühlkreisläufe und Erdsonden- bzw. Erdkollektorenkreisläufe übertragen werden. Ebenso sind weitere experimentelle systematische Anlagen- bzw. Komponentenuntersuchungen zum Verhalten von Gasen zu empfehlen. 



\section{Literaturverzeichnis}

\section{Normen und Regelwerke}

ASTM D1177-17 (2007). ASTM D1177-17 Standard Test Method for Freezing Point of Aqueous Engine Coolants. doi: 10.1520/d1177-17.

DIN 1343 (Jan. 1990). DIN 1343 Referenzzustand, Normzustand, Normvolumen; Begriffe und Werte. DIN-Normenausschuss Technische Grundlagen, Fundamental Technical Standards Committee.

DIN EN ISO 12185 (1996). DIN EN ISO 12185 Bestimmung der Dichte.

DIN EN ISO 13443 (2007). DIN EN ISO 13443 Erdgas - Standardbezugsbedingungen. DIN-Normenausschuss Gastechnik (NAGas).

DIN V ENV 13005 (1999). DIN V ENV 13005 Leitfaden zur Angabe der Unsicherheit beim Messen.

DKD-R 5-1 (2010). DKD-R 5-1 Kalibrierung von Widerstandsthermometern. Deutschen Akkreditierungsstelle $\mathrm{GmbH}$ (DAkkS).

DKD-R 6-1 (2014). DKD-R 6-1 Kalibrierung vvon Druckmessgeräten. PhysikalischTechnischen Bundesanstalt (PTB).

EURAMET (2009). Guidelines on the Calibration of Non-Automatic Weighing Instruments. Euramet.

FW 442 (Dezember 2011). Druckhaltung in Heizwasser-Fernwärmesystemen.

FW 510 (Nov. 2003). Anforderungen an das Kreislaufwasser von Industrie- und Fernwärmeheizanlagen sowie Hinweise für deren Betrieb.

GUM (2008). ISO/IEC Guide 98-3:2008 - Uncertainty of measurement - Part 3: Guide to the expression of uncertainty in measurement (GUM:1995). Genf.

VDI 2035-2 (Aug. 2009). VDI 2035 Blatt 2 Vermeidung von Schäden in WarmwasserHeizungsanlagen; Heizwasserseitige Korrosion. Verein Deutscher Ingenieure.

VDI 4708 - Druckhaltung, Entlüftung, Entgasung (Jan. 2011). VDI-Richtlinie. url: https: //katalogbeta.slub-dresden.de/id/dswarm-201-REUONTY2NTAONg/\#detail.

\section{Weitere Literatur}

Abdi, Majid Abedinzadegan, Arshad Hussain, Kelly Hawboldt und Erika Beronich (2007). „Experimental Study of Solubility of Natural Gas Components in Aqueous Solutions of Ethylene Glycol at Low-Temperature and High-Pressure Conditions". In: Journal of Chemical \& Engineering Data 52.5, S. 1741-1746. doi: 10.1021/je700134r. eprint: http: //dx.doi.org/10.1021/je700134r. url: http://dx.doi.org/10.1021/je700134r.

Air Liquide (Juni 2009). Alphagaz - Produkt-Portfolio - v1.4. Air Liquide.

Air Liquide 2 (2009). Datenblatt Synthetische Luft KW-Frei- v2. Air Liquide. url: http: //gasekatalog.airliquide.de/.

Alvarez, Jorge, Rosa Crovetto und Roberto Fernández-Prini (Aug. 1988). „The Dissolution of $\mathrm{N} 2$ and of $\mathrm{H} 2$ in Water from Room Temperature to 640 K". In: Berichte der Bunsengesellschaft für physikalische Chemie 92.8, S. 935-940. doi: 10.1002/bbpc. 198800223. 
Alvarez, Jorge und Roberto Fernandez-Prini (1991). „A semiempirical procedure to describe the thermodynamics of dissolution of non-polar gases in water". In: Fluid Phase Equilibria 66.3, S. 309-326. issn: 0378-3812. doi: http://dx . doi .org/10 .1016/03783812 (91) 85063 -Z. url: http : / www . sciencedirect . com/science/article/pii/ $037838129185063 Z$.

Ambrose, D. und D.J. Hall (1981). „Thermodynamic properties of organic oxygen compounds $\mathrm{L}$. The vapour pressures of 1,2-ethanediol (ethylene glycol) and bis(2-hydroxyethyl) ether (diethylene glycol)". In: The Journal of Chemical Thermodynamics 13.1, S. 61-66. issn: 0021-9614. doi: http://dx . doi .org/10 .1016/S0021-9614(81)80009-2. url: http: //www.sciencedirect.com/science/article/pii/s0021961481800092.

Bell, Ian H., Jorrit Wronski, Sylvain Quoilin und Vincent Lemort (2014). „Pure and Pseudopure Fluid Thermophysical Property Evaluation and the Open-Source Thermophysical Property Library CoolProp“. In: Industrial \& Engineering Chemistry Research 53.6, S. 24982508. doi: 10.1021/ie4033999. eprint: http://pubs . acs .org/doi/pdf/10 .1021/ ie4033999. url: http://pubs.acs.org/doi/abs/10.1021/ie4033999.

Chapoy, Antonin, Amir H. Mohammadi, Bahman Tohidi und Dominique Richon (Juli 2004). "Gas Solubility Measurement and Modeling for the Nitrogen Water System from 274.18 K to 363.02 K". In: Journal of Chemical \& Engineering Data 49.4, S. 1110-1115. doi: 10.1021/je049869d.

Chiavone-Filho, Osvaldo, Patricio Proust und Peter Rasmussen (1993). „Vapor-liquid equilibria for glycol ether + water systems". In: Journal of Chemical \& Engineering Data 38.1, S. 128-131. doi: 10 . 1021/ je00009a031. eprint: http://dx . doi .org/10 .1021/ je00009a031. url: http://dx.doi.org/10.1021/je00009a031.

D’Ans, J. und E. Lax (1997). Taschenbuch für Chemiker und Physiker: Band 3: Elemente, anorganische Verbindungen und Materialien, Minerale (German Edition). Springer. isbn: 3540600353.

Denbigh, K. (1. Jan. 1974). Prinzipien des Chemischen Gleichgewichts. Steinkopff. 416 S. isbn: 3798503621. url: http://www. ebook.de/de/product/17681328/k_denbigh_ prinzipien_des_chemischen_gleichgewichts.html.

Dlugokencky, Ed und Pieter Tans (2017). ESRL Global Monitoring Division. Earth System Research Laboratory. National Oceanic \& Atmospheric Administration.

Duchardt, T., G. Andersohn, M. Oechsner und R. Müller (2016). „Anforderungen an Kühlmittel auf Basis alternativer Gefrierpunkterniedrigungsmittel". In: MTZ. 12. Ser. 77. Jahrgang.

Eismann, R. (2017). Thermohydraulische Dimensionierung von Solaranlagen: Theorie und Praxis der kostenoptimierenden Anlagenplanung. Berlin Heidelberg New York: SpringerVerlag. isbn: 978-3-658-07125-7. doi: 10 . 1007/978-3-658-07125-7. url: https : //link. springer. com/content/pdf/10.1007\%2F978-3-658-07125-7.pdf.

Fendu, Elena M. und Florin Oprea (Feb. 2014). „Vapor-Liquid Equilibria for Water Propylene Glycols Binary Systems: Experimental Data and Regression“. In: Journal of Chemical \& Engineering Data 59.3, S. 792-801. doi: 10.1021/je4009014.

Fernández-Prini, Roberto, Jorge L. Alvarez und Allan H. Harvey (Juni 2003). „Henry's Constants and Vapor-Liquid Distribution Constants for Gaseous Solutes in H2O and D2O at High Temperatures". In: Journal of Physical and Chemical Reference Data 32.2, S. 903916. doi: $10.1063 / 1.1564818$.

Fischer, Kai und Juergen Gmehling (1994). „P-x and .gamma..infin. Data for the Different Binary Butanol-Water Systems at 50.degree.C". In: Journal of Chemical \& Engineering Data 39.2, S. 309-315. doi: 10 .1021/je00014a026. eprint: http://dx . doi .org/10. 1021/je00014a026. url: http://dx.doi.org/10.1021/je00014a026. 
Fischer, Kai und Michael Wilken (2001). „Experimental determination of oxygen and nitrogen solubility in organic solvents up to $10 \mathrm{MPa}$ at temperatures between $298 \mathrm{~K}$ and $398 \mathrm{~K}$ ". In: J. Chem. Thermodynamics. Bd. 33, S. 1285-1308. doi: 10.1006/jcht.2001.0837.

Fogg P.G.T.; Gerrard, W. (1991). Solubility of Gases in Liquids. John Wiley \& Sons Ltd.

Gamsjäger, Heinz u. a. (Juni 2010). „The IUPAC-NIST Solubility Data Series: A Guide to Preparation and Use of Compilations and Evaluations". In: Journal of Physical and Chemical Reference Data 39.2, S. 023101. doi: 10.1063/1.3380845.

Gernert, Georg Johannes (2013). „A New Helmholtz Energy Model for Humid Gases and CCS Mixtures". Diss. Ruhr-Universität Bochum.

Grabe, M. (2000). „Gedanken zur Revision der Gauß'schen Fehlerrechnung“. In: tm Technisches Messen 6 6, S. 283-288.

Grabe, M. (2010). Generalized Gaussian Error Calculus. Springer-Verlag Berlin Heidelberg.

Guignon, Bérengère, Cristina Aparicio und Pedro D. Sanz (2010). „Volumetric Properties of Pressure-Transmitting Fluids up to $350 \mathrm{MPa}$ : Water, Ethanol, Ethylene Glycol, Propylene Glycol, Castor Oil, Silicon Oil, and Some of Their Binary Mixture". In: Journal of Chemical \& Engineering Data 55.9, S. 3017-3023. doi: 10.1021/je9010568. eprint: http://dx. doi.org/10.1021/je9010568. url: http://dx.doi.org/10.1021/je9010568.

Harvey, A. H., S. G. Kaplan und J. H. Burnett (Sep. 2005). „Effect of Dissolved Air on the Density and Refractive Index of Water". In: International Journal of Thermophysics 26.5, S. 1495-1514. issn: 1572-9567. doi: 10.1007/s10765-005-8099-0. url: https: //doi.org/10.1007/s10765-005-8099-0.

Hefter, G.T. und R.P.T. Tomkins, Hrsg. (März 2003). The Experimental Determination of Solubilities. John Wiley \& Sons, Ltd. doi: 10.1002/0470867833.

Heller, Winfried (2005). „Habilitationsschrift: Hydrodynamische Effekte unter besonderer Berücksichtigung der Wasserqualität und ihre Messverfahren“. Habilitationsschrift. TU Dresden. url: http : / / www . qucosa . de / fileadmin / data / qucosa / documents / 1474 / 1141215758714-7391.pdf.

Henry, William (1803). „III. Experiments on the quantity of gases absorbed by water, at different temperatures, and under different pressures". In: Philosophical Transactions of the Royal Society of London 93, S. 29-274. doi: 10.1098/rstl .1803 .0004. eprint: http: //rstl . royalsocietypublishing . org/content/93/29.full . pdf+html. url: http://rstl. royalsocietypublishing.org/content/93/29. short.

Heymann, M. und K. Rühling (2013). Technikumsversuche zum Vergleich von Entlüftung und Entgasung + Vergleich von Befülltechnologien und Wirksamkeit von Entgasern (Kapitel $6+7$ in Endbericht Solarthermie2000plus - Entgasung von Solarkreisläufen und Bestimmung der zur Auslegung erforderlichen Stoffdaten). Techn. Ber. TU Dresden, Institut für Energietechnik. url: https : / / tu-dresden . de/ing/maschinenwesen/iet / gewv / ressourcen/dateien / forschung _ und _ projekte/projekte/berichte/ab_ entgasung_solarkreislaeufe?lang=de.

Heymann, Martin, Felix Panitz, Karin Rühling und Clemens Felsmann (2014). „Solubility Coefficients for Solar Liquids, a New Method to Quantify Undissolved Gases and Practical Recommendations“. In: Energy Procedia 48.0. Proceedings of the 2nd International Conference on Solar Heating and Cooling for Buildings and Industry (SHC 2013), S. 721-730. issn: 1876-6102. doi: http : / / dx . doi .org/10 .1016/ j . egypro. 2014 .02 .084. url: http://www.sciencedirect.com/science/article/pii/S1876610214003464.

Hildebrand, Joel Henry und Robert L. Scott (1950). The solubility of nonelectrolytes. American Chemical Society. Monograph series,no. 17. New York: Reinhold Pub. Corp., x, 488 p. url: https://hdl.handle.net/2027/wu .89077805729.

Hillerns (Juni 2013). E-Mail vom 13.06.2013 Reinheit der Propylenglykolgemische. 
Hillerns, Frank (2002). „Untersuchungen zur thermischen Dauerbelastbarkeit von Solarflüssigkeiten. Solarfluid muss zahlreiche Anforderungen erfüllen." In: Erneuerbare Energien. 1. Ser., 49ff.

Holfeld, Anna (Apr. 2008). „Thermodynamisch-hydraulische Charakterisierung von WasserFrostschutzmittel-Gemischen“. Großer Beleg. TU Dresden, Institut für Energietechnik, Professur Gebäudeenergietechnik und Wärmeversorgung.

Hunt, Sarah (2016). „Auswertung der Monitoring-Daten der Liegenschaft HENK im BMWiProjekt "FAkS - Feldtest Absorptionskälteanlagen für KWKK-Systeme"".. In: Technische Universität Berlin, Fachgebiet Maschinen- und Energieanlagentechnik.

IAPWS, Hrsg. (2004). Guideline on the Henry's Constant and Vapor-Liquid Distribution Constant for Gases in $\mathrm{H} 2 \mathrm{O}$ and D2O at High Temperatures. IAPWS (International Association for the Properties of Water und Steam). url: www. iapws . org.

IAPWS IF-97 (Aug. 2007). IAPWS IF-97 (Revised Release 2007) on the IAPWS Industrial Formulation 1997 for the Thermodynamic Properties of Water and Steam. IAPWS (International Association for the Properties of Water und Steam). url: www . iapws .org.

Kosmowski, I. und P. Hergt (1990). „Förderung gasbeladener Medien mit Hilfe von Normalund Sonderausführungen von Kreiselpumpen“. In: KSB Technische Berichte 2626.

Kretzschmar, H.-J. (2010). FluidLAB - Property Software - LibHuGas and LiblF97. Techn. Ber. Hochschule Zittau/Görlitz.

LS-SDB (Oktober 2009). EG - Sicherheitsdatenblatt Tyfocor LS-Fertigmischung. Metasol Chemie $\mathrm{GmbH}$.

Lüdecke, Christa und Dorothea Lüdecke (2000). Thermodynamik. Physikalisch-chemische Grundlagen der thermischen Verfahrenstechnik. Springer-Verlag Berlin Heidelberg New York.

Marchetti, Andrea u. a. (2000). „Variation of volumic properties with temperature and composition of 2-butanone + 1,2-propanediol binary mixtures". In: Journal of Molecular Liquids 88.2, S. 183-195. issn: 0167-7322. doi: http://dx.doi.org/10.1016/S01677322 (00)00153-7. url: http://www. sciencedirect.com/science/article/pii/ S0167732200001537.

Melinder, Åke (2010). Properties of Secondary Working Fluids for Indirect Systems. IIF-IIR Publishing.

Melzer, W.-M. (1990). „Ein Beitrag zur experimentellen Untersuchung von Gas-FlüssigkeitsPhasengleichgewichten". Diss. TU Berlin.

Meyer, E. (2002). „Untersuchungen zur Thermodynamik der Absorption von $\mathrm{CO}_{2}, \mathrm{SO}_{2}$ und $\mathrm{NH}_{3}$ in wässrigen, elektrolythaltigen Lösungen“. Diss. Universität Kaiserslautern.

Moore, Joseph C. u.a. (1982). „Partial Molar Volumes of "Gases” at Infinite Dilution in Water at 298.15 K". In: J. Chem. Eng. Data 27, S. 22-24.

Morrison, T. J. und F. Billett (1952). „730. The salting-out of non-electrolytes. Part II. The effect of variation in non-electrolyte". In: J. Chem. Soc. (0), S. 3819-3822. doi: 10.1039/JR9520003819. url: http://dx.doi .org/10.1039/JR9520003819.

Murray, C.N. und J.P. Riley (Juni 1969). „The solubility of gases in distilled water and sea water-II. Oxygen". In: Deep Sea Research and Oceanographic Abstracts 16.3, S. 311320. doi: 10.1016/0011-7471(69)90021-7.

Murray, C.N., J.P. Riley und T.R.S. Wilson (Juni 1969). „The solubility of gases in distilled water and sea water-I. Nitrogen“. In: Deep Sea Research and Oceanographic Abstracts 16.3, S. 297-310. doi: 10.1016/0011-7471(69)90020-5.

O'Sullivan, Thomas D. und Norman O. Smith (1970). „The Solubility and Partial Molar Volume of Nitrogen and Methane in Water and in Aqueous Sodium Chloride from 50 to $125{ }^{\circ} \mathrm{C}$ and 100 to 600 Atm". In: Journal of Physical Chemistry 74/7, S. 1460-1466. 
Panitz, F. und K. Rühling (Mai 2016). „Löslichkeit von Stickstoff und Sauerstoff in WasserGlykol-Kreisläufen“. In: 26. Symposium Thermische Solarenergie. OTTI e.V. Staffelstein. isbn: 978-3-943891-65-2.

Panitz, F. und K. Rühling (14. März 2017). Gasgehaltsanalyse Rückkühlkreis HENK. Kurzbericht. TU Dresden, Institut für Energietechnik.

Panitz, F., K. Rühling und M. Wagner (2013). Bestimmung von Löslichkeitskoeffizienten (Kapitel 3 in Endbericht Solarthermie2000plus - Entgasung von Solarkreisläufen und Bestimmung der zur Auslegung erforderlichen Stoffdaten). Techn. Ber. TU Dresden, Institut für Energietechnik. url: https : / / tu-dresden . de/ing/maschinenwesen/iet / gewv / ressourcen/dateien / forschung _ und _ projekte/projekte/berichte / ab_ entgasung_solarkreislaeufe?lang=de.

Pfennig, Andreas (2003). Thermodynamik der Gemische (German Edition). Springer. isbn: 3-540-02776-9.

Pham, M. (2015). Löslichkeitsuntersuchungen für Wasser-Glykol-Gemische. Diplomarbeit. TU Dresden.

Rane, Nilesh Vijay, Alka Kumari, J. Soujanya und B. Satyavathi (2016). „Excess properties and isobaric (vapor + liquid) equilibrium at sub-atmospheric pressures of binary (1,2propanediol + 1,3-propanediol) system: Measurement and modelling". In: The Journal of Chemical Thermodynamics 97, S. 142-157. issn: 0021-9614. doi: https://doi.org/10. 1016/j.jct.2016.01.019. url: http://www. sciencedirect.com/science/article/ $\mathrm{pii/S0021961416000410.}$

Rettich, T. R., Rubin Battino und Emmerich Wilhelm (1984). „Solubility of gases in liquids. $\mathrm{XVI}$. Henry's law coefficients for nitrogen in water at 5 to $50^{\circ} \mathrm{C}^{\prime \prime}$. In: Journal of Solution Chemistry 13.5, S. 335-348. issn: 1572-8927. doi: 10.1007/BF00645706. url: http: //dx.doi.org/10.1007/BF00645706.

Rühling, K. (März 2002). Gase in kleinen und mittleren Wasserheiznetzen und Kältekreisläufen. Schlussbericht zum AiF-Forschungsvorhaben 12086 B. Techn. Ber. TU Dresden, Institut für Energietechnik.

Rühling, K., M. Heymann und F. Panitz (Mai 2012a). „Entgasung von Solarkreisläufen und Bestimmung der zur Auslegung erforderlichen Stoffdaten“. In: 22. Symposium Thermische Solarenergie. Staffelstein: OTTI e.V. isbn: 978-3-941785-89-2.

Rühling, K., M. Heymann, F. Panitz und R. Eismann (Mai 2011a). „Entlüftung und Entgasung von Solaranlagen“. In: 21. Symposium Thermische Solarenergie. Staffelstein: OTTI e.V. isbn: 978-3-941785-57-1.

Rühling, K., M. Heymann, F. Panitz und R. Eismann (Mai 2011b). „Entlüftung und Entgasung von Solaranlagen“. In: Sonderveröffentlichung der AGFW.

Rühling, K., M. Heymann, F. Panitz und T. Rosemann (Apr. 2013a). „Vergleich von Befülltechnologien und Entgasungsszenarien für Solaranlagen“. In: 23. Symposium Thermische Solarenergie. Staffelstein: OTTI e.V. isbn: 978-3-943891-10-2.

Rühling, K. und D. Uhlmann (1998). Gase in kleinen und mittleren Wasserheiznetzen. Schlussbericht zum AiF-Forschungsvorhaben 11103-B. Techn. Ber. TU Dresden, Institut für Energietechnik.

Rühling, K. u. a. (2013b). Endbericht Solarthermie2000plus - Entgasung von Solarkreisläufen und Bestimmung der zur Auslegung erforderlichen Stoffdaten. Techn. Ber. TU Dresden, Institut für Energietechnik. url: https://tu-dresden.de/ing/maschinenwesen/iet/ gewv / ressourcen / dateien / forschung_ und_ projekte / projekte / berichte / ab_ entgasung_solarkreislaeufe?lang=de.

Rühling, Karin, Martin Heymann und Felix Panitz (2012b). „Venting and degasification of solar circuits". In: Energy Procedia 30.0. 1st International Conference on Solar Heating 
and Cooling for Buildings and Industry (SHC 2012), S. 815-823. issn: 1876-6102. doi: 10.1016/j .egypro.2012.11.092.

Saddington, Arthur W. und Norman W. Krase (Feb. 1934). „Vapor-Liquid Equilibria in the System Nitrogen-Water". In: Journal of the American Chemical Society 56.2, S. 353361. doi: 10.1021/ja01317a024.

Sander, R. (Apr. 2015). "Compilation of Henry's law constants (version 4.0) for water as solvent". In: Atmospheric Chemistry and Physics 15.8, S. 4399-4981. doi: 10.5194/acp$15-4399-2015$.

Sander, Rolf (Jan. 1999). „Modeling Atmospheric Chemistry: Interactions between GasPhase Species and Liquid Cloud/Aerosol Particles". In: Surveys in Geophysics 20.1, S. $1-$ 31. issn: 1573-0956. doi: 10.1023/A:1006501706704. url: https://doi.org/10.1023/ A : 1006501706704 .

Sittiho, Mutchima (2011). „Quantitative Beurteilung des Gaseintrags in thermische Energieversorgungssysteme aufgrund der Gaspermeation“. Diss. TU Chemnitz.

Span, R. u. a. (2016). „TREND - Thermodynamic Reference and Engineering Data 3.0“. In: Lehrstuhl für Thermodynamik, Ruhr-Universität Bochum.

Stephan, Peter, Karlheinz Schaber, Karl Stephan und Franz Mayinger (2010). Thermodynamik - Grundlagen und technische Anwendungen, Band 2: Mehrstoffsysteme und chemische Reaktionen. 15. Springer-Verlag Berlin Heidelberg. doi: 10.1007/978-3-540-36855-7.

Stichel, W. (1998). „Einsatz und Alterung von Frostschutzmitteln in Heizanlagen“. In: Materials and Corrosion 49.2, S. 88-97. issn: 1521-4176. doi: 10 . 1002 / (SICI ) 1521 4176 (199802) 49:2<88: : AID-MAC088>3 . 0 . C0 ; 2 - 9. url: http ://dx . doi .org/10 . 1002/(SICI) 1521-4176 (199802) 49:2\%3C88: : AID-MAC088\%3E3. 0.C0;2-9.

Tanaka, M. u. a. (2001). „Recommended table for the density of water between $0{ }^{\circ} \mathrm{C}$ and 40 ${ }^{\circ} \mathrm{C}$ based on recent experimental reports". In: Metrologica 38, S. 301-309.

Tulegenov, A.S., R.J. Wheatley, M.P. Hodges und A.H. Harvey (2008). „Intermolecular potential and second virial coefficient of the water-nitrogen complex". In: J. Chem. Phys. 126, S. 094305. doi: 10.1063/1.2446843.

Tyfocor (2015). Tyfocor, Technische Information. Metasol Chemie GmbH.

TyfocorLS (Oktober 2001). Tyfocor LS, Technische Information. Metasol Chemie GmbH.

Umbreit, L. (Dezember 2014). „Untersuchungen zum Einfluss von atmosphärischen Gasen auf Wirkungsgrad und Druckverlust solarthermischer Kollektoren“. Diplomarbeit. TU Dresden.

Unger, S. (2013). Löslichkeitsuntersuchungen für Solarflüssigkeiten - Diffusions- und Absorptionsverhalten. Großer Beleg. TU Dresden, Fakultät Maschinenwesen, Institut für Energietechnik.

Planungshandbuch Solarthermie (2008). Handbuch. Allendorf (Eder): Viessmann Deutschland $\mathrm{GmbH}$. url: https://www.google.de/url?sa=t\&rct=j\&q=\&esrc=s\&source=web\& $c d=2 \& v e d=0$ ahUKEwi iqq_EsaDZAhXRxqQKHdFNCxgQFgg5MAE\&url=https $\% 3 \mathrm{~A} \% 2 \mathrm{~F} \% 2 \mathrm{Fwww}$. viessmann - community . com $\% 2 \mathrm{Fviessmann} \% 2 \mathrm{Fattachments} \% 2 \mathrm{Fviessmann} \% 2 \mathrm{Fqanda}$ anlagenbetreiber \% 2F37301\%2F2\%2F29555 . pdf \& usg=ADvVaw1KAasKI6CtAGsqGs2 jd9G.

Wagner, M. (2010). „Aufbau einer Apparatur zur Bestimmung technischer Löslichkeitskoeffizienten für Wasser-Glykol-Gemische“. Diplomarbeit. TU Dresden, Institut für Energietechnik.

Wallace, J.J.M. und P.V. Hobbs (2006). Atmospheric Science: An Introductory Survey. International Geophysics Series. Elsevier Academic Press. isbn: 9780127329512. url: https: //books . google.de/books?id=j0C4lAEACAAJ.

Walther, G. und F. W. Günthert (1998). „Neue Untersuchungen zur Selbstentlüftungsgeschwindigkeit in Trinkwasserleitungen". In: Wasser Abwasser 08, S. 475-481. 
Wilcock, J. Robert und Rubin Battino (Dez. 1974). „Solubility of oxygen-nitrogen-mixture in water". In: Nature 252, S. 614-615.

Wolf, Fabian (2018). „Gaskavitation in kohlenwasserstoffhaltigen Flüssigkeiten: Einfluss von ruhendem, strömendem und hydrodynamisch kavitierendem Zustand“. Diss. TU Dresden. isbn: 978-3-95908-130-6.

Yamamoto, H., H. Kamei und J. Tokunaga (1994). „Solubilities of Argon, Oxygen and Nitrogen in 1,2- Propanediol+water mixed solvent at $298.15 \mathrm{~K}$ and $101.33 \mathrm{kPa}$ “. In: Journal of chemical engineering of Japan 27.4, S. 455-459.

Yamamoto, H. und J. Tokunaga (1994). „Solubilities of Nitrogen and Oxygen in 1,2- Ethanediol + Water at $298.15 \mathrm{~K}$ and 101.33 kPa“. In: J. Chem. Eng. Data 39, S. 544-547.

Young, C.L., R. Battino und H.L. Clever (1987). „The Solubility of gases in liquids. Introductory Information". In: Methane. Elsevier, S. xii-xviii. doi: 10.1016/b978-0-08-0292007.50007-2.

Zhou, Tongliang und Rubin Battino (2001). „Partial Molar Volumes of 13 Gases in Water at $298.15 \mathrm{~K}$ and $303.15 \mathrm{~K}$ ". In: J. Chem. Eng. Data 46, S. 331-332.

Hinweis: Teile der Arbeit sind dem vom Autor eigenständig verfassten Teil [Panitz u. a., 2013] des Schlussberichts [Rühling u. a., 2013b], der auf Vorarbeiten dieser Dissertation beruht, wortwörtlich übernommen. Ein Teil der Versuchsreihen wurde basierend auf den Arbeiten des Autors zu dieser Dissertation bereits durch [Unger, 2013] und [Pham, 2015] im Rahmen ihrer studentischen Arbeiten vereinfacht ausgewertet. 



\section{A Anhang}

A.1 Tabelle der verbleibenden Isofugazitäts-Gleichungen entsprechend der Vereinfachungen

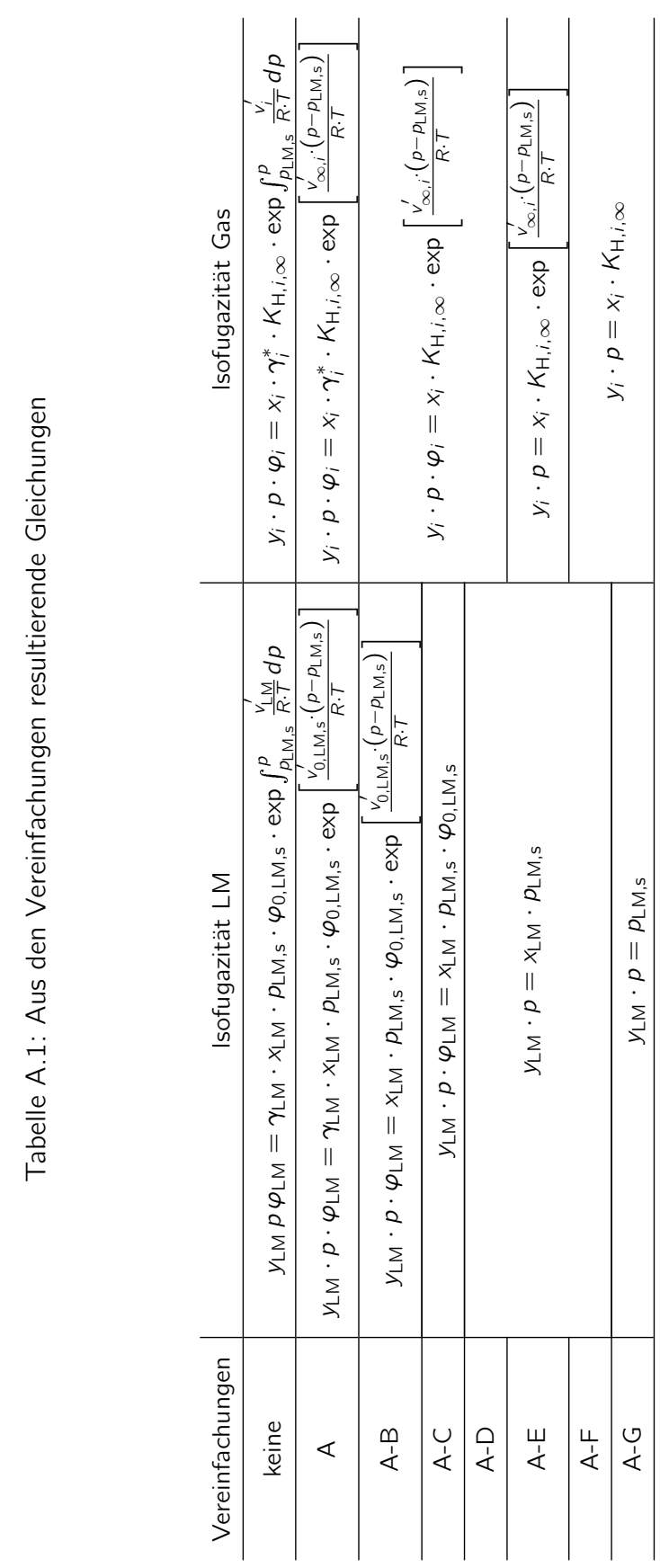




\section{A.2 Herleitung Zusammenhang der Vereinfachungen A-F und A-G}

Aus der Gleichung

$$
x_{i, \mathrm{~A}-\mathrm{F}}=p_{i, \mathrm{~A}-\mathrm{F}} \cdot \frac{1}{K_{\mathrm{H}, i, \infty}}
$$

mit $p_{i, \mathrm{~A}-\mathrm{F}}=p-\left(1-x_{i, \mathrm{~A}-\mathrm{F}}\right) \cdot p_{\mathrm{LM}, \mathrm{s}}$ ergibt sich

$$
x_{i, \mathrm{~A}-\mathrm{F}}=\left(p-\left(1-x_{i, \mathrm{~A}-\mathrm{F}}\right) \cdot p_{\mathrm{LM}, \mathrm{s}}\right) \cdot \frac{1}{K_{\mathrm{H}, i, \infty}}
$$

Dies lässt sich umformen zu

$$
x_{i, \mathrm{~A}-\mathrm{F}}=\left(p-p_{\mathrm{LM}, \mathrm{s}}\right) \cdot \frac{1}{K_{\mathrm{H}, i, \infty}}+\frac{x_{i, \mathrm{~A}-\mathrm{F}} \cdot p_{\mathrm{LM}, \mathrm{s}}}{K_{\mathrm{H}, i, \infty}} .
$$

Der erste Summand entspricht resultierenden Gleichung der Vereinfachungen A-G

$$
x_{i, \mathrm{~A}-\mathrm{G}}=p_{i, \mathrm{~A}-\mathrm{G}} \cdot \frac{1}{K_{\mathrm{H}, i, \infty}}
$$

mit $p_{i, \mathrm{~A}-\mathrm{G}}=p-p_{\mathrm{LM}, \mathrm{s}}$. Es ergibt sich also

$$
x_{i, \mathrm{~A}-\mathrm{F}}=x_{i, \mathrm{~A}-\mathrm{G}}+\frac{x_{i, \mathrm{~A}-\mathrm{F}} \cdot p_{\mathrm{LM}, \mathrm{s}}}{K_{\mathrm{H}, i, \infty}}
$$

und damit

$$
x_{i, \mathrm{~A}-\mathrm{F}} \cdot\left(1-\frac{p_{\mathrm{LM}, \mathrm{s}}}{K_{\mathrm{H}, i, \infty}}\right)=x_{i, \mathrm{~A}-\mathrm{G}} .
$$

\section{A.3 Angaben zum Aufbau}




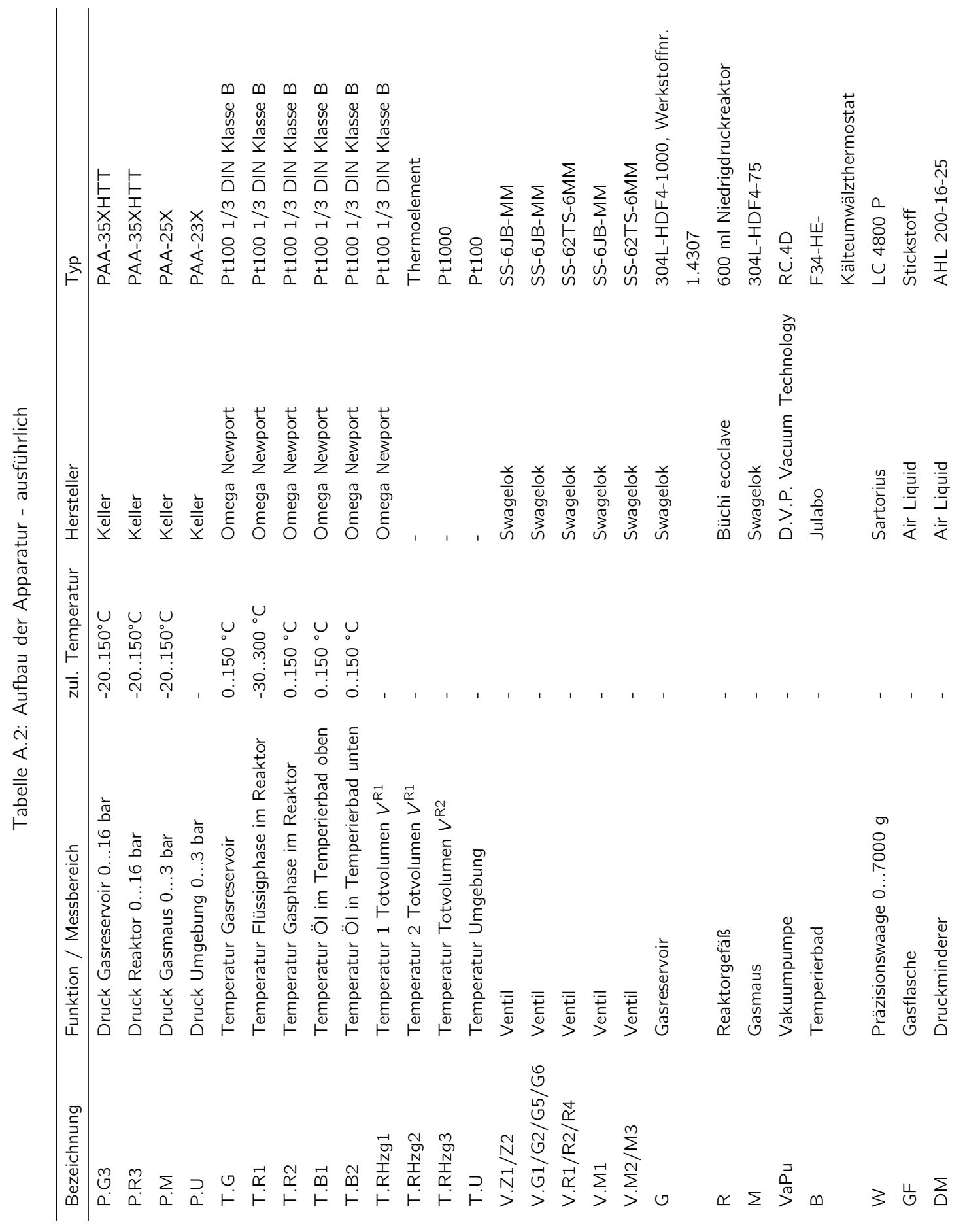




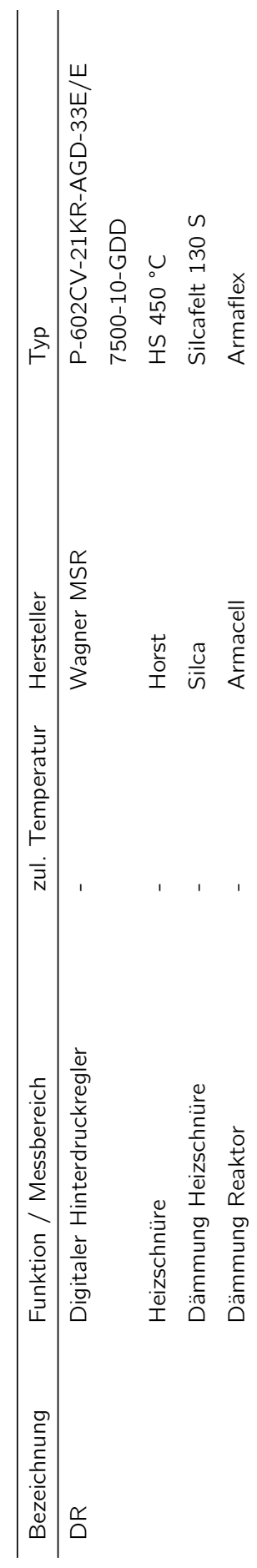




\section{A.4 Charakterisierung der Versuchskonfigurationen}

Neben den entsprechend Tabelle A.3 leicht veränderten Versuchsvolumina sind folgende Unterschiede zu nennen, die sich durch sukzessive Optimierung oder auch Materialausfälle ergeben haben.

Konfiguration A:

- Ausgangszustand luftgesättigt

- Rühren mit $1000 \mathrm{~min}^{-1}$

- Trocknung durch Evakuierung, Totvolumina werden dabei auf $115{ }^{\circ} \mathrm{C}$ beheizt

Änderungen in Konfiguration B:

- Rühren mit 1100 min $^{-1}$ anlässlich hoher Zähigkeit von $100 \mathrm{G}$

- Trocknung des Gefäßes durch iterative Evakuierungen und anschließendes Einsaugen von Luft, vorherige Temperierung des Reaktors auf $80{ }^{\circ} \mathrm{C}$ (Totvolumina auf $115{ }^{\circ} \mathrm{C}$ )

- Umbau des Vakuum-Anschlusses, Einbau des Ventils V.R5

Änderungen in Konfiguration C:

- Abbau von P.R1 und P.R2, Umbau Totraumtemperierung, T.RHzg2 außer Betrieb gesetzt

- Anbau P.V zur besseren Bestimmung des Vakuums

Änderungen in Konfiguration D:

- Änderung der Regler-Konstanten des Thermostaten

- Ausgangszustand entgast

- für Luftversuche: Installation Probenahme-Zylinder und Drucksensor P.P 


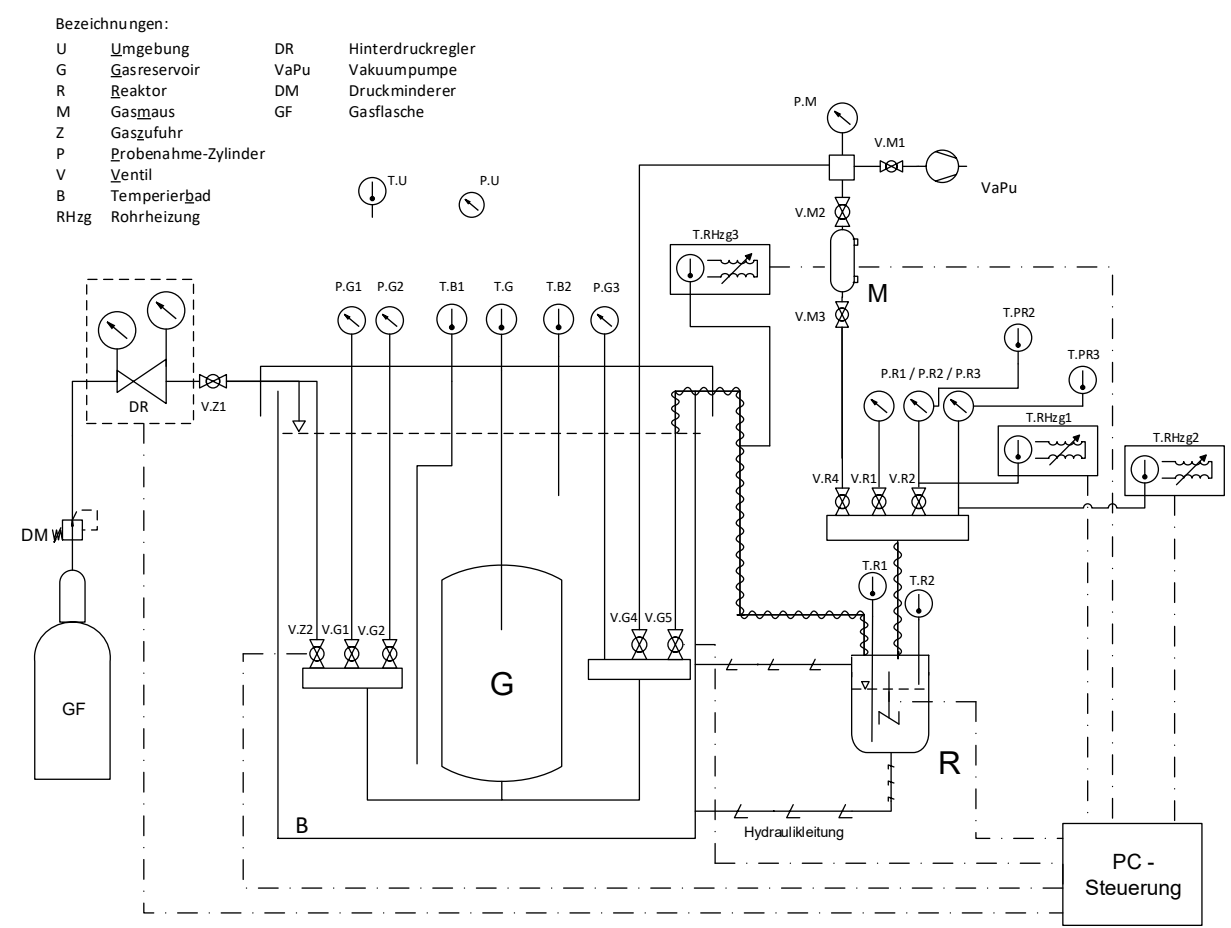

Abbildung A.1: Detailaufbau der Apparatur - Konfiguration A

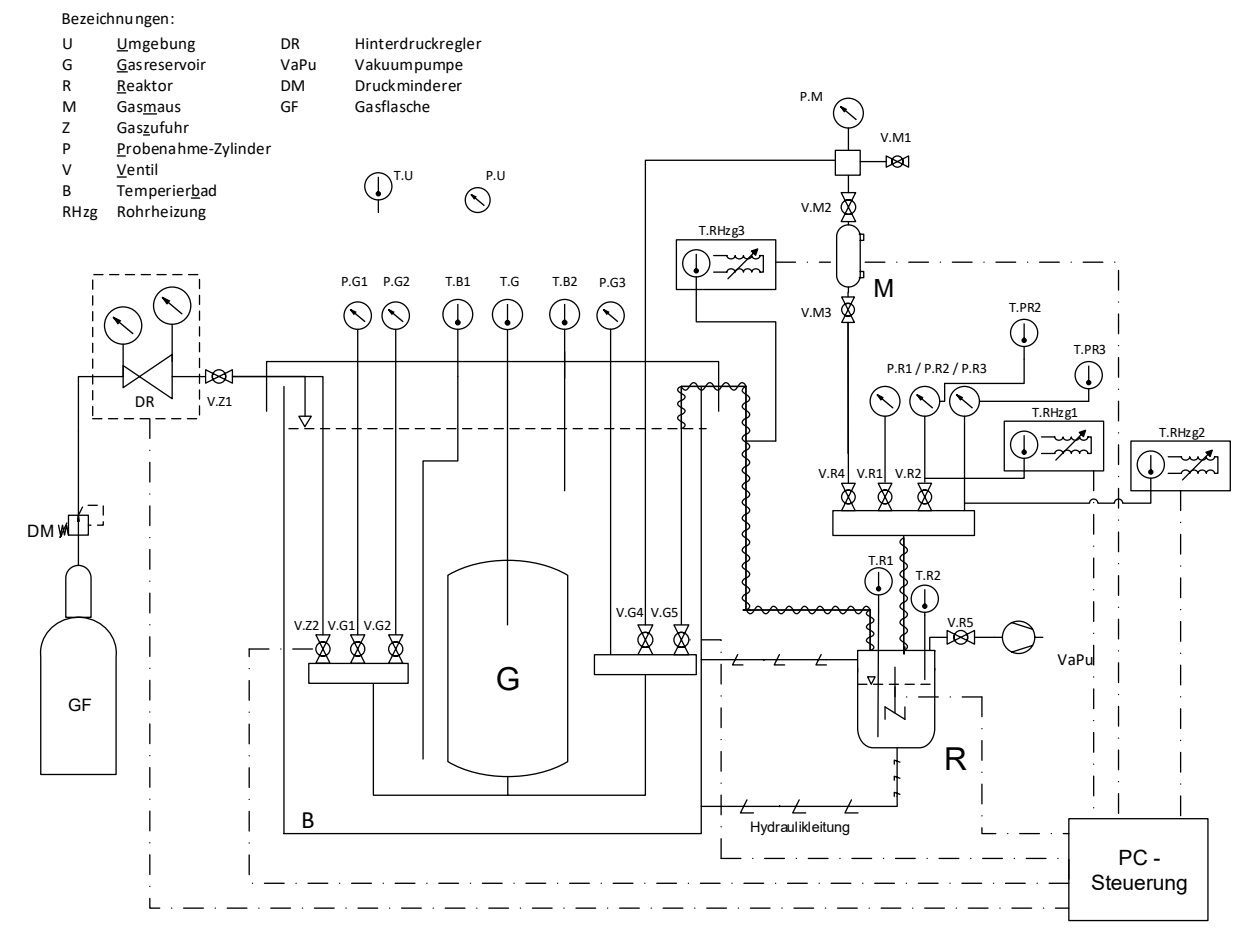

Abbildung A.2: Detailaufbau der Apparatur - Konfiguration B 


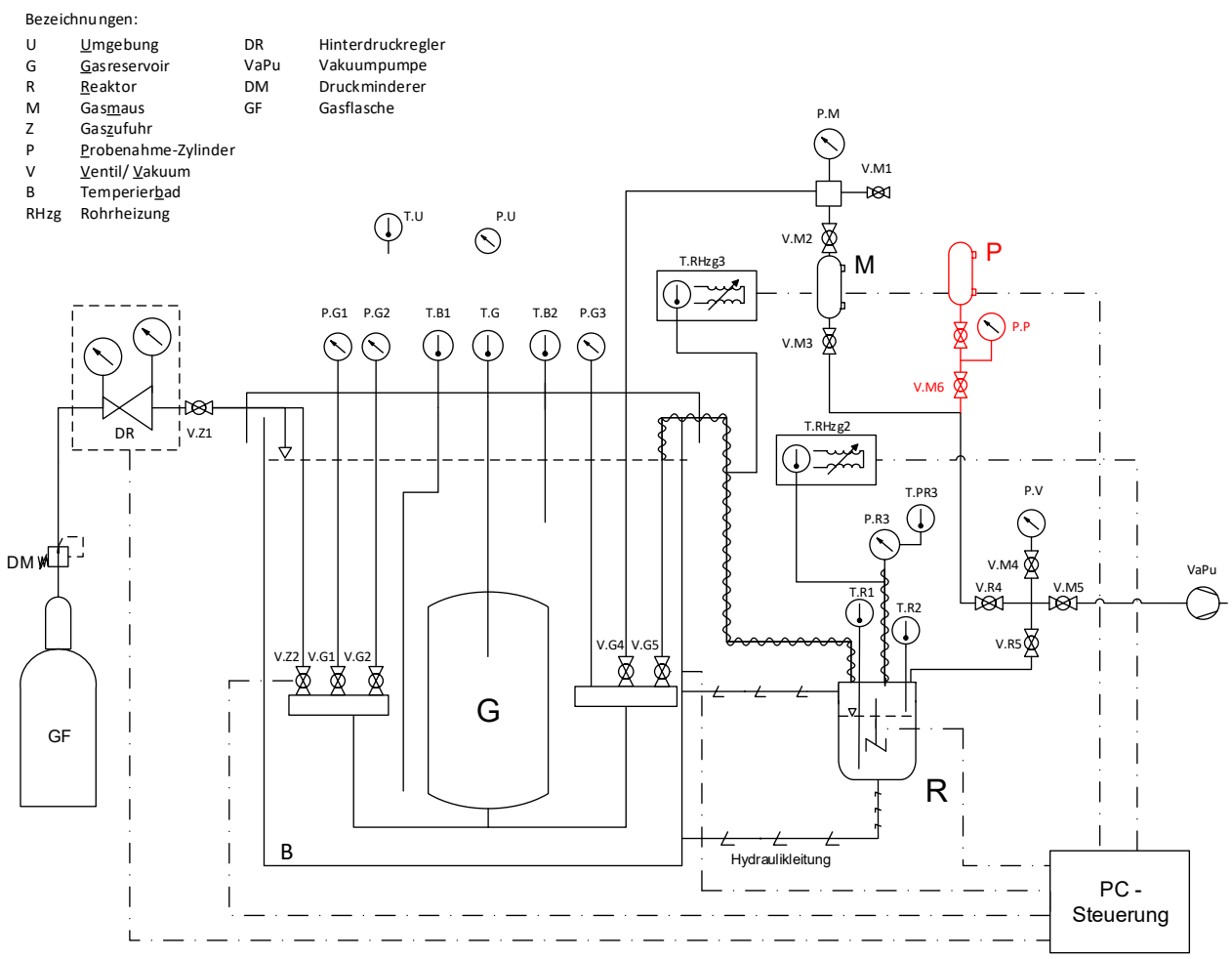

Abbildung A.3: Detailaufbau der Apparatur - Konfiguration C, Konfiguration D (Ergänzung ggü. Konfiguration $C$ rot dargestellt) 


\section{A.5 Abschätzung des Lösungsmittelmasse-Verlusts bei entgastem Ausgangszustand}

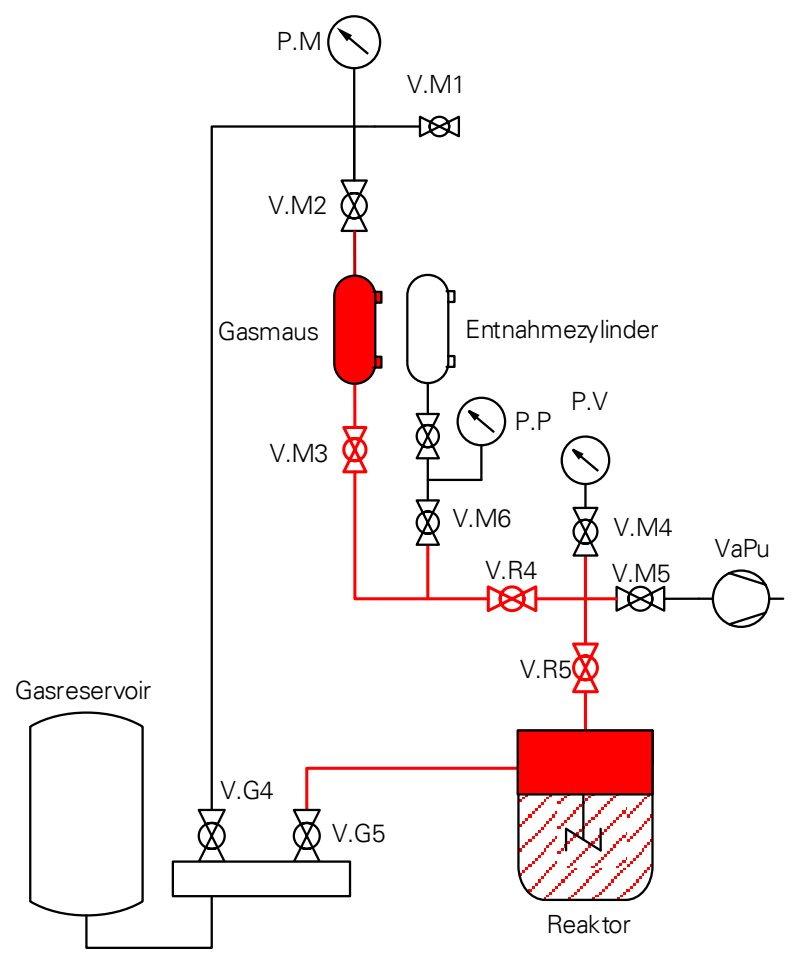

Abbildung A.4: Verbundenes Volumen nach Druckausgleich zwischen evakuiertem Hilfsvolumen und Reaktor

Die in Abschnitt 4.4.1.1 beschriebene Entgasung des Lösungsmittels findet mittels des evakuierten Hilfs-Volumens statt, das von V.R5 bis V.M2 reicht und die Gasmaus einschließt (vgl. Abbildung A.4). Dieses Volumen kann abgeschätzt werden zu ca. $V_{\text {Hilf }}=100 \mathrm{ml}$. Beim Druckausgleich wird auch im Reaktor in Dampfform vorliegendes Lösungsmittel teilweise in das Hilfs-Volumen ausgeschoben. Da das Hilfsvolumen annähernd die gleiche Temperatur besitzt, wird davon ausgegangen, dass der ausgeschobene Dampf dort nicht kondensiert. Die bei jedem Druckausgleich ausgeschobene Dampfmenge für jeden Zyklus kann also mit Idealgasverhalten ${ }^{1}$ durch den im gesättigten Zustand vorliegenden Partialdruck $p_{\mathrm{LM}, \mathrm{s}}$ nach oben abgeschätzt werden zu

$$
m_{\mathrm{LM}, \mathrm{V}, \text { Zyklus }} \leq M_{\mathrm{LM}}^{\prime \prime} \cdot \frac{p_{\mathrm{LM}, \mathrm{s}} \cdot V_{\text {Hilf }}}{R \cdot T} .
$$

Für die einzelnen Lösungsmittelkomponenten $k$ ergibt sich nach Raoult

$$
m_{k, \mathrm{~V}, \text { Zyklus }} \leq M_{k} \cdot \frac{x_{k} \cdot p_{k, \mathrm{~s}} \cdot V_{\text {Hilf }}}{R \cdot T}
$$

Der stoffspezifische Term $M_{k}^{\prime \prime} \cdot \frac{x_{k} \cdot p_{k, s}}{R \cdot T}$ ist dabei die Konzentration des Lösungsmitteldampfes in $\mathrm{kg} / \mathrm{m}^{3}$. Bei einer angenommenen Temperatur von $20{ }^{\circ} \mathrm{C}$ beträgt diese für reines Was-

${ }^{1}$ Auch für die Glykoldampf mit relativ großen Molekülen muss hier, aufgrund fehlender anderer Daten, der Idealgasansatz zur Abschätzung genügen. 
ser $0,0173 \mathrm{~kg} / \mathrm{m}^{3}$. Für reines Propylenglykol bzw. Ethylenglykol ergeben sich aufgrund der deutlich geringeren Partialdrücke trotz der höheren molaren Massen deutlich geringere Werte von $5,8 \cdot 10^{-4} \mathrm{~kg} / \mathrm{m}^{3}$ bzw. $1,9 \cdot 10^{-4} \mathrm{~kg} / \mathrm{m}^{3}{ }^{2}$ Bei reinem Wasser ist also der höchste Lösungsmittelaustrag zu erwarten.

Die Anzahl der Entgasungszyklen übersteigt in keinem der Wasserversuche den Wert 10. Der Gesamtmasseverlust beträgt somit maximal

$$
m_{\mathrm{LM}, \mathrm{V}, \text { ges }} \ll 10 \cdot 0,0173 \mathrm{~kg} / \mathrm{m}^{3} \cdot 100 \mathrm{ml}=0,0173 \mathrm{~g} .
$$

Die ausgeschobene Gasmenge liegt gerade bei den ersten Ausschüben nicht lösungmittelgesättigt vor, also sind real ausgetragene Mengen noch deutlich unter dem Maximalwert. Damit liegt der Fehler bei Nichtbeachtung des Lösungsmittelmasseverlusts maximal in der Größenordnung der Messunsicherheit bei der vorangegangenen Wägung des Lösungsmittels, dessen Einfluss auf die Unsicherheit des Gesamtergebnisses, wie später in Abschnitt 4.9 zu sehen, marginal ist. In der Berechnung und Messunsicherheitsbetrachtung wurde daher der Lösungsmittelmasse-Verlust vernachlässigt.

\section{A.6 Bestimmung der Volumina $V_{0}^{G}$ und $V_{0}$}

\section{A.6.1 Volumen Gasreservoir $V_{0}^{G}$}

Die Bestimmung des Volumens $V_{0}^{G}$ erfolgt über die bekannte Zustandsgleichung von Stickstoff. Im Zustand A befindet sich die definierte Stickstoffmenge nur im Gasreservoir und die vorher mit Stickstoff gespülte Gasmaus ist evakuiert. Im Zustand B werden die beiden Volumina verbunden. Es erfolgt ein Druckausgleich. Die Gesamtmenge Stickstoff in Reaktor und Gasvolumen bleibt die gleiche bei beiden Zuständen. Bei bekanntem Volumen der Gasmaus $V^{\mathrm{M}}$ und bekannten Drücken im Zustand $A$ und $B$ lässt sich das Volumen $V_{0}^{G}$ ermitteln. Es gilt

$$
m_{\mathrm{N} 2, \mathrm{~A}}^{\mathrm{G}}+m_{\mathrm{N} 2, \mathrm{~A}}^{\mathrm{M}}=m_{\mathrm{N} 2, \mathrm{~B}}^{\mathrm{G}}+m_{\mathrm{N} 2, \mathrm{~B}}^{\mathrm{M}} .
$$

Bei bekanntem Dichteverhalten von Stickstoff und gemessenen Drücken und Temperaturen folgt

$$
\rho\left(p_{\mathrm{M}}^{\mathrm{G}}, t_{\mathrm{A}}^{\mathrm{G}}\right) \cdot V_{0}^{\mathrm{G}}+\rho\left(p_{\mathrm{A}}^{\mathrm{M}}, t_{\mathrm{A}}^{\mathrm{M}}\right) \cdot V^{\mathrm{M}}=\rho\left(p_{\mathrm{B}}^{\mathrm{G}}, t_{\mathrm{B}}^{\mathrm{G}}\right) \cdot V_{0}^{\mathrm{G}}+\rho\left(p_{\mathrm{B}}^{\mathrm{G}}, t_{\mathrm{B}}^{\mathrm{M}}\right) \cdot V^{\mathrm{M}}
$$

und umgestellt

$$
V_{0}^{G}=V^{\mathrm{M}} \cdot\left(\frac{\rho\left(p_{\mathrm{B}}^{\mathrm{G}}, t_{\mathrm{B}}^{\mathrm{M}}\right)-\rho\left(p_{\mathrm{A}}^{\mathrm{M}}, t_{\mathrm{A}}^{\mathrm{M}}\right)}{\rho\left(p_{\mathrm{A}}^{\mathrm{G}}, t_{\mathrm{A}}^{\mathrm{G}}\right)-\rho\left(p_{\mathrm{B}}^{G}, t_{\mathrm{B}}^{\mathrm{G}}\right)}\right) .
$$

\section{A.6.2 Volumen Reaktor $V_{0}$}

Das Volumen des Reaktorgefäßes geht deutlich sensibler in die Rechnung ein. Daher ist das für $V_{0}^{G}$ gewählte Verfahren hier nicht anwendbar. Es kann gezeigt werden, dass der absolute

\footnotetext{
${ }^{2}$ Aufgrund der noch geringeren Dampfdrücke der Inhibitoren wird erwartet, dass deren Konzentrationen noch geringer sind.
} 
Fehler zur Berechnung des Volumens nach dem bei $V_{0}^{G}$ angewandten Verfahren bei kleineren Volumina stark sinkt.

Daher wurde hier das Volumen des Reaktorgefäßes durch Auffüllen mit vorher gewogenem Wasser „verkleinert“. Die Gasmaus wird mit Stickstoff im Überdruck gefüllt. Im Zustand 2 wird in das Reaktorgefäß entspannt. Hier ist jedoch die Löslichkeit von Stickstoff im Wasser zu beachten.

Das Verfahren entspricht dem Vorgehen für die Bestimmung der Gaslöslichkeit, wobei jedoch hier die Gasmaus die Funktion des Gasreservoirs ersetzt. Die gesuchte Größe ist hier nicht die Gaslöslichkeit (für Wasser bekannt), sondern das Volumen des Reaktorgefäßes.

Eine Umstellung des Lösungsalgorithmus zur Bestimmung der Löslichkeit ist aufwendig. Daher wird die bekannte Berechnung der Löslichkeit (siehe Abschnitt 4.5)

$$
f_{\text {Löslichkeit }}\left(\vec{x}, V_{0}\right)
$$

mit den Eingangsgrößen $\vec{x}$ (Messwerte) und $V_{0}$ genutzt und die Bedingung

$$
\lambda_{\mathrm{H}_{2} \mathrm{O}} \text {, Tabelle } \stackrel{!}{=} f_{\text {Löslichkeit }}\left(\vec{x}, V_{0}\right)
$$

aufgestellt und $V_{0}$ numerisch bestimmt durch die nichtlineare Gleichung

$$
0=f_{\text {Löslichkeit }}\left(\vec{x}, V_{0}\right)-\lambda_{\mathrm{H}_{2} \mathrm{O}} \text {, Tabelle } .
$$

Auch für die Messunsicherheitsbetrachtung $U\left(V_{0}\right)$ kann aufgrund der Schnelligkeit des Solvers diese Funktion genutzt werden. Unsicherheiten werden die gleichen genutzt wie beim Hauptversuch. Temperaturmessungen haben jedoch aufgrund der Messungen bei annähernd Umgebungstemperatur deutlich geringere Fehlerbandbreiten, insbesondere für die sonst unsicher temperierten Toträume. Für eine geringe Messunsicherheit ist (im Gegensatz zu den Löslichkeitsversuchen) eine sehr große, in Standardversuchen jedoch unpraktikable, Wassermenge eingefüllt worden. 


\section{A.6.3 Ermittelte Volumina}

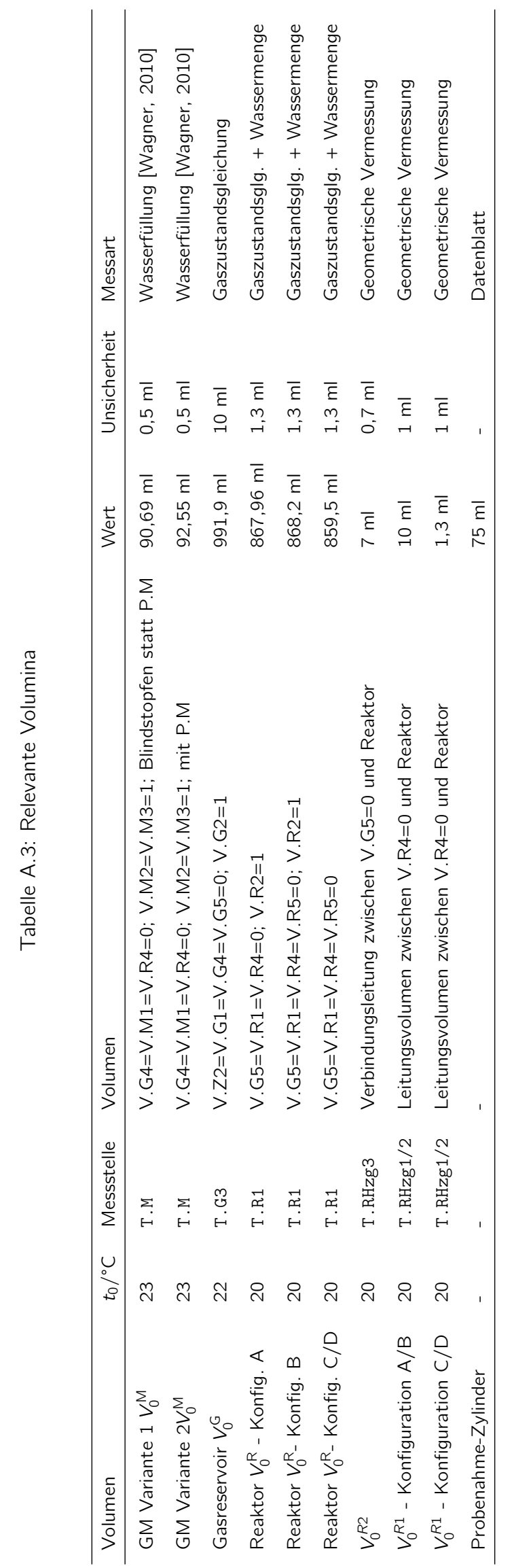




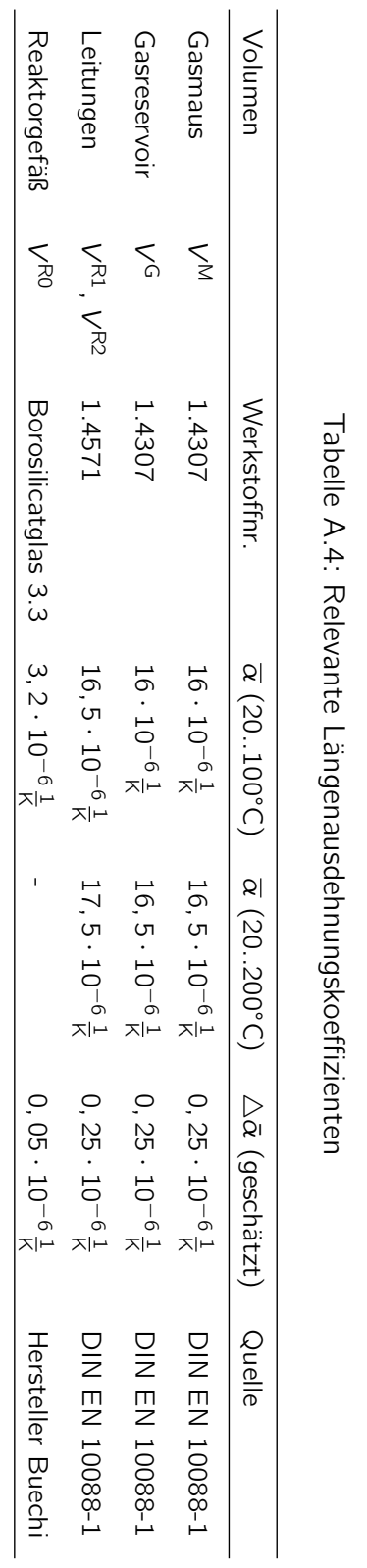




\section{A.7 Kalibrierkurven und deren Unsicherheit - Theorie}

\section{A.7.1 Hintergrund / Einordnung}

Für Druck- und Temperatursensorik wurden eigene Kalibrierungen vorgenommen. Dazu werden die Messwerte der Sensoren mit einer geeigneten Referenz verglichen. Hier wird die Erstellung von Kalibrierkurven und deren Unsicherheit diskutiert unter der Bedingung, dass die gemessenen Korrektionen nicht nur eine statistische Unsicherheit, sondern auch eine signifikante systematische Unsicherheit besitzen.

Ursache sind Referenzsensoren, die selbst signifikante Unsicherheiten aufweisen. Im Sinne eines rationellen Einsatzes finanzieller und personeller Ressourcen wurde auf eine Realisierung geringerer systematischer Unsicherheiten der Korrektionen verzichtet. Aufgrund der Effekte durch

- mehrere in die Gesamtrechnung eingehende Messungen mit dem gleichen Sensor und

- Kalibrierung der Apparatur mit Wasser

verringern sich jedoch für das Messergebnis deutlich die Messunsicherheitsbeiträge durch die Kalibrierkurvenunsicherheiten. Im Folgenden werden dafür die mathematischen Grundlagen beschrieben.

\section{A.7.2 Kalibrierkurve / Korrekturfunktion}

Der Anzeigewert (Rohwert) $x_{\text {raw }}$ des Sensors soll in den korrigierten/kalibrierten Messwert $x_{\text {korr }}$ überführt werden mit

$$
x_{\text {korr }}=x_{\text {raw }}+K\left(x_{\text {raw }}\right)
$$

wobei $K\left(x_{\text {raw }}\right)$ die Korrekturfunktion ist. Als "wahre" Korrekturkurve wird ein Polynom

$$
K\left(x_{\mathrm{Anz}}\right)=c_{1}+c_{2} \cdot x_{\mathrm{raw}}+c_{3} \cdot x_{\mathrm{raw}}^{2}
$$

angenommen.

Eine Kalibrierung führt zu Tupeln aus Anzeigewert $x_{\text {raw,i }}$ des Sensors und zu-

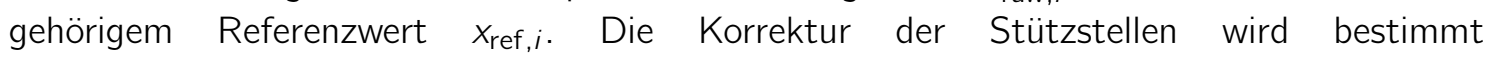
aus

$$
K_{i}=x_{\text {ref }, i}-x_{\text {raw }, i}
$$

Es ergeben sich also $i=1 . . m$ Tupel $\left[x_{\text {raw }, i}, K_{i}\right]$ als Stützstellen für das Korrekturpolynom $K\left(x_{\text {raw }}\right)$ 


\section{A.7.3 Bestimmung der Koeffizienten der Kalibrierkurve}

Aus den Tupeln $\left(x_{\text {raw }, i}, K\right)$, im Folgenden nur noch $\left(x_{i}, y_{i}\right)$ genannt, wird mittels "Methode der kleinsten Abweichungsquadrate" das Polynom $K\left(x_{\text {raw }}\right)$ bestimmt. In [Grabe, 2010] wird dazu die orthogonale Projektion benutzt.

Definiert man folgende Größen

$$
\boldsymbol{A}=\left(\begin{array}{ccc}
1 & x_{1} & x_{1}^{2} \\
1 & x_{2} & x_{2}^{2} \\
& \cdots & \\
1 & x_{m} & x_{m}^{2}
\end{array}\right), \quad \boldsymbol{c}=\left(\begin{array}{c}
c_{1} \\
c_{2} \\
c_{3}
\end{array}\right), \quad \boldsymbol{y}=\left(\begin{array}{c}
K_{1} \\
K_{2} \\
\ldots \\
K_{m}
\end{array}\right)
$$

und

$$
\boldsymbol{B}=\boldsymbol{A}\left(\boldsymbol{A}^{\top} \boldsymbol{A}\right)^{-1}=\left(b_{i j}\right)
$$

erhält man die Koeffizienten durch

$$
c=B^{\top} y
$$

bzw.

$$
c_{j}=\sum_{i=1}^{m} b_{i j} K_{i}
$$

\section{A.7.4 Unsicherheit der Stützstellen}

Die Messunsicherheit $U_{K_{i}}$ der Stützstellen $K_{i}$ besitzt

- zufällige Anteile (Auflösung der Messwerte, Wiederholpräzision) und

- einen signifikanten systematischen Anteil.

Dabei muss davon ausgegangen werden, dass die unbekannte systematische Messabweichung für jede Stützstelle $K_{i}$ unterschiedlich sein kann.

\section{A.7.5 Unsicherheit der Koeffizienten}

Aus Gründen der Vereinfachung der Rechnung wird der Gesamtfehler (inkl. zufälliger, eigentlich nicht korrelierter Anteile) pro Stützstelle als maximal korreliert betrachtet. Diese Vereinfachung liefert eine etwas gröbere Abschätzung und wird hier durch das sonst wesentlich komplexeres Formelwerk für zufällige Fehler und den deutlich überwiegenden systematischen Teil der Unsicherheit im Unsicherheitsbudget von $K_{i}$ begründet. 
Allgemein ergibt sich also der Gesamtfehler eines Koeffizienten $c_{j}$ der Kalibrierkurve $\mathrm{zu}$

$$
U_{c_{j}}=\sum_{i=1}^{m}\left|\frac{\partial c_{j}}{\partial K_{i}}\right| \cdot U_{K_{i}}=\sum_{i=1}^{m}\left|b_{i j}\right| \cdot U_{K_{i}}
$$

\section{A.7.6 Unsicherheit der Kalibrierkurve}

Da die Messunsicherheiten $U_{c_{j}}$ der Koeffizienten durch gleiche Eingangsgrößen $U_{K_{i}}$ untereinander korreliert sind, wird der Fehler der gesamten Kalibrierkurve auch direkt aus den Messwerten bestimmt. An der Stelle $x_{\text {raw }}$ ergibt sich die Unsicherheit der Korrekturfunktion $K$ zu

$$
U_{K}\left(x_{\text {raw }}\right)=\sum_{i=1}^{m}\left|\frac{\partial K\left(x_{\text {raw }}\right)}{\partial K_{i}}\right| \cdot U_{K_{i}} .
$$

Für die Korrekturfunktion $K\left(x_{\text {raw }}\right)=c_{1}+c_{2} \cdot x_{\text {raw }}+c_{3} \cdot x_{\text {raw }}^{2}$ gelangt man durch Einsetzen der Gleichungen A.22 für die Koeffzienten zu

$$
K\left(x_{\text {raw }}\right)=\sum_{i=1}^{m}\left(b_{i 1}+b_{i 2} \cdot x_{\text {raw }}+b_{i 3} \cdot x_{\text {raw }}^{2}\right) \cdot K_{i}
$$

Damit ergibt sich für die Gesamtunsicherheit

$$
U_{K}\left(x_{\text {raw }}\right)=\sum_{i=1}^{m}\left|b_{i 1}+b_{i 2} \cdot x_{\text {raw }}+b_{i 3} \cdot x_{\text {raw }}^{2}\right| \cdot U_{K_{i}} .
$$

\section{A.7.7 Integration in Berechnungsformel}

In einer Gesamtformel $f(x)$ gehen neben der Kalibrierkurve weitere Eingangsgrößen $x_{1} \ldots x_{j}$ ein

Dafür soll nun die Gesamtunsicherheit berechnet werden. Dabei sind alle $x_{j}$ unkorreliert, während alle $K_{i}$ untereinander ungünstigst korreliert sein können. Die Eingangsgrößen sind

$$
\boldsymbol{x}=\left[x_{1} \ldots x_{n} K_{1} \ldots K_{m}\right]
$$

Nach dem Gauss'schen Unsicherheitsfortpflanzungsgesetz gilt

$$
U_{f}^{2}=\sum_{j=1}^{n}\left(\frac{\partial f}{\partial x_{j}}\right)^{2} \cdot U_{x_{j}}^{2}+\sum_{i=1}^{m}\left(\frac{\partial f}{\partial K_{i}}\right)^{2} \cdot U_{K_{i}}^{2}+\sum_{\substack{k_{i} l=1 \\ k \neq 1}}^{m}\left(\frac{\partial f}{\partial K_{k}} \cdot \frac{\partial f}{\partial K_{l}}\right) \cdot U_{K_{k l}}
$$


Für maximale Korrelation der Unsicherheiten der $K_{i}$ (von der hier durch den hohen systematischen Anteil vereinfacht ausgegangen wird) lässt sich dies auch schreiben als (Herleitung in Abschnitt A.8)

$$
U_{f}^{2}=\sum_{j=1}^{n}\left(\frac{\partial f}{\partial x_{j}}\right)^{2} \cdot U_{x_{j}}^{2}+\sum_{i=1}^{m}\left(\frac{\partial f}{\partial K_{i}}\right)^{2} \cdot U_{K_{i}}^{2}+\sum_{\substack{k, l=1 \\ k \neq 1}}^{m}\left|\frac{\partial f}{\partial K_{k}}\right| \cdot\left|\frac{\partial f}{\partial K_{l}}\right| U_{K_{k}} \cdot U_{K_{l}}
$$

bzw.

$$
U_{f}^{2}=\sum_{j=1}^{n}\left(\frac{\partial f}{\partial x_{j}}\right)^{2} \cdot U_{x_{j}}^{2}+\left(\sum_{i=1}^{m}\left|\frac{\partial f}{\partial K_{i}}\right| \cdot U_{K_{i}}\right)^{2}
$$

Nach dieser Gleichung kann also der Messunsicherheitsbeitrag der kalibrierten Messwerte eines jeden Sensors jeweils durch Worst-Case-Abschätzung separat bestimmt werden zu $U_{\sum K}=\sum_{i=1}^{m}\left|\frac{\partial f}{\partial K_{i}}\right| \cdot U_{K_{i}}$ und geht dann entsprechend als quadratischer Summand $U_{\sum K}^{2}$ in die quadrierte Gesamtmessunsicherheit $U_{f}^{2}$ ein.

\section{A.8 Herleitung Unsicherheitsfortpflanzung für maximal korrelierte Unsicherheiten}

Im Folgenden wird die maximale resultierende Gesamtunsicherheit für den Fall korrelierter Einzelunsicherheiten abgeschätzt.

Es gilt allgemein

$$
-\sigma_{k} \cdot \sigma_{l} \geq \sigma_{k l} \geq \sigma_{k} \cdot \sigma_{l}
$$

Gesucht ist im zulässigen Bereich der $\sigma_{k l}$ die maximale Gesamtunsicherheit

$$
\sigma_{f, \text { worst }}^{2}=\max \left(\sum_{i=1}^{m}\left(\frac{\partial f}{\partial x_{i}}\right)^{2} \cdot \sigma_{i}^{2}+\sum_{\substack{k, l=1 \\ k \neq l}}^{m}\left(\frac{\partial f}{\partial x_{k}} \cdot \frac{\partial f}{\partial x_{l}}\right) \cdot \sigma_{k l}\right)
$$

Es folgt

$$
\sigma_{f, \text { worst }}^{2}=\sum_{i=1}^{m}\left(\frac{\partial f}{\partial x_{i}}\right)^{2} \cdot \sigma_{i}^{2}+\max \left(\sum_{\substack{k, l=1 \\ k \neq l}}^{m}\left(\frac{\partial f}{\partial x_{k}} \cdot \frac{\partial f}{\partial x_{l}}\right) \cdot \sigma_{k l}\right)
$$




$$
\sigma_{f, \text { worst }}^{2}=\sum_{i=1}^{m}\left(\frac{\partial f}{\partial x_{i}}\right)^{2} \cdot \sigma_{i}^{2}+\sum_{\substack{k, l=1 \\ k \neq l}}^{m}\left|\frac{\partial f}{\partial x_{k}}\right| \cdot\left|\frac{\partial f}{\partial x_{l}}\right| \cdot \sigma_{k} \cdot \sigma_{l}
$$

Dies lässt sich mit der Binomischen Formel rückformen zu

$$
\sigma_{f, \text { worst }}=\sum_{i=1}^{m}\left|\frac{\partial f}{\partial x_{i}}\right| \cdot \sigma_{i}
$$

und entspricht damit der oft benutzten "Worst-Case“Abschätzung.

\section{A.9 Unsicherheit der Kalibrierung - Anwendung}

\section{A.9.1 Temperaturkalibrierung}

Die Kalibrierung der Sensoren T.G, T.R1 und T.R2 lehnt sich an [DKD-R 5-1] an. Als Normal wurde ein Präzisionsthermometer Typ F250 MKII der Firma ASL verwendet, welches bei $0 / 50 / 95 / 100 / 150{ }^{\circ} \mathrm{C}$ vom Kalibrierlabor kalibriert wurde.

Tabelle A.5: Unsicherheitsbudget der Korrektur $K_{i}$ der Temperaturkalibrierung

\begin{tabular}{lllll}
\hline Größe & Kurzbezeichnung & Verteilung & halbe Breite & Sensitivität in $K_{i}$ \\
\hline$t_{\text {ref }}$ & Streuung der Messwerte des Normals & t-Verteilung & - & 1 \\
$\delta t_{\text {sys }}$ & $\begin{array}{l}\text { Systematische Unsicherheit des } \\
\text { Normals } \\
\text { (Kalibrierfehler und Kurvenfehler) }\end{array}$ & Rechteck & $0,13 \mathrm{~K}$ & 1 \\
$t_{\text {Anz }}$ & $\begin{array}{l}\text { Streuung der Messwerte des } \\
\text { Kalibriergegenstands }\end{array}$ & t-Verteilung & - & 1 \\
$\delta t_{\text {Anz }}$ & $\begin{array}{l}\text { Auflösung der Messwerte des } \\
\text { Kalibriergegenstands }\end{array}$ & Rechteck & $0,005 \mathrm{~K}$ & 1 \\
\hline
\end{tabular}

Die Gesamtunsicherheit $U_{K_{i}}$ wurde entsprechend [GUM] bestimmt.

\section{A.9.2 Drucksensorkalibrierung}

Zur Kalibrierung der Drucksensoren wird ein an der Professur vorhandener Druckkalibrator Typ CPH 6400 der Firma Wika verwendet. Die zum Druckkalibrator erworbenen Referenzzellen ( 1 Stück: 0 bis 6 bar bzw. 0 bis 16 bar) besitzen eine Genauigkeit von 0,025\% vom Messbereich bzw. 0,1\% vom Messwert. Die Drucksensorkalibrierung erfolgt angelehnt an [DKD-R 6-1].

Die Gesamtunsicherheit $U_{K_{i}}$ wurde entsprechend [GUM] bestimmt. Für die unterschiedlichen Temperaturniveaus zeigen sich leicht unterschiedliche Korrekturen $K_{i}$. Vereinfachend wurde eine gemeinsame Kalibrierkurve $K$ für alle Temperaturen gewählt. Das ermittelte Messunsicherheitsbudget der $K_{i}$ wurde daher noch so erweitert, dass mit einer Korrekturfunktion das 
Tabelle A.6: Unsicherheitsbudget der Korrektur $K_{i}$ der Druckkalibrierung

\begin{tabular}{lllll}
\hline Größe & Kurzbezeichnung & Verteilung & halbe Breite der Verteilung & Sensitvität in $K_{i}$ \\
\hline$p_{\text {ref }}$ & Auflösung des Messwerts der Referenz & - & vernachlässigbar & 1 \\
$\delta p_{\text {sys }}$ & Systematischer Fehler des Normals & Normal & 4 mbar (erweitert) & 1 \\
& (Kalibrierfehler und Kurvenfehler) & & & \\
$\delta p_{\text {wdh }}$ & Wiederholpräzision & Rechteck & 1 mbar & 1 \\
$\delta p_{\text {Anz }}$ & Auflösung der Messwerte des & Rechteck & 0,5 mbar & 1 \\
& Kalibriergegenstands & & & \\
\hline
\end{tabular}

ganze genutzte Temperaturspektrum abdeckt werden kann $\left(U_{K_{i}}=8 \mathrm{mbar}\right)$. Diese Vereinfachung erhöht die Unsicherheit, jedoch fällt diese durch die Kalibrierung mit vorgelagerten Wasserversuchen nur wenig ins Gewicht.

\section{A.10 Herleitung der Kalibrierungsformel}

Im Folgenden wird die Löslichkeit $\lambda$ ersetzt durch das allgemeinere

$$
y=f(\vec{x})
$$

wobei $\vec{x}$ den Vektor der Eingangsgrößen der Berechnung der Löslichkeit darstellt. Jede Messung der Löslichkeit ist durch die Abweichung der Eingangsgrößen selbst abweichungsbehaftet. Die abweichungsbehafteten Größen $x$ bzw. $y$ setzen sich aus wahren Größen $\widetilde{x}$ bzw. $\widetilde{y}$ und den wahren Messabweichungen $\widetilde{\triangle} x$ bzw. $\tilde{\triangle} y$ zusammen:

$$
x=\tilde{x}+\tilde{\triangle} x
$$

Hier sind also nun wirklich die Abweichungen und nicht die Unsicherheiten gemeint.

Die Löslichkeiten von Solarflüssigkeit (Versuch I) und Wasser (Versuch II) werden in getrennten Versuchen bestimmt. Stellt man diese Versuche gegenüber, so gibt es in den Berechnungen

- Eingangsgrößen $x_{\mathrm{I}, \mathrm{var}, i}$ bzw. $x_{I I, v a r, i}$ mit sich unterscheidenden (unbekannten aber abschätzbaren) Abweichungen (z.B. Druckanzeige, Dichte), im Folgenden zusammengefasst in den Vektoren $\vec{x}_{I, v a r}$ und $\vec{x}_{I I, v a r}$ und

- Eingangsgrößen $x_{\mathrm{con}, i}=x_{\mathrm{I}, \text { con }, i}=x_{\|, \text {con }, i}$ mit gleichen (unbekannten aber abschätzbaren) Abweichungen (z.B. Volumina $V_{0}^{\mathrm{G}}$ und $V_{0}^{\mathrm{Rj}}$, Abweichungen der Kalibrierkoeffizienten etc.), im Folgenden zusammengefasst im Vektor $\vec{x}_{\text {con }}$.

Besitzt eine Größe sowohl konstante als auch unterscheidende Abweichungen bzw. Messwerte, muss man diese aufsplitten in zwei Werte, z.B. $y_{\mathrm{O} 2}$ in N2+O2,2 $=y_{\mathrm{O}} 2$ in N2+O2,2, Mess $+a_{y} \mathrm{O} 2,2$ wobei $y_{\mathrm{O} 2}$ in N2+O2,2, Mess dann zu den Eingangsgrößen mit variablen Abweichungen $\vec{x}_{\mathrm{I}, \text { var }}$ und $a_{y} \mathrm{O} 2,2$ zu den Eingangsgrößen mit gleichem Wert und gleicher Abweichung $\vec{x}_{\text {con }}$ gehört. 
Versuch I ergibt für die Löslichkeit $y_{1}=f\left(\vec{x}_{1, v a r}, \vec{x}_{\text {con }}\right)$ und lässt sich mit Hilfe der Abweichungen $\tilde{\triangle} x$ in linearer Näherung wie folgt zum wahren Wert $\tilde{y}=f\left(\overrightarrow{\tilde{x}}_{\text {l,var }}, \overrightarrow{\tilde{x}}_{\text {con }}\right)$ in Bezug setzen

$$
y_{1}=\tilde{y}_{1}+\tilde{\triangle} y_{1, \text { var }}+\left.\sum_{j=1}^{n} \frac{\partial f}{\partial x_{\mathrm{con}, j}}\right|_{\vec{x}_{\mid}} \cdot \tilde{\triangle} x_{\mathrm{con}, j} .
$$

Die Abweichung für die Eingangsgrößen $x_{\mathrm{l}, \text { var }}(i)$ wird hier direkt zusammengefasst zu $\widetilde{\triangle} y_{1, \text { var }}$.

Analog ergibt Versuch II

$$
y_{\|}=f\left(\vec{x}_{\|, v a r}, \vec{x}_{\text {con }}\right)=\widetilde{y}_{\|}+\tilde{\triangle} y_{\|, \text {var }}+\left.\sum_{j=1}^{n} \frac{\partial f}{\partial x_{\text {con }, j}}\right|_{\overrightarrow{x \|}_{\|}} \tilde{\triangle} x_{\text {con }, j}
$$

und unter der Annahme, dass der tabellierte Wert für Wasser $y_{\|}$,Tabelle annähernd abweichungsfrei ist, gilt

$$
y_{\|}=f\left(\vec{x}_{\|, v a r}, \vec{x}_{\text {con }}\right)=y_{\|, \text {Tabelle }}+\tilde{\triangle} y_{\| I, v a r}+\left.\sum_{j=1}^{n} \frac{\partial f}{\partial x_{\text {con }, j}}\right|_{\overrightarrow{x_{\|}}} \tilde{\triangle} x_{\text {con }, j}
$$

Diese Gleichung kann interpretiert werden als zusätzliche Nebenbedingung für Gleichung A.38, die die Abweichung, verursacht durch $\vec{x}_{\text {con }}$ einschränkt. Zum Einsetzen in obige Gleichung A.38 wird diese umgestellt nach der $n$-ten wahren Abweichung $\widetilde{\triangle} x_{\text {con, } n}$ zu

$$
\tilde{\triangle} x_{\text {con }, n}=\frac{y_{\| I}-y_{\|, \text {Tabelle }}-\tilde{\triangle} y_{\|, \text {var }}-\left.\sum_{j=1}^{n-1} \frac{\partial f}{\partial x_{\text {con }, j}}\right|_{\overrightarrow{x_{\|}}} \tilde{\triangle} x_{\text {con }, j}}{\left.\frac{\partial f}{\partial x_{\mathrm{con}, n}}\right|_{\overrightarrow{x_{\|}}}}
$$

und eingesetzt in Gleichung A.38 ergibt sich

$$
\begin{aligned}
& y_{1}-\frac{\left.\frac{\partial f}{\partial x_{c o n}, n}\right|_{\vec{x}_{1}}}{\left.\frac{\partial f}{\partial x_{c o n}, n}\right|_{\overrightarrow{\|}_{\|}}} \cdot\left(y_{\mid l}-y_{1 \mid, \text { Tabelle }}\right)=y_{1, \text { kalibriert }}=\widetilde{y}_{\mid}+\tilde{\triangle} y_{1, \text { var }}
\end{aligned}
$$

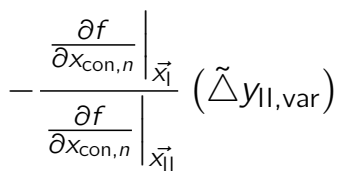

$$
\begin{aligned}
& +\sum_{j=1}^{n-1}\left(\left.\frac{\partial f}{\partial x_{\mathrm{con}, j}}\right|_{\overrightarrow{x_{1}}}-\left.\frac{\left.\frac{\partial f}{\partial x_{\mathrm{con}, n}}\right|_{\overrightarrow{x_{1}}}}{\left.\frac{\partial f}{\partial x_{\mathrm{con}, n}}\right|_{\overrightarrow{x_{\|}}}} \frac{\partial f}{\partial x_{\mathrm{con}, j}}\right|_{\overrightarrow{x_{\|}}}\right) \tilde{\triangle} x_{\mathrm{con}, j}
\end{aligned}
$$

Diese Gleichung könnte jetzt bereits für eine bessere Abschätzung des Ergebnisses durch $y_{1}$,kalibriert statt $y_{1}$ genutzt werden. Jedoch wird für die weitere Vereinfachung der Gleichung 
angenommen, dass die Eingangsgröße $x_{c o n, n}$ wie folgt in die Formel eingeht (konstanter Abweichungsbeitrag auf Ergebnis):

$$
y=f\left(\vec{x}_{\mathrm{var}}, \vec{x}_{\mathrm{con}}\right)=f^{*}\left(\vec{x}_{\mathrm{var}}, x_{\mathrm{con}, 1}, \ldots, x_{\mathrm{con}, n-1}\right)+x_{\mathrm{con}, n} .
$$

Diese kann interpretiert werden als zusätzlicher unbekannter, konstanter, additiver Einfluss, der neben den $n-1$ bekannten Einflüssen berücksichtigt wird. Im Folgenden wird gezeigt, dass dieser Einfluss bei kalibrierter Berechnung keine Rolle mehr spielt.

Für den jetzt vorliegenden Spezialfall für $x_{\text {con, } n}$ gilt

$$
\left.\frac{\partial f}{\partial x_{\text {con }, n}}\right|_{\vec{x}_{1}}=\left.\frac{\partial f}{\partial x_{\text {con }, n}}\right|_{\vec{x}_{\|}}
$$

und es folgt eingesetzt in Gleichung A.42 und durch Umstellung nach dem wahren Wert $\tilde{y}_{1}$

$$
\begin{aligned}
\tilde{y}_{l}= & f\left(\overrightarrow{\tilde{x}}_{l, \mathrm{var}}, \overrightarrow{\tilde{x}}_{\mathrm{con}}\right)=y_{1}-y_{\| I}+y_{\| I, \text { Tabelle }}-\tilde{\triangle} y_{\mathrm{l}, \mathrm{var}} \\
& +\widetilde{\triangle} y_{\|, \mathrm{var}}-\sum_{j=1}^{n-1}\left(\left.\frac{\partial f}{\partial x_{\mathrm{con}, j}}\right|_{\vec{x}_{1}}-\left.\frac{\partial f}{\partial x_{\mathrm{con}, j}}\right|_{\vec{x}_{\|}}\right) \tilde{\triangle} x_{\mathrm{con}, j} .
\end{aligned}
$$

Zusammengefasst ergibt sich

$$
\begin{aligned}
& \tilde{y}_{l}=y_{1}-y_{\mid l}+y_{\mid l, \text { Tabelle }}-\tilde{\Delta} y_{\mid}+\tilde{\Delta} y_{\| \mid} \\
& =y_{\|}-y_{\|}+y_{\|, \text {Tabelle }}-\tilde{\triangle}\left(y_{\|}-y_{\| \prime}\right)
\end{aligned}
$$

im Gegensatz zu (ohne Kalibrierung):

$$
\tilde{y}_{1}=y_{1}-\tilde{\triangle} y_{1}
$$

Der kalibrierte Wert ergibt sich also aus

$$
y_{l, \text { kalibriert }}=y_{l}-y_{\| I}+y_{l \mid, \text { Tabelle }}
$$

Für eine Sinnhaftigkeit der Kalibrierung muss $\widetilde{\triangle}\left(y_{\mid}-y_{\| I}\right)$ kleiner abgeschätzt werden können als $\tilde{\triangle} y_{1}$.

Es gilt

$$
\tilde{\triangle} y_{1}=\tilde{\triangle} y_{l, v a r}+\sum_{j=1}^{n}\left|\frac{\partial f}{\partial x_{\text {con }, j}}\right|_{\vec{x}_{\mid}} \tilde{\triangle} x_{\text {con }, j}
$$


und für den kalibrierten Wert (aus Gleichung A.45)

$$
\tilde{\triangle}\left(y_{1}-y_{\mid I}\right)=\tilde{\triangle} y_{l_{\text {, var }}}-\tilde{\triangle} y_{\text {II, var }}+\sum_{j=1}^{n-1}\left(\left.\frac{\partial f}{\partial x_{\mathrm{con}, j}}\right|_{\vec{x}_{\mid}}-\left.\frac{\partial f}{\partial x_{\mathrm{con}, j}}\right|_{\vec{x}_{\mid l}}\right) \tilde{\triangle} x_{\mathrm{con}, j} .
$$

Zwar kommt als Abweichung $\triangle y_{\| I, v a r}$ hinzu, jedoch kann bei günstiger Beschaffenheit der Gleichung, der Einfluss der $\widetilde{\triangle} x_{\text {con }, j}$ deutlich minimiert werden. Dies ist der Fall, wenn sowohl der Versuchsablauf als auch die eingesetzten Stoffe sich ähnlich sind, da sich dann auch die Eingangsgrößen $\vec{x}_{\text {var }}$ (und damit $\vec{x}_{I}$ und $\vec{x}_{\| I}$ ) und damit die partiellen Ableitungen $\left.\frac{\partial f}{\partial x_{\operatorname{con}, j}}\right|_{\vec{x}}$ ähnlich sind. Daher ist der Einsatz von Wasser als Referenzflüssigkeit hier durchaus sinnvoll. Außerdem kann die Einflussgröße $x_{\text {con, } n}$ gänzlich eliminiert werden.

Die Formel für den kalibrierten Messwert lautet also

$$
y_{1, \text { kalibriert }}=f^{*}\left(\vec{x}_{\mathrm{l}, \mathrm{var}}, \vec{x}_{\mathrm{con}}^{*}\right)-f\left(\vec{x}_{\mathrm{var}, \mathrm{II}}, \vec{x}_{\mathrm{con}}^{*}\right)+y_{\mathrm{ll}} \text {,Tabelle }
$$

mit

$$
\vec{x}_{\text {con }}^{*}=\left(x_{i}\right), i=1 . . n-1
$$

und der zugehörigen Abweichung

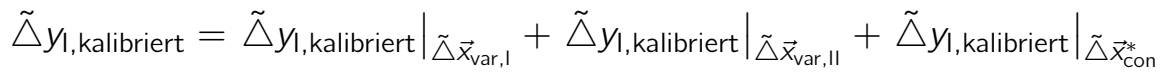

und der zugehörigen Unsicherheit

$$
U^{2}\left(y_{1, \text { kalibriert }}\right)=\sum\left(\frac{\partial y_{1, \text { kalibriert }}}{\partial x_{j}} \cdot U_{j}\right)^{2}
$$

oder getrennt in Variablen mit variablen und konstanten Werten

$$
\begin{aligned}
U^{2}\left(y_{1, \text { kalibriert }}\right)= & \sum_{i}\left(\left.\frac{\partial f}{\partial x_{\mathrm{var}, i}} \cdot U_{i}\right|_{\overrightarrow{x_{1}}}\right)^{2}+\sum_{i}\left(\left.\frac{\partial f}{\partial x_{\mathrm{var}, i}} \cdot U_{i}\right|_{\overrightarrow{x_{\|}}}\right)^{2}+ \\
& \sum_{j}\left(\left(\left.\frac{\partial f}{\partial x_{\mathrm{con}, j}}\right|_{\overrightarrow{x_{1}}}-\left.\frac{\partial f}{\partial x_{\mathrm{con}, j}}\right|_{\overrightarrow{x_{\|}}}\right) \cdot U_{j}\right)^{2}+U^{2}\left(y_{\mid l}, \text { Tabelle }\right) .
\end{aligned}
$$

Hier kann man ebenso erkennen, dass sich der Unsicherheitsbeitrag für konstante Größen durch die Differenz $\left.\frac{\partial f}{\partial x_{\mathrm{con}, j}}\right|_{\overrightarrow{x_{1}}}-\left.\frac{\partial f}{\partial x_{\mathrm{con}, j}}\right|_{\vec{x}_{\|}}$als kleiner erweisen kann als $\frac{\partial f}{\partial x_{\mathrm{con}, j}} \mid$ nur für Versuch I. Hinzu kommt der Unsicherheitsbeitrag für die sich unterscheidenen Eingangsgrößen des Versuchs II und der Unsicherheitsbeitrag des Tabellenwertes $y_{I I}$,Tabelle. 


\section{A.11 Gasphasenzusammensetzung bei gegebener}

Flüssigphasenzusammensetzung

Aus Gleichung 6.8 folgt

$$
\frac{y_{j, \mathrm{tr}}}{y_{i, \mathrm{tr}}}=\frac{\lambda_{i}}{\lambda_{j}} \cdot \frac{w_{j}}{w_{i}} .
$$

Außerdem gilt für alle Gase $j$

$$
\sum y_{j, \mathrm{tr}}=1 \text {. }
$$

Daher ist

$$
\begin{aligned}
& y_{i, \mathrm{tr}}=1-\sum_{j \neq i} y_{j, \mathrm{tr}} \\
& y_{i, \mathrm{tr}}=1-\sum_{j \neq i} \frac{\lambda_{i}}{\lambda_{j}} \cdot \frac{w_{j}}{w_{i}} \cdot y_{i, \mathrm{tr}} \\
& y_{i, \mathrm{tr}}=\frac{1}{1+\sum_{j \neq i} \frac{\lambda_{i}}{\lambda_{j}} \cdot \frac{w_{j}}{w_{i}}}
\end{aligned}
$$




\section{A.12 Herleitung des Temperaturverhaltens bei statischer Druckhaltung mit MAG}

Druckhaltung ohne Zusatzvolumen $V_{z}$

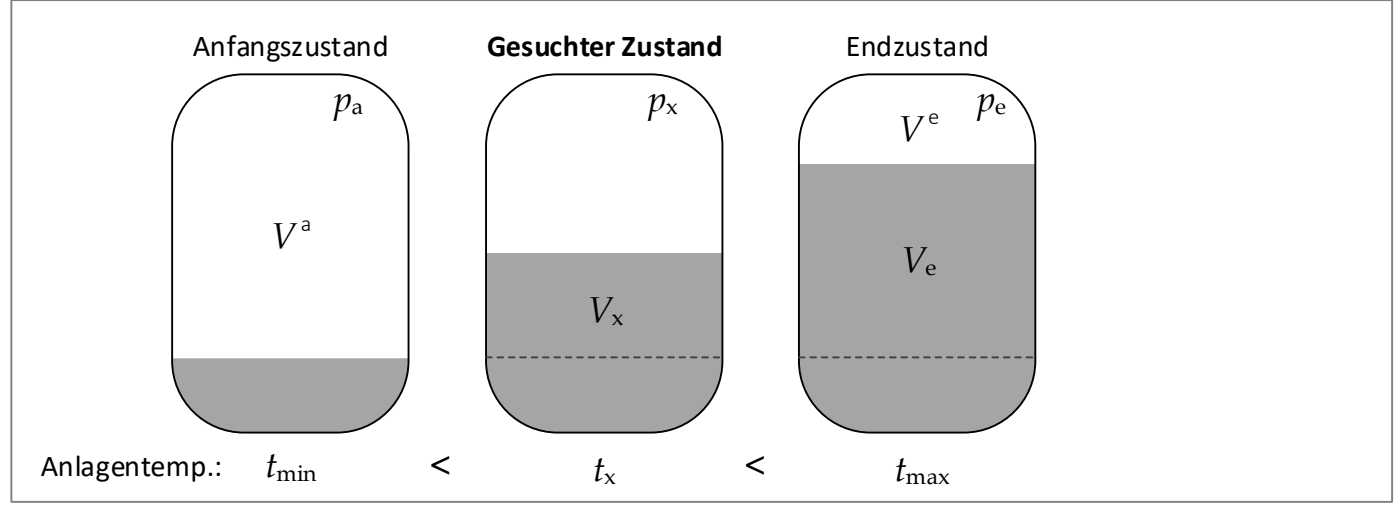

Druckhaltung mit Zusatzvolumen $V_{z}$

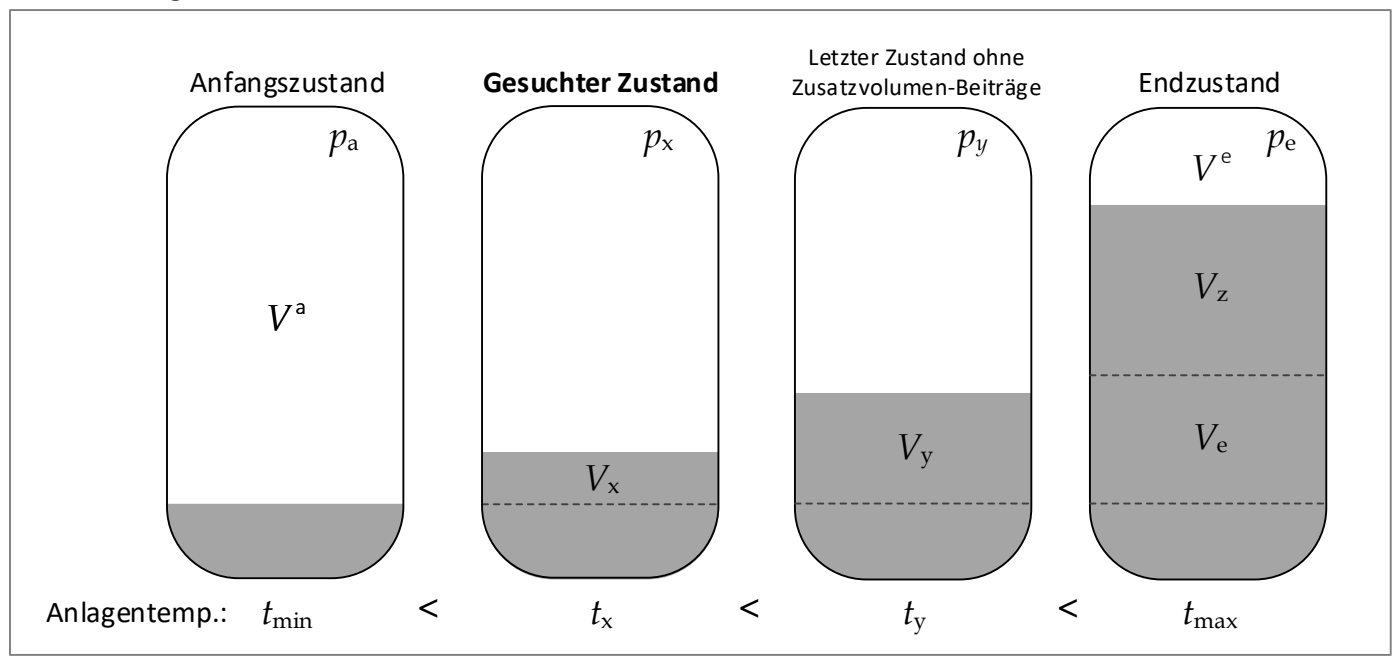

Abbildung A.5: Schematische Darstellung des Gasblasen- und Flüssigkeitsvolumens im MAG (Membran idealisiert waagerecht und spannungsfrei) für die möglichen Zustände der Anlage; oben: Anlage ohne auftretende Zusatzvolumina; unten Anlage mit auftretenden Zusatzvolumina (Annahme: ein Teil des Zusatzvolumens $V_{z}$ tritt bereits bei $t>t_{y}$ und damit vor Erreichen der Maximaltemperatur $t_{\max }$ auf)

Das Gasvolumen des MAG im Ausgangszustand sei $V^{\mathrm{a}}$, im Endzustand $V^{\mathrm{e}}$. Das Volumen im Endzustand ergibt sich, wie in Abbildung A.5 dargestellt, durch Aufnahme des Volumens der Temperaturdehnung der Flüssigkeit $V_{\mathrm{e}}$ und etwaiger prozessbedingter zusätzlicher Volumina V zu

$$
V^{\mathrm{e}}=V^{\mathrm{a}}-\left(V_{\mathrm{e}}+V_{\mathrm{Z}}\right)
$$


Für das Gasvolumen im MAG gilt unter der vereinfachenden Annahme, dass das MAG stets (konstante) Raumtemperatur hat,

$$
p \cdot V=\text { const }
$$

und damit für Anfangs- und Endzustand

$$
\text { I: } p_{\mathrm{a}} \cdot V^{\mathrm{a}}=p_{\mathrm{e}} \cdot\left(V^{\mathrm{a}}-\left(V_{\mathrm{e}}+V_{\mathrm{Z}}\right)\right)
$$

und für jeden Zwischenzustand bei $p_{x}$, bei dem sich das Anlagenvolumen um $V_{x}$ geändert hat

$$
\text { II } p_{a} \cdot V^{a}=p_{x} \cdot\left(V^{a}-V_{x}\right) .
$$

Stellt man Gleichung I um zu

$$
V^{\mathrm{a}}=\frac{V_{\mathrm{e}}+V_{\mathrm{Z}}}{1-\frac{p_{a}}{p_{e}}}
$$

formt man Gleichung II um zu

$$
p_{\mathrm{a}}=p_{\mathrm{x}} \cdot\left(1-\frac{V_{x}}{V^{\mathrm{a}}}\right)
$$

und setzt Gleichung A.66 ein, ergibt sich

$$
p_{\mathrm{a}}=p_{\mathrm{x}} \cdot\left(1-\frac{V_{x}}{V_{\mathrm{e}}+V_{\mathrm{Z}}} \cdot\left(1-\frac{p_{a}}{p_{e}}\right)\right)
$$

und nach der gesuchten Größe umgestellt

$$
p_{x}=\frac{p_{a}}{1-\frac{V_{x}}{V_{e}+V_{z}} \cdot\left(1-\frac{p_{a}}{p_{e}}\right)}
$$

Für Anlagen ohne Prozess- und Verdampfungsvolumina $V_{Z}$ wie Rückkühlanlagen ergibt sich das Gesamt-Anlagenänderungsvolumen nur durch Wärmedehnung. Es ergibt sich mit dem Wärmeträgerinhalt der Anlage $V_{\mathrm{A}}$

$$
V_{e}=V_{\mathrm{A}} \cdot\left(\frac{\rho\left(t_{\min }\right)}{\rho\left(t_{\max }\right)}-1\right)
$$


und für das Anlagenänderungsvolumen bei $t_{x}$

$$
V_{x}=V_{\mathrm{A}} \cdot\left(\frac{\rho\left(t_{\min }\right)}{\rho\left(t_{x}\right)}-1\right)
$$

Damit ergibt sich für eine beliebige mittlere Anlagentemperatur $t_{x}$ mit $t_{\min } \leq t_{x} \leq$ $t_{\max }$

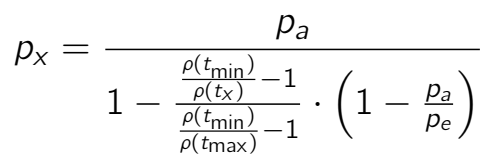

Für Solarthermieanlagen treten Verdampfungsvolumina auf. Geht man aber davon aus, dass es keine Effekte partieller Stagnation im Anlagenbetrieb gibt, gilt bis zu Beginn der Stagnationsphase, also bis zum ersten Auftreten von Verdampfungsvolumina, also bis zum Zustand $y$ in Abbildung A.5 unten, ebenso

$$
V_{x}=V_{A} \cdot\left(\frac{\rho\left(t_{\min }\right)}{\rho\left(t_{x}\right)}-1\right)
$$

Das Gesamtanlagen-Änderungsvolumen sei um den Faktor $f_{1}>1$ größer als das Volumen der Temperaturdehnung.

$$
V_{\mathrm{e}}+V_{\mathrm{Z}}=f_{1} \cdot V_{\mathrm{e}}=f_{1} \cdot V_{\mathrm{A}} \cdot\left(\frac{\rho\left(t_{\min }\right)}{\rho\left(t_{\max }\right)}-1\right)
$$

Damit ergibt sich für den Druck bei einer beliebigen mittleren Temperatur $t_{x}$ der Anlage unterhalb der Verdampfung/Stagnation, also bei $t_{\min } \leq t_{x} \leq t_{y}$

$$
p_{x}=\frac{p_{\mathrm{a}}}{1-\frac{\frac{\rho\left(t_{\min }\right)}{\rho\left(t_{x}\right)}-1}{f_{1} \cdot\left(\frac{\rho\left(t_{\min }\right)}{\rho\left(t_{\max }\right)}-1\right)} \cdot\left(1-\frac{p_{a}}{p_{e}}\right)}
$$




\section{A.13 Auswertung}

\section{A.13.1 Drucksprungdiagramme}

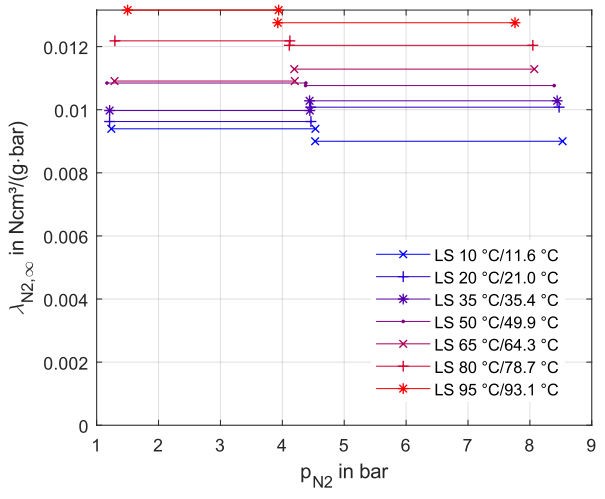

Abbildung A.6: Tyfocor ${ }^{\circledR}$ LS (kalibriert)

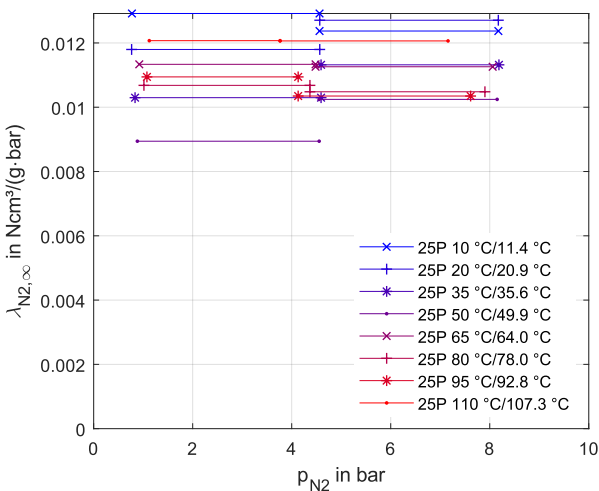

Abbildung A.8: Propylenglykol 25 Gew.-\% (kalibriert)

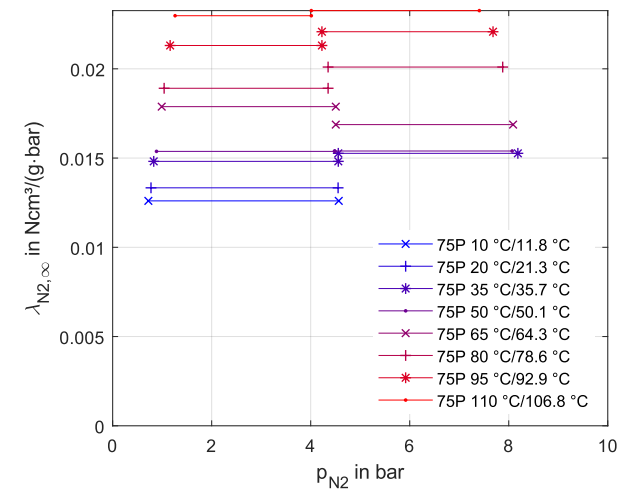

Abbildung A.10: Propylenglykol 75 Gew.-\% (kalibriert)

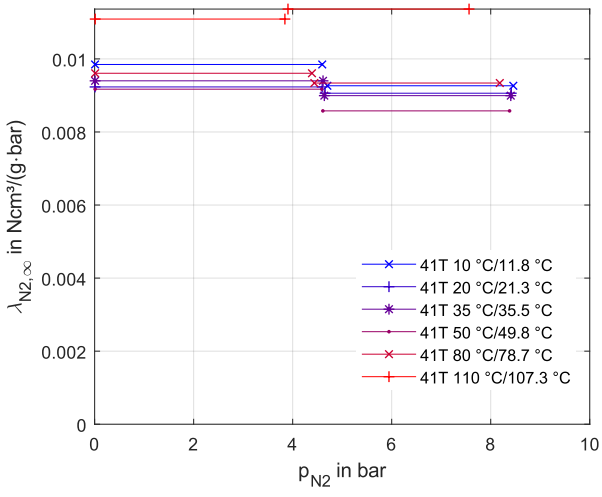

Abbildung A.7: Tyfocor ${ }^{\circledR} 41$ Gew.-\% (kalibriert)

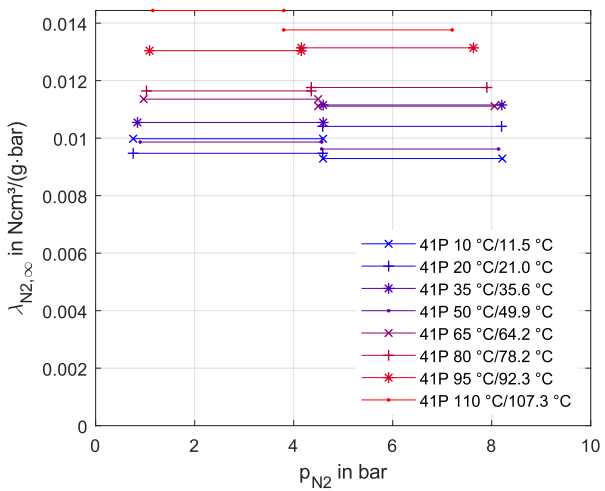

Abbildung A.9: Propylenglykol 41,84 Gew.-\% (kalibriert)

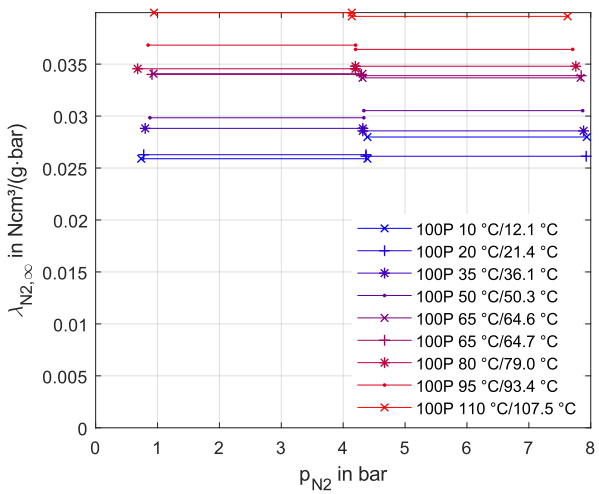

Abbildung A.11: Propylenglykol $100 \%$ (kalibriert) 


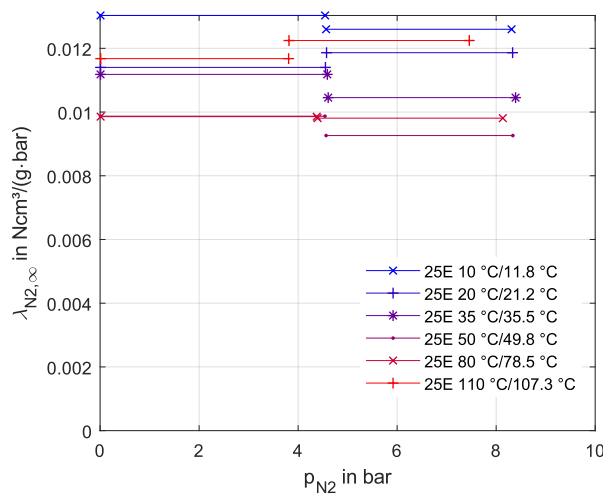

Abbildung A.12: Ethylenglykol 25 Gew.-\% (kalibriert)

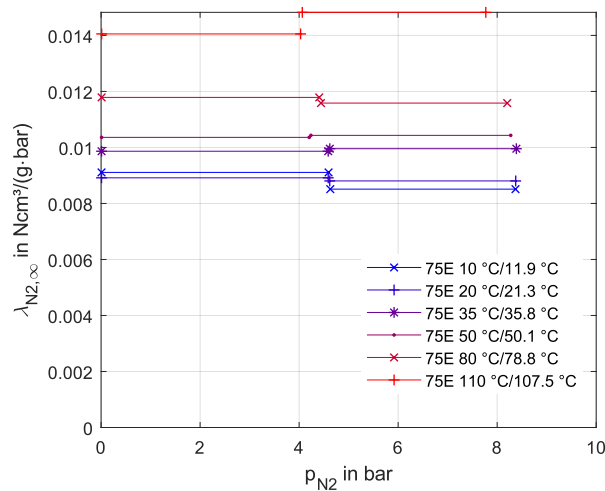

Abbildung A.14: Ethylenglykol 75 Gew.-\% (kalibriert)

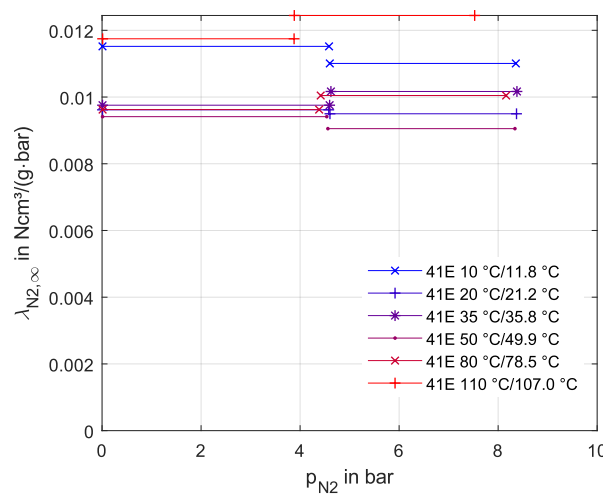

Abbildung A.13: Ethylenglykol 41,84 Gew.-\% (kalibriert)

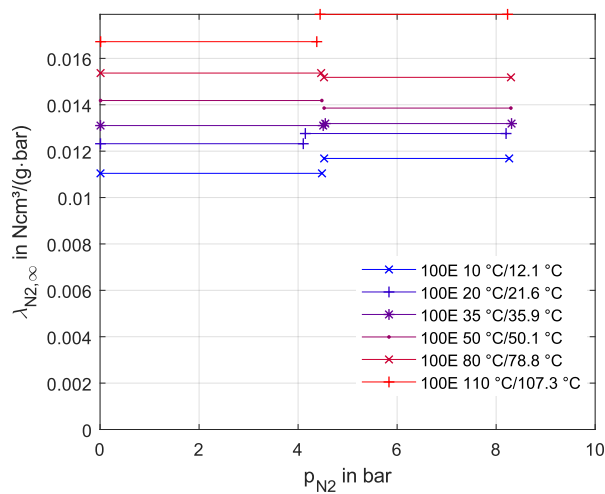

Abbildung A.15: Ethylenglykol $100 \%$ (kalibriert)

\section{A.13.2 Ermittelte Löslichkeiten aus den Versuchen}

\section{A.13.2.1 Stickstoff-Versuche}

Tabelle A.7: Messwerte $\mathrm{N}_{2}$ in 25P

\begin{tabular}{lllll}
\hline$t^{\prime} /{ }^{\circ} \mathrm{C}$ & $p_{1} /$ bar & $p_{2} /$ bar & $\lambda_{\infty, \text { kalibriert }} / \frac{\mathrm{cm}^{3}}{\mathrm{~g} \cdot \text { bar }}$ & $U(\lambda) / \frac{\mathrm{cm}^{3}}{\mathrm{~g} \cdot \mathrm{bar}}$ \\
\hline 11.2 & 0.9796 & 4.7685 & 0.0129 & 0.0015 \\
11.2 & 4.7685 & 8.3804 & 0.0124 & 0.0017 \\
20.7 & 0.9896 & 4.7895 & 0.0118 & 0.0014 \\
20.7 & 4.7895 & 8.3964 & 0.0127 & 0.0016 \\
35.4 & 1.0997 & 4.8566 & 0.0103 & 0.0014 \\
35.4 & 4.8566 & 8.4494 & 0.0113 & 0.0015 \\
49.7 & 1.2158 & 4.8876 & 0.0089 & 0.0013 \\
49.7 & 4.8876 & 8.4824 & 0.0102 & 0.0014 \\
63.8 & 1.3709 & 4.9316 & 0.0113 & 0.0013 \\
63.8 & 4.9316 & 8.5084 & 0.0113 & 0.0014 \\
77.7 & 1.6561 & 5.0086 & 0.0107 & 0.0013 \\
77.7 & 5.0086 & 8.5434 & 0.0105 & 0.0013 \\
92.8 & 2.0433 & 5.1097 & 0.0115 & 0.0013 \\
92.9 & 5.1097 & 8.6004 & 0.0115 & 0.0013
\end{tabular}




\begin{tabular}{lllll}
\hline$t^{\prime} /{ }^{\circ} \mathrm{C}$ & $p_{1} /$ bar & $p_{2} /$ bar & $\lambda_{\infty, \text { kalibriert }} / \frac{\mathrm{cm}^{3}}{\mathrm{~g} \text { bar }}$ & $U(\lambda) / \frac{\mathrm{cm}^{3}}{\mathrm{~g} \cdot \text { bar }}$ \\
\hline 92.6 & 2.0443 & 5.0987 & 0.0109 & 0.0013 \\
92.6 & 5.0987 & 8.5814 & 0.0103 & 0.0013 \\
107.1 & 2.5886 & 5.2307 & 0.0121 & 0.0013 \\
107.1 & 5.2307 & 8.6224 & 0.0121 & 0.0013 \\
\hline
\end{tabular}

Tabelle A.8: Messwerte $\mathrm{N}_{2}$ in 41P

\begin{tabular}{lllll}
\hline$t^{\prime} /{ }^{\circ} \mathrm{C}$ & $p_{1} /$ bar & $p_{2} /$ bar & $\lambda_{\infty, \text { kalibriert }} / \frac{\mathrm{cm}^{3}}{\mathrm{~g} \cdot \text { bar }}$ & $U(\lambda) / \frac{\mathrm{cm}^{3}}{\mathrm{~g} \cdot \text { bar }}$ \\
\hline 11.3 & 0.9626 & 4.7995 & 0.0100 & 0.0015 \\
11.3 & 4.7995 & 8.4194 & 0.0093 & 0.0017 \\
20.8 & 0.9806 & 4.8075 & 0.0095 & 0.0014 \\
20.7 & 4.8075 & 8.4204 & 0.0104 & 0.0016 \\
35.4 & 1.1047 & 4.8556 & 0.0105 & 0.0014 \\
35.3 & 4.8556 & 8.4624 & 0.0112 & 0.0015 \\
49.7 & 1.2228 & 4.8906 & 0.0099 & 0.0013 \\
49.7 & 4.8906 & 8.4624 & 0.0096 & 0.0014 \\
63.9 & 1.4029 & 4.9286 & 0.0114 & 0.0013 \\
63.9 & 4.9286 & 8.4894 & 0.0111 & 0.0014 \\
78.0 & 1.6450 & 4.9706 & 0.0116 & 0.0013 \\
77.9 & 4.9706 & 8.5154 & 0.0118 & 0.0013 \\
92.0 & 1.9882 & 5.0536 & 0.0130 & 0.0013 \\
92.0 & 5.0536 & 8.5304 & 0.0131 & 0.0013 \\
107.0 & 2.5235 & 5.1717 & 0.0144 & 0.0013 \\
107.0 & 5.1717 & 8.5744 & 0.0138 & 0.0013 \\
\hline
\end{tabular}

Tabelle A.9: Messwerte $\mathrm{N}_{2}$ in 75P

\begin{tabular}{lllll}
\hline$t^{\prime} /{ }^{\circ} \mathrm{C}$ & $p_{1} /$ bar & $p_{2} /$ bar & $\lambda_{\infty, \text { kalibriert }} / \frac{\mathrm{cm}^{3}}{\mathrm{~g} \cdot \text { bar }}$ & $U(\lambda) / \frac{\mathrm{cm}}{\mathrm{g} \cdot \text { bar }}$ \\
\hline 11.6 & 0.9236 & 4.7685 & 0.0126 & 0.0015 \\
21.1 & 0.9896 & 4.7685 & 0.0133 & 0.0014 \\
35.5 & 1.0737 & 4.8015 & 0.0148 & 0.0014 \\
35.5 & 4.8015 & 8.4284 & 0.0153 & 0.0015 \\
49.8 & 1.1778 & 4.7745 & 0.0154 & 0.0013 \\
49.8 & 4.7745 & 8.3604 & 0.0154 & 0.0015 \\
64.1 & 1.3629 & 4.8786 & 0.0179 & 0.0013 \\
64.1 & 4.8786 & 8.4594 & 0.0169 & 0.0014 \\
78.4 & 1.5430 & 4.8546 & 0.0189 & 0.0013 \\
78.3 & 4.8546 & 8.3804 & 0.0201 & 0.0014 \\
92.6 & 1.8722 & 4.9376 & 0.0213 & 0.0013 \\
92.6 & 4.9376 & 8.3934 & 0.0221 & 0.0014 \\
106.5 & 2.2824 & 5.0336 & 0.0230 & 0.0013 \\
106.5 & 5.0336 & 8.4274 & 0.0233 & 0.0014 \\
\hline
\end{tabular}

Tabelle A.10: Messwerte $\mathrm{N}_{2}$ in 100P

\begin{tabular}{lllll}
\hline$t^{\prime} /{ }^{\circ} \mathrm{C}$ & $p_{1} /$ bar & $p_{2} /$ bar & $\lambda_{\infty, \text { kalibriert }} / \frac{\mathrm{cm}^{3}}{\mathrm{~g} \cdot \mathrm{bar}}$ & $U(\lambda) / \frac{\mathrm{cm}}{\mathrm{g} \cdot \mathrm{bar}}$ \\
\hline 11.9 & 0.9306 & 4.5865 & 0.0259 & 0.0015 \\
11.9 & 4.5865 & 8.1314 & 0.0280 & 0.0020
\end{tabular}




\begin{tabular}{lllll}
\hline$t^{\prime} /{ }^{\circ} \mathrm{C}$ & $p_{1} /$ bar & $p_{2} /$ bar & $\lambda_{\infty, \text { kalibriert }} / \frac{\mathrm{cm}^{3}}{\mathrm{~g} \cdot \text { bar }}$ & $U(\lambda) / \frac{\mathrm{cm}^{3}}{\mathrm{~g} \cdot \text { bar }}$ \\
\hline 21.2 & 0.9776 & 4.5685 & 0.0263 & 0.0015 \\
21.2 & 4.5685 & 8.1274 & 0.0261 & 0.0018 \\
35.9 & 1.0117 & 4.5284 & 0.0288 & 0.0014 \\
35.9 & 4.5284 & 8.0954 & 0.0286 & 0.0018 \\
50.0 & 1.0987 & 4.5555 & 0.0298 & 0.0014 \\
50.0 & 4.5555 & 8.0914 & 0.0305 & 0.0017 \\
64.3 & 1.1688 & 4.5465 & 0.0341 & 0.0014 \\
64.3 & 4.5465 & 8.0704 & 0.0337 & 0.0017 \\
64.5 & 1.1447 & 4.5274 & 0.0340 & 0.0014 \\
64.5 & 4.5274 & 8.0794 & 0.0339 & 0.0017 \\
78.7 & 0.9286 & 4.4474 & 0.0346 & 0.0014 \\
78.7 & 4.4474 & 8.0084 & 0.0348 & 0.0017 \\
93.2 & 1.1197 & 4.4724 & 0.0368 & 0.0014 \\
93.1 & 4.4724 & 7.9804 & 0.0364 & 0.0017 \\
107.3 & 1.2468 & 4.4424 & 0.0400 & 0.0014 \\
107.3 & 4.4424 & 7.9293 & 0.0396 & 0.0017 \\
\hline
\end{tabular}

Tabelle A.11: Messwerte $\mathrm{N}_{2}$ in 25E

\begin{tabular}{lllll}
\hline$t^{\prime} /{ }^{\circ} \mathrm{C}$ & $p_{1} /$ bar & $p_{2} /$ bar & $\lambda_{\infty, \text { kalibriert }} / \frac{\mathrm{cm}^{3}}{\mathrm{~g} \cdot \mathrm{bar}}$ & $U(\lambda) / \frac{\mathrm{cm}}{\mathrm{g} \cdot \text { bar }}$ \\
\hline 11.6 & 0.0430 & 4.5785 & 0.0130 & 0.0014 \\
11.6 & 4.5785 & 8.3244 & 0.0126 & 0.0017 \\
20.9 & 0.0550 & 4.5975 & 0.0114 & 0.0013 \\
20.9 & 4.5975 & 8.3554 & 0.0119 & 0.0016 \\
35.3 & 0.0810 & 4.6605 & 0.0112 & 0.0013 \\
35.3 & 4.6605 & 8.4444 & 0.0105 & 0.0015 \\
49.6 & 0.1441 & 4.6765 & 0.0099 & 0.0012 \\
49.6 & 4.6765 & 8.4484 & 0.0093 & 0.0014 \\
78.3 & 0.4313 & 4.7975 & 0.0099 & 0.0011 \\
78.3 & 4.7975 & 8.5394 & 0.0098 & 0.0013 \\
107.1 & 1.2038 & 5.0036 & 0.0117 & 0.0011 \\
107.1 & 5.0036 & 8.6454 & 0.0122 & 0.0012 \\
\hline
\end{tabular}

Tabelle A.12: Messwerte $\mathrm{N}_{2}$ in $41 \mathrm{E}$

\begin{tabular}{lllll}
\hline$t^{\prime} /{ }^{\circ} \mathrm{C}$ & $p_{1} /$ bar & $p_{2} /$ bar & $\lambda_{\infty, \text { kalibriert }} / \frac{\mathrm{cm}}{\mathrm{g} \cdot \text { bar }}$ & $U(\lambda) / \frac{\mathrm{cm}}{\mathrm{g} \cdot \text { bar }}$ \\
\hline 11.6 & 0.0370 & 4.6125 & 0.0115 & 0.0014 \\
11.6 & 4.6125 & 8.3694 & 0.0110 & 0.0016 \\
20.9 & 0.0550 & 4.6245 & 0.0096 & 0.0013 \\
20.9 & 4.6245 & 8.3924 & 0.0095 & 0.0015 \\
35.5 & 0.0850 & 4.6715 & 0.0098 & 0.0012 \\
35.5 & 4.6715 & 8.4284 & 0.0102 & 0.0014 \\
49.6 & 0.1341 & 4.6625 & 0.0094 & 0.0012 \\
49.6 & 4.6625 & 8.4404 & 0.0091 & 0.0014 \\
78.2 & 0.4093 & 4.7835 & 0.0096 & 0.0011 \\
78.2 & 4.7835 & 8.5284 & 0.0100 & 0.0013 \\
106.7 & 1.0807 & 4.9506 & 0.0117 & 0.0011 \\
106.7 & 4.9506 & 8.5934 & 0.0124 & 0.0012 \\
\hline
\end{tabular}


Tabelle A.13: Messwerte $\mathrm{N}_{2}$ in 75E

\begin{tabular}{lllll}
\hline$t^{\prime} /{ }^{\circ} \mathrm{C}$ & $p_{1} /$ bar & $p_{2} /$ bar & $\lambda_{\infty, \text { kalibriert }} / \frac{\mathrm{cm}^{3}}{\mathrm{~g} \cdot \mathrm{bar}}$ & $U(\lambda) / \frac{\mathrm{cm}}{\mathrm{g} \cdot \mathrm{bar}}$ \\
\hline 11.7 & 0.0520 & 4.6375 & 0.0091 & 0.0014 \\
11.7 & 4.6375 & 8.3784 & 0.0085 & 0.0016 \\
21.0 & 0.0540 & 4.6325 & 0.0089 & 0.0013 \\
21.1 & 4.6325 & 8.3884 & 0.0088 & 0.0015 \\
35.5 & 0.0740 & 4.6515 & 0.0099 & 0.0012 \\
35.5 & 4.6515 & 8.4194 & 0.0100 & 0.0014 \\
49.8 & 0.1111 & 4.3044 & 0.0104 & 0.0012 \\
49.8 & 4.3044 & 8.3404 & 0.0104 & 0.0014 \\
78.6 & 0.2892 & 4.6845 & 0.0118 & 0.0011 \\
78.5 & 4.6845 & 8.4454 & 0.0116 & 0.0013 \\
107.2 & 0.7595 & 4.7775 & 0.0141 & 0.0011 \\
107.2 & 4.7775 & 8.4834 & 0.0148 & 0.0012 \\
\hline
\end{tabular}

Tabelle A.14: Messwerte $\mathrm{N}_{2}$ in $100 \mathrm{E}$

\begin{tabular}{lllll}
\hline$t^{\prime} /{ }^{\circ} \mathrm{C}$ & $p_{1} /$ bar & $p_{2} /$ bar & $\lambda_{\infty, \text { kalibriert }} / \frac{\mathrm{cm}^{3}}{\mathrm{~g} \cdot \text { bar }}$ & $U(\lambda) / \frac{\mathrm{cm}^{3}}{\mathrm{~g} \cdot \text { bar }}$ \\
\hline 11.9 & 0.0540 & 4.5274 & 0.0110 & 0.0014 \\
11.9 & 4.5274 & 8.2644 & 0.0117 & 0.0016 \\
21.4 & 0.0520 & 4.1473 & 0.0123 & 0.0013 \\
21.4 & 4.1473 & 8.2014 & 0.0128 & 0.0015 \\
35.7 & 0.0530 & 4.5505 & 0.0131 & 0.0012 \\
35.7 & 4.5505 & 8.3124 & 0.0132 & 0.0014 \\
49.9 & 0.0580 & 4.5274 & 0.0142 & 0.0012 \\
49.8 & 4.5274 & 8.2994 & 0.0139 & 0.0014 \\
78.5 & 0.0760 & 4.5304 & 0.0154 & 0.0011 \\
78.5 & 4.5304 & 8.3074 & 0.0152 & 0.0013 \\
107.0 & 0.1111 & 4.4764 & 0.0167 & 0.0011 \\
107.0 & 4.4764 & 8.2624 & 0.0179 & 0.0012 \\
\hline
\end{tabular}

Tabelle A.15: Messwerte $\mathrm{N}_{2}$ in Tyfocor LS

\begin{tabular}{lllll}
\hline$t^{\prime} /{ }^{\circ} \mathrm{C}$ & $p_{1} /$ bar & $p_{2} /$ bar & $\lambda_{\infty, \text { kalibriert }} / \frac{\mathrm{cm}^{3}}{\mathrm{~g} \cdot \text { bar }}$ & $U(\lambda) / \frac{\mathrm{cm}^{3}}{\mathrm{~g} \text { bar }}$ \\
\hline 11.3 & 1.4439 & 4.7415 & 0.0094 & 0.0016 \\
11.3 & 4.7375 & 8.7354 & 0.0090 & 0.0016 \\
20.8 & 1.4319 & 4.6855 & 0.0096 & 0.0015 \\
20.8 & 4.6825 & 8.6954 & 0.0101 & 0.0015 \\
35.2 & 1.4709 & 4.7055 & 0.0100 & 0.0014 \\
35.2 & 4.7015 & 8.7024 & 0.0103 & 0.0014 \\
49.7 & 1.4950 & 4.7085 & 0.0108 & 0.0014 \\
49.7 & 4.7045 & 8.7214 & 0.0108 & 0.0014 \\
64.1 & 1.7351 & 4.6475 & 0.0109 & 0.0014 \\
64.1 & 4.6395 & 8.5174 & 0.0113 & 0.0013 \\
78.4 & 1.9372 & 4.7645 & 0.0122 & 0.0013 \\
78.4 & 4.7555 & 8.6904 & 0.0120 & 0.0013 \\
92.8 & 2.4575 & 4.8976 & 0.0132 & 0.0014 \\
92.9 & 4.8846 & 8.7174 & 0.0128 & 0.0012 \\
\hline
\end{tabular}


Tabelle A.16: Messwerte $\mathrm{N}_{2}$ in $41 \mathrm{~T}$

\begin{tabular}{lllll}
\hline$t^{\prime} /{ }^{\circ} \mathrm{C}$ & $p_{1} /$ bar & $p_{2} /$ bar & $\lambda_{\infty, \text { kalibriert }} / \frac{\mathrm{cm}^{3}}{\mathrm{~g} \cdot \text { bar }}$ & $U(\lambda) / \frac{\mathrm{cm}}{\mathrm{g} \cdot \text { bar }}$ \\
\hline 11.6 & 0.0500 & 4.6375 & 0.0098 & 0.0014 \\
11.6 & 4.6375 & 8.3924 & 0.0093 & 0.0016 \\
21.1 & 0.0610 & 4.6475 & 0.0092 & 0.0013 \\
21.1 & 4.6475 & 8.4074 & 0.0091 & 0.0015 \\
35.2 & 0.0830 & 4.6865 & 0.0094 & 0.0012 \\
35.2 & 4.6865 & 8.4524 & 0.0090 & 0.0014 \\
49.6 & 0.1321 & 4.7105 & 0.0092 & 0.0012 \\
49.5 & 4.7105 & 8.4814 & 0.0086 & 0.0014 \\
78.4 & 0.4153 & 4.7945 & 0.0096 & 0.0011 \\
78.4 & 4.7945 & 8.5394 & 0.0093 & 0.0013 \\
107.0 & 1.1297 & 4.9626 & 0.0111 & 0.0011 \\
107.0 & 4.9626 & 8.6194 & 0.0114 & 0.0012 \\
\hline
\end{tabular}

\section{A.13.2.2 Luft-Versuche}

Tabelle A.17: Messwerte $\mathrm{O}_{2}$ in 41P

\begin{tabular}{lllll}
\hline$t^{\prime} /{ }^{\circ} \mathrm{C}$ & $p_{1} /$ bar & $p_{2} /$ bar & $\lambda_{\infty, \text { kalibriert }} / \frac{\mathrm{cm}}{\mathrm{g} \cdot \mathrm{bar}}$ & $U(\lambda) / \frac{\mathrm{cm}}{\mathrm{g} \cdot \mathrm{bar}}$ \\
\hline 11.5 & 0.0770 & 4.5885 & 0.0220 & 0.0055 \\
20.9 & 0.0560 & 4.5795 & 0.0203 & 0.0053 \\
35.0 & 0.0850 & 4.6115 & 0.0196 & 0.0049 \\
49.2 & 0.1361 & 4.6045 & 0.0216 & 0.0045 \\
78.1 & 0.4163 & 4.7085 & 0.0212 & 0.0040 \\
92.2 & 0.7005 & 4.7765 & 0.0237 & 0.0038 \\
106.7 & 1.1467 & 4.8796 & 0.0263 & 0.0037 \\
\hline
\end{tabular}

Tabelle A.18: Messwerte $\mathrm{O}_{2}$ in 41E

\begin{tabular}{lllll}
\hline$t^{\prime} /{ }^{\circ} \mathrm{C}$ & $p_{1} /$ bar & $p_{2} /$ bar & $\lambda_{\infty, \text { kalibriert }} / \frac{\mathrm{cm}^{3}}{\mathrm{~g} \cdot \text { bar }}$ & $U(\lambda) / \frac{\mathrm{cm}^{3}}{\mathrm{~g} \cdot \text { bar }}$ \\
\hline 11.5 & 0.1021 & 4.5705 & 0.0227 & 0.0055 \\
21.0 & 0.0620 & 4.5945 & 0.0180 & 0.0053 \\
35.2 & 0.0810 & 4.6165 & 0.0186 & 0.0048 \\
49.4 & 0.1351 & 4.6305 & 0.0188 & 0.0045 \\
78.0 & 0.4003 & 4.7215 & 0.0171 & 0.0040 \\
106.5 & 1.0757 & 4.9326 & 0.0214 & 0.0035 \\
\hline
\end{tabular}

Tabelle A.19: Messwerte $\mathrm{O}_{2}$ in $41 \mathrm{~T}$

\begin{tabular}{lllll}
\hline$t^{\prime} /{ }^{\circ} \mathrm{C}$ & $p_{1} /$ bar & $p_{2} /$ bar & $\lambda_{\infty, \text { kalibriert } / \frac{\mathrm{cm}^{3}}{\mathrm{~g} \cdot \text { bar }}}$ & $U(\lambda) / \frac{\mathrm{cm}^{3}}{\mathrm{~g} \cdot \mathrm{bar}}$ \\
\hline 11.4 & 0.0710 & 4.5755 & 0.0224 & 0.0055 \\
20.9 & 0.0570 & 4.6005 & 0.0183 & 0.0053 \\
35.0 & 0.0830 & 4.6345 & 0.0179 & 0.0048 \\
49.2 & 0.1321 & 4.6265 & 0.0186 & 0.0045 \\
78.1 & 0.4053 & 4.7495 & 0.0162 & 0.0040
\end{tabular}




\begin{tabular}{lllll}
\hline$t^{\prime} /{ }^{\circ} \mathrm{C}$ & $p_{1} /$ bar & $p_{2} /$ bar & $\lambda_{\infty, \text { kalibriert }} / \frac{\mathrm{cm}^{3}}{\mathrm{~g} \cdot \mathrm{bar}}$ & $U(\lambda) / \frac{\mathrm{cm}^{3}}{\mathrm{~g} \cdot \mathrm{bar}}$ \\
\hline 106.9 & 1.1217 & 4.9326 & 0.0208 & 0.0035 \\
\hline
\end{tabular}

\section{A.14 Dichtemessungen}
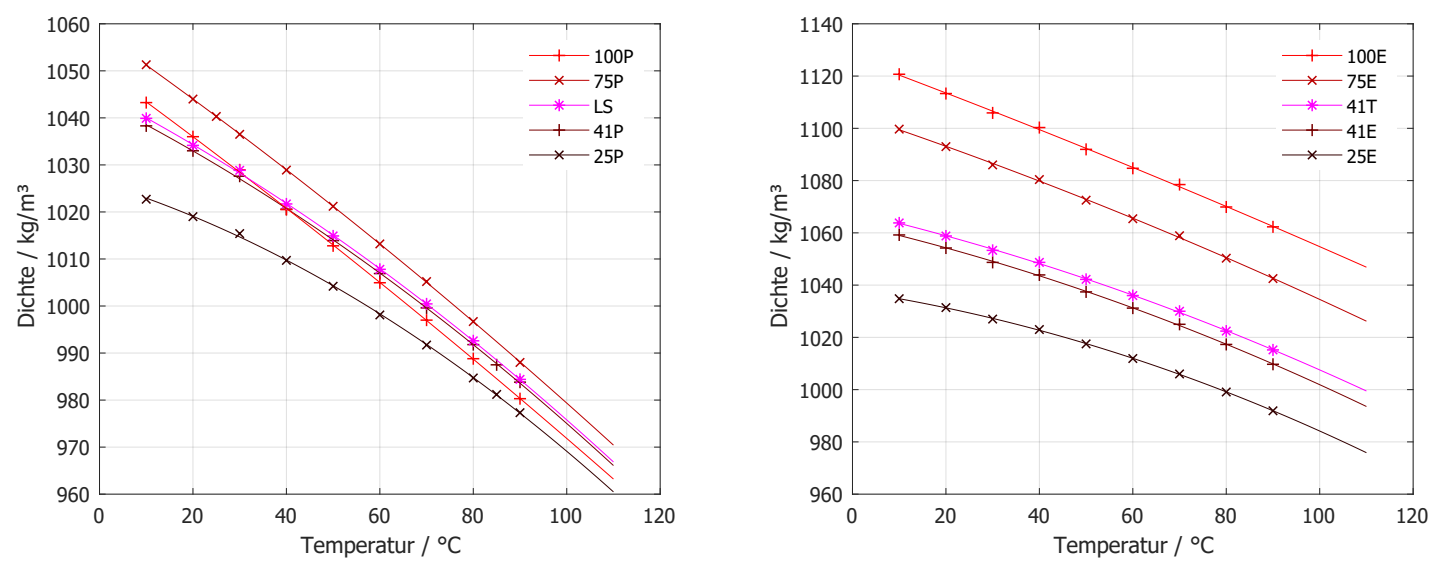

Abbildung A.16: Propylenglykol-Wasser: Dichtemes-
sungen und verwendete Regressionen

Abbildung A.17: Ethylenglykol-Wasser:

Dichtemessungen und verwendete Regressionen

Tabelle A.20: Dichte der eingesetzten Fertiggemische bei Umgebungsdruck

\begin{tabular}{llll}
\hline & & LS & $41 T$ \\
Temperatur & $\begin{array}{l}\text { Messung } \\
\mathrm{kg} / \mathrm{m}^{3}\end{array}$ & $\begin{array}{l}\text { Referenz } \\
\mathrm{kg} / \mathrm{m}^{3}\end{array}$ & $\begin{array}{l}\text { Messung } \\
\mathrm{kg} / \mathrm{m}^{3}\end{array}$ \\
\hline 10 & 1039,9 & - & 1063,8 \\
20 & 1034,2 & 1034,26 & 1058,8 \\
30 & 1028,9 & - & 1053,3 \\
40 & 1021,7 & - & 1048,7 \\
50 & 1014,9 & - & 1042,2 \\
60 & 1007,8 & - & 1036,0 \\
70 & 1000,5 & - & 1030,0 \\
80 & 992,6 & - & 1022,4 \\
90 & 984,4 & - & 1015,2 \\
\hline
\end{tabular}

1) Messungen mit Mettler Toledo DE40; Gesamtmessunsicherheit $0,3 \mathrm{~kg} / \mathrm{m}^{3}$

2) Messungen bei Tyforop Chemie AG 
Tabelle A.21: Dichte der Propylenglykol-Wasser-Gemische für die untersuchten Glykolanteile bei Umgebungsdruck

\begin{tabular}{|c|c|c|c|c|c|c|c|}
\hline \multirow[b]{2}{*}{$\begin{array}{l}\text { Temp. } \\
{\left[{ }^{\circ} \mathrm{C}\right]} \\
\end{array}$} & \multicolumn{2}{|c|}{25 Gew.- $\%$} & \multicolumn{2}{|c|}{41,84 Gew.- $\%$} & \multirow{2}{*}{$\begin{array}{l}75 \text { Gew.- } \% \\
\text { Messung } \\
1 \text { ) } \\
{\left[\mathrm{kg} / \mathrm{m}^{3}\right]}\end{array}$} & \multicolumn{2}{|r|}{100 Gew.- $\%$} \\
\hline & $\begin{array}{l}\text { Messung }^{1)} \\
{\left[\mathrm{kg} / \mathrm{m}^{3}\right]}\end{array}$ & $\begin{array}{l}\text { Referenz }{ }^{2)} \\
{\left[\mathrm{kg} / \mathrm{m}^{3}\right]}\end{array}$ & $\begin{array}{l}\text { Messung }^{1)} \\
{\left[\mathrm{kg} / \mathrm{m}^{3}\right]}\end{array}$ & $\begin{array}{l}\text { Referenz }{ }^{2)} \\
{\left[\mathrm{kg} / \mathrm{m}^{3}\right]}\end{array}$ & & $\begin{array}{l}\text { Messung }^{1)} \\
{\left[\mathrm{kg} / \mathrm{m}^{3}\right]}\end{array}$ & $\begin{array}{l}\text { [Marchetti u. a., 2000] } \\
{\left[\mathrm{kg} / \mathrm{m}^{3}\right]}\end{array}$ \\
\hline 10 & 1022,7 & - & 1038,3 & - & 1051,3 & 1043,3 & 1043,26 \\
\hline 20 & 1019,0 & 1019,1 & 1033,0 & 1033 & 1044,0 & 1036,0 & 1035,94 \\
\hline 25 & - & - & - & - & 1040,3 & - & - \\
\hline 30 & 1015,4 & - & 1027,6 & - & 1036,5 & 1029,0 & 1028,50 \\
\hline 40 & 1009,7 & - & 1020,6 & - & 1028,9 & 1020,5 & 1020,95 \\
\hline 50 & 1004,2 & - & 1013,9 & - & 1021,2 & 1012,8 & 1013,27 \\
\hline 60 & 998,1 & - & 1006,9 & - & 1013,2 & 1005,0 & 1005,49 \\
\hline 70 & 991,7 & - & 999,6 & - & 1005,2 & 997,0 & 997,56 \\
\hline 80 & 984,7 & 984,8 & 991,8 & 991,9 & 996,7 & 988,8 & 989,56 \\
\hline 85 & 981,2 & - & 987,5 & - & - & - & - \\
\hline 90 & 977,3 & - & 983,8 & 983,9 & 988,0 & 980,3 & - \\
\hline
\end{tabular}

1) Messungen mit Mettler Toledo DE40; Gesamtmessunsicherheit $0,3 \mathrm{~kg} / \mathrm{m}^{3}$

2) Messungen bei SGS Germany GmbH nach [DIN EN ISO 12185]

Tabelle A.22: Dichte der Ethylenglykol-Wasser-Gemische für die untersuchten Glykolanteile bei Umgebungsdruck

\begin{tabular}{|c|c|c|c|c|c|}
\hline \multirow{3}{*}{$\begin{array}{l}\text { Temperatur } \\
{\left[{ }^{\circ} \mathrm{C}\right]} \\
\end{array}$} & \multirow{3}{*}{$\begin{array}{l}25 \text { Gew.- } \% \\
\text { Messung }^{1)} \\
{\left[\mathrm{kg} / \mathrm{m}^{3}\right]}\end{array}$} & \multirow{3}{*}{$\begin{array}{l}41,84 \text { Gew.- } \% \\
\text { Messung }{ }^{1)} \\
{\left[\mathrm{kg} / \mathrm{m}^{3}\right]}\end{array}$} & \multirow{3}{*}{$\begin{array}{l}75 \text { Gew.- } \% \\
\text { Messung }{ }^{1)} \\
{\left[\mathrm{kg} / \mathrm{m}^{3}\right]}\end{array}$} & \multirow{3}{*}{\multicolumn{2}{|c|}{$\begin{array}{l}100 \text { Gew.-\% } \\
\text { [Chiavone-Filho u. a., 1993 } \\
{\left[\mathrm{kg} / \mathrm{m}^{3}\right]}\end{array}$}} \\
\hline & & & & & \\
\hline & & & & & \\
\hline 10 & 1034,8 & 1059,2 & 1099,7 & 1120,7 & - \\
\hline 20 & 1031,4 & 1054,2 & 1093,0 & 1113,3 & 1113,47 \\
\hline 30 & 1027,0 & 1048,7 & 1086,0 & 1105,9 & - \\
\hline 40 & 1023,0 & 1043,9 & 1080,4 & 1100,3 & - \\
\hline 50 & 1017,5 & 1037,4 & 1072,5 & 1091,9 & - \\
\hline 60 & 1011,9 & 1031,2 & 1065,4 & 1084,7 & - \\
\hline 70 & 1006,0 & 1025,0 & 1058,9 & 1078,5 & - \\
\hline 80 & 999,1 & 1017,3 & 1050,3 & 1069,9 & - \\
\hline 90 & 991,9 & 1009,7 & 1042,5 & 1062,3 & - \\
\hline
\end{tabular}

1) Messungen mit Mettler Toledo DE40; Gesamtmessunsicherheit $0,3 \mathrm{~kg} / \mathrm{m}^{3}$ 


\section{A.15 Henry-Diagramme der untersuchten Gemische mit Additiven}
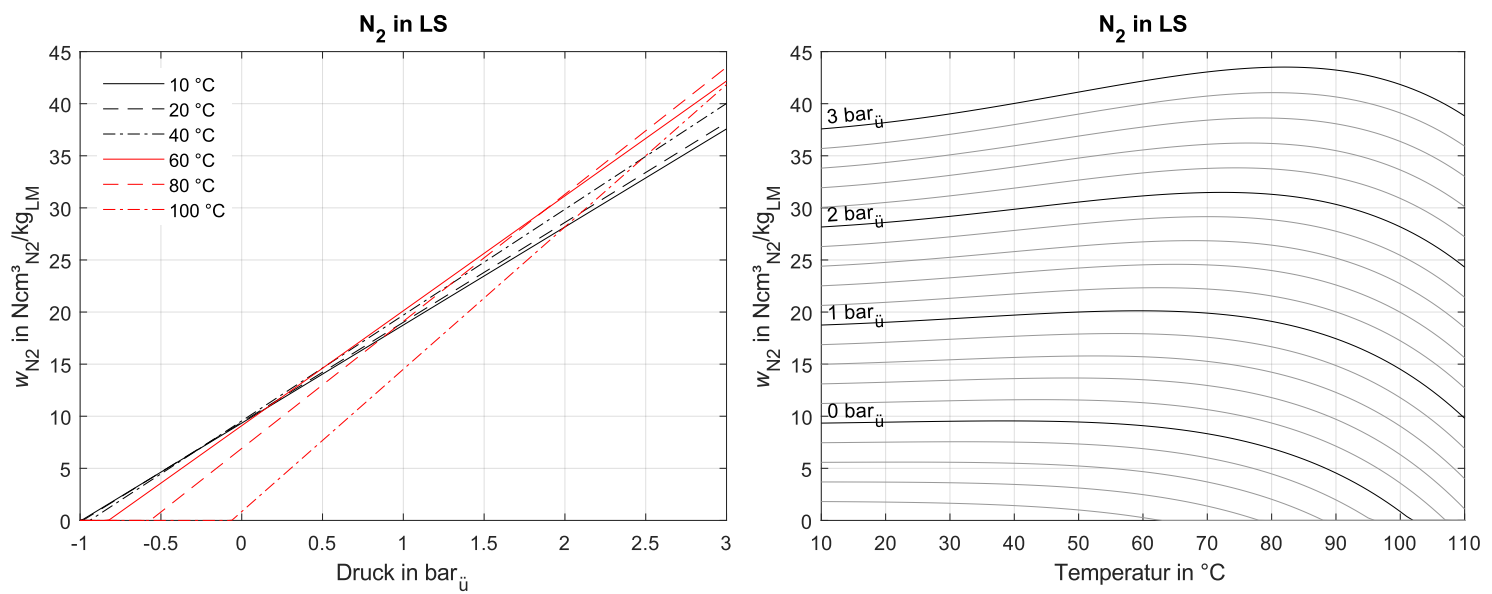

Abbildung A.18: Löslichkeit von Stickstoff in Tyfocor ${ }^{\circledR}$

LS; links: Isothermen-, rechts: Isobaren-Darstellung
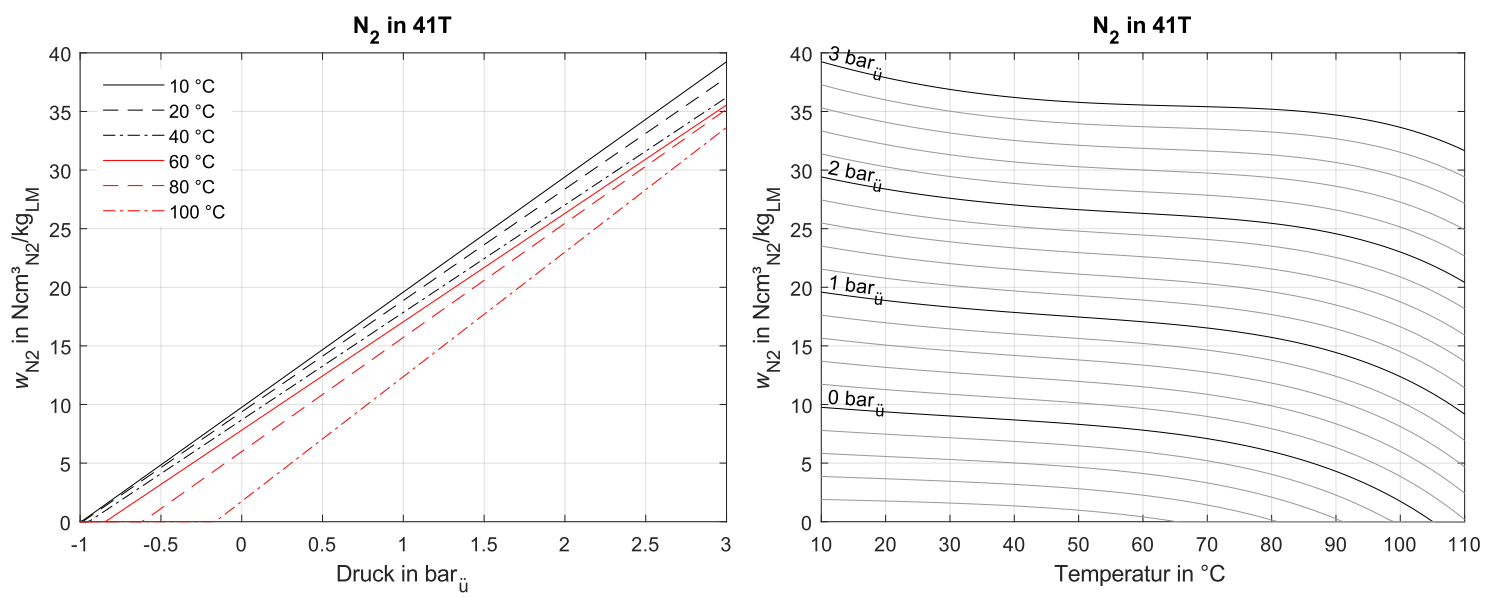

Abbildung A.19: Löslichkeit von Stickstoff in 41T; links: Isothermen-, rechts: Isobaren-Darstellung 